\title{
Strategies for Patient, Family, and Caregiver Engagement
}


Number 36

\section{Strategies for Patient, Family, and Caregiver Engagement}

Prepared for:

Agency for Healthcare Research and Quality

U.S. Department of Health and Human Services

5600 Fishers Lane

Rockville, MD 20857

www.ahrq.gov

Contract No. 290-2015-00006-I

Prepared by:

Johns Hopkins University Evidence-based Practice Center

Baltimore, MD

Investigators:

Wendy L. Bennett, M.D., M.P.H.

Samantha Pitts, M.D., M.P.H.

Hanan Aboumatar, M.D., M.P.H.

Ritu Sharma, B.Sc.

Brandon M. Smith, M.D.

Asar Das, M.P.H., M.B.B.S.

Jeff Day, M.D., M.A.

Katherine Holzhauer, B.A.

Eric B. Bass, M.D., M.P.H.

AHRQ Publication No. 20-EHC017

August 2020 


\section{Addendum}

The literature search was updated for the manuscript related to this Technical Brief, "Patient Engagement Strategies for Adults With Chronic Conditions," published in Systematic Reviews Journal, focusing on patient and family engagement strategies for adults with chronic conditions. We found 131 (19 from an updated search) reviews of direct patient-care strategies in adults, no additional reviews to add to the previously identified 5 reviews of health-system strategies, and no reviews of community-policy strategies. Similar to the Technical Brief, most direct patientcare reviews focused on self-management support (updated $n=85$ ) and shared decision making (updated $n=43$ ). Overall, 49 reviews reported positive effects, 35 reported potential benefits, 37 reported unclear benefits, and 4 reported no benefits. The findings from the updated search did not change the Technical Brief's findings. 
This report is based on research conducted by the Johns Hopkins University Evidence-based Practice Center (EPC) under contract to the Agency for Healthcare Research and Quality (AHRQ), Rockville, MD (Contract No. 290-2015-00006-I). The findings and conclusions in this document are those of the authors, who are responsible for its contents; the findings and conclusions do not necessarily represent the views of AHRQ. Therefore, no statement in this report should be construed as an official position of AHRQ or of the U.S. Department of Health and Human Services.

\section{None of the investigators have any affiliations or financial involvement that conflicts with the material presented in this report.}

The information in this report is intended to help healthcare decision makers—patients and clinicians, health system leaders, and policymakers, among others-make well-informed decisions and thereby improve the quality of healthcare services. This report is not intended to be a substitute for the application of clinical judgment. Anyone who makes decisions concerning the provision of clinical care should consider this report in the same way as any medical reference and in conjunction with all other pertinent information, i.e., in the context of available resources and circumstances presented by individual patients.

This report is made available to the public under the terms of a licensing agreement between the author and the Agency for Healthcare Research and Quality. This report may be used and reprinted without permission except those copyrighted materials that are clearly noted in the report. Further reproduction of those copyrighted materials is prohibited without the express permission of copyright holders.

AHRQ or U.S. Department of Health and Human Services endorsement of any derivative products that may be developed from this report, such as clinical practice guidelines, other quality enhancement tools, or reimbursement or coverage policies may not be stated or implied.

AHRQ appreciates appropriate acknowledgment and citation of its work. Suggested language for acknowledgment: This work was based on an evidence report, Strategies for Patient, Family and Caregiver Engagement, by the Evidence-based Practice Center Program at the Agency for Healthcare Research and Quality (AHRQ).

Suggested citation: Bennett WL, Pitts S, Aboumatar H, Sharma R, Smith BM, Das A, Day J, Holzhauer K, Bass EB. Strategies for Patient, Family and Caregiver Engagement. Technical Brief. (Prepared by the Johns Hopkins University Evidence-based Practice Center under Contract No. 290-2015-00006-I.) AHRQ Publication No. 20-EHC017. Rockville, MD: Agency for Healthcare Research and Quality; August 2020. DOI: https://doi.org/10.23970/AHRQEPCTB36. Posted final reports are located on the Effective Health Care Program search page. 


\section{Preface}

The Agency for Healthcare Research and Quality (AHRQ), through its Evidence-based Practice Centers (EPCs), sponsors the development of evidence reports and technology assessments to assist public- and private-sector organizations in their efforts to improve the quality of healthcare in the United States. The reports and assessments provide organizations with comprehensive, science-based information on common, costly medical conditions and new healthcare technologies and strategies. The EPCs systematically review the relevant scientific literature on topics assigned to them by AHRQ and conduct additional analyses when appropriate prior to developing their reports and assessments.

This EPC evidence report is a Technical Brief. A Technical Brief is a rapid report, typically on an emerging medical technology, strategy or intervention. It provides an overview of key issues related to the intervention-for example, current indications, relevant patient populations and subgroups of interest, outcomes measured, and contextual factors that may affect decisions regarding the intervention. Although Technical Briefs generally focus on interventions for which there are limited published data and too few completed protocol-driven studies to support definitive conclusions, the decision to request a Technical Brief is not solely based on the availability of clinical studies. The goals of the Technical Brief are to provide an early objective description of the state of the science, a potential framework for assessing the applications and implications of the intervention, a summary of ongoing research, and information on future research needs. In particular, through the Technical Brief, AHRQ hopes to gain insight on the appropriate conceptual framework and critical issues that will inform future research.

AHRQ expects that the EPC evidence reports and technology assessments will inform individual health plans, providers, and purchasers as well as the healthcare system as a whole by providing important information to help improve healthcare quality.

If you have comments on this Technical Brief, they may be sent by mail to the Task Order Officer named below at: Agency for Healthcare Research and Quality, 5600 Fishers Lane, Rockville, MD 20857, or by email to epc@ahrq.hhs.gov.

Gopal Khanna, M.B.A.

Director

Agency for Healthcare Research and Quality

Stephanie Chang, M.D., M.P.H.

Director

Evidence-based Practice Center Program

Center for Evidence and Practice Improvement

Agency for Healthcare Research and Quality
Arlene Bierman, M.D., M.S.

Director

Center for Evidence and

Practice Improvement

Agency for Healthcare Research and Quality

Elise Berliner, Ph.D.

Task Order Officer

Center for Evidence and

Practice Improvement

Agency for Healthcare Research and Quality 


\section{Acknowledgments}

We would like to thank the Key Informants, Peer Reviewers, and the Task Order Officers.

We also thank Jeanette Edelstein, M.A., for her services in copyediting this report.

\section{Key Informants}

In designing the study questions, the EPC consulted a panel of Key Informants who represent subject experts and end-users of research. Key Informant input can inform key issues related to the topic of the Technical Brief. Key Informants are not involved in the analysis of the evidence or the writing of the report. Therefore, in the end, study questions, design, methodological approaches, and/or conclusions do not necessarily represent the views of individual Key Informants.

Key Informants must disclose any financial conflicts of interest greater than $\$ 5,000$ and any other relevant business or professional conflicts of interest. Because of their role as end-users, individuals with potential conflicts may be retained. The TOO and the EPC work to balance, manage, or mitigate any conflicts of interest.

The list of Key Informants who provided input to this report follows:

James E. Bailey, M.D., M.P.H., FACP*

University of Tennessee Health Sciences Center

Memphis, TN

Giselle Corbie-Smith, M.D., M.Sc.

UNC School of Medicine

Chapel Hill, NC

Deborah L. Dokken, M.P.A.*

Institute for Patient- and Family-Centered Care

Bethesda, MD

J. Greg Merritt, Ph.D.*

Patient is Partner, LLC

Brighton, MI

Danielle McQuigg, Ph.D., M.B.A.

Howard County General Hospital

Columbia, MD

Julie Mitchell, M.D., M.S., FACP*

Medical College of Wisconsin

Anthem Blue Cross Blue Shield

Milwaukee, WI 
Margaret R. Riley, CMA-AC*

Health Occupation Instructor

Ellicott City, MD

Carrie Rubenstein, M.D.

Swedish Medical Center

Seattle, WA

Lane Stiles, M.A.*

Vanderbilt University Medical Center

Nashville, TN

Erin Welch*

Johns Hopkins Children's Center Pediatric Family Advisory Council

Baltimore, MD

Tom Workman, Ph.D., M.A.

American Institutes for Research

Washington, DC

*Also contributed as a peer reviewer

\section{Peer Reviewers}

Prior to publication of the final evidence report, EPCs sought input from independent Peer Reviewers without financial conflicts of interest. However, the conclusions and synthesis of the scientific literature presented in this report does not necessarily represent the views of individual reviewers.

Peer Reviewers must disclose any financial conflicts of interest greater than $\$ 5,000$ and any other relevant business or professional conflicts of interest. Because of their unique clinical or content expertise, individuals with potential non-financial conflicts may be retained. The TOO and the EPC work to balance, manage, or mitigate any potential non-financial conflicts of interest identified.

The list of Peer Reviewers follows:

Kristin L. Carman, Ph.D., M.A.

Patient-Centered Outcomes Research Institute

Washington, DC

Anjana Sharma, M.D.

University of California

San Francisco, CA 


\section{Structured Abstract}

Objective: The widely applied Chronic Care Model emphasizes the need for an "informed activated patient" and a "prepared and proactive team" to improve patient outcomes. This Technical Brief applies a framework to create a map of the currently available evidence on patient and family engagement strategies that have been used to help people manage chronic conditions.

Methods: We adapted a framework for patient, family, and caregiver engagement. Key Informants provided input on the framework for categorizing different types of engagement strategies. We searched PubMed and CINAHL from January 2015 to January 2020 to identify relevant systematic reviews and to identify original research articles focused on health system and community/policy levels, which were not covered well by systematic reviews. Our gray literature search focused on reports by national organizations.

Results: Guided by our framework, we categorized patient and family engagement strategies into direct patient care, health system, and community/policy levels. The search yielded 134 systematic reviews. Of those, 126 reviews focused on the direct patient care level, 5 on the health system level, and none on the community level. Eight reviews (five with studies having comparison groups and three with studies lacking comparison groups) reported implementation outcomes. The number of studies per review ranged from 0 to 488 studies for the direct patient care level. Reviews on direct patient care level engagement mostly focused on self-management support (88) and shared decision making (34), and many used mobile health and electronic health record tools to improve engagement. The majority included studies of adults (96), while only 14 focused on children. Self-management support strategies were mostly tested as part of multicomponent interventions. Multiple technology-based modalities were used. The most frequently reported clinical outcomes were adherence to medication/self-care plans, and measures of chronic disease control (e.g., hemoglobin A1c and blood pressure control). For self-management in adults, 26 reviews reported positive effects, 18 reported potential benefits, and 19 reported unclear benefits. None reported any harms. Most reviews of shared decision making also described multicomponent interventions. Seven showed positive effects, ten showed potential benefits, eight showed unclear benefits, and one showed no benefits. Health system-level strategies most commonly involved patients and family caregivers serving on patient and community advisory councils and participating in meetings or project teams. No rigorous evaluations were reported on these strategies. One original article was identified that described patient engagement at the community level and focused on a neighborhood-clinic partnership.

Conclusions: Patient and family engagement strategies with the greatest evidence pertain to self-management support for adults with chronic conditions. Use of technology to facilitate patient and family engagement is a promising approach. Few studies examined advanced care planning or interventions for patients with multiple chronic conditions. More research is needed to address a big gap in evidence on patient and family engagement at the health system and community/policy levels. 


\section{Contents}

Evidence Summary ................................................................................................................................ 1

Introduction ................................................................................................................................. 1

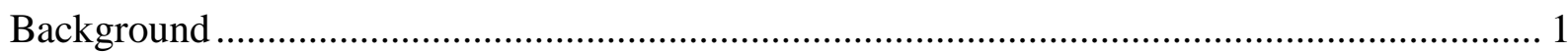

Engagement at Organizational and Policy Level ................................................................. 1

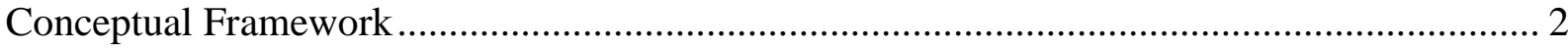

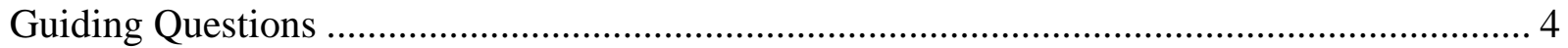

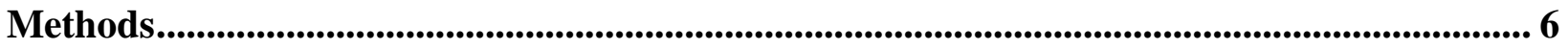

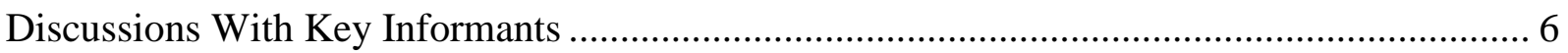

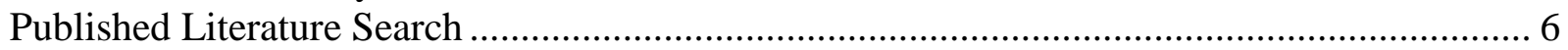

Search, Study Selection, and Data Abstraction ................................................................. 8

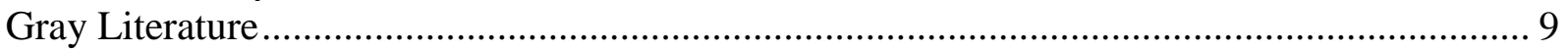

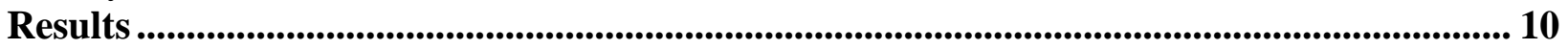

Results of the Key Informant Interviews ......................................................................... 10

Results of the Published Literature Search .............................................................................. 12

Overview of Direct Patient Care Strategies (Guiding Question 1.a-1.c) ............................... 14

Direct Patient Care Strategies - In Reviews Including Adults With Chronic Conditions

(Guiding Question 1.a-1.c) .............................................................................................. 17

Direct Patient Care Strategies - In Reviews Including Children With Chronic Conditions

(Guiding Question 1.a-1.c) ............................................................................................. 26

Direct Patient Care Strategies - In Reviews Including Both Adults and Children With

Chronic Conditions (Guiding Question 1.a-1.c) .................................................................. 32

Implementation of Direct Patient Care Strategies (Guiding Question 1.d-1.f)..................... 36

Health System and Organization Strategies - In Patients With Chronic Conditions (Guiding

Question 1.a-1.c) ............................................................................................................. 37

Implementation of Health System and Organization Strategies - In Patients With Chronic

Conditions (Guiding Question 1.d-1.f) ........................................................................... 38

Community/Policy Engagement Strategies .......................................................................... 39

Results From the Gray Literature: Innovative Patient and Family Engagement Strategies

Among Patients With Chronic Conditions ……………......................................................... 39

Discussion and Implications............................................................................................... 44

Summary of Main Findings .................................................................................................. 44

Strengths and Limitations of Our Evidence Map ………………………................................. 45

Implications for Clinical Practice, Education, and Health Policy ………………………........... 46

Future Research Needs ............................................................................................... 46

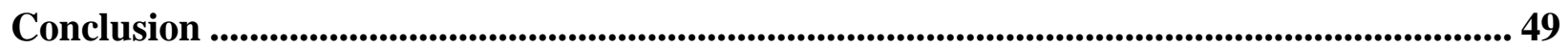

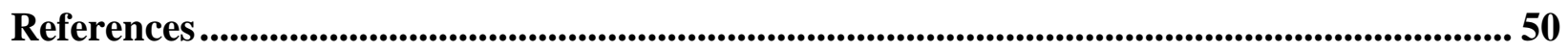

Tables

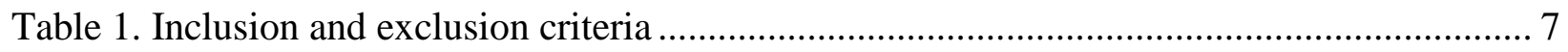

Table 2. Number of systematic reviews reporting benefits among adults, stratified by interventions and conditions .................................................................................................... 23

Table 3. Number of systematic reviews reporting benefits among children and adolescents, stratified by interventions and conditions .................................................................................. 31 Table 4. Number of systematic reviews reporting benefits among adults and children stratified by interventions and conditions. 


\section{Figures}

Figure 1. Patient, family, and caregiver engagement conceptual framework............................. 4

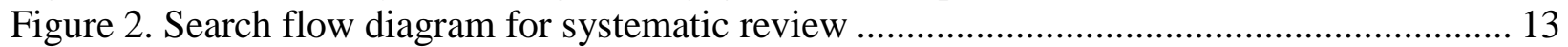

Figure 3. Search flow diagram for original studies.......................................................... 13

Figure 4. Number of systematic reviews addressing direct patient care and family engagement

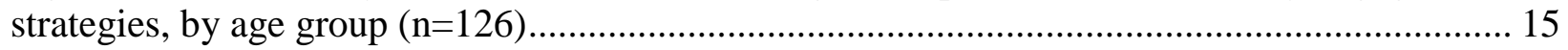

Figure 5. Intervention modality by direct patient engagement strategies (self-management

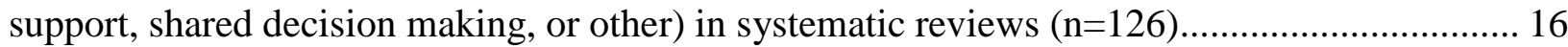

Figure 6. Systematic reviews on direct patient care strategies, by year and focus on technology

$(\mathrm{n}=126)$.

Figure 7. Chronic medical conditions targeted in systematic reviews of different types of interventions for improving patient and family engagement at the direct patient care level among adults $(\mathrm{n}=96)$

Figure 8. Patient and family engagement outcomes assessed for different types of interventions

at the direct patient care level, among adults, as reported in systematic reviews $(n=96) \ldots \ldots . . . . . .20$

Figure 9. Percentage of systematic reviews reporting benefits of different types of direct patient care interventions for improving patient and family engagement, among adults, as reported in systematic reviews $(\mathrm{n}=96)$

Figure 10. Chronic medical conditions targeted in systematic reviews of different types of interventions for improving patient and family engagement at the direct patient care level, among children and adolescents ( $\mathrm{n}=14)$.

Figure 11. Patient and family engagement outcomes assessed for different types of direct patient care interventions, among children and adolescents, as reported in systematic reviews (n=14).. 28 Figure 12. Percentage of systematic reviews reporting benefits of different types of direct patient care interventions for improving patient and family engagement, among children and adolescents, as reported in systematic reviews $(\mathrm{n}=14)$

Figure 13. Chronic medical conditions targeted in systematic reviews of different types of interventions for improving patient and family engagement at the direct patient care level, among reviews of studies of adults and children combined $(n=16)$.............................................. 33 Figure 14. Patient and family engagement outcomes assessed for different types of direct patient care interventions, among adults and children, as reported in systematic reviews $(n=16) \ldots \ldots \ldots . . .34$ Figure 15. Percentage of systematic reviews reporting benefits of different types of direct care patient interventions for improving patient and family engagement, among adults and children,

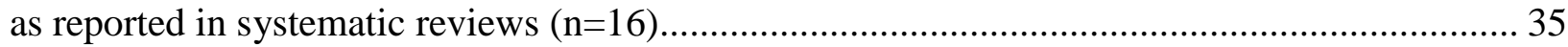

Figure 16. Map of the evidence on patient and family engagement strategies by level

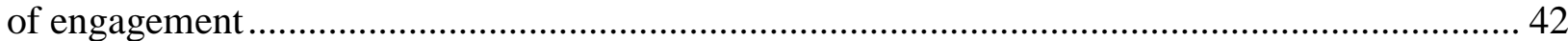
Figure 17. Map of the evidence on direct patient and family engagement strategies by reported level of benefit for different types of outcomes....

\section{Boxes}

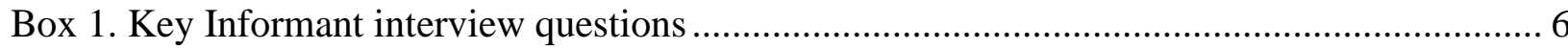

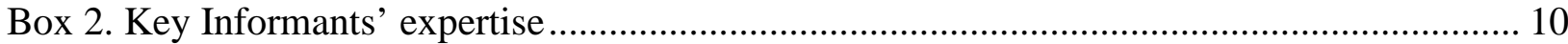

Box 3. Key messages from the Key Informants .............................................................. 11

\section{Appendixes}

Appendix A. Search Strategies

Appendix B. Evidence Tables 
Appendix C. Summary Table for Gray Literature Search

Appendix D. List of Excluded Studies for Systematic Reviews

Appendix E. List of Excluded Studies for Original Articles

Appendix F. List of Included Studies for Original Articles

Appendix G. Word Cloud 


\section{Evidence Summary}

\section{Key Points}

- The majority of systematic reviews on patient and family engagement for the management of chronic conditions focused on direct patient care engagement strategies. For this report, direct patient care strategies are defined as strategies that directly inform the patients' own treatment decisions, health behaviors, or outcomes (e.g., self-management support, shared decision making, and communication strategies).

- The direct patient care engagement strategies most commonly included team-based care to support patient self-management, patient-provider communication using shared decision making, and mobile health and electronic health record tools to improve engagement.

- The direct patient care engagement strategies with the highest volume of evidence (i.e., several large randomized controlled trials (RCTs)) included group-based educational programs to promote chronic disease self-management by peers and other healthcare professionals; web-based and short message service interventions for cancer survivors; promising telehealth programs to promote communication, self-monitoring, and counseling; and mobile health to promote weight loss.

- Few systematic reviews or original articles focused on patient and family engagement strategies at the health system or community/policy levels. For this report, we defined a "health system level strategy" as a strategy that has an impact beyond the individual patient's care (e.g., informing changes to the services of the clinic and health care system). We defined a "community or policy strategy" as a strategy that engages patients, consumers, or citizens in policymaking or that engages communities in health care policies. We did not identify any RCTs or high-quality observational studies of health system interventions. Most existing studies at the health system level examined the impact of patient and family engagement on care processes or service delivery, policy or planning documents, and educational materials or tool development.

- Patient and family advisory councils and having patients serve on committees are the most commonly studied health system level patient and family engagement strategies.

- Health system level patient engagement strategies demonstrated some benefits, such as improvements in health care processes, development of organizational plans and policies, and education or tools.

- The single article addressing a community level patient engagement strategy described a neighborhood-clinic partnership in the Navajo Nation aimed at improving care for people living with diabetes.

\section{Background and Purpose}

Patient engagement is increasingly described as essential to improving outcomes. The objective of this Technical Brief is to apply a logical conceptual framework to create a map of the currently available evidence on patient and family engagement strategies used to help people with chronic conditions. Report findings can inform decisions of healthcare leaders as well as highlight the areas in need of more research. 


\section{Methods}

We followed processes established by the Evidence-based Practice Center Program for Technical Briefs, including interviewing Key Informants. Our protocol is posted on the program's website (https://effectivehealthcare.ahrq.gov/products/family-engagement/protocol). The searches were conducted in January 2020. Details of the methodology can be found in the full report. Given the broad definition of patient and family engagement and the large body of evidence, we focused our search on systematic reviews, with supplemental searches for original research articles and gray literature in areas having a paucity of reviews.

\section{Results}

Building on the conceptualization of patient and family engagement by Carman et al, we categorized patient and family engagement strategies into strategies at the direct patient care, health system, and community/policy levels. The search yielded 134 systematic reviews. Of those, 126 focused on the direct patient care level, 5 on the health system level, and none on the community level. Eight reviews (five with studies having comparison groups and three with studies lacking comparison groups) reported implementation outcomes. The reviews included a large number of studies (4,111 studies for direct patient care level, without excluding duplicates). Given the low number of reviews at the health system and community/policy levels, we searched for original articles and identified three studies on engagement at the health system level and one at the community level.

Reviews on direct patient care level engagement most commonly focused on self-management support (88) and shared decision making (34), and many used mobile health and electronic health record tools to improve engagement. The majority included studies of adults (96), while 14 focused on children. Many reviews focused on single medical conditions, most commonly among people with diabetes for self-management support, followed by patients with cancer and with cancer screening needs for shared decision making. Very few reviews focused on patients with multiple chronic conditions.

Self-management support strategies were mostly tested within multicomponent interventions. These strategies included (1) education and information sharing on chronic conditions and treatment options, (2) helping patients achieve behavior change via goal setting, self-monitoring and symptom management, use of action plans, problem solving, tracking data, and feedback, (3) facilitating communication with healthcare providers and adherence to self-care plans via reminders/alerts, remote monitoring, and decision support, and (4) providing psychosocial support including healthcare navigation assistance, connection to social services and peers, counseling, and cognitive behavioral therapy. Multiple delivery methods were used, including individual and group education programs. While some strategies incorporated technology as the sole means for connecting with patients, others included in-person approaches or a mix. Technology-based approaches included devices, sensor-based technologies, gaming, videoconferencing, remote monitoring, and texting. The most frequently reported outcomes were chronic disease management measures (e.g., hemoglobin A1c and blood pressure control) followed by health-related quality of life and medication adherence. For adult self-management, 26 reviews showed positive effects, 18 showed potential benefits, and 19 showed unclear benefits. None reported any harms. More details are provided in the report on studies of children and adolescents. The two reviews that focused exclusively on studies of patients with multiple 
chronic conditions showed unclear benefits, citing difficulty in operationalizing self-management for multiple chronic conditions and reduced ability to help these patients.

Most shared decision-making reviews described multicomponent interventions, including provider training and patient education, technology-enabled delivery modes (e.g., video and web-based tools), and decision support tools. Reported outcomes mainly included patient knowledge, activation, decisional conflict, and satisfaction. Few reviews reported on clinical outcomes. Seven showed positive effects, ten showed potential benefits, eight showed unclear benefits, and one showed no benefits. Within the two reviews that focused on use of patient portals, one reported provider perceptions that releasing abnormal or sensitive test results to patients could cause confusion or excess worry for patients, but there was no systematic measurement of harm.

Health system level engagement strategies most commonly included having patients and family caregivers serving on patient and family advisory councils and other committees within the health system; participating in meetings, on project teams, or forums and workshops to provide patients with skills to support engagement; or serving as instructors for healthcare professionals in training or as consultants. No rigorous evaluations reported on these strategies, and the studies were mainly limited to case reports and observational studies. Impacts of patient engagement have been reported on care processes and service delivery, priority setting, educational materials or tool development, physical space design, trainings for staff, and increasing staff awareness of patient perspectives.

No reviews and only one original article was identified that described patient engagement at the community level. The study evaluated efforts to strengthen engagement between community members and the Indian Health Service.

\section{Limitations}

This report has several limitations, including (1) use of systematic reviews rather than original studies for direct patient-care strategies due to the large body of evidence, (2) exclusion of studies on patient engagement in research, (3) focus on engagement of patients with chronic health conditions, excluding reviews on chronic disease prevention, and (4) no assessment of the risk of bias in the original studies.

\section{Implications and Conclusions}

Patient and family engagement strategies with the highest volume of evidence among adults with chronic conditions pertain to direct patient care using self-management support strategies. However, we identified inconsistent findings among reviews of self-management strategies, even within the same chronic condition. This is in part due to the heterogeneity of tested interventions, different measures, and low quality of the original studies. The evidence on engagement strategies in the pediatric population is limited by a small number of systematic reviews with few reporting on clinical outcomes. Use of technology as part of the patient and family engagement strategy is emerging as a promising approach. Few studies examined engagement strategies for advanced care planning or for patients with multiple chronic conditions. More research is needed to address a large gap in evidence on patient and family engagement at the health system and community/policy levels. 


\section{Introduction}

\section{Background}

Patient and family engagement refers to patients, families, and health care providers working in active partnership across various levels to help improve healthcare outcomes. ${ }^{1}$ While a patient may engage with their healthcare provider devising strategies to manage their own health, they may also engage at a system level with other healthcare providers and leaders in efforts to improve the care provided to all other patients. Successful patient and family engagement has potential to reduce costs, improve care processes, reduce provider burnout and improve patient outcomes. ${ }^{2,3}$ The widely applied and accepted Chronic Care Model emphasizes the need for having both an "informed activated patient" and a "prepared and proactive team" to improve patient outcomes. ${ }^{4,5}$ Patient activation refers to the level at which a patient have the knowledge, skills, willingness and ability to manage their own health. ${ }^{6}$ Multiple studies have demonstrated that activated patients have better health outcomes and lower utilization of emergency health care services. ${ }^{7}$ Conversely, reduced patient engagement in health care is associated with significant, serious, or life-threatening adverse events. ${ }^{8}$

In the U.S. the prevalence of chronic diseases has been increasing, necessitating a shift towards care in the ambulatory and community settings that enable long-term, sustainable strategies for preventing and managing chronic disease. ${ }^{9-11}$ Interventions, such as tailored coaching that increased patient activation, have been associated with improved intermediate outcomes, including chronic disease self-management behavior and reduced health care utilization. ${ }^{12}$ Self-management education and support interventions have also improved outcomes and function among patients with single and multiple chronic diseases. ${ }^{13-19}$

Despite the benefits of patient engagement, not all patients have the capacity to get engaged in their care, including children and patients with dementia or disability. Therefore, family and caregiver engagement strategies are needed to support vulnerable patients including children, the elderly, people at the end of life, and people with disabilities. ${ }^{20,21}$

Clinicians and healthcare systems have key roles in facilitating patient and family engagement. ${ }^{22}$ Many tested interventions aim to improve clinicians' communication skills and shared decision-making techniques, ${ }^{23-27}$ in part because clinicians use complicated medical jargon, limiting patients' understanding of their care. ${ }^{28}$ Communication studies show that clinicians quickly interrupt patients, allowing less opportunity for listening to concerns and building rapport. ${ }^{29}$ Patients and families may not feel empowered to speak up about their concerns in health care environments, with greater risks among marginalized patients and families, including those with limited English language proficiency. ${ }^{30,31}$ Interventions that target patient-provider communication have improved patient satisfaction, as well as patient-centered health outcomes (e.g., symptoms of depression, anxiety, pain; management of blood pressure; and improvement in functional status). ${ }^{23-27,32,33}$

\section{Engagement at Organizational and Policy Level}

Increasingly, health care systems are implementing strategies to engage patients, families, and caregivers in efforts to improve care delivery, efficiency, outcomes, and patient experience, and reduce health care costs. ${ }^{22,34,35}$ Part of the motivation was the Patient Protection and Affordable Care Act (2010), which tied Medicare reimbursements with scores on a patient experience survey. ${ }^{36}$ A 2016 survey of over 1,450 hospitals demonstrated that most hospitals had 
policies supporting patient and family engagement, including some that established patient and family advisory councils, but there was wide variation in strategy and implementation. ${ }^{34}$ Patient and family engagement strategies have been integrated into advanced primary care models, including Patient Centered Medical Homes and the Comprehensive Primary Care Initiative of the Centers for Medicare \& Medicaid Services. ${ }^{37,38}$ Diverse organizations have developed tools to promote engagement of patients and families at the organizational level, including the Agency for Healthcare Research and Quality (AHRQ), and the Institute for Patient- and Family-Centered Care, resulting in implementation guides and workshops. ${ }^{39-42}$ An early review of patient and family engagement in health care design identified case studies, which demonstrated positive effects on patients' perspectives, changes to services available to patients, and improved staff attitudes toward patient engagement, but found little evidence on quality or effectiveness of care. ${ }^{43} \mathrm{~A}$ more recent review identified process improvements, but few studies examined clinical outcomes. ${ }^{44}$

The objective of this Technical Brief is to apply a logical conceptual framework to map the currently available evidence on patient and family engagement strategies that have been used to help patients, families, and caregivers manage chronic conditions. The long-term goal is to inform decision making by health systems on which engagement strategies to deploy to improve outcomes.

\section{Conceptual Framework}

In this Technical Brief, we adapted a widely used conceptual framework on patient and family engagement interventions by Carman and colleagues, that categorizes strategies into the direct patient care level, health system or organizational level, or community/policy level (Figure 1). ${ }^{1}$ We applied the definition of patient and family engagement from this framework as "patients, families, their representatives, and health professionals working in active partnership at various levels across the health care system and the community - direct care, organizational design and governance, and policymaking — to improve health and health care." "We applied the adapted framework to create an evidence map displaying the evidence for patient and family engagement strategies. ${ }^{1}$ We expanded the model related to examples of strategies and highlighting potential measures and outcomes. However, in recognition of the importance of the "continuum of engagement" (i.e., levels of how active the patient is in communication and health care decisions, and how involved the patient is in health care organization decisions) described in the original model, we focused our review on patient and family engagement that requires “involvement” and "partnership and shared leadership" (i.e., not solely consultation or one way communication) (see PICOTS, Table 1).

For the purposes of this report, we defined "direct patient care strategies" as those strategies that directly inform the patients' own treatment decisions, health behaviors, or outcomes (e.g., self-management support, shared decision making, and communication strategies). Direct patient care strategies may be delivered by the patient's primary care practice and provider, communitybased organizations or the patient's health insurer through case management and population health programs. We defined a "health system level strategy" as a strategy that engages patients and families in organizational activities and/or decision making and informs the delivery of care within a health care system, beyond the individual patient's care (e.g., participation in an advisory committee or board membership). Examples include patient and family advisory councils, in which the patients and families provide feedback about how to improve the care processes and quality of care and patient experience to improve care for all patients, not just 
themselves. We defined a "community or policy strategy" as a strategy that engages patients, consumers, or citizens in policymaking or that engages communities in health care policies (e.g., a hospital-neighborhood partnership to address community's concerns, disease-specific group of patients lobbying for more funding to study a rare disease, or a community group advocating for sugar-sweetened beverage or tobacco-related local policy changes to improve neighborhood public health). We acknowledge that many of these levels overlap, as it is possible that direct care engagement strategies could also yield improvements at the system-level, and patients engaged in hospital committees could also benefit directly by improving their own care practices.

In addition, in this report we used the term "patient and family engagement" to represent engagement of the patient and family, as well as non-family caregivers, who the patient deems part of his or her care team. In addition, we intended the term "patient and family engagement" to broadly include the engagement of other consumers and citizens for the purposes of improving the quality of patient care and health outcomes for people living in the community and served by the health care system. 
Figure 1. Patient, family, and caregiver engagement conceptual framework

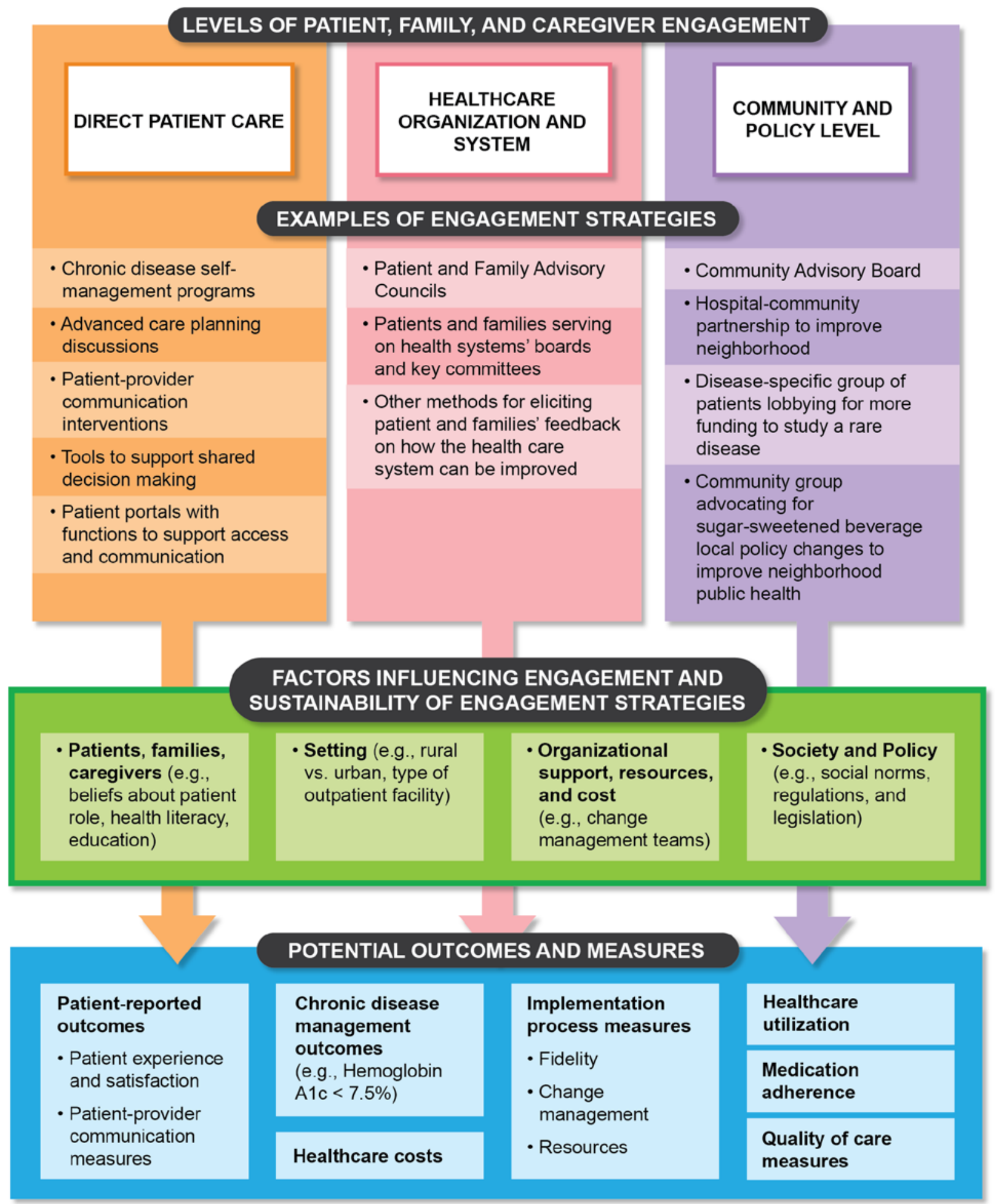

\section{Guiding Questions}

This Technical Brief was guided by the following questions: 
1. What patient engagement strategies have been studied to help patients, families, and caregivers manage their chronic conditions and improve patient health outcomes?

a. What are the characteristics of the patients/conditions? What is the specific role for families and caregivers? Have the subpopulations of interest been studied in the literature?

b. What are the characteristics of these patient and family engagement strategies?

c. What outcomes, including harms, have been studied?

d. Which elements must be implemented to have fidelity? Which elements can be adapted to reflect the local context without losing fidelity?

e. What resources and costs are required to implement these strategies?

f. What change management strategies support sustainment after implementation?

2. What gaps exist in the current research?

a. Which patient engagement strategies identified by experts as currently relevant have no research evidence or inadequate evidence?

b. For which patient engagement strategies are additional primary research studies needed to answer questions important to policy and practice of self-management?

c. For which patient engagement strategies are there sufficient primary research studies that a new systematic review would add to current knowledge? 


\section{Methods}

We addressed Guiding Question 1 through literature review and discussion with Key Informants. We conducted a systematic literature search to identify systematic reviews on patient engagement strategies for chronic conditions. Guiding Question 2 was informed by the findings from Guiding Question 1.

\section{Discussions With Key Informants}

Key Informants were selected for their specific perspectives, including caregivers, patient representatives (e.g., from the Institute for Patient- and Family-Centered Care), health system leaders, primary care providers, nurses, payers (e.g., from Anthem, Inc.), and researchers testing patient and family engagement strategies. We solicited input on what to emphasize in the methodologic approach to the Technical Brief (e.g., strategies, methods, outcomes) and what is most important from each of their perspectives. We asked Key Informants to share their perspectives on topics that pertain to patient and family engagement and the Guiding Questions. These interviews allowed us to characterize different engagement strategies that may not appear in either the gray or published literature.

We developed interview guides, separate for each type of Key Informant, as appropriate. Box 1 lists the full set of Key Informant interview questions.

\section{Box 1. Key Informant interview questions}

From your experience or clinical practice, please identify an example of a patient, family or family engagement strategy.

We will ask you to discuss this strategy on the call and consider some of these aspects of the strategy:

- Who/what was the focus?

- What was the setting? Who was involved?

- Was it successful - why or why not?

- What contributed to it being successful or not?

Call 1: Word Cloud Brainstorm as a tool to enhance discussion: please share your words/phrases on what you think is most important to measure and know about whether a patient, family and caregiver engagement strategy is working (such as patient satisfaction, better quality of care, etc.).

Call 2: Word Cloud Brainstorm as a tool to enhance discussion: please share your words/phrases on some of the challenges that exist to implement patient, family and caregiver engagement strategies into the health system (such as costs, or lack of evidence of what works best).

We conducted interviews in small groups on the telephone. Notes were drafted for each call. Calls were recorded to assist with ensuring complete and accurate documentation.

\section{Published Literature Search}

We defined the criteria for inclusion in the review of published literature, using the PICOTS framework (Population, Intervention, Comparison, Outcomes, Timing, and Setting). Table 1 lists the eligibility criteria. 
Table 1. Inclusion and exclusion criteria

\begin{tabular}{|c|c|c|}
\hline PICOTS & Include & Exclude \\
\hline Population & $\begin{array}{l}\text { - Patients (children and adults) with chronic } \\
\text { medical conditions* (e.g., DM, HTN, ESRD) and } \\
\text { their families and caregivers } \\
\text { Patient representatives/caregivers serving on } \\
\text { committees/councils aimed at improving care } \\
\text { delivery to patients with chronic health } \\
\text { conditions and those that over-utilize care } \\
\text { - Subpopulations, including } \\
\bullet \quad \text { Ethnic and racial minority } \\
\text { - Limited language skills } \\
\bullet \quad \text { Low literacy/low health literacy } \\
\bullet \quad \text { Cognitive impairment }\end{array}$ & None \\
\hline Interventions & 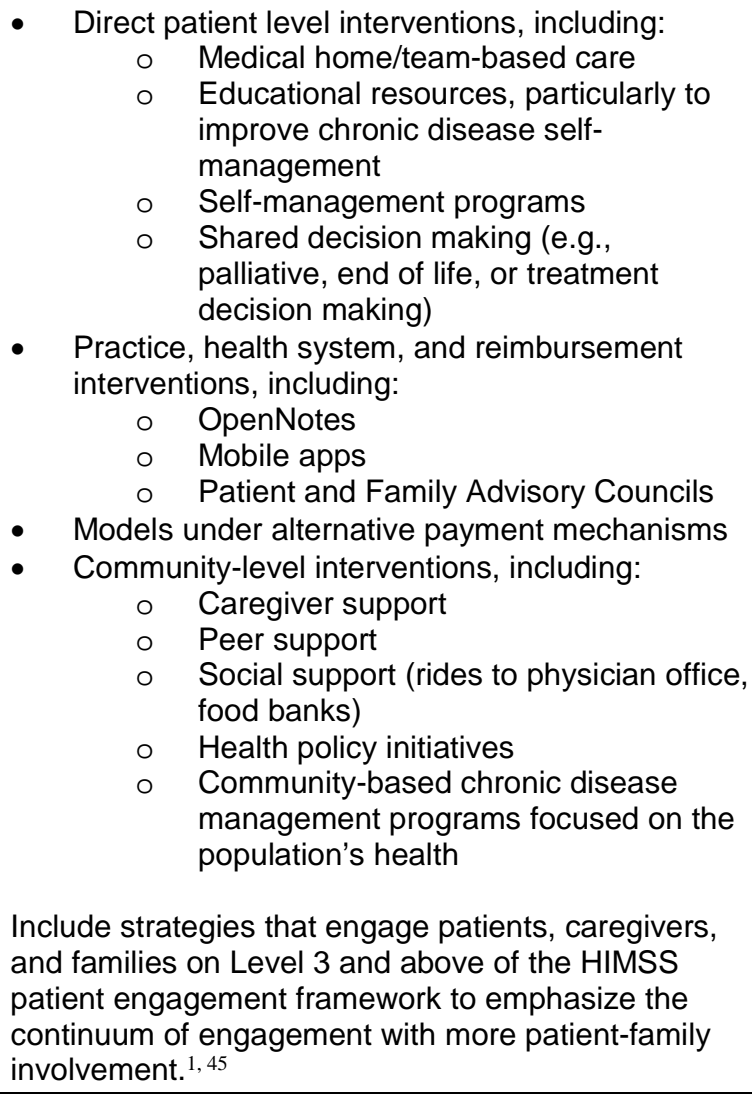 & $\begin{array}{l}\text { - One-time education-only or } \\
\text { information-providing intervention } \\
\text { (e.g., providing a handout) } \\
\text { Without 2-way interaction or ability } \\
\text { for patient to ask questions (e.g., } \\
\text { providing access to web-based } \\
\text { educational program) } \\
\text { Exclude strategies Level } 1 \text { and } 2 \text { of } \\
\text { the HIMSS patient engagement } \\
\text { framework. }{ }^{45}\end{array}$ \\
\hline Comparators & $\begin{array}{l}\text { Any comparator (pre/post, concurrent) } \\
\text { Note: For reviews addressing implementation of } \\
\text { engagement strategies, we will not require a } \\
\text { comparison group }\end{array}$ & $\begin{array}{l}\text { At systematic review level - reviews } \\
\text { without studies that had comparison } \\
\text { groups (e.g. qualitative studies). } \\
\text { At individual study level - studies without } \\
\text { comparison group. }\end{array}$ \\
\hline
\end{tabular}




\begin{tabular}{|c|c|c|}
\hline PICOTS & Include & Exclude \\
\hline Outcomes & 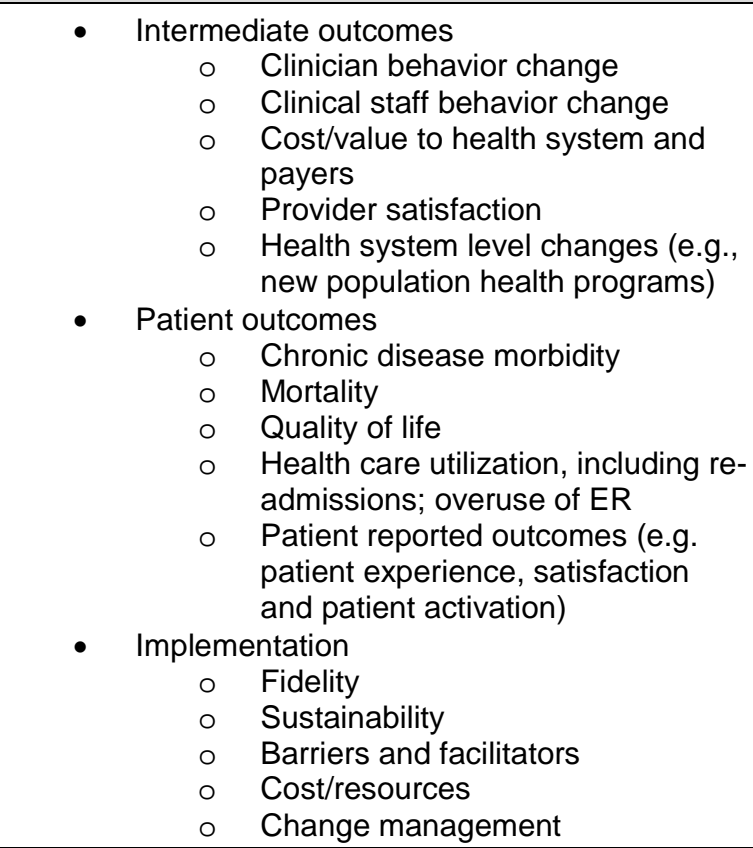 & None \\
\hline Timing & 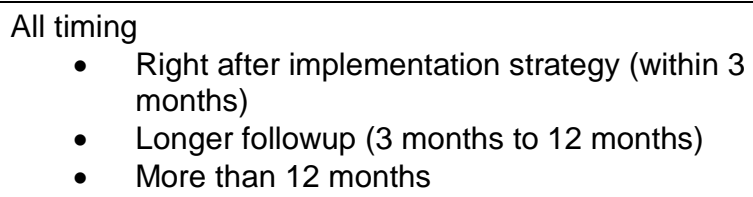 & \\
\hline Setting & $\begin{array}{l}\text { All settings where self-management occurs (e.g., } \\
\text { home/community/clinic/assisted living) }\end{array}$ & $\begin{array}{l}\text { At systematic review level - If majority of } \\
\text { articles in review were non-US-based } \\
\text { studies. } \\
\text { At individual study level - Non-US } \\
\text { studies }\end{array}$ \\
\hline
\end{tabular}

*Chronic diseases are defined broadly as conditions that last 1 year or more and require ongoing medical attention or limit activities of daily living or both.

DM = diabetes mellitus; ER = emergency room; ESRD = end-stage renal disease; HIMSS = Health Information and Management Systems Society; HTN = hypertension; IT = information technology

\section{Search, Study Selection, and Data Abstraction}

Our search strategies are in Appendix A. We first searched PubMed and CINAHL from January 2015 through January 2020 to identify systematic reviews. Due to the large volume of published studies, we focused on reviews published since January 2015, which would capture original articles published prior to 2015. Once we identified systematic reviews, we augmented the search by using the same search strategy (i.e., January 2015 through January 2020 using PubMed and CINAHL) to identify relevant original studies that might not have been included in the systematic reviews.

Search results were screened first at the abstract level, and then at the full-text level using the same relevant inclusion criteria. We selected original articles that focused on health system and community/policy level engagement strategies, where we had identified gaps in the search for reviews. We updated the search while the Technical Brief was posted for public comment. 
Search results were screened independently by two team members, first at the abstract level, and then at the full-text level. We abstracted author, publication year, end search dates, and number and type of included studies, populations, interventions, outcomes, results, and findings from each eligible systematic review. We used the term modality to describe the interventions with regard to how they are being delivered and by whom, with categories of technology (e.g. telehealth, m health , patient portal), peer/lay support, community health worker/patient navigator, nurse/case managers, team based care, and other options (as specified). These categories were chosen based on expert input on features of commonly tested interventions. The team summarized the findings from the systematic reviews based on the assessment by the reviews' authors in their conclusions. The conclusions and degree of benefit was categorized as the following: No benefit, Unclear Benefit (mix of both positive and no or unclear benefit studies), potential (more positive studies than and no or unclear benefit studies) and positive benefit (most studies showed positive benefits in most outcomes). One reviewer categorized the conclusions and they were verified by a second reviewer.

\section{Gray Literature}

We designed the gray literature search to identify reports on health care organization/system and community strategies that might fill in gaps not covered by the published literature. We targeted the gray literature search on reports from national organizations, including the Patient Centered Outcomes Research Institute (PCORI), the Institute for Patient- and Family-Centered Care, the Institute for Healthcare Improvement, and governmental agencies such as AHRQ and the National Institute of Nursing Research, as well as information received from our Key Informants. No materials were submitted through the Supplemental Evidence and Data for Systematic Reviews (SEADS) portal. 


\section{Results}

To organize the results, first, we presented the findings from the Key Informant interviews. Following the interviews, we presented the findings by Guiding Question. Within Guiding Question 1, we presented the results by level of engagement (see Figure 1). Within the direct patient care engagement level, we presented Guiding Question 1.a through 1.c by the population included in the reviews (i.e., adults, children, and then reviews that included both adults and children) followed by Guiding Question 1.d through 1.f, focused on implementation outcomes. Guiding Question 2 includes the overall evidence map to summarize the evidence and gaps.

\section{Results of the Key Informant Interviews}

We completed three 1-hour interviews with eleven Key Informants. Box 2 describes the expertise of the Key Informants.

\section{Box 2. Key Informants' expertise}

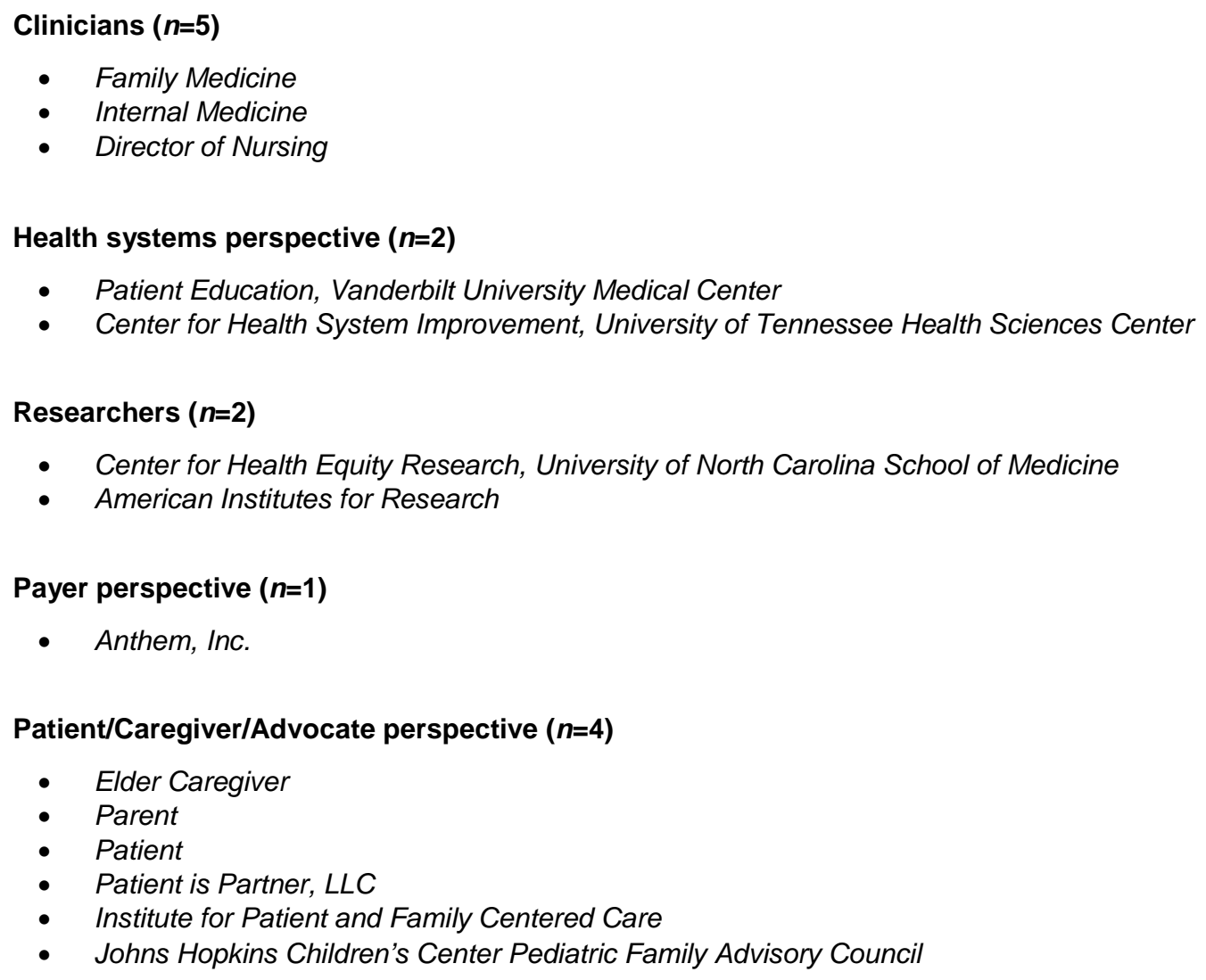

Box 3 provides the key messages identified during the interviews. Key Informants highlighted the challenge of defining what patient and family engagement means, and how to implement and measure it in a variety of clinical and community settings. Key Informants were generally in agreement about the proposed conceptual model (see Methods) for this review. Key Informants described a broad range of strategies that could be considered patient, family, and caregiver engagement, and noted that sometimes the strategies overlapped with each other. 
Box 3. Key messages from the Key Informants

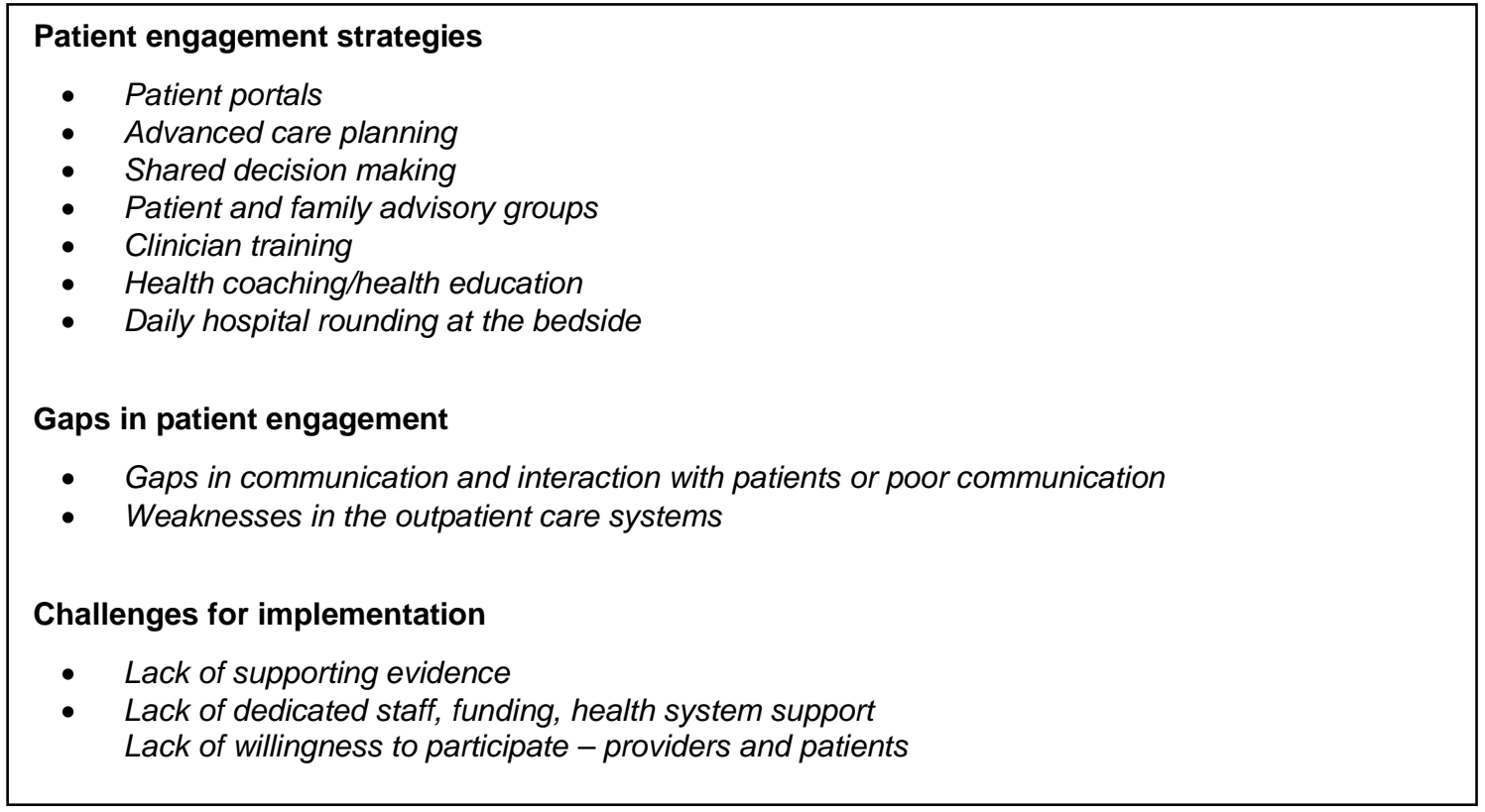

For direct patient care engagement, Key Informants shared their opinions about several models that are "well studied but not yet widely implemented," including daily inpatient multidisciplinary team rounding at the patient's bedside to review the plan of care with the patient and family, embedding of diabetes health educators into federally qualified health centers to support patients with low health literacy, and tools to facilitate shared decision making and advanced care planning.

For the level of health system engagement, Key Informants described the impact of the patient portal within the electronic health record that facilitates electronic communication with providers. Patient stakeholders valued the access to providers and quick responses they receive. Providers highlighted the challenges involved with managing the types and volume of patient requests and the possibility that patients with lower literacy or technology skills may become more marginalized. In addition, Key Informants highlighted the trend that health systems are widely implementing Patient and Family Advisory Councils to advise about a wide range of programs and initiatives. The challenge of these councils continues to be engaging diverse patients with a wide range of health care needs and including the voice of vulnerable populations. Finally, insurance companies and other payers (e.g. Medicaid and Medicare plans) are also working to incentivize patient and family engagement through value-based purchasing models and the enhancement of care coordination through their own population health programs. $^{46}$

Using our "word cloud” brainstorming tool (Appendix G), Key Informants highlighted the challenges to implementing these strategies, including: a medical culture that does not prioritize patient and family input; lack of evidence for return on investment; and lack of devoted resources for implementation, evaluation, and evidence generation/dissemination. Thus, discussions with Key Informants helped to identify important aspects of strategies to examine in this review. 


\section{Results of the Published Literature Search}

Figure 2 shows the search flow diagram for our main search focused on systematic reviews. The search for systematic reviews identified 868 references published between 2015 and 2020. Title and abstract review selected 325 references for full text review. One hundred thirty-four systematic reviews met the eligibility criteria. One hundred and twenty six reviews addressed direct patient care strategy, five reviews addressed health care system level strategy, and eight reviews (five with studies having comparison groups and 3 with studies lacking comparison groups) reported implementation outcomes (e.g., barriers, facilitators, resources, costs, change management). A listing of excluded studies is included in Appendix D.

Figure 3 shows the search flow diagram for original studies not included in the systematic reviews that described health system or community-level patient and family engagement strategies. A list of original studies focused on direct patient care strategy is included in Appendix F. We identified 4 original studies focused on these strategies. A listing of excluded studies is included in Appendix E. 
Figure 2. Search flow diagram for systematic review

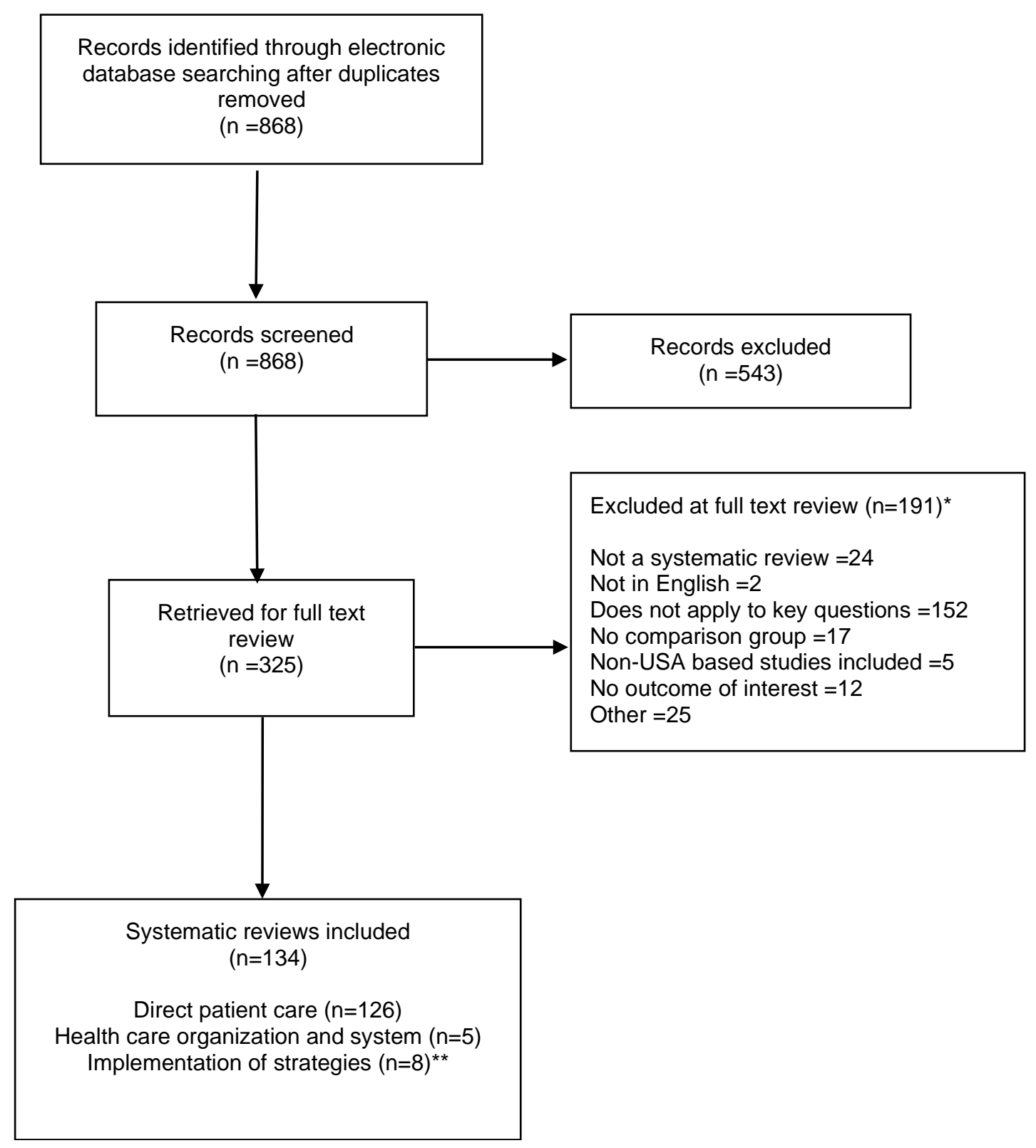

* Total exceeds the number of citations in the exclusion box, because citations could be excluded for more than one reason

**Overlapping with direct patient care and health care organization and system 
Figure 3. Search flow diagram for original studies

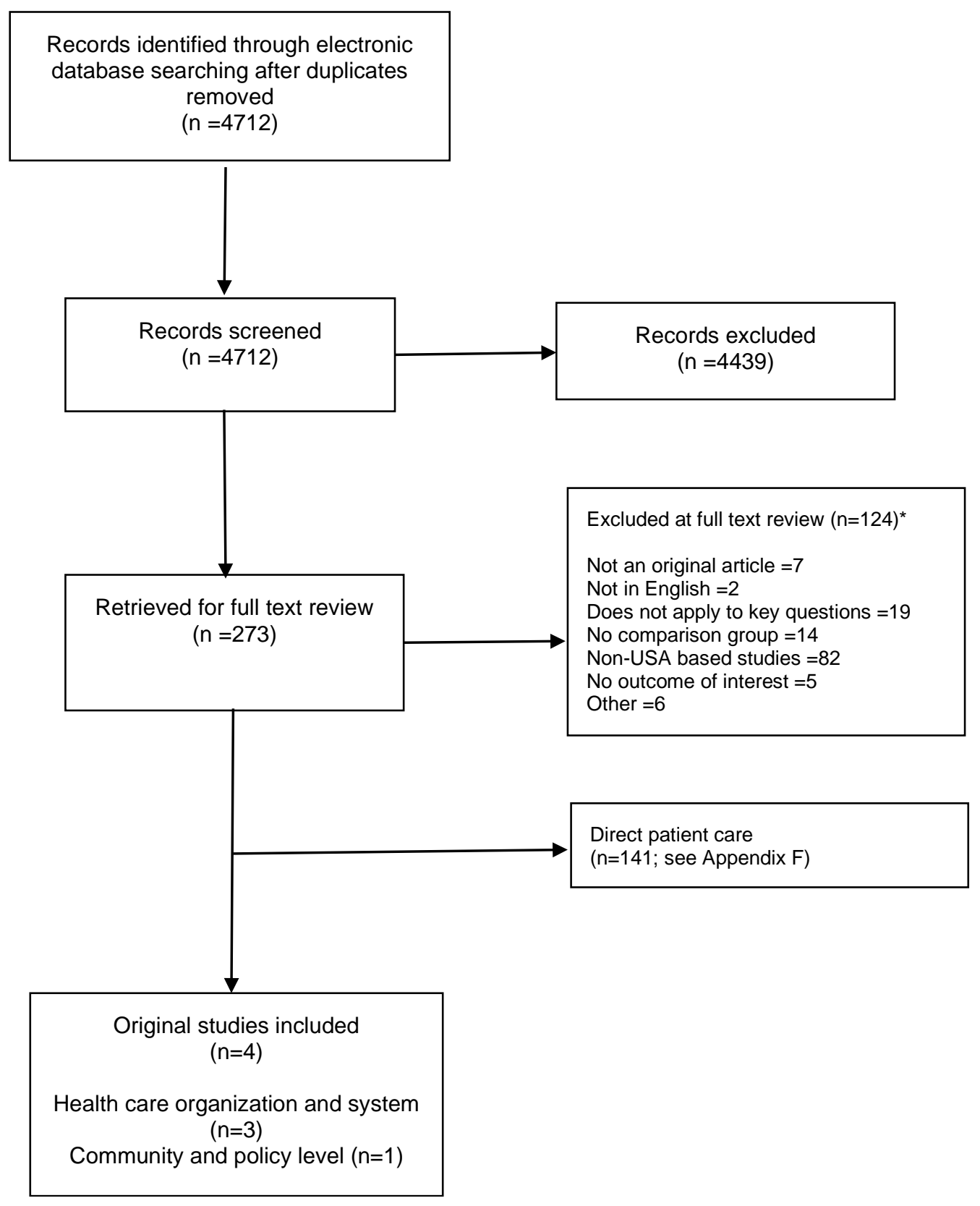

*Total exceeds the number of citations in the exclusion box, because citations could be excluded for more than one reason 
Guiding Question 1: What patient engagement strategies have been studied to help patients, families, and caregivers manage their chronic conditions and improve patient health outcomes?

We identified 131 systematic reviews addressing patient engagement strategies to help patients, families and caregivers manage their chronic conditions and improve patient health outcomes. Of these, 126 focused on direct patient care strategies and five focused on health system strategies. We did not find any systematic reviews that primarily focused on community/policy strategy. The search for original research not included in the reviews identified three articles for health system strategies and one for community/policy strategy.

We presented our results for Guiding Question 1 regarding patient and family engagement strategies using the following format:

- $\quad$ Direct Patient Care Strategies addressing Guiding Question 1.a-1.c

o Overview in patients with chronic conditions

o In adults with chronic conditions

o In children with chronic conditions

o In adults and children with chronic conditions

o Implementation of direct patient care strategies addressing Guiding Question 1.d.-1.f.

- Health System Strategies addressing Guiding Question 1.a-1.c

o In patients with chronic conditions

o Implementation of health system strategies addressing Guiding Question 1.d-1.f

- Community/Policy Strategies addressing Guiding Question 1.a-1.c

o In patients with chronic conditions

o Implementation of community/policy strategies addressing Guiding Question 1.d-1.f

\section{Overview of Direct Patient Care Strategies (Guiding Question 1.a-} 1.c)

Definition: Direct patient care strategy is defined as directly informing patients' own treatment decisions, health behaviors, or outcomes (e.g. self-management support, shared decision making, and communication strategies.

We identified 126 systematic reviews evaluating direct patient care engagement strategies, with the number of included studies per review ranging from 0 to 488 studies. The range of RCTs included in these reviews were 0 to 105 . We described the systematic reviews by three categories of strategies (self-management support, shared decision making/communication, and other).

Figure 4 shows the number of systematic reviews by study population (adults, children, adults and children) and across the three categories of direct patient care strategies. The majority $(n=88)$ of reviews focused on self-management support (e.g., counseling, team-based care) and 34 reviews focused on shared decision making or enhanced patient-provider communication. Four reviews focused on other direct patient care engagement strategies. Of the 126 systematic reviews, most $(n=96)$ studied strategies used with adults, 16 studied strategies used with children, and 14 included strategies used with both children and/or adolescents/adults (Figure 4). 
Figure 4. Number of systematic reviews addressing direct patient care and family engagement strategies, by age group $(n=126)$

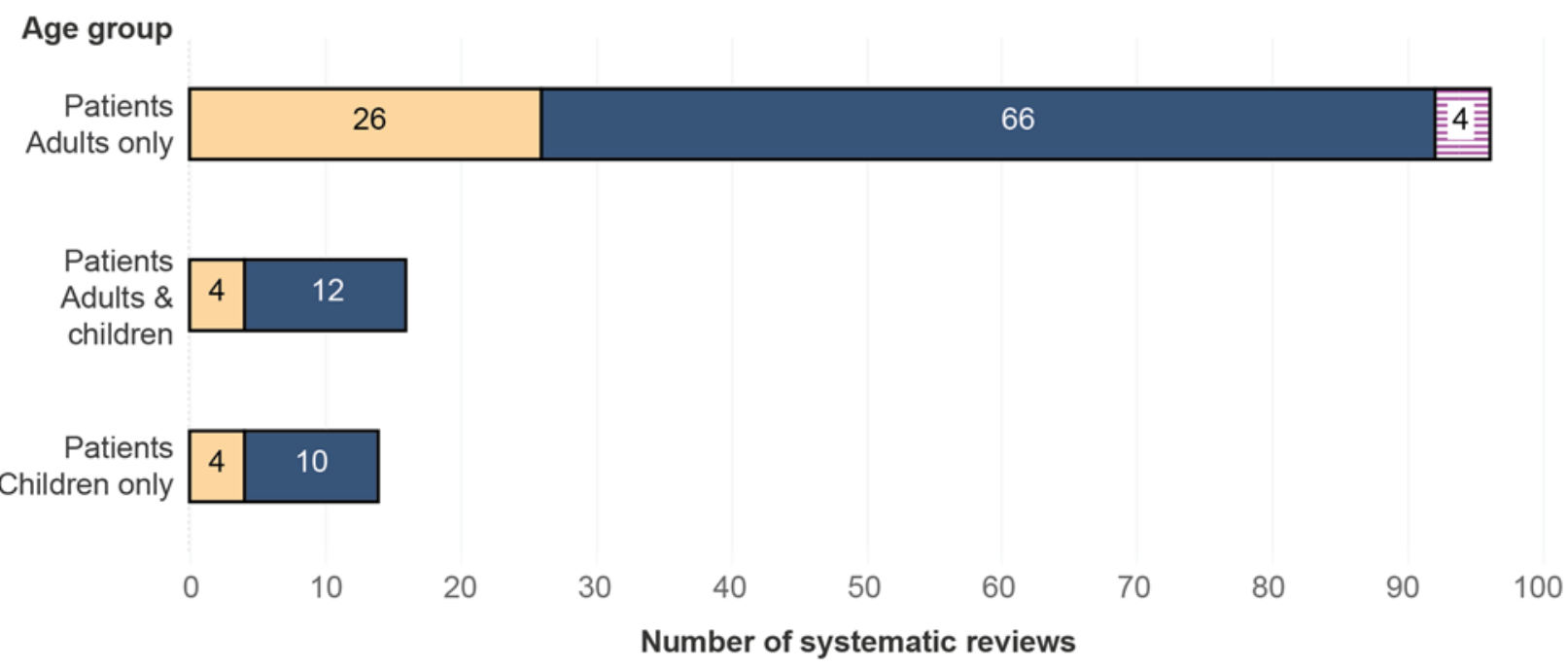

\footnotetext{
Engagement strategy

$\square$ Shared decision making

Self-management support

Other
}

Figure 5 shows the modality (e.g., types of teams, tools or technology) for each type of direct care engagement strategy across all 126 systematic reviews. Across all age groups, the majority of reviews focused on self-management support, and among those, most reviews included nurses or case managers delivering the engagement intervention. The next most frequent modality for delivering the engagement intervention was mobile health. Among the shared decision-making interventions, most reviews focused on education and counseling, followed by engagement by nurses or case managers. 
Figure 5. Intervention modality by direct patient engagement strategies (self-management support, shared decision making, or other) in systematic reviews $(n=126)$

\begin{tabular}{|c|c|c|}
\hline Intervention & Modality & Number of systematic reviews \\
\hline $\begin{array}{l}\text { Self-management } \\
\text { support (including } \\
\text { health behavior } \\
\text { change) }\end{array}$ & $\begin{array}{l}\text { Nurse/case managers } \\
\text { Mobile Health } \\
\text { Multiple technologies } \\
\text { Education/Counseling } \\
\text { multiple modalities } \\
\text { Patient Portal } \\
\text { Peer/lay support } \\
\text { Telehealth } \\
\text { Other technology } \\
\text { Team based care } \\
\text { Community health worker }\end{array}$ & $8^{9}{ }^{11}{ }^{13}{ }^{14}$ \\
\hline $\begin{array}{l}\text { Shared } \\
\text { decision making } \\
\text { (or patient- } \\
\text { provider } \\
\text { communication) }\end{array}$ & $\begin{array}{l}\text { Education/Counseling } \\
\text { Nurse/case managers } \\
\text { Multiple modalities } \\
\text { Other technology } \\
\text { Multiple technologies } \\
\text { Telehealth } \\
\text { Patient Portal }\end{array}$ & ${ }_{1}^{2}{ }^{4}$ \\
\hline Other & $\begin{array}{l}\text { Education/Counseling } \\
\text { Nurse/case managers } \\
\text { Multiple modalities }\end{array}$ & $\begin{array}{l}1 \\
1\end{array}$ \\
\hline
\end{tabular}

Figure 6 shows the distribution of reviews by year of publication and the number of reviews focused on technology (e.g., mobile health, electronic health record). We described the reviews that assessed the role of the patient portal, electronic health record, or mobile health within their respective sections. In 2015, 14 published reviews focused on technology, and many of these specifically assessed the role of the patient portal or secure messaging within the electronic health record on patient self-management and communication. 
Figure 6. Systematic reviews on direct patient care strategies, by year and focus on technology $(n=126)$

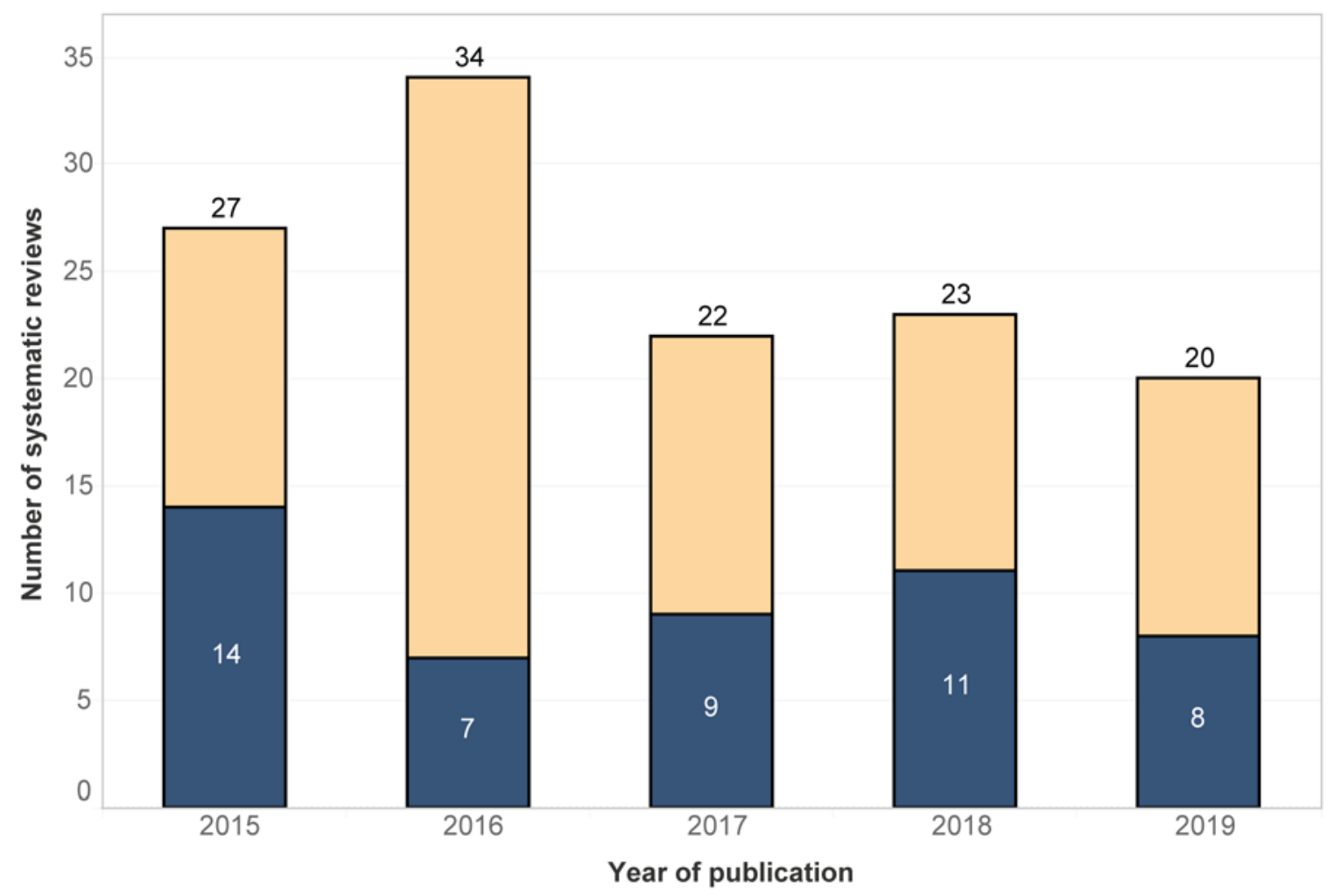

Measure Names

Total number of reviews

Number of reviews focused on technology

\section{Direct Patient Care Strategies - In Reviews Including Adults With Chronic Conditions (Guiding Question 1.a-1.c)}

We found 96 systematic reviews that reported on direct patient care engagement strategies among adults. Of these, 66 reviews focused on self-management support (e.g., counseling, teambased care) and 26 focused on shared decision making or enhanced patient-provider communication. Four reviews focused on other direct patient care engagement strategies. (Appendix B and Figure 4).

\section{Self-Management Support}

We identified 66 systematic reviews (included studies ranged from 2 to 350; with 2 to 67 RCTs) evaluating self-management support strategies for adults.

Figure 7 shows the distribution of chronic medical conditions in these reviews. The systematic reviews focused on a range of chronic conditions including diabetes mellitus, cardiovascular disease, cancer or cancer screening, kidney disease, mental health, neurologic disease, respiratory disorders, and multiple chronic conditions. The most commonly targeted conditions were diabetes mellitus $(n=26)$, cardiovascular disease and hypertension $(n=20)$, respiratory disorders $(n=17)$, and mixed chronic conditions $(n=20)$. 
Figure 7. Chronic medical conditions targeted in systematic reviews of different types of interventions for improving patient and family engagement at the direct patient care level among adults $(n=96)$

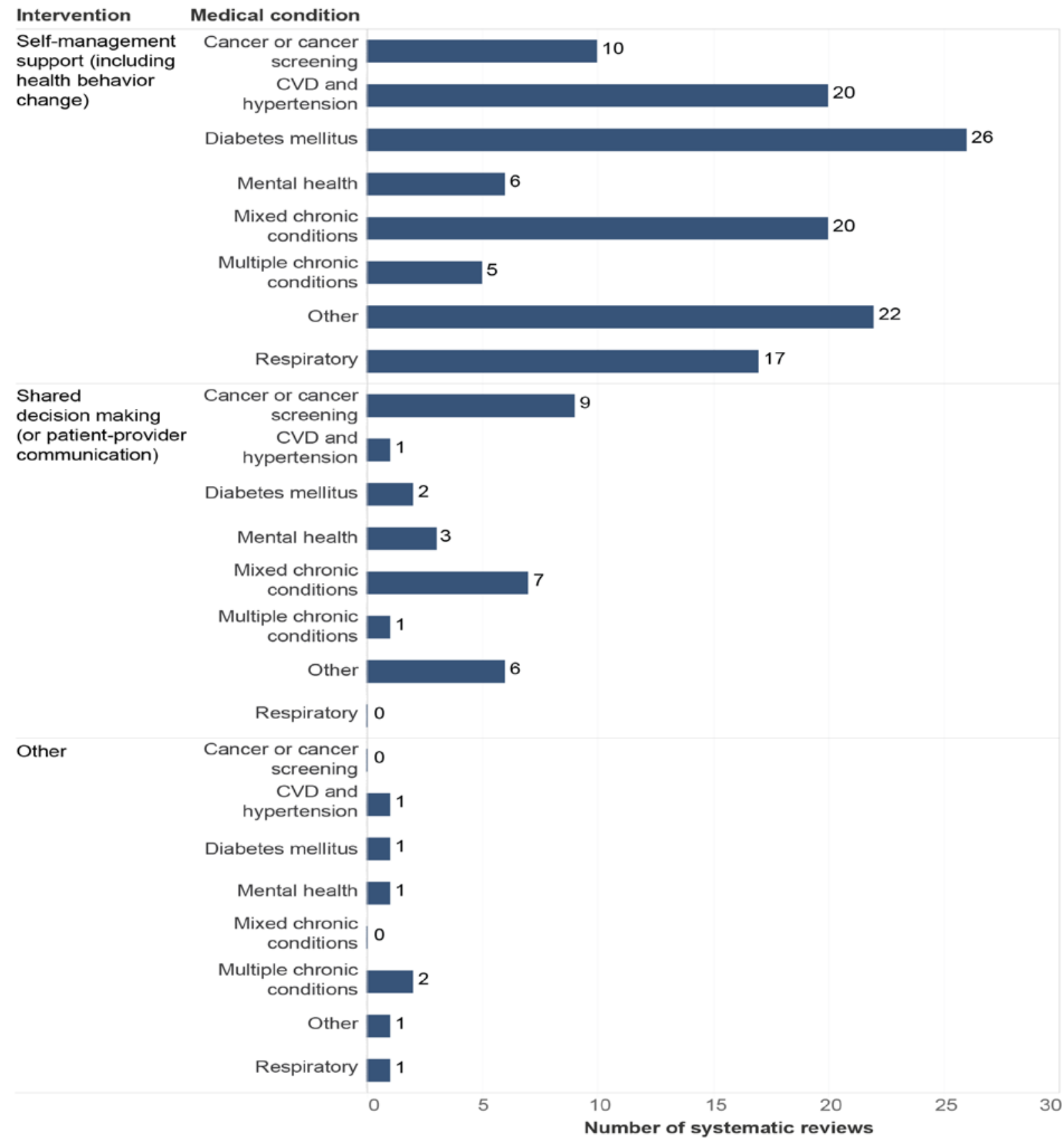

$\mathrm{CVD}=$ cardiovascular disease

Other category included = kidney disease, end of life, epilepsy, HIV, cerebral palsy, musculoskeletal, chronic pain, spina bifida, sickle cell, neurologic diseases, osteoarthritis, rheumatologic diseases

The 66 systematic reviews focused on self-management support tested a wide range of engagement strategies to help patients engage in their healthcare and support them in selfmanagement of their chronic conditions. The strategies and interventions frequently incorporated 
multiple components. These components included the following: (1) education and provision of information on health conditions and treatment options; (2) helping patients achieve behavior change via coaching and motivational interviewing, goal-setting, self-monitoring and symptom management, using action plans, problem-solving, tracking data on status and progress, and feedback provision; (3) facilitating communication with healthcare providers and adherence to treatment and self-care plans via reminders, alerts, logging, remote monitoring, and decision support; and (4) providing psychosocial support including healthcare navigation assistance, connection to social services and peers, counseling, and cognitive behavioral therapy interventions. Multiple delivery methods were used, including individual and group education programs that used face-to-face, telephonic, computer-based, and other online platforms. While some strategies incorporated technology as the sole means for connecting with patients (e.g., text messaging and digital coaching), others included in-person approaches (e.g., nurses doing home visits) or a mix of technology-based and in-person approaches (e.g., initial in-person sessions followed by e-mail and text followup). Technology-based approaches included: computer- and mobile-based devices, with or without internet connections; sensor-based technologies; gaming technology; videoconferencing; remote monitoring; and text messaging. The people involved in delivery of these strategies included physicians, nurses, occupational therapists, social workers, health educators, dieticians, psychologists, physiotherapists and other healthcare professionals, health coaches (often nurses with additional coach training), community healthcare workers, and peers (lay people with similar conditions).

Seven systematic reviews examined use of the engagement modality of the electronic health record's patient portal or secure messaging system for supporting patient self-management. ${ }^{47-53}$

Figure 8 shows the distribution of the outcomes addressed in the systematic reviews of selfmanagement among adults, which included the following outcomes: chronic disease clinical outcomes (e.g., hemoglobin A1c, blood pressure), health care utilization (e.g., re-admissions and emergency department use), adherence to medication or self-management tasks, patient satisfaction or experience, quality of life, decisional support outcome, and mortality. The most frequently reported outcomes were adherence to medication or self-management tasks (77\%), chronic disease clinical outcomes (66\%), quality of life (44\%), and health care utilization (23\%). 
Figure 8. Patient and family engagement outcomes assessed for different types of interventions at the direct patient care level, among adults, as reported in systematic reviews ( $n=96)$ Outcomes

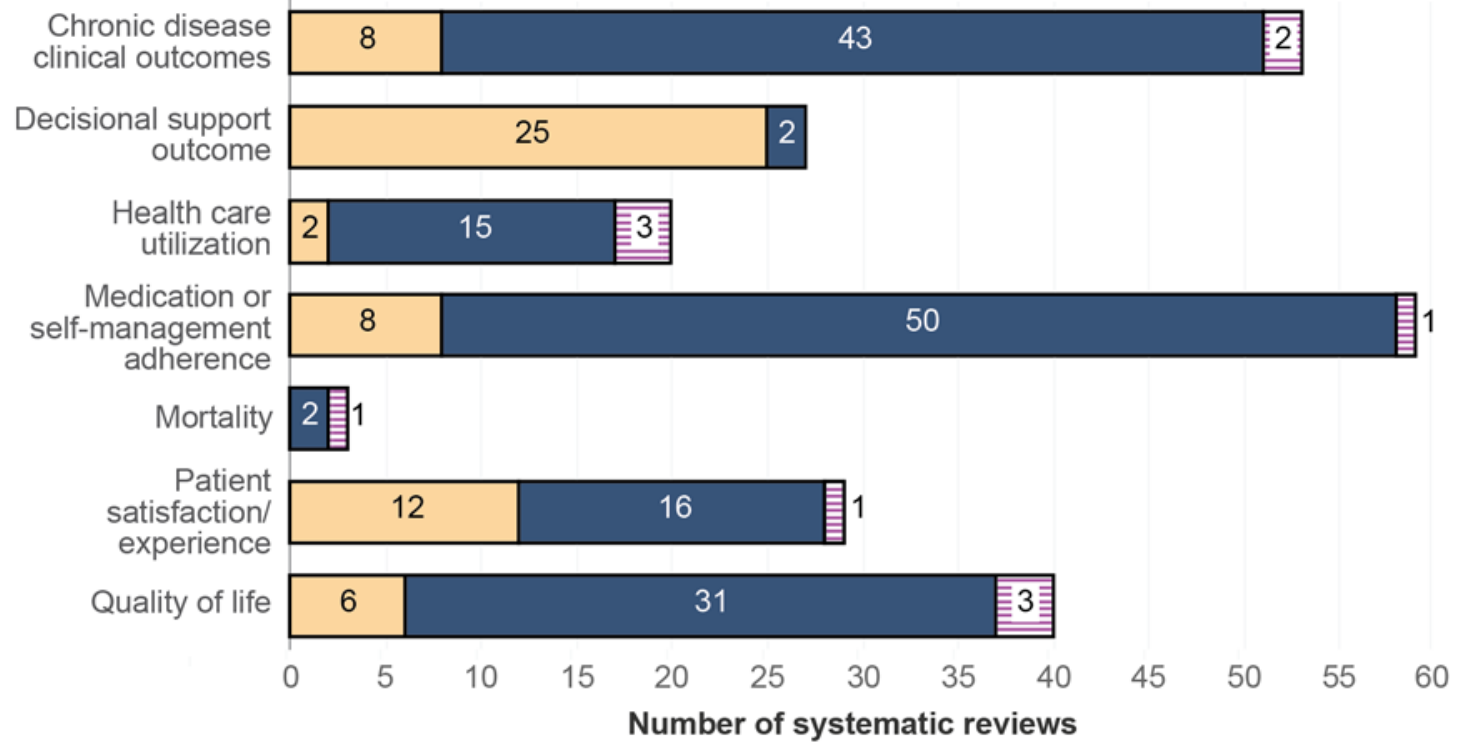

Intervention

$\square$ Shared decision making (or patient-provider communication)

Self-management support (including health behavior change)

Other

Figure 9 shows the findings by strategy. While 40 percent of the systematic reviews showed definite positive effects, 28 percent showed potential benefits, and 28 percent could not make any conclusions about benefit. No reviews reported any harm.

Table 2 reports the findings by strategy and health condition. Reviews of self-management support for diabetes and cardiovascular disease, and reviews of shared decision- making for cancer and cancer screening commonly reported benefits. We found two reviews that focused on transitional care for adults with chronic conditions and both reported benefits.

Thirteen systematic reviews focused on self-management support for diabetes mellitus and reported on diabetes outcomes. Of those, four showed benefits for measures of glycemic control and diabetes knowledge, ${ }^{54-57}$ and one showed cost benefits for educational support interventions. ${ }^{58}$ Benefits were unclear for the impact on quality of life and utilization. Six systematic reviews focused on self-management support interventions among cancer patients, and three showed potential and clear benefits. ${ }^{59-61}$ One of those was a systematic review and meta-analysis of 34 RCTs of web-based self-management support interventions for cancer survivors. The meta-analysis showed positive effects on fatigue, depression, anxiety, and overall quality of life. The communicative functions of the web-based interventions had showed benefits, particularly access to other peers. ${ }^{59}$ Out of three systematic reviews focused on selfmanagement support for cardiovascular disease, one showed no benefits ${ }^{62}$ and two showed mixed findings. ${ }^{63,64}$ Self-management interventions showed promising benefits for improving overall risk factor control among patients with stroke. ${ }^{65}$ For adults with asthma, one review showed low to moderate quality evidence for improvement of asthma-specific quality of life, asthma severity, and lung function tests with chronic disease management programs of at least 3 
months duration with self-management support as a component along with healthcare professional support, care coordination, and/or system level components. ${ }^{66}$

Two large systematic reviews showed benefits to low-income, underserved, and racial and ethnic minority patients from interventions delivered by community health workers, specifically in the area of diabetes control, hypertension, and cancer screening behaviors. ${ }^{54,67}$ Two systematic reviews that only included studies of patients with multiple chronic conditions showed unclear benefits, citing difficulty in operationalizing self-management for multiple chronic conditions and a reduced ability to help these patients. ${ }^{68,69}$

One systematic review reported findings of a pooled analysis including 2,742 patients in which phone text messaging interventions doubled the odds of medication adherence. However, the authors cautioned that more research is needed given short study durations and use of selfreported medication adherence measures. ${ }^{70}$

Two reviews focused on studies involving caregivers. One study, which looked at a broad range of patient- and family-centered self-care interventions for patients with several types of chronic conditions, ${ }^{71}$ included nine RCTs and showed positive impact on reduced rehospitalizations with varied impact on health-related quality of life (HRQOL). The other systematic review focused on studies of self-management support interventions for patients with chronic obstructive pulmonary disease (COPD) and chronic heart failure. The review did not find evidence of additional improvement in patient HRQOL among those studies that involved caregivers compared with the rest of the studies. ${ }^{72}$ Among the eight systematic reviews focused on patient portals as engagement modality, one study focused on a clinical outcome (hemoglobin $\mathrm{A} 1 \mathrm{C}^{53}$ ) and the other studies focused on patient experience, patient knowledge, and patient empowerment. The systematic review by Kuo and colleagues included 11 studies that addressed the role of the patient portal to support diabetes self-management and found that 7 of the 11 studies showed improvement in patients' hemoglobin A1c with the use of secure messaging. ${ }^{53}$ 
Figure 9. Percentage of systematic reviews reporting benefits of different types of direct patient care interventions for improving patient and family engagement, among adults, as reported in systematic reviews $(n=96)^{*}$

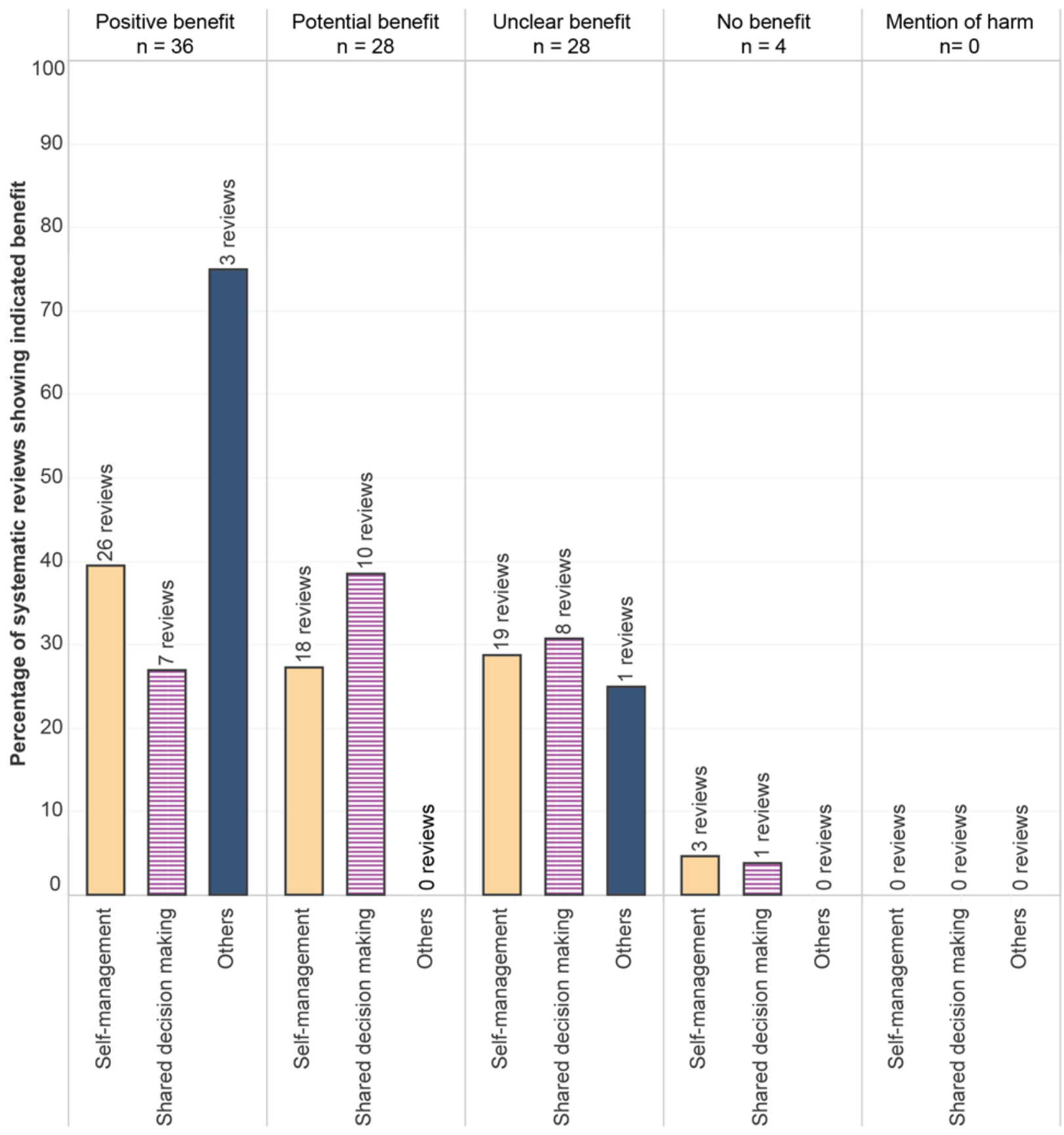

Direct patient care engagement interventions

Self-management (total $n=66$ reviews)

Shared decision making (total $n=26$ reviews)

Others (total $n=4$ reviews)

*Each percentage is calculated based on the total number of reviews on a given type of intervention

Unclear benefit = In five reviews of self-management, the review question was not about evaluating outcomes 
Table 2. Number of systematic reviews reporting benefits among adults, stratified by interventions and conditions

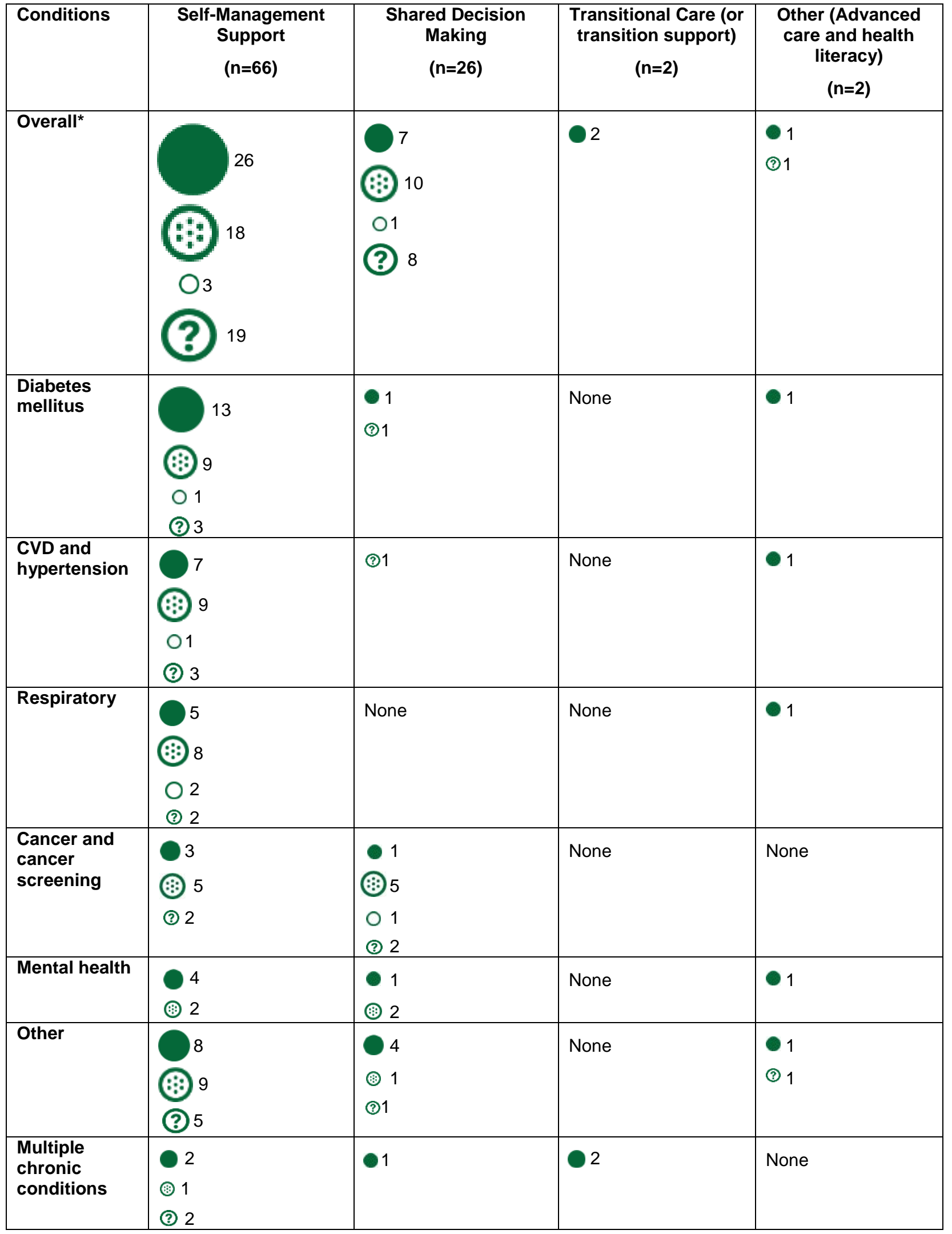




\begin{tabular}{|c|c|c|c|c|}
\hline Conditions & $\begin{array}{c}\text { Self-Management } \\
\text { Support } \\
(n=66)\end{array}$ & $\begin{array}{c}\text { Shared Decision } \\
\text { Making } \\
(n=26)\end{array}$ & $\begin{array}{l}\text { Transitional Care (or } \\
\text { transition support) } \\
\qquad(n=2)\end{array}$ & $\begin{array}{l}\text { Other (Advanced } \\
\text { care and health } \\
\text { literacy) } \\
(n=2)\end{array}$ \\
\hline $\begin{array}{l}\text { Mixed chronic } \\
\text { conditions }\end{array}$ & $\begin{array}{l}3 \\
\text { (ㄱ) } \\
\text { ? } 8\end{array}$ & $\begin{array}{l}2 \\
\text { 우 } 2 \\
\text { (2) } 3\end{array}$ & None & None \\
\hline
\end{tabular}

Positive benefits

(7) Potential benefit

No benefit

(?) Unclear

Circle size corresponds to number of reviews reporting the type of benefit for a specific health condition.

$\mathrm{CVD}=$ cardiovascular disease

* Total exceeds the number for overall because reviews could be included for more than one specific condition as reported in the systematic review.

\section{Shared Decision Making or Patient-Provider Communication}

We identified 26 systematic reviews evaluating shared decision making or patient-provider communication engagement strategies for adults with chronic disease (Appendix B). The systematic reviews that evaluated shared decision making or patient-provider communication included between 4 and 120 studies each (with a range of 1 to 105 RCTs). The shared decision-making reviews focused on a range of chronic conditions, including diabetes mellitus, cardiovascular disease, cancer or cancer screening, kidney disease, mental health, neurologic, respiratory, and multiple chronic conditions.

The systematic reviews most often focused on cancer/cancer screening $(n=9)$, mixed chronic conditions $(n=7)$, mental health conditions $(n=3)$, and diabetes $(n=2)$. For example, the systematic review by Stovell and colleagues identified 11 RCTs that reported on shared decision-making outcomes in patients with psychosis (Figure 7). ${ }^{73}$

Most shared decision-making interventions involved multiple components, including provider trainings and patient education tools, which included technology-enabled delivery modes (e.g., video, web-based tools) and decision supports. The largest systematic review ( $n=105$ studies), a Cochrane review by Stacey and colleagues, broadly assessed decision aids for people facing health treatment or screening decisions. ${ }^{74}$ This review also evaluated whether studies had considered the health literacy of their patient populations, such as adapting tools for lower literacy patients. ${ }^{74}$ Most interventions included guidance and coaching for patients and training for physicians, as well as education for patients. ${ }^{74}$ Two systematic reviews specifically evaluated shared decision-making engagement strategies for advanced care planning. ${ }^{75,76}$ The systematic review by Vermunt and colleagues focused on collaborative goal setting for older patients with chronic diseases ${ }^{75}$ and reported on five interventions included in eight studies, most commonly reporting on tools and team-based discussions. Two systematic reviews examined the role of the electronic health record's patient portal or secure messaging system as an engagement strategy to enhance patient-provider communication. ${ }^{52,77}$ One systematic review focused on patient engagement using an inpatient portal for communication, ${ }^{52}$ and the other review focused on the outpatient clinical setting. ${ }^{77}$ 
Figure 8 shows the frequency of outcomes reported in the studies involving the engagement strategy. Most of these systematic reviews focused on patient-reported outcomes including patient activation, patient knowledge, patient's decisional conflict, and satisfaction with the decision-making process. Few reviews reported on clinical outcomes. The systematic review by Kashaf and colleagues reported on shared decision making among patients with type 2 diabetes and showed a lack of association with shared decision making and outcomes of glycemic control, patient satisfaction, quality of life, medication adherence, or trust in physician. ${ }^{78}$ The systematic review by Vermunt and colleagues included eight studies and showed statistically significant improvements in process measures, including application of goal setting for older patients with chronic diseases in the four intervention studies.

Figure 9 shows the findings on benefits of shared decision-making interventions, as reported by the systematic reviews. Overall, systematic reviews showed an unclear or potential benefit of the interventions on the patient-reported and documentation outcomes, with few studies reporting clinical benefits. Among the two studies focusing on the patient portal to enhance patientprovider communication, the outcomes focused on patient experience, patient knowledge, and patient-provider communication. One study noted, but did not systematically measure, harms, including provider perception that releasing abnormal or sensitive test results to patients could cause confusion or excess worry for patients.

\section{Other Interventions}

We identified four systematic reviews that focused on engagement around care transitions, ${ }^{79,80}$ advanced care planning, ${ }^{81}$ and specific educational programming for patients undergoing peritoneal dialysis. ${ }^{82}$

The two reviews on transitional care support strategies among adults with chronic disease included a range of engagement interventions with patient education and support (e.g., motivational interview/individualized face-to-face coaching, follow up phone calls, and home visits), as well as care coordination components (e.g., coordination of hospital and primary care, medication management). ${ }^{79,80}$ Both reviews showed positive impact on outcomes. The largest, a systematic review and meta-analysis that included 92 studies from Europe, Asia, North America, and Oceania, ${ }^{79}$ focused on patients 65 years of age and older with at least one chronic disease. The review showed that, compared with usual care, interventions to help support these patients after hospitalization reduced mortality and healthcare utilization (number needed to treat to reduce mortality at 3 months was 50; to reduce readmissions at 3 months was 7), without significant differences in quality of life.

A Cochrane review by Coulter and colleagues examined 19 studies (16 RCTs) focused on advanced care planning for adults with long-term or chronic health conditions. The engagement interventions included a variety of tools to facilitate goal setting and action planning, including patient information packets (e.g. digital versatile discs or books), structured consultations with health coaches, and individual and group visits. Fifteen of the 19 studies showed a positive effect in at least one outcome, such as physical health, psychological health and health behaviors, and three studies reported on hospital readmissions, medication usage and cost-effectiveness. ${ }^{81}$

Among the systematic reviews of direct patient care interventions in adults, 8 reviews included patients with multiple chronic conditions (5 with self-management support interventions, one with shared decision-making intervention and 2 with transitional care interventions). Five of the 8 were deemed to have positive benefit (see Table 2). 


\section{Direct Patient Care Strategies - In Reviews Including Children With Chronic Conditions (Guiding Question 1.a-1.c)}

We identified 14 systematic reviews evaluating direct patient care engagement strategies for children and adolescents with chronic disease (Figure 4). One review focused on adolescents alone (12 to 18 years of age); ${ }^{83}$ six on children and adolescents ( 0 to 18 years of age); ${ }^{84-89}$ one on adolescents and young adults (11 to 25 years of age) $;{ }^{90}$ and, six on children, adolescents, and young adults (0 to 28 years of age). ${ }^{91-96}$ The 14 reviews included studies with a range of 0 to 93 studies (included RCTs ranged from 0 to 93).

Figure 10 shows the chronic health conditions reported in the 14 systematic reviews that reported on direct patient care engagement strategies in children and adolescents. The most frequently examined conditions were diabetes mellitus (9 reviews) ${ }^{83,84,86,91-96}$ and asthma (8 reviews), ${ }^{84,86,91-96}$ followed by cystic fibrosis (5 reviews), ${ }^{83,84,91-93}$ cancer (5 reviews), ${ }^{84,86,93-95}$ and blood disorders (4 reviews). ${ }^{84,86,89} 93$ Five systematic reviews included studies with family caregivers (i.e., parents/guardians), ${ }^{84,86,87,89,96}$ including one in which caregivers were the primary population of interest. ${ }^{84}$ No systematic review in this group specifically intended to examine a vulnerable patient population. Of the 14 systematic reviews, three examined strategies primarily used in the home, ${ }^{84,91,94}$ two focused on strategies primarily used in the clinic setting, ${ }^{87,89}$ three reported on strategies primarily used in the inpatient setting, ${ }^{83,86,90}$ and four focused on strategies used in multiple settings. ${ }^{92,93,95,96}$ Three reviews included school and/or camp settings. ${ }^{92,93,96}$ 
Figure 10. Chronic medical conditions targeted in systematic reviews of different types of interventions for improving patient and family engagement at the direct patient care level, among children and adolescents $(n=14)$

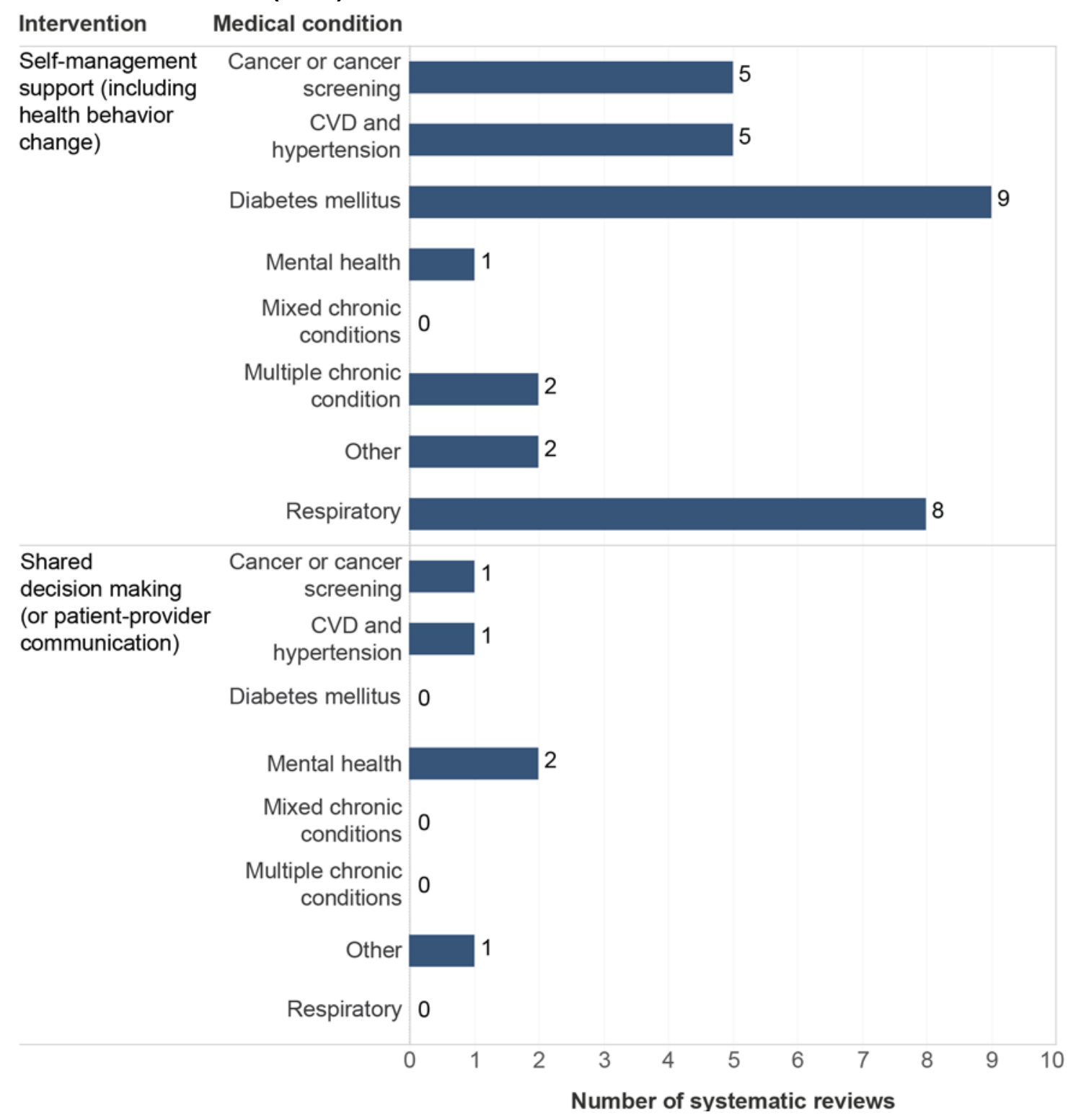

CVD = cardiovascular disease

In the 14 systematic reviews of direct patient care engagement strategies in children and adolescents, the only engagement strategies evaluated were self-management support (10 reviews) and shared decision making (4 reviews) (Figure 5). Three reviews evaluated selfmanagement engagement strategies in the context of transitions of care, including team-based

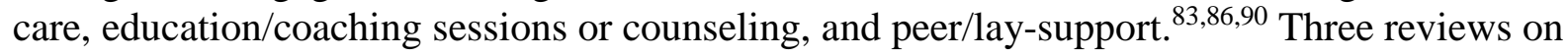
direct patient care engagement strategies focused on technology (i.e., mobile applications, webbased care, and/or video games) and four reviews described studies where at least one component of education/coaching sessions was delivered in combination with technology. For example, a systematic review by Charlier and colleagues reviewed the effectiveness of healthrelated video games on the self-management skills of children, adolescents, and young adults. ${ }^{95}$ 
Among the four systematic reviews in children and adolescents that evaluated shared decision-making strategies, two reported on interventions with education/coaching sessions and decision aids. ${ }^{87,89}$ The systematic review by Cheng and colleagues examined the use of shared decision making in children and adolescents with mental health disorders. Most of the RCTs included in the review showed that parents using shared decision making with providers had lower decisional conflict, and more engagement with treatment, and more of the parents' priorities were addressed. ${ }^{87}$ Wyatt and colleagues also demonstrated a significant reduction in decisional conflict in a meta-analysis of nine studies that contained a heterogenous population of children with and without chronic disease. ${ }^{89}$

The 14 systematic reviews of direct patient care engagement strategies in children and adolescents reported on the following engagement outcomes: quality of life (8 reviews), medication or self-management adherence measures (7 reviews), and chronic disease clinical outcomes (6 reviews) (Figure 11). Two reviews reported healthcare utilization, decisional support, and patient experience/satisfaction measures. In the largest review for this population ( $\mathrm{n}=93$ studies), Knafl and colleagues described the nature of family engagement interventions for children with chronic disease. ${ }^{84}$ This review reported that engagement strategies promoting family function (i.e., problem solving, communication skills, cohesion) improved measures of child well-being, condition control, and adherence measures. In another example, the systematic review by Hamline and colleagues evaluated hospital to home interventions in 31 studies of children with chronic disease. ${ }^{86}$ In this review, family engagement interventions, along with care coordination, were associated with a more than 50 percent reduction in hospital readmissions and a 25 percent reduction in emergency room visits following inpatient discharge. The review found that parent education by the engagement strategy of "teach backs" and the use of contingency plans were the most consistently effective in reducing post-discharge utilization.

Figure 11. Patient and family engagement outcomes assessed for different types of direct patient care interventions, among children and adolescents, as reported in systematic reviews ( $n=14)$ Outcomes

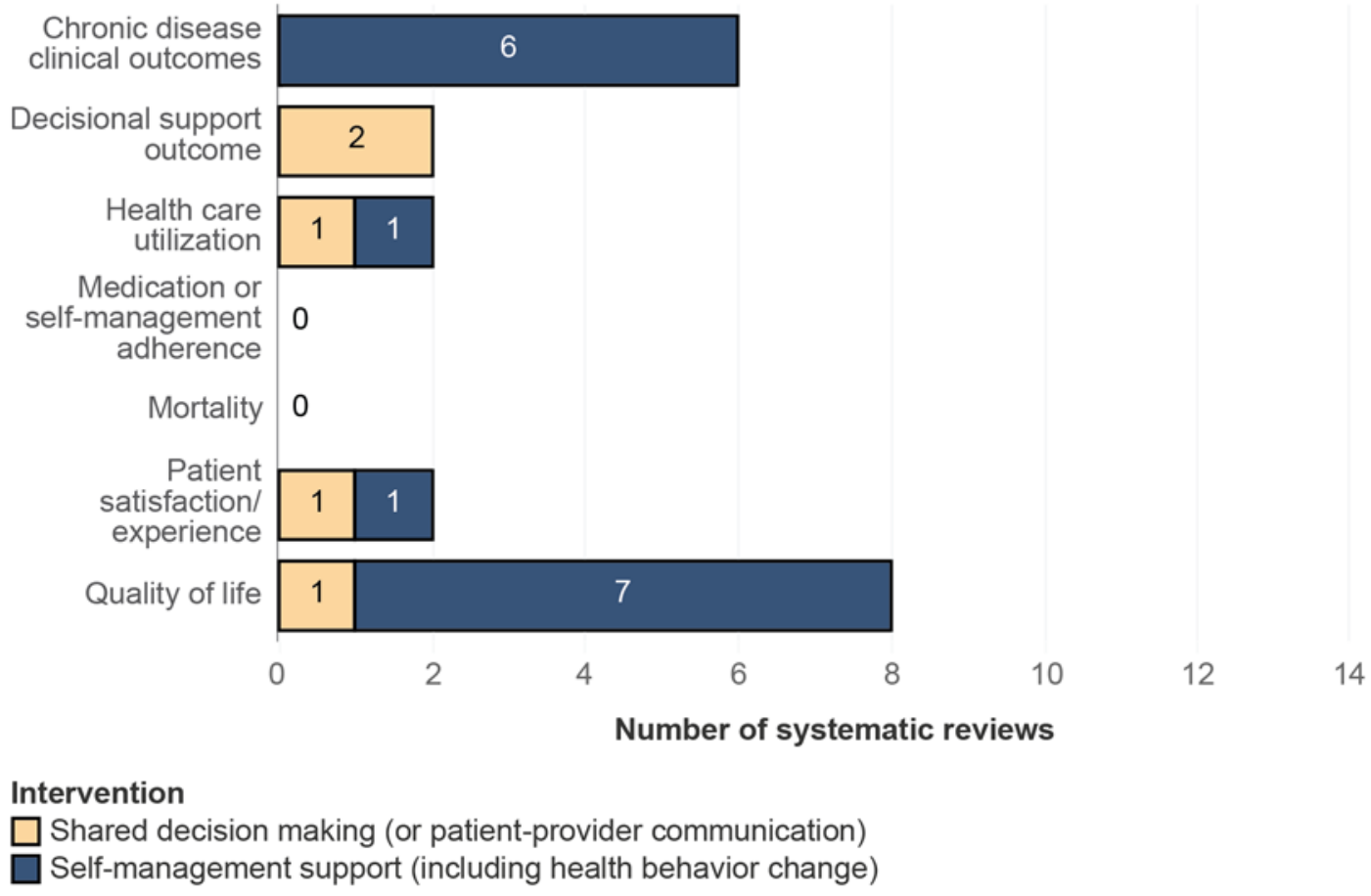


Overall, the 14 systematic reviews of direct patient care engagement strategies in children and adolescents showed positive effects in 3 reviews or potential benefit in 5 reviews. Three reviews showed unclear benefit, ${ }^{83,94,96}$ one described no benefit, ${ }^{91}$ and two did not report any findings. ${ }^{85,88}$ No reviews reported harms associated with patient and family engagement strategies (Figure 12).

Figure 12. Percentage of systematic reviews reporting benefits of different types of direct patient care interventions for improving patient and family engagement, among children and adolescents, as reported in systematic reviews $(n=14)^{*}$

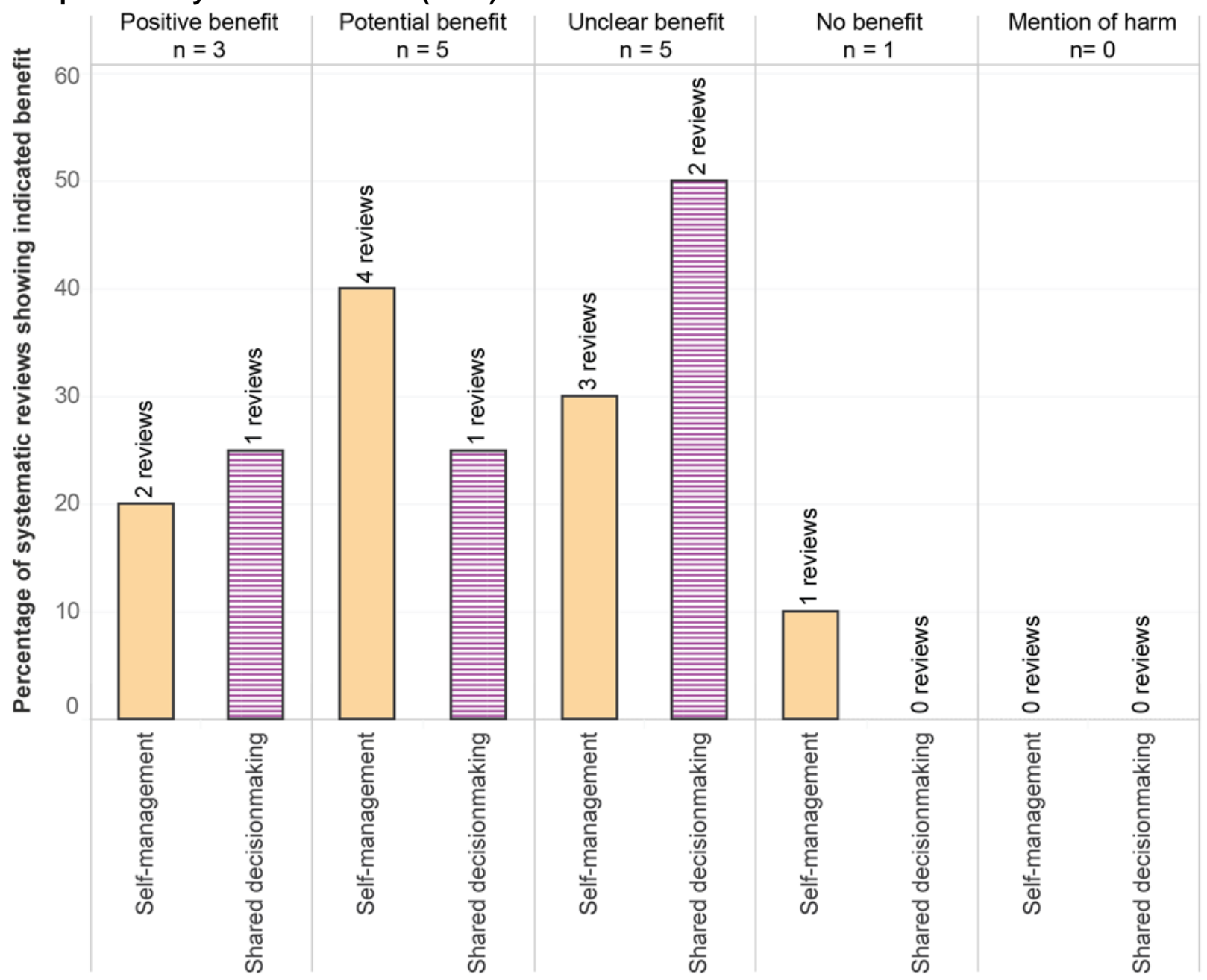

Direct patient care engagement interventions

Self-management (total $n=10$ reviews)

Shared decision making (total $n=4$ reviews)

*Each percentage is calculated based on the total number of reviews on a given type of intervention

Unclear benefit = In one review of shared decision making, the review question was not about evaluating outcomes 
Table 3 depicts the findings from reviews for specific chronic health condition by type of engagement strategy in children and adolescents. Reviews that focused on studies of patients with diabetes, cardiovascular disease, and respiratory diseases commonly reported benefits with self-management support interventions. Shared decision making interventions were reported to be beneficial among patients with mental health conditions. 
Table 3. Number of systematic reviews reporting benefits among children and adolescents, stratified by interventions and conditions

\begin{tabular}{|c|c|c|c|c|}
\hline Conditions & $\begin{array}{l}\text { Self-Management } \\
\text { Support } \\
(n=10)\end{array}$ & $\begin{array}{l}\text { Shared Decision } \\
\text { Making } \\
(n=4)\end{array}$ & $\begin{array}{l}\text { Transitional Care (or } \\
\text { transition support) } \\
\qquad(n=0)\end{array}$ & $\begin{array}{l}\text { Other (Advanced } \\
\text { care and health } \\
\text { literacy) } \\
(\mathrm{n}=0)\end{array}$ \\
\hline Overall* & $\begin{array}{l}02 \\
: 84 \\
01 \\
\text { ? } 3\end{array}$ & $\begin{array}{l}1 \\
\text { (:) } 1 \\
\text { (2) } 2\end{array}$ & None & None \\
\hline $\begin{array}{l}\text { Diabetes } \\
\text { mellitus }\end{array}$ & $\begin{array}{l}2 \\
\text { :P } 3 \\
\text { ○ } 1 \\
\text { ?? } 3\end{array}$ & None & None & None \\
\hline $\begin{array}{l}\text { CVD and } \\
\text { hypertension }\end{array}$ & 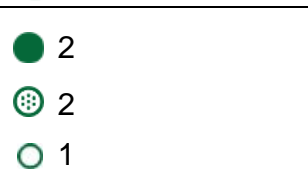 & (3) 1 & None & None \\
\hline Respiratory & $\begin{array}{l}\text { O } 2 \\
\text { () } 3 \\
\text { ○ } 1 \\
\text { (3) } 2\end{array}$ & None & None & None \\
\hline $\begin{array}{l}\text { Cancer and } \\
\text { cancer } \\
\text { screening }\end{array}$ & $\begin{array}{l}1 \\
\text { 유 } 3 \\
\text { (3) } 1\end{array}$ & (3) 1 & None & None \\
\hline Mental health & - 1 & $\begin{array}{l}-1 \\
\text { - } 1\end{array}$ & None & None \\
\hline Other & $\begin{array}{l}\text { - } 1 \\
\text { (3) } 1\end{array}$ & (3) 1 & None & None \\
\hline $\begin{array}{l}\text { Multiple } \\
\text { chronic } \\
\text { conditions }\end{array}$ & $\begin{array}{l}0 \\
0 \\
0\end{array}$ & None & None & None \\
\hline $\begin{array}{l}\text { Mixed chronic } \\
\text { conditions }\end{array}$ & None & None & None & None \\
\hline
\end{tabular}

Positive benefits

(7) Potential benefit

No benefit

(?) Unclear

Circle size corresponds to number of reviews reporting the type of benefit for a specific health condition.

CVD = cardiovascular disease

*Total exceeds the number for overall because reviews could be included for more than one specific condition as reported in the systematic review. 


\section{Direct Patient Care Strategies - In Reviews Including Both Adults and Children With Chronic Conditions (Guiding Question 1.a-1.c)}

We identified 16 systematic reviews evaluating direct patient care engagement strategies that included children, adolescents, and adults with chronic disease. Overall, this increased the total number of reviews that included studies in pediatric populations to 30. However, given distinctions in eligibility criteria, the 16 systematic reviews presented here were analyzed separately as a "mixed" population of children, adolescents, and adults. Similar to the reviews with only children and adolescents, the most commonly studied conditions were asthma and diabetes mellitus, and the most frequently studied engagement strategies were self-management support and shared decision making. Yet, a smaller percentage of reviews (25\%) included family or caregivers in their population of interest compared with those including children and adolescents alone (35.7\%).

The 16 systematic reviews that reported on direct patient care engagement strategies in children, adolescents, and adults focused on a total of ten chronic diseases that spanned all age groups (Figure 13). Asthma (6 reviews) ${ }^{97-102}$ and diabetes (3 reviews) ${ }^{103-105}$ were the most frequently studied. Four reviews included family members or caregivers, ${ }^{101,105-107}$ but only the systematic review by Chi and colleagues identified caregivers as their target population. ${ }^{107}$ The review by Chi and colleagues was also the only systematic review in this group to specifically mention a vulnerable patient population in their results, with 23 percent of the studies focused on patients living in rural settings. ${ }^{107}$ In addition, two systematic reviews included one study each that centered on vulnerable populations (rural patients or incarcerated patients). ${ }^{103,108}$

Of the 16 reviews, engagement strategies primarily involved self-management support (13 reviews) and shared decision making (4 reviews). ${ }^{101,102,109,110}$ One review examined health literacy, ${ }^{111}$ and one specifically mentioned advanced care planning. ${ }^{110}$ Four reviews cited multiple engagement strategies. ${ }^{102,104,110,111}$ Technology was the most frequently cited intervention modality (11 reviews). Nurses or case managers ${ }^{104,105}$ and coaching/educational sessions ${ }^{101,106}$ were examined in two reviews, each. Shared decision-making reviews primarily looked at coaching/educational sessions, although Winston and colleagues examined the use of video-based decision aids in a review of 488 studies. ${ }^{110}$ However, most of those studies focused on cancer or cancer screenings in adults, with only 9.5 percent of studies performed in a pediatric population. 
Figure 13. Chronic medical conditions targeted in systematic reviews of different types of interventions for improving patient and family engagement at the direct patient care level, among reviews of studies of adults and children combined $(n=16)$

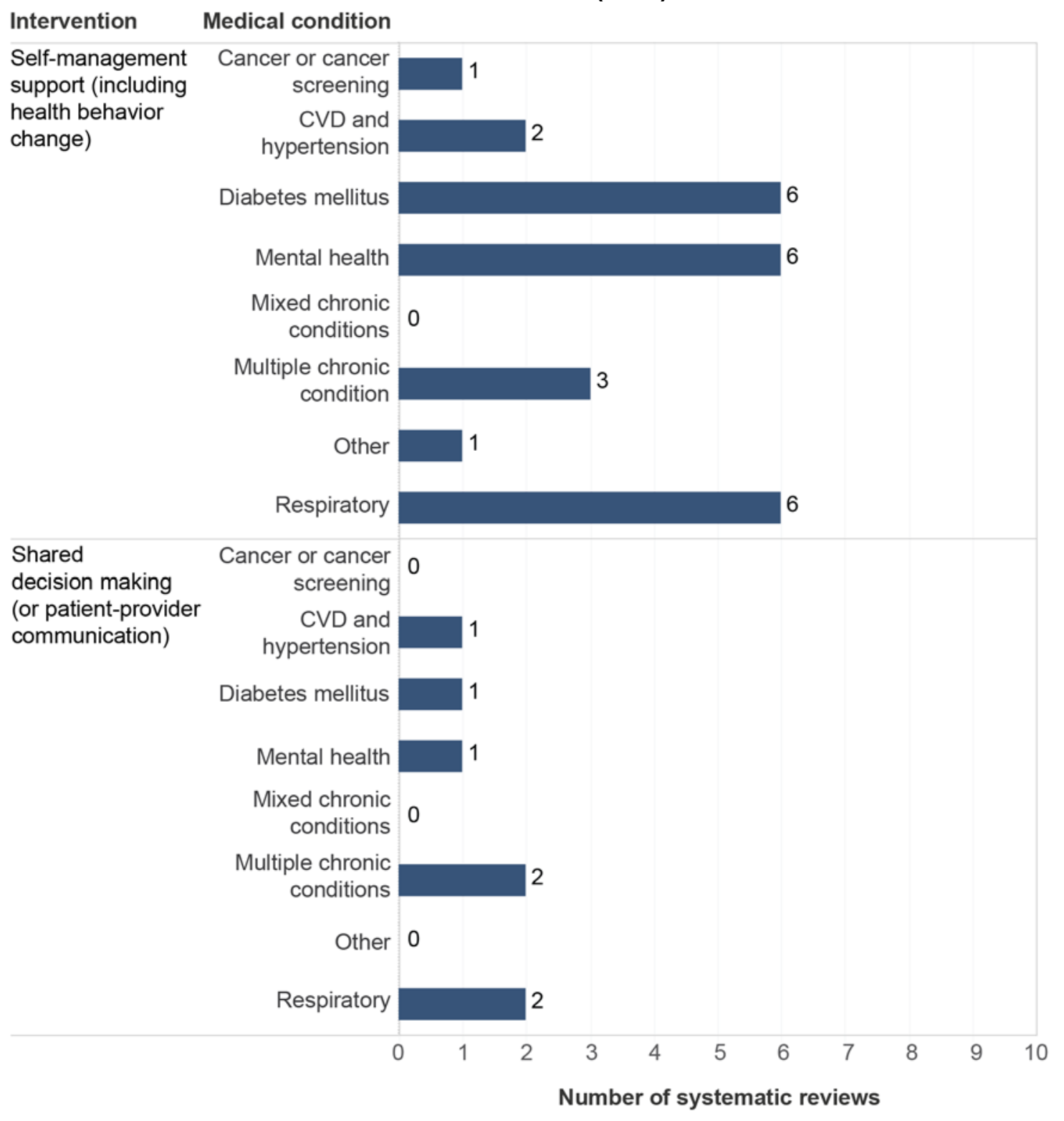

CVD $=$ cardiovascular disease

The 16 systematic reviews of direct patient care engagement strategies in children and adolescents reported on the following engagement outcomes: medication or self-management adherence measures (13 reviews), chronic disease clinical outcomes (11 reviews), quality of life measures (7 reviews), patient satisfaction or experience (5 reviews), decisional support (2 reviews), and healthcare utilization (2 reviews) (Figure 14). As an example of a common engagement strategy for self-management support using technology, a systematic review by Kew and colleagues ${ }^{100}$ examined the use of home telemonitoring on asthma symptoms for pediatric and adult patients between clinic visits with feedback by clinicians. The authors looked at 18 RCTs with multiple outcome measures for adherence, clinical outcomes, utilization, and quality of life. Only a small effect size was seen for improvement in quality of life. At least one RCT 
within the review examined pediatric patients and did not find any difference in control of their asthma between home telemonitoring and controls.

Figure 14. Patient and family engagement outcomes assessed for different types of direct patient care interventions, among adults and children, as reported in systematic reviews $(n=16)$

Outcomes

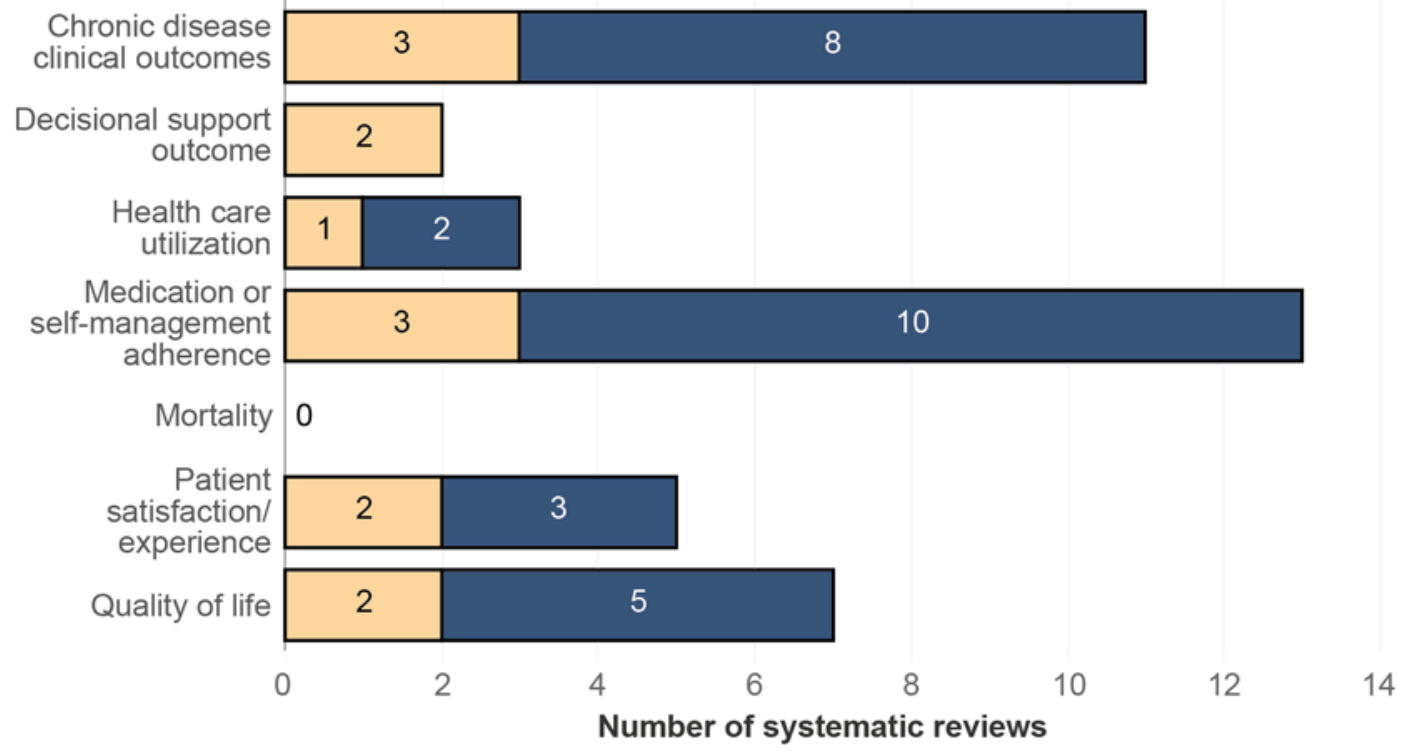

Intervention

Shared decision making (or patient-provider communication)

Self-management support (including health behavior change)

Overall, the 16 systematic reviews of direct patient care engagement strategies in children, adolescents, and adults showed positive effect in 9 reviews or potential benefit in 2 reviews. Five reviews showed unclear benefit. ${ }^{97,100,101,108,112}$ No reviews reported harms associated with patient and family engagement strategies (Figure 15). 
Figure 15. Percentage of systematic reviews reporting benefits of different types of direct care patient interventions for improving patient and family engagement, among adults and children, as reported in systematic reviews $(n=16)^{*}$

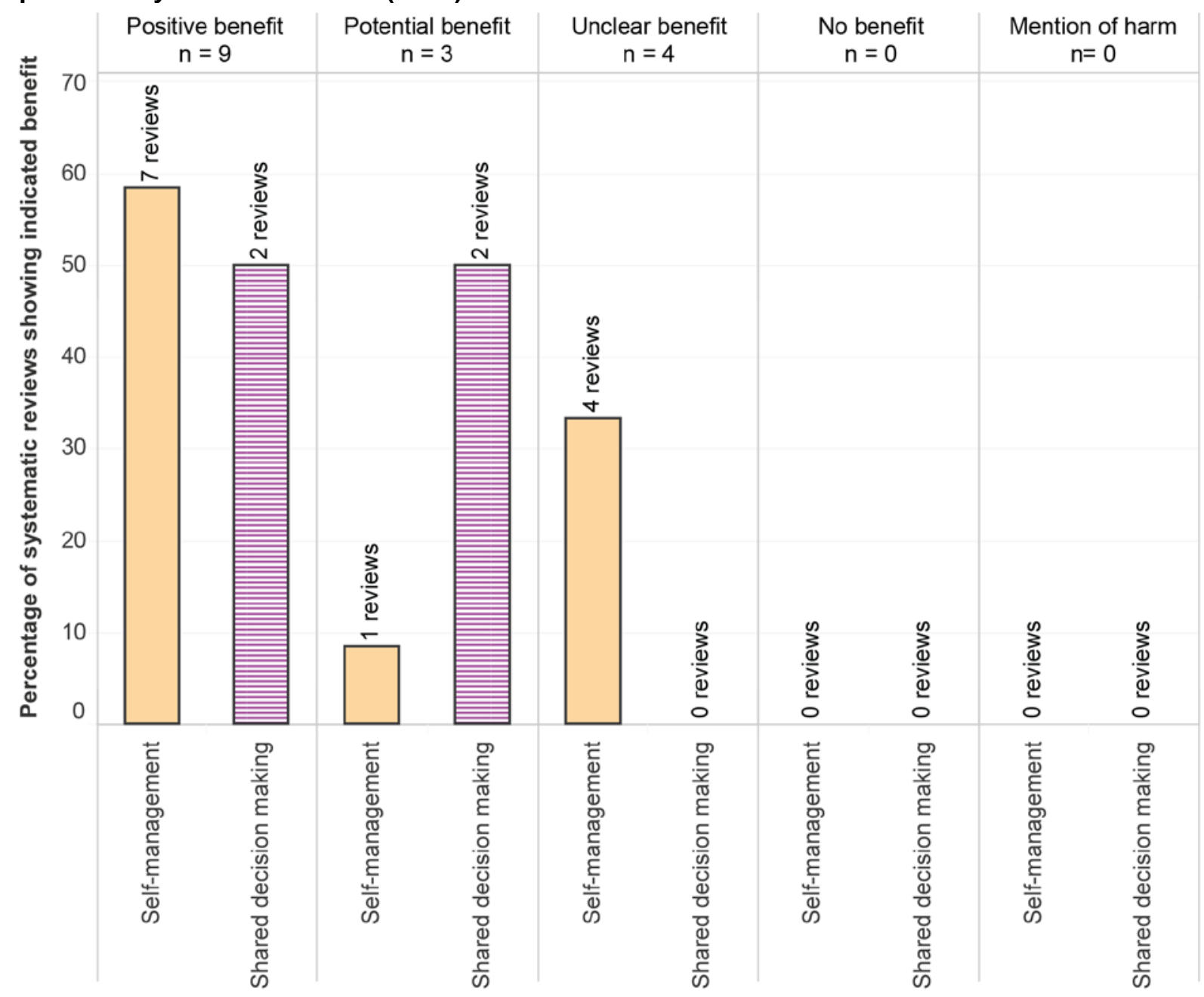

Direct patient care engagement interventions

Self-management (total $n=12$ reviews)

Shared decision making (total $n=4$ reviews)

*Each percentage is calculated based on the total number of reviews on a given type of intervention

Table 4 depicts the findings from reviews for specific chronic health condition by type of engagement strategy in mixed population (children and adults). Reviews that focused on studies of patients with diabetes, cardiovascular disease and respiratory diseases commonly reported benefits with self-management support interventions. 
Table 4. Number of systematic reviews reporting benefits among adults and children stratified by interventions and conditions

\begin{tabular}{|c|c|c|c|c|}
\hline Conditions & $\begin{array}{l}\text { Self-Management } \\
\text { Support } \\
(n=12)\end{array}$ & $\begin{array}{l}\text { Shared Decision } \\
\text { Making } \\
(n=4)\end{array}$ & $\begin{array}{l}\text { Transitional Care (or } \\
\text { transition support) } \\
\qquad(n=0)\end{array}$ & $\begin{array}{l}\text { Other (Advanced } \\
\text { care and health } \\
\text { literacy) } \\
(n=0)\end{array}$ \\
\hline Overall* & $\begin{array}{l}7 \\
\text { (3) } 1 \\
\text { (2) } 4\end{array}$ & $\begin{array}{l}2 \\
\text { (우 } 2\end{array}$ & None & None \\
\hline Diabetes & $\begin{array}{l}4 \\
\text { (i) } 1 \\
\text { (3) } 1\end{array}$ & - 1 & None & None \\
\hline $\begin{array}{l}\text { CVD and } \\
\text { Hypertension }\end{array}$ & $\begin{array}{l}1 \\
\text { (3) } 1\end{array}$ & - 1 & None & None \\
\hline Respiratory & $\begin{array}{l}3 \\
\text { (3) } 1 \\
\text { (2) } 2\end{array}$ & $\begin{array}{l}\text {. } 1 \\
\text { (장 } 1\end{array}$ & None & None \\
\hline $\begin{array}{l}\text { Cancer and } \\
\text { cancer } \\
\text { screening }\end{array}$ & - 1 & None & None & None \\
\hline Mental health & $\begin{array}{l}3 \\
\text { (3) } \\
\text { (2) }\end{array}$ & - 1 & None & None \\
\hline Other & 1 & None & None & None \\
\hline $\begin{array}{l}\text { Multiple } \\
\text { chronic } \\
\text { conditions }\end{array}$ & $\begin{array}{l}2 \\
\text { (31 }\end{array}$ & $\begin{array}{l}\cdot 1 \\
\text { (아 } 1\end{array}$ & None & None \\
\hline $\begin{array}{l}\text { Mixed chronic } \\
\text { conditions }\end{array}$ & None & None & None & None \\
\hline
\end{tabular}

Positive benefits

(1) Potential benefit

No benefit

(?) Unclear

Circle size corresponds to number of reviews reporting the type of benefit for a specific health condition.

CVD $=$ cardiovascular disease

* Total exceeds the number for overall because reviews could be included for more than one specific condition as reported in the systematic review.

\section{Implementation of Direct Patient Care Strategies (Guiding Question 1.d-1.f)}

Three of the systematic reviews addressed implementation of direct patient care engagement strategies in adults. ${ }^{50,112,113}$ The systematic review by Scholl and colleagues specifically addressed organizational- and system-level characteristics that influence implementation of shared decision-making strategies. ${ }^{113}$ Only one study in the review had a comparison group, 
which was at the pre-intervention phase. The review described six categories of organizational characteristics that promote implementation of engagement strategies: organizational leadership, culture, teamwork, resources, priorities, and workflow.

Of the nine systematic reviews examining the patient portal as a modality for patient and family engagement, two highlighted the implementation process for the portal. ${ }^{50,112}$ For example, the systematic review by Dendere and colleagues focused on the inpatient portal and identified 22 studies addressing the design and usability testing of the portal and 36 articles addressing process outcomes, such as portal adoption. They reported fewer studies that highlighted the organizational factors (e.g., leadership) that led to portal implementation. ${ }^{50}$ The systematic review by Kruse and colleagues also highlighted the costs associated with implementation of patient portals for patient engagement and communication. ${ }^{112}$

In addition to the included systematic reviews, we identified three systematic reviews that reported on implementation outcomes. ${ }^{114-116}$ Systematic reviews by Anderson and Legare reported on communication (Anderson on end of life communication ${ }^{115}$, and Legare on shared decision making $\left.{ }^{116}\right)$. They reported barriers including the payment model, which is linked to the amount of time a provider spends with the patients, as well as six categories of "organizational leadership, culture, teamwork, resources, priorities, and workflows." 113 The systematic review by McBain and colleagues described the effect of self-management support interventions on healthcare utilization in 17 articles among patients with COPD, hypertension, and chronic heart failure, and showed increased outpatient and home visit utilization, as well as a possible decrease in hospital admissions. ${ }^{114}$

\section{Health System and Organization Strategies - In Patients With Chronic Conditions (Guiding Question 1.a-1.c)}

Definition: Health system level strategy is defined as a strategy that engages patients and families in organizational activities and/or decision-making and informs the delivery of care within a health care system, beyond the individual patient's care (e.g., participation in an advisory committee or board membership).

We identified five systematic reviews ${ }^{117-121}$ and three additional original articles ${ }^{97,122,123}$ that reported on patient and caregiver engagement at the health system level. The articles described specific strategies, facilitators and barriers to implementation, ${ }^{119,120}$ and the impact of patient engagement on health care delivery and outcomes. ${ }^{117-121}$

The health system patient and caregiver engagement strategies reported in the five systematic reviews addressed a variety of chronic conditions, most commonly mental health, ${ }^{117,119,121}$ cancer, ${ }^{119,121}$ diabetes mellitus, ${ }^{117,119}$ and neurologic conditions, ${ }^{119,121}$ as well as priority setting and improvement of care processes that were not disease-specific. ${ }^{117,121}$ Patient and caregiver engagement strategies most often included patients and family members, but also included representatives of community-based organizations ${ }^{117}$ and other community members, ${ }^{121}$ "consumers,"118 and "well members of the public." "120 Most systematic reviews examined engagement within a variety of health care settings, ${ }^{117-119,121}$ such as hospitals or outpatient settings, although these settings were often not well described. One systematic review examined patient engagement in health care delivery in ambulatory, emergency department, or inpatient settings in hospitals. ${ }^{120}$

Patient and caregiver engagement strategies included patient and community advisory councils, ${ }^{117,119-121}$ service as members of committees, participation in meetings or on project 
teams, ${ }^{119-121}$ forums and workshops to provide patients with skills to support engagement, ${ }^{118,119}$ patients serving as instructors of trainees, ${ }^{118}$ and patients providing consultative input (e.g., through surveys, focus groups, or interviews). ${ }^{119,120}$

These systematic reviews provide different lenses through which system level patient and family engagement can be viewed. The review by Sharma and colleagues on the impact of patient advisors ${ }^{121}$ allowed for a broad range of study designs, including qualitative research and case studies. However, the review also required an assessment of impact for one of three primary outcomes (clinical care, patient safety, or patient satisfaction) or a secondary outcome (including the impact on clinic processes, priorities, physical space, or staff or patients as advisors). This systematic review did not identify any "rigorous, prospective RCTs that assessed our primary outcomes of patient clinical care, patient safety, or patient satisfaction," but found one cluster RCT in which "patient advisors helped clinics set priorities that were better aligned with the Patient Centered Medical Home and chronic care models." ${ }^{121}$ Most of the included studies were case reports and observational studies which primarily described the development of material for patient education or self-management (17 studies), physical space design (15 studies), trainings for staff or trainees developed with patient engagement (10 studies), workflow or service changes (7 studies), and changes in staff awareness of patient perspectives (5 studies). Similarly, in the systematic review by Bombard and colleagues ${ }^{119}$ which broadly examined patient engagement strategies, the most commonly reported outcomes of patient engagement were care processes or service delivery (35 studies), policy or planning documents (15 studies), and educational materials or tool development (11 studies).

In contrast to the approaches of Sharma and Bombard, the systematic review by Oldfield and colleagues on patient, family, and community advisory councils ${ }^{117}$ only included studies with a comparator group, and had no limitation on the outcomes evaluated. Studies in this systematic review were categorized by the intent of the patient engagement, whether it was to inform direct care (3 studies), organizational design (3 studies), policymaking (5 studies), or health-related research (5 studies). Oldfield and colleagues similarly identified a "paucity of RCTs or highquality observational studies.” However, they developed three "guiding principles” based on six studies that compared different aspects of patient engagement: (1) in-person and "collective" engagement is more effective than surveys, phone calls, or individual meetings; (2) patients with leadership roles in the community are more effective participants; and (3) organizational or policy recommendations made with advisory council input required a longer time and greater resources to achieve results (based on 2 studies) and might be of lower quality (based on 1 study).

\section{Implementation of Health System and Organization Strategies - In Patients With Chronic Conditions (Guiding Question 1.d-1.f)}

Two of the systematic reviews described implementation measures related to health system or organization-level patient and family engagement strategies. ${ }^{119,120}$ Both of these systematic reviews included multiple patient and family engagement strategies, although one was limited to care delivered in hospital-based settings. ${ }^{120}$

Both reviews reported the importance of defining clear roles for patients and training of patients and providers or staff. ${ }^{119,120}$ These systematic reviews also identified provider skepticism or "negative beliefs and attitudes about patient roles and input" as barriers to successful patient engagement. Similarly, staff, provider, and/or practice awareness, interest, and engagement were significant barriers in a cluster RCT of feedback to primary care providers from patients with 
significant physical disability or severe mental illness, ${ }^{122}$ and in a national collaborative study on patient and family collaboration in intensive care units. ${ }^{97}$

Based on the barriers and facilitators in their systematic review, ${ }^{120}$ Bombard and colleagues identified distinct techniques to improve patient engagement during different components of the process, including design, patient recruitment, patient involvement, creating a receptive context, and leadership actions. These techniques included ensuring diversity and representation, providing incentives to participation, using flexibility in approaches to patient and family engagement, enacting strategies to "level the playing field and [support] staff in their efforts to be partners," and demonstrating executive or institutional commitment. ${ }^{120}$

Neither the identified reviews nor the original studies addressed fidelity in implementation or strategies to specifically support sustainment.

\section{Community/Policy Engagement Strategies}

Definition: Community or policy strategy is defined as a strategy that engages patients, consumers, or citizens in policymaking or that engages communities in health care policies (e.g., a hospital-neighborhood partnership to address community's concerns, disease-specific group of patients lobbying for more funding to study a rare disease, or a community group advocating for sugar-sweetened beverage or tobacco-related local policy changes to improve neighborhood public health).

We did not find any systematic reviews on community or policy level engagement strategies, but we identified one original article that described community and policy level engagement. ${ }^{124}$ King and colleagues described a mixed methods evaluation of a longitudinal cohort study to evaluate efforts to strengthen engagement between the Navajo National Community Health Representatives Program and the Navajo Area Indian Health Services that serve the Navajo Nation in three U.S. states. The Community Outreach and Patient Empowerment Program, in partnership with the Navajo Nation, developed a community-health system engagement intervention to improve communication and care coordination between the clinics and the community through its community health workers, with a focus on people living with uncontrolled diabetes. The program included two community advisory councils. Intermediate outcomes suggested that community health representatives perceived greater engagement with clinics through access to the client health information via the electronic health record, care coordination efforts, and direct referrals/communication with providers. ${ }^{124}$ We did not identify any articles that described implementation of community and policy engagement strategies.

\section{Results From the Gray Literature: Innovative Patient and Family Engagement Strategies Among Patients With Chronic Conditions}

Appendix C summarizes the findings from the gray literature search, which we designed to address gaps in our review of published literature, with a particular focus on health care organization/system and community level engagement. Most of the gray literature we found focused on health system strategies that included toolkits for health care systems to facilitate the implementation of patient and family engagement strategies, including patient and family advisory councils (e.g., "Strategically Advancing Patient and Family Advisory Councils in New York State Hospitals”). In addition, we identified several tools for health care providers and 
systems to use to engage patients and families in conversations with their providers (e.g., "Supporting the Supporters: What Family Caregivers Need to Care for a Loved One with Cancer" from the Institute for Healthcare Improvement).

\section{Guiding Question 2: What gaps exist in the current research?}

In this section, we report the gaps in current research by highlighting which engagement strategies had little or no available evidence but had been identified as promising by our experts (Figure 1). In the discussion section, we will comment on the engagement strategies for which additional research is needed, or for which a new systematic review would help to synthesize current knowledge.

Figure 16 provides an evidence map that highlights the overall findings of this systematic review of patient and family engagement strategies. Figure 17 shows map of the evidence on direct patient and family engagement strategies by reported level of benefit for different types of outcomes.

We identified several major gaps. First, relatively few reviews addressed system and community/policy level strategies (5 out of 131 reviews) and even when we augmented the search to identify original studies, only three met our inclusion criteria (i.e., with a comparison group). Second, within these reviews, authors noted the absence of RCTs or high-quality observational studies of health system interventions. Third, most existing studies examined the impact of system level patient and family engagement strategies on care processes or service delivery, policy or planning documents, or educational materials or tool development. As one review noted, "objective clinical outcomes, including quality, safety, and patient satisfaction, should be assessed in order to provide a stronger evidence base for system-level patient engagement." ${ }^{121}$ Finally, tools for standardized measurement of patient engagement would facilitate evaluation of implementation success. ${ }^{117}$ Despite gaps in the evidence around health system strategies, in the gray literature, we identified several toolkits aimed at increasing the uptake of these strategies (Appendix C). Second, regarding the direct patient care strategies, our Key Informants highlighted the importance of advanced care planning, but we identified relatively few $(n=4)$ reviews focused on patient and family engagement strategies for advanced care planning. ${ }^{75,76,81,110}$ Third, most reviews focused on direct patient care strategies for people living with diabetes $(n=45)$, and fewer studies focused on patients with chronic mental health conditions or multiple chronic conditions, given that 4 in 10 adults have more than 1 chronic health condition. ${ }^{125}$ The most common chronic health conditions in the U.S. are cancer, diabetes, heart disease, Alzheimer's disease, chronic lung disease, chronic kidney disease and stroke. ${ }^{125}$ We identified very few studies in patients with dementia, stroke, or chronic kidney disease. The majority of systematic reviews addressing shared decision making focused on cancer screening and treatment $(n=9)$. Fourth, few $(n=13)$ reviews of direct patient care strategies focused on addressing their effectiveness among vulnerable populations, including urban or rural, minority, low income, or older adults. Fifth, we identified gaps in the systematic reviews reporting on implementation outcomes, health care services utilization, or cost. These outcomes are of high importance to a health system focused on value-based care and measuring cost and hospital readmissions, but few studies measured implementation or utilization outcomes. Finally, the majority of measured outcomes involved patient surveys to assess satisfaction or HRQOL, but many reviews also addressed chronic disease clinical outcomes, particularly in the area of diabetes management. Fewer reviews addressed caregiver-related measures, even among 
pediatric studies where many more interventions engaged caregivers and parents of children with chronic health conditions.

Based on our evidence map we identified a need for primary research studies to (1) develop valid and reliable measures for patient engagement, and measures for assessing the patient experience that span the continuum of care rather than assessing separate care episodes; (2) test engagement strategies with roles for family caregivers to advance self-management among both adults and children with chronic conditions; (3) test interventions for engaging patients and families in advanced care planning and end of life care; (4) develop patient portal and other technology tools that are adapted for patients with lower literacy or low technology skills to facilitate communication with medical providers; (5) measure cost-effectiveness of patient and caregiver engagement strategies to support self-management; (6) identify approaches to improve access to care, and ensure effective and quick responses to patients and family caregivers; (7) develop interventions to engage patients with multiple chronic conditions and their family caregivers in self-management of their multiple conditions.

Based on our evidence map we identified a need for more primary research studies in children and adolescents living with chronic disease to focus on (1) effective use of technology to facilitate engagement in self-management; (2) impact of engagement strategies on clinical outcomes as few studies reported clinical outcomes important to patients and families.

Based on our evidence map we identified a need for high quality primary research studies to engage patients and family caregivers at the health system and community level as overall few studies have been published. For example, studies are needed to test approaches to help (1) change medical culture so that patient and family input is prioritized and acted upon; and (2) engage patients and caregivers from diverse backgrounds and vulnerable populations and making their voice more heard within Patient and Family Advisory Councils and other platforms within healthcare organizations.

Finally, our evidence map highlights a need for both systematic review and original studies to examine implementation outcomes related to scaling and implementing direct and health system level engagement strategies. The learning health system needs to understand the fidelity, implementation, cost, and sustainability of engagement strategies. 
Figure 16. Map of the evidence on patient and family engagement strategies by level of engagement

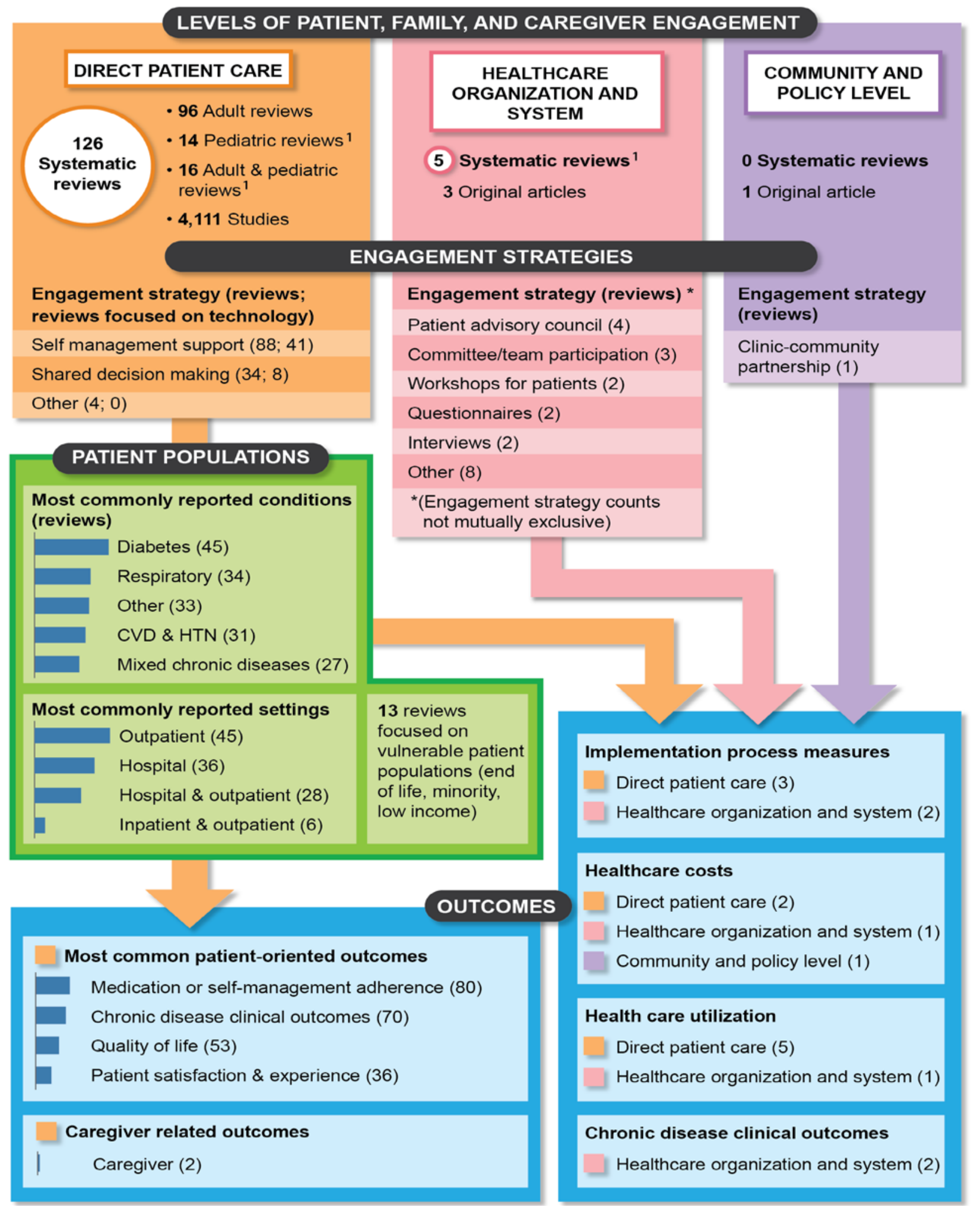

${ }^{1}$ Systematic review included family caregivers. The numbers in the green box (Patient populations) represent reviews. 
Figure 17. Map of the evidence on direct patient and family engagement strategies by reported level of benefit for different types of outcomes

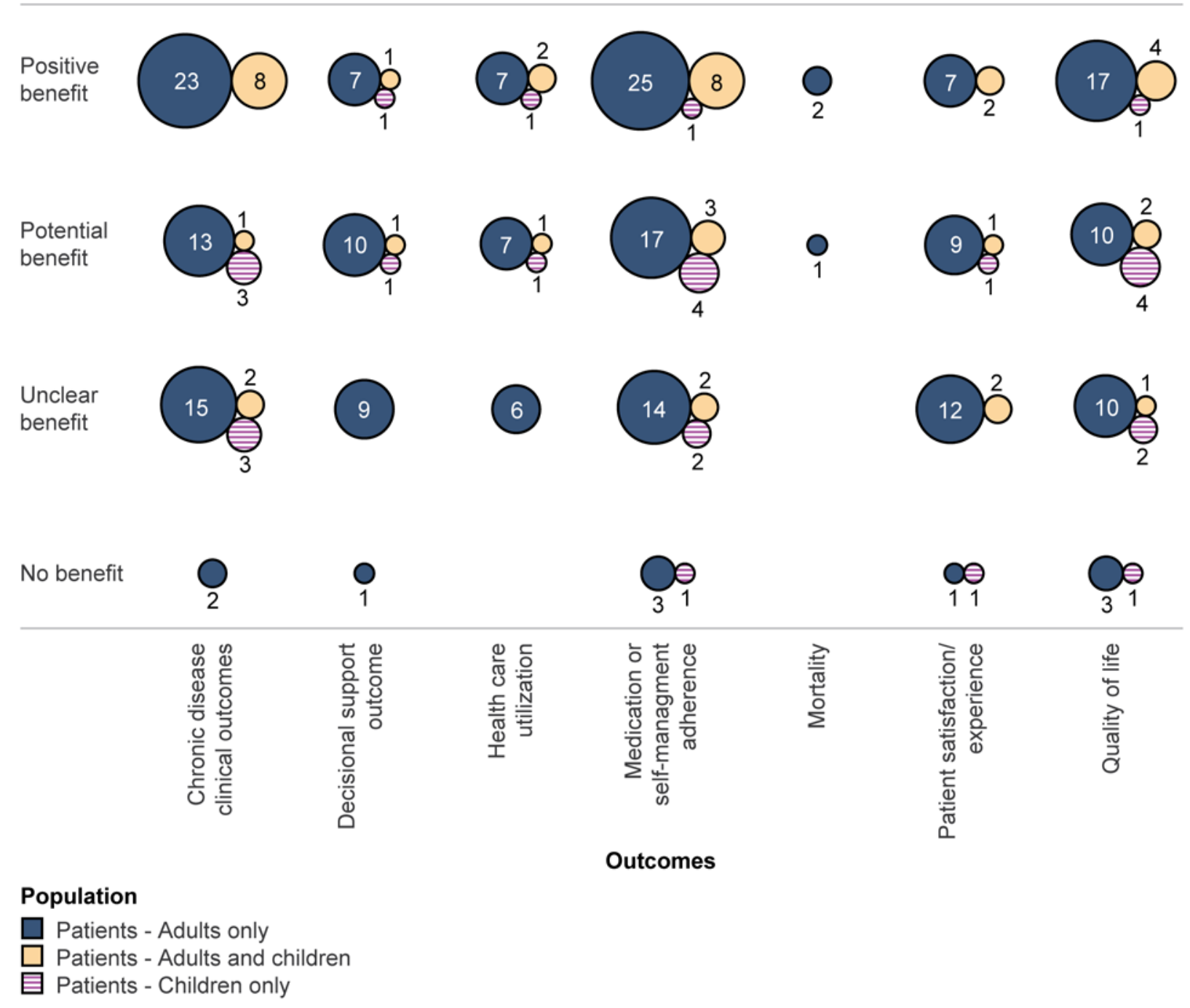




\section{Discussion and Implications}

\section{Summary of Main Findings}

Increasingly, patients, families, and caregivers play key roles not only in managing their own health and health care, but also in contributing to the development and improvement of the health care delivery system. In this Technical Brief, we built on a conceptual framework on patient and family engagement from Carman and colleagues to categorize engagement strategies into direct patient care (i.e., strategies that directly impacted individual patients' treatment or decision making), health system level (i.e., strategies with a health system impact beyond the individual patient's care), and community/policy level (i.e., strategies that engage consumers and communities in health care policies) (Figure 1). ${ }^{1}$ In selecting articles for inclusion, we applied the broad definition of patient and family engagement by Carman and colleagues, as "patients, families, their representatives, and health professionals working in active partnership at [these] various levels across the health care system." Although this definition is commonly used, the Key Informants highlighted the challenges of applying such a broad definition of patient and family engagement to the current evidence base. Their comments were consistent with the recent position paper by the American College of Physicians, "Principles for Patient and Family Partnership in Care," as what it means to do patient and family engagement vastly differs by setting, scale, and intended outcomes. ${ }^{35}$ Thus, it is not surprising that we found a great deal of heterogeneity in systematic reviews of patient and family engagement strategies.

Overall, our review included 131 systematic reviews. Of these reviews, 126 focused on direct patient care (with 34 reviews on shared decision making, 88 on self-management support, and 4 on other engagement strategies). We identified only five systematic reviews and three original studies that assessed patient and caregiver engagement at the health system level. These health system-level strategies most commonly included patient and family advisory councils and committees. We found only one original study and no systematic reviews on patient and family engagement at the community level. Of the five systematic reviews and three original articles focused on patient and family engagement at the health system level, patient and family advisors or advisory councils were the focus of two systematic reviews, ${ }^{117,121}$ while three reviews examined system level patient and family engagement through a variety of other strategies, including participating in meetings, committees, and project teams, ${ }^{119-121}$ or providing consultative input through surveys, focus groups, or interviews. ${ }^{119,120}$ Health system level reviews demonstrated some benefits, such as improvements in health care processes, development of organizational plans and policies, and education or tool development primarily in descriptive studies. The one article focused on a community patient engagement strategy highlighted a neighborhood-clinic partnership in the Navajo Nation. The partnership aimed to increase community health worker engagement with healthcare services to improve care for patients with diabetes, which has a high burden of disease on their community. ${ }^{124}$

The 126 systematic reviews on direct-care patient engagement strategies varied in terms of populations (14 in children, 16 in adults/children, 96 in adults only) and chronic diseases (diabetes was most commonly studied), and clinical settings and modalities for engagement (e.g., health coaches, mobile applications). Despite a high volume of studies addressing direct-patient care engagement strategies, fewer systematic reviews focused on the engagement of vulnerable patient populations. The most common direct patient care engagement strategies included teambased care to support patient self-management, patient-provider communication using shared decision making, as well as mobile health and electronic health record tools to improve engagement. Among adult patients, the most promising and innovative strategies with the highest volume of evidence (i.e., several large RCTs) included group-based educational programs to 
promote chronic disease self-management by peers and other healthcare professionals, ${ }^{126}$ webbased and short message service interventions for cancer survivors, ${ }^{59}$ promising telehealth programs to promote communication, self-monitoring and counseling, ${ }^{127}$ as well as mobile health to promote weight loss. ${ }^{128}$ Among pediatric patients, the most promising and innovative directpatient care engagement strategies included: eHealth tools for symptom control in adolescents with asthma; ${ }^{98}$ transitions of care support through combined care coordination and family education sessions at hospital discharge ${ }^{86}$ and shared decision making with decision aids, ${ }^{89}$ especially for mental health treatment. ${ }^{87}$ Importantly, these strategies required consideration of the child's age and developmental level, but also standard educational components as reported by Saxby and colleagues. ${ }^{92}$

\section{Strengths and Limitations of Our Evidence Map}

To our knowledge, this is the first systematic review to address patient and family engagement strategies focused on health system level and community/policy level strategies, in addition to direct patient and family engagement strategies. Similar to our review, the systematic review by Coulter and colleagues included direct-patient care engagement strategies. ${ }^{129}$ Like the Coulter review, we identified many patient and family engagement reviews focused on selfmanagement support and education, as well as clinical decision making through patient-provider communication strategies. ${ }^{129}$ Sharma and colleagues conducted a review of patient and family engagement strategies specifically related to patient safety and identified only one review with a health system level engagement strategy. ${ }^{130}$ Despite the large number of direct patient care engagement studies, we identified inconsistent findings for the benefits of some selfmanagement and communication strategies, even within the same chronic disease condition. The inconsistency was in part owing to the heterogeneity of tested interventions, different measures, and low quality of the original studies.

Given the widespread implementation of electronic health records and the proliferation of mobile phone applications in recent years, a major contribution of our review was the identification of 49 systematic reviews focused on using technology (mobile health, electronic health records, Web-based programs) as part of the engagement strategy. One systematic review, by Irizarry and colleagues, ${ }^{77}$ included 122 articles (14 RCTs) and focused on patient engagement using the electronic health record. This descriptive review identified five major topics related to patient engagement using the electronic medical record (patient adoption, provider endorsement, health literacy, usability, and utility) and, highlighted that use of patient portals was influenced by patients' age, ethnicity, education level, health literacy, health status, and role as a caregiver. Health care delivery factors, mainly provider endorsement and patient portal usability, also contributed to patients' ability to engage through and with the patient portal. ${ }^{77}$ Only one systematic review, by Kuo and colleagues, reported on a clinical outcome related to the effect of secure messaging in the patient portal on diabetes outcomes. ${ }^{53}$ This review identified one RCT, which showed a decrease in hemoglobin A1c among patients with uncontrolled diabetes at 6 months but not at 12 months. ${ }^{53,131}$

We also have identified several limitations of our review and evidence map. First, because of the broad definition of patient and family engagement, we focused on identifying and synthesizing findings from systematic reviews, rather than original articles for direct patient-care strategies, which made it less feasible to capture details about each study's population, intervention, and barriers to implementation. We were not able to extract the detail information from the studies included in the systematic reviews. This is particularly important because we were unable to directly capture whether vulnerable populations were included within each study and had to rely on the information provided by the review. In addition, this resulted in heterogeneity of interventions along the continuum of engagement within some reviews, which 
may account for some of the inconsistent findings in our results. Also, at the direct care level, we were also unable to provide a granular description of the tested interventions, and instead summarized them by modality and disease area. Second, we excluded articles and reviews explicitly focused on patient engagement for the purposes of research, especially around research prioritization or on community advisory boards focused on research. This exclusion has important implications as, increasingly, the learning health system is blending research with continuous quality improvement with similar goals of improving health care quality, safety, and delivery. In addition, community-based participatory research has a long tradition of engaging patients, communities, and stakeholders in research that is driven by and sustained within the community. We acknowledge that many patient and family engagement strategies are being used in community-based participatory research, and that there is often a blending between engagement for patient care and for research on improving the delivery of patient care. Third, we focused on engagement of patients with chronic health conditions, and therefore excluded reviews and articles that only focused on chronic disease prevention (e.g., increasing exercise by using a mobile application). Owing to the importance of population health initiatives focused on wellness and prevention, future reviews could address this gap by understanding the patient and family engagement strategies for staying well and preventing chronic disease. Fourth, we did not assess the risk of bias in the original studies included in the systematic reviews or in the additional original studies we found. Fifth, because of the scope of this project including direct patient care to community level engagement strategies, we may have missed examples of community and policy level engagement, as few of these articles met inclusion criteria for having a comparison group or including outcomes of interest. ${ }^{132-141}$

\section{Implications for Clinical Practice, Education, and Health Policy}

The learning health system is defined by the AHRQ as "a health system in which internal data and experience are systematically integrated with external evidence, and that knowledge is put into practice." 142 Because one of the principles of the learning health system is to "promote the inclusion of patients as vital members of the learning team," it is important to identify best practices and high quality evidence to select strategies that not only engage patients but also lead to improvements in care quality and value. The American Institute for Research created a Roadmap for Patient and Family Engagement in Healthcare Practice and Research to assist health care systems and providers in partnering with patients and families. ${ }^{143}$ They described best practices of strategies that healthcare systems are currently using. Despite the enthusiasm about increasing patient engagement at a systems level and calls to make it an expectation, our review identified a paucity of rigorous studies about the effectiveness and implementation of health system strategies, making it challenging to recommend wide uptake. In addition, our review aimed to describe barriers and facilitators to implementing patient and family engagement strategies in clinics and hospitals. However, because few systematic reviews specifically addressed or reported implementation outcomes, it is unclear which are the best and most effective processes for engaging patients who have diverse voices, ultimately to inform improvements in health care delivery. Explicit development of a theoretical framework for understanding the key elements of a system level patient and family engagement strategy could help guide implementation, measurement development, and evaluation.

\section{Future Research Needs}

More reviews are needed that are focused on patients with chronic mental health conditions and those with multiple chronic conditions particularly given the vulnerability and risk of poor 
outcomes for these patients. Also, more reviews are needed that focus on children and adolescents living with chronic disease. The systematic reviews at the health system level consistently highlighted a need for high quality studies with robust study designs to evaluate patient and organizational level outcomes. Some studies highlighted barriers to high quality evidence, which included the heterogeneity of both the intervention and outcomes studied, as well as limited tools for measuring patient engagement. In fact, a recent systematic review, by Dukhanin, ${ }^{144}$ reported on measurement and evaluation tools for the assessments of patient, public, consumer, and community engagement in organization-, community-, and system-level healthcare decision making. Most of these diverse 23 tools used surveys that assessed the process of engagement, as well as the impact of engagement participants (e.g., improved knowledge) on the services provided by the organization or system (e.g., improved quality or decreased utilization of services) and on the organization (e.g., redesign of staff roles, or staff training policies). ${ }^{144}$ There is a need for measures to assess to what extent health systems are supporting the engagement of patients and caregivers. In terms of measures of patient experience, there are models for engaging patients and families in the development of these measures in order to include questions that address what patients care about most and how they would like to be engaged in their care. ${ }^{145}$ In addition, few studies rigorously evaluated health system interventions using health services design methods that included a comparison group. Future study design and analysis methods could include interrupted time series and propensity score approaches. ${ }^{146}$ As more evidence emerges on patient and family engagement strategies at the health system level, it will become necessary to synthesize that evidence. Synthesis will be challenging because the strategies are likely to be highly variable in the nature of the populations, interventions, comparisons, and outcomes studied.

We also identified future research needs to improve direct patient care strategies. The majority of the evidence on direct patient care strategies for patient and family engagement among patients with chronic disease is in the area of self-management support. Further study is needed to assess impact of strategies to improve shared decision making and patient-provider communications on clinical outcomes. In addition, despite a great deal of discussion by the Key Informants about the importance of patient and family engagement to support advanced care planning, we found a large gap in evidence on the effectiveness of engagement strategies focused on advanced care planning for patients with chronic conditions.

Among the systematic reviews of direct patient care interventions in adults, 8 reviews included patients with multiple chronic conditions (5 with self-management support interventions, one with shared decision-making intervention and 2 with transitional care interventions) (Table 2 and Figure 7). Five of the 8 were deemed to have positive benefit, indicating an important area for future research in identifying which of these interventions have the highest level of evidence and could potentially be more widely implemented.

The role of family and caregivers is particularly relevant to efforts to improve engagement among subpopulations including older adults and people with impaired decision-making capacity (e.g. patients with dementia). However, few reviews were able to examine the sub-populations included in the studies. Future studies need to provide details about their target populations to permit better assessment of the applicability of strategies to all patients and communities. Future studies are needed to assess risks and benefits of engagement including patient anxiety (increase or decrease), as well as disproportionate access to engagement methods, which could worsen health disparities. However, outside of the pediatric articles, few studies in adults included family caregivers or measured caregiver-related outcomes, highlighting an important research gap. In the pediatric population, more studies are needed to assess the effectiveness of engagement strategies on clinical outcomes, as most studies focused only on patient-reported outcomes. More pediatric studies are needed to examine the effect of technology among children 
and adolescents living with chronic disease, as these strategies were not as well studied in children, compared with the adult population. In addition, most pediatric studies addressed children with lower medical complexity or single chronic diseases (like asthma), indicating that more rigorous studies are needed to study patient and family engagement among families with children that have medically complex health conditions. New evidence synthesis will be needed as evidence grows on the effectiveness of patient and family engagement strategies for pediatric conditions and advanced care planning. As indicated above, synthesis will be challenging because of expected heterogeneity in the populations, interventions, comparisons, and outcomes studied. 


\section{Conclusion}

In conclusion, we identified a wealth and diversity of evidence on direct patient care engagement strategies for adults with chronic conditions, but a dearth of evidence for strategies at the health system and community/policy levels. Patient and family engagement strategies with the greatest evidence pertain to self- management support. We identified inconsistent findings among reviews of self-management strategies, even within the same chronic condition. This is in part due to the heterogeneity of tested interventions, different measures, and low quality of the original studies. Use of technology to facilitate patient and family engagement is a promising approach. Few studies examined engagement strategies for advanced care planning or interventions for patients with multiple chronic conditions. The evidence on engagement strategies in the pediatric population is limited by a small number of systematic reviews and few reporting on clinical outcomes. More research is needed to address a big gap in evidence on patient and family engagement at the health system and community/policy levels. Such research should use robust study designs, such as cluster RCTs, assessing the impact on clinical outcomes and patient satisfaction, and using standardized tools to measure the impact on patient and family engagement. 


\section{References}

1. Carman KL, Dardess P, Maurer M, et al. Patient and family engagement: a framework for understanding the elements and developing interventions and policies. Health affairs (Project Hope). 2013 Feb;32(2):223-31. doi: 10.1377/hlthaff.2012.1133. PMID: 23381514.

2. Pozdnyakova A, Laiteerapong N, Volerman A, et al. Impact of Medical Scribes on Physician and Patient Satisfaction in Primary Care. Journal of general internal medicine. 2018 Jul;33(7):1109-15. doi: 10.1007/s11606-018-4434-6. PMID: 29700790 .

3. Boissy A, Windover AK, Bokar D, et al. Communication Skills Training for Physicians Improves Patient Satisfaction. Journal of general internal medicine. 2016 Jul;31(7):755-61. doi: 10.1007/s11606-0163597-2. PMID: 26921153.

4. Von Korff M, Gruman J, Schaefer J, et al. Collaborative management of chronic illness. Annals of internal medicine. 1997 Dec 15;127(12):1097-102. doi: 10.7326/0003-4819-127-12-19971215000008. PMID: 9412313.

5. Wagner EH, Bennett SM, Austin BT, et al. Finding common ground: patientcenteredness and evidence-based chronic illness care. Journal of alternative and complementary medicine (New York, NY). 2005;11 Suppl 1:S7-15. doi: 10.1089/acm.2005.11.s-7. PMID: 16332190.

6. Hibbard JH, Stockard J, Mahoney ER, et al. Development of the Patient Activation Measure (PAM): conceptualizing and measuring activation in patients and consumers. Health services research. 2004 Aug;39(4 Pt 1):1005-26. doi: 10.1111/j.1475-6773.2004.00269.x. PMID: 15230939.
7. Remmers C, Hibbard J, Mosen DM, et al. Is patient activation associated with future health outcomes and healthcare utilization among patients with diabetes? The Journal of ambulatory care management. 2009 OctDec;32(4):320-7. doi: 10.1097/JAC.0b013e3181ba6e77. PMID: 19888008.

8. Weingart SN, Zhu J, Chiappetta L, et al. Hospitalized patients' participation and its impact on quality of care and patient safety. International journal for quality in health care : journal of the International Society for Quality in Health Care. 2011 Jun;23(3):26977. doi: 10.1093/intqhe/mzr002. PMID: 21307118.

9. Anderson G. Chronic Care: Making the Case for Ongoing Care, Princeton, N.J.: Robert Wood Johnson Foundation, 2010. . 2010.

10. RAND - www.rand.org/t/TL221

11. Hussey PS, Schneider EC, Rudin RS, et al. Continuity and the costs of care for chronic disease. JAMA internal medicine. 2014 May;174(5):742-8. doi: 10.1001/jamainternmed.2014.245. PMID: 24638880 .

12. Hibbard JH, Greene J, Tusler M. Improving the outcomes of disease management by tailoring care to the patient's level of activation. The American journal of managed care. 2009 Jun;15(6):353-60. PMID: 19514801.

13. Rost K, Nutting P, Smith JL, et al. Managing depression as a chronic disease: a randomised trial of ongoing treatment in primary care. BMJ (Clinical research ed). 2002 Oct 26;325(7370):934. doi: 10.1136/bmj.325.7370.934. PMID: 12399343.

14. Lorig KR, Sobel DS, Stewart AL, et al. Evidence suggesting that a chronic disease self-management program can improve health status while reducing hospitalization: a randomized trial. Medical care. 1999 Jan;37(1):5-14. doi: 10.1097/00005650199901000-00003. PMID: 10413387. 
15. Greenfield S, Kaplan SH, Ware JE, Jr., et al. Patients' participation in medical care: effects on blood sugar control and quality of life in diabetes. Journal of general internal medicine. 1988 Sep-Oct;3(5):448-57. doi: 10.1007/bf02595921. PMID: 3049968.

16. Funnell MM, Brown TL, Childs BP, et al. National standards for diabetes selfmanagement education. Diabetes care. 2012 Jan;35 Suppl 1:S101-8. doi: 10.2337/dc12s101. PMID: 22187467.

17. Fitzpatrick SL, Schumann KP, Hill-Briggs

F. Problem solving interventions for diabetes self-management and control: a systematic review of the literature. Diabetes research and clinical practice. 2013 May;100(2):145-61. doi: 10.1016/j.diabres.2012.12.016. PMID: 23312614.

18. Effing T, Monninkhof EM, van der Valk $\mathrm{PD}$, et al. Self-management education for patients with chronic obstructive pulmonary disease. The Cochrane database of systematic reviews. 2007 Oct 17(4):Cd002990. doi: 10.1002/14651858.CD002990.pub2. PMID: 17943778.

19. Bayliss EA, Ellis JL, Steiner JF. Barriers to self-management and quality-of-life outcomes in seniors with multimorbidities. Annals of family medicine. 2007 SepOct;5(5):395-402. doi: 10.1370/afm.722. PMID: 17893380.

20. Patient- and family-centered care and the pediatrician's role. Pediatrics. 2012 Feb;129(2):394-404. doi: 10.1542/peds.2011-3084. PMID: 22291118.

21. Conway JB, Edgman-Levitan S, Schlucter J, et al. Partnering with Patients and Families To Design a Patient- and Family-Centered Health Care System: A Roadmap for the Future, A Work in Progress. Institute for Family-Centered Care, Bethesda, MD: 2006.

22. Institute of $\mathrm{M}$. The National Academies Collection: Reports funded by National Institutes of Health. In: Olsen LA, Saunders RS, McGinnis JM, eds. Patients Charting the Course: Citizen Engagement and the Learning Health System: Workshop Summary. Washington (DC): National Academies Press (US) National Academy of Sciences.; 2011.
23. Entwistle VA, McCaughan D, Watt IS, et al. Speaking up about safety concerns: multisetting qualitative study of patients' views and experiences. Quality \& safety in health care. 2010 Dec;19(6):e33. doi: 10.1136/qshc.2009.039743. PMID: 21127092.

24. Griffin SJ, Kinmonth AL, Veltman MW, et al. Effect on health-related outcomes of interventions to alter the interaction between patients and practitioners: a systematic review of trials. Annals of family medicine. 2004 Nov-Dec;2(6):595-608. doi: 10.1370/afm.142. PMID: 15576546.

25. Kaplan SH, Greenfield S, Ware JE, Jr. Assessing the effects of physician-patient interactions on the outcomes of chronic disease. Medical care. 1989 Mar;27(3 Suppl):S110-27. doi: 10.1097/00005650198903001-00010. PMID: 2646486.

26. Peat M, Entwistle V, Hall J, et al. Scoping review and approach to appraisal of interventions intended to involve patients in patient safety. Journal of health services research \& policy. 2010 Jan;15 Suppl 1:1725. doi: 10.1258/jhsrp.2009.009040. PMID: 20075123.

27. Schillinger D, Piette J, Grumbach K, et al. Closing the loop: physician communication with diabetic patients who have low health literacy. Archives of internal medicine. 2003 Jan 13;163(1):83-90. doi: 10.1001/archinte.163.1.83. PMID: 12523921.

28. Castro CM, Wilson C, Wang F, et al. Babel babble: physicians' use of unclarified medical jargon with patients. American journal of health behavior. 2007 Sep-Oct;31 Suppl 1:S85-95. doi: 10.5555/ajhb.2007.31.supp.S85. PMID: 17931142.

29. Marvel MK, Epstein RM, Flowers K, et al. Soliciting the patient's agenda: have we improved? Jama. 1999 Jan 20;281(3):283-7. doi: 10.1001/jama.281.3.283. PMID: 9918487. 
30. Brugge D, Edgar T, George K, et al. Beyond literacy and numeracy in patient provider communication: focus groups suggest roles for empowerment, provider attitude and language. BMC public health. 2009 Sep 21;9:354. doi: 10.1186/1471-2458-9-354. PMID: 19772555.

31. Wilson E, Chen AH, Grumbach K, et al. Effects of limited English proficiency and physician language on health care comprehension. Journal of general internal medicine. 2005 Sep;20(9):800-6. doi: 10.1111/j.1525-1497.2005.0174.x. PMID: 16117746.

32. Rao JK, Anderson LA, Inui TS, et al. Communication interventions make a difference in conversations between physicians and patients: a systematic review of the evidence. Medical care. 2007 Apr;45(4):340-9. doi: 10.1097/01.mlr.0000254516.04961.d5. PMID: 17496718.

33. Cooper LA, Roter DL, Carson KA, et al. A randomized trial to improve patient-centered care and hypertension control in underserved primary care patients. Journal of general internal medicine. 2011 Nov;26(11):1297-304. doi: 10.1007/s11606011-1794-6. PMID: 21732195.

34. Herrin J, Harris KG, Kenward K, et al. Patient and family engagement: a survey of US hospital practices. BMJ quality \& safety. 2016 Mar;25(3):182-9. doi: 10.1136/bmjqs2015-004006. PMID: 26082560.

35. Nickel WK, Weinberger SE, Guze PA. Principles for Patient and Family Partnership in Care: An American College of Physicians Position Paper. Annals of internal medicine. 2018 Dec 4;169(11):7969. doi: 10.7326/m18-0018. PMID: 30476985.

36. Millenson M, Macri J. Will the Affordable Care Act Move Patient-Centeredness to Center Stage? Timely Analysis of Immediate Health Policy Issues. 2002.

37. Center for Medicare \& Medicaid Services Comprehensive Primary Care Initiative https://innovation.cms.gov/initiatives /comprehensive-primary-careinitiative/.
38. PCMH Standards and Guidelines. 2017. https://www.kentuckyrec.com/wpcontent/uploads/2017/10/02.-PCMHStandards-and-Guidelines-2017Edition-Version-2-9-30-17.pdf.

39. Working With Patient and Families as Advisors (Implementation Handbook) Agency for Healthcare Research and Quality. 2008.

40. Creating a Patient and Family Advisory Council: A Toolkit for Pediatric Practices National Institute for Children's Health Quality. 2012.

41. CPC Patient and Family Engagement Resources from the National Partnership for Women \& Families 2013. https://innovation.cms.gov/Files/x/cp ci-patientfamengresource.pdf.

42. Moving Forward with Patient- and FamilyCentered Care: Partnerships for Quality and Safety. 2019.

43. Crawford MJ, Rutter D, Manley C, et al. Systematic review of involving patients in the planning and development of health care. BMJ (Clinical research ed). 2002 Nov 30;325(7375):1263. doi: 10.1136/bmj.325.7375.1263. PMID: 12458240.

44. Johnson KE, Mroz TM, Abraham M, et al. Promoting Patient and Family Partnerships in Ambulatory Care Improvement: A Narrative Review and Focus Group Findings. Advances in therapy. 2016 Aug;33(8):1417-39. doi: 10.1007/s12325016-0364-z. PMID: 27352378.

45. Healthcare Information and Management Systems Society: HIMSS patient engagement framework. Chicago, IL. 2014 [updated 2017].

http://www.himss.org/himss-patientengagement-framework.

46. Sandy LG, Tuckson RV, Stevens SL. UnitedHealthcare experience illustrates how payers can enable patient engagement. Health affairs (Project Hope). 2013 Aug;32(8):1440-5. doi: 10.1377/hlthaff.2012.1082. PMID: 23918489. 
47. Price M, Bellwood P, Kitson N, et al. Conditions potentially sensitive to a personal health record (PHR) intervention, a systematic review. BMC medical informatics and decision making. $2015 \mathrm{Apr}$ 18;15:32. doi: 10.1186/s12911-015-0159-1. PMID: 25927384.

48. Kruse CS, Bolton K, Freriks G. The effect of patient portals on quality outcomes and its implications to meaningful use: a systematic review. Journal of medical Internet research. 2015 Feb 10;17(2):e44. doi: 10.2196/jmir.3171. PMID: 25669240.

49. Ammenwerth E, Hoerbst A, Lannig S, et al. Effects of Adult Patient Portals on Patient Empowerment and Health-Related Outcomes: A Systematic Review. Studies in health technology and informatics. 2019 Aug 21;264:1106-10. doi: 10.3233/shti190397. PMID: 31438096.

50. Dendere R, Slade C, Burton-Jones A, et al. Patient Portals Facilitating Engagement With Inpatient Electronic Medical Records: A Systematic Review. Journal of medical Internet research. 2019 Apr 11;21(4):e12779. doi: 10.2196/12779. PMID: 30973347.

51. Risling T, Martinez J, Young J, et al. Evaluating Patient Empowerment in Association With eHealth Technology: Scoping Review. Journal of medical Internet research. 2017 Sep 29;19(9):e329. doi: 10.2196/jmir.7809. PMID: 28963090.

52. Kelly MM, Coller RJ, Hoonakker PL. Inpatient Portals for Hospitalized Patients and Caregivers: A Systematic Review. Journal of hospital medicine. 2018 Jun 1;13(6):405-12. doi: 10.12788/jhm.2894. PMID: 29261819.

53. Kuo A, Dang S. Secure Messaging in Electronic Health Records and Its Impact on Diabetes Clinical Outcomes: A Systematic Review. Telemedicine journal and e-health : the official journal of the American Telemedicine Association. 2016 Sep;22(9):769-77. doi: 10.1089/tmj.2015.0207. PMID: 27027337.
54. Han HR, McKenna S, Nkimbeng M, et al. A Systematic Review of Community Health Center Based Interventions for People with Diabetes. Journal of community health. 2019 Jul 6. doi: 10.1007/s10900-019-00693y. PMID: 31280431.

55. Levengood TW, Peng Y, Xiong KZ, et al. Team-Based Care to Improve Diabetes Management: A Community Guide Metaanalysis. American journal of preventive medicine. 2019 Jul;57(1):e17-e26. doi: 10.1016/j.amepre.2019.02.005. PMID: 31227069.

56. Zhao FF, Suhonen R, Koskinen S, et al. Theory-based self-management educational interventions on patients with type 2 diabetes: a systematic review and metaanalysis of randomized controlled trials. Journal of advanced nursing. 2017 Apr;73(4):812-33. doi: 10.1111/jan.13163. PMID: 27681948.

57. CADTH Rapid Response Reports. Patientand Family-Centered Care Initiatives in Acute Care Settings: A Review of the Clinical Evidence, Safety and Guidelines. Ottawa (ON): Canadian Agency for Drugs and Technologies in Health; 2015.

58. Teljeur C, Moran PS, Walshe S, et al. Economic evaluation of chronic disease selfmanagement for people with diabetes: a systematic review. Diabetic medicine : a journal of the British Diabetic Association. 2017 Aug;34(8):1040-9. doi: 10.1111/dme.13281. PMID: 27770591.

59. Kim AR, Park HA. Web-based Selfmanagement Support Interventions for Cancer Survivors: A Systematic Review and Meta-analyses. Studies in health technology and informatics. 2015;216:142-7. PMID: 26262027.

60. Hammer MJ, Ercolano EA, Wright F, et al. Self-management for adult patients with cancer: an integrative review. Cancer nursing. 2015 Mar-Apr;38(2):E10-26. doi: 10.1097/ncc.0000000000000122. PMID: 25692736. 
61. Escriva Boulley G, Leroy T, Bernetiere C, et al. Digital health interventions to help living with cancer: A systematic review of participants' engagement and psychosocial effects. Psycho-oncology. 2018 Dec;27(12):2677-86. doi: 10.1002/pon.4867. PMID: 30152074.

62. Clarkesmith DE, Pattison HM, Khaing PH, et al. Educational and behavioural interventions for anticoagulant therapy in patients with atrial fibrillation. The Cochrane database of systematic reviews. 2017 Apr 5;4:Cd008600. doi: 10.1002/14651858.CD008600.pub3. PMID: 28378924.

63. Alessa T, Abdi S, Hawley MS, et al. Mobile Apps to Support the Self-Management of Hypertension: Systematic Review of Effectiveness, Usability, and User Satisfaction. JMIR mHealth and uHealth. 2018 Jul 23;6(7):e10723. doi: 10.2196/10723. PMID: 30037787.

64. Palacios J, Lee GA, Duaso M, et al. InternetDelivered Self-management Support for Improving Coronary Heart Disease and Selfmanagement-Related Outcomes: A Systematic Review. The Journal of cardiovascular nursing. 2017

Jul/Aug;32(4):E9-e23. doi: 10.1097/jcn.0000000000000392. PMID: 28107251.

65. Sakakibara BM, Kim AJ, Eng JJ. A Systematic Review and Meta-Analysis on Self-Management for Improving Risk Factor Control in Stroke Patients. International journal of behavioral medicine. 2017 Feb;24(1):42-53. doi: 10.1007/s12529-0169582-7. PMID: 27469998.

66. Peytremann-Bridevaux I, Arditi C, Gex G, et al. Chronic disease management programmes for adults with asthma. The Cochrane database of systematic reviews. 2015 May 27(5):Cd007988. doi: 10.1002/14651858.CD007988.pub2. PMID: 26014500 .
67. Kim K, Choi JS, Choi E, et al. Effects of Community-Based Health Worker Interventions to Improve Chronic Disease Management and Care Among Vulnerable Populations: A Systematic Review. American journal of public health. 2016 Apr;106(4):e3-e28. doi: 10.2105/ajph.2015.302987. PMID: 26890177.

68. Ko D, Bratzke LC, Roberts T. Selfmanagement assessment in multiple chronic conditions: A narrative review of literature. International journal of nursing studies. 2018 Jul;83:83-90. doi: 10.1016/j.ijnurstu.2018.04.009. PMID: 29709734.

69. Smith SM, Wallace E, O'Dowd T, et al. Interventions for improving outcomes in patients with multimorbidity in primary care and community settings. The Cochrane database of systematic reviews. 2016 Mar 14;3:Cd006560. doi: 10.1002/14651858.CD006560.pub3. PMID: 26976529.

70. Thakkar J, Kurup R, Laba TL, et al. Mobile Telephone Text Messaging for Medication Adherence in Chronic Disease: A Metaanalysis. JAMA internal medicine. 2016 Mar;176(3):340-9. doi: 10.1001/jamainternmed.2015.7667. PMID: 26831740.

71. Deek H, Hamilton S, Brown N, et al. Family-centred approaches to healthcare interventions in chronic diseases in adults: a quantitative systematic review. Journal of advanced nursing. 2016 May;72(5):968-79. doi: 10.1111/jan.12885. PMID: 26751971.

72. Noonan MC, Wingham J, Dalal HM, et al. Involving caregivers in self-management interventions for patients with heart failure and chronic obstructive pulmonary disease. A systematic review and meta-analysis. Journal of advanced nursing. 2019 Aug 22. doi: 10.1111/jan.14172. PMID: 31441088.

73. Stovell D, Morrison AP, Panayiotou M, et al. Shared treatment decision-making and empowerment-related outcomes in psychosis: systematic review and metaanalysis. The British journal of psychiatry : the journal of mental science. 2016 Jul;209(1):23-8. doi: 10.1192/bjp.bp.114.158931. PMID: 27198483. 
74. Stacey D, Legare F, Lewis K, et al. Decision aids for people facing health treatment or screening decisions. The Cochrane database of systematic reviews. $2017 \mathrm{Apr}$

12;4:Cd001431. doi: 10.1002/14651858.CD001431.pub5. PMID: 28402085.

75. Vermunt N, Harmsen M, Westert GP, et al. Collaborative goal setting with elderly patients with chronic disease or multimorbidity: a systematic review. BMC geriatrics. 2017 Jul 31;17(1):167. doi: 10.1186/s12877-017-0534-0. PMID: 28760149 .

76. Cardona-Morrell M, Benfatti-Olivato G, Jansen J, et al. A systematic review of effectiveness of decision aids to assist older patients at the end of life. Patient education and counseling. 2017 Mar;100(3):425-35. doi: 10.1016/j.pec.2016.10.007. PMID: 27765378.

77. Irizarry T, DeVito Dabbs A, Curran CR. Patient Portals and Patient Engagement: A State of the Science Review. Journal of medical Internet research. 2015 Jun 23;17(6):e148. doi: 10.2196/jmir.4255. PMID: 26104044.

78. Saheb Kashaf M, McGill ET, Berger ZD. Shared decision-making and outcomes in type 2 diabetes: A systematic review and meta-analysis. Patient education and counseling. 2017 Dec;100(12):2159-71. doi: 10.1016/j.pec.2017.06.030. PMID: 28693922.

79. Le Berre M, Maimon G, Sourial N, et al. Impact of Transitional Care Services for Chronically Ill Older Patients: A Systematic Evidence Review. Journal of the American Geriatrics Society. 2017 Jul;65(7):1597-608. doi: 10.1111/jgs.14828. PMID: 28403508.

80. Sendall M, McCosker L, Crossley K, et al. A structured review of chronic care model components supporting transition between healthcare service delivery types for older people with multiple chronic diseases. Health information management : journal of the Health Information Management Association of Australia. 2017 May;46(2):58-68. doi: 10.1177/1833358316681687. PMID: 27923916 .
81. Coulter A, Entwistle VA, Eccles A, et al. Personalised care planning for adults with chronic or long-term health conditions. The Cochrane database of systematic reviews. 2015 Mar 3(3):Cd010523. doi: 10.1002/14651858.CD010523.pub2. PMID: 25733495.

82. Schaepe C, Bergjan M. Educational interventions in peritoneal dialysis: a narrative review of the literature.

International journal of nursing studies. 2015 Apr;52(4):882-98. doi: 10.1016/j.ijnurstu.2014.12.009. PMID: 25616708.

83. Campbell F, Biggs K, Aldiss SK, et al. Transition of care for adolescents from paediatric services to adult health services. The Cochrane database of systematic reviews. 2016 Apr 29;4:Cd009794. doi: 10.1002/14651858.CD009794.pub2. PMID: 27128768.

84. Knafl KA, Havill NL, Leeman J, et al. The Nature of Family Engagement in Interventions for Children With Chronic Conditions. Western journal of nursing research. 2017 May;39(5):690-723. doi: 10.1177/0193945916664700. PMID: 27596106.

85. Malone H, Biggar S, Javadpour S, et al. Interventions for promoting participation in shared decision-making for children and adolescents with cystic fibrosis. The Cochrane database of systematic reviews. 2019 May 23;5:Cd012578. doi: 10.1002/14651858.CD012578.pub2. PMID: 31119726.

86. Hamline MY, Speier RL, Vu PD, et al. Hospital-to-Home Interventions, Use, and Satisfaction: A Meta-analysis. Pediatrics. 2018 Nov;142(5). doi: 10.1542/peds.20180442. PMID: 30352792.

87. Cheng H, Hayes D, Edbrooke-Childs J, et al. What approaches for promoting shared decision-making are used in child mental health? A scoping review. Clinical psychology \& psychotherapy. 2017 Nov;24(6):O1495-o511. doi: 10.1002/cpp.2106. PMID: 28752631. 
88. Coyne I, O'Mathuna DP, Gibson F, et al. Interventions for promoting participation in shared decision-making for children with cancer. The Cochrane database of systematic reviews. 2016 Nov 29;11:Cd008970. doi: 10.1002/14651858.CD008970.pub3. PMID: 27898175.

89. Wyatt KD, List B, Brinkman WB, et al. Shared Decision Making in Pediatrics: A Systematic Review and Meta-analysis. Academic pediatrics. 2015 NovDec;15(6):573-83. doi: 10.1016/j.acap.2015.03.011. PMID: 25983006.

90. Clemente D, Leon L, Foster H, et al. Systematic review and critical appraisal of transitional care programmes in rheumatology. Seminars in arthritis and rheumatism. 2016 Dec;46(3):372-9. doi: 10.1016/j.semarthrit.2016.06.003. PMID: 27496195.

91. Low JK, Manias E. Use of TechnologyBased Tools to Support Adolescents and Young Adults With Chronic Disease: Systematic Review and Meta-Analysis. JMIR mHealth and uHealth. 2019 Jul 18;7(7):e12042. doi: 10.2196/12042. PMID: 31322129 .

92. Saxby N, Beggs S, Battersby M, et al. What are the components of effective chronic condition self-management education interventions for children with asthma, cystic fibrosis, and diabetes? A systematic review. Patient education and counseling. 2019 Apr;102(4):607-22. doi: 10.1016/j.pec.2018.11.001. PMID: 30471988.

93. Bal MI, Sattoe JN, Roelofs PD, et al. Exploring effectiveness and effective components of self-management interventions for young people with chronic physical conditions: A systematic review. Patient education and counseling. 2016 Aug;99(8):1293-309. doi: 10.1016/j.pec.2016.02.012. PMID: 26954345.
94. Majeed-Ariss R, Baildam E, Campbell M, et al. Apps and Adolescents: A Systematic Review of Adolescents' Use of Mobile Phone and Tablet Apps That Support Personal Management of Their Chronic or Long-Term Physical Conditions. Journal of medical Internet research. 2015 Dec 23;17(12):e287. doi: 10.2196/jmir.5043. PMID: 26701961.

95. Charlier N, Zupancic N, Fieuws S, et al. Serious games for improving knowledge and self-management in young people with chronic conditions: a systematic review and meta-analysis. Journal of the American Medical Informatics Association : JAMIA. 2016 Jan;23(1):230-9. doi: 10.1093/jamia/ocv100. PMID: 26186934.

96. Sattoe JN, Bal MI, Roelofs PD, et al. Selfmanagement interventions for young people with chronic conditions: A systematic overview. Patient education and counseling. 2015 Jun;98(6):704-15. doi: 10.1016/j.pec.2015.03.004. PMID: 25819373.

97. Kleinpell R, Zimmerman J, Vermoch KL, et al. Promoting Family Engagement in the ICU: Experience From a National Collaborative of 63 ICUs. Critical care medicine. 2019 Dec;47(12):1692-8. doi: 10.1097/ccm.0000000000004009. PMID: 31567354 .

98. Lancaster K, Abuzour A, Khaira M, et al. The Use and Effects of Electronic Health Tools for Patient Self-Monitoring and Reporting of Outcomes Following Medication Use: Systematic Review. Journal of medical Internet research. 2018 Dec 18;20(12):e294. doi: 10.2196/jmir.9284. PMID: 30563822.

99. Lycett HJ, Raebel EM, Wildman EK, et al. Theory-Based Digital Interventions to Improve Asthma Self-Management Outcomes: Systematic Review. Journal of medical Internet research. 2018 Dec 12;20(12):e293. doi: 10.2196/jmir.9666. PMID: 30541741.

100. Kew KM, Cates CJ. Home telemonitoring and remote feedback between clinic visits for asthma. The Cochrane database of systematic reviews. 2016 Aug 3(8):Cd011714. doi: 10.1002/14651858.CD011714.pub2. PMID: 27486836. 
101. Kew KM, Malik P, Aniruddhan K, et al. Shared decision-making for people with asthma. The Cochrane database of systematic reviews. 2017 Oct 3;10:Cd012330. doi: 10.1002/14651858.CD012330.pub2. PMID: 28972652.

102. Clayman ML, Bylund CL, Chewning B, et al. The Impact of Patient Participation in Health Decisions Within Medical Encounters: A Systematic Review. Medical decision making : an international journal of the Society for Medical Decision Making. 2016 May;36(4):427-52. doi: 10.1177/0272989x15613530. PMID: 26585293.

103. Niznik JD, He H, Kane-Gill SL. Impact of clinical pharmacist services delivered via telemedicine in the outpatient or ambulatory care setting: A systematic review. Research in social \& administrative pharmacy : RSAP. 2018 Aug;14(8):707-17. doi: 10.1016/j.sapharm.2017.10.011. PMID: 29100941.

104. Hill B, Richardson B, Skouteris H. Do we know how to design effective health coaching interventions: a systematic review of the state of the literature. American journal of health promotion : AJHP. 2015 May-Jun;29(5):e158-68. doi: 10.4278/ajhp.130510-LIT-238. PMID: 24720388 .

105. Pamungkas RA, Chamroonsawasdi K, Vatanasomboon P. A Systematic Review: Family Support Integrated with Diabetes Self-Management among Uncontrolled Type II Diabetes Mellitus Patients. Behavioral sciences (Basel, Switzerland). 2017 Sep 15;7(3). doi: 10.3390/bs7030062. PMID: 28914815.

106. Graham CD, Gouick J, Krahe C, et al. A systematic review of the use of Acceptance and Commitment Therapy (ACT) in chronic disease and long-term conditions. Clinical psychology review. 2016 Jun;46:46-58. doi: 10.1016/j.cpr.2016.04.009. PMID: 27176925.

107. Chi NC, Demiris G. A systematic review of telehealth tools and interventions to support family caregivers. Journal of telemedicine and telecare. 2015 Jan;21(1):37-44. doi: 10.1177/1357633x14562734. PMID: 25475220 .
108. Barello S, Triberti S, Graffigna G, et al. eHealth for Patient Engagement: A Systematic Review. Frontiers in psychology. 2015;6:2013. doi: 10.3389/fpsyg.2015.02013. PMID: 26779108.

109. Voruganti T, Grunfeld E, Makuwaza T, et al. Web-Based Tools for Text-Based Patient-Provider Communication in Chronic Conditions: Scoping Review. Journal of medical Internet research. 2017 Oct 27;19(10):e366. doi: 10.2196/jmir.7987. PMID: 29079552.

110. Winston K, Grendarova P, Rabi D. Videobased patient decision aids: A scoping review. Patient education and counseling. 2018 Apr;101(4):558-78. doi: 10.1016/j.pec.2017.10.009. PMID: 29102063.

111. Bashi N, Fatehi F, Fallah M, et al. SelfManagement Education Through mHealth: Review of Strategies and Structures. JMIR mHealth and uHealth. 2018 Oct 19;6(10):e10771. doi: 10.2196/10771. PMID: 30341042.

112. Kruse CS, Argueta DA, Lopez L, et al. Patient and provider attitudes toward the use of patient portals for the management of chronic disease: a systematic review. Journal of medical Internet research. 2015 Feb 20;17(2):e40. doi: 10.2196/jmir.3703. PMID: 25707035.

113. Scholl I, LaRussa A, Hahlweg P, et al. Organizational- and system-level characteristics that influence implementation of shared decision-making and strategies to address them - a scoping review. Implementation science : IS. 2018 Mar 9;13(1):40. doi: 10.1186/s13012-018-0731z. PMID: 29523167.

114. McBain H, Shipley M, Newman S. The impact of self-monitoring in chronic illness on healthcare utilisation: a systematic review of reviews. BMC health services research. 2015 Dec 18;15:565. doi: 10.1186/s12913-015-1221-5. PMID: 26684011. 
115. Anderson RJ, Bloch S, Armstrong M, et al. Communication between healthcare professionals and relatives of patients approaching the end-of-life: A systematic review of qualitative evidence. Palliative medicine. 2019 Sep;33(8):926-41. doi: 10.1177/0269216319852007. PMID: 31184529 .

116. Legare F, Adekpedjou R, Stacey D, et al. Interventions for increasing the use of shared decision making by healthcare professionals. The Cochrane database of systematic reviews. $2018 \mathrm{Jul}$ 19;7:Cd006732. doi: 10.1002/14651858.CD006732.pub4. PMID: 30025154.

117. Oldfield BJ, Harrison MA, Genao I, et al. Patient, Family, and Community Advisory Councils in Health Care and Research: a Systematic Review. Journal of general internal medicine. 2019 Jul;34(7):1292-303. doi: 10.1007/s11606-018-4565-9. PMID: 30051331.

118. McCarron TL, Moffat K, Wilkinson G, et al. Understanding patient engagement in health system decision-making: a co-designed scoping review. Systematic reviews. 2019 Apr 18;8(1):97. doi: 10.1186/s13643-0190994-8. PMID: 30999950.

119. Bombard Y, Baker GR, Orlando E, et al. Engaging patients to improve quality of care: a systematic review. Implementation science : IS. 2018 Jul 26;13(1):98. doi: 10.1186/s13012-018-0784-z. PMID: 30045735 .

120. Liang L, Cako A, Urquhart R, et al. Patient engagement in hospital health service planning and improvement: a scoping review. BMJ open. 2018 Jan 30;8(1):e018263. doi: 10.1136/bmjopen2017-018263. PMID: 29382676.

121. Sharma AE, Knox M, Mleczko VL, et al. The impact of patient advisors on healthcare outcomes: a systematic review. BMC health services research. 2017 Oct 23;17(1):693. doi: 10.1186/s12913-017-2630-4. PMID: 29058625.
122. Iezzoni LI, Chang Y, Matulewicz H, et al. Health plan enrollees with disability informing primary care practices and providers about their quality of care: A randomized trial. Disability and health journal. 2018 Oct;11(4):537-44. doi: 10.1016/j.dhjo.2018.05.006. PMID: 30054227.

123. Unaka N, Statile A, Jerardi K, et al. Improving the Readability of Pediatric Hospital Medicine Discharge Instructions. Journal of hospital medicine. 2017 Jul;12(7):551-7. doi: 10.12788/jhm.2770. PMID: 28699944.

124. King C, Goldman A, Gampa V, et al. Strengthening the role of Community Health Representatives in the Navajo Nation. BMC public health. 2017 Apr 21;17(1):348. doi: 10.1186/s12889-017-4263-2. PMID: 28431541.

125. Chronic Diseases in America -National Center for Chronic Disease Prevention and Health Promotion.

https://www.cdc.gov/chronicdisease/ resources/infographic/chronicdiseases.htm.

126. Stenberg U, Haaland-Overby M, Fredriksen $\mathrm{K}$, et al. A scoping review of the literature on benefits and challenges of participating in patient education programs aimed at promoting self-management for people living with chronic illness. Patient education and counseling. 2016 Nov;99(11):1759-71. doi: 10.1016/j.pec.2016.07.027. PMID: 27461944.

127. Totten AM, Womack DM, Eden KB, et al. AHRQ Comparative Effectiveness Technical Briefs. Telehealth: Mapping the Evidence for Patient Outcomes From Systematic Reviews. Rockville (MD): Agency for Healthcare Research and Quality (US); 2016.

128. Dounavi K, Tsoumani O. Mobile Health Applications in Weight Management: A Systematic Literature Review. American journal of preventive medicine. 2019 Jun;56(6):894-903. doi: 10.1016/j.amepre.2018.12.005. PMID: 31003801. 
129. Coulter A, Ellins J. Effectiveness of strategies for informing, educating, and involving patients. BMJ (Clinical research ed). 2007 Jul 7;335(7609):24-7. doi: 10.1136/bmj.39246.581169.80. PMID: 17615222.

130. Sharma AE, Rivadeneira NA, Barr-Walker J, et al. Patient Engagement In Health Care Safety: An Overview Of Mixed-Quality Evidence. Health affairs (Project Hope). 2018 Nov;37(11):1813-20. doi: 10.1377/hlthaff.2018.0716. PMID: 30395509.

131. Tang PC, Overhage JM, Chan AS, et al. Online disease management of diabetes: engaging and motivating patients online with enhanced resources-diabetes (EMPOWER-D), a randomized controlled trial. Journal of the American Medical Informatics Association : JAMIA. 2013 May 1;20(3):526-34. doi: 10.1136/amiajnl-2012001263. PMID: 23171659.

132. Zittleman L, Emsermann C, Dickinson M, et al. Increasing colon cancer testing in rural Colorado: evaluation of the exposure to a community-based awareness campaign. BMC public health. 2009 Aug 10;9:288. doi: 10.1186/1471-2458-9-288. PMID: 19664277.

133. Bender BG, Dickinson P, Rankin A, et al. The Colorado Asthma Toolkit Program: a practice coaching intervention from the High Plains Research Network. Journal of the American Board of Family Medicine : JABFM. 2011 May-Jun;24(3):240-8. doi: 10.3122/jabfm.2011.03.100171. PMID: 21551395.

134. Norman N, Bennett C, Cowart S, et al. Boot camp translation: a method for building a community of solution. Journal of the American Board of Family Medicine : JABFM. 2013 May-Jun;26(3):254-63. doi: 10.3122/jabfm.2013.03.120253. PMID: 23657693.

135. Westfall JM, Zittleman L, Sutter C, et al. Testing to prevent colon cancer: results from a rural community intervention. Annals of family medicine. 2013 Nov-Dec;11(6):5007. doi: 10.1370/afm.1582. PMID: 24218373.
136. Kiran T, Davie S, MacLeod P. Citizen Engagement in Primary Care. Annals of family medicine. 2018 Mar;16(2):175. doi: 10.1370/afm.2185. PMID: 29531113.

137. Roland KB, Milliken EL, Rohan EA, et al. Use of Community Health Workers and Patient Navigators to Improve Cancer Outcomes Among Patients Served by Federally Qualified Health Centers: A Systematic Literature Review. Health equity. 2017;1(1):61-76. doi: 10.1089/heq.2017.0001. PMID: 28905047.

138. Radhakrishnan K, Xie B, Berkley A, et al. Barriers and Facilitators for Sustainability of Tele-Homecare Programs: A Systematic Review. Health services research. 2016 Feb;51(1):48-75. doi: 10.1111/14756773.12327. PMID: 26119048.

139. Dogba MJ, Dossa AR, Breton E, et al. Using information and communication technologies to involve patients and the public in health education in rural and remote areas: a scoping review. BMC health services research. 2019 Feb 19;19(1):128. doi: 10.1186/s12913-019-3906-7. PMID: 30782147.

140. Young A, Menon D, Street J, et al. Exploring patient and family involvement in the lifecycle of an orphan drug: a scoping review. Orphanet journal of rare diseases. 2017 Dec 22;12(1):188. doi: 10.1186/s13023-017-0738-6. PMID: 29273068.

141. Menon D, Stafinski T, Dunn A, et al. Involving patients in reducing decision uncertainties around orphan and ultraorphan drugs: a rare opportunity? The patient. 2015 Feb;8(1):29-39. doi: 10.1007/s40271-014-0106-8. PMID: 25516506.

142. About Learning Health Systems. Content last reviewed May 2019. Agency for Healthcare Research and Quality, Rockville, MD. 2019.

https://www.ahrq.gov/learninghealth-systems/about.html. 
143. A Roadmap for Patient and Family Engagement in Healthcare- Practice and Research Practical strategies for advancing engagement in healthcare-starting today. https://www.air.org/sites/default/files /Roadmap-Patient-FamilyEngagement.pdf.

144. Dukhanin V, Topazian R, DeCamp M. Metrics and Evaluation Tools for Patient Engagement in Healthcare Organizationand System-Level Decision-Making: A Systematic Review. International journal of health policy and management. 2018 Oct 1;7(10):889-903. doi:

10.15171/ijhpm.2018.43. PMID: 30316241.
145. Currie SR, Liu P, Adamyk-Simpson J, et al. Validation of a Comprehensive Patient Experience Survey for Addiction and Mental Health that was Co-designed with Service Users. Community mental health journal. 2020 Jan 1. doi: 10.1007/s10597019-00534-1. PMID: 31893327.

146. Knapp EA, Bennett WL, Wilson RF, et al. Methods and Risks of Bias in Natural Experiments in Obesity: Opportunities for the Future Informed by a Systematic

Review. Obesity (Silver Spring, Md). 2019 Dec;27(12):1950-7. doi: 10.1002/oby.22645. PMID: 31693802. 


\section{Appendix A. Search Strategies}

\section{PubMed Search}

\begin{tabular}{|c|c|}
\hline \# & ring \\
\hline $\begin{array}{l}\text { 1. } \\
\text { Population }\end{array}$ & $\begin{array}{l}\text { Patient Participation[Mesh] OR "patient participation"[tiab] OR "patient engagement" [tiab] } \\
\text { OR "patient involvement" [tiab] OR "patient empowerment" [tiab] OR "patient partnership" } \\
\text { [tiab] OR "patient activation" [tiab] OR "patient-activated" [tiab] OR "family participation"[tiab] } \\
\text { OR "family engagement" [tiab] OR "family involvement" [tiab] OR "family empowerment" [tiab] } \\
\text { OR "family partnership" [tiab] OR "family activation" [tiab] OR "consumer participation"[tiab] } \\
\text { OR "consumer engagement" [tiab] OR "consumer involvement" [tiab] OR "consumer } \\
\text { empowerment" [tiab] OR "consumer partnership" [tiab] OR "consumer activation" [tiab] OR } \\
\text { "caregiver participation"[tiab] OR "caregiver engagement" [tiab] OR "caregiver involvement" } \\
\text { [tiab] OR "caregiver empowerment" [tiab] OR "caregiver activation" [tiab] OR "patient context" } \\
\text { [tiab] OR "patient capacity" [tiab] OR "patients capacity" [tiab] }\end{array}$ \\
\hline $\begin{array}{l}2 . \\
\text { Intervention }\end{array}$ & $\begin{array}{l}\text { Advisory Committees [Mesh] OR PFAC [tiab] OR "patient council" [tiab] OR "patient } \\
\text { committee"[tiab] OR "patient advisor" [tiab] OR "family council" [tiab] OR "consumer council" } \\
\text { [tiab] OR "family advisor" [tiab] OR "advisory council" [tiab] OR "community advisory" [tiab] } \\
\text { OR Self-Management [mh] OR "Self-Management" [tiab] OR "Self Management" [tiab] OR } \\
\text { Shared Decision Making [mh] OR "decision making"[tiab] OR "decision-making" [tiab] OR } \\
\text { "Speaking up" [tiab] OR telemedicine [mh] OR Electronic Health Records [mh] OR "Electronic } \\
\text { Health Records" [tiab] OR "Medical Informatics"[mh] OR "health informatics"[tiab] OR "mobile } \\
\text { health" [tiab] OR "eHealth" [tiab] OR "digital health"[tiab] OR "smart phone"[tiab] OR "mobile } \\
\text { app"[tiab] OR "mobile applications"[tiab] OR "mHealth"[tiab] OR "smartphones" [tiab] OR } \\
\text { Patient Portals [mh] OR "Patient Portals" [tiab] OR Patient Access to Records [mh] OR } \\
\text { "Patient Access to Records"[tiab] OR "Opennotes" [tiab] OR Health Literacy [mh] OR literacy } \\
\text { [tiab] OR hospice [tiab] OR palliative [tiab] OR "end-of-life" [tiab] OR "end of life"[tiab] OR } \\
\text { terminal care [mh] OR Palliative Care [mh] OR "patient safety"[tiab] OR "decision } \\
\text { support"[tiab] OR Advance Care Planning [mh] OR "Advance Care Planning" [tiab] OR } \\
\text { "Advance Directives" [tiab] OR "peer support"[tiab] OR "social support"[tiab] OR "family } \\
\text { support"[tiab] OR "healthcare professional support" [tiab] OR "patient navigator" [tiab] OR } \\
\text { Accountable Care Organizations[mh] OR Population Health Management [MH] OR } \\
\text { "Accountable Care Organizations"[tiab] OR "Population Health Management" [tiab] }\end{array}$ \\
\hline
\end{tabular}




\begin{tabular}{|c|c|}
\hline \# & String \\
\hline 1 AND 2 & 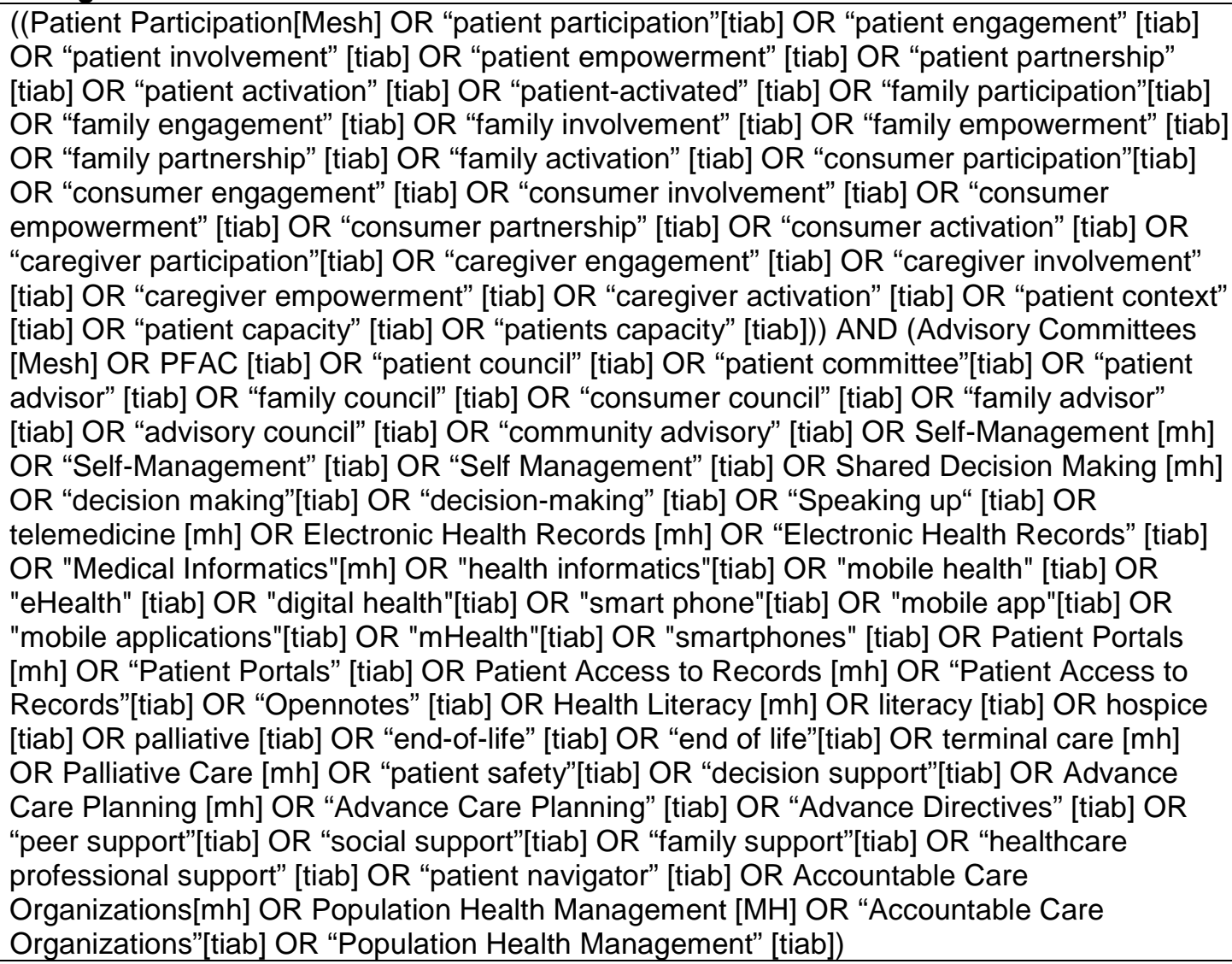 \\
\hline $\begin{array}{l}\text { 4. Limit } \\
2015 \text { - } \\
\text { present }\end{array}$ & Filters activated: Publication date from 2015/01/01 to Present \\
\hline $\begin{array}{l}\text { 5. Limit to } \\
\text { systematic } \\
\text { reviews }\end{array}$ & \#4 AND Cochrane validated systematic review search \\
\hline $\begin{array}{l}\text { 6. Original } \\
\text { articles } \\
\text { without } \\
\text { systematic } \\
\text { reviews }\end{array}$ & \#4 AND without Cochrane validated systematic review search \\
\hline
\end{tabular}




\section{Appendix B. Evidence Tables}

\section{Direct Patient Care}

Evidence Table 1. Study characteristics of included systematic reviews addressing direct patient care

\begin{tabular}{|c|c|c|c|c|c|c|c|}
\hline $\begin{array}{l}\text { Type of } \\
\text { Strategy }\end{array}$ & Author, Year & $\begin{array}{l}\text { Objective of } \\
\text { Review }\end{array}$ & $\begin{array}{l}\text { Literature } \\
\text { Start - } \\
\text { End Year }\end{array}$ & Inclusion Criteria & Exclusion Criteria & $\begin{array}{l}\text { Number of } \\
\text { Included Studies }\end{array}$ & Settings \\
\hline $\begin{array}{l}\text { Advanced } \\
\text { Care } \\
\text { Planning - } \\
\text { Adult Only }\end{array}$ & $\begin{array}{l}\text { Coulter, } \\
2015^{1}\end{array}$ & $\begin{array}{l}\text { To assess the } \\
\text { effects of } \\
\text { personalized care } \\
\text { planning for adults } \\
\text { with long-term } \\
\text { health conditions } \\
\text { compared to usual } \\
\text { care (i.e. forms of } \\
\text { care in which } \\
\text { active involvement } \\
\text { of patients in } \\
\text { treatment and } \\
\text { management } \\
\text { decisions is not } \\
\text { explicitly attempted } \\
\text { or achieved). }\end{array}$ & $\begin{array}{l}\text { Inception- } \\
2013\end{array}$ & $\begin{array}{l}\text { We included } \\
\text { randomized controlled } \\
\text { trials and cluster- } \\
\text { randomized trials } \\
\text { involving adults with } \\
\text { long-term conditions } \\
\text { where the intervention } \\
\text { included collaborative } \\
\text { (between individual } \\
\text { patients and clinicians) } \\
\text { goal setting and action } \\
\text { planning }\end{array}$ & $\begin{array}{l}\text { We excluded } \\
\text { studies where there } \\
\text { was little or no } \\
\text { opportunity for the } \\
\text { patient to have } \\
\text { meaningful } \\
\text { influence on goal } \\
\text { selection, choice of } \\
\text { treatment or } \\
\text { support package, } \\
\text { or both. }\end{array}$ & $\begin{array}{l}\text { Total: } 19 \\
\text { RCTs: } 19 \\
\text { Non-RCTs with } \\
\text { comparison: } 0 \\
\text { Observational: } 0\end{array}$ & Outpatient clinics \\
\hline
\end{tabular}




\begin{tabular}{|c|c|c|c|c|c|c|c|}
\hline $\begin{array}{l}\text { Type of } \\
\text { Strategy }\end{array}$ & Author, Year & $\begin{array}{l}\text { Objective of } \\
\text { Review }\end{array}$ & $\begin{array}{l}\text { Literature } \\
\text { Start - } \\
\text { End Year }\end{array}$ & Inclusion Criteria & Exclusion Criteria & $\begin{array}{l}\text { Number of } \\
\text { Included Studies }\end{array}$ & Settings \\
\hline $\begin{array}{l}\text { Health } \\
\text { Literacy - } \\
\text { Adult Only }\end{array}$ & $\begin{array}{l}\text { Schaepe, } \\
2015^{2}\end{array}$ & $\begin{array}{l}\text { To review the } \\
\text { current literature } \\
\text { on educational } \\
\text { interventions used } \\
\text { in peritoneal } \\
\text { dialysis (PD). }\end{array}$ & $2006-2013$ & $\begin{array}{l}\text { (1) Types of } \\
\text { participants: adult } \\
\text { participants (>18 } \\
\text { years), (a) training } \\
\text { others in PD and (b) } \\
\text { receiving PD training } \\
\text { themselves; (2) Types } \\
\text { of intervention: } \\
\text { educational } \\
\text { interventions; (3) } \\
\text { Types of outcome } \\
\text { measures: no } \\
\text { limitations were } \\
\text { imposed on outcome } \\
\text { measures (4) Study } \\
\text { design: systematic } \\
\text { reviews, meta- } \\
\text { analyses, primary } \\
\text { qualitative and } \\
\text { quantitative research } \\
\text { studies (5) Setting: } \\
\text { clinical or home (6) } \\
\text { Publication: academic } \\
\text { journal (peer reviewed) } \\
\text { (7) Language: English } \\
\text { or German }\end{array}$ & $\begin{array}{l}\text { (1) Studies } \\
\text { focusing on } \\
\text { hemodialysis, } \\
\text { studies focusing on } \\
\text { both hemodialysis } \\
\text { and peritoneal } \\
\text { dialysis; (2) Case } \\
\text { studies or very } \\
\text { small samples n < } \\
\text { 6; (3) Quantitative } \\
\text { studies using } \\
\text { descriptive } \\
\text { methods and non- } \\
\text { statistical methods; } \\
\text { (4) Studies without } \\
\text { education }\end{array}$ & $\begin{array}{l}\text { Total: } 18 \\
\text { RCTs: } 2 \\
\text { Non-RCTs with } \\
\text { comparison: } 0 \\
\text { Observational:16 }\end{array}$ & $\begin{array}{l}\text { Inpatient, outpatient } \\
\text { clinics, home }\end{array}$ \\
\hline
\end{tabular}




\begin{tabular}{|c|c|c|c|c|c|c|c|}
\hline $\begin{array}{l}\text { Type of } \\
\text { Strategy }\end{array}$ & Author, Year & $\begin{array}{l}\text { Objective of } \\
\text { Review }\end{array}$ & $\begin{array}{l}\text { Literature } \\
\text { Start - } \\
\text { End Year }\end{array}$ & Inclusion Criteria & Exclusion Criteria & $\begin{array}{l}\text { Number of } \\
\text { Included Studies }\end{array}$ & Settings \\
\hline \multirow[t]{2}{*}{$\begin{array}{l}\text { Self- } \\
\text { Management } \\
\text { Support - } \\
\text { Children } \\
\text { Only }\end{array}$} & Bal, $2015^{3}$ & $\begin{array}{l}\text { To systematically } \\
\text { explore the } \\
\text { effectiveness and } \\
\text { effective } \\
\text { components of } \\
\text { self-management } \\
\text { interventions }\end{array}$ & $2000-2015$ & $\begin{array}{l}\text { RCT; English, 2003- } \\
\text { 2015; studies focusing } \\
\text { on the evaluation of } \\
\text { SMl and describing the } \\
\text { SMI or referring to } \\
\text { previous description(s) } \\
\text { of the intervention; } \\
\text { Outcome measures: } \\
\text { studies considering } \\
\text { clearly defined } \\
\text { outcome measures; } \\
\text { Participants: studies } \\
\text { focusing on young } \\
\text { people aged 7-25 } \\
\text { years with somatic } \\
\text { chronic conditions or } \\
\text { physical disability. The } \\
\text { age of seven years is } \\
\text { considered a } \\
\text { developmentally } \\
\text { appropriate age to start } \\
\text { the development of } \\
\text { independence. As } \\
\text { young adults are still } \\
\text { developing their full } \\
\text { potential, the age } \\
\text { range was extended } \\
\text { from } 18 \text { to } 25 \text { years. }\end{array}$ & None listed & $\begin{array}{l}\text { Total:42 } \\
\text { RCTs:42 } \\
\text { Non-RCTs with } \\
\text { comparison:0 } \\
\text { Observational:0 }\end{array}$ & $\begin{array}{l}\text { Clinics, home, } \\
\text { school, camps }\end{array}$ \\
\hline & Low, $2019^{4}$ & $\begin{array}{l}\text { This study aimed } \\
\text { to evaluate the } \\
\text { current evidence } \\
\text { on Web- or mobile- } \\
\text { based } \\
\text { interventions } \\
\text { designed for } \\
\text { adolescent and } \\
\text { young adults. }\end{array}$ & $1967-2019$ & $\begin{array}{l}\text { i.No restrictions on the } \\
\text { study design, ii. } \\
\text { Intervention must be } \\
\text { freely available on } \\
\text { device or web page iii. } \\
\text { Participants aged }<18 \\
\text { years or } \geq 18 \text { years with } \\
\text { chronic diseases }\end{array}$ & $\begin{array}{l}\text { Unclear indication } \\
\text { for pediatrics vs } \\
\text { adults } \\
\text { interventions. }\end{array}$ & $\begin{array}{l}\text { Total:29 } \\
\text { RCTs:6 } \\
\text { Non-RCTs with } \\
\text { comparison:2 } \\
\text { Observational:21 }\end{array}$ & Home \\
\hline
\end{tabular}




\begin{tabular}{|c|c|c|c|c|c|c|c|}
\hline $\begin{array}{l}\text { Type of } \\
\text { Strategy }\end{array}$ & Author, Year & $\begin{array}{l}\text { Objective of } \\
\text { Review }\end{array}$ & $\begin{array}{l}\text { Literature } \\
\text { Start - } \\
\text { End Year }\end{array}$ & Inclusion Criteria & Exclusion Criteria & $\begin{array}{l}\text { Number of } \\
\text { Included Studies }\end{array}$ & Settings \\
\hline & Saxby, $2018^{5}$ & $\begin{array}{l}\text { To articulate the } \\
\text { components of } \\
\text { educational } \\
\text { interventions that } \\
\text { help children learn } \\
\text { about managing } \\
\text { their asthma, cystic } \\
\text { fibrosis, or } \\
\text { diabetes, relevant } \\
\text { to their } \\
\text { age/developmental } \\
\text { status }\end{array}$ & $\begin{array}{l}\text { Not } \\
\text { mentioned- } \\
2018\end{array}$ & $\begin{array}{l}\text { Studies were included } \\
\text { if they were published } \\
\text { before } 27 \text { January } \\
2018 \text {, and if they } \\
\text { focused on children } \\
\text { and adolescents (aged } \\
20 \text { years) with asthma, } \\
\text { T1DM, or CF as active } \\
\text { participants in the } \\
\text { CCSM education } \\
\text { strategy. }\end{array}$ & $\begin{array}{l}\text { Studies were } \\
\text { excluded if they } \\
\text { focused on adults, } \\
\text { other childhood } \\
\text { conditions, or if } \\
\text { they did not contain } \\
\text { age/ } \\
\text { developmentally } \\
\text { based CCSM } \\
\text { education } \\
\text { components. }\end{array}$ & $\begin{array}{l}\text { Total:30 } \\
\text { RCTs:23 } \\
\text { Non-RCTs with } \\
\text { comparison:1 } \\
\text { Observational:6 }\end{array}$ & $\begin{array}{l}\text { School, hospital, } \\
\text { home }\end{array}$ \\
\hline & Knafl, $2017^{6}$ & $\begin{array}{l}\text { Based on a sample } \\
\text { of } 70 \text { interventions } \\
\text { for families in } \\
\text { which there was a } \\
\text { child with a chronic } \\
\text { physical condition, } \\
\text { this analysis } \\
\text { examined the } \\
\text { nature of family } \\
\text { engagement in the } \\
\text { interventions }\end{array}$ & $2000-2014$ & $\begin{array}{l}\text { (a) Randomized clinical } \\
\text { trial (RCT) design; (b) } \\
\text { published in an } \\
\text { English- language } \\
\text { journal between } \\
\text { January } 1,2000 \text {, and } \\
\text { March } 31,2014 \text {; and } \\
\text { (c) intervention } \\
\text { included families in } \\
\text { which a child less than } \\
19 \text { years old had a } \\
\text { CPC. }\end{array}$ & None listed & $\begin{array}{l}\text { Total:93 } \\
\text { RCTs:93 } \\
\text { Non-RCTs with } \\
\text { comparison:0 } \\
\text { Observational:0 }\end{array}$ & Home \\
\hline
\end{tabular}




\begin{tabular}{|c|c|c|c|c|c|c|c|}
\hline $\begin{array}{l}\text { Type of } \\
\text { Strategy }\end{array}$ & Author, Year & $\begin{array}{l}\text { Objective of } \\
\text { Review }\end{array}$ & $\begin{array}{l}\text { Literature } \\
\text { Start - } \\
\text { End Year }\end{array}$ & Inclusion Criteria & Exclusion Criteria & $\begin{array}{l}\text { Number of } \\
\text { Included Studies }\end{array}$ & Settings \\
\hline & $\begin{array}{l}\text { Campbell, } \\
2016^{7}\end{array}$ & $\begin{array}{l}\text { To evaluate the } \\
\text { effectiveness of } \\
\text { interventions } \\
\text { designed to } \\
\text { improve the } \\
\text { transition of care } \\
\text { for adolescents } \\
\text { from pediatric to } \\
\text { adult health } \\
\text { services }\end{array}$ & $\begin{array}{l}\text { Inception- } \\
2015\end{array}$ & $\begin{array}{l}\text { Randomized controlled } \\
\text { trials (RCTs), } \\
\text { controlled before- and } \\
\text { after-studies (CBAs), } \\
\text { and interrupted time- } \\
\text { series studies (ITSs); } \\
\text { We included } \\
\text { adolescents with } \\
\text { conditions that } \\
\text { required ongoing } \\
\text { clinical care, who } \\
\text { would be leaving } \\
\text { pediatric services and } \\
\text { would require on- } \\
\text { going services in adult } \\
\text { healthcare units, or } \\
\text { had already transferred } \\
\text { to adult services, and } \\
\text { their families, parents, } \\
\text { or guardians }\end{array}$ & None listed & $\begin{array}{l}\text { Total:4 } \\
\text { RCTs:4 } \\
\text { Non-RCTs with } \\
\text { comparison:0 } \\
\text { Observational:0 }\end{array}$ & Hospital \\
\hline
\end{tabular}




\begin{tabular}{|c|c|c|c|c|c|c|c|}
\hline $\begin{array}{l}\text { Type of } \\
\text { Strategy }\end{array}$ & Author, Year & $\begin{array}{l}\text { Objective of } \\
\text { Review }\end{array}$ & $\begin{array}{l}\text { Literature } \\
\text { Start - } \\
\text { End Year }\end{array}$ & Inclusion Criteria & Exclusion Criteria & $\begin{array}{l}\text { Number of } \\
\text { Included Studies }\end{array}$ & Settings \\
\hline & $\begin{array}{l}\text { Majeed-Ariss, } \\
2015^{8}\end{array}$ & $\begin{array}{l}\text { To systematically } \\
\text { review the } \\
\text { literature on the } \\
\text { effectiveness of } \\
\text { mobile apps } \\
\text { designed to } \\
\text { support } \\
\text { adolescents' } \\
\text { management of } \\
\text { their physical } \\
\text { chronic or long- } \\
\text { term conditions. }\end{array}$ & $2003-2015$ & $\begin{array}{l}\text { Adolescents aged 10- } \\
24 \text { years; diagnosed } \\
\text { with chronic physical } \\
\text { conditions in any } \\
\text { setting; Any app for a } \\
\text { mobile phone or tablet } \\
\text { that could be } \\
\text { considered a } \\
\text { management } \\
\text { intervention (or a } \\
\text { component of an } \\
\text { intervention) in terms } \\
\text { of content and/or } \\
\text { delivery; Intervention } \\
\text { versus usual care or } \\
\text { intervention variant } \\
\text { versus intervention } \\
\text { variant or pre and post; } \\
\text { Randomized controlled } \\
\text { trial (RCT) or controlled } \\
\text { clinical trial or cohort } \\
\text { analytic study or case- } \\
\text { control study or cohort } \\
\text { study or interrupted } \\
\text { time series. }\end{array}$ & $\begin{array}{l}\text { Non-English- } \\
\text { language } \\
\text { publications and } \\
\text { studies that } \\
\text { focused on } \\
\text { adolescents with } \\
\text { mental health } \\
\text { problems, learning } \\
\text { disabilities, and/or } \\
\text { cognitive } \\
\text { impairment were } \\
\text { excluded due to } \\
\text { resource } \\
\text { limitations. } \\
\text { Interventions using } \\
\text { mobile phone } \\
\text { technology only in } \\
\text { the context of } \\
\text { delivering/receiving } \\
\text { text messages or } \\
\text { phone calls were } \\
\text { also excluded. }\end{array}$ & $\begin{array}{l}\text { Total:4 } \\
\text { RCTs:1 } \\
\text { Non-RCTs with } \\
\text { comparison:0 } \\
\text { Observational:3 }\end{array}$ & Home \\
\hline
\end{tabular}




\begin{tabular}{|c|c|c|c|c|c|c|c|}
\hline $\begin{array}{l}\text { Type of } \\
\text { Strategy }\end{array}$ & Author, Year & $\begin{array}{l}\text { Objective of } \\
\text { Review }\end{array}$ & $\begin{array}{l}\text { Literature } \\
\text { Start - } \\
\text { End Year }\end{array}$ & Inclusion Criteria & Exclusion Criteria & $\begin{array}{l}\text { Number of } \\
\text { Included Studies }\end{array}$ & Settings \\
\hline & $\begin{array}{l}\text { Charlier, } \\
2015^{9}\end{array}$ & $\begin{array}{l}\text { To conduct a } \\
\text { systematic review } \\
\text { and meta-analysis } \\
\text { of randomized } \\
\text { controlled trials } \\
\text { assessing the } \\
\text { effectiveness of } \\
\text { serious games in } \\
\text { improving } \\
\text { knowledge and/or } \\
\text { self-management } \\
\text { behaviors in young } \\
\text { people with chronic } \\
\text { conditions. }\end{array}$ & $1990-2004$ & $\begin{array}{l}\text { 1) RCTs that compared } \\
\text { a digital game (serious } \\
\text { game or commercial) } \\
\text { with either standard } \\
\text { education or no } \\
\text { specific education, 2) a } \\
\text { study population of } \\
\text { children or adolescents } \\
\text { with chronic conditions } \\
\text { at any stage of } \\
\text { disease, and 3) a } \\
\text { quantitative } \\
\text { assessment of } \\
\text { patients' knowledge } \\
\text { and/or self- } \\
\text { management as one of } \\
\text { the outcomes } \\
\text { variables. }\end{array}$ & $\begin{array}{l}\text { Articles referring to } \\
\text { computer game } \\
\text { interventions in } \\
\text { relation to health } \\
\text { promotion } \\
\text { programs in } \\
\text { preventive } \\
\text { healthcare; } \\
\text { focusing on } \\
\text { symptom } \\
\text { management or } \\
\text { distraction without } \\
\text { measuring } \\
\text { behaviors; focusing } \\
\text { on measurement } \\
\text { and diagnostic } \\
\text { methods; and on } \\
\text { game theory, game } \\
\text { development, and } \\
\text { evaluation were } \\
\text { excluded. }\end{array}$ & $\begin{array}{l}\text { Total:9 } \\
\text { RCTs:9 } \\
\text { Non-RCTs with } \\
\text { comparison:0 } \\
\text { Observational:0 }\end{array}$ & Hospital and home \\
\hline & $\begin{array}{l}\text { Sattoe, } \\
2015^{10}\end{array}$ & $\begin{array}{l}\text { To provide a } \\
\text { systematic } \\
\text { overview of self- } \\
\text { management } \\
\text { interventions (SMI) } \\
\text { for young people } \\
\text { with chronic } \\
\text { conditions with } \\
\text { respect to content, } \\
\text { formats, theories, } \\
\text { and evaluated } \\
\text { outcomes. }\end{array}$ & $2003-2014$ & $\begin{array}{l}\text { Original research } \\
\text { articles in English, } \\
\text { published from 2003- } \\
\text { 2014; ages 7-25 with } \\
\text { somatic chronic } \\
\text { disease or disability; } \\
\text { studies focusing on the } \\
\text { evaluation of an SMI } \\
\text { and describing the SMI } \\
\text { or referring to previous } \\
\text { description(s) of the } \\
\text { intervention }\end{array}$ & None listed & $\begin{array}{l}\text { Total:86 } \\
\text { RCTs:45 } \\
\text { Non-RCTs with } \\
\text { comparison:29 } \\
\text { Observational:12 }\end{array}$ & $\begin{array}{l}\text { Home, clinic, } \\
\text { hospital, camp, } \\
\text { online }\end{array}$ \\
\hline
\end{tabular}




\begin{tabular}{|c|c|c|c|c|c|c|c|}
\hline $\begin{array}{l}\text { Type of } \\
\text { Strategy }\end{array}$ & Author, Year & $\begin{array}{l}\text { Objective of } \\
\text { Review }\end{array}$ & $\begin{array}{l}\text { Literature } \\
\text { Start - } \\
\text { End Year }\end{array}$ & Inclusion Criteria & Exclusion Criteria & $\begin{array}{l}\text { Number of } \\
\text { Included Studies }\end{array}$ & Settings \\
\hline & $\begin{array}{l}\text { Hamline, } \\
2018^{11}\end{array}$ & $\begin{array}{l}\text { Which pediatric } \\
\text { hospital discharge } \\
\text { interventions affect } \\
\text { health care use or } \\
\text { parental } \\
\text { satisfaction with } \\
\text { care }\end{array}$ & $\begin{array}{l}\text { Inception- } \\
2017\end{array}$ & $\begin{array}{l}\text { Inclusion criteria were: } \\
\text { (1) available in English, } \\
\text { (2) focused on children } \\
<18 \text { years of age, (3) } \\
\text { pediatric data reported } \\
\text { separately from adult } \\
\text { data, (4) not focused } \\
\text { on normal newborns or } \\
\text { pregnancy, (5) } \\
\text { discharge intervention } \\
\text { implemented in the } \\
\text { inpatient setting, and } \\
\text { (6) outcomes of health } \\
\text { care use or caregiver } \\
\text { satisfaction. }\end{array}$ & $\begin{array}{l}\text { Reviews, case } \\
\text { studies, and } \\
\text { commentaries were } \\
\text { excluded. }\end{array}$ & $\begin{array}{l}\text { Total:31 } \\
\text { RCTs:5 } \\
\text { Non-RCTs with } \\
\text { comparison:25 } \\
\text { Observational:1 }\end{array}$ & Hospital \\
\hline & $\begin{array}{l}\text { Clemente, } \\
2016^{12}\end{array}$ & $\begin{array}{l}\text { Identify existing } \\
\text { models of } \\
\text { transitional care in } \\
\text { rheumatic and } \\
\text { musculoskeletal } \\
\text { diseases (RMD), } \\
\text { describe their } \\
\text { strengths and } \\
\text { weaknesses, and } \\
\text { provide support to } \\
\text { a consensus } \\
\text { initiative to develop } \\
\text { recommendations } \\
\text { for transitional } \\
\text { care. }\end{array}$ & $\begin{array}{l}\text { Inception- } \\
2014\end{array}$ & $\begin{array}{l}\text { Studies were selected } \\
\text { if they described valid } \\
\text { transition programs in } \\
\text { jRMDs. The term valid } \\
\text { referred to programs } \\
\text { that had been } \\
\text { described in sufficient } \\
\text { details as to be } \\
\text { reproducible and had } \\
\text { been implemented and } \\
\text { tested in real life } \\
\text { conditions). }\end{array}$ & None listed & Total:27 & Hospital \\
\hline
\end{tabular}




\begin{tabular}{|c|c|c|c|c|c|c|c|}
\hline $\begin{array}{l}\text { Type of } \\
\text { Strategy }\end{array}$ & Author, Year & $\begin{array}{l}\text { Objective of } \\
\text { Review }\end{array}$ & $\begin{array}{l}\text { Literature } \\
\text { Start - } \\
\text { End Year }\end{array}$ & Inclusion Criteria & Exclusion Criteria & $\begin{array}{l}\text { Number of } \\
\text { Included Studies }\end{array}$ & Settings \\
\hline \multirow[t]{2}{*}{$\begin{array}{l}\text { Self- } \\
\text { Management } \\
\text { Support - } \\
\text { Adults and } \\
\text { Children }\end{array}$} & Niznik, $2018^{13}$ & $\begin{array}{l}\text { Identify the impact } \\
\text { of clinical } \\
\text { pharmacist } \\
\text { telemedicine } \\
\text { interventions on } \\
\text { clinical outcomes, } \\
\text { subsequently } \\
\text { defined as clinical } \\
\text { disease } \\
\text { management, } \\
\text { patient self- } \\
\text { management, and } \\
\text { adherence, in } \\
\text { outpatient or } \\
\text { ambulatory } \\
\text { settings }\end{array}$ & $\begin{array}{l}\text { Inception- } \\
2016\end{array}$ & $\begin{array}{l}\text { Pharmacist } \\
\text { interventions; } \\
\text { outpatient our } \\
\text { ambulatory settings }\end{array}$ & $\begin{array}{l}\text { Studies of non- } \\
\text { clinical outcomes } \\
\text { (i.e. dispensing or } \\
\text { product } \\
\text { preparation) and } \\
\text { with no comparator } \\
\text { were excluded }\end{array}$ & $\begin{array}{l}\text { Total:34 } \\
\text { RCTs:17 } \\
\text { Non-RCTs with } \\
\text { comparison:17 } \\
\text { Observational:0 }\end{array}$ & Hospital \\
\hline & $\begin{array}{l}\text { Barello, } \\
2016^{14}\end{array}$ & $\begin{array}{l}\text { This paper aimed } \\
\text { at reviewing } \\
\text { findings from the } \\
\text { literature about the } \\
\text { use of eHealth in } \\
\text { engaging patients } \\
\text { in their own care } \\
\text { process }\end{array}$ & 2004-2014 & $\begin{array}{l}\text { The eHealth actions } \\
\text { described must have } \\
\text { been performed for the } \\
\text { engagement of } \\
\text { patients (technologies } \\
\text { applied to engage } \\
\text { other health } \\
\text { stakeholders such as } \\
\text { medical staff, hospital } \\
\text { managers, or others } \\
\text { were excluded); The } \\
\text { intervention had to } \\
\text { feature at least one } \\
\text { group of participants } \\
\text { (single cases } \\
\text { excluded); both } \\
\text { between and within } \\
\text { groups designs were } \\
\text { considered; The } \\
\text { intervention had to } \\
\text { assess one or more } \\
\text { variables connected to } \\
\text { patient engagement. }\end{array}$ & $\begin{array}{l}\text { The interventions } \\
\text { used not well- } \\
\text { specified } \\
\text { technologies, or the } \\
\text { technologies used } \\
\text { were not clearly } \\
\text { internet-based (i.e., } \\
\text { telephone); (2) the } \\
\text { terms "patient } \\
\text { engagement," or } \\
\text { "patient activation" } \\
\text { were actually } \\
\text { present in the } \\
\text { paper, but there } \\
\text { were not } \\
\text { referenced }\end{array}$ & $\begin{array}{l}\text { Total:11 } \\
\text { RCTs:3 } \\
\text { Non-RCTs with } \\
\text { comparison:1 } \\
\text { Observational:7 }\end{array}$ & Home \\
\hline
\end{tabular}




\begin{tabular}{|c|c|c|c|c|c|c|c|}
\hline $\begin{array}{l}\text { Type of } \\
\text { Strategy }\end{array}$ & Author, Year & $\begin{array}{l}\text { Objective of } \\
\text { Review }\end{array}$ & $\begin{array}{l}\text { Literature } \\
\text { Start - } \\
\text { End Year }\end{array}$ & Inclusion Criteria & Exclusion Criteria & $\begin{array}{l}\text { Number of } \\
\text { Included Studies }\end{array}$ & Settings \\
\hline & Bashi, $2018^{15}$ & $\begin{array}{l}\text { To investigate } \\
\text { smartphone-based } \\
\text { educational } \\
\text { interventions and } \\
\text { their structures and } \\
\text { strategies for } \\
\text { patient self- } \\
\text { management. }\end{array}$ & $2006-2016$ & $\begin{array}{l}\text { Peer-reviewed studies, } \\
\text { Primary or secondary } \\
\text { studies reporting } \\
\text { clinical trials. }\end{array}$ & $\begin{array}{l}\text { Conference } \\
\text { abstracts, book } \\
\text { reviews, letters, } \\
\text { editorials, case } \\
\text { reports and } \\
\text { unpublished } \\
\text { studies were } \\
\text { excluded }\end{array}$ & $\begin{array}{l}\text { Total:15 } \\
\text { RCTs:6 } \\
\text { Non-RCTs with } \\
\text { comparison:0 } \\
\text { Observational:11 }\end{array}$ & Home \\
\hline & Lycett, $2018^{16}$ & $\begin{array}{l}\text { Examine the use } \\
\text { and application of } \\
\text { theory in the } \\
\text { development of } \\
\text { digital interventions } \\
\text { to enhance asthma } \\
\text { self-management } \\
\text { and to evaluate the } \\
\text { effectiveness of } \\
\text { theory-based } \\
\text { interventions in } \\
\text { improving } \\
\text { adherence, self- } \\
\text { management, and } \\
\text { clinical outcomes }\end{array}$ & $\begin{array}{l}\text { Not } \\
\text { mentioned- } \\
2017\end{array}$ & $\begin{array}{l}\text { Paper in English; } \\
\text { Patients with asthma; } \\
\text { Empirical study (pilot, } \\
\text { feasibility, or evaluative } \\
\text { study); Intervention } \\
\text { focused on patient } \\
\text { (rather than physician } \\
\text { or carer); Digital } \\
\text { intervention (eg, online } \\
\text { intervention, smart } \\
\text { phone app, electronic } \\
\text { monitor, short } \\
\text { message service } \\
\text { (SMS), interactive } \\
\text { voice recognition, or } \\
\text { wearable; Intervention } \\
\text { designed to enhance } \\
\text { adherence or } \\
\text { persistence with } \\
\text { asthma medication or } \\
\text { self- management; } \\
\text { Explicit mention of the } \\
\text { use of theory to design } \\
\text { the self-management } \\
\text { intervention or to } \\
\text { increase engagement } \\
\text { with the intervention }\end{array}$ & $\begin{array}{l}\text { Conference } \\
\text { abstracts; Paper } \\
\text { not in English; } \\
\text { Review or letter; } \\
\text { Intervention is } \\
\text { delivered to } \\
\text { parent(s) of } \\
\text { children with } \\
\text { asthma Not an } \\
\text { empirical study; } \\
\text { Clinician focus } \\
\text { (clinician attitude, } \\
\text { behavior, or } \\
\text { diagnostic tool); } \\
\text { Intervention not } \\
\text { designed to } \\
\text { enhance }\end{array}$ & $\begin{array}{l}\text { Total:14 } \\
\text { RCTs:10 } \\
\text { Non-RCTs with } \\
\text { comparison:0 } \\
\text { Observational:4 }\end{array}$ & Home \\
\hline
\end{tabular}




\begin{tabular}{|c|c|c|c|c|c|c|c|}
\hline $\begin{array}{l}\text { Type of } \\
\text { Strategy }\end{array}$ & Author, Year & $\begin{array}{l}\text { Objective of } \\
\text { Review }\end{array}$ & $\begin{array}{l}\text { Literature } \\
\text { Start - } \\
\text { End Year }\end{array}$ & Inclusion Criteria & Exclusion Criteria & $\begin{array}{l}\text { Number of } \\
\text { Included Studies }\end{array}$ & Settings \\
\hline & $\begin{array}{l}\text { Lancaster, } \\
2018^{17}\end{array}$ & $\begin{array}{l}\text { Determine the } \\
\text { impact of patients' } \\
\text { use of eHealth } \\
\text { tools on self- } \\
\text { reporting adverse } \\
\text { effects and } \\
\text { symptoms that } \\
\text { promote changes } \\
\text { to medication use }\end{array}$ & $2000-2018$ & $\begin{array}{l}\text { eHealth tool must have } \\
\text { allowed patients (or } \\
\text { caregivers) to enter } \\
\text { information directly (as } \\
\text { opposed to information } \\
\text { being entered by a } \\
\text { health care provider); } \\
\text { included self-reporting } \\
\text { functionalities focusing } \\
\text { on medication } \\
\text { monitoring, contain a } \\
\text { medication monitoring } \\
\text { or use component, or } \\
\text { specifically } \\
\text { incorporating the } \\
\text { option for the patient or } \\
\text { caregiver to enter } \\
\text { symptoms including } \\
\text { adverse effects; and } \\
\text { needed to focus } \\
\text { specifically on } \\
\text { medication use, clinical } \\
\text { outcomes, or symptom } \\
\text { reporting following use } \\
\text { of the eHealth tool }\end{array}$ & $\begin{array}{l}\text { Exclusion criteria } \\
\text { were conference } \\
\text { abstracts; } \\
\text { qualitative studies; } \\
\text { without a } \\
\text { comparator group; } \\
\text { did not report on at } \\
\text { least one } \\
\text { medication-related } \\
\text { outcome; self- } \\
\text { management } \\
\text { strategies focused } \\
\text { on lifestyle } \\
\text { modification, } \\
\text { behavioral } \\
\text { interventions, or } \\
\text { nondrug } \\
\text { interventions; } \\
\text { focused solely on } \\
\text { the validation of an } \\
\text { eHealth tool; } \\
\text { focused on } \\
\text { methodological or } \\
\text { technical aspects of } \\
\text { eHealth } \\
\text { interventions; } \\
\text { containing } \\
\text { nonempirical } \\
\text { information; } \\
\text { synthesized } \\
\text { information about } \\
\text { multiple eHealth } \\
\text { tools in an article; } \\
\text { and eHealth tools } \\
\text { used } \\
\text { by regulatory } \\
\text { agencies to report } \\
\text { adverse drug } \\
\text { events (ADEs) }\end{array}$ & $\begin{array}{l}\text { Total:14 } \\
\text { RCTs:13 } \\
\text { Non-RCTs with } \\
\text { comparison:1 } \\
\text { Observational:0 }\end{array}$ & Home \\
\hline
\end{tabular}




\begin{tabular}{|c|c|c|c|c|c|c|c|}
\hline $\begin{array}{l}\text { Type of } \\
\text { Strategy }\end{array}$ & Author, Year & $\begin{array}{l}\text { Objective of } \\
\text { Review }\end{array}$ & $\begin{array}{l}\text { Literature } \\
\text { Start - } \\
\text { End Year }\end{array}$ & Inclusion Criteria & Exclusion Criteria & $\begin{array}{l}\text { Number of } \\
\text { Included Studies }\end{array}$ & Settings \\
\hline & Kew, $2016^{18}$ & $\begin{array}{l}\text { To assess the } \\
\text { efficacy and safety } \\
\text { of home } \\
\text { telemonitoring with } \\
\text { healthcare } \\
\text { professional } \\
\text { feedback between } \\
\text { clinic visits, } \\
\text { compared with } \\
\text { usual care for } \\
\text { asthma }\end{array}$ & $1992-2016$ & $\begin{array}{l}\text { We included parallel } \\
\text { randomized controlled } \\
\text { trials (RCTs) of adults } \\
\text { or children with asthma } \\
\text { in which any form of } \\
\text { technology was used } \\
\text { to measure and share } \\
\text { asthma monitoring } \\
\text { data with a healthcare } \\
\text { provider between clinic } \\
\text { visits, compared with } \\
\text { other monitoring or } \\
\text { usual care. }\end{array}$ & $\begin{array}{l}\text { We excluded trials } \\
\text { in which } \\
\text { technologies were } \\
\text { used for monitoring } \\
\text { with no input from a } \\
\text { doctor or nurse }\end{array}$ & $\begin{array}{l}\text { Total:18 } \\
\text { RCTs:18 } \\
\text { Non-RCTs with } \\
\text { comparison:0 } \\
\text { Observational:0 }\end{array}$ & Home \\
\hline & $\begin{array}{l}\text { Graham, } \\
2016^{19}\end{array}$ & $\begin{array}{l}\text { This systematic } \\
\text { review aimed to: } \\
\text { collate all ACT } \\
\text { interventions with } \\
\text { chronic } \\
\text { disease/long-term } \\
\text { conditions, } \\
\text { evaluate their } \\
\text { quality, and } \\
\text { comment on } \\
\text { efficacy }\end{array}$ & $\begin{array}{l}\text { Inception- } \\
2015\end{array}$ & $\begin{array}{l}\text { Studies were included } \\
\text { if they described an } \\
\text { ACT intervention } \\
\text { applied to a long-term } \\
\text { condition }\end{array}$ & $\begin{array}{l}\text { 1) were not } \\
\text { published in } \\
\text { English; 2) } \\
\text { described a } \\
\text { hypothetical } \\
\text { intervention; 3) did } \\
\text { not clearly use ACT } \\
\text { techniques; 4) were } \\
\text { undertaken with a } \\
\text { chronic pain } \\
\text { population or } \\
\text { mental health } \\
\text { population; 5) were } \\
\text { designed to prevent } \\
\text { illness in a group } \\
\text { without a long-term } \\
\text { condition; or 6) } \\
\text { were used to } \\
\text { manage symptoms } \\
\text { where } \\
\text { evidence for } \\
\text { causative biological } \\
\text { pathology is } \\
\text { unclear }\end{array}$ & $\begin{array}{l}\text { Total:18 } \\
\text { RCTs:10 } \\
\text { Non-RCTs with } \\
\text { comparison:0 } \\
\text { Observational:8 }\end{array}$ & Hospital \\
\hline
\end{tabular}




\begin{tabular}{|c|c|c|c|c|c|c|c|}
\hline $\begin{array}{l}\text { Type of } \\
\text { Strategy }\end{array}$ & Author, Year & $\begin{array}{l}\text { Objective of } \\
\text { Review }\end{array}$ & $\begin{array}{l}\text { Literature } \\
\text { Start - } \\
\text { End Year }\end{array}$ & Inclusion Criteria & Exclusion Criteria & $\begin{array}{l}\text { Number of } \\
\text { Included Studies }\end{array}$ & Settings \\
\hline & Yin, $2019^{20}$ & $\begin{array}{l}\text { This study aimed } \\
\text { to characterize the } \\
\text { different tasks and } \\
\text { contexts in which } \\
\text { context-aware } \\
\text { systems for patient } \\
\text { work were used as } \\
\text { well as to assess } \\
\text { any existing } \\
\text { evidence about the } \\
\text { impact of such } \\
\text { systems on health- } \\
\text { related process or } \\
\text { outcome } \\
\text { measures. }\end{array}$ & $\begin{array}{l}\text { Not } \\
\text { mentioned- } \\
2017\end{array}$ & $\begin{array}{l}\text { Chronic conditions, } \\
\text { involved the use of a } \\
\text { context-aware system } \\
\text { to support patients' } \\
\text { health-related } \\
\text { activities, and reported } \\
\text { the evaluation of the } \\
\text { systems by the users }\end{array}$ & $\begin{array}{l}\text { Not in English; } \\
\text { focused on health } \\
\text { care providers } \\
\text { instead of } \\
\text { consumers; } \\
\text { interventions that } \\
\text { merely gathered } \\
\text { and displayed } \\
\text { context information, } \\
\text { without using it to } \\
\text { adapt system } \\
\text { behavior (passive } \\
\text { context awareness) }\end{array}$ & $\begin{array}{l}\text { Total:6 } \\
\text { RCTs:0 } \\
\text { Non-RCTs with } \\
\text { comparison:0 } \\
\text { Observational:6 }\end{array}$ & Home \\
\hline & Chi, $2015^{21}$ & $\begin{array}{l}\text { The present study } \\
\text { aimed to } \\
\text { systematically } \\
\text { review evidence on } \\
\text { the effect of } \\
\text { telehealth } \\
\text { applications on } \\
\text { family caregivers }\end{array}$ & $\begin{array}{l}\text { Not } \\
\text { mentioned- } \\
2014\end{array}$ & $\begin{array}{l}\text { Studies were included } \\
\text { if they used any } \\
\text { telehealth interventions } \\
\text { and focused on family } \\
\text { caregivers' outcomes }\end{array}$ & $\begin{array}{l}\text { Studies were } \\
\text { excluded if they } \\
\text { were not published } \\
\text { in English, did not } \\
\text { involve human } \\
\text { subjects or did not } \\
\text { employ a telehealth } \\
\text { intervention }\end{array}$ & $\begin{array}{l}\text { Total:65 } \\
\text { RCTs:19 } \\
\text { Non-RCTs with } \\
\text { comparison:33 } \\
\text { Observational:14 }\end{array}$ & Hospital and home \\
\hline & Hill, $2015^{22}$ & $\begin{array}{l}\text { To systematically } \\
\text { review health } \\
\text { coaching } \\
\text { interventions } \\
\text { regarding } \\
\text { effectiveness of } \\
\text { health coaching for } \\
\text { specific outcomes, } \\
\text { optimal } \\
\text { intervention } \\
\text { approaches, and } \\
\text { identification of } \\
\text { specific techniques } \\
\text { associated with } \\
\text { effectiveness. }\end{array}$ & $2000-2012$ & $\begin{array}{l}\text { We included RCTs that } \\
\text { used health coaching } \\
\text { to influence health- } \\
\text { related outcomes or } \\
\text { processes. Participants } \\
\text { of any age were } \\
\text { included and outcome } \\
\text { measures were not } \\
\text { limited in any way, nor } \\
\text { was the method in } \\
\text { which health coaching } \\
\text { was administered (e.g., } \\
\text { via telephone, Internet, } \\
\text { in person). }\end{array}$ & $\begin{array}{l}\text { Studies that did not } \\
\text { report their } \\
\text { outcome measures } \\
\text { were excluded } \\
\text { (e.g., feasibility } \\
\text { studies). Papers } \\
\text { were limited to } \\
\text { those in English } \\
\text { published between } \\
\text { January } 2000 \text { and } \\
\text { October } 2012 \text {. }\end{array}$ & $\begin{array}{l}\text { Total:16 } \\
\text { RCTs:16 } \\
\text { Non-RCTs with } \\
\text { comparison:0 } \\
\text { Observational:0 }\end{array}$ & Hospital and home \\
\hline
\end{tabular}




\begin{tabular}{|c|c|c|c|c|c|c|c|}
\hline $\begin{array}{l}\text { Type of } \\
\text { Strategy }\end{array}$ & Author, Year & $\begin{array}{l}\text { Objective of } \\
\text { Review }\end{array}$ & $\begin{array}{l}\text { Literature } \\
\text { Start - } \\
\text { End Year }\end{array}$ & Inclusion Criteria & Exclusion Criteria & $\begin{array}{l}\text { Number of } \\
\text { Included Studies }\end{array}$ & Settings \\
\hline & $\begin{array}{l}\text { Pamungkas, } \\
2017^{23}\end{array}$ & $\begin{array}{l}\text { This SR aimed to } \\
\text { describe the } \\
\text { impact of Diabetes } \\
\text { Self Management } \\
\text { Education that } \\
\text { involves family } \\
\text { members on } \\
\text { patient outcomes } \\
\text { related to patient } \\
\text { health behaviors } \\
\text { such as blood } \\
\text { glucose } \\
\text { monitoring, } \\
\text { medication } \\
\text { adherence, lifestyle } \\
\text { changes, and } \\
\text { physiological } \\
\text { markers including } \\
\text { body mass index, } \\
\text { blood pressure, } \\
\text { cholesterol level } \\
\text { and glycemic } \\
\text { control in patients } \\
\text { with uncontrolled } \\
\text { type } 2 \text { DM. }\end{array}$ & $2008-2016$ & $\begin{array}{l}\text { PICO (Participant- } \\
\text { Intervention- } \\
\text { Comparison- } \\
\text { Outcomes) format, } \\
\text { based on the Joanna } \\
\text { Briggs Institute (JBI) } \\
\text { (2014) [14], was used. } \\
\text { Adults with } \\
\text { uncontrolled type } 2 \\
\text { DM, studies utilizing } \\
\text { any treatment } \\
\text { strategies (e.g., usual } \\
\text { care, didactic method, } \\
\text { participatory learning, } \\
\text { internet-based } \\
\text { methods) were } \\
\text { included in this review }\end{array}$ & $\begin{array}{l}\text { Excluded Studies: } \\
\text { diabetes } \\
\text { medication alone or } \\
\text { intervention that did } \\
\text { not include a family } \\
\text { component, Non- } \\
\text { RCTs or studies } \\
\text { without control } \\
\text { group, not } \\
\text { published in an } \\
\text { academic journal } \\
\text { (e.g., unpublished } \\
\text { dissertation), and } \\
\text { studies focused on } \\
\text { diabetes prevention }\end{array}$ & $\begin{array}{l}\text { Total:23 } \\
\text { RCTs:19 } \\
\text { Non-RCTs with } \\
\text { comparison:3 } \\
\text { Observational:2 }\end{array}$ & Hospital and home \\
\hline & Kruse, $2015^{24}$ & $\begin{array}{l}\text { Describe } \\
\text { characteristics of } \\
\text { portals associated } \\
\text { with positive } \\
\text { perception by } \\
\text { patients and } \\
\text { providers. }\end{array}$ & 2004-2014 & $\begin{array}{l}\text { Attitudes of patients } \\
\text { and providers re: } \\
\text { patient portal. }\end{array}$ & $\begin{array}{l}\text { Did not report } \\
\text { patient \& provider } \\
\text { attitudes }\end{array}$ & $\begin{array}{l}\text { Total:27 } \\
\text { RCTs:0 }\end{array}$ & Outpatient \\
\hline
\end{tabular}




\begin{tabular}{|c|c|c|c|c|c|c|c|}
\hline $\begin{array}{l}\text { Type of } \\
\text { Strategy }\end{array}$ & Author, Year & $\begin{array}{l}\text { Objective of } \\
\text { Review }\end{array}$ & $\begin{array}{l}\text { Literature } \\
\text { Start - } \\
\text { End Year }\end{array}$ & Inclusion Criteria & Exclusion Criteria & $\begin{array}{l}\text { Number of } \\
\text { Included Studies }\end{array}$ & Settings \\
\hline \multirow[t]{2}{*}{$\begin{array}{l}\text { Self- } \\
\text { Management } \\
\text { Support - } \\
\text { Adults Only }\end{array}$} & $\begin{array}{l}\text { Whitehead, } \\
2016^{25}\end{array}$ & $\begin{array}{l}\text { To assess the } \\
\text { effectiveness of } \\
\text { mobile phone and } \\
\text { tablet apps in self- } \\
\text { management of } \\
\text { key symptoms of } \\
\text { long-term } \\
\text { conditions. (Only } \\
\text { one RCT from US) }\end{array}$ & $2005-2016$ & $\begin{array}{l}\text { Original research } \\
\text { published in peer } \\
\text { reviewed journals that } \\
\text { evaluated self- } \\
\text { management apps for } \\
\text { their effect on disease- } \\
\text { specific clinical } \\
\text { outcomes such as } \\
\text { HbA1C, BP, FEV1. } \\
\text { RCTs with comparison } \\
\text { or control group }\end{array}$ & $\begin{array}{l}\text { (1) they reported on } \\
\text { primary prevention } \\
\text { among healthy or } \\
\text { at-risk groups, (2) } \\
\text { the focus lay } \\
\text { outside of the self- } \\
\text { management } \\
\text { domain, (3) review } \\
\text { papers, editorials, } \\
\text { commentaries, (4) } \\
\text { no chronic } \\
\text { diseases (5) } \\
\text { language other } \\
\text { than English. }\end{array}$ & $\begin{array}{l}\text { Total:9 } \\
\text { RCTs:9 } \\
\text { Non-RCTs with } \\
\text { comparison:0 } \\
\text { Observational:0 }\end{array}$ & Home \\
\hline & Kim, $2015^{26}$ & $\begin{array}{l}\text { This systematic } \\
\text { review is aimed at } \\
\text { identifying the } \\
\text { general } \\
\text { characteristics of } \\
\text { web-based self- } \\
\text { management } \\
\text { support } \\
\text { interventions for } \\
\text { cancer survivors } \\
\text { and to perform the } \\
\text { corresponding } \\
\text { meta-analyses to } \\
\text { assess the effects } \\
\text { of these } \\
\text { interventions }\end{array}$ & $2000-2014$ & $\begin{array}{l}\text { Patients diagnosed } \\
\text { with cancer or their } \\
\text { caregivers; Web-based } \\
\text { self-management } \\
\text { support interventions; } \\
\text { behavioral or health } \\
\text { outcomes; randomized } \\
\text { controlled trials or } \\
\text { quasi- experimental } \\
\text { designs; Original } \\
\text { research articles } \\
\text { published in English in } \\
\text { peer-reviewed journals } \\
\text { between January } 2000 \\
\text { and June } 2014 \text {. }\end{array}$ & None listed & $\begin{array}{l}\text { Total:37 } \\
\text { RCTs:34 } \\
\text { Non-RCTs with } \\
\text { comparison:0 } \\
\text { Observational:3 }\end{array}$ & Setting not included \\
\hline
\end{tabular}




\begin{tabular}{|c|c|c|c|c|c|c|c|}
\hline $\begin{array}{l}\text { Type of } \\
\text { Strategy }\end{array}$ & Author, Year & $\begin{array}{l}\text { Objective of } \\
\text { Review }\end{array}$ & $\begin{array}{l}\text { Literature } \\
\text { Start - } \\
\text { End Year }\end{array}$ & Inclusion Criteria & Exclusion Criteria & $\begin{array}{l}\text { Number of } \\
\text { Included Studies }\end{array}$ & Settings \\
\hline & $\begin{array}{l}\text { Teljeur, } \\
2016^{27}\end{array}$ & $\begin{array}{l}\text { To systematically } \\
\text { review the } \\
\text { evidence on the } \\
\text { costs and cost- } \\
\text { effectiveness of } \\
\text { self-management } \\
\text { support } \\
\text { interventions for } \\
\text { people with } \\
\text { diabetes. }\end{array}$ & $\begin{array}{l}\text { Not } \\
\text { mentioned } \\
-2015\end{array}$ & $\begin{array}{l}\text { P: adults with Type I } \\
\text { \&ll diabetes, I: } \\
\text { substantial component } \\
\text { of self management } \\
\text { support, C: compared } \\
\text { with routine care, O: } \\
\text { costs and cost- } \\
\text { effectiveness of self- } \\
\text { management support } \\
\text { interventions for } \\
\text { people with diabetes. } \\
\text { RCTs, observational } \\
\text { studies or economic } \\
\text { modelling studies were } \\
\text { eligible for inclusion. }\end{array}$ & $\begin{array}{l}\text { Part of the study } \\
\text { population was } \\
\text { from a nursing } \\
\text { home or non- } \\
\text { community dwelling } \\
\text { setting; } \\
\text { Excluded if only } \\
\text { published as } \\
\text { abstracts, people } \\
\text { aged < } 18 \text { years; } \\
\text { cost data were not } \\
\text { clearly reported; or } \\
\text { they compared } \\
\text { blood glucose self- } \\
\text { monitoring }\end{array}$ & $\begin{array}{l}\text { Total:37 } \\
\text { RCTs:6 } \\
\text { Non-RCTs with } \\
\text { comparison:11 } \\
\text { Observational:20 }\end{array}$ & Hospital and home \\
\hline & $\begin{array}{l}\text { Hecke, } \\
2016^{28}\end{array}$ & $\begin{array}{l}\text { This SR aims to } \\
\text { assess the quality } \\
\text { of evidence and } \\
\text { determine the } \\
\text { effect of patient- } \\
\text { related and } \\
\text { economic } \\
\text { outcomes of self- } \\
\text { management } \\
\text { support } \\
\text { interventions in } \\
\text { chronically ill } \\
\text { patients with a low } \\
\text { socio-economic } \\
\text { status. }\end{array}$ & $2000-2013$ & $\begin{array}{l}\text { Patients age }>18 \text { yrs } \\
\text { with chronic diseases } \\
\text { having low SES, } \\
\text { studies reporting on } \\
\text { Self Management } \\
\text { Support Interventions } \\
\text { with a focus on patient- } \\
\text { related outcomes } \\
\text { and/or economic } \\
\text { outcomes. No } \\
\text { language restriction. }\end{array}$ & None listed & $\begin{array}{l}\text { Total:27 } \\
\text { RCTs:18 } \\
\text { Non-RCTs with } \\
\text { comparison:1 } \\
\text { Observational:8 }\end{array}$ & Hospital \\
\hline
\end{tabular}




\begin{tabular}{|c|c|c|c|c|c|c|c|}
\hline $\begin{array}{l}\text { Type of } \\
\text { Strategy }\end{array}$ & Author, Year & $\begin{array}{l}\text { Objective of } \\
\text { Review }\end{array}$ & $\begin{array}{l}\text { Literature } \\
\text { Start - } \\
\text { End Year }\end{array}$ & Inclusion Criteria & Exclusion Criteria & $\begin{array}{l}\text { Number of } \\
\text { Included Studies }\end{array}$ & Settings \\
\hline & Hooft, $2016^{29}$ & $\begin{array}{l}\text { Realist Review: } \\
\text { The aim of this } \\
\text { study was to } \\
\text { examine how } \\
\text { nurse-led } \\
\text { interventions that } \\
\text { support self- } \\
\text { management of } \\
\text { outpatients with } \\
\text { chronic conditions } \\
\text { work and in what } \\
\text { contexts they work } \\
\text { successfully }\end{array}$ & $2000-2015$ & $\begin{array}{l}\text { Only studies using a } \\
\text { comparison between } \\
\text { 'standard care' and } \\
\text { self-management } \\
\text { support interventions } \\
\text { (e.g. RCT, before-after } \\
\text { design and qualitative } \\
\text { and quantitative } \\
\text { methods). Self- } \\
\text { management support } \\
\text { interventions with a } \\
\text { prominent role for } \\
\text { nurses, outpatient } \\
\text { clinic setting, adults } \\
\text { with chronic condition, } \\
\text { evaluation study and } \\
\text { written in the English } \\
\text { language. }\end{array}$ & $\begin{array}{l}\text { If results were not } \\
\text { measured at a } \\
\text { patient level, if the } \\
\text { setting was a } \\
\text { palliative care, } \\
\text { primary care, or } \\
\text { psychiatric care. }\end{array}$ & $\begin{array}{l}\text { Total:38 } \\
\text { RCTs:21 } \\
\text { Non-RCTs with } \\
\text { comparison:3 } \\
\text { Observational:14 }\end{array}$ & Hospital \\
\hline & $\begin{array}{l}\text { Stenberg, } \\
2016^{30}\end{array}$ & $\begin{array}{l}\text { Scoping Review: } \\
\text { To give a } \\
\text { comprehensive } \\
\text { overview of } \\
\text { benefits and } \\
\text { challenges from } \\
\text { participating in } \\
\text { group based } \\
\text { patient education } \\
\text { programs that are } \\
\text { carried out by } \\
\text { health care } \\
\text { professionals and } \\
\text { lay participants, } \\
\text { aimed at promoting } \\
\text { self-management } \\
\text { for people living } \\
\text { with chronic illness. }\end{array}$ & $2008-2015$ & $\begin{array}{l}\text { Articles in English and } \\
\text { Scandinavian in peer- } \\
\text { reviewed journals that } \\
\text { had investigated the } \\
\text { benefits and } \\
\text { challenges from } \\
\text { participating in patient } \\
\text { education programs } \\
\text { aimed at promoting } \\
\text { self-management for } \\
\text { people older than } 18 \\
\text { years of age, living with } \\
\text { chronic illness. }\end{array}$ & $\begin{array}{l}\text { Studies excluded if } \\
\text { lay participants } \\
\text { were not involved } \\
\text { in planning or } \\
\text { carrying out the } \\
\text { programs, not } \\
\text { reporting on } \\
\text { benefits or } \\
\text { challenges from } \\
\text { participation as } \\
\text { reported by } \\
\text { participants, and } \\
\text { not chronic illness. }\end{array}$ & $\begin{array}{l}\text { Total:47 } \\
\text { RCTs:28 } \\
\text { Non-RCTs with } \\
\text { comparison:0 } \\
\text { Observational:19 }\end{array}$ & $\begin{array}{l}\text { Hospital, home, } \\
\text { community settings, } \\
\text { CHCs }\end{array}$ \\
\hline
\end{tabular}




\begin{tabular}{|c|c|c|c|c|c|c|c|}
\hline $\begin{array}{l}\text { Type of } \\
\text { Strategy }\end{array}$ & Author, Year & $\begin{array}{l}\text { Objective of } \\
\text { Review }\end{array}$ & $\begin{array}{l}\text { Literature } \\
\text { Start - } \\
\text { End Year }\end{array}$ & Inclusion Criteria & Exclusion Criteria & $\begin{array}{l}\text { Number of } \\
\text { Included Studies }\end{array}$ & Settings \\
\hline & Plow, $2016^{31}$ & $\begin{array}{l}\text { The purpose of this } \\
\text { scoping review } \\
\text { was to describe } \\
\text { randomized } \\
\text { controlled trials } \\
\text { (RCTs) of tailored } \\
\text { self-management } \\
\text { interventions in } \\
\text { adults with } \\
\text { neurological and } \\
\text { musculoskeletal } \\
\text { conditions that } \\
\text { characteristically } \\
\text { result in mobility } \\
\text { impairments. We } \\
\text { focused on } \\
\text { summarizing the } \\
\text { outcomes of these } \\
\text { RCTs and the } \\
\text { strategies used to } \\
\text { promote behavior } \\
\text { change. }\end{array}$ & $1980-2015$ & $\begin{array}{l}\text { RCTs in English } \\
\text { language with } \\
\text { community-dwelling } \\
\text { adults who acquire } \\
\text { diseases or } \\
\text { impairments in } \\
\text { neurological or } \\
\text { musculoskeletal } \\
\text { systems and have an } \\
\text { outcome measure of } \\
\text { medication adherence, } \\
\text { physical activity, } \\
\text { nutrition, sleep } \\
\text { hygiene, smoking } \\
\text { cessation, or alcohol } \\
\text { use. }\end{array}$ & $\begin{array}{l}\text { (1) primarily } \\
\text { evaluating the } \\
\text { beneficial effects of } \\
\text { exercise programs, } \\
\text { medications, or } \\
\text { vocational } \\
\text { rehabilitation } \\
\text { programs } \\
\text { (2) including } \\
\text { children or under } \\
18 \text { years old, adults } \\
\text { living in a nursing } \\
\text { home or receiving } \\
\text { the entire } \\
\text { intervention during } \\
\text { inpatient care, older } \\
\text { adults without } \\
\text { needing to have a } \\
\text { condition as } \\
\text { defined above, and } \\
\text { adults } \\
\text { with a primary } \\
\text { diagnosis of } \\
\text { cardiovascular } \\
\text { disease, epilepsy, } \\
\text { cancer, endocrine } \\
\text { disease, mental } \\
\text { health disorder, or } \\
\text { Alzheimer's } \\
\text { disease } \\
\text { (3) Studies on } \\
\text { motivational } \\
\text { interviewing } \\
\text { (4) Conference } \\
\text { proceedings, } \\
\text { abstracts, and } \\
\text { review articles }\end{array}$ & $\begin{array}{l}\text { Total:13 } \\
\text { RCTs:13 } \\
\text { Non-RCTs with } \\
\text { comparison:0 } \\
\text { Observational:0 }\end{array}$ & Hospital \\
\hline
\end{tabular}




\begin{tabular}{|c|c|c|c|c|c|c|c|}
\hline $\begin{array}{l}\text { Type of } \\
\text { Strategy }\end{array}$ & Author, Year & $\begin{array}{l}\text { Objective of } \\
\text { Review }\end{array}$ & $\begin{array}{l}\text { Literature } \\
\text { Start - } \\
\text { End Year }\end{array}$ & Inclusion Criteria & Exclusion Criteria & $\begin{array}{l}\text { Number of } \\
\text { Included Studies }\end{array}$ & Settings \\
\hline & $\begin{array}{l}\text { Noonan, } \\
2019^{32}\end{array}$ & $\begin{array}{l}\text { To quantify the } \\
\text { impact of involving } \\
\text { caregivers in self- } \\
\text { management } \\
\text { interventions on } \\
\text { health-related } \\
\text { quality of life of } \\
\text { patients with heart } \\
\text { failure or chronic } \\
\text { obstructive } \\
\text { pulmonary } \\
\text { disease. }\end{array}$ & 1990-2018 & $\begin{array}{l}\text { RCTs involving } \\
\text { caregivers in self- } \\
\text { management } \\
\text { interventions ( } \geq 2 \\
\text { components) } \\
\text { compared with usual } \\
\text { care for adult patients } \\
\text { with heart failure or } \\
\text { chronic obstructive } \\
\text { pulmonary disease. } \\
\text { Primary outcome of } \\
\text { analysis was patient } \\
\text { health-related quality } \\
\text { of life. Intervention: } \\
\text { Self-management } \\
\text { intervention programs } \\
\text { which were comprised } \\
\text { of two or more } \\
\text { intervention } \\
\text { components. }\end{array}$ & $\begin{array}{l}\text { We excluded: (a) } \\
\text { studies in long-term } \\
\text { residential care } \\
\text { setting; (b) studies } \\
\text { where caregivers } \\
\text { were not explicitly } \\
\text { part of the } \\
\text { intervention } \\
\text { delivery. }\end{array}$ & $\begin{array}{l}\text { Total:26 } \\
\text { RCTs:26 } \\
\text { Non-RCTs with } \\
\text { comparison:0 } \\
\text { Observational:0 }\end{array}$ & Hospital and home \\
\hline & Kuo, $2016^{33}$ & $\begin{array}{l}\text { Assess the clinical } \\
\text { evidence } \\
\text { supporting the use } \\
\text { of secure } \\
\text { messaging in } \\
\text { EHRs in self- } \\
\text { management of } \\
\text { diabetes. }\end{array}$ & $\begin{array}{l}\text { Not } \\
\text { mentioned- } \\
2015\end{array}$ & $\begin{array}{l}\text { Patients with diabetes; } \\
\text { secure messaging in } \\
\text { EHR }\end{array}$ & $\begin{array}{l}\text { No online secure } \\
\text { messaging to } \\
\text { communicate with } \\
\text { providers }\end{array}$ & $\begin{array}{l}\text { Total:11 } \\
\text { RCTs:2 } \\
\text { Non-RCTs with } \\
\text { comparison:1 } \\
\text { Observational:8 }\end{array}$ & Home \\
\hline
\end{tabular}




\begin{tabular}{|c|c|c|c|c|c|c|c|}
\hline $\begin{array}{l}\text { Type of } \\
\text { Strategy }\end{array}$ & Author, Year & $\begin{array}{l}\text { Objective of } \\
\text { Review }\end{array}$ & $\begin{array}{l}\text { Literature } \\
\text { Start - } \\
\text { End Year }\end{array}$ & Inclusion Criteria & Exclusion Criteria & $\begin{array}{l}\text { Number of } \\
\text { Included Studies }\end{array}$ & Settings \\
\hline & $\begin{array}{l}\text { Sakakibara, } \\
2016^{34}\end{array}$ & $\begin{array}{l}\text { To describe the } \\
\text { self management } \\
\text { interventions used } \\
\text { to improve risk } \\
\text { factor control in } \\
\text { stroke patients and } \\
\text { quantitatively } \\
\text { assess their effects } \\
\text { overall risk factor } \\
\text { control from } \\
\text { lifestyle behavior } \\
\text { and individual risk } \\
\text { factors. }\end{array}$ & $\begin{array}{l}\text { Inception- } \\
2015\end{array}$ & $\begin{array}{l}\text { RCTs were included if } \\
\text { they involved a self- } \\
\text { management } \\
\text { intervention to improve } \\
\text { risk factors in adults } \\
\text { (aged } 18 \text { years and } \\
\text { older) who have had a } \\
\text { stroke or transient } \\
\text { ischemic attack (TIA). } \\
\text { Also active } \\
\text { participation required. } \\
\text { Clear definition of } \\
\text { intervention and } \\
\text { control treatments. } \\
\text { English language. }\end{array}$ & $\begin{array}{l}\text { Excluded if no } \\
\text { comparison or if } \\
\text { more than half of } \\
\text { the study sample } \\
\text { included individuals } \\
\text { without a stroke } \\
\text { diagnosis }\end{array}$ & $\begin{array}{l}\text { Total:14 } \\
\text { RCTs:14 } \\
\text { Non-RCTs with } \\
\text { comparison:0 } \\
\text { Observational:0 }\end{array}$ & Hospital and home \\
\hline & $\begin{array}{l}\text { Whiteman, } \\
2016^{35}\end{array}$ & $\begin{array}{l}\text { Aimed to: review } \\
\text { the evidence of the } \\
\text { effect of self- } \\
\text { management } \\
\text { interventions } \\
\text { targeting both } \\
\text { medical and } \\
\text { psychiatric } \\
\text { illnesses and } \\
\text { evaluate the } \\
\text { potential for } \\
\text { implementation. }\end{array}$ & $1946-2015$ & $\begin{array}{l}\text { P: Adults with a } \\
\text { diagnosis of } \\
\text { schizophrenia, } \\
\text { schizoaffective } \\
\text { disorder, or bipolar } \\
\text { disorder and a chronic } \\
\text { medical illness I: self- } \\
\text { management } \\
\text { intervention studies } \\
\text { that address both } \\
\text { medical and psychiatric } \\
\text { self-management C: } \\
\text { self-management skills } \\
\text { and behaviors, self- } \\
\text { management attitudes, } \\
\text { biological outcomes, } \\
\text { services utilization, and } \\
\text { functional status. No } \\
\text { restriction on } \\
\text { language, and studies } \\
\text { included randomized } \\
\text { control trials, pre/post } \\
\text { designs. }\end{array}$ & $\begin{array}{l}\text { We excluded } \\
\text { preventative } \\
\text { interventions and } \\
\text { health promotion or } \\
\text { lifestyle } \\
\text { interventions } \\
\text { targeting substance } \\
\text { use, smoking } \\
\text { cessation, weight } \\
\text { loss, weight-gain } \\
\text { prevention, } \\
\text { physical activity, or } \\
\text { fitness. }\end{array}$ & $\begin{array}{l}\text { Total:15 } \\
\text { RCTs:9 } \\
\text { Non-RCTs with } \\
\text { comparison:6 } \\
\text { Observational:0 }\end{array}$ & Hospital and home \\
\hline
\end{tabular}




\begin{tabular}{|c|c|c|c|c|c|c|c|}
\hline $\begin{array}{l}\text { Type of } \\
\text { Strategy }\end{array}$ & Author, Year & $\begin{array}{l}\text { Objective of } \\
\text { Review }\end{array}$ & $\begin{array}{l}\text { Literature } \\
\text { Start - } \\
\text { End Year }\end{array}$ & Inclusion Criteria & Exclusion Criteria & $\begin{array}{l}\text { Number of } \\
\text { Included Studies }\end{array}$ & Settings \\
\hline & Sokol, $2016^{36}$ & $\begin{array}{l}\text { To assess the } \\
\text { reach and } \\
\text { effectiveness of } \\
\text { peer support } \\
\text { among those who } \\
\text { are hardly reached, } \\
\text { along with peer } \\
\text { support strategies } \\
\text { (conceptual and } \\
\text { operational) used. }\end{array}$ & $2000-2015$ & $\begin{array}{l}\text { Studies had to include } \\
\text { prioritization of a hardly } \\
\text { reached population, } \\
\text { ongoing support from a } \\
\text { nonprofessional, } \\
\text { assistance in applying } \\
\text { behavior change plans, } \\
\text { and at least } 1 \text { of the } 2 \\
\text { following components: } \\
\text { social and emotional } \\
\text { support or } \\
\text { encouragement of } \\
\text { recommended care. }\end{array}$ & $\begin{array}{l}\text { Studies were } \\
\text { excluded if } \\
\text { they addressed } \\
\text { temporally isolated } \\
\text { behaviors rather } \\
\text { than complex } \\
\text { behaviors, were } \\
\text { limited to protocol } \\
\text { classes, were } \\
\text { group taught or } \\
\text { facilitated, included } \\
\text { peer support as the } \\
\text { dependent variable, } \\
\text { did not include } \\
\text { statistical tests of } \\
\text { significance, or } \\
\text { included } \\
\text { comparison } \\
\text { conditions that } \\
\text { involved substantial } \\
\text { social support. }\end{array}$ & $\begin{array}{l}\text { Total:47 } \\
\text { RCTs:13 } \\
\text { Observational:34 }\end{array}$ & $\begin{array}{l}\text { Hospital, } \\
\text { Community } \\
\text { settings, CHCs }\end{array}$ \\
\hline & $\begin{array}{l}\text { Zomahoun, } \\
2016^{37}\end{array}$ & $\begin{array}{l}\text { This SR aimed to } \\
\text { assess whether } \\
\text { Motivational } \\
\text { Interview } \\
\text { interventions are } \\
\text { effective to } \\
\text { enhance } \\
\text { medication } \\
\text { adherence in } \\
\text { adults with chronic } \\
\text { diseases and to } \\
\text { explore the effect } \\
\text { of individual MI } \\
\text { intervention } \\
\text { characteristics. }\end{array}$ & $2012-2016$ & $\begin{array}{l}\text { RCTs that assessed MI } \\
\text { intervention } \\
\text { effectiveness on } \\
\text { medication adherence } \\
\text { in adults with chronic } \\
\text { diseases. Comparator } \\
\text { groups had to include } \\
\text { individuals who were } \\
\text { not receiving Ml. } \\
\text { Outcomes: medication } \\
\text { adherence had to be } \\
\text { explicitly stated as the } \\
\text { primary or secondary } \\
\text { outcome of the MI } \\
\text { intervention. }\end{array}$ & None listed & $\begin{array}{l}\text { Total:19 } \\
\text { RCTs:19 } \\
\text { Non-RCTs with } \\
\text { comparison:0 } \\
\text { Observational:0 }\end{array}$ & Hospital and home \\
\hline
\end{tabular}




\begin{tabular}{|c|c|c|c|c|c|c|c|}
\hline $\begin{array}{l}\text { Type of } \\
\text { Strategy }\end{array}$ & Author, Year & $\begin{array}{l}\text { Objective of } \\
\text { Review }\end{array}$ & $\begin{array}{l}\text { Literature } \\
\text { Start - } \\
\text { End Year }\end{array}$ & Inclusion Criteria & Exclusion Criteria & $\begin{array}{l}\text { Number of } \\
\text { Included Studies }\end{array}$ & Settings \\
\hline & $\begin{array}{l}\text { Palacio, } \\
2016^{38}\end{array}$ & $\begin{array}{l}\text { To evaluate the } \\
\text { impact of } \\
\text { motivational } \\
\text { interviewing (MI) } \\
\text { and of the MI } \\
\text { delivery format, } \\
\text { fidelity } \\
\text { assessment, } \\
\text { fidelity-based } \\
\text { feedback, } \\
\text { counselors' } \\
\text { background and MI } \\
\text { exposure time on } \\
\text { adherence. }\end{array}$ & 1966-2015 & $\begin{array}{l}\text { Randomized trials that } \\
\text { compared Ml to one or } \\
\text { more control groups, } \\
\text { reported a measure of } \\
\text { medication adherence } \\
\text { in numerical form, had } \\
\text { a follow-up period of } \\
\text { any length and were } \\
\text { published in English. }\end{array}$ & $\begin{array}{l}\text { Studies that used } \\
\text { MI in combination } \\
\text { with other } \\
\text { strategies since the } \\
\text { isolated effect of MI } \\
\text { could not be } \\
\text { determined, were } \\
\text { excluded. }\end{array}$ & $\begin{array}{l}\text { Total:17 } \\
\text { RCTs:17 } \\
\text { Non-RCTs with } \\
\text { comparison:0 } \\
\text { Observational:0 }\end{array}$ & Hospital \\
\hline & $\begin{array}{l}\text { Eeden, } \\
2016^{39}\end{array}$ & $\begin{array}{l}\text { Objective was to } \\
\text { systematically } \\
\text { review the } \\
\text { literature of full } \\
\text { economic } \\
\text { evaluation studies } \\
\text { of self- } \\
\text { management } \\
\text { interventions in } \\
\text { adult chronic } \\
\text { patients and to } \\
\text { investigate their } \\
\text { methodological } \\
\text { quality and cost- } \\
\text { effectiveness. }\end{array}$ & 1990-2014 & $\begin{array}{l}\text { Full economic } \\
\text { evaluation studies } \\
\text { reporting on SMls in } \\
\text { line with Barlow's } \\
\text { definition (2) of self- } \\
\text { management of } \\
\text { chronic diseases were } \\
\text { eligible for inclusion. }\end{array}$ & $\begin{array}{l}\text { Studies were } \\
\text { Excluded if patients } \\
\text { could not be } \\
\text { classified as having } \\
\text { a chronic disease } \\
\text { or receiving chronic } \\
\text { care, if participants } \\
\text { were younger than } \\
18 \text { years of age, if } \\
\text { the study was not } \\
\text { written in English or } \\
\text { Dutch and/or was } \\
\text { published before } \\
1990 .\end{array}$ & $\begin{array}{l}\text { Total:22 } \\
\text { RCTs:6 } \\
\text { Non-RCTs with } \\
\text { comparison:13 } \\
\text { Observational:3 }\end{array}$ & Hospital and home \\
\hline
\end{tabular}




\begin{tabular}{|c|c|c|c|c|c|c|c|}
\hline $\begin{array}{l}\text { Type of } \\
\text { Strategy }\end{array}$ & Author, Year & $\begin{array}{l}\text { Objective of } \\
\text { Review }\end{array}$ & $\begin{array}{l}\text { Literature } \\
\text { Start - } \\
\text { End Year }\end{array}$ & Inclusion Criteria & Exclusion Criteria & $\begin{array}{l}\text { Number of } \\
\text { Included Studies }\end{array}$ & Settings \\
\hline & $\begin{array}{l}\text { Jacelon, } \\
2016^{40}\end{array}$ & $\begin{array}{l}\text { The purpose of this } \\
\text { scoping review of } \\
\text { literature is to } \\
\text { explore the types } \\
\text { of computer-based } \\
\text { systems used for } \\
\text { self-management } \\
\text { of chronic disease, } \\
\text { the goals and } \\
\text { success of these } \\
\text { systems, the value } \\
\text { added by } \\
\text { technology } \\
\text { integration and the } \\
\text { target audience for } \\
\text { these systems. }\end{array}$ & $2006-2016$ & $\begin{array}{l}\text { The research articles } \\
\text { included were focused } \\
\text { on technology for self- } \\
\text { management of } \\
\text { chronic disease. }\end{array}$ & $\begin{array}{l}\text { Excluded if they } \\
\text { were not focused } \\
\text { on self- } \\
\text { management, were } \\
\text { not primary } \\
\text { research, were not } \\
\text { technology- based } \\
\text { or were integrative } \\
\text { reviews; they were } \\
\text { also excluded if the } \\
\text { intervention } \\
\text { provided feedback } \\
\text { only to the clinician } \\
\text { and not to the } \\
\text { person with the } \\
\text { disease }\end{array}$ & Total:30 & Hospital and home \\
\hline & Smith, $2017^{41}$ & $\begin{array}{l}\text { This review aimed } \\
\text { to identify and } \\
\text { summarize the } \\
\text { existing evidence } \\
\text { on the } \\
\text { effectiveness of } \\
\text { interventions to } \\
\text { improve clinical } \\
\text { and mental health } \\
\text { outcomes and } \\
\text { patient-reported } \\
\text { outcomes including } \\
\text { health-related } \\
\text { quality of life for } \\
\text { people with multi- } \\
\text { morbidity in } \\
\text { primary care and } \\
\text { community } \\
\text { settings. }\end{array}$ & $1990-2015$ & $\begin{array}{l}\text { P: Any people or } \\
\text { populations with multi- } \\
\text { morbidity receiving } \\
\text { care in a primary or } \\
\text { community care setting } \\
\text { only. I: any type of } \\
\text { intervention that was } \\
\text { specifically directed } \\
\text { towards a group of } \\
\text { people defined as } \\
\text { having multi-morbidity. } \\
\text { O: clinical or mental } \\
\text { health outcomes (e.g. } \\
\text { blood pressure, } \\
\text { symptom scores, } \\
\text { depression scores), } \\
\text { Patient-reported } \\
\text { outcome measures } \\
\text { (e.g. quality of life, } \\
\text { well-being, measures } \\
\text { of disability or } \\
\text { functional status), } \\
\text { Utilization of health } \\
\text { services }\end{array}$ & $\begin{array}{l}\text { Studies where } \\
\text { interventions were } \\
\text { directed at } \\
\text { communities of } \\
\text { people based on } \\
\text { location or age of } \\
\text { participants in } \\
\text { which participants } \\
\text { could be presumed } \\
\text { to have } \\
\text { multimorbidity on } \\
\text { the basis of their } \\
\text { age or residence in } \\
\text { a nursing home but } \\
\text { interventions were } \\
\text { not designed to } \\
\text { specifically target } \\
\text { multimorbidity. }\end{array}$ & $\begin{array}{l}\text { Total:18 } \\
\text { RCTs:18 } \\
\text { Non-RCTs with } \\
\text { comparison:0 } \\
\text { Observational:0 }\end{array}$ & Hospital \\
\hline
\end{tabular}




\begin{tabular}{|c|c|c|c|c|c|c|c|}
\hline $\begin{array}{l}\text { Type of } \\
\text { Strategy }\end{array}$ & Author, Year & $\begin{array}{l}\text { Objective of } \\
\text { Review }\end{array}$ & $\begin{array}{l}\text { Literature } \\
\text { Start - } \\
\text { End Year }\end{array}$ & Inclusion Criteria & Exclusion Criteria & $\begin{array}{l}\text { Number of } \\
\text { Included Studies }\end{array}$ & Settings \\
\hline & $\begin{array}{l}\text { Hammer, } \\
2015^{42}\end{array}$ & $\begin{array}{l}\text { The purpose of this } \\
\text { integrative review } \\
\text { is to evaluate } \\
\text { intervention studies } \\
\text { led by nurse } \\
\text { principal } \\
\text { investigators for } \\
\text { self-care } \\
\text { management in } \\
\text { patients with } \\
\text { cancer }\end{array}$ & $2000-2012$ & $\begin{array}{l}\text { Adults/older adults with } \\
\text { cancer who received } \\
\text { an intervention to help } \\
\text { with self-management } \\
\text { from disease and } \\
\text { treatment-related } \\
\text { effects, with } \\
\text { comparison to same } \\
\text { patient populations } \\
\text { receiving usual care, } \\
\text { and through an } \\
\text { experimental design } \\
\text { study }\end{array}$ & $\begin{array}{l}\text { Studies that } \\
\text { addressed the } \\
\text { following areas } \\
\text { without also } \\
\text { containing a self- } \\
\text { care intervention: } \\
\text { medications, } \\
\text { surgical } \\
\text { procedures, } \\
\text { treatment decision } \\
\text { making, perception, } \\
\text { needs assessment, } \\
\text { evaluations of } \\
\text { patient-provider } \\
\text { communication, } \\
\text { evaluations of } \\
\text { screenings, } \\
\text { evaluation of } \\
\text { barriers to } \\
\text { symptom } \\
\text { management, } \\
\text { validation of } \\
\text { assessment } \\
\text { questionnaires. } \\
\text { Non-English } \\
\text { articles were also } \\
\text { excluded. }\end{array}$ & $\begin{array}{l}\text { Total:46 } \\
\text { RCTs:35 } \\
\text { Non-RCTs with } \\
\text { comparison:0 } \\
\text { Observational:11 }\end{array}$ & $\begin{array}{l}\text { Outpatient clinics, } \\
\text { home }\end{array}$ \\
\hline
\end{tabular}




\begin{tabular}{|c|c|c|c|c|c|c|c|}
\hline $\begin{array}{l}\text { Type of } \\
\text { Strategy }\end{array}$ & Author, Year & $\begin{array}{l}\text { Objective of } \\
\text { Review }\end{array}$ & $\begin{array}{l}\text { Literature } \\
\text { Start - } \\
\text { End Year }\end{array}$ & Inclusion Criteria & Exclusion Criteria & $\begin{array}{l}\text { Number of } \\
\text { Included Studies }\end{array}$ & Settings \\
\hline & Kim, $2016^{43}$ & $\begin{array}{l}\text { A systematic } \\
\text { review to } \\
\text { synthesize } \\
\text { evidence } \\
\text { concerning the } \\
\text { types of CBHW } \\
\text { interventions, the } \\
\text { qualification and } \\
\text { characteristics of } \\
\text { CBHWs, and } \\
\text { patient outcomes } \\
\text { and cost- } \\
\text { effectiveness of } \\
\text { such interventions } \\
\text { in vulnerable } \\
\text { populations with } \\
\text { chronic, non- } \\
\text { communicable } \\
\text { conditions }\end{array}$ & $\begin{array}{l}\text { Not } \\
\text { mentioned- } \\
2014\end{array}$ & $\begin{array}{l}\text { (1) Randomized } \\
\text { controlled trials } \\
\text { published in English in } \\
\text { peer-reviewed journals, } \\
\text { (2) studies testing } \\
\text { CBHW-led } \\
\text { interventions, (3) } \\
\text { studies focused on } \\
\text { adults, and (4) studies } \\
\text { focused on chronic } \\
\text { conditions. }\end{array}$ & $\begin{array}{l}\text { (1) Studies focused } \\
\text { on children, (2) } \\
\text { non-data-based } \\
\text { articles (e.g., } \\
\text { editorials, } \\
\text { commentaries), } \\
\text { and (3) studies } \\
\text { focused on non- } \\
\text { vulnerable } \\
\text { populations. }\end{array}$ & $\begin{array}{l}\text { Total:67 } \\
\text { RCTs:67 } \\
\text { Non-RCTs with } \\
\text { comparison:0 } \\
\text { Observational:0 }\end{array}$ & $\begin{array}{l}\text { Home, } \\
\text { hospital/clinic/CHC, } \\
\text { community centers, } \\
\text { churches }\end{array}$ \\
\hline & $\begin{array}{l}\text { Thakkar, } \\
2016^{44}\end{array}$ & $\begin{array}{l}\text { To conduct a meta- } \\
\text { analysis of } \\
\text { randomized clinical } \\
\text { trials to assess the } \\
\text { effect of mobile } \\
\text { telephone text } \\
\text { messaging on } \\
\text { medication } \\
\text { adherence in } \\
\text { chronic disease. }\end{array}$ & $\begin{array}{l}\text { Inception- } \\
2015\end{array}$ & $\begin{array}{l}\text { (1) The trial studied } \\
\text { adult patients ( } \geq 18 \\
\text { years) with chronic } \\
\text { disease, (2) the } \\
\text { patients received a } \\
\text { mobile telephone text } \\
\text { message intervention } \\
\text { designed to promote } \\
\text { medication adherence, } \\
\text { (3) the design was a } \\
\text { randomized clinical trial } \\
\text { (RCT) with at least } 4 \\
\text { weeks' follow- up, and } \\
\text { (4) the trial reported } \\
\text { quantitative measures } \\
\text { of the effect of text } \\
\text { messaging on } \\
\text { medication adherence }\end{array}$ & $\begin{array}{l}\text { (1) primary } \\
\text { intervention under } \\
\text { consideration was } \\
\text { not limited to text } \\
\text { messages, (2) the } \\
\text { focus was solely } \\
\text { disease } \\
\text { management or } \\
\text { education and did } \\
\text { not report } \\
\text { medication } \\
\text { adherence or } \\
\text { reported only } \\
\text { surrogate } \\
\text { outcomes } \\
\text { (3) the study } \\
\text { involved } \\
\text { psychiatric, military, } \\
\text { or institutionalized } \\
\text { patients. }\end{array}$ & $\begin{array}{l}\text { Total:16 } \\
\text { RCTs:0 } \\
\text { Non-RCTs with } \\
\text { comparison:0 } \\
\text { Observational:0 }\end{array}$ & $\begin{array}{l}\text { Inpatient, outpatient } \\
\text { clinics }\end{array}$ \\
\hline
\end{tabular}




\begin{tabular}{|c|c|c|c|c|c|c|c|}
\hline $\begin{array}{l}\text { Type of } \\
\text { Strategy }\end{array}$ & Author, Year & $\begin{array}{l}\text { Objective of } \\
\text { Review }\end{array}$ & $\begin{array}{l}\text { Literature } \\
\text { Start - } \\
\text { End Year }\end{array}$ & Inclusion Criteria & Exclusion Criteria & $\begin{array}{l}\text { Number of } \\
\text { Included Studies }\end{array}$ & Settings \\
\hline & Deek, $2016^{45}$ & $\begin{array}{l}\text { To identify } \\
\text { elements of } \\
\text { effective family- } \\
\text { centered self-care } \\
\text { interventions that } \\
\text { are likely to } \\
\text { improve outcomes } \\
\text { of adults living with } \\
\text { chronic conditions }\end{array}$ & $2000-2014$ & $\begin{array}{l}\text { Quantitative studies } \\
\text { targeting patient } \\
\text { outcomes through } \\
\text { family- centered } \\
\text { interventions in adults } \\
\text { with chronic disease }\end{array}$ & $\begin{array}{l}\text { Studies were } \\
\text { excluded if they } \\
\text { were not } \\
\text { intervention } \\
\text { studies, caregiver- } \\
\text { focused outcome } \\
\text { interventions, had } \\
\text { no family } \\
\text { involvement, } \\
\text { centered on } \\
\text { pediatric and } \\
\text { adolescent } \\
\text { populations, review } \\
\text { papers, pilot } \\
\text { studies, protocols } \\
\text { or concerned non- } \\
\text { chronic conditions }\end{array}$ & $\begin{array}{l}\text { Total:10 } \\
\text { RCTs:9 } \\
\text { Non-RCTs with } \\
\text { comparison:1 } \\
\text { Observational:0 }\end{array}$ & Hospital and home \\
\hline & $\begin{array}{l}\text { Bolscher- } \\
\text { Niehuis, } \\
2016^{46}\end{array}$ & $\begin{array}{l}\text { To gain insight into } \\
\text { the evidence of the } \\
\text { effects of self- } \\
\text { management } \\
\text { support programs } \\
\text { on the activities of } \\
\text { daily living of older } \\
\text { adults living at } \\
\text { home. }\end{array}$ & $1998-2015$ & $\begin{array}{l}\text { Studies that described } \\
\text { a self-management } \\
\text { support program } \\
\text { directed at adults of on } \\
\text { average } 65 \text { years or } \\
\text { older, and living in the } \\
\text { community, used RCT } \\
\text { design and presented } \\
\text { information about the } \\
\text { effects on activities of } \\
\text { daily living. }\end{array}$ & None listed & $\begin{array}{l}\text { Total:12 } \\
\text { RCTs:12 } \\
\text { Non-RCTs with } \\
\text { comparison:0 } \\
\text { Observational:0 }\end{array}$ & Hospital \\
\hline
\end{tabular}




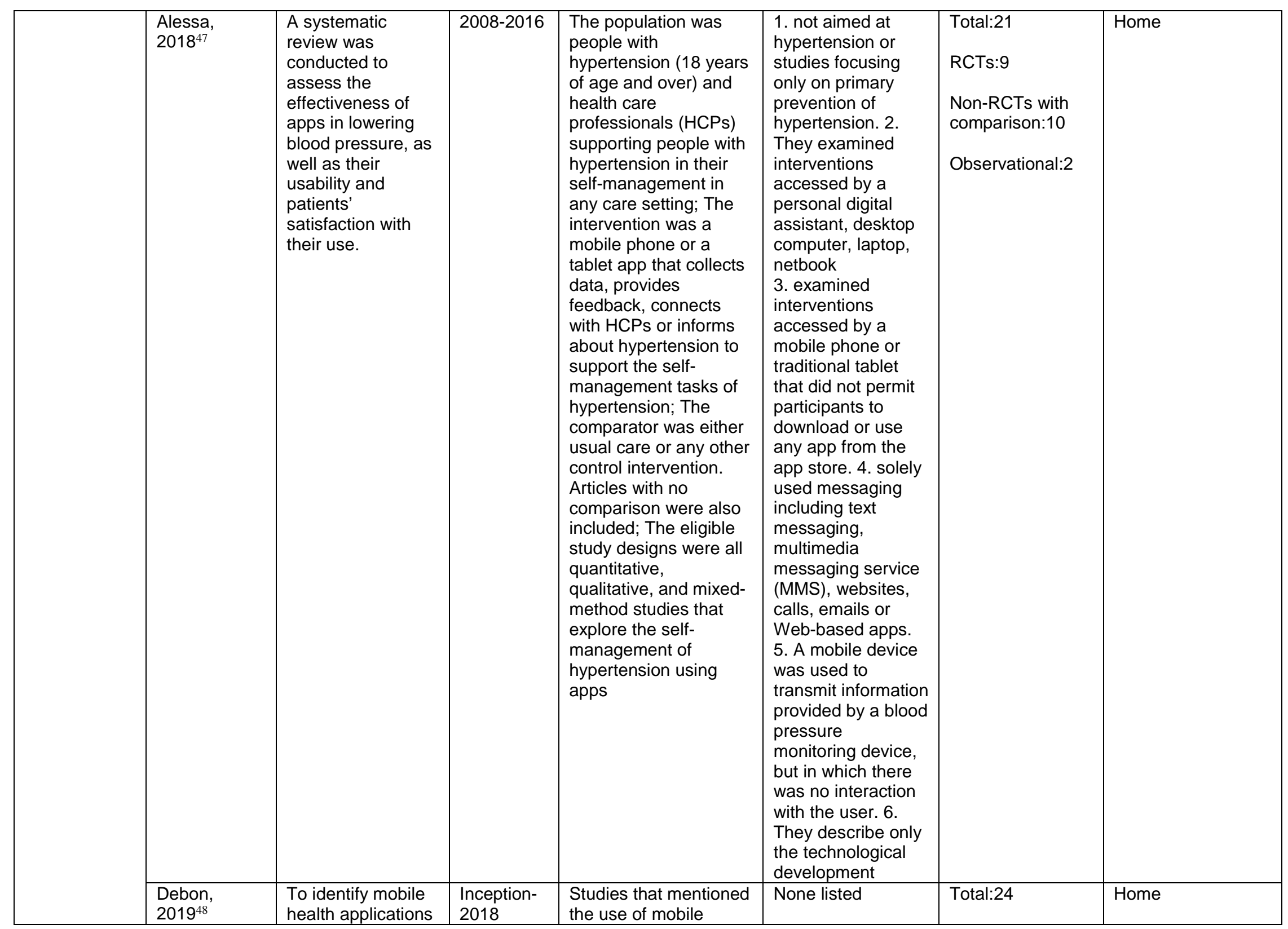




\begin{tabular}{|c|c|c|c|c|c|c|c|}
\hline $\begin{array}{l}\text { Type of } \\
\text { Strategy }\end{array}$ & Author, Year & $\begin{array}{l}\text { Objective of } \\
\text { Review }\end{array}$ & $\begin{array}{l}\text { Literature } \\
\text { Start - } \\
\text { End Year }\end{array}$ & Inclusion Criteria & Exclusion Criteria & $\begin{array}{l}\text { Number of } \\
\text { Included Studies }\end{array}$ & Settings \\
\hline & & $\begin{array}{l}\text { with features for } \\
\text { improving the } \\
\text { lifestyle of patients } \\
\text { with chronic } \\
\text { diseases. }\end{array}$ & & $\begin{array}{l}\text { APPs allowing user } \\
\text { interaction or testing } \\
\text { for the change in } \\
\text { lifestyle of patients with } \\
\text { chronic disease and } \\
\text { addressed at least one } \\
\text { routine or treatment } \\
\text { task for monitoring } \\
\text { chronic diseases. }\end{array}$ & & RCTs:0 & \\
\hline & $\begin{array}{l}\text { Luedke, } \\
2019^{49}\end{array}$ & $\begin{array}{l}\text { For adults with } \\
\text { epilepsy, i. What } \\
\text { are the most } \\
\text { commonly } \\
\text { employed } \\
\text { components of } \\
\text { self-management } \\
\text { interventions } \\
\text { evaluated in } \\
\text { comparative } \\
\text { studies? li. Effects } \\
\text { of self- } \\
\text { management } \\
\text { interventions on } \\
\text { self-management } \\
\text { skills and self- } \\
\text { efficacy, clinical } \\
\text { outcomes, and } \\
\text { health care } \\
\text { utilization iii. } \\
\text { Barriers and } \\
\text { facilitators }\end{array}$ & $\begin{array}{l}\text { Inception- } \\
2018\end{array}$ & $\begin{array}{l}\text { Randomized or quasi- } \\
\text { experimental studies } \\
\text { that enrolled adults } \\
\text { with epilepsy, } \\
\text { evaluated self- } \\
\text { management } \\
\text { interventions, and } \\
\text { reported a relevant } \\
\text { clinical, process, or } \\
\text { economic outcome. } \\
\text { Plus additional } \\
\text { observational designs } \\
\text { and qualitative studies } \\
\text { addressing facilitators } \\
\text { or barriers to adoption } \\
\text { or implementation. }\end{array}$ & None listed & $\begin{array}{l}\text { Total:28 } \\
\text { RCTs:13 } \\
\text { Non-RCTs with } \\
\text { comparison:2 } \\
\text { Observational:13 }\end{array}$ & Hospital \\
\hline
\end{tabular}




\begin{tabular}{|c|c|c|c|c|c|c|c|}
\hline $\begin{array}{l}\text { Type of } \\
\text { Strategy }\end{array}$ & Author, Year & $\begin{array}{l}\text { Objective of } \\
\text { Review }\end{array}$ & $\begin{array}{l}\text { Literature } \\
\text { Start - } \\
\text { End Year }\end{array}$ & Inclusion Criteria & Exclusion Criteria & $\begin{array}{l}\text { Number of } \\
\text { Included Studies }\end{array}$ & Settings \\
\hline & $\begin{array}{l}\text { Warner, } \\
2019^{50}\end{array}$ & $\begin{array}{l}\text { To identify whether } \\
\text { community-based } \\
\text { Self-Management } \\
\text { Programs (SMPs) } \\
\text { actively engaged, } \\
\text { or taught, } \\
\text { individuals patient- } \\
\text { oriented strategies; } \\
\text { and whether } \\
\text { having these } \\
\text { attributes led to } \\
\text { significant } \\
\text { differences in } \\
\text { outcomes. }\end{array}$ & 1986-2016 & $\begin{array}{l}\text { adults } 65 \text { years of age } \\
\text { and older (of any } \\
\text { ethnicity) who had one } \\
\text { or more chronic } \\
\text { conditions, I: } \\
\text { Community-based Self } \\
\text { Management } \\
\text { Programs, C: usual } \\
\text { care or an attention } \\
\text { control condition (e.g. } \\
\text { equivalent time } \\
\text { engagement but } \\
\text { without an } \\
\text { intervention), O: } \\
\text { patient-oriented self- } \\
\text { management } \\
\text { strategies, Studies: } \\
\text { RCTs, C-RCT }\end{array}$ & $\begin{array}{l}\text { Chronic conditions } \\
\text { that were not } \\
\text { relevant to older } \\
\text { adults or had } \\
\text { minimal impact on } \\
\text { everyday life. } \\
\text { Trials in which the } \\
\text { participants' age } \\
\text { could not be } \\
\text { assessed from the } \\
\text { report or after } \\
\text { contacting the } \\
\text { authors, or where } \\
\text { the mean age of } \\
\text { participants in the } \\
\text { trial was less than } \\
65 \text { years. Programs } \\
\text { directed at } \\
\text { clinicians or } \\
\text { caregivers. }\end{array}$ & $\begin{array}{l}\text { Total:31 } \\
\text { RCTs:31 } \\
\text { Non-RCTs with } \\
\text { comparison:0 } \\
\text { Observational:0 }\end{array}$ & $\begin{array}{l}\text { Community settings } \\
\text { or } \mathrm{CHCs}\end{array}$ \\
\hline & Han, $2019^{51}$ & $\begin{array}{l}\text { Effects of } \\
\text { community health } \\
\text { center } \\
\text { interventions in } \\
\text { people with } \\
\text { diabetes }\end{array}$ & $\begin{array}{l}\text { Inception- } \\
2018\end{array}$ & $\begin{array}{l}\text { Articles were included } \\
\text { in this review if the } \\
\text { study was: about } \\
\text { diabetes mellitus, } \\
\text { published in the } \\
\text { English language and } \\
\text { involved participants } \\
\text { who were } 18 \text { years and } \\
\text { older. Additionally, only } \\
\text { intervention studies } \\
\text { that were conducted } \\
\text { within a CHC set- ting } \\
\text { in the U.S., and studies } \\
\text { that reported patient } \\
\text { outcomes were } \\
\text { included }\end{array}$ & None listed & $\begin{array}{l}\text { Total:29 } \\
\text { RCTs:17 } \\
\text { Non-RCTs with } \\
\text { comparison:2 } \\
\text { Observational:8 }\end{array}$ & Outpatient clinic \\
\hline
\end{tabular}




\begin{tabular}{|c|c|c|c|c|c|c|c|}
\hline $\begin{array}{l}\text { Type of } \\
\text { Strategy }\end{array}$ & Author, Year & $\begin{array}{l}\text { Objective of } \\
\text { Review }\end{array}$ & $\begin{array}{l}\text { Literature } \\
\text { Start - } \\
\text { End Year }\end{array}$ & Inclusion Criteria & Exclusion Criteria & $\begin{array}{l}\text { Number of } \\
\text { Included Studies }\end{array}$ & Settings \\
\hline & $\begin{array}{l}\text { Levengood, } \\
2019^{52}\end{array}$ & $\begin{array}{l}\text { Examine the } \\
\text { effectiveness of } \\
\text { team based care in } \\
\text { improving health } \\
\text { outcomes of } \\
\text { people living with } \\
\text { diabetes }\end{array}$ & 2003-2015 & $\begin{array}{l}\text { Studies were included } \\
\text { if the following criteria } \\
\text { were met: focused on } \\
\text { people diagnosed with } \\
\text { Type } 1 \text { or } 2 \text { diabetes; } \\
\text { conducted in a World } \\
\text { Bank-designated high- } \\
\text { income economy10 } \\
\text { and published in } \\
\text { English; RCT; team } \\
\text { consisted of patient, } \\
\text { primary care provider } \\
\text { (not necessarily a } \\
\text { physician), and one or } \\
\text { more healthcare } \\
\text { professionals; team } \\
\text { members aware of } \\
\text { each other's roles and } \\
\text { responsibilities; relied } \\
\text { on multidirectional flow } \\
\text { of information to } \\
\text { manage patient care; } \\
\text { care was ongoing, } \\
\text { longitudinal (two or } \\
\text { more contacts between } \\
\text { patients and added } \\
\text { team members); and } \\
\text { included one or more } \\
\text { outcomes of interest }\end{array}$ & None listed & $\begin{array}{l}\text { Total:35 } \\
\text { RCTs:35 } \\
\text { Non-RCTs with } \\
\text { comparison:0 } \\
\text { Observational:0 }\end{array}$ & $\begin{array}{l}\text { Hospital, outpatient } \\
\text { clinic, home }\end{array}$ \\
\hline
\end{tabular}




\begin{tabular}{|c|c|c|c|c|c|c|c|}
\hline $\begin{array}{l}\text { Type of } \\
\text { Strategy }\end{array}$ & Author, Year & $\begin{array}{l}\text { Objective of } \\
\text { Review }\end{array}$ & $\begin{array}{l}\text { Literature } \\
\text { Start - } \\
\text { End Year }\end{array}$ & Inclusion Criteria & Exclusion Criteria & $\begin{array}{l}\text { Number of } \\
\text { Included Studies }\end{array}$ & Settings \\
\hline & Zhao, $2015^{53}$ & $\begin{array}{l}\text { To examine the } \\
\text { effectiveness of } \\
\text { telemedicine in } \\
\text { relieving asthma } \\
\text { symptoms. }\end{array}$ & $\begin{array}{l}\text { Inception- } \\
2013\end{array}$ & $\begin{array}{l}\text { P: Adults } 18 \text { years of } \\
\text { age with Asthma, I: } \\
\text { intervention involved } \\
\text { any format of } \\
\text { telemedicine such as } \\
\text { text messaging, } \\
\text { telephone, Internet, or } \\
\text { mobile phone, C: usual } \\
\text { care, O: clinical } \\
\text { outcome of controlling } \\
\text { asthma symptoms, } \\
\text { Study design: } \\
\text { randomized controlled } \\
\text { trials }\end{array}$ & $\begin{array}{l}\text { Exclusion criteria } \\
\text { were as follows: (1) } \\
\text { participants had a } \\
\text { diagnosis of a lung } \\
\text { disease other than } \\
\text { asthma; (2) the } \\
\text { study did not } \\
\text { evaluate changes } \\
\text { of asthma } \\
\text { symptoms; (3) non- } \\
\text { English publication; } \\
\text { and (4) the } \\
\text { publication was a } \\
\text { letter, comment, } \\
\text { editorial, or case } \\
\text { report }\end{array}$ & $\begin{array}{l}\text { Total:11 } \\
\text { RCTs:11 } \\
\text { Non-RCTs with } \\
\text { comparison:0 } \\
\text { Observational:0 }\end{array}$ & Hospital and home \\
\hline & $\begin{array}{l}\text { Lederle, } \\
2019^{54}\end{array}$ & $\begin{array}{l}\text { Our review and } \\
\text { meta-analysis } \\
\text { sheds light on the } \\
\text { relationship } \\
\text { between lay-led } \\
\text { self-management } \\
\text { programs and } \\
\text { health care } \\
\text { utilization. }\end{array}$ & $2006-2017$ & $\begin{array}{l}\text { Lay-led strategies for } \\
\text { self-management, } 1 \text { or } \\
\text { more chronic disease, } \\
\text { health care utilization } \\
\text { outcomes, in person } \\
\text { component }\end{array}$ & $\begin{array}{l}\text { Children and } \\
\text { adolescents }\end{array}$ & $\begin{array}{l}\text { Total:49 } \\
\text { RCTs:33 } \\
\text { Non-RCTs with } \\
\text { comparison:0 } \\
\text { Observational:16 }\end{array}$ & Outpatient clinic \\
\hline & $\begin{array}{l}\text { Nazarov, } \\
2019^{55}\end{array}$ & $\begin{array}{l}\text { Identify studies of } \\
\text { interventions that } \\
\text { support the } \\
\text { maintenance of } \\
\text { work and return to } \\
\text { work (RTW) } \\
\text { among workers } \\
\text { with chronic } \\
\text { illnesses. }\end{array}$ & $\begin{array}{l}\text { Not } \\
\text { mentioned- } \\
2018\end{array}$ & $\begin{array}{l}\text { RCTs and controlled } \\
\text { clinical trials (CCTs) } \\
\text { were included. Studies } \\
\text { were selected if they } \\
\text { described factors } \\
\text { related to RTW of } \\
\text { employed adults (aged } \\
\text { 18+) with chronic } \\
\text { disease }\end{array}$ & $\begin{array}{l}\text { Any other study } \\
\text { form without a } \\
\text { comparison group }\end{array}$ & $\begin{array}{l}\text { Total:15 } \\
\text { RCTs:15 } \\
\text { Non-RCTs with } \\
\text { comparison:0 } \\
\text { Observational:0 }\end{array}$ & Workplace \\
\hline
\end{tabular}




\begin{tabular}{|c|c|c|c|c|c|c|c|}
\hline $\begin{array}{l}\text { Type of } \\
\text { Strategy }\end{array}$ & Author, Year & $\begin{array}{l}\text { Objective of } \\
\text { Review }\end{array}$ & $\begin{array}{l}\text { Literature } \\
\text { Start - } \\
\text { End Year }\end{array}$ & Inclusion Criteria & Exclusion Criteria & $\begin{array}{l}\text { Number of } \\
\text { Included Studies }\end{array}$ & Settings \\
\hline & $\begin{array}{l}\text { Sangrar, } \\
2019^{56}\end{array}$ & $\begin{array}{l}\text { To review the } \\
\text { literature on } \\
\text { chronic disease } \\
\text { self-management } \\
\text { programs that } \\
\text { blend face-to-face } \\
\text { and } \\
\text { online/computer- } \\
\text { based education } \\
\text { design and } \\
\text { delivery. }\end{array}$ & $2004-2019$ & $\begin{array}{l}\text { Included studies where } \\
\text { participants had } \\
\text { cancer, cardiovascular } \\
\text { disease and/or mental } \\
\text { illnesses in addition to } \\
\text { the chronic diseases of } \\
\text { interest; Only patient } \\
\text { education programs } \\
\text { that combined face-to- } \\
\text { face and online/ } \\
\text { computer-based } \\
\text { strategies delivered or } \\
\text { mediated by } \\
\text { healthcare } \\
\text { professionals in at } \\
\text { least one study arm } \\
\text { were included }\end{array}$ & $\begin{array}{l}\text { We excluded } \\
\text { studies focused } \\
\text { only on patients } \\
\text { with cancers, } \\
\text { cardiovascular } \\
\text { conditions, and } \\
\text { serious mental } \\
\text { illnesses }\end{array}$ & $\begin{array}{l}\text { Total:12 } \\
\text { RCTs:6 } \\
\text { Non-RCTs with } \\
\text { comparison:1 } \\
\text { Observational:5 }\end{array}$ & $\begin{array}{l}\text { Outpatient Clinic, } \\
\text { home }\end{array}$ \\
\hline & Zhao, $2016^{57}$ & $\begin{array}{l}\text { To synthesize the } \\
\text { effects of theory- } \\
\text { based self- } \\
\text { management } \\
\text { educational } \\
\text { interventions on } \\
\text { patients with type } 2 \\
\text { diabetes (T2DM) in } \\
\text { randomized } \\
\text { controlled trials. }\end{array}$ & $1980-2015$ & $\begin{array}{l}\text { Population: targeted } \\
\text { participants } \geq 18 \text { years } \\
\text { old and with T2DM. I: } \\
\text { educational } \\
\text { intervention was based } \\
\text { on a theory/model, with } \\
\text { a clear description in } \\
\text { the study, or used a } \\
\text { structured method } \\
\text { which had a theory as } \\
\text { the background, C: } \\
\text { compared with routine } \\
\text { care. O: HbA1c and } \\
\text { BMI, Self efficacy. } \\
\text { Studies: RCTs }\end{array}$ & $\begin{array}{l}\text { Studies including } \\
\text { children, no } \\
\text { comparison or non- } \\
\text { RCTs }\end{array}$ & $\begin{array}{l}\text { Total:20 } \\
\text { RCTs:20 } \\
\text { Non-RCTs with } \\
\text { comparison:0 } \\
\text { Observational:0 }\end{array}$ & Hospital \\
\hline
\end{tabular}




\begin{tabular}{|c|c|c|c|c|c|c|c|}
\hline $\begin{array}{l}\text { Type of } \\
\text { Strategy }\end{array}$ & Author, Year & $\begin{array}{l}\text { Objective of } \\
\text { Review }\end{array}$ & $\begin{array}{l}\text { Literature } \\
\text { Start - } \\
\text { End Year }\end{array}$ & Inclusion Criteria & Exclusion Criteria & $\begin{array}{l}\text { Number of } \\
\text { Included Studies }\end{array}$ & Settings \\
\hline & $\begin{array}{l}\text { Skrabal } \\
\text { Ross, } 2018^{58}\end{array}$ & $\begin{array}{l}\text { aims to explore } \\
\text { what is known } \\
\text { about mobile } \\
\text { phone-delivered } \\
\text { interventions } \\
\text { designed to } \\
\text { enhance } \\
\text { adherence to oral } \\
\text { chemotherapy, to } \\
\text { examine the } \\
\text { reported findings } \\
\text { on the utility of } \\
\text { these interventions } \\
\text { in increasing oral } \\
\text { chemotherapy } \\
\text { adherence, and to } \\
\text { identify } \\
\text { opportunities for } \\
\text { development of } \\
\text { future interventions }\end{array}$ & $\begin{array}{l}\text { Not } \\
\text { mentioned- } \\
2018\end{array}$ & $\begin{array}{l}\text { (1) research-based } \\
\text { studies on } \\
\text { interventions that aim } \\
\text { to increase adherence } \\
\text { to oral chemotherapy } \\
\text { or endocrine therapy, } \\
\text { (2) targets cancer } \\
\text { patients taking oral } \\
\text { chemotherapy or } \\
\text { endocrine therapy, (3) } \\
\text { use of mobile phones } \\
\text { as a main tool to } \\
\text { deliver the intervention, } \\
\text { and (4) articles written } \\
\text { in English. }\end{array}$ & None listed & $\begin{array}{l}\text { Total:5 } \\
\text { RCTs:2 } \\
\text { Non-RCTs with } \\
\text { comparison:0 } \\
\text { Observational:3 }\end{array}$ & Home \\
\hline
\end{tabular}




\begin{tabular}{|c|c|c|c|c|c|c|c|}
\hline $\begin{array}{l}\text { Type of } \\
\text { Strategy }\end{array}$ & Author, Year & $\begin{array}{l}\text { Objective of } \\
\text { Review }\end{array}$ & $\begin{array}{l}\text { Literature } \\
\text { Start - } \\
\text { End Year }\end{array}$ & Inclusion Criteria & Exclusion Criteria & $\begin{array}{l}\text { Number of } \\
\text { Included Studies }\end{array}$ & Settings \\
\hline & $\begin{array}{l}\text { Ha Dinh, } \\
2016^{59}\end{array}$ & $\begin{array}{l}\text { This systematic } \\
\text { review examined } \\
\text { the evidence on } \\
\text { using the teach- } \\
\text { back method in } \\
\text { health education } \\
\text { programs for } \\
\text { improving } \\
\text { adherence and } \\
\text { self-management } \\
\text { of people with } \\
\text { chronic disease }\end{array}$ & $\begin{array}{l}\text { Inception- } \\
2013\end{array}$ & $\begin{array}{l}\text { Adult patients (aged } 18 \\
\text { years and over) in any } \\
\text { healthcare setting with } \\
\text { one or more chronic } \\
\text { disease including heart } \\
\text { failure, diabetes, } \\
\text { cardiovascular } \\
\text { disease, cancer, } \\
\text { asthma, chronic } \\
\text { obstructive pulmonary } \\
\text { disease, chronic } \\
\text { kidney disease, } \\
\text { arthritis, epilepsy or a } \\
\text { mental health } \\
\text { condition; Eligible } \\
\text { studies were those } \\
\text { which reported on the } \\
\text { use of the teach-back } \\
\text { method alone or in } \\
\text { combination with other } \\
\text { supporting educational } \\
\text { strategies; This review } \\
\text { considered quantitative } \\
\text { studies including } \\
\text { randomized controlled } \\
\text { trials (RCTs), non- } \\
\text { randomized controlled } \\
\text { trials, quasi- } \\
\text { experimental studies, } \\
\text { case-controlled } \\
\text { studies, cohort studies, } \\
\text { and before and after } \\
\text { studies; Studies } \\
\text { published in English } \\
\text { were considered for } \\
\text { inclusion in this review. }\end{array}$ & $\begin{array}{l}\text { Studies that } \\
\text { included seriously } \\
\text { ill patients, and/or } \\
\text { those with } \\
\text { impairments in } \\
\text { verbal } \\
\text { communication and } \\
\text { cognitive function } \\
\text { were excluded. }\end{array}$ & $\begin{array}{l}\text { Total:10 } \\
\text { RCTs:8 } \\
\text { Non-RCTs with } \\
\text { comparison:1 } \\
\text { Observational:1 }\end{array}$ & $\begin{array}{l}\text { Inpatient, outpatient } \\
\text { clinics, home }\end{array}$ \\
\hline
\end{tabular}




\begin{tabular}{|c|c|c|c|c|c|c|c|}
\hline $\begin{array}{l}\text { Type of } \\
\text { Strategy }\end{array}$ & Author, Year & $\begin{array}{l}\text { Objective of } \\
\text { Review }\end{array}$ & $\begin{array}{l}\text { Literature } \\
\text { Start - } \\
\text { End Year }\end{array}$ & Inclusion Criteria & Exclusion Criteria & $\begin{array}{l}\text { Number of } \\
\text { Included Studies }\end{array}$ & Settings \\
\hline & Ko, $2018^{60}$ & $\begin{array}{l}\text { This review aimed } \\
\text { to examine how } \\
\text { self-management } \\
\text { has been } \\
\text { operationalized in } \\
\text { the context of } \\
\text { multiple chronic } \\
\text { conditions. }\end{array}$ & 2006-2017 & $\begin{array}{l}\text { Peer-reviewed } \\
\text { research articles which } \\
\text { operationalized self- } \\
\text { management in adults } \\
\text { with at least two or } \\
\text { more chronic illnesses } \\
\text { in adults > } 18 \text { Y were } \\
\text { selected. }\end{array}$ & $\begin{array}{l}\text { Articles were } \\
\text { excluded if they } \\
\text { used instruments } \\
\text { that operationalized } \\
\text { self-management } \\
\text { for specific single } \\
\text { chronic illness } \\
\text { populations such } \\
\text { as diabetes or } \\
\text { heart failure. }\end{array}$ & $\begin{array}{l}\text { Total:7 } \\
\text { RCTs:4 } \\
\text { Non-RCTs with } \\
\text { comparison:0 } \\
\text { Observational:3 }\end{array}$ & Hospital and home \\
\hline & $\begin{array}{l}\text { Changizi, } \\
2017^{61}\end{array}$ & $\begin{array}{l}\text { The present study } \\
\text { was conducted } \\
\text { aiming to assess } \\
\text { the effectiveness of } \\
\text { mHealth in } \\
\text { improving health } \\
\text { behaviors among } \\
\text { an elderly } \\
\text { population }\end{array}$ & $2012-2016$ & $\begin{array}{l}\text { Use of mHealth for } \\
\text { promoting health } \\
\text { behavior in elderly } \\
\text { populations (age of } 60 \\
\text { and over), with a main } \\
\text { focus on authentic } \\
\text { experiments and } \\
\text { clinical trials }\end{array}$ & $\begin{array}{l}\text { Irrelevance to the } \\
\text { main subject, } \\
\text { younger age- } \\
\text { group, } \\
\text { methodology, study } \\
\text { design (review } \\
\text { article, descriptive, } \\
\text { cross-sectional } \\
\text { study, survey } \\
\text { research) and lack } \\
\text { of originality }\end{array}$ & $\begin{array}{l}\text { Total:12 } \\
\text { RCTs:10 } \\
\text { Non-RCTs with } \\
\text { comparison:0 } \\
\text { Observational:2 }\end{array}$ & $\begin{array}{l}\text { Home, outpatient } \\
\text { clinic }\end{array}$ \\
\hline
\end{tabular}




\begin{tabular}{|c|c|c|c|c|c|c|c|}
\hline $\begin{array}{l}\text { Type of } \\
\text { Strategy }\end{array}$ & Author, Year & $\begin{array}{l}\text { Objective of } \\
\text { Review }\end{array}$ & $\begin{array}{l}\text { Literature } \\
\text { Start - } \\
\text { End Year }\end{array}$ & Inclusion Criteria & Exclusion Criteria & $\begin{array}{l}\text { Number of } \\
\text { Included Studies }\end{array}$ & Settings \\
\hline & Kelly, $2018^{62}$ & $\begin{array}{l}\text { To assess the } \\
\text { efficacy, cost- } \\
\text { effectiveness and } \\
\text { adverse effects of } \\
\text { self-management } \\
\text { interventions for } \\
\text { adults and children } \\
\text { with non-cystic } \\
\text { fibrosis } \\
\text { bronchiectasis. }\end{array}$ & $1937-2018$ & $\begin{array}{l}\text { Randomized controlled } \\
\text { trials of any duration } \\
\text { that included adults or } \\
\text { children with a } \\
\text { diagnosis of non-cystic } \\
\text { fibrosis bronchiectasis } \\
\text { assessing self- } \\
\text { management } \\
\text { interventions delivered } \\
\text { in any form. Self- } \\
\text { management } \\
\text { interventions included } \\
\text { at least two of the } \\
\text { following elements: } \\
\text { patient education, } \\
\text { airway clearance } \\
\text { techniques, adherence } \\
\text { to medication, exercise } \\
\text { (including pulmonary } \\
\text { rehabilitation) and } \\
\text { action plans. }\end{array}$ & $\begin{array}{l}\text { We excluded } \\
\text { participants with a } \\
\text { diagnosis of cystic } \\
\text { fibrosis (CF), } \\
\text { sarcoidosis or } \\
\text { active allergic } \\
\text { bronchopulmonary } \\
\text { aspergillosis. We } \\
\text { also excluded } \\
\text { studies of other } \\
\text { long-term health } \\
\text { conditions unless } \\
\text { results for people } \\
\text { with bronchiectasis } \\
\text { were reported s }\end{array}$ & $\begin{array}{l}\text { Total:2 } \\
\text { RCTs:2 } \\
\text { Non-RCTs with } \\
\text { comparison:0 } \\
\text { Observational:0 }\end{array}$ & $\begin{array}{l}\text { Home, outpatient } \\
\text { clinic }\end{array}$ \\
\hline
\end{tabular}




\begin{tabular}{|c|c|c|c|c|c|c|c|}
\hline $\begin{array}{l}\text { Type of } \\
\text { Strategy }\end{array}$ & Author, Year & $\begin{array}{l}\text { Objective of } \\
\text { Review }\end{array}$ & $\begin{array}{l}\text { Literature } \\
\text { Start - } \\
\text { End Year }\end{array}$ & Inclusion Criteria & Exclusion Criteria & $\begin{array}{l}\text { Number of } \\
\text { Included Studies }\end{array}$ & Settings \\
\hline & Jeddi, $2017^{63}$ & $\begin{array}{l}\text { We conducted a } \\
\text { systematic review } \\
\text { of randomized } \\
\text { controlled trials } \\
\text { (RCTs) to assess } \\
\text { the features and } \\
\text { effects of IT-based } \\
\text { interventions on } \\
\text { self-management } \\
\text { outcomes of CKD } \\
\text { patients }\end{array}$ & $\begin{array}{l}\text { Not } \\
\text { mentioned- } \\
2016\end{array}$ & $\begin{array}{l}\text { (1) IT-based } \\
\text { interventions in } \\
\text { patients with CKD } \\
\text { stages } 1 \text { to 5; (2) } \\
\text { Interventions with all } \\
\text { kinds of IT-based tools, } \\
\text { such as a smart } \\
\text { phone, tablet, smart TV } \\
\text { or computer, that } \\
\text { support all or part of } \\
\text { the intervention } \\
\text { consisting of the self- } \\
\text { management; (3) The } \\
\text { study design being a } \\
\text { randomized controlled } \\
\text { trial (RCT); (4) Having } \\
\text { a control group } \\
\text { receiving standard/ } \\
\text { usual care without IT- } \\
\text { based systems. }\end{array}$ & $\begin{array}{l}\text { (1) Studies in which } \\
\text { healthcare } \\
\text { providers were the } \\
\text { consumers of the } \\
\text { intervention; (2) } \\
\text { Studies with the IT- } \\
\text { based intervention } \\
\text { performed only } \\
\text { through the direct } \\
\text { involvement of } \\
\text { healthcare } \\
\text { providers; (3) } \\
\text { Studies focused on } \\
\text { the feasibility, } \\
\text { validity, } \\
\text { acceptability, or } \\
\text { description of IT- } \\
\text { based tools and } \\
\text { systems; (4) } \\
\text { Descriptive studies } \\
\text { without comparison } \\
\text { group, case } \\
\text { reports, } \\
\text { commentaries, } \\
\text { reviews, study } \\
\text { protocols, surveys, } \\
\text { conference } \\
\text { proceedings, and } \\
\text { before-after trials. }\end{array}$ & $\begin{array}{l}\text { Total:8 } \\
\text { RCTs:8 } \\
\text { Non-RCTs with } \\
\text { comparison:0 } \\
\text { Observational:0 }\end{array}$ & Home \\
\hline
\end{tabular}




\begin{tabular}{|c|c|c|c|c|c|c|c|}
\hline $\begin{array}{l}\text { Type of } \\
\text { Strategy }\end{array}$ & Author, Year & $\begin{array}{l}\text { Objective of } \\
\text { Review }\end{array}$ & $\begin{array}{l}\text { Literature } \\
\text { Start - } \\
\text { End Year }\end{array}$ & Inclusion Criteria & Exclusion Criteria & $\begin{array}{l}\text { Number of } \\
\text { Included Studies }\end{array}$ & Settings \\
\hline & $\begin{array}{l}\text { Conway, } \\
2017^{64}\end{array}$ & $\begin{array}{l}\text { The objective of } \\
\text { this integrative } \\
\text { review was to } \\
\text { examine the types } \\
\text { of digital health } \\
\text { technologies that } \\
\text { targeted } \\
\text { medication } \\
\text { adherence in the } \\
\text { adult population } \\
\text { with diabetes or } \\
\text { hypertension }\end{array}$ & $2006-2016$ & $\begin{array}{l}\text { (1) English-language, } \\
\text { peer-reviewed } \\
\text { randomized controlled } \\
\text { trials (RCTs) with } \\
\text { quasi-experimental, } \\
\text { observational, or } \\
\text { qualitative design; (2) } \\
\text { studies containing } \\
\text { digital health } \\
\text { interventions to } \\
\text { improve medication } \\
\text { adherence to } \\
\text { prescription } \\
\text { medications in adults } \\
\text { (ie, } 18 \text { years or older); } \\
\text { and (3) studies } \\
\text { focused on diabetes or } \\
\text { hypertension. }\end{array}$ & $\begin{array}{l}\text { (1) studies that did } \\
\text { not include results } \\
\text { of medication } \\
\text { adherence rates or } \\
\text { (2) pilot studies }\end{array}$ & $\begin{array}{l}\text { Total:13 } \\
\text { RCTs:9 } \\
\text { Non-RCTs with } \\
\text { comparison:0 } \\
\text { Observational:4 }\end{array}$ & Home \\
\hline & Kim, $2017^{65}$ & $\begin{array}{l}\text { Scoping review to } \\
\text { (1) understand the } \\
\text { nature, extent, and } \\
\text { range of smart } \\
\text { device-based } \\
\text { research activities, } \\
\text { (2) identify the } \\
\text { limitations of the } \\
\text { current research } \\
\text { and knowledge } \\
\text { gap, (3) } \\
\text { recommend future } \\
\text { research directions }\end{array}$ & $2010-2016$ & $\begin{array}{l}\text { All study designs, only } \\
\text { articles published in } \\
2010 \text { or after were } \\
\text { selected to } \\
\text { accommodate the } \\
\text { introduction of tablets } \\
\text { and the wide adoption } \\
\text { of smartphones, } \\
\text { average age of } \\
\text { participants was } 50 \\
\text { years or older, aimed } \\
\text { to support chronic } \\
\text { disease management. }\end{array}$ & $\begin{array}{l}\text { SMS or interactive } \\
\text { voice response- } \\
\text { based mHealth } \\
\text { interventions, (2) } \\
\text { studies that } \\
\text { validated electronic } \\
\text { versions of scales } \\
\text { or questionnaire } \\
\text { forms of existing } \\
\text { instruments, (3) } \\
\text { smart device-based } \\
\text { interventions for } \\
\text { postoperative } \\
\text { monitoring }\end{array}$ & $\begin{array}{l}\text { Total:51 } \\
\text { RCTs:13 } \\
\text { Non-RCTs with } \\
\text { comparison:5 } \\
\text { Observational:33 }\end{array}$ & Home \\
\hline
\end{tabular}




\begin{tabular}{|c|c|c|c|c|c|c|c|}
\hline $\begin{array}{l}\text { Type of } \\
\text { Strategy }\end{array}$ & Author, Year & $\begin{array}{l}\text { Objective of } \\
\text { Review }\end{array}$ & $\begin{array}{l}\text { Literature } \\
\text { Start - } \\
\text { End Year }\end{array}$ & Inclusion Criteria & Exclusion Criteria & $\begin{array}{l}\text { Number of } \\
\text { Included Studies }\end{array}$ & Settings \\
\hline & Cho, $2017^{66}$ & $\begin{array}{l}\text { SR: Aim of this SR } \\
\text { was to assess the } \\
\text { impact of } \\
\text { technology- } \\
\text { mediated } \\
\text { interventions on } \\
\text { QoL and to identify } \\
\text { the instruments } \\
\text { used to measure } \\
\text { the QoL of persons } \\
\text { living with } \\
\text { HIVIAIDS (PLWH). }\end{array}$ & $1994-2016$ & $\begin{array}{l}\text { 1) manuscript; 2) RCTs } \\
\text { that assessed the } \\
\text { impact of technology- } \\
\text { mediated interventions } \\
\text { as compared to usual } \\
\text { care or any other } \\
\text { intervention; 3) } \\
\text { population of interest } \\
\text { as PLWH; 4) examined } \\
\text { QoL as a health } \\
\text { outcome. }\end{array}$ & $\begin{array}{l}\text { Excluded poster } \\
\text { sessions, } \\
\text { presentations, } \\
\text { protocols, letters, } \\
\text { comments, } \\
\text { editorials, } \\
\text { correspondences } \\
\text { or grey literature } \\
\text { (e.g., blogs, } \\
\text { newsletters, } \\
\text { videos). }\end{array}$ & $\begin{array}{l}\text { Total:10 } \\
\text { RCTs:10 } \\
\text { Non-RCTs with } \\
\text { comparison:0 } \\
\text { Observational:0 }\end{array}$ & Hospital and home \\
\hline & $\begin{array}{l}\text { Clarkesmith, } \\
2017^{67}\end{array}$ & $\begin{array}{l}\text { SR: Synthesizing } \\
\text { the evidence about } \\
\text { the effects of } \\
\text { educational and } \\
\text { behavioral } \\
\text { interventions in } \\
\text { patients with atrial } \\
\text { fibrillation who are } \\
\text { taking oral } \\
\text { anticoagulant } \\
\text { medication. }\end{array}$ & $1806-2016$ & $\begin{array}{l}\text { Randomized controlled } \\
\text { trials (RCTs) of } \\
\text { educational or } \\
\text { behavioral } \\
\text { interventions with any } \\
\text { length of follow-up and } \\
\text { in any language } \\
\text { included. Adults (aged } \\
18 \text { years or older) with } \\
\text { AF, categorized } \\
\text { according to the } \\
\text { European Society of } \\
\text { Cardiology (ESC) } \\
\text { guidelines. } \\
\text { Interventions and } \\
\text { outcomes as } \\
\text { mentioned. }\end{array}$ & None listed & $\begin{array}{l}\text { Total:11 } \\
\text { RCTs:10 } \\
\text { Non-RCTs with } \\
\text { comparison:0 } \\
\text { Observational:0 }\end{array}$ & Hospital \\
\hline
\end{tabular}




\begin{tabular}{|c|c|c|c|c|c|c|c|}
\hline $\begin{array}{l}\text { Type of } \\
\text { Strategy }\end{array}$ & Author, Year & $\begin{array}{l}\text { Objective of } \\
\text { Review }\end{array}$ & $\begin{array}{l}\text { Literature } \\
\text { Start - } \\
\text { End Year }\end{array}$ & Inclusion Criteria & Exclusion Criteria & $\begin{array}{l}\text { Number of } \\
\text { Included Studies }\end{array}$ & Settings \\
\hline & $\begin{array}{l}\text { Massimi, } \\
2017^{68}\end{array}$ & $\begin{array}{l}\text { The aim of this } \\
\text { systematic review } \\
\text { and meta-analysis } \\
\text { is to assess the } \\
\text { efficacy of the } \\
\text { nurse-led self- } \\
\text { management } \\
\text { support versus } \\
\text { usual care } \\
\text { evaluating patient } \\
\text { outcomes in } \\
\text { chronic care } \\
\text { community } \\
\text { programs. }\end{array}$ & $1990-2016$ & $\begin{array}{l}\text { "P: adults } 65 \text { years of } \\
\text { age and older (of any } \\
\text { ethnicity) who had one } \\
\text { or more chronic } \\
\text { conditions, I: nurse-led } \\
\text { self-management } \\
\text { support intervention, C: } \\
\text { compared to the usual } \\
\text { care, to improve O: } \\
\text { observer-reported } \\
\text { outcomes (OROs) } \\
\text { particularly clinical } \\
\text { outcomes and patient- } \\
\text { reported outcomes } \\
\text { (PROs). Studies } \\
\text { design: RCTs" }\end{array}$ & $\begin{array}{l}\text { In-hospital based } \\
\text { care and discharge } \\
\text { planning program } \\
\text { from hospital were } \\
\text { excluded. Non RCT } \\
\text { design or without } \\
\text { comparison } \\
\text { excluded }\end{array}$ & $\begin{array}{l}\text { Total:29 } \\
\text { RCTs:13 }\end{array}$ & $\begin{array}{l}\text { Patient home and } \\
\text { community based } \\
\text { facilities }\end{array}$ \\
\hline & $\begin{array}{l}\text { Dounavi, } \\
2019^{69}\end{array}$ & $\begin{array}{l}\text { Identify existing } \\
\text { evidence on the } \\
\text { efficacy of mobile } \\
\text { health technology } \\
\text { in facilitating } \\
\text { weight } \\
\text { management } \\
\text { behaviors, such as } \\
\text { healthy food } \\
\text { consumption and } \\
\text { physical activity }\end{array}$ & $2012-2017$ & $\begin{array}{l}\text { (1) adult population; (2) } \\
\text { typical intellectual } \\
\text { ability; (3) dependent } \\
\text { variable: weight } \\
\text { management } \\
\text { behaviors; (4) } \\
\text { independent variable: } \\
\text { use of mobile } \\
\text { technology including } \\
\text { self-monitoring } \\
\text { strategies; and (5) } \\
\text { primary study }\end{array}$ & $\begin{array}{l}\text { (1) age <18 years; } \\
\text { (2) diagnosis of } \\
\text { intellectual } \\
\text { disability; (3) weight } \\
\text { gain control in } \\
\text { pregnancy or } \\
\text { postpartum weight } \\
\text { loss; (4) use of } \\
\text { mobile technology } \\
\text { for } \\
\text { education/provision } \\
\text { of information } \\
\text { versus interactive } \\
\text { use for self- } \\
\text { management }\end{array}$ & $\begin{array}{l}\text { Total:39 } \\
\text { RCTs:22 } \\
\text { Non-RCTs with } \\
\text { comparison:2 } \\
\text { Observational:15 }\end{array}$ & $\begin{array}{l}\text { Outpatient Clinic, } \\
\text { home }\end{array}$ \\
\hline & Price, $2015^{70}$ & $\begin{array}{l}\text { Describe evidence } \\
\text { for benefit from } \\
\text { patient health } \\
\text { record-enabled } \\
\text { management, by } \\
\text { health condition. }\end{array}$ & $2008-2014$ & $\begin{array}{l}\text { Intervention =self-care } \\
\text { activities; Use pf PHR; } \\
\text { Outpatient }\end{array}$ & $\begin{array}{l}\text { Patients not using } \\
\text { PHR; usability } \\
\text { testing }\end{array}$ & $\begin{array}{l}\text { Total:23 } \\
\text { RCTs:7 } \\
\text { Non-RCTs with } \\
\text { comparison:2 } \\
\text { Observational:14 }\end{array}$ & Outpatient \\
\hline
\end{tabular}




\begin{tabular}{|c|c|c|c|c|c|c|c|}
\hline $\begin{array}{l}\text { Type of } \\
\text { Strategy }\end{array}$ & Author, Year & $\begin{array}{l}\text { Objective of } \\
\text { Review }\end{array}$ & $\begin{array}{l}\text { Literature } \\
\text { Start - } \\
\text { End Year }\end{array}$ & Inclusion Criteria & Exclusion Criteria & $\begin{array}{l}\text { Number of } \\
\text { Included Studies }\end{array}$ & Settings \\
\hline & $\begin{array}{l}\text { Aquino, } \\
2017^{71}\end{array}$ & $\begin{array}{l}\text { To identify and } \\
\text { evaluate the } \\
\text { effectiveness of } \\
\text { individual } \\
\text { empowerment } \\
\text { strategies } \\
\text { inpatients with } \\
\text { diabetes mellitus } \\
\text { (DM). }\end{array}$ & $\begin{array}{l}\text { Not } \\
\text { mentioned- } \\
2016\end{array}$ & $\begin{array}{l}\text { "P" (problem): patients } \\
\text { with diabetes mellitus; } \\
\text { "I" (intervention): } \\
\text { individual intervention } \\
\text { strategies for } \\
\text { empowerment; "C" } \\
\text { (control): patients } \\
\text { without intervention for } \\
\text { empowerment; "O" } \\
\text { (outcomes): reduction } \\
\text { of HbA1c;"S" (study } \\
\text { design): randomized } \\
\text { controlled trials. }\end{array}$ & $\begin{array}{l}\text { Review articles, } \\
\text { editorials, letters to } \\
\text { the editor, news } \\
\text { reports, comments, } \\
\text { as well as the } \\
\text { results of } \\
\text { dissertations, } \\
\text { theses or abstracts } \\
\text { published in annals } \\
\text { of congress or } \\
\text { scientific journals, } \\
\text { articles with } \\
\text { collective strategies } \\
\text { or using both } \\
\text { strategies }\end{array}$ & $\begin{array}{l}\text { Total:11 } \\
\text { RCTs:11 } \\
\text { Non-RCTs with } \\
\text { comparison:0 } \\
\text { Observational:0 }\end{array}$ & Hospital and home \\
\hline & $\begin{array}{l}\text { Ammenwerth, } \\
2019^{72}\end{array}$ & $\begin{array}{l}\text { Assess the effect } \\
\text { of patient portals } \\
\text { on patient } \\
\text { empowerment and } \\
\text { health-related } \\
\text { outcomes. }\end{array}$ & $2000-2017$ & $\begin{array}{l}\text { Applied patient portal } \\
\text { taxonomy - access, } \\
\text { remind, request, } \\
\text { communicate, share, } \\
\text { manage, educate. }\end{array}$ & $\begin{array}{l}\text { Exclude age }<18 \text { or } \\
\text { wen caregivers } \\
\text { were targeted }\end{array}$ & $\begin{array}{l}\text { Total:10 } \\
\text { RCTs:10 } \\
\text { Non-RCTs with } \\
\text { comparison:0 } \\
\text { Observational:0 }\end{array}$ & Outpatient \\
\hline & $\begin{array}{l}\text { Donald, } \\
2018^{73}\end{array}$ & $\begin{array}{l}\text { To systematically } \\
\text { identify and } \\
\text { describe self } \\
\text { management } \\
\text { interventions for } \\
\text { adult patients with } \\
\text { chronic kidney } \\
\text { disease (CKD). }\end{array}$ & $\begin{array}{l}\text { Not } \\
\text { mentioned- } \\
2016\end{array}$ & $\begin{array}{l}\text { All studies included } \\
\text { (RCTs, Non RCTs, } \\
\text { Quasi, Prepost, Obs), } \\
\text { self management } \\
\text { interventions for adult } \\
\text { patients with chronic } \\
\text { kidney disease (CKD). } \\
\text { Outcomes included } \\
\text { behaviors, cognitions, } \\
\text { physiological } \\
\text { measures, symptoms, } \\
\text { health status and } \\
\text { healthcare. }\end{array}$ & & $\begin{array}{l}\text { Total:50 } \\
\text { RCTs:19 } \\
\text { Non-RCTs with } \\
\text { comparison:13 }\end{array}$ & Community-based \\
\hline
\end{tabular}




\begin{tabular}{|c|c|c|c|c|c|c|c|}
\hline $\begin{array}{l}\text { Type of } \\
\text { Strategy }\end{array}$ & Author, Year & $\begin{array}{l}\text { Objective of } \\
\text { Review }\end{array}$ & $\begin{array}{l}\text { Literature } \\
\text { Start - } \\
\text { End Year }\end{array}$ & Inclusion Criteria & Exclusion Criteria & $\begin{array}{l}\text { Number of } \\
\text { Included Studies }\end{array}$ & Settings \\
\hline & $\begin{array}{l}\text { Boulley, } \\
2018^{74}\end{array}$ & $\begin{array}{l}\text { This study aims to } \\
\text { highlight the } \\
\text { components of } \\
\text { Digital health } \\
\text { interventions (DI), } \\
\text { investigate patient } \\
\text { engagement with } \\
\text { DI, and explore the } \\
\text { effects of DI on } \\
\text { psychosocial } \\
\text { variables. }\end{array}$ & $\begin{array}{l}\text { Inception- } \\
2017\end{array}$ & $\begin{array}{l}\text { (1) reviewed and } \\
\text { published in English, } \\
\text { (2) tested one or more } \\
\text { DI, (3) assessed } \\
\text { psychosocial variables } \\
\text { or engagement with DI, } \\
\text { (4) presented DI } \\
\text { focused on helping } \\
\text { patients or survivors to } \\
\text { autonomously manage } \\
\text { their health condition } \\
\text { on a daily basis (5) } \\
\text { presented a main } \\
\text { study, a pilot study, or } \\
\text { an exploratory study, } \\
\text { and, (6) presented a } \\
\text { study which had one of } \\
\text { the following design } \\
\text { types: RCT, cross- } \\
\text { sectional study or } \\
\text { pretest-posttest study. }\end{array}$ & $\begin{array}{l}\text { Studies were } \\
\text { excluded when (1) } \\
\text { the DI presented } \\
\text { aimed at preventing } \\
\text { or detecting cancer, } \\
\text { (2) participants did } \\
\text { not actually use D- } \\
\text { tools, (3) } \\
\text { psychosocial } \\
\text { variables, or } \\
\text { engagement with } \\
\text { DI were not } \\
\text { assessed, (4) the } \\
\text { DI presented aimed } \\
\text { at improving self- } \\
\text { care }\end{array}$ & $\begin{array}{l}\text { Total:29 } \\
\text { RCTs:15 } \\
\text { Non-RCTs with } \\
\text { comparison:8 } \\
\text { Observational:6 }\end{array}$ & Hospital and home \\
\hline & $\begin{array}{l}\text { Peytremann- } \\
\text { Bridevaux, } \\
2015^{75}\end{array}$ & $\begin{array}{l}\text { To evaluate the } \\
\text { effectiveness of } \\
\text { chronic disease } \\
\text { management } \\
\text { programs for adults } \\
\text { with asthma. }\end{array}$ & $\begin{array}{l}\text { Inception- } \\
2014\end{array}$ & $\begin{array}{l}\text { We included individual } \\
\text { or cluster-randomized } \\
\text { controlled trials, non- } \\
\text { randomized controlled } \\
\text { trials, and controlled } \\
\text { before-after studies } \\
\text { com- paring chronic } \\
\text { disease management } \\
\text { programs with usual } \\
\text { care in adults over } 16 \\
\text { years of age with a } \\
\text { diagnosis of asthma }\end{array}$ & $\begin{array}{l}\text { We excluded } \\
\text { studies in which } \\
\text { patients with other } \\
\text { significant } \\
\text { pulmonary chronic } \\
\text { disease (like } \\
\text { moderate or severe } \\
\text { COPD or } \\
\text { bronchiectasis) } \\
\text { represented a } \\
\text { significant } \\
\text { proportion of } \\
\text { participants, unless } \\
\text { subgroup analysis } \\
\text { was available. }\end{array}$ & $\begin{array}{l}\text { Total:20 } \\
\text { RCTs:15 } \\
\text { Non-RCTs with } \\
\text { comparison:5 } \\
\text { Observational:0 }\end{array}$ & $\begin{array}{l}\text { Inpatient, outpatient } \\
\text { clinics }\end{array}$ \\
\hline
\end{tabular}




\begin{tabular}{|c|c|c|c|c|c|c|c|}
\hline $\begin{array}{l}\text { Type of } \\
\text { Strategy }\end{array}$ & Author, Year & $\begin{array}{l}\text { Objective of } \\
\text { Review }\end{array}$ & $\begin{array}{l}\text { Literature } \\
\text { Start - } \\
\text { End Year }\end{array}$ & Inclusion Criteria & Exclusion Criteria & $\begin{array}{l}\text { Number of } \\
\text { Included Studies }\end{array}$ & Settings \\
\hline & $\begin{array}{l}\text { Laukner, } \\
2016^{76}\end{array}$ & $\begin{array}{l}\text { A scoping review } \\
\text { was undertaken to } \\
\text { discover } \\
\text { community-based } \\
\text { peer support } \\
\text { initiatives for adults } \\
\text { in rural settings } \\
\text { living with chronic } \\
\text { conditions }\end{array}$ & $2000-2014$ & $\begin{array}{l}\text { Interventions/programs } \\
\text { (rather than opinion } \\
\text { pieces); the years } \\
2000-\text { January } 2014 \text { (to } \\
\text { ensure } \\
\text { currency);English only } \\
\text { (due to language } \\
\text { limitations of the } \\
\text { research group); adults } \\
\text { ( } \geq 18 \text { years); explicit } \\
\text { involvement of peers } \\
\text { who work with people } \\
\text { who have a chronic } \\
\text { condition the peer is } \\
\text { familiar with; } \\
\text { community-based } \\
\text { (rather than hospital- } \\
\text { based) with an } \\
\text { emphasis on } \\
\text { community } \\
\text { involvement (rather } \\
\text { than medical } \\
\text { management); explicit } \\
\text { reference to being } \\
\text { located in rural } \\
\text { settings. }\end{array}$ & $\begin{array}{l}\text { professional-led } \\
\text { initiatives with no } \\
\text { focus on the } \\
\text { development of } \\
\text { peer supports; } \\
\text { initiatives that only } \\
\text { focus on friendship } \\
\text { development } \\
\text { without reference to } \\
\text { community } \\
\text { involvement; } \\
\text { initiatives that focus } \\
\text { on caregivers of } \\
\text { people with chronic } \\
\text { conditions }\end{array}$ & Total:13 & $\begin{array}{l}\text { Outpatient Clinics, } \\
\text { home }\end{array}$ \\
\hline
\end{tabular}




\begin{tabular}{|c|c|c|c|c|c|c|c|}
\hline $\begin{array}{l}\text { Type of } \\
\text { Strategy }\end{array}$ & Author, Year & $\begin{array}{l}\text { Objective of } \\
\text { Review }\end{array}$ & $\begin{array}{l}\text { Literature } \\
\text { Start - } \\
\text { End Year }\end{array}$ & Inclusion Criteria & Exclusion Criteria & $\begin{array}{l}\text { Number of } \\
\text { Included Studies }\end{array}$ & Settings \\
\hline & Lee, $2018^{77}$ & $\begin{array}{l}\text { We conducted a } \\
\text { systematic review } \\
\text { to examine the } \\
\text { effectiveness of } \\
\text { mHealth } \\
\text { interventions on } \\
\text { process measures } \\
\text { as well as health } \\
\text { outcomes in } \\
\text { randomized } \\
\text { controlled trials } \\
\text { (RCTs) to improve } \\
\text { chronic disease } \\
\text { management. }\end{array}$ & $2005-2016$ & $\begin{array}{l}\text { The inclusion criteria } \\
\text { were RCTs that } \\
\text { conducted an } \\
\text { intervention using } \\
\text { mobile devices such as } \\
\text { smartphones or tablets } \\
\text { for adult patients with } \\
\text { chronic diseases to } \\
\text { examine disease } \\
\text { management or health } \\
\text { promotion. }\end{array}$ & $\begin{array}{l}\text { The exclusion } \\
\text { criteria were as } \\
\text { follows: studies that } \\
\text { focused on a } \\
\text { healthy population, } \\
\text { pregnant women, } \\
\text { non-adults (i.e., } \\
\text { adolescents and } \\
\text { children), or } \\
\text { healthcare } \\
\text { providers (e.g., } \\
\text { apps, for } \\
\text { physicians' or } \\
\text { nurses' use only); } \\
\text { studies that used } \\
\text { only qualitative } \\
\text { methods }\end{array}$ & $\begin{array}{l}\text { Total:12 } \\
\text { RCTs:12 } \\
\text { Non-RCTs with } \\
\text { comparison:0 } \\
\text { Observational:0 }\end{array}$ & Hospital and home \\
\hline & $\begin{array}{l}\text { Warrington, } \\
2019^{78}\end{array}$ & $\begin{array}{l}\text { This review aimed } \\
\text { to (1) describe the } \\
\text { features and } \\
\text { functions of } \\
\text { existing electronic } \\
\text { symptom reporting } \\
\text { systems (eg, } \\
\text { symptom } \\
\text { monitoring, tailored } \\
\text { self-management } \\
\text { advice), and (2) } \\
\text { explore which } \\
\text { features may be } \\
\text { associated with } \\
\text { patient } \\
\text { engagement and } \\
\text { patient-centered } \\
\text { outcomes. }\end{array}$ & $2000-2016$ & $\begin{array}{l}\text { "P: Male and female } \\
\text { adults }>18 \text {, I: Online } \\
\text { systems for patients to } \\
\text { report or manage } \\
\text { symptoms and side } \\
\text { effects during cancer } \\
\text { treatment from home; } \\
\text { Internet-based or - } \\
\text { enabled systems, } \\
\text { including mobile apps. } \\
\text { C: Stage } 2 \text { only: The } \\
\text { review included studies } \\
\text { with any comparator } \\
\text { and non comparator, } \\
\text { O: Monitoring of } \\
\text { symptoms by health } \\
\text { care professionals } \\
\text { (HCPs) and patients, } \\
\text { QoL measures; self- } \\
\text { efficacy measures } \\
\text { including patient } \\
\text { activation, patient } \\
\text { empowerment, } \\
\text { mastery; and patient } \\
\text { satisfaction." }\end{array}$ & $\begin{array}{l}\text { Systems designed } \\
\text { to be accessed at } \\
\text { one time point only } \\
\text { were excluded; } \\
\text { access to the } \\
\text { system had to be } \\
\text { ongoing. }\end{array}$ & $\begin{array}{l}\text { Total:29 } \\
\text { RCTs:7 } \\
\text { Non-RCTs with } \\
\text { comparison:1 } \\
\text { Observational:21 }\end{array}$ & Home \\
\hline
\end{tabular}




\begin{tabular}{|c|c|c|c|c|c|c|c|}
\hline $\begin{array}{l}\text { Type of } \\
\text { Strategy }\end{array}$ & Author, Year & $\begin{array}{l}\text { Objective of } \\
\text { Review }\end{array}$ & $\begin{array}{l}\text { Literature } \\
\text { Start - } \\
\text { End Year }\end{array}$ & Inclusion Criteria & Exclusion Criteria & $\begin{array}{l}\text { Number of } \\
\text { Included Studies }\end{array}$ & Settings \\
\hline & $\begin{array}{l}\text { Palacios, } \\
2017^{79}\end{array}$ & $\begin{array}{l}\text { To conduct a } \\
\text { systematic review } \\
\text { to (1) determine } \\
\text { the effectiveness of } \\
\text { Internet-delivered } \\
\text { CHD self- } \\
\text { management } \\
\text { support for } \\
\text { improving CHD, } \\
\text { mood, and self- } \\
\text { management } \\
\text { related outcomes } \\
\text { and (2) identify and } \\
\text { describe essential } \\
\text { components for } \\
\text { effectiveness. } \\
\text { (Note: only one } \\
\text { study from the US } \\
\text { out of seven RCTs } \\
\text { included in this } \\
\text { review) }\end{array}$ & $2000-2015$ & $\begin{array}{l}\text { Studies with following } \\
\text { format. P: Adults with a } \\
\text { diagnosis (clinician or } \\
\text { self-reported) of CHD, } \\
\text { I: Tested the } \\
\text { effectiveness of } \\
\text { Internet-delivered self } \\
\text { management support } \\
\text { for CHD and } \\
\text { addressed wellbeing } \\
\text { outcomes, such as } \\
\text { mood, quality of life, or } \\
\text { functional status. C: } \\
\text { comparison groups: } \\
\text { usual care, waiting list, } \\
\text { attention, information, } \\
\text { or online discussion } \\
\text { group O: Clinical } \\
\text { outcomes, lifestyles } \\
\text { changes, QoL, mental } \\
\text { health. }\end{array}$ & $\begin{array}{l}\text { Studies not in } \\
\text { English and without } \\
\text { RCT design or } \\
\text { comparison } \\
\text { excluded }\end{array}$ & $\begin{array}{l}\text { Total:7 } \\
\text { RCTs:7 } \\
\text { Non-RCTs with } \\
\text { comparison:0 } \\
\text { Observational:0 }\end{array}$ & Home \\
\hline & $\begin{array}{l}\text { Warner, } \\
2015^{80}\end{array}$ & $\begin{array}{l}\text { SR to identify self- } \\
\text { management } \\
\text { support strategies } \\
\text { in stroke self } \\
\text { management } \\
\text { interventions and } \\
\text { effectively } \\
\text { improved } \\
\text { outcomes, focusing } \\
\text { specifically on } \\
\text { function and } \\
\text { participation } \\
\text { outcomes. }\end{array}$ & $1986-2012$ & $\begin{array}{l}\text { Pre-post, quasi- } \\
\text { experimental and } \\
\text { randomized controlled } \\
\text { trial study designs with } \\
\text { comparison } \\
\text { group/usual care, self- } \\
\text { management support } \\
\text { strategies in stroke } \\
\text { patients, were included }\end{array}$ & $\begin{array}{l}\text { Excluded if they } \\
\text { only disseminated } \\
\text { information (e.g. } \\
\text { self-help } \\
\text { workbooks, } \\
\text { provision of written } \\
\text { materials, tapes or } \\
\text { DVDs) or reported } \\
\text { on a single } \\
\text { intervention } \\
\text { strategy [cognitive } \\
\text { behavioral therapy } \\
\text { (CBT), exercise, } \\
\text { self-help group, } \\
\text { relaxation, } \\
\text { information] }\end{array}$ & $\begin{array}{l}\text { Total:95 } \\
\text { RCTs:6 } \\
\text { Non-RCTs with } \\
\text { comparison:3 } \\
\text { Observational:86 }\end{array}$ & Hospital, CHC \\
\hline
\end{tabular}




\begin{tabular}{|c|c|c|c|c|c|c|c|}
\hline $\begin{array}{l}\text { Type of } \\
\text { Strategy }\end{array}$ & Author, Year & $\begin{array}{l}\text { Objective of } \\
\text { Review }\end{array}$ & $\begin{array}{l}\text { Literature } \\
\text { Start - } \\
\text { End Year }\end{array}$ & Inclusion Criteria & Exclusion Criteria & $\begin{array}{l}\text { Number of } \\
\text { Included Studies }\end{array}$ & Settings \\
\hline & $\begin{array}{l}\text { Dendere, } \\
2019^{81}\end{array}$ & $\begin{array}{l}\text { Assess effect of } \\
\text { inpatient patient } \\
\text { portals on patient } \\
\text { engagement, } \\
\text { health care } \\
\text { delivery }\end{array}$ & $2005-2017$ & $\begin{array}{l}\text { Hospital, inpatient; } \\
\text { hospital EMR with } \\
\text { patient portal }\end{array}$ & None listed & Total:58 & Hospital \\
\hline & $\begin{array}{l}\text { Almutairi, } \\
2019^{82}\end{array}$ & $\begin{array}{l}\text { To assess the } \\
\text { effectiveness of } \\
\text { patient activation } \\
\text { intervention on } \\
\text { T2DM glycemic } \\
\text { control and Self- } \\
\text { management } \\
\text { behaviors SMBs. }\end{array}$ & $2004-2018$ & $\begin{array}{l}\text { We included } \\
\text { randomized controlled } \\
\text { trials with sample size } \\
\geq 120 \text { and follow up } \\
\text { period of } \geq 12 \text { months } \\
\text { and assess the } \\
\text { effectiveness of patient } \\
\text { activation intervention } \\
\text { on T2DM glycemic } \\
\text { control and SMBs }\end{array}$ & $\begin{array}{l}\text { Excluded if the } \\
\text { intervention was } \\
\text { not based on } \\
\text { patient activation, } \\
\text { the participants } \\
\text { were less than } 18 \\
\text { years of age, had } \\
\text { other types of } \\
\text { diabetes, the } \\
\text { outcomes were not } \\
\text { self-management } \\
\text { behavior } \\
\text { and glycemic } \\
\text { control, the design } \\
\text { was not RCT, } \\
\text { sample size was } \\
\text { less than } 120 \text {, and } \\
\text { lastly, the duration } \\
\text { of follow-up was } \\
\text { less than } 12 \\
\text { months }\end{array}$ & $\begin{array}{l}\text { Total:10 } \\
\text { RCTs:10 } \\
\text { Non-RCTs with } \\
\text { comparison:0 } \\
\text { Observational:0 }\end{array}$ & Hospital \\
\hline
\end{tabular}




\begin{tabular}{|c|c|c|c|c|c|c|c|}
\hline $\begin{array}{l}\text { Type of } \\
\text { Strategy }\end{array}$ & Author, Year & $\begin{array}{l}\text { Objective of } \\
\text { Review }\end{array}$ & $\begin{array}{l}\text { Literature } \\
\text { Start - } \\
\text { End Year }\end{array}$ & Inclusion Criteria & Exclusion Criteria & $\begin{array}{l}\text { Number of } \\
\text { Included Studies }\end{array}$ & Settings \\
\hline & Kelly, $2018^{83}$ & $\begin{array}{l}\text { Review the } \\
\text { literature } \\
\text { evaluating the } \\
\text { design, use, and } \\
\text { impact of inpatient } \\
\text { portals, which are } \\
\text { patient portals } \\
\text { designed to give } \\
\text { hospitalized } \\
\text { patients and } \\
\text { caregivers } \\
\text { inpatient EHR } \\
\text { clinical information } \\
\text { for the purpose of } \\
\text { engaging them in } \\
\text { hospital care. }\end{array}$ & 2006-2017 & Hospital EMR & $\begin{array}{l}\text { Exclude of only ER } \\
\text { or ambulatory } \\
\text { portal. }\end{array}$ & $\begin{array}{l}\text { Total:9 } \\
\text { RCTs:0 } \\
\text { Non-RCTs with } \\
\text { comparison:1 } \\
\text { Observational:8 }\end{array}$ & Hospital \\
\hline & $\begin{array}{l}\text { Risling, } \\
2017^{84}\end{array}$ & $\begin{array}{l}\text { explore the } \\
\text { concept of patient } \\
\text { empowerment } \\
\text { within the } \\
\text { electronic health } \\
\text { (eHealth) context }\end{array}$ & $2000-2016$ & $\begin{array}{l}\text { EHR, PHR or patient } \\
\text { portal }\end{array}$ & not on EHR & $\begin{array}{l}\text { Total:19 } \\
\text { RCTs:1 } \\
\text { Non-RCTs with } \\
\text { comparison:2 } \\
\text { Observational:16 }\end{array}$ & Outpatient \\
\hline
\end{tabular}




\begin{tabular}{|c|c|c|c|c|c|c|c|}
\hline $\begin{array}{l}\text { Type of } \\
\text { Strategy }\end{array}$ & Author, Year & $\begin{array}{l}\text { Objective of } \\
\text { Review }\end{array}$ & $\begin{array}{l}\text { Literature } \\
\text { Start - } \\
\text { End Year }\end{array}$ & Inclusion Criteria & Exclusion Criteria & $\begin{array}{l}\text { Number of } \\
\text { Included Studies }\end{array}$ & Settings \\
\hline & $\begin{array}{l}\text { Hamine, } \\
2015^{85}\end{array}$ & $\begin{array}{l}\text { We conducted a } \\
\text { systematic review } \\
\text { of the literature to } \\
\text { evaluate the } \\
\text { effectiveness of } \\
\text { mHealth in } \\
\text { supporting the } \\
\text { adherence of } \\
\text { patients to chronic } \\
\text { diseases } \\
\text { management } \\
\text { ("mAdherence"), } \\
\text { and the usability, } \\
\text { feasibility, and } \\
\text { acceptability of } \\
\text { mAdherence tools } \\
\text { and platforms in } \\
\text { chronic disease } \\
\text { management } \\
\text { among patients } \\
\text { and health care } \\
\text { providers. }\end{array}$ & $1980-2014$ & $\begin{array}{l}\text { We included original } \\
\text { research published in } \\
\text { peer-reviewed journals } \\
\text { that evaluated mHealth } \\
\text { tools for effect on } \\
\text { patient adherence to } \\
\text { chronic disease } \\
\text { management, disease- } \\
\text { specific clinical } \\
\text { outcomes, and } \\
\text { usability, feasibility, } \\
\text { and acceptability } \\
\text { features. Studies that } \\
\text { focused on clinical } \\
\text { measures, such as } \\
\text { hemoglobin A1c } \\
\text { (HbA1c) or blood } \\
\text { pressure (BP), were } \\
\text { included; Only articles } \\
\text { reporting that the } \\
\text { mAdherence } \\
\text { intervention was } \\
\text { designed for } \\
\text { secondary prevention } \\
\text { targeting chronic } \\
\text { disease patients were } \\
\text { included }\end{array}$ & $\begin{array}{l}\text { Studies on primary } \\
\text { prevention among } \\
\text { healthy or at-risk } \\
\text { groups. We also } \\
\text { excluded articles } \\
\text { regarding } \\
\text { interventions that } \\
\text { were not tested in a } \\
\text { sample population } \\
\text { with clearly } \\
\text { described methods } \\
\text { and results. In } \\
\text { addition, review } \\
\text { articles, editorials, } \\
\text { commentaries }\end{array}$ & $\begin{array}{l}\text { Total:107 } \\
\text { RCTs:50 } \\
\text { Non-RCTs with } \\
\text { comparison:17 } \\
\text { Observational:40 }\end{array}$ & Setting not included \\
\hline
\end{tabular}




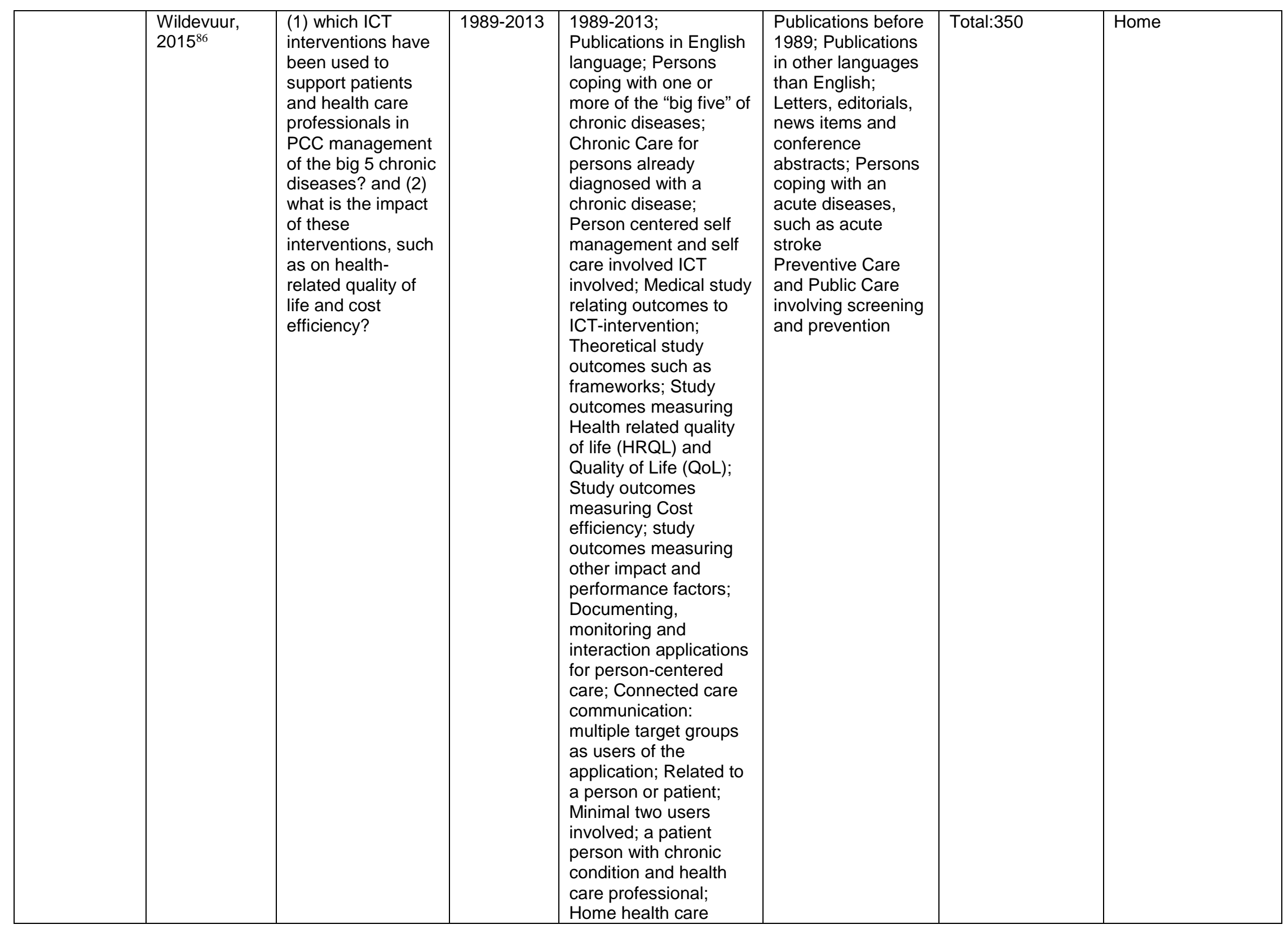




\begin{tabular}{|c|c|c|c|c|c|c|c|}
\hline $\begin{array}{l}\text { Type of } \\
\text { Strategy }\end{array}$ & Author, Year & $\begin{array}{l}\text { Objective of } \\
\text { Review }\end{array}$ & $\begin{array}{l}\text { Literature } \\
\text { Start - } \\
\text { End Year }\end{array}$ & Inclusion Criteria & Exclusion Criteria & $\begin{array}{l}\text { Number of } \\
\text { Included Studies }\end{array}$ & Settings \\
\hline & & & & $\begin{array}{l}\text { setting: care activities } \\
\text { at home connected to } \\
\text { care activities at other } \\
\text { health care settings }\end{array}$ & & & \\
\hline
\end{tabular}




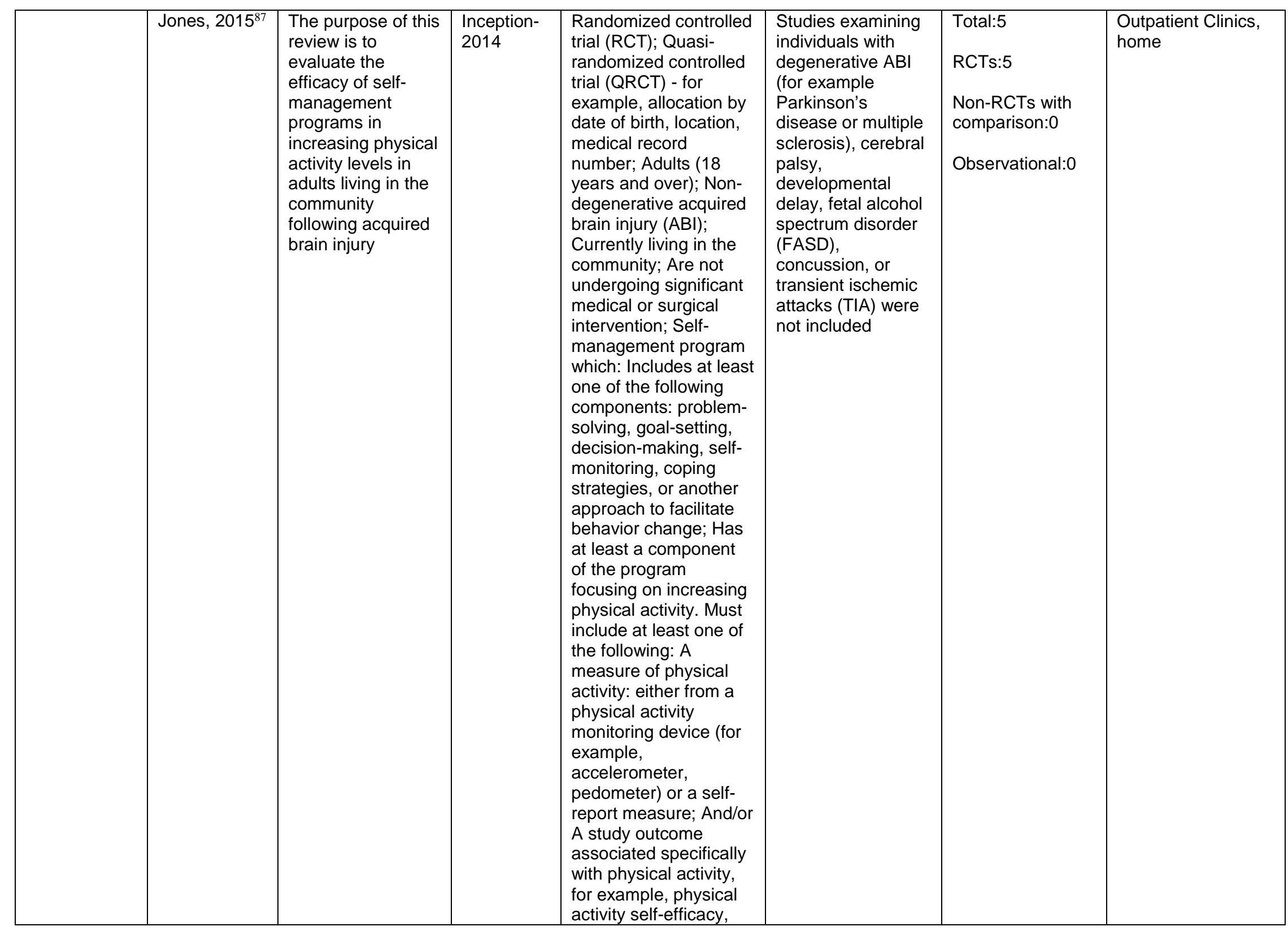




\begin{tabular}{|c|c|c|c|c|c|c|c|}
\hline $\begin{array}{l}\text { Type of } \\
\text { Strategy }\end{array}$ & Author, Year & $\begin{array}{l}\text { Objective of } \\
\text { Review }\end{array}$ & $\begin{array}{l}\text { Literature } \\
\text { Start - } \\
\text { End Year }\end{array}$ & Inclusion Criteria & Exclusion Criteria & $\begin{array}{l}\text { Number of } \\
\text { Included Studies }\end{array}$ & Settings \\
\hline & & & & $\begin{array}{l}\text { physical self-concept, } \\
\text { or stages of change in } \\
\text { relation to physical } \\
\text { activity. }\end{array}$ & & & \\
\hline & $\begin{array}{l}\text { Pereira, } \\
2015^{88}\end{array}$ & $\begin{array}{l}\text { The purpose of this } \\
\text { article is to review } \\
\text { various delivery } \\
\text { methods of Internet } \\
\text { diabetes education } \\
\text { that have been } \\
\text { evaluated, as well } \\
\text { as their } \\
\text { effectiveness in } \\
\text { improving } \\
\text { diabetes-related } \\
\text { outcomes. } \\
\text { (Diabetes self- } \\
\text { management } \\
\text { education) }\end{array}$ & $2004-2013$ & $\begin{array}{l}\text { Publications in English, } \\
\text { type } 2 \text { diabetes } \\
\text { education interventions } \\
\text { (including those } \\
\text { focused on health } \\
\text { maintenance and } \\
\text { prevention of } \\
\text { complications), adult } \\
\text { participants (age } 18 \\
\text { years or older), and } \\
\text { specifically programs } \\
\text { offered via the Internet. } \\
\text { Randomized trials, } \\
\text { exploratory studies, } \\
\text { and comparative } \\
\text { effectiveness designs } \\
\text { were included. }\end{array}$ & $\begin{array}{l}\text { Healthcare provider } \\
\text { education } \\
\text { programs, face-to- } \\
\text { face or non- } \\
\text { Internet-based } \\
\text { diabetes education } \\
\text { interventions, } \\
\text { studies focused on } \\
\text { multiple diseases } \\
\text { or other types of } \\
\text { diabetes, pediatric } \\
\text { samples (age < } 18 \\
\text { years), and studies } \\
\text { focused on primary } \\
\text { outcomes relate }\end{array}$ & $\begin{array}{l}\text { Total:14 } \\
\text { RCTs:9 } \\
\text { Non-RCTs with } \\
\text { comparison:1 } \\
\text { Observational:4 }\end{array}$ & Hospital and home \\
\hline & Kruse, $2015^{89}$ & $\begin{array}{l}\text { Describe effect of } \\
\text { patient portals on } \\
\text { quality of care and } \\
\text { chronic disease } \\
\text { outcomes }\end{array}$ & 2011-2014 & $\begin{array}{l}\text { All study designs } \\
\text { included }\end{array}$ & $\begin{array}{l}\text { Did not report } \\
\text { portal. }\end{array}$ & Total:27 & Outpatient \\
\hline & Yin, $2019^{90}$ & $\begin{array}{l}\text { How mobile health } \\
\text { apps for IBD care } \\
\text { have fit into a } \\
\text { clinical care } \\
\text { framework and the } \\
\text { challenges that } \\
\text { clinicians and } \\
\text { technologists face } \\
\text { in approaching } \\
\text { future } \\
\text { opportunities. }\end{array}$ & $2010-2019$ & $\begin{array}{l}\text { Articles exploring the } \\
\text { use of a digital health } \\
\text { intervention in the care } \\
\text { of IBD, or CD or UC } \\
\text { specifically. }\end{array}$ & $\begin{array}{l}\text { Excluded if they did } \\
\text { not involve IBD, } \\
C D \text {, or UC or } \\
\text { teleconferencing or } \\
\text { video chatting as } \\
\text { the sole } \\
\text { intervention. }\end{array}$ & $\begin{array}{l}\text { Total:28 } \\
\text { RCTs:14 }\end{array}$ & Home \\
\hline
\end{tabular}




\begin{tabular}{|c|c|c|c|c|c|c|c|}
\hline $\begin{array}{l}\text { Type of } \\
\text { Strategy }\end{array}$ & Author, Year & $\begin{array}{l}\text { Objective of } \\
\text { Review }\end{array}$ & $\begin{array}{l}\text { Literature } \\
\text { Start - } \\
\text { End Year }\end{array}$ & Inclusion Criteria & Exclusion Criteria & $\begin{array}{l}\text { Number of } \\
\text { Included Studies }\end{array}$ & Settings \\
\hline $\begin{array}{l}\text { Shared } \\
\text { Decision } \\
\text { Making - } \\
\text { Children } \\
\text { Only }\end{array}$ & $\begin{array}{l}\text { Malone, } \\
2019^{91}\end{array}$ & $\begin{array}{l}\text { To assess the } \\
\text { effectiveness of } \\
\text { interventions that } \\
\text { promote } \\
\text { participation in } \\
\text { shared decision- } \\
\text { making for children } \\
\text { and adolescents } \\
\text { (aged between four } \\
\text { and } 18 \text { years) with } \\
\text { CF. }\end{array}$ & $\begin{array}{l}\text { Inception- } \\
2019\end{array}$ & $\begin{array}{l}\text { Randomized controlled } \\
\text { trials (RCTs) (but not } \\
\text { cross-over RCTs) of } \\
\text { interventions } \\
\text { promoting shared } \\
\text { decision-making for } \\
\text { children and } \\
\text { adolescents with CF } \\
\text { aged between four and } \\
\text { 18 years, such as } \\
\text { information provision, } \\
\text { booklets, two-way } \\
\text { interaction, checking } \\
\text { understanding (by the } \\
\text { participant), } \\
\text { preparation to } \\
\text { participate in a } \\
\text { healthcare decision, } \\
\text { decision-aids, and } \\
\text { training interventions } \\
\text { or educational } \\
\text { programs. We planned } \\
\text { to include interventions } \\
\text { aimed at children or } \\
\text { adolescents (or both), } \\
\text { parents or healthcare } \\
\text { professionals or any } \\
\text { combination of these } \\
\text { groups provided that } \\
\text { the focus was aimed at } \\
\text { promoting shared } \\
\text { decision-making for } \\
\text { children and } \\
\text { adolescents with CF }\end{array}$ & None listed & $\begin{array}{l}\text { Total:0 } \\
\text { RCTs:0 } \\
\text { Non-RCTs with } \\
\text { comparison:0 } \\
\text { Observational:0 }\end{array}$ & \\
\hline
\end{tabular}




\begin{tabular}{|c|c|c|c|c|c|c|c|}
\hline $\begin{array}{l}\text { Type of } \\
\text { Strategy }\end{array}$ & Author, Year & $\begin{array}{l}\text { Objective of } \\
\text { Review }\end{array}$ & $\begin{array}{l}\text { Literature } \\
\text { Start - } \\
\text { End Year }\end{array}$ & Inclusion Criteria & Exclusion Criteria & $\begin{array}{l}\text { Number of } \\
\text { Included Studies }\end{array}$ & Settings \\
\hline & $\begin{array}{l}\text { Cheng, } \\
2017^{92}\end{array}$ & $\begin{array}{l}\text { The aim of this } \\
\text { scoping review } \\
\text { was to identify and } \\
\text { describe SDM } \\
\text { approaches (tools, } \\
\text { techniques, and } \\
\text { technologies) used } \\
\text { in child and youth } \\
\text { mental health }\end{array}$ & $\begin{array}{l}\text { Inception- } \\
2016\end{array}$ & $\begin{array}{l}\text { English language; } \\
\text { described an SDM } \\
\text { approach (tool, } \\
\text { technique, or } \\
\text { technology); included } \\
\text { sufficient detail on the } \\
\text { SDM approach for } \\
\text { quality assessment; did } \\
\text { not use only a } \\
\text { questionnaire to } \\
\text { provide feedback on } \\
\text { SDM or related } \\
\text { concepts (e.g., } \\
\text { therapeutic alliance) } \\
\text { without another SDM } \\
\text { approach; child or } \\
\text { adolescent population } \\
\text { (up to } 18 \text { years); carers } \\
\text { of children or } \\
\text { adolescents; and } \\
\text { mental health setting }\end{array}$ & $\begin{array}{l}\text { Did not describe an } \\
\text { SDM approach but } \\
\text { merely used the } \\
\text { term "SDM" in the } \\
\text { record; adult } \\
\text { population not in } \\
\text { the context of } \\
\text { caring for children } \\
\text { or adolescents; and } \\
\text { were not based in } \\
\text { mental health } \\
\text { services or settings }\end{array}$ & $\begin{array}{l}\text { Total:15 } \\
\text { RCTs:4 } \\
\text { Non-RCTs with } \\
\text { comparison:0 } \\
\text { Observational:11 }\end{array}$ & Clinics \\
\hline & $\begin{array}{l}\text { Coyne, } \\
2016^{93}\end{array}$ & $\begin{array}{l}\text { To examine the } \\
\text { effects of SDM } \\
\text { interventions on } \\
\text { the process of } \\
\text { SDM for children } \\
\text { with cancer who } \\
\text { are aged four to } 18 \\
\text { years. }\end{array}$ & $\begin{array}{l}\text { Inception- } \\
2016\end{array}$ & $\begin{array}{l}\text { Randomized controlled } \\
\text { trials (RCTs) and } \\
\text { controlled clinical trials } \\
\text { (CCTs) of SDM } \\
\text { interventions for } \\
\text { children with cancer } \\
\text { aged four to } 18 \text { years. } \\
\text { The types of decisions } \\
\text { included were: } \\
\text { treatment, health care } \\
\text { and research } \\
\text { participation decisions. }\end{array}$ & None listed & $\begin{array}{l}\text { Total:0 } \\
\text { RCTs:0 } \\
\text { Non-RCTs with } \\
\text { comparison:0 } \\
\text { Observational:0 }\end{array}$ & \\
\hline
\end{tabular}




\begin{tabular}{|c|c|c|c|c|c|c|c|}
\hline $\begin{array}{l}\text { Type of } \\
\text { Strategy }\end{array}$ & Author, Year & $\begin{array}{l}\text { Objective of } \\
\text { Review }\end{array}$ & $\begin{array}{l}\text { Literature } \\
\text { Start - } \\
\text { End Year }\end{array}$ & Inclusion Criteria & Exclusion Criteria & $\begin{array}{l}\text { Number of } \\
\text { Included Studies }\end{array}$ & Settings \\
\hline & Wyatt, $2015^{94}$ & $\begin{array}{l}\text { To summarize the } \\
\text { efficacy of SDM } \\
\text { interventions in } \\
\text { pediatrics on } \\
\text { patient-centered } \\
\text { outcomes. }\end{array}$ & $\begin{array}{l}\text { Inception- } \\
2013\end{array}$ & $\begin{array}{l}\text { We broadly defined } \\
\text { SDM as the process of } \\
\text { involving patients or } \\
\text { their } \\
\text { caregivers/surrogates } \\
\text { in medical decision } \\
\text { making with clinicians. } \\
\text { As such, methods or } \\
\text { approaches (including } \\
\text { tools) designed to } \\
\text { facilitate involvement in } \\
\text { the process of medical } \\
\text { decision making } \\
\text { involving patients }<18 \\
\text { years of age, their } \\
\text { parents, or both and } \\
\text { reported in English } \\
\text { were eligible for } \\
\text { inclusion }\end{array}$ & $\begin{array}{l}\text { We excluded } \\
\text { studies on } \\
\text { antenatal/perinatal } \\
\text { care and research } \\
\text { participation } \\
\text { decisions }\end{array}$ & $\begin{array}{l}\text { Total:6 } \\
\text { RCTs:4 } \\
\text { Non-RCTs with } \\
\text { comparison:1 } \\
\text { Observational:1 }\end{array}$ & Clinics \\
\hline
\end{tabular}




\begin{tabular}{|c|c|c|c|c|c|c|c|}
\hline $\begin{array}{l}\text { Shared } \\
\text { Decision } \\
\text { Making - } \\
\text { Adults and } \\
\text { Children }\end{array}$ & $\begin{array}{l}\text { Voruganti, } \\
2017^{95}\end{array}$ & $\begin{array}{l}\text { (1) Conduct a } \\
\text { systematic search } \\
\text { of the published } \\
\text { literature and the } \\
\text { Internet for Web- } \\
\text { based tools for } \\
\text { text-based } \\
\text { communication } \\
\text { between patients } \\
\text { and providers; (2) } \\
\text { map tool } \\
\text { characteristics, } \\
\text { their intended use, } \\
\text { contexts in which } \\
\text { they were used, } \\
\text { and by whom; (3) } \\
\text { describe the nature } \\
\text { of their evaluation; } \\
\text { and (4) understand } \\
\text { the terminology } \\
\text { used to describe } \\
\text { the tools. }\end{array}$ & $\begin{array}{l}\text { Not } \\
\text { mentioned- } \\
2016\end{array}$ & $\begin{array}{l}\text { Supports Web-based } \\
\text { communication } \\
\text { between patients and } \\
\text { health professionals for } \\
\text { within-tool } \\
\text { communication (ie, } \\
\text { messages sent within } \\
\text { the tool are responded } \\
\text { to using the tool rather } \\
\text { than via phone call } \\
\text { outside the tool } \\
\text { environment); Uses a } \\
\text { text-based form of } \\
\text { dialogue (including text } \\
\text { messages via cell } \\
\text { phone); one or more } \\
\text { chronic conditions, } \\
\text { used in the health care } \\
\text { context; Is intended for } \\
\text { patients and health } \\
\text { care providers } \\
\text { (physician, nurse, } \\
\text { pharmacist, social } \\
\text { worker, etc) to } \\
\text { communicate } \\
\text { regarding direct patient } \\
\text { care; Communication } \\
\text { may be guided but not } \\
\text { restricted (ie, patient } \\
\text { should have the } \\
\text { opportunity to ask any } \\
\text { question); Involves } \\
\text { communication } \\
\text { between a minimum of } \\
\text { one patient and one } \\
\text { health care } \\
\text { professional (ie, at } \\
\text { least two end users }\end{array}$ & $\begin{array}{l}\text { Tools that function } \\
\text { for information } \\
\text { transfer but not } \\
\text { communication } \\
\text { - Audio or video- } \\
\text { based forms of } \\
\text { communication that } \\
\text { do not include text- } \\
\text { based } \\
\text { communication } \\
\text { - Electronic medical } \\
\text { records, patient } \\
\text { health data } \\
\text { repositories, and } \\
\text { portals that do not } \\
\text { have a } \\
\text { communication } \\
\text { component } \\
\text { - Online support } \\
\text { forums, even if they } \\
\text { support } \\
\text { communication } \\
\text { between many } \\
\text { patients and many } \\
\text { health } \\
\text { professionals } \\
\text { - Tools for } \\
\text { communication } \\
\text { exclusively } \\
\text { between patients } \\
\text { - Theoretical or } \\
\text { conceptual papers, } \\
\text { frameworks, and } \\
\text { descriptions } \\
\text { - Offline native } \\
\text { apps for mobile } \\
\text { devices (ie, those } \\
\text { which are not } \\
\text { connected to the } \\
\text { Internet) } \\
\text { - Tools to support } \\
\text { behavior change } \\
\text { interventions in } \\
\text { otherwise healthy } \\
\text { patients (ie, without }\end{array}$ & $\begin{array}{l}\text { Total:54 } \\
\text { RCTs:25 } \\
\text { Non-RCTs with } \\
\text { comparison:23 } \\
\text { Observational:6 }\end{array}$ & Home \\
\hline
\end{tabular}

B-56 


\begin{tabular}{|c|c|c|c|c|c|c|c|}
\hline $\begin{array}{l}\text { Type of } \\
\text { Strategy }\end{array}$ & Author, Year & $\begin{array}{l}\text { Objective of } \\
\text { Review }\end{array}$ & $\begin{array}{l}\text { Literature } \\
\text { Start - } \\
\text { End Year }\end{array}$ & Inclusion Criteria & Exclusion Criteria & $\begin{array}{l}\text { Number of } \\
\text { Included Studies }\end{array}$ & Settings \\
\hline & & & & & $\begin{array}{l}\text { a chronic condition; } \\
\text { eg, smoking } \\
\text { cessation, diet, and } \\
\text { alcoholism) }\end{array}$ & & \\
\hline & Kew, $2017^{96}$ & $\begin{array}{l}\text { To assess benefits } \\
\text { and potential } \\
\text { harms of shared } \\
\text { decision-making } \\
\text { for adults and } \\
\text { children with } \\
\text { asthma. }\end{array}$ & $\begin{array}{l}\text { Not } \\
\text { mentioned- } \\
2016\end{array}$ & $\begin{array}{l}\text { We included studies of } \\
\text { individual or cluster } \\
\text { parallel randomized } \\
\text { controlled design } \\
\text { conducted to compare } \\
\text { an SDM intervention } \\
\text { for adults and children } \\
\text { with asthma versus a } \\
\text { control intervention. No } \\
\text { restrictions on place, } \\
\text { date, or language of } \\
\text { publication. } \\
\text { Interventions targeting } \\
\text { healthcare } \\
\text { professionals or } \\
\text { patients, their families } \\
\text { or care-givers, or both. }\end{array}$ & $\begin{array}{l}\text { We excluded } \\
\text { studies of } \\
\text { interventions that } \\
\text { involved multiple } \\
\text { components other } \\
\text { than the SDM } \\
\text { intervention unless } \\
\text { the control group } \\
\text { also received these } \\
\text { interventions. }\end{array}$ & $\begin{array}{l}\text { Total:4 } \\
\text { RCTs:4 } \\
\text { Non-RCTs with } \\
\text { comparison:0 } \\
\text { Observational:0 }\end{array}$ & Hospital and home \\
\hline & $\begin{array}{l}\text { Clayman, } \\
2015^{97}\end{array}$ & $\begin{array}{l}\text { Assess the extent } \\
\text { to which patient } \\
\text { participation in } \\
\text { decision making } \\
\text { within medical } \\
\text { encounters is } \\
\text { associated with } \\
\text { measured patient } \\
\text { outcomes. }\end{array}$ & $\begin{array}{l}\text { Inception- } \\
2015\end{array}$ & $\begin{array}{l}\text { Participants = all } \\
\text { patients; Interventions } \\
\text { (if applicable) = } \\
\text { attempt to increase } \\
\text { patient participation in } \\
\text { decisions; } \\
\text { Comparators (if } \\
\text { applicable) = usual } \\
\text { care, attention control, } \\
\text { or no choice; } \\
\text { Outcomes = any } \\
\text { measured patient } \\
\text { outcome; and Study } \\
\text { design = randomized } \\
\text { and nonrandomized } \\
\text { studies. }\end{array}$ & $\begin{array}{l}\text { Citations with no } \\
\text { abstract were } \\
\text { excluded only if } \\
\text { they clearly were } \\
\text { not about the topic, } \\
\text { if they were not in } \\
\text { English, or if they } \\
\text { were non-research } \\
\text { articles (e.g., } \\
\text { editorials). }\end{array}$ & $\begin{array}{l}\text { Total:116 } \\
\text { RCTs:11 } \\
\text { Observational:105 }\end{array}$ & Hospital \\
\hline
\end{tabular}




\begin{tabular}{|c|c|c|c|c|c|c|c|}
\hline $\begin{array}{l}\text { Type of } \\
\text { Strategy }\end{array}$ & Author, Year & $\begin{array}{l}\text { Objective of } \\
\text { Review }\end{array}$ & $\begin{array}{l}\text { Literature } \\
\text { Start - } \\
\text { End Year }\end{array}$ & Inclusion Criteria & Exclusion Criteria & $\begin{array}{l}\text { Number of } \\
\text { Included Studies }\end{array}$ & Settings \\
\hline & $\begin{array}{l}\text { Winston, } \\
2017^{98}\end{array}$ & $\begin{array}{l}\text { This study reviews } \\
\text { the published } \\
\text { literature on the } \\
\text { use of video-based } \\
\text { decision aids (DA) } \\
\text { for patients. }\end{array}$ & NR-2016 & $\begin{array}{l}\text { Studies were included } \\
\text { for full text review if } \\
\text { they contained original } \\
\text { data and the study } \\
\text { included the use of a } \\
\text { pre-produced video, a } \\
\text { medically relevant } \\
\text { behavioral outcome, } \\
\text { and evidence of patient } \\
\text { choice. Population } \\
\text { adults and children } \\
\text { both. }\end{array}$ & $\begin{array}{l}\text { Exclusion criteria } \\
\text { were: the use of } \\
\text { live broadcasts, } \\
\text { including } \\
\text { videoconferencing } \\
\text { and live television; } \\
\text { exclusively static } \\
\text { presentations such } \\
\text { as Powerpoint; } \\
\text { interactive visual } \\
\text { aids such as video } \\
\text { games. outcomes } \\
\text { restricted to } \\
\text { knowledge or } \\
\text { attitude change }\end{array}$ & Total:488 & Hospital and home \\
\hline $\begin{array}{l}\text { Shared } \\
\text { Decision } \\
\text { Making - } \\
\text { Adults Only }\end{array}$ & $\begin{array}{l}\text { Nathan, } \\
2016^{99}\end{array}$ & $\begin{array}{l}\text { We conducted a } \\
\text { systematic re- view } \\
\text { to characterize the } \\
\text { application and } \\
\text { effectiveness of } \\
\text { DAs in racial, } \\
\text { ethnic, sexual, and } \\
\text { gender minorities }\end{array}$ & $2004-2013$ & $\begin{array}{l}\text { Our search included } \\
\text { English RCTs that } \\
\text { evaluated DAs within } \\
\text { minority populations. } \\
\text { Based on established } \\
\text { models of SDM and } \\
\text { DAs, } 14 \text { we included } \\
\text { studies with an } \\
\text { intervention that } \\
\text { included 1) information } \\
\text { sharing or education } \\
\text { and 2) risks and } \\
\text { benefits of treatment } \\
\text { options, to enable } \\
\text { SDM. }\end{array}$ & $\begin{array}{l}\text { Studies in which < } \\
50 \% \text { of the } \\
\text { participants } \\
\text { identified as part of } \\
\text { a racial (African } \\
\text { American, Asian } \\
\text { American), ethnic } \\
\text { (Hispanic/Latino), } \\
\text { sexual (LGB) or } \\
\text { gender } \\
\text { (Transgender) } \\
\text { minority were } \\
\text { excluded.12,16 } \\
\text { Studies with } \\
\text { individuals younger } \\
\text { than } 18 \text { years old }\end{array}$ & $\begin{array}{l}\text { Total:19 } \\
\text { RCTs:19 } \\
\text { Non-RCTs with } \\
\text { comparison:0 } \\
\text { Observational:0 }\end{array}$ & Outpatient clinic \\
\hline
\end{tabular}




\begin{tabular}{|c|c|c|c|c|c|c|c|}
\hline $\begin{array}{l}\text { Type of } \\
\text { Strategy }\end{array}$ & Author, Year & $\begin{array}{l}\text { Objective of } \\
\text { Review }\end{array}$ & $\begin{array}{l}\text { Literature } \\
\text { Start - } \\
\text { End Year }\end{array}$ & Inclusion Criteria & Exclusion Criteria & $\begin{array}{l}\text { Number of } \\
\text { Included Studies }\end{array}$ & Settings \\
\hline & $\begin{array}{l}\text { Wagner, } \\
2019^{100}\end{array}$ & $\begin{array}{l}\text { This review } \\
\text { analyzes } \\
\text { intervention and } \\
\text { evaluation studies } \\
\text { on patient } \\
\text { education and } \\
\text { continuing medical } \\
\text { education which } \\
\text { aim to enhance } \\
\text { shared decision- } \\
\text { making. }\end{array}$ & $2006-2016$ & $\begin{array}{l}\text { We included (1) } \\
\text { studies on patient } \\
\text { education with the aim } \\
\text { of advancing patients' } \\
\text { communication skills; } \\
\text { (2) studies on } \\
\text { continuing medical } \\
\text { education, in which } \\
\text { physicians received } \\
\text { training on } \\
\text { communication skills } \\
\text { and how to implement } \\
\text { shared decision- } \\
\text { making; (3) RCTs and } \\
\text { evaluation studies, and } \\
\text { published between } \\
2006 \text { and June } 2016 \\
\text { and be in German } \\
\text { and/or English } \\
\text { language. }\end{array}$ & $\begin{array}{l}\text { We excluded study } \\
\text { protocols and } \\
\text { publications with } \\
\text { predominantly } \\
\text { methodological } \\
\text { content, published } \\
\text { before } 2006 \text { and } \\
\text { languages other } \\
\text { than German or } \\
\text { English }\end{array}$ & $\begin{array}{l}\text { Total:16 } \\
\text { RCTs:11 } \\
\text { Non-RCTs with } \\
\text { comparison:4 } \\
\text { Observational:1 }\end{array}$ & Hospital \\
\hline & $\begin{array}{l}\text { Goodridge, } \\
2019^{101}\end{array}$ & $\begin{array}{l}\text { Scoping review to } \\
\text { map the existing } \\
\text { literature and } \\
\text { describe } \\
\text { interventions } \\
\text { aimed at building } \\
\text { the capacity of } \\
\text { patients to } \\
\text { participate in care } \\
\text { during } \\
\text { hospitalization by: } \\
\text { (1) describing and } \\
\text { categorizing the } \\
\text { aspects of care } \\
\text { targeted by these } \\
\text { interventions and } \\
\text { (2) identifying the } \\
\text { behavior change } \\
\text { techniques (BCTs) } \\
\text { used in these } \\
\text { interventions. }\end{array}$ & $\begin{array}{l}\text { Inception- } \\
2017\end{array}$ & $\begin{array}{l}\text { Studies reporting } \\
\text { primary research } \\
\text { studies on building the } \\
\text { capacity of hospitalized } \\
\text { adult patients to } \\
\text { participate in care } \\
\text { which described or } \\
\text { included one or more } \\
\text { structured or } \\
\text { systematic } \\
\text { interventions and } \\
\text { described the } \\
\text { outcomes for at least } \\
\text { the key stakeholder } \\
\text { group were included. } \\
\text { All study designs were } \\
\text { included. only English }\end{array}$ & $\begin{array}{l}\text { Excluded studies: } \\
\text { having children and } \\
\text { adolescents; } \\
\text { community or home } \\
\text { settings; oncology } \\
\text { patients, and } \\
\text { emergency } \\
\text { department } \\
\text { settings. We also } \\
\text { excluded papers } \\
\text { focused on patient } \\
\text { participation in } \\
\text { research, } \\
\text { databases, quality } \\
\text { improvement }\end{array}$ & $\begin{array}{l}\text { Total:87 } \\
\text { RCTs:6 } \\
\text { Non-RCTs with } \\
\text { comparison:21 } \\
\text { Observational:60 }\end{array}$ & Hospital \\
\hline
\end{tabular}




\begin{tabular}{|c|c|c|c|c|c|c|c|}
\hline $\begin{array}{l}\text { Type of } \\
\text { Strategy }\end{array}$ & Author, Year & $\begin{array}{l}\text { Objective of } \\
\text { Review }\end{array}$ & $\begin{array}{l}\text { Literature } \\
\text { Start - } \\
\text { End Year }\end{array}$ & Inclusion Criteria & Exclusion Criteria & $\begin{array}{l}\text { Number of } \\
\text { Included Studies }\end{array}$ & Settings \\
\hline & $\begin{array}{l}\text { Irizarry, } \\
2015^{102}\end{array}$ & $\begin{array}{l}\text { Describe evidence } \\
\text { for encouragement } \\
\text { and support of } \\
\text { patient } \\
\text { engagement } \\
\text { through the patient } \\
\text { portal }\end{array}$ & 2006-2014 & $\begin{array}{l}\text { Reports on patient } \\
\text { experience; electronic } \\
\text { PHR }\end{array}$ & No patient portal & $\begin{array}{l}\text { Total:120 } \\
\text { RCTs:14 } \\
\text { Non-RCTs with } \\
\text { comparison:4 }\end{array}$ & Outpatient \\
\hline & $\begin{array}{l}\text { Violette, } \\
2015^{103}\end{array}$ & $\begin{array}{l}\text { Aims to summarize } \\
\text { and critically } \\
\text { appraise the } \\
\text { randomized control } \\
\text { trials (RCTs) that } \\
\text { have addressed } \\
\text { the impact of } \\
\text { decision aids on } \\
\text { decisional } \\
\text { outcomes } \\
\text { (including } \\
\text { decisional conflict } \\
\text { and regret), } \\
\text { patients' treatment } \\
\text { choices, health } \\
\text { outcomes, and } \\
\text { health care use in } \\
\text { the context of } \\
\text { localized prostate } \\
\text { cancer. }\end{array}$ & $1974-2014$ & $\begin{array}{l}\text { RCTs of a decision aid } \\
\text { intervention for } \\
\text { localized prostate } \\
\text { cancer without } \\
\text { language restrictions. }\end{array}$ & $\begin{array}{l}\text { RCTs that included } \\
\text { patients with } \\
\text { metastatic or locally } \\
\text { advanced disease } \\
\text { were excluded. }\end{array}$ & $\begin{array}{l}\text { Total:14 } \\
\text { RCTs:14 } \\
\text { Non-RCTs with } \\
\text { comparison:0 } \\
\text { Observational:0 }\end{array}$ & Hospital \\
\hline
\end{tabular}




\begin{tabular}{|c|c|c|c|c|c|c|c|}
\hline $\begin{array}{l}\text { Type of } \\
\text { Strategy }\end{array}$ & Author, Year & $\begin{array}{l}\text { Objective of } \\
\text { Review }\end{array}$ & $\begin{array}{l}\text { Literature } \\
\text { Start - } \\
\text { End Year }\end{array}$ & Inclusion Criteria & Exclusion Criteria & $\begin{array}{l}\text { Number of } \\
\text { Included Studies }\end{array}$ & Settings \\
\hline & Jain, $2015^{104}$ & $\begin{array}{l}\text { To evaluate the } \\
\text { impact of video } \\
\text { decision aids on } \\
\text { patients' } \\
\text { preferences } \\
\text { regarding life- } \\
\text { sustaining } \\
\text { treatments } \\
\text { (primary outcome) } \\
\text { compared with } \\
\text { non-video-based } \\
\text { interventions. }\end{array}$ & $1980-2014$ & $\begin{array}{l}\text { "Study design, RCT, } \\
\text { enrolled adult patients } \\
\text { (age } 18 \text { years or older) } \\
\text { in an inpatient or } \\
\text { outpatient setting, } \\
\text { included an arm } \\
\text { evaluating an ACP } \\
\text { video decision aid to } \\
\text { assist with choices } \\
\text { about future use of life- } \\
\text { sustaining treatments } \\
\text { and included a } \\
\text { comparator arm with } \\
\text { no ACP video decision } \\
\text { aid and reported data } \\
\text { on at least one } \\
\text { outcome of interest." }\end{array}$ & $\begin{array}{l}\text { Non RCTs with no } \\
\text { comparison or did } \\
\text { not fit the inclusion } \\
\text { criteria }\end{array}$ & $\begin{array}{l}\text { Total:10 } \\
\text { RCTs:10 } \\
\text { Non-RCTs with } \\
\text { comparison:0 } \\
\text { Observational:0 }\end{array}$ & Hospital \\
\hline & $\begin{array}{l}\text { Mclntyre, } \\
2015^{105}\end{array}$ & $\begin{array}{l}\text { This review aims to } \\
\text { establish whether } \\
\text { computer-based } \\
\text { learning systems } \\
\text { (CBLSs) should } \\
\text { replace standard } \\
\text { education for } \\
\text { cancer } \\
\text { populations. }\end{array}$ & $2009-2013$ & $\begin{array}{l}\text { Adult populations of } \\
\text { patients with a } \\
\text { pathological diagnosis } \\
\text { of cancer, Studies } \\
\text { comparing CBLS with } \\
\text { SE as defined by this } \\
\text { research, outcomes: } \\
\text { Knowledge, } \\
\text { satisfaction, Decision } \\
\text { making and the effect } \\
\text { of patient } \\
\text { characteristics. }\end{array}$ & $\begin{array}{l}\text { Excluded studies: } \\
\text { Patients <18 years, } \\
\text { investigating } \\
\text { cognitively impaired } \\
\text { and/or palliative } \\
\text { patients and/or } \\
\text { patients with } \\
\text { language } \\
\text { difficulties, CBLS } \\
\text { alone without a SE } \\
\text { control for } \\
\text { comparison, } \\
\text { development of a } \\
\text { CBLS without } \\
\text { reporting } \\
\text { outcomes. }\end{array}$ & $\begin{array}{l}\text { Total:8 } \\
\text { RCTs:6 } \\
\text { Non-RCTs with } \\
\text { comparison:1 } \\
\text { Observational:1 }\end{array}$ & Hospital and home \\
\hline
\end{tabular}




\begin{tabular}{|c|c|c|c|c|c|c|c|}
\hline $\begin{array}{l}\text { Type of } \\
\text { Strategy }\end{array}$ & Author, Year & $\begin{array}{l}\text { Objective of } \\
\text { Review }\end{array}$ & $\begin{array}{l}\text { Literature } \\
\text { Start - } \\
\text { End Year }\end{array}$ & Inclusion Criteria & Exclusion Criteria & $\begin{array}{l}\text { Number of } \\
\text { Included Studies }\end{array}$ & Settings \\
\hline & $\begin{array}{l}\text { Kashaf, } \\
2015^{106}\end{array}$ & $\begin{array}{l}\text { Aims was to review } \\
\text { the literature, } \\
\text { exploring the } \\
\text { association } \\
\text { between SDM with } \\
\text { regard to treatment } \\
\text { and QOL } \\
\text { outcomes in } \\
\text { cancer, and to } \\
\text { identify the } \\
\text { variables that } \\
\text { moderate this } \\
\text { association. }\end{array}$ & $1992-2014$ & $\begin{array}{l}\text { Different study } \\
\text { designs, adults ( } 18 \\
\text { years) with a first-time } \\
\text { diagnosis of cancer, } \\
\text { option of decision } \\
\text { making, studies } \\
\text { measure patient } \\
\text { participation in cancer } \\
\text { treatment decision } \\
\text { making and outcome } \\
\text { as QoL, and articles } \\
\text { published in English }\end{array}$ & None listed & $\begin{array}{l}\text { Total:17 } \\
\text { RCTs:1 } \\
\text { Non-RCTs with } \\
\text { comparison:2 } \\
\text { Observational:14 }\end{array}$ & Home \\
\hline & $\begin{array}{l}\text { Friedrichs, } \\
2016^{107}\end{array}$ & $\begin{array}{l}\text { The aim is } \\
\text { therefore to give a } \\
\text { systematic } \\
\text { overview of the } \\
\text { literature of patient } \\
\text { preferences and } \\
\text { SDM in the } \\
\text { treatment of } \\
\text { patients with SUD }\end{array}$ & $1980-2013$ & $\begin{array}{l}\text { Randomized controlled } \\
\text { trials (RCTs), } \\
\text { quantitative studies } \\
\text { and qualitative studies } \\
\text { were included; Studies } \\
\text { that evaluated } \\
\text { interventions with } \\
\text { either health care } \\
\text { professionals or } \\
\text { patients were included } \\
\text { in this review. Studies } \\
\text { were screened using } \\
\text { the following inclusion } \\
\text { criteria: 1) substance } \\
\text { related disorder, 2) } \\
\text { adults, 3) patient } \\
\text { preference or 4) } \\
\text { Shared Decision } \\
\text { Making }\end{array}$ & $\begin{array}{l}\text { 1) Patients with } \\
\text { cognitive } \\
\text { impairments, 2) } \\
\text { children and } \\
\text { adolescents, 3) } \\
\text { evaluation of } \\
\text { patients' reasons or } \\
\text { choices for } \\
\text { treatment, and 4) } \\
\text { Motivational } \\
\text { Interviewing } \\
\text { interventions. }\end{array}$ & $\begin{array}{l}\text { Total:25 } \\
\text { RCTs:9 } \\
\text { Non-RCTs with } \\
\text { comparison:0 } \\
\text { Observational:16 }\end{array}$ & Outpatient clinic \\
\hline
\end{tabular}




\begin{tabular}{|c|c|c|c|c|c|c|c|}
\hline $\begin{array}{l}\text { Type of } \\
\text { Strategy }\end{array}$ & Author, Year & $\begin{array}{l}\text { Objective of } \\
\text { Review }\end{array}$ & $\begin{array}{l}\text { Literature } \\
\text { Start - } \\
\text { End Year }\end{array}$ & Inclusion Criteria & Exclusion Criteria & $\begin{array}{l}\text { Number of } \\
\text { Included Studies }\end{array}$ & Settings \\
\hline & Baik, 2018 & $\begin{array}{l}\text { The aim of this } \\
\text { systematic } \\
\text { literature review is } \\
\text { to detail and } \\
\text { compare } \\
\text { interventions } \\
\text { supporting SDM } \\
\text { over the last } 10 \\
\text { years (January } \\
2008 \text { to December } \\
2017 \text { ) and to } \\
\text { analyze } \\
\text { patient/caregiver } \\
\text { outcomes at the } \\
\text { end of life. }\end{array}$ & $2008-2017$ & $\begin{array}{l}\text { Studies included if they } \\
\text { met the following } \\
\text { criteria: (1) published } \\
\text { between January } 2008 \\
\text { and December } 2017, \\
\text { (2) written in English, } \\
\text { (3) original research } \\
\text { study with data, (4) } \\
\text { studies in which all } \\
\text { patients were receiving } \\
\text { palliative care, and (5) } \\
\text { studies where an } \\
\text { intervention to support } \\
\text { SDM was conducted. }\end{array}$ & $\begin{array}{l}\text { Studies were } \\
\text { excluded if they (1) } \\
\text { provided } \\
\text { discussion, opinion, } \\
\text { commentary, } \\
\text { review, editorial, or } \\
\text { protocol only, (2) } \\
\text { were a published } \\
\text { conference abstract } \\
\text { only or presentation } \\
\text { slides, (3) involved } \\
\text { patients who were } \\
\text { less than } 18 \text { years } \\
\text { old, or (4) } \\
\text { examined the SDM } \\
\text { intervention for only } \\
\text { healthcare } \\
\text { professionals }\end{array}$ & $\begin{array}{l}\text { Total:12 } \\
\text { RCTs:6 } \\
\text { Non-RCTs with } \\
\text { comparison:1 } \\
\text { Observational:5 }\end{array}$ & Hospital and home \\
\hline & $\begin{array}{l}\text { van Weert, } \\
2016^{109}\end{array}$ & $\begin{array}{l}\text { We sought to } \\
\text { systematically } \\
\text { review randomized } \\
\text { controlled trials } \\
\text { (RCTs) and clinical } \\
\text { controlled trials } \\
\text { (CCTs) evaluating } \\
\text { the efficacy of } \\
\text { decision aids as } \\
\text { compared to usual } \\
\text { care or alternative } \\
\text { intervention(s) for } \\
\text { older adults facing } \\
\text { treatment, } \\
\text { screening or care } \\
\text { decisions. }\end{array}$ & $\begin{array}{l}\text { Inception- } \\
2014\end{array}$ & $\begin{array}{l}\text { We included published } \\
\text { RCTs/CCTs of } \\
\text { interventions designed } \\
\text { to improve shared } \\
\text { decision making (SDM) } \\
\text { by older adults (aged } \\
65+\text { ) and RCTs/CCTs } \\
\text { that analyzed the effect } \\
\text { of the intervention in a } \\
\text { subgroup with a mean } \\
\text { age of } 65\end{array}$ & $\begin{array}{l}\text { We excluded } \\
\text { decision aid studies } \\
\text { focusing on: } \\
\text { decisions about } \\
\text { lifestyle changes, } \\
\text { clinical trial entry, } \\
\text { general advance } \\
\text { directives, general } \\
\text { education } \\
\text { programs; and } \\
\text { decision aids to } \\
\text { promote a } \\
\text { recommended } \\
\text { option. }\end{array}$ & $\begin{array}{l}\text { Total:22 } \\
\text { RCTs:22 } \\
\text { Non-RCTs with } \\
\text { comparison:0 } \\
\text { Observational:0 }\end{array}$ & $\begin{array}{l}\text { Outpatient clinic, } \\
\text { home }\end{array}$ \\
\hline
\end{tabular}




\begin{tabular}{|c|c|c|c|c|c|c|c|}
\hline $\begin{array}{l}\text { Type of } \\
\text { Strategy }\end{array}$ & Author, Year & $\begin{array}{l}\text { Objective of } \\
\text { Review }\end{array}$ & $\begin{array}{l}\text { Literature } \\
\text { Start - } \\
\text { End Year }\end{array}$ & Inclusion Criteria & Exclusion Criteria & $\begin{array}{l}\text { Number of } \\
\text { Included Studies }\end{array}$ & Settings \\
\hline & $\begin{array}{l}\text { Berlin, } \\
2018^{110}\end{array}$ & $\begin{array}{l}\text { This systematic } \\
\text { review and meta- } \\
\text { analysis assesses } \\
\text { the feasibility and } \\
\text { efficacy of } \\
\text { preoperative } \\
\text { decision aids (DAs) } \\
\text { to improve the } \\
\text { patient decision- } \\
\text { making process for } \\
\text { breast } \\
\text { reconstruction. }\end{array}$ & $\begin{array}{l}\text { Inception- } \\
2018\end{array}$ & $\begin{array}{l}\text { Studies that met } \\
\text { eligibility for inclusion } \\
\text { assessed the feasibility } \\
\text { or efficacy of a DA to } \\
\text { improve patient } \\
\text { decision making about } \\
\text { post mastectomy } \\
\text { breast reconstruction. } \\
\text { Prospective and } \\
\text { retrospective studies } \\
\text { were eligible for } \\
\text { inclusion. }\end{array}$ & $\begin{array}{l}\text { Studies not } \\
\text { reporting or } \\
\text { assessing } \\
\text { outcomes related to } \\
\text { DAs to improve } \\
\text { patient decision } \\
\text { making about post } \\
\text { mastectomy breast } \\
\text { reconstruction, } \\
\text { abstracts without } \\
\text { an accompanying } \\
\text { published } \\
\text { manuscript and } \\
\text { editorials were } \\
\text { excluded. }\end{array}$ & $\begin{array}{l}\text { Total:17 } \\
\text { RCTs:8 } \\
\text { Non-RCTs with } \\
\text { comparison:3 } \\
\text { Observational:6 }\end{array}$ & Hospital \\
\hline & $\begin{array}{l}\text { Johnson, } \\
2018^{111}\end{array}$ & $\begin{array}{l}\text { To determine the } \\
\text { effectiveness of } \\
\text { interventions to } \\
\text { support shared } \\
\text { decision making in } \\
\text { hypertension. }\end{array}$ & $\begin{array}{l}\text { Inception- } \\
2017\end{array}$ & $\begin{array}{l}\text { Controlled studies } \\
\text { evaluating the effects } \\
\text { of shared decision- } \\
\text { making interventions } \\
\text { for adults with } \\
\text { hypertension } \\
\text { compared with any } \\
\text { comparator in any } \\
\text { setting and reporting } \\
\text { any outcome } \\
\text { measures. (Only one } \\
\text { study from the US, } \\
\text { other from Europe) }\end{array}$ & $\begin{array}{l}\text { Excluded studies } \\
\text { reporting } \\
\text { interventions } \\
\text { unrelated to health- } \\
\text { care decisions. } \\
\text { Excluded } \\
\text { interventions that } \\
\text { aimed to increase } \\
\text { the involvement of } \\
\text { patients in their } \\
\text { own care } \\
\text { generally, but not in } \\
\text { health-care } \\
\text { decisions } \\
\text { specifically }\end{array}$ & $\begin{array}{l}\text { Total:11 } \\
\text { RCTs:5 } \\
\text { Non-RCTs with } \\
\text { comparison:6 } \\
\text { Observational:0 }\end{array}$ & Hospital \\
\hline
\end{tabular}




\begin{tabular}{|c|c|c|c|c|c|c|c|}
\hline $\begin{array}{l}\text { Type of } \\
\text { Strategy }\end{array}$ & Author, Year & $\begin{array}{l}\text { Objective of } \\
\text { Review }\end{array}$ & $\begin{array}{l}\text { Literature } \\
\text { Start - } \\
\text { End Year }\end{array}$ & Inclusion Criteria & Exclusion Criteria & $\begin{array}{l}\text { Number of } \\
\text { Included Studies }\end{array}$ & Settings \\
\hline & $\begin{array}{l}\text { Martínez- } \\
\text { Alonso, } \\
2017^{112}\end{array}$ & $\begin{array}{l}\text { The aim of this } \\
\text { systematic review } \\
\text { and meta-analysis } \\
\text { of randomized } \\
\text { controlled trials } \\
\text { (RCTs) and } \\
\text { observational } \\
\text { studies is to } \\
\text { assess the effect of } \\
\text { decision aids (DAs) } \\
\text { in women aged } 50 \\
\text { and below facing } \\
\text { the decision to be } \\
\text { screened for breast } \\
\text { cancer. }\end{array}$ & $\begin{array}{l}\text { Not } \\
\text { mentioned- } \\
2016\end{array}$ & $\begin{array}{l}\text { Participants were } \\
\text { women facing } \\
\text { decisions about } \\
\text { screening in a } \\
\text { population-based } \\
\text { screening or } \\
\text { opportunistic case } \\
\text { finding framework } \\
\text { within the age interval } \\
\text { of recommended } \\
\text { mammography } \\
\text { screening. } \\
\text { Interventions: DAs } \\
\text { were defined as } \\
\text { interventions aimed to } \\
\text { help women make a } \\
\text { deliberative choice } \\
\text { regarding participation } \\
\text { in mammography } \\
\text { screening. }\end{array}$ & $\begin{array}{l}\text { We excluded } \\
\text { studies aimed at } \\
\text { elderly women } \\
\text { only, and studies } \\
\text { where participants } \\
\text { were asked to } \\
\text { make hypothetical } \\
\text { choices. We also } \\
\text { excluded studies } \\
\text { aimed at } \\
\text { increasing } \\
\text { participation or } \\
\text { promoting } \\
\text { adherence, and } \\
\text { studies not carried } \\
\text { out in the context of } \\
\text { women }\end{array}$ & $\begin{array}{l}\text { Total:4 } \\
\text { RCTs:3 } \\
\text { Non-RCTs with } \\
\text { comparison:1 } \\
\text { Observational:0 }\end{array}$ & Hospital and home \\
\hline & $\begin{array}{l}\text { Légaré, } \\
2018^{113}\end{array}$ & $\begin{array}{l}\text { To determine the } \\
\text { effectiveness of } \\
\text { interventions for } \\
\text { increasing the use } \\
\text { of SDM by } \\
\text { healthcare } \\
\text { professionals. We } \\
\text { considered } \\
\text { interventions } \\
\text { targeting patients, } \\
\text { interventions } \\
\text { targeting } \\
\text { healthcare } \\
\text { professionals, and } \\
\text { interventions } \\
\text { targeting both. }\end{array}$ & $1974-2017$ & $\begin{array}{l}\text { Randomized and non- } \\
\text { randomized trials, } \\
\text { controlled before-after } \\
\text { studies and interrupted } \\
\text { time series studies } \\
\text { evaluating } \\
\text { interventions for } \\
\text { increasing the use of } \\
\text { SDM in which the } \\
\text { primary outcomes were } \\
\text { evaluated using } \\
\text { observer-based or } \\
\text { patient-reported } \\
\text { measures. Participants } \\
\text { could be any } \\
\text { healthcare professional } \\
\text { and patients }\end{array}$ & Not listed & $\begin{array}{l}\text { Total:87 } \\
\text { RCTs:83 } \\
\text { Non-RCTs with } \\
\text { comparison:3 } \\
\text { Observational:1 }\end{array}$ & Hospital \\
\hline
\end{tabular}




\begin{tabular}{|c|c|c|c|c|c|c|c|}
\hline $\begin{array}{l}\text { Type of } \\
\text { Strategy }\end{array}$ & Author, Year & $\begin{array}{l}\text { Objective of } \\
\text { Review }\end{array}$ & $\begin{array}{l}\text { Literature } \\
\text { Start - } \\
\text { End Year }\end{array}$ & Inclusion Criteria & Exclusion Criteria & $\begin{array}{l}\text { Number of } \\
\text { Included Studies }\end{array}$ & Settings \\
\hline & $\begin{array}{l}\text { Stovell, } \\
2016^{114}\end{array}$ & $\begin{array}{l}\text { To examine the } \\
\text { effects of shared } \\
\text { decision-making on } \\
\text { indices of } \\
\text { treatment-related } \\
\text { empowerment of } \\
\text { people with } \\
\text { psychosis }\end{array}$ & $1806-2015$ & $\begin{array}{l}\text { Trials were included if } \\
\text { they compared a } \\
\text { psychosocial } \\
\text { intervention designed } \\
\text { to enhance shared } \\
\text { decision-making in the } \\
\text { planning of treatment } \\
\text { for psychosis with } \\
\text { usual care or a non- } \\
\text { specific control } \\
\text { treatment. We } \\
\text { included trials where } \\
\text { assessing the effects } \\
\text { of promoting shared } \\
\text { decision-making was } \\
\text { either a primary or a } \\
\text { secondary aim of the } \\
\text { study. }\end{array}$ & $\begin{array}{l}\text { Studies of advance } \\
\text { statements or care } \\
\text { planning not } \\
\text { involving promotion } \\
\text { of shared decision- } \\
\text { making were } \\
\text { excluded, as were } \\
\text { studies providing } \\
\text { interventions to } \\
\text { family members or } \\
\text { carers. }\end{array}$ & $\begin{array}{l}\text { Total:11 } \\
\text { RCTs:11 } \\
\text { Non-RCTs with } \\
\text { comparison:0 } \\
\text { Observational:0 }\end{array}$ & Hospital and home \\
\hline & $\begin{array}{l}\text { Porter, } \\
2016^{115}\end{array}$ & $\begin{array}{l}\text { The published } \\
\text { literature was } \\
\text { systematically } \\
\text { reviewed to } \\
\text { determine the } \\
\text { effect of using } \\
\text { mobile electronic } \\
\text { devices to record } \\
\text { food or nutrient } \\
\text { intake on diabetes } \\
\text { control and } \\
\text { nutrition outcomes }\end{array}$ & $\begin{array}{l}\text { Inception- } \\
2016\end{array}$ & $\begin{array}{l}\text { Original research } \\
\text { among people with } \\
\text { type } 1 \text { or } 2 \text { diabetes } \\
\text { mellitus or gestational } \\
\text { diabetes (excluding } \\
\text { pre-diabetes or } \\
\text { diabetes prevention) } \\
\text { with any treatment } \\
\text { regimen, using mobile } \\
\text { electronic devices } \\
\text { where food or nutrient } \\
\text { intake was recorded } \\
\text { (alone or in addition to } \\
\text { other parameters) and } \\
\text { compared with usual } \\
\text { care or alternative } \\
\text { treatment models was } \\
\text { considered. }\end{array}$ & $\begin{array}{l}\text { Interventions } \\
\text { consisting of text } \\
\text { messages, phone } \\
\text { calls, and access to } \\
\text { internet or websites } \\
\text { only were ineligible, } \\
\text { although these } \\
\text { were acceptable if } \\
\text { delivered in } \\
\text { addition to the } \\
\text { intervention of } \\
\text { interest described } \\
\text { above }\end{array}$ & $\begin{array}{l}\text { Total:9 } \\
\text { RCTs:8 } \\
\text { Non-RCTs with } \\
\text { comparison:1 } \\
\text { Observational:0 }\end{array}$ & $\begin{array}{l}1 \text { inpatient, } \\
\text { remainder } \\
\text { outpatient clinics }\end{array}$ \\
\hline
\end{tabular}




\begin{tabular}{|c|c|c|c|c|c|c|c|}
\hline $\begin{array}{l}\text { Type of } \\
\text { Strategy }\end{array}$ & Author, Year & $\begin{array}{l}\text { Objective of } \\
\text { Review }\end{array}$ & $\begin{array}{l}\text { Literature } \\
\text { Start - } \\
\text { End Year }\end{array}$ & Inclusion Criteria & Exclusion Criteria & $\begin{array}{l}\text { Number of } \\
\text { Included Studies }\end{array}$ & Settings \\
\hline & $\begin{array}{l}\text { Morrell, } \\
2016^{116}\end{array}$ & $\begin{array}{l}\text { To describe the } \\
\text { range of decision } \\
\text { aids (DAs) } \\
\text { available to enable } \\
\text { informed choice for } \\
\text { older patients at } \\
\text { the end of life } \\
\text { (EOL) and assess } \\
\text { their effectiveness } \\
\text { or acceptability. }\end{array}$ & $1995-2015$ & $\begin{array}{l}\text { "All study types in } \\
\text { English language } \\
\text { including any modality } \\
\text { of DAs for end of life } \\
\text { were eligible for } \\
\text { inclusion. Participant } \\
\text { older patients (aged } 60 \\
\text { years and above) with } \\
\text { advanced or terminal } \\
\text { illness. Outcome of } \\
\text { interest: Effectiveness } \\
\text { of DAs (Change in } \\
\text { knowledge of condition } \\
\text { or prognosis, } \\
\text { Reduction in decisional } \\
\text { conflict, and } \\
\text { satisfaction with the } \\
\text { DA, treatment } \\
\text { decision)." }\end{array}$ & $\begin{array}{l}\text { Excluded case } \\
\text { studies due to their } \\
\text { inability to } \\
\text { demonstrate } \\
\text { effectiveness but } \\
\text { considered } \\
\text { conference } \\
\text { abstracts eligible to } \\
\text { prevent publication } \\
\text { bias as it is known } \\
\text { that over a third of } \\
\text { these do not result } \\
\text { in full publication. }\end{array}$ & $\begin{array}{l}\text { Total:17 } \\
\text { RCTs:6 } \\
\text { Non-RCTs with } \\
\text { comparison:8 } \\
\text { Observational:3 }\end{array}$ & $\begin{array}{l}\text { Outpatients, } \\
\text { inpatients and } \\
\text { nursing homes }\end{array}$ \\
\hline & $\begin{array}{l}\text { Baptista, } \\
2018^{117}\end{array}$ & $\begin{array}{l}\text { This meta-analysis } \\
\text { aimed to } \\
\text { investigate the } \\
\text { impact of using } \\
\text { Web-based } \\
\text { decision aids to } \\
\text { support men's } \\
\text { prostate cancer } \\
\text { screening } \\
\text { decisions in } \\
\text { comparison with } \\
\text { usual care and } \\
\text { other formats of } \\
\text { decision aids. }\end{array}$ & $\begin{array}{l}\text { Inception- } \\
2016\end{array}$ & $\begin{array}{l}\text { We included studies } \\
\text { comparing Web-based } \\
\text { decision aids to } \\
\text { several parameters: (1) } \\
\text { no intervention/usual } \\
\text { care or (2) alternative } \\
\text { decision aids formats. } \\
\text { Web-based menas } \\
\text { accessed over internet. } \\
\text { Outcome: at least one } \\
\text { quality of decision- } \\
\text { making outcome (eg, } \\
\text { knowledge, decisional } \\
\text { conflict, and } \\
\text { involvement in decision } \\
\text { making). }\end{array}$ & $\begin{array}{l}\text { Excluded if they did } \\
\text { not include web- } \\
\text { based DAs or they } \\
\text { used computer } \\
\text { based without } \\
\text { internet access. } \\
\text { Not reporting SDM } \\
\text { outcome. }\end{array}$ & $\begin{array}{l}\text { Total:7 } \\
\text { RCTs:7 } \\
\text { Non-RCTs with } \\
\text { comparison:0 } \\
\text { Observational:0 }\end{array}$ & Home \\
\hline
\end{tabular}




\begin{tabular}{|c|c|c|c|c|c|c|c|}
\hline $\begin{array}{l}\text { Type of } \\
\text { Strategy }\end{array}$ & Author, Year & $\begin{array}{l}\text { Objective of } \\
\text { Review }\end{array}$ & $\begin{array}{l}\text { Literature } \\
\text { Start - } \\
\text { End Year }\end{array}$ & Inclusion Criteria & Exclusion Criteria & $\begin{array}{l}\text { Number of } \\
\text { Included Studies }\end{array}$ & Settings \\
\hline & $\begin{array}{l}\text { Spronk, } \\
2018^{118}\end{array}$ & $\begin{array}{l}\text { The aim of this } \\
\text { review was to } \\
\text { assess the } \\
\text { availability and } \\
\text { effectiveness of } \\
\text { tools supporting } \\
\text { SDM in metastatic } \\
\text { breast cancer care. }\end{array}$ & $2006-2017$ & $\begin{array}{l}\text { Studies needed to } \\
\text { focus on the } \\
\text { development and/or } \\
\text { evaluation of an } \\
\text { initiative or tool that } \\
\text { focused on i) } \\
\text { information provision } \\
\text { about decisions, ii) } \\
\text { decision making } \\
\text { process, or iii) eliciting } \\
\text { treatment preferences } \\
\text { in metastatic breast } \\
\text { cancer care. Outcomes } \\
\text { included in the studies } \\
\text { had to be any i) } \\
\text { patient-reported } \\
\text { outcome, or ii) health } \\
\text { outcome. }\end{array}$ & Not listed & $\begin{array}{l}\text { Total:7 } \\
\text { RCTs:2 } \\
\text { Non-RCTs with } \\
\text { comparison:2 } \\
\text { Observational:3 }\end{array}$ & Hospital \\
\hline & $\begin{array}{l}\text { Stacey D, } \\
2017^{119}\end{array}$ & $\begin{array}{l}\text { SR to assess the } \\
\text { effects of decision } \\
\text { aids in people } \\
\text { facing treatment or } \\
\text { screening } \\
\text { decisions. } \\
\text { Participants } \\
\text { include adults aged } \\
18 \text { years or older } \\
\text { who were making } \\
\text { decisions about } \\
\text { screening or } \\
\text { treatment options } \\
\text { for themselves, a } \\
\text { child, or an } \\
\text { incapacitated } \\
\text { significant other. }\end{array}$ & $2012-2015$ & $\begin{array}{l}\text { Published randomized } \\
\text { controlled trials } \\
\text { comparing decision } \\
\text { aids to usual care } \\
\text { and/or alternative } \\
\text { interventions in adults. }\end{array}$ & $\begin{array}{l}\text { Excluded studies } \\
\text { comparing detailed } \\
\text { versus simple } \\
\text { decision aid, non } \\
\text { RCT design, } \\
\text { qualitative studies }\end{array}$ & $\begin{array}{l}\text { Total:105 } \\
\text { RCTs:105 } \\
\text { Non-RCTs with } \\
\text { comparison:0 } \\
\text { Observational:0 }\end{array}$ & Hospital \\
\hline
\end{tabular}




\begin{tabular}{|c|c|c|c|c|c|c|c|}
\hline $\begin{array}{l}\text { Type of } \\
\text { Strategy }\end{array}$ & Author, Year & $\begin{array}{l}\text { Objective of } \\
\text { Review }\end{array}$ & $\begin{array}{l}\text { Literature } \\
\text { Start - } \\
\text { End Year }\end{array}$ & Inclusion Criteria & Exclusion Criteria & $\begin{array}{l}\text { Number of } \\
\text { Included Studies }\end{array}$ & Settings \\
\hline & $\begin{array}{l}\text { Vermunt, } \\
2017^{120}\end{array}$ & $\begin{array}{l}\text { This SR aims to } \\
\text { identify and } \\
\text { evaluate studies on } \\
\text { the effects of } \\
\text { interventions that } \\
\text { support } \\
\text { collaborative goal } \\
\text { setting or health } \\
\text { priority setting } \\
\text { compared to usual } \\
\text { care for elderly } \\
\text { people with a } \\
\text { chronic health } \\
\text { condition or multi- } \\
\text { morbidity. }\end{array}$ & 1990-2015 & $\begin{array}{l}\text { RCTs, NRCTs, } \\
\text { controlled before-after } \\
\text { (CBA) studies, } \\
\text { interrupted time series } \\
\text { (ITS) and repeated } \\
\text { measures studies. } \\
\text { Patients with multi- } \\
\text { morbidity or at least } \\
\text { one chronic disease } \\
\text { (mean age } \pm \text { standard } \\
\text { deviation incl. age } 65 \text { ). } \\
\text { Both single and } \\
\text { multifactorial } \\
\text { interventions } \\
\text { supporting } \\
\text { collaborative goal } \\
\text { setting or health priority } \\
\text { setting were included. }\end{array}$ & Not listed & $\begin{array}{l}\text { Total:8 } \\
\text { RCTs:5 } \\
\text { Non-RCTs with } \\
\text { comparison:0 } \\
\text { Observational:3 }\end{array}$ & Hospital \\
\hline & $\begin{array}{l}\text { Kashaf, } \\
2017^{121}\end{array}$ & $\begin{array}{l}\text { This review } \\
\text { systematically } \\
\text { examines, } \\
\text { summarizes and, } \\
\text { where possible, } \\
\text { quantitatively } \\
\text { synthesizes the } \\
\text { evidence } \\
\text { association } \\
\text { between treatment } \\
\text { shared decision- } \\
\text { making (SDM) and } \\
\text { outcomes in } \\
\text { diabetes. }\end{array}$ & 1990-2016 & $\begin{array}{l}\text { population composed } \\
\text { of adults }>18 \text { years, } \\
\text { study concern } \\
\text { decision-making within } \\
\text { any context of diabetes } \\
\text { treatment or } \\
\text { management with } \\
\text { patient participation } \\
\text { and studies measuring } \\
\text { the measure process } \\
\text { of care or clinical } \\
\text { outcomes relevant to } \\
\text { diabetes management. }\end{array}$ & $\begin{array}{l}\text { Non-comparative } \\
\text { designs such as } \\
\text { case series and } \\
\text { exploratory } \\
\text { research and Non- } \\
\text { longitudinal studies } \\
\text { such as cross- } \\
\text { sectional surveys } \\
\text { were excluded. }\end{array}$ & $\begin{array}{l}\text { Total:16 } \\
\text { RCTs:13 } \\
\text { Non-RCTs with } \\
\text { comparison:1 } \\
\text { Observational:2 }\end{array}$ & Hospital \\
\hline
\end{tabular}




\begin{tabular}{|c|c|c|c|c|c|c|c|}
\hline $\begin{array}{l}\text { Type of } \\
\text { Strategy }\end{array}$ & Author, Year & $\begin{array}{l}\text { Objective of } \\
\text { Review }\end{array}$ & $\begin{array}{l}\text { Literature } \\
\text { Start - } \\
\text { End Year }\end{array}$ & Inclusion Criteria & Exclusion Criteria & $\begin{array}{l}\text { Number of } \\
\text { Included Studies }\end{array}$ & Settings \\
\hline & $\begin{array}{l}\text { Samalin, } \\
2018^{122}\end{array}$ & $\begin{array}{l}\text { This study aims to } \\
\text { provide a review of } \\
\text { the randomized } \\
\text { controlled studies } \\
\text { evaluating the } \\
\text { effects of share } \\
\text { decision-making } \\
\text { (SDM) intervention } \\
\text { in comparison to } \\
\text { care as usual in } \\
\text { patients with mood } \\
\text { disorders. }\end{array}$ & $2000-2017$ & $\begin{array}{l}\text { P: Adults with mood } \\
\text { disorders: dysthymia, } \\
\text { MDD, or BD type I and } \\
\text { type II., I: SDM } \\
\text { intervention or } \\
\text { collaborative care, } \\
\text { which contained at } \\
\text { least one module using } \\
\text { a SDM process. } \\
\text { Outcome: clinical and } \\
\text { health related QoL, } \\
\text { Study design: RCTs }\end{array}$ & Not listed & $\begin{array}{l}\text { Total:14 } \\
\text { RCTs:14 } \\
\text { Non-RCTs with } \\
\text { comparison:0 } \\
\text { Observational:0 }\end{array}$ & Hospital, PCC \\
\hline & $\begin{array}{l}\text { Martínez- } \\
\text { González, } \\
2018^{123}\end{array}$ & $\begin{array}{l}\text { We systematically } \\
\text { reviewed the } \\
\text { extent of SDM } \\
\text { implementation in } \\
\text { interventions to } \\
\text { facilitate SDM for } \\
\text { prostate cancer } \\
\text { screening and } \\
\text { treatment. }\end{array}$ & $\begin{array}{l}\text { Inception- } \\
2015\end{array}$ & $\begin{array}{l}\text { Included studies: RCTs } \\
\text { conducted in primary } \\
\text { and specialized care, } \\
\text { addressing } \\
\text { interventions aiming to } \\
\text { facilitate SDM for } \\
\text { prostate cancer } \\
\text { screening and } \\
\text { treatment. Comparing } \\
\text { SDM interventions to } \\
\text { one or more alternative } \\
\text { interventions, and/or } \\
\text { usual care. }\end{array}$ & $\begin{array}{l}\text { We excluded } \\
\text { studies conducted } \\
\text { in non-clinical } \\
\text { settings and } \\
\text { community studies } \\
\text { in which } \\
\text { discussions were } \\
\text { not intended or } \\
\text { could not occur. }\end{array}$ & $\begin{array}{l}\text { Total:36 } \\
\text { RCTs:36 } \\
\text { Non-RCTs with } \\
\text { comparison:0 } \\
\text { Observational:0 }\end{array}$ & $\begin{array}{l}\text { Hospital, outpatient } \\
\text { clinics, PCC }\end{array}$ \\
\hline & $\begin{array}{l}\text { Martínez- } \\
\text { González, } \\
2018^{124}\end{array}$ & $\begin{array}{l}\text { We sought to } \\
\text { evaluate the } \\
\text { evidence on the } \\
\text { effectiveness of } \\
\text { SDM as compared } \\
\text { to current clinical } \\
\text { practice for patient- } \\
\text { and SDM-related } \\
\text { outcomes. We } \\
\text { focused on the } \\
\text { population of men } \\
\text { facing preference- } \\
\text { sensitive decisions } \\
\text { for Prostate } \\
\text { Cancer screening. }\end{array}$ & $\begin{array}{l}\text { Inception- } \\
2015\end{array}$ & $\begin{array}{l}\text { We included RCTs } \\
\text { published in English, } \\
\text { from any country, } \\
\text { investigating SDM for } \\
\text { PC screening in } \\
\text { primary or specialized } \\
\text { care compared SDM to } \\
\text { usual care regardless } \\
\text { of the intervention } \\
\text { target. }\end{array}$ & Not listed & $\begin{array}{l}\text { Total:4 } \\
\text { RCTs:4 } \\
\text { Non-RCTs with } \\
\text { comparison:0 } \\
\text { Observational:0 }\end{array}$ & Hospital \\
\hline
\end{tabular}




\begin{tabular}{|c|c|c|c|c|c|c|c|}
\hline $\begin{array}{l}\text { Type of } \\
\text { Strategy }\end{array}$ & Author, Year & $\begin{array}{l}\text { Objective of } \\
\text { Review }\end{array}$ & $\begin{array}{l}\text { Literature } \\
\text { Start - } \\
\text { End Year }\end{array}$ & Inclusion Criteria & Exclusion Criteria & $\begin{array}{l}\text { Number of } \\
\text { Included Studies }\end{array}$ & Settings \\
\hline $\begin{array}{l}\text { Transitional } \\
\text { Care - Adult } \\
\text { Only }\end{array}$ & $\begin{array}{l}\text { Sendall, } \\
2016^{125}\end{array}$ & $\begin{array}{l}\text { This structured } \\
\text { review will identify } \\
\text { the components of } \\
\text { the chronic care } \\
\text { model (CCM) } \\
\text { required to support } \\
\text { healthcare that } \\
\text { transitions } \\
\text { seamlessly } \\
\text { between hospital } \\
\text { and ambulatory } \\
\text { settings for people } \\
\text { over } 65 \text { years of } \\
\text { age who have two } \\
\text { or more chronic } \\
\text { diseases }\end{array}$ & $\begin{array}{l}\text { Inception- } \\
2015\end{array}$ & $\begin{array}{l}\text { (i) involved a cohort of } \\
\text { older people (aged }>65 \\
\text { years) with two or more } \\
\text { chronic diseases; (ii) } \\
\text { involved healthcare } \\
\text { delivery between } \\
\text { hospital and non- } \\
\text { hospital (i.e. primary } \\
\text { healthcare, outpatient } \\
\text { or community) } \\
\text { services; (iii) involved } \\
\text { at least two } \\
\text { components of } \\
\text { Wagner's CCM (i.e. } \\
\text { healthcare } \\
\text { organization, self- } \\
\text { management support, } \\
\text { delivery system design, } \\
\text { decision support, } \\
\text { clinical information } \\
\text { system and community } \\
\text { capacity); (iv) reported } \\
\text { at least one of the } \\
\text { following outcomes: } \\
\text { emergency } \\
\text { presentations, hospital } \\
\text { admissions, health out- } \\
\text { comes for patients or } \\
\text { patient and clinician } \\
\text { satisfaction with the } \\
\text { integrated healthcare } \\
\text { system; and (v) } \\
\text { published in English } \\
\text { language }\end{array}$ & $\begin{array}{l}\text { (i) were not original } \\
\text { research articles, } \\
\text { (ii) integrated } \\
\text { services for one } \\
\text { chronic disease or } \\
\text { within a health } \\
\text { setting or (iii) did } \\
\text { not assess an } \\
\text { outcome of interest. }\end{array}$ & $\begin{array}{l}\text { Total:4 } \\
\text { RCTs:3 } \\
\text { Non-RCTs with } \\
\text { comparison:1 } \\
\text { Observational:0 }\end{array}$ & $\begin{array}{l}\text { Inpatient, outpatient } \\
\text { clinics }\end{array}$ \\
\hline
\end{tabular}




\begin{tabular}{|c|c|c|c|c|c|c|c|}
\hline $\begin{array}{l}\text { Type of } \\
\text { Strategy }\end{array}$ & Author, Year & $\begin{array}{l}\text { Objective of } \\
\text { Review }\end{array}$ & $\begin{array}{l}\text { Literature } \\
\text { Start - } \\
\text { End Year }\end{array}$ & Inclusion Criteria & Exclusion Criteria & $\begin{array}{l}\text { Number of } \\
\text { Included Studies }\end{array}$ & Settings \\
\hline & $\begin{array}{l}\text { Berre, } \\
2017^{126}\end{array}$ & $\begin{array}{l}\text { To determine the } \\
\text { effectiveness of } \\
\text { interventions } \\
\text { targeting } \\
\text { transitions from } \\
\text { hospital to the } \\
\text { primary care } \\
\text { setting for } \\
\text { chronically ill older } \\
\text { patients. }\end{array}$ & 1995-2016 & $\begin{array}{l}\text { P: } 65 \text { years old or older } \\
\text { with at least one CD } \\
\text { who have been } \\
\text { hospitalized and are } \\
\text { being discharged back } \\
\text { to home. I: TC } \\
\text { interventions, C: Usual } \\
\text { care (UC) (non- } \\
\text { structured follow up), } \\
\text { O: All-cause mortality, } \\
\text { Health utilization, QoL. } \\
\text { Study design: RCTs. } \\
\text { English or French }\end{array}$ & Not listed & $\begin{array}{l}\text { Total:92 } \\
\text { RCTs:92 } \\
\text { Non-RCTs with } \\
\text { comparison:0 } \\
\text { Observational:0 }\end{array}$ & Hospital and home \\
\hline
\end{tabular}


Evidence Table 2. Population characteristics of included systematic reviews addressing direct patient care

\begin{tabular}{|c|c|c|c|c|c|c|c|c|c|}
\hline Type of Strategy & Author, Year & $\begin{array}{l}\text { Multiple } \\
\text { Chronic } \\
\text { Condition } \\
\end{array}$ & $\begin{array}{l}\text { Mixed } \\
\text { Chronic } \\
\text { Conditions } \\
\end{array}$ & Diabetes & $\begin{array}{l}\text { Cardiovascular } \\
\text { Disease and } \\
\text { Hypertension }\end{array}$ & Respiratory & $\begin{array}{l}\text { Cancer or } \\
\text { Cancer } \\
\text { Screening }\end{array}$ & $\begin{array}{l}\text { Mental } \\
\text { Health }\end{array}$ & Other \\
\hline $\begin{array}{l}\text { Health Literacy - } \\
\text { Adult Only }\end{array}$ & $\begin{array}{l}\text { Schaepe, } \\
2015^{2}\end{array}$ & & & & & & & & Kidney disease \\
\hline $\begin{array}{l}\text { Advanced Care } \\
\text { Planning - Adult } \\
\text { Only }\end{array}$ & Coulter, $2015^{1}$ & & & $x$ & $x$ & $x$ & & $\mathrm{x}$ & Kidney disease \\
\hline \multirow{2}{*}{$\begin{array}{l}\text { Transitional Care - } \\
\text { Adult Only }\end{array}$} & Berre, $2017^{126}$ & $\mathrm{X}$ & & & & & & & \\
\hline & $\begin{array}{l}\text { Sendall, } \\
2016^{125}\end{array}$ & $\mathrm{X}$ & & & & & & & \\
\hline \multirow{16}{*}{$\begin{array}{l}\text { Shared Decision } \\
\text { Making - Adult } \\
\text { Only }\end{array}$} & $\begin{array}{l}\text { Kashaf, } \\
2015^{106}\end{array}$ & & & & & & $x$ & & \\
\hline & $\begin{array}{l}\text { Martínez- } \\
\text { Alonso, } \\
2017^{112} \\
\end{array}$ & & & & & & $x$ & & \\
\hline & $\begin{array}{l}\text { Stacey D, } \\
2017^{119}\end{array}$ & & $\mathrm{X}$ & & & & & & \\
\hline & $\begin{array}{l}\text { Morrell, } \\
2016^{116}\end{array}$ & & & & & & & & End of life \\
\hline & Porter, $2016^{115}$ & & & $\mathrm{X}$ & & & & & \\
\hline & Stovell, $2016^{114}$ & & & & & & & $\mathrm{X}$ & \\
\hline & $\begin{array}{l}\text { van Weert, } \\
2016^{109}\end{array}$ & & $\mathrm{X}$ & & & & & & \\
\hline & $\begin{array}{l}\text { Spronk, } \\
2018^{118}\end{array}$ & & & & & & $X$ & & \\
\hline & Jain, $2015^{104}$ & & $\mathrm{X}$ & & & & & & \\
\hline & $\begin{array}{l}\text { Irizarry, } \\
2015^{102}\end{array}$ & & $\mathrm{X}$ & & & & & & \\
\hline & $\begin{array}{l}\text { Violette, } \\
2015^{103}\end{array}$ & & & & & & $\mathrm{X}$ & & \\
\hline & $\begin{array}{l}\text { Nathan , } \\
2016^{99}\end{array}$ & & $\mathrm{X}$ & & & & $\mathrm{X}$ & & Kidney disease \\
\hline & $\begin{array}{l}\text { Samalin, } \\
2018^{122}\end{array}$ & & & & & & & $X$ & $\begin{array}{l}\text { Depression and } \\
\text { Bipolar disorder }\end{array}$ \\
\hline & $\begin{array}{l}\text { Mclntyre, } \\
2015^{105}\end{array}$ & & & & & & $\mathrm{X}$ & & \\
\hline & $\begin{array}{l}\text { Baptista, } \\
2018^{117}\end{array}$ & & & & & & $\mathrm{X}$ & & \\
\hline & $\begin{array}{l}\text { Légaré, } \\
2018^{113}\end{array}$ & & $\mathrm{X}$ & & & & & & \\
\hline
\end{tabular}




\begin{tabular}{|c|c|c|c|c|c|c|c|c|c|}
\hline Type of Strategy & Author, Year & $\begin{array}{l}\text { Multiple } \\
\text { Chronic } \\
\text { Condition }\end{array}$ & $\begin{array}{l}\text { Mixed } \\
\text { Chronic } \\
\text { Conditions }\end{array}$ & Diabetes & $\begin{array}{l}\text { Cardiovascular } \\
\text { Disease and } \\
\text { Hypertension }\end{array}$ & Respiratory & $\begin{array}{l}\text { Cancer or } \\
\text { Cancer } \\
\text { Screening }\end{array}$ & $\begin{array}{l}\text { Mental } \\
\text { Health }\end{array}$ & Other \\
\hline & $\begin{array}{l}\text { Martínez- } \\
\text { González, } \\
2018^{124}\end{array}$ & & & & & & $x$ & & \\
\hline & $\begin{array}{l}\text { Johnson, } \\
2018^{111}\end{array}$ & & & & $x$ & & & & \\
\hline & Berlin, 2018 ${ }^{110}$ & & & & & & & & Surgical patients \\
\hline & Baik, $2018^{108}$ & & & & & & & & End of life \\
\hline & $\begin{array}{l}\text { Goodridge, } \\
2019^{101}\end{array}$ & & & & & & & & $\begin{array}{l}\text { Hospitalized } \\
\text { patients }\end{array}$ \\
\hline & $\begin{array}{l}\text { Wagner, } \\
2019^{100}\end{array}$ & & $x$ & & & & & & \\
\hline & $\begin{array}{l}\text { Kashaf, } \\
2017^{121} \\
\end{array}$ & & & $\mathrm{X}$ & & & & & \\
\hline & $\begin{array}{l}\text { Vermunt, } \\
2017^{120}\end{array}$ & $\mathrm{X}$ & & & & & & & \\
\hline & $\begin{array}{l}\text { Martínez- } \\
\text { González, } \\
2018^{123} \\
\end{array}$ & & & & & & $x$ & & \\
\hline & $\begin{array}{l}\text { Friedrichs, } \\
2016^{107}\end{array}$ & & & & & & & $x$ & \\
\hline \multirow{10}{*}{$\begin{array}{l}\text { Self-Management } \\
\text { Support - Adult } \\
\text { Only }\end{array}$} & Kruse, $2015^{89}$ & & $\mathrm{X}$ & & & & & & \\
\hline & Yin, $2019^{90}$ & & $\mathrm{X}$ & & & & & & IBD \\
\hline & $\begin{array}{l}\text { Jacelon, } \\
2016^{40} \\
\end{array}$ & & $x$ & & & & & & \\
\hline & $\begin{array}{l}\text { Sangrar, } \\
2019^{56}\end{array}$ & & & $x$ & & $x$ & & & $\begin{array}{l}\text { diabetes, asthma, } \\
\text { COPD }\end{array}$ \\
\hline & $\begin{array}{l}\text { Nazarov, } \\
2019^{55}\end{array}$ & & & $\mathrm{x}$ & $\mathrm{X}$ & $\mathrm{X}$ & & $\mathrm{X}$ & Neuro \\
\hline & Lederle, $2019^{54}$ & & & $x$ & $\mathrm{X}$ & $X$ & $x$ & $x$ & $\begin{array}{l}\text { Any chronic } \\
\text { disease (lung, } \\
\text { heart, arthritis, } \\
\text { diabetes, mental } \\
\text { illness, stroke, } \\
\text { back pain, cancer) }\end{array}$ \\
\hline & $\begin{array}{l}\text { Levengood, } \\
2019^{52}\end{array}$ & & & $x$ & & & & & \\
\hline & Han, $2019^{51}$ & & & $\mathrm{X}$ & & & & & \\
\hline & Warner, $2019^{50}$ & & $x$ & $x$ & $x$ & $x$ & & & $\begin{array}{l}\text { Osteoarthritis, } \\
\text { physical rehab }\end{array}$ \\
\hline & $\begin{array}{l}\text { Whitehead, } \\
2016^{25}\end{array}$ & & & $\mathrm{X}$ & $X$ & $X$ & & & \\
\hline
\end{tabular}




\begin{tabular}{|c|c|c|c|c|c|c|c|c|c|}
\hline Type of Strategy & Author, Year & $\begin{array}{l}\text { Multiple } \\
\text { Chronic } \\
\text { Condition }\end{array}$ & $\begin{array}{l}\text { Mixed } \\
\text { Chronic } \\
\text { Conditions }\end{array}$ & Diabetes & $\begin{array}{l}\text { Cardiovascular } \\
\text { Disease and } \\
\text { Hypertension }\end{array}$ & Respiratory & $\begin{array}{l}\text { Cancer or } \\
\text { Cancer } \\
\text { Screening }\end{array}$ & $\begin{array}{l}\text { Mental } \\
\text { Health }\end{array}$ & Other \\
\hline & Palacio, $2016^{38}$ & & $\mathrm{X}$ & & & & & & \\
\hline & Eeden, $2016^{39}$ & & $\mathrm{X}$ & $\mathrm{X}$ & & $\mathrm{X}$ & & & \\
\hline & Kuo, $2016^{33}$ & & & $x$ & & & & & \\
\hline & Alessa, $2018^{47}$ & & & & $\mathrm{X}$ & & & & \\
\hline & Smith, $2017^{41}$ & $\mathrm{X}$ & & & & & & & \\
\hline & $\begin{array}{l}\text { Laukner, } \\
2016^{76}\end{array}$ & $X$ & & $X$ & $\mathrm{X}$ & & & $\mathrm{X}$ & \\
\hline & Kim, $2016^{43}$ & & & & $\mathrm{X}$ & $\mathrm{X}$ & $\mathrm{X}$ & $\mathrm{X}$ & \\
\hline & $\begin{array}{l}\text { Ha Dinh, } \\
2016^{59}\end{array}$ & & & $\mathrm{X}$ & $\mathrm{X}$ & $\mathrm{X}$ & & & \\
\hline & $\begin{array}{l}\text { Thakkar, } \\
2016^{44}\end{array}$ & & & & $x$ & $\mathrm{X}$ & & & $\begin{array}{l}\text { HIV, allergies, } \\
\text { neuro }\end{array}$ \\
\hline & Deek, $2016^{45}$ & & & $\mathrm{X}$ & $\mathrm{X}$ & $\mathrm{X}$ & $\mathrm{X}$ & & Musculoskeletal \\
\hline & Kim, $2015^{26}$ & & & & & & $\mathrm{X}$ & & \\
\hline & Debon, $2019^{48}$ & & $\mathrm{X}$ & & & & & & \\
\hline & $\begin{array}{l}\text { Peytremann- } \\
\text { Bridevaux, } \\
2015^{75}\end{array}$ & & & & & $\mathrm{X}$ & & & Asthma \\
\hline & Jones, $2015^{87}$ & & & & & & & & $\begin{array}{l}\text { Neuro, Post-TBI, } \\
\text { stroke patients }\end{array}$ \\
\hline & Luedke, $2019^{49}$ & & & & & & & & Epilepsy \\
\hline & $\begin{array}{l}\text { Zomahoun, } \\
2016^{37}\end{array}$ & & $\mathrm{X}$ & & & & & & \\
\hline & $\begin{array}{l}\text { Sakakibara, } \\
2016^{34}\end{array}$ & & & & & & & & $\begin{array}{l}\text { Neuro, Stroke } \\
\text { patients only }\end{array}$ \\
\hline & Plow, $2016^{31}$ & & & & & & & & Neuro \\
\hline & $\begin{array}{l}\text { Stenberg, } \\
2016^{30}\end{array}$ & & $\mathrm{X}$ & $\mathrm{X}$ & $\mathrm{X}$ & & $\mathrm{X}$ & $\mathrm{X}$ & \\
\hline & $\begin{array}{l}\text { Bolscher- } \\
\text { Niehuis, } 2016^{46}\end{array}$ & & $\mathrm{X}$ & & & & & & \\
\hline & Sokol, $2016^{36}$ & & $\mathrm{X}$ & & & & & & $\begin{array}{l}\text { Maternal and } \\
\text { health issues }\end{array}$ \\
\hline & Hooft, $2016^{29}$ & & $\mathrm{X}$ & & & & & & \\
\hline & Hecke, $2016^{28}$ & & $x$ & $x$ & & & & & \\
\hline & Teljeur, 2016 27 & & & $\mathrm{X}$ & & & & & \\
\hline & Pereira, $2015^{88}$ & & & $x$ & & & & & \\
\hline & Zhao, $2016^{57}$ & & & $x$ & & & & & \\
\hline & $\begin{array}{l}\text { Dounavi, } \\
2019^{69}\end{array}$ & & & & & & & & Obesity \\
\hline
\end{tabular}




\begin{tabular}{|c|c|c|c|c|c|c|c|c|c|}
\hline Type of Strategy & Author, Year & $\begin{array}{l}\text { Multiple } \\
\text { Chronic } \\
\text { Condition }\end{array}$ & $\begin{array}{l}\text { Mixed } \\
\text { Chronic } \\
\text { Conditions }\end{array}$ & Diabetes & $\begin{array}{l}\text { Cardiovascular } \\
\text { Disease and } \\
\text { Hypertension }\end{array}$ & Respiratory & $\begin{array}{l}\text { Cancer or } \\
\text { Cancer } \\
\text { Screening }\end{array}$ & $\begin{array}{l}\text { Mental } \\
\text { Health }\end{array}$ & Other \\
\hline & $\begin{array}{l}\text { Whiteman, } \\
2016^{35}\end{array}$ & $x$ & & & & & & $\mathrm{X}$ & \\
\hline & $\begin{array}{l}\text { Skrabal Ross, } \\
2018^{58}\end{array}$ & & & & & & $\mathrm{X}$ & & \\
\hline & $\begin{array}{l}\text { Massimi, } \\
2017^{68}\end{array}$ & $\mathrm{X}$ & & $\mathrm{X}$ & $\mathrm{X}$ & & & & \\
\hline & $\begin{array}{l}\text { Clarkesmith, } \\
2017^{67}\end{array}$ & & & & $\mathrm{X}$ & & & & $\begin{array}{l}\text { patients with atrial } \\
\text { fibrillation }\end{array}$ \\
\hline & Cho, $2017^{66}$ & & & & & & & & $\begin{array}{l}\text { Persons living with } \\
\text { HIVIAIDS (PLWH) }\end{array}$ \\
\hline & Kim, $2017^{65}$ & & $\mathrm{X}$ & $\mathrm{X}$ & $\mathrm{X}$ & $\mathrm{X}$ & & & \\
\hline & $\begin{array}{l}\text { Conway, } \\
2017^{64}\end{array}$ & & & $x$ & $\mathrm{X}$ & & & & \\
\hline & Jeddi, $2017^{63}$ & & & & & & & & Kidney disease \\
\hline & Kelly, $2018^{62}$ & & & & & $\mathrm{X}$ & & & \\
\hline & $\begin{array}{l}\text { Changizi, } \\
2017^{61}\end{array}$ & & & $\mathrm{X}$ & $\mathrm{X}$ & & & & Obesity \\
\hline & Ko, $2018^{60}$ & $\mathrm{X}$ & & & & & & & \\
\hline & $\begin{array}{l}\text { Noonan, } \\
2019^{32} \\
\end{array}$ & & & & $x$ & $\mathrm{X}$ & & & \\
\hline & Price, $2015^{70}$ & & $\mathrm{X}$ & $\mathrm{X}$ & & & & & \\
\hline & $\begin{array}{l}\text { Palacios, } \\
2017^{79}\end{array}$ & & & & $x$ & & & & $\begin{array}{l}\text { Patients with } \\
\text { coronary heart } \\
\text { disease (CHD) }\end{array}$ \\
\hline & $\begin{array}{l}\text { Wildevuur, } \\
2015^{86}\end{array}$ & & & $\mathrm{X}$ & $\mathrm{X}$ & $\mathrm{X}$ & $\mathrm{X}$ & & Neuro \\
\hline & Risling, $2017^{84}$ & & $\mathrm{X}$ & & & & & & \\
\hline & $\begin{array}{l}\text { Almutairi, } \\
2019^{82}\end{array}$ & & & $x$ & & & & & \\
\hline & Lee, $2018^{77}$ & & $\mathrm{X}$ & & & & & & \\
\hline & Donald, $2018^{73}$ & & & & & & & & Kidney disease \\
\hline & Aquino, $2017^{71}$ & & & $\mathrm{X}$ & & & & & \\
\hline & Kelly, $2018^{83}$ & & $\mathrm{X}$ & & & & & & \\
\hline & $\begin{array}{l}\text { Warrington, } \\
2019^{78}\end{array}$ & & & & & & $\mathrm{X}$ & & \\
\hline & $\begin{array}{l}\text { Dendere, } \\
2019^{81} \\
\end{array}$ & & $x$ & & & & & & \\
\hline & $\begin{array}{l}\text { Ammenwerth, } \\
2019^{72}\end{array}$ & & $\mathrm{X}$ & & & & & & \\
\hline
\end{tabular}




\begin{tabular}{|c|c|c|c|c|c|c|c|c|c|}
\hline Type of Strategy & Author, Year & $\begin{array}{l}\text { Multiple } \\
\text { Chronic } \\
\text { Condition }\end{array}$ & $\begin{array}{l}\text { Mixed } \\
\text { Chronic } \\
\text { Conditions }\end{array}$ & Diabetes & $\begin{array}{l}\text { Cardiovascular } \\
\text { Disease and } \\
\text { Hypertension }\end{array}$ & Respiratory & $\begin{array}{l}\text { Cancer or } \\
\text { Cancer } \\
\text { Screening }\end{array}$ & $\begin{array}{l}\text { Mental } \\
\text { Health }\end{array}$ & Other \\
\hline & $\begin{array}{l}\text { Hamine, } \\
2015^{85}\end{array}$ & & & $\mathrm{X}$ & $x$ & $\mathrm{X}$ & & & \\
\hline & Zhao, $2015^{53}$ & & & & & $\mathrm{X}$ & & & \\
\hline & $\begin{array}{l}\text { Hammer, } \\
2015^{42}\end{array}$ & & & & & & $x$ & & \\
\hline & Boulley, $2018^{74}$ & & & & & & $\mathrm{X}$ & & \\
\hline & Warner, $2015^{80}$ & & & & & & & & Stroke, neuro \\
\hline \multirow[t]{4}{*}{$\begin{array}{l}\text { Shared Decision } \\
\text { Making - Adults } \\
\text { and Children }\end{array}$} & $\begin{array}{l}\text { Voruganti, } \\
2017^{95}\end{array}$ & & & $\mathrm{X}$ & $\mathrm{X}$ & $x$ & & $\mathrm{X}$ & $\begin{array}{l}\text { chronic pain, } \\
\text { cerebral palsy, } \\
\text { eczema }\end{array}$ \\
\hline & $\begin{array}{l}\text { Winston, } \\
2017^{98}\end{array}$ & $\mathrm{X}$ & & & & & & & $\begin{array}{l}\text { Non-chronic } \\
\text { conditions }\end{array}$ \\
\hline & Kew, $2017^{96}$ & & & & & $\mathrm{X}$ & & & \\
\hline & $\begin{array}{l}\text { Clayman, } \\
2015^{97}\end{array}$ & $\mathrm{X}$ & & & & & & & $\begin{array}{l}\text { Non-chronic } \\
\text { conditions }\end{array}$ \\
\hline \multirow{12}{*}{$\begin{array}{l}\text { Self Management } \\
\text { Support - Adults } \\
\text { and Children }\end{array}$} & $\begin{array}{l}\text { Pamungkas, } \\
2017^{23}\end{array}$ & & & $\mathrm{X}$ & & & & & \\
\hline & Kew, $2016^{18}$ & & & & & $\mathrm{X}$ & & & \\
\hline & Hill, $2015^{22}$ & $\mathrm{X}$ & & & & & & & \\
\hline & Barello, 2016 14 & & & & & & & $x$ & HIV \\
\hline & $\begin{array}{l}\text { Graham, } \\
2016^{19}\end{array}$ & & & $x$ & & $X$ & & $\mathrm{X}$ & $\begin{array}{l}\text { Kidney disease, } \\
\text { Cerebral Palsy, } \\
\text { Brain Injury }\end{array}$ \\
\hline & Niznik, $2018^{13}$ & & & $\mathrm{X}$ & $\mathrm{X}$ & $\mathrm{X}$ & & $\mathrm{X}$ & $\begin{array}{l}\text { Kidney disease, } \\
\text { hyperlipidemia }\end{array}$ \\
\hline & Bashi, $2018^{15}$ & $\mathrm{X}$ & & & & & & $\mathrm{X}$ & \\
\hline & Lycett, $2018^{16}$ & & & & & $\mathrm{X}$ & & & \\
\hline & $\begin{array}{l}\text { Lancaster, } \\
2018^{17}\end{array}$ & & & $x$ & & $X$ & $X$ & & \\
\hline & Yin, $2019^{20}$ & & & $\mathrm{X}$ & $\mathrm{X}$ & $\mathrm{X}$ & & $\mathrm{X}$ & $\begin{array}{l}\text { Kidney disease, } \\
\text { Parkinson disease }\end{array}$ \\
\hline & Chi, $2015^{21}$ & & & $\mathrm{X}$ & & & & $\mathrm{X}$ & $\begin{array}{l}\text { Kidney disease, } \\
\text { Parkinson disease }\end{array}$ \\
\hline & Kruse, $2015^{24}$ & $\mathrm{X}$ & & & & & & & \\
\hline \multirow[t]{3}{*}{$\begin{array}{l}\text { Shared Decision } \\
\text { Making - Children } \\
\text { Only }\end{array}$} & Wyatt, $2015^{94}$ & & & & & & & $\mathrm{X}$ & $\begin{array}{l}\text { ADHD, Heme/Onc, } \\
\text { HIV, } \\
\text { developmental } \\
\text { disabilities }\end{array}$ \\
\hline & Coyne, $2016^{93}$ & & & & & & $X$ & & \\
\hline & Cheng, $2017^{92}$ & & & & & & & $x$ & \\
\hline
\end{tabular}




\begin{tabular}{|c|c|c|c|c|c|c|c|c|c|}
\hline Type of Strategy & Author, Year & $\begin{array}{l}\text { Multiple } \\
\text { Chronic } \\
\text { Condition }\end{array}$ & $\begin{array}{l}\text { Mixed } \\
\text { Chronic } \\
\text { Conditions }\end{array}$ & Diabetes & $\begin{array}{l}\text { Cardiovascular } \\
\text { Disease and } \\
\text { Hypertension }\end{array}$ & Respiratory & $\begin{array}{l}\text { Cancer or } \\
\text { Cancer } \\
\text { Screening }\end{array}$ & $\begin{array}{l}\text { Mental } \\
\text { Health }\end{array}$ & Other \\
\hline & Malone, $2019^{91}$ & & & & $x$ & & & & $\mathrm{CF}$ \\
\hline \multirow{10}{*}{$\begin{array}{l}\text { Self Management } \\
\text { Support - Children } \\
\text { Only }\end{array}$} & $\begin{array}{l}\text { Clemente, } \\
2016^{12}\end{array}$ & & & & & & & & $\begin{array}{l}\text { Rheumatologic/MS } \\
\mathrm{K}\end{array}$ \\
\hline & $\begin{array}{l}\text { Hamline, } \\
2018^{11} \\
\end{array}$ & $\mathrm{X}$ & & $\mathrm{X}$ & $\mathrm{X}$ & $\mathrm{X}$ & $\mathrm{X}$ & $\mathrm{x}$ & $\begin{array}{l}\text { sickle cell disease, } \\
\text { CCF, epilepsy }\end{array}$ \\
\hline & Sattoe, $2015^{10}$ & & & $\mathrm{X}$ & & $\mathrm{X}$ & & & \\
\hline & Charlier, $2015^{9}$ & & & $\mathrm{X}$ & & $\mathrm{X}$ & $\mathrm{X}$ & & \\
\hline & $\begin{array}{l}\text { Majeed-Ariss, } \\
2015^{8}\end{array}$ & & & $x$ & & $\mathrm{X}$ & $x$ & & \\
\hline & $\begin{array}{l}\text { Campbell, } \\
2016^{7}\end{array}$ & & & $x$ & & & & & $\begin{array}{l}\text { cystic fibrosis, } \\
\text { inflammatory bowel } \\
\text { disease, spina } \\
\text { bifida }\end{array}$ \\
\hline & Knafl, $2017^{6}$ & & & $x$ & $x$ & $\mathrm{X}$ & $\mathrm{X}$ & & $\begin{array}{l}\text { cystic fibrosis, } \\
\text { blood disorders, } \\
\text { fibromyalgia }\end{array}$ \\
\hline & Saxby, $2018^{5}$ & & & $\mathrm{X}$ & $\mathrm{X}$ & $\mathrm{X}$ & & & \\
\hline & Low, $2019^{4}$ & $\mathrm{X}$ & & $\mathrm{X}$ & $\mathrm{X}$ & $\mathrm{X}$ & & & Rheumatic \\
\hline & Bal, $2015^{3}$ & & & $\mathrm{X}$ & $\mathrm{X}$ & $\mathrm{X}$ & $\mathrm{X}$ & & $\begin{array}{l}\text { cystic fibrosis, } \\
\text { sickle cell disease, } \\
\text { spina bifida }\end{array}$ \\
\hline
\end{tabular}


Evidence Table 3. Intervention characteristics of included systematic reviews addressing direct patient care

\begin{tabular}{|c|c|c|c|}
\hline Type of Strategy & Author, Year & Description of Intervention & Modality \\
\hline $\begin{array}{l}\text { Advanced Care } \\
\text { Planning - Adults } \\
\text { Only }\end{array}$ & Coulter, $2015^{1}$ & $\begin{array}{l}\text { All } 19 \text { studies included components that were intended to support behavior } \\
\text { change among patients, involving either face-to- face support or telephone } \\
\text { support; A variety of tools and techniques were used in the interventions, } \\
\text { including patient information }\end{array}$ & Education/counseling \\
\hline $\begin{array}{l}\text { Health Literacy - } \\
\text { Adults Only }\end{array}$ & Schaepe, $2015^{2}$ & $\begin{array}{l}\text { Most of the } 18 \text { included studies focused on educational intervention programs for } \\
\text { people undergoing peritoneal dialysis. }\end{array}$ & Education/counseling \\
\hline \multirow[t]{2}{*}{$\begin{array}{l}\text { Transitional Care - } \\
\text { Adults Only }\end{array}$} & Sendall, $2016^{125}$ & $\begin{array}{l}\text { Each of the studies used clinical information sharing, community linkages and } \\
\text { supported self-management components of the CCM to create a seamless } \\
\text { transition across services for older people with two or more chronic diseases. }\end{array}$ & Multiple modalities \\
\hline & Berre, $2017^{126}$ & $\begin{array}{l}\text { Educational components (e.g., motivational interview/individualized face-to-face } \\
\text { coaching, brochures, videotape) }(94.6 \%) \text {, and medication management }(55.4 \%) \text {. } \\
\text { multidisciplinary coordination process. Phone contacts }(59.2 \%) \text { or home visits } \\
(59.8 \%) \text {, and provide }\end{array}$ & Nurse/case managers \\
\hline \multirow{10}{*}{$\begin{array}{l}\text { Self Management } \\
\text { Support - Adults } \\
\text { Only }\end{array}$} & Yin, $2019^{90}$ & Mobile health Apps directed education and management of IBD & Mobile health \\
\hline & Kruse, $2015^{89}$ & & Patient portal \\
\hline & Whiteman, $2016^{35}$ & $\begin{array}{l}\text { Automated Telehealth, Health and Recovery Peer program (HARP), Integrated } \\
\text { Illness Management and Recovery, HOPES, All these interventions targeted a } \\
\text { heterogeneous set of serious mental illnesses and medical illnesses that require } \\
\text { ongoing treatment. }\end{array}$ & Nurse/case managers \\
\hline & Nazarov, $2019^{55}$ & $\begin{array}{l}\text { Workplace oriented intervention programs; Cognitive behavioral therapy } \\
\text { interventions; Self-management programs; Vocational rehabilitation programs; } \\
\text { Coaching interventions; Comparative intervention strategies; Interventions that } \\
\text { prevent or slow down chronic }\end{array}$ & Multiple modalities \\
\hline & Zhao, $2016^{57}$ & $\begin{array}{l}\text { Theory of empowerment (Presentation, discussion and Reflection), Extended } \\
\text { health belief model ( Pamphlets and face to face lecture, question and answer), } \\
\text { Diabetes conversation maps program (goal settings, group discussion), Problem- } \\
\text { based learning }\end{array}$ & Nurse/case managers \\
\hline & Teljeur, $2016^{27}$ & $\begin{array}{l}\text { Education with self management training and with computer-assisted self care, } \\
\text { Lifestyle modification program, Printed materials and telephone counselling, } 6-\mathrm{h} \\
\text { structured group education program, Automated uploading of blood, } \\
\text { Telemedicine - blood glucose }\end{array}$ & Nurse/case managers \\
\hline & Hecke, $2016^{28}$ & $\begin{array}{l}\text { Self Management Support Interventions ( lifestyle advice, education for diseases } \\
\text { and symptom management, Problem-solving, goal-setting delivered through face } \\
\text { to face, telephone, video conference) }\end{array}$ & Nurse/case managers \\
\hline & Hooft, $2016^{29}$ & $\begin{array}{l}\text { Educational and counselling components; some involved physical exercises, } \\
\text { Family involvement sessions, interventions were provided in group sessions, } \\
\text { sometimes combined with individual sessions. Mostly in person. }\end{array}$ & Nurse/case managers \\
\hline & Stenberg, $2016^{30}$ & $\begin{array}{l}\text { In person counseling programs such as Diabetes Peer to Peer program, Learning } \\
\text { and mastery/Educational lifestyle courses, self-management programs }\end{array}$ & Peer/lay support' \\
\hline & Plow, $2016^{31}$ & $\begin{array}{l}\text { Interventions = emotion and pain management strategies, physical activity } \\
\text { training, Behavior Change Techniques delivered through One-to-one, in-person } \\
\text { then distant follow up through web and phone. }\end{array}$ & Nurse/case managers \\
\hline
\end{tabular}

\section{B-79}




\begin{tabular}{|c|c|c|c|}
\hline Type of Strategy & Author, Year & Description of Intervention & Modality \\
\hline \multirow[t]{15}{*}{ dits } & Sakakibara, $2016^{34}$ & $\begin{array}{l}\text { Self management interventions, feedback on performance, goal setting/action } \\
\text { planning and problem solving through in person and telephone sessions. both } \\
\text { individual and group formats }\end{array}$ & Nurse/case managers \\
\hline & Massimi, $2017^{68}$ & $\begin{array}{l}\text { Health educational program } \pm \text { LAY (Look After Yourself), "Care Guide" (care plan) } \\
\text { and an Action Plane (patient's self-care plane) delivered face-to-face, face-to- } \\
\text { face/telephone, nurse visits at patients home or nurse specific clinics, CHCs }\end{array}$ & Nurse/case managers \\
\hline & $\begin{array}{l}\text { Bolscher-Niehuis, } \\
2016^{46}\end{array}$ & $\begin{array}{l}\text { Core elements of the self-management support program were: health promotion } \\
\text { and information about the disease, education aimed at knowledge, skills and } \\
\text { strategies to manage the consequences of the disease/disability, coaching of } \\
\text { health behavior change }\end{array}$ & Nurse/case managers \\
\hline & Sokol, $2016^{36}$ & $\begin{array}{l}\text { Regular communication between peer supporter and participant usually in } 2 \\
\text { weeks, feedback, contact monitoring and record keeping. Community } \\
\text { partnerships. Tailored content was also reported }(n=8) \text {, with peer supporters } \\
\text { recognizing participants' needs and }\end{array}$ & Peer/lay support' \\
\hline & Whitehead, $2016^{25}$ & $\begin{array}{l}\text { An app plus feedback or contact with participants either by text or phone } \\
\text { conversation, App based diseases specific education, App based teleconsultation } \\
\text { intervention. }\end{array}$ & Mobile Health \\
\hline & Palacio, $2016^{38}$ & $\begin{array}{l}\text { Motivational Interviewing, counseling, (Face to face, phone calls, both, computer- } \\
\text { based, individual and in group sessions) }\end{array}$ & Nurse/case managers \\
\hline & Debon, $2019^{48}$ & $\begin{array}{l}\text { Reminders for follow up through messages, sending alerts, logs of blood glucose, } \\
\text { eating habits, lifestyle change education. }\end{array}$ & Mobile Health \\
\hline & Eeden, $2016^{39}$ & $\begin{array}{l}\text { Nurse led Educational program. Guided SMI with education and training of skills } \\
\text { provided by a family physician. Internet based SMI including online/group-based } \\
\text { education according to a computerized personal action plan. }\end{array}$ & Nurse/case managers \\
\hline & Kuo, $2016^{33}$ & Secure messaging in electronic health records & Patient Portal \\
\hline & Jacelon, $2016^{40}$ & $\begin{array}{l}\text { SMI: Disease Focused education, Peripherals, Feedbacks, Diary, Provider } \\
\text { access, User education. Provider consultations, Sensor based technology use }\end{array}$ & Multiple technologies \\
\hline & Noonan, $2019^{32}$ & $\begin{array}{l}\text { Behavior change in adults (Digital weighing scale, Medication box. Calibrated } \\
\text { bottle, Diary), Model of health promotion and Bandura's self-efficacy theory } \\
\text { (Computer \& CD ROM Written teaching materials), Dialogue guides, Content of } \\
\text { conversation, Education }\end{array}$ & Nurse/case managers \\
\hline & Warner, $2019^{50}$ & $\begin{array}{l}\text { Arthritis Self-management Program (Goals, Problem solving, Action planning, } \\
\text { Skill Building). Guided Care Goals (Problem solving, Action planning, Education, } \\
\text { Skill building, Education, Monitor). Strategies included Process, Health Behavior } \\
\text { change }\end{array}$ & Nurse/case managers \\
\hline & Changizi, $2017^{61}$ & Call cell phone, text messages, mobile app & Mobile Health \\
\hline & Kelly, $2018^{62}$ & $\begin{array}{l}\text { We specified that interventions should include collaborative interaction be- tween } \\
\text { participants and healthcare providers, involving goal setting and feedback, with at } \\
\text { least two points of contact, and that specific program should include at least two } \\
\text { of }\end{array}$ & Education/Counseling \\
\hline & Levengood, $2019^{52}$ & Team based care - patient education and counseling, either in person or remote & Team based care \\
\hline
\end{tabular}




\begin{tabular}{|c|c|c|c|}
\hline \multirow{14}{*}{ Iype or strategy } & Author, Year & Description of Intervention & Modality \\
\hline & Jeddi, $2017^{63}$ & $\begin{array}{l}\text { Interventions were delivered via smartphones/personal digital assistants (PDAs) } \\
\text { (three studies), wearable devices (three studies), computerized systems (one } \\
\text { study), and multiple component (one study). }\end{array}$ & Mobile Health \\
\hline & Kim, $2015^{26}$ & $\begin{array}{l}\text { Automated functions in } 16 \text { interventions. Supplementary modes were used in } 16 \\
\text { interventions. The most widely used function in the automated functions category } \\
\text { was "the use of an enriched information environment" ( } n=17) \text {, and the most } \\
\text { widely used function in }\end{array}$ & Other technology \\
\hline & Alessa, $2018^{47}$ & $\begin{array}{l}\text { The intervention was a mobile phone or a tablet app that collects data, provides } \\
\text { feedback, connects with HCPs or informs about hypertension to support the self- } \\
\text { management tasks of hypertension }\end{array}$ & Mobile Health \\
\hline & Smith, $2017^{41}$ & $\begin{array}{l}\text { At organizational level (Case management or coordination of care, } \\
\text { Reorganization of care/team working, Nurse training ), At individual level ( } \\
\text { Individual management plans Support for self management, Personalized goals } \\
\text { and participant workbooks, Individual }\end{array}$ & Nurse/case managers \\
\hline & Zomahoun, $2016^{37}$ & $\begin{array}{l}\text { Motivational Interviewing (Face to face, phone calls, both, computer-based, } \\
\text { individual and in group sessions) }\end{array}$ & Nurse/case managers \\
\hline & $\begin{array}{l}\text { Skrabal Ross, } \\
2018^{58}\end{array}$ & $\begin{array}{l}\text { mobile phone-delivered interventions - SMS text messages and mobile apps; } \\
\text { Despite the variety of adherence-enhancing strategies in the interventions, } 2 \\
\text { strategies were common to most studies: drug-related symptom management } \\
\text { advice and reporting and medical }\end{array}$ & Mobile Health \\
\hline & Sangrar, $2019^{56}$ & $\begin{array}{l}\text { education programs that combined face-to-face and online/ computer-based } \\
\text { strategies delivered or mediated by healthcare professionals }\end{array}$ & Multiple modalities \\
\hline & Luedke, $2019^{49}$ & $\begin{array}{l}\text { Educational ( sign/symptom monitoring; enhancing problem-solving, Medications } \\
\text { management and decision-making skills) and psychosocial therapy interventions } \\
\text { (eg, cognitive behavioral therapy, problem solving therapy, progressive muscle } \\
\text { relaxation) }\end{array}$ & Nurse/case managers \\
\hline & Kim, $2017^{65}$ & $\begin{array}{l}\text { self-monitoring, automated feedback, patient education and decision making } \\
\text { using smartphone Apps }\end{array}$ & Mobile Health \\
\hline & Lederle, $2019^{54}$ & $\begin{array}{l}\text { Structured program for individuals with chronic diseases administered by trained } \\
\text { affected persons who are helping patients to improve their own health }\end{array}$ & Peer/lay support' \\
\hline & Dounavi, $2019^{69}$ & $\begin{array}{l}\text { mHealth apps - Main app components were the provision of health-related } \\
\text { information, feedback, reminders, peer support groups, goal setting, food and } \\
\text { physical activity logging, weight self-monitoring, digital coaching, and exceptional } \\
\text { blood pressure tagging }\end{array}$ & Mobile Health \\
\hline & Conway, $2017^{64}$ & $\begin{array}{l}\text { Strategies used to improve medication adherence included four primary } \\
\text { approaches: IVR (with or without human interaction), SMS text messaging, } \\
\text { telemonitoring and/or tailored care management, and Web-based software }\end{array}$ & Multiple technologies \\
\hline & Cho, $2017^{66}$ & $\begin{array}{l}\text { eHealth (computer-based system, Internet-based program, and Health } \\
\text { Information Exchange), mHealth (Apps), Telehealth (phone calls and } \\
\text { telemedicine), Web-based decision support system + telephone-based } \\
\text { monitoring }\end{array}$ & Multiple technologies \\
\hline
\end{tabular}




\begin{tabular}{|c|c|c|c|}
\hline Type of Strategy & Author, Year & Description of Intervention & Modality \\
\hline \multirow[t]{16}{*}{ sing } & Clarkesmith, $2017^{67}$ & $\begin{array}{l}\text { Self Monitoring and education ( one to one, group training sessions, Video } \\
\text { component, educational booklet), decision support aid employed pictograms and } \\
\text { computerized version }\end{array}$ & Nurse/case managers \\
\hline & Ko, $2018^{60}$ & $\begin{array}{l}\text { Collaborative care, psychiatric illness self-management, nurse led self } \\
\text { management sessions, communications with physicians }\end{array}$ & Nurse/case managers \\
\hline & Han, $2019^{51}$ & $\begin{array}{l}\text { Individual and group education sessions with some studies including } \\
\text { telephone/electronic follow ups }\end{array}$ & Education/Counseling \\
\hline & Kelly, $2018^{83}$ & Patient portals for hospitalized patients & Patient Portal \\
\hline & Hamine, $2015^{85}$ & $\begin{array}{l}\text { mHealth included any mobile device or service, such as mobile phones, SMS, } \\
\text { smartphones, personal digital assistants, and devices that work on wireless } \\
\text { technology or Bluetooth-compatible devices }\end{array}$ & Mobile Health \\
\hline & Hammer, $2015^{42}$ & $\begin{array}{l}\text { There were various types of educational and/or counseling sessions that guided } \\
\text { patients toward self-assessment and management of cancer/treatment-related } \\
\text { challenges. The major commonality of these programs included cognitive- } \\
\text { behavioral type therapies of }\end{array}$ & Education/Counseling \\
\hline & Zhao, $2015^{53}$ & $\begin{array}{l}\text { Internet-based management (electronic diary, treatment decision support, } \\
\text { monitoring support by a study nurse) plus physician office visits, SMS monitoring } \\
\text { to assist managing asthma control, Interactive voice response, Internet-based } \\
\text { self management program }\end{array}$ & Multiple technologies \\
\hline & Almutairi, $2019^{82}$ & $\begin{array}{l}\text { Interventions based on patient activation concept which focuses on patient } \\
\text { knowledge, skills and confidence building. Includes Motivational interviewing, } \\
\text { Patient empowerment, patient-centered tailored care, PRECEDE- PROCEED } \\
\text { model, health action process app }\end{array}$ & Nurse/case managers \\
\hline & Dendere, $2019^{81}$ & Patient portals tethered to an electronic medical record in inpatient settings & Patient Portal \\
\hline & Wildevuur, $2015^{86}$ & $\begin{array}{l}\text { Initially defined PCC-ICT as a category of Internet technology that connects } \\
\text { patients to health care professionals and enables them to interact and exchange } \\
\text { information, including multimedia data such as audio (voice), video, and images. }\end{array}$ & Multiple technologies \\
\hline & Boulley, $2018^{74}$ & $\begin{array}{l}\text { live video conferencing, online interactive workshops, websites including social } \\
\text { networks for synchronous or asynchronous communication, text messages, } \\
\text { automated voice response, PA tracking devices or Wi-Fi weighing scales } \\
\text { associated with an app. Follow-up }\end{array}$ & Multiple technologies \\
\hline & $\begin{array}{l}\text { Ammenwerth, } \\
2019^{72}\end{array}$ & Patient portals with electronic health record access. & Patient Portal \\
\hline & Aquino, $2017^{71}$ & $\begin{array}{l}\text { individual consultations, phone calls, sessions via a website and use of a booklet, } \\
\text { daily sessions through a tablet and also shared their blood glucose data and } \\
\text { received feedback through individual messages via tablet }\end{array}$ & Nurse/case managers \\
\hline & Donald, $2018^{73}$ & $\begin{array}{l}\text { intervention were face to face (80\%), telephone, email, print version (64\%), } \\
\text { Electronic i-e website, mobile applications (16\%) }\end{array}$ & Multiple modalities \\
\hline & Lee, $2018^{77}$ & $\begin{array}{l}\text { real-time or regular basis symptom assessments, monitoring of disease, pre- } \\
\text { programed reminders, or feedbacks tailored specifically to the data provided by } \\
\text { participants via mHealth devices or mobile apps. Training of mHealth systems } \\
\text { was provided to participants }\end{array}$ & Mobile Health \\
\hline & Risling, $2017^{84}$ & Tethered patient portals providing access to electronic health records & Patient Portal \\
\hline
\end{tabular}

\section{B-82}




\begin{tabular}{|c|c|c|c|}
\hline Type of Strategy & Author, Year & Description of Intervention & Modality \\
\hline \multirow[t]{12}{*}{ 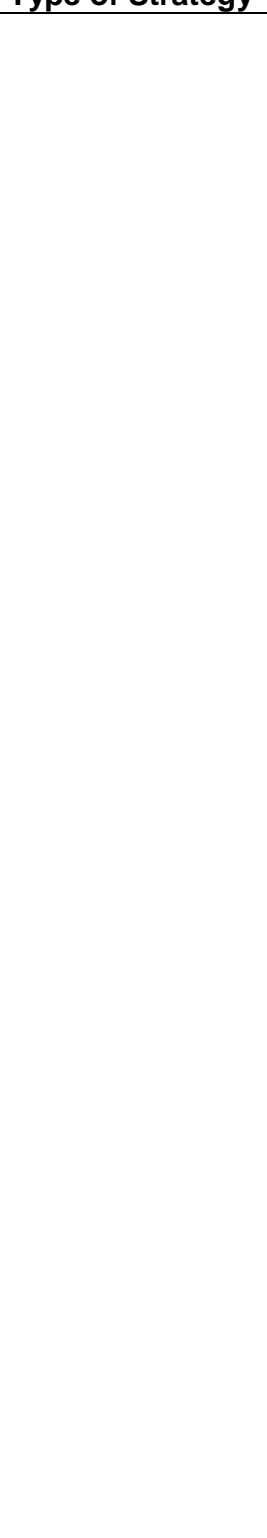 } & Palacios, $2017^{79}$ & $\begin{array}{l}\text { 1. Web site and online forums plus tailored intervention Vs Generic version of the } \\
\text { Web site and an online forum. } 2 \text {. Internet-delivered cognitive behavior therapy Vs } \\
\text { Internet-delivered attention control health information package. 3. text messages }\end{array}$ & Multiple technologies \\
\hline & Pereira, $2015^{88}$ & $\begin{array}{l}\text { Internet education Vs face-to-face diabetes education comparison, online DSME } \\
\text { program, Diabetes coach Web program (interactive, personalized profile, } \\
\text { nurse/patient e-mail), interactive Web program for tracking food intake and } \\
\text { glucose levels; e-mail feature }\end{array}$ & Telehealth \\
\hline & Warrington, $2019^{78}$ & $\begin{array}{l}\text { Main Interventions: } 1 \text {. communication with HCPs, 2. Disease monitoring ( HCPs } \\
\text { to remotely access and monitor patient reported data, give feedback) 3. Symptom } \\
\text { management (provide tailored advice for symptom or side effect management) }\end{array}$ & Multiple technologies \\
\hline & Deek, $2016^{45}$ & $\begin{array}{l}\text { All studies involved face to face educational sessions with the patients and their } \\
\text { caregivers. However, the type of sessions and the follow-up varied across the } \\
\text { studies. Three categories of interventions were identified: self-care strategies, } \\
\text { coping ski }\end{array}$ & Education/Counseling \\
\hline & Laukner, $2016^{76}$ & $\begin{array}{l}\text { Four programs using telecommunications only (including one or more of the } \\
\text { following: websites, discussion boards, emails, telephone and/or telehealth), four } \\
\text { using in-person meetings only and two combining telecommunication and in- } \\
\text { person; Peer leaders in }\end{array}$ & Peer/lay support' \\
\hline & Price, $2015^{70}$ & $\begin{array}{l}\text { Range of PHR functions: Access medical record; record personal health data, } \\
\text { communicate with support group, communicate with providers, manage care } \\
\text { plan. }\end{array}$ & Patient portal \\
\hline & Kim, $2016^{43}$ & $\begin{array}{l}\text { The roles assumed by CBHWs included health education }(n=48) \text {, counseling }(n \\
=36) \text {, navigation assistance }(n=21) \text {, case management }(n=4) \text {, social services } \\
(n=7) \text {, and social support }(n=18) \text {. }\end{array}$ & $\begin{array}{l}\text { Community health } \\
\text { worker/patient navigator }\end{array}$ \\
\hline & Ha Dinh, $2016^{59}$ & $\begin{array}{l}\text { There was little consistency among studies in relation to delivery method, } \\
\text { duration, educational components and persons who conducted the health } \\
\text { education programs; Interventions involved educational content delivered with the } \\
\text { teach- back method in one-on }\end{array}$ & Education/Counseling \\
\hline & Thakkar, $2016^{44}$ & $\begin{array}{l}\text { Text messaging systems - Fifteen studies sent text messages at a fixed } \\
\text { predetermined frequency; One study used real-time medication monitoring in } \\
\text { which patients were sent a text message reminder only if the participant failed to } \\
\text { open the medication dispenser }\end{array}$ & Mobile Health \\
\hline & Jones, $2015^{87}$ & $\begin{array}{l}\text { All the interventions included at least some element of face-to- face delivery; } \\
\text { however, two studies delivered the majority of their intervention remotely via } \\
\text { telephone; The three studies that utilized only face-to-face delivery all did this via } \\
\text { group session }\end{array}$ & Education/Counseling \\
\hline & $\begin{array}{l}\text { Peytremann- } \\
\text { Bridevaux, 201575 }\end{array}$ & $\begin{array}{l}\text { we considered the following five criteria for our operational definition of CDM: } 1 . \\
\text { at least one organizational component (i.e., elements that interfere with the care } \\
\text { process or that aim to improve continuity of care) targeting patients (Steuten } \\
2007 a \text {; }\end{array}$ & multiple modalities \\
\hline & Warner, $2015^{80}$ & $\begin{array}{l}\text { goal setting, follow-up, and an individualized approach using structured } \\
\text { information and professional support }\end{array}$ & Nurse/case managers \\
\hline
\end{tabular}




\begin{tabular}{|c|c|c|c|}
\hline Type of Strategy & Author, Year & Description of Intervention & Modality \\
\hline \multirow[t]{12}{*}{$\begin{array}{l}\text { Self Management } \\
\text { Support - Adults } \\
\text { and Children }\end{array}$} & Lancaster, $2018^{17}$ & $\begin{array}{l}\text { eHealth tool was considered to be any internet-based intervention, including } \\
\text { mobile health apps, used by patients for clinical purposes that focused on } \\
\text { improving patient health and clinical outcomes; patients and/or caregivers can } \\
\text { directly enter or edit h }\end{array}$ & Multiple technologies \\
\hline & Barello, $2016^{14}$ & $\begin{array}{l}\text { Heterogeneous group - Web based portals, some monitoring capabilities, } \\
\text { communication tools, counseling/therapy, patient specific information - some self } \\
\text { reported }\end{array}$ & Multiple technologies \\
\hline & Kruse, $2015^{24}$ & Patient portals for the management of chronic disease & Patient Portal \\
\hline & Yin, $2019^{20}$ & $\begin{array}{l}\text { Patient-facing technologies: Acceleration sensors, Earphones and wearable } \\
\text { computer, Wireless BP monitor, Mobile App, Environmental sensors, Exhaled air } \\
\text { sensor }\end{array}$ & Multiple technologies \\
\hline & Pamungkas, $2017^{23}$ & $\begin{array}{l}\text { Individual or group sessions including personalized counseling, goal-setting, } \\
\text { problem solving, and explanation of ways in which family members can support } \\
\text { self-care practice and follow-up sessions (computer-based, phone call, short } \\
\text { message service (mail) }\end{array}$ & multiple modalities \\
\hline & Chi, $2015^{21}$ & $\begin{array}{l}\text { There were six main categories of interventions delivered via technologies: } \\
\text { education }(37 \%) \text {, consultation (including decision support aid) (37\%), } \\
\text { psychosocial/cognitive behavioral therapy (including problem solving training) } \\
(35 \%) \text {, social support ( } 23 \%) \text {, }\end{array}$ & Multiple technologies \\
\hline & Niznik, $2018^{13}$ & $\begin{array}{l}\text { Clinical pharmacy telemedicine interventions in the outpatient or ambulatory } \\
\text { setting; telephone }(n=25) \text {, video consultation }(n=4) \text {, text or electronic } \\
\text { messaging }(n=3) \text {, e-mail }(n=2) \text {, automated electronic reports }(n=1) \text {, and fax }(n \\
=1) \text {. }\end{array}$ & Telehealth \\
\hline & Kew, $2016^{18}$ & $\begin{array}{l}\text { We included studies comparing home telemonitoring of asthma between clinic } \\
\text { visits, using any form of technology (e.g. telephone calls, emails, text messages, } \\
\text { online software), with a form of monitoring that does not include ongoing remote } \\
\text { professional fee }\end{array}$ & Telehealth \\
\hline & Lycett, $2018^{16}$ & $\begin{array}{l}\text { Interventions were classified as fully digital or partly digital (digital and non digital } \\
\text { components). Data were extracted on the type of digital platform (eg, SMS, smart } \\
\text { device app) and the type of non digital component (eg, telephone call, paper- } \\
\text { based). }\end{array}$ & Multiple modalities \\
\hline & Hill, $2015^{22}$ & $\begin{array}{l}\text { Motivational interviewing, Cognitive behavioral therapy and social cognitive } \\
\text { theory based approaches. Included behavior change techniques were Goal } \\
\text { settings, action planning, barrier identification/problem solving, prompt self- } \\
\text { monitoring of behavior }\end{array}$ & Nurse/case managers \\
\hline & Bashi, $2018^{15}$ & $\begin{array}{l}\text { Text and Video clips in smartphone App delivered educational material about } \\
\text { causes of diseases, monitoring signs and symptoms, exercise instructions, diet } \\
\text { recommendations, and coping strategies. }\end{array}$ & Mobile Health \\
\hline & Graham, $2016^{19}$ & Acceptance and commitment therapy - either individual or group & Education/Counseling \\
\hline $\begin{array}{l}\text { Self Management } \\
\text { Support - Children } \\
\text { Only }\end{array}$ & Knafl, $2017^{6}$ & $\begin{array}{l}\text { intervention had to be psychologically, socially, or behaviorally oriented and } \\
\text { include participation by the child's parent(s) or primary caregiver(s) (hereafter } \\
\text { referred to as parents); Interventions were predominantly psychoeducational ( } \mathrm{n}= \\
55 ; 79 \%) \text {, wit }\end{array}$ & Education/Counseling \\
\hline
\end{tabular}




\begin{tabular}{|c|c|c|c|}
\hline Type of Strategy & Author, Year & Description of Intervention & Modality \\
\hline \multirow[t]{9}{*}{ sits } & Sattoe, $2015^{10}$ & $\begin{array}{l}\text { Medical management, role management (communicating, decision-making, } \\
\text { assertiveness, and keeping up with peers), emotional management (building self- } \\
\text { confidence, developing a positive body image, self- appreciation, maintaining } \\
\text { positive thinking, stress man }\end{array}$ & Education/Counseling \\
\hline & Low, $2019^{4}$ & $\begin{array}{l}\text { Static text to audiovisual materials, games using mHealth Apps and Web-based } \\
\text { technology }\end{array}$ & Multiple technologies \\
\hline & Hamline, $2018^{11}$ & $\begin{array}{l}\text { (1) interventions involving communication between the inpatient and outpatient } \\
\text { health care providers (provider communication interventions [PCIs]), (2) } \\
\text { interventions involving care coordination (CCls, defined as those that involved } \\
\text { intentional organization }\end{array}$ & multiple modalities \\
\hline & Clemente, $2016^{12}$ & $\begin{array}{l}\text { All transitional care programs had a written transition policy and protocol agreed } \\
\text { by the pediatric and adult teams that described in detail the transition process. } \\
\text { Two components of these programs were of particular importance: (1) } \\
\text { information and e }\end{array}$ & Education/Counseling \\
\hline & Bal, $2015^{3}$ & $\begin{array}{l}\text { medical management (61.9\%), e.g. self- monitoring of blood glucose values in } \\
\text { diabetes, accessing healthcare, but also child-parent sharing or teamwork related } \\
\text { to medical management tasks. Interventions included education ( } 88.1 \%) \text {, peer- } \\
\text { support ( } 23.8 \%), \text { pro }\end{array}$ & multiple modalities \\
\hline & Campbell, $2016^{7}$ & $\begin{array}{l}\text { The four studies explored different types of interventions: transition- preparation } \\
\text { training (TPT) delivered in a two-day workshop for adolescents with spina bifida; } \\
\text { a web- and SMS-based educational intervention for adolescents with a range of } \\
\text { different c }\end{array}$ & multiple modalities \\
\hline & Charlier, $2015^{9}$ & $\begin{array}{l}\text { Within the game intervention group, patients played a video game with } \\
\text { educational content aiming at knowledge improvement or promotion of self- } \\
\text { management behaviors. Games were software packages that run on a personal } \\
\text { computer, console, or mobile phone. }\end{array}$ & Other technology \\
\hline & Saxby, $2018^{5}$ & $\begin{array}{l}\text { Education interventions grouped in to the following: Directive learning (i.e., } \\
\text { involves the use of a structured evidence- based curriculum to teach skills and } \\
\text { knowledge to children); Active and experiential learning }\end{array}$ & Education/Counseling \\
\hline & Majeed-Ariss, $2015^{8}$ & $\begin{array}{l}\text { The commonality among all apps studied was that they aimed to support the } \\
\text { adolescent in the medical management of their physical condition. Cafazzo et al's } \\
\text { [55] and Frøisland et al's [56] apps for type } 1 \text { diabetes management focused on } \\
\text { increasing the number }\end{array}$ & Mobile Health \\
\hline \multirow[t]{2}{*}{$\begin{array}{l}\text { Shared Decision } \\
\text { Making - Adult Only }\end{array}$} & Baik, $2018^{108}$ & $\begin{array}{l}\text { Technology-enabled delivery modes (e.g., video, DVD, web-based tool), print } \\
\text { materials, palliative care consultation and structured meeting between patients/ } \\
\text { caregivers and inter professional care team. Other were Goals of Care video } \\
\text { decision aid, booklet a }\end{array}$ & Nurse/case managers \\
\hline & $\begin{array}{l}\text { Martínez-González, } \\
2018^{123}\end{array}$ & $\begin{array}{l}\text { Face-to-face, by telephone, consultations, questionnaires, paper based material } \\
\text { and web-based format. Elements of interventions were weighing up benefits and } \\
\text { harms", "risks", "pros and cons of options", "patients' values", "preferences" }\end{array}$ & Multiple modalities \\
\hline
\end{tabular}




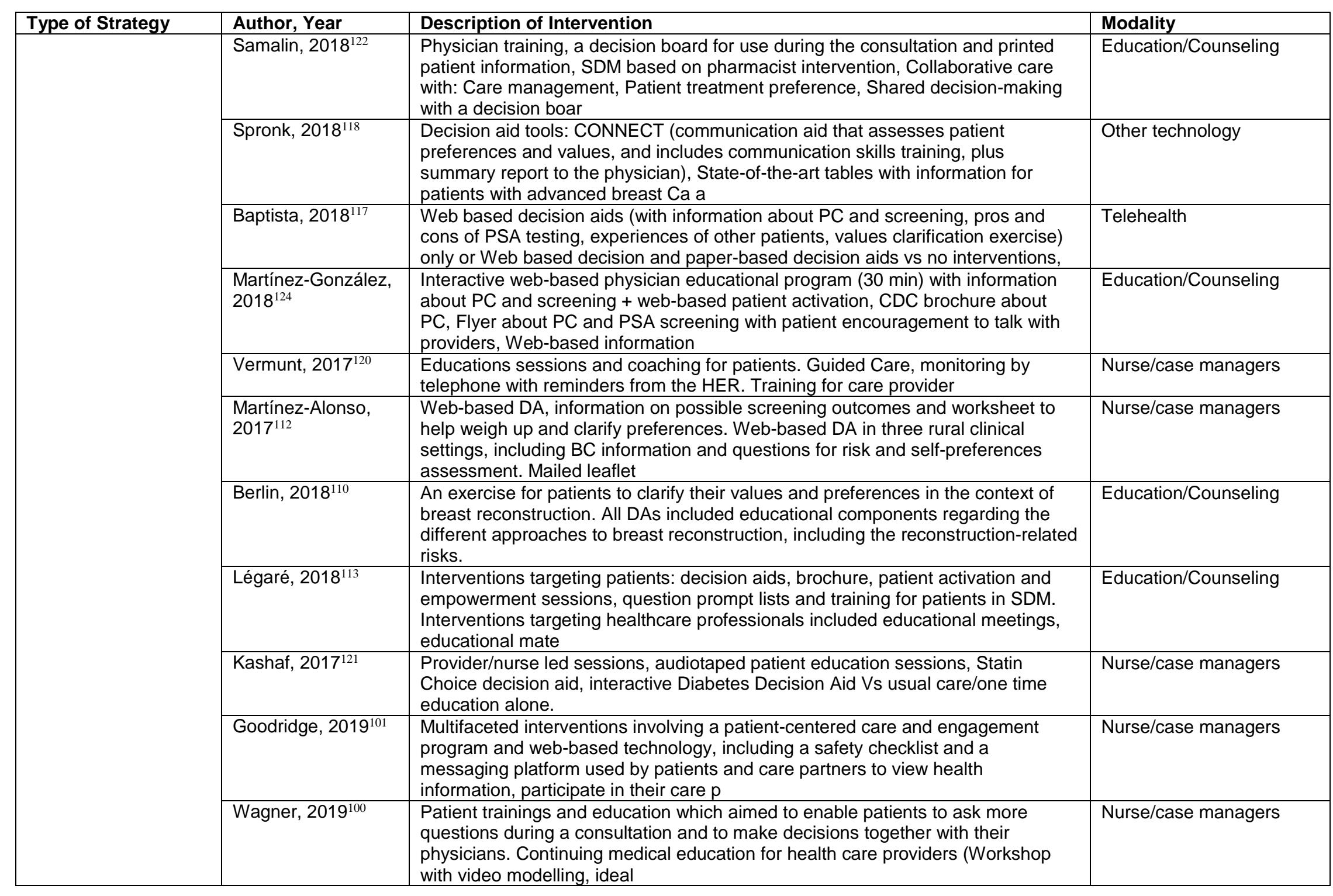




\begin{tabular}{|c|c|c|c|}
\hline Type of Strategy & \multirow[b]{2}{*}{ Johnson, $2018^{111}$} & \multirow[b]{2}{*}{$\begin{array}{l}\text { Description of Intervention } \\
\text { Intervention components included training interventions for clinicians, coaching } \\
\text { for patients, decision aids and written materials for patients. shared decision- } \\
\text { making training program for general practitioners. Previsit coaching, by } \\
\text { community health wo }\end{array}$} & \multirow{2}{*}{$\begin{array}{l}\text { Modality } \\
\text { Education/Counseling }\end{array}$} \\
\hline & & & \\
\hline & Kashaf, $2015^{106}$ & $\begin{array}{l}\text { Decision aid (booklet and audiotape/disc facilitating SDM), Perceived treatment } \\
\text { choice, }\end{array}$ & Education/Counseling \\
\hline & Violette, $2015^{103}$ & $\begin{array}{l}\text { Audio recording of clinical encounter, Written information printout, CCE } \\
\text { information booklet, Communication strategy intervention via DVD and } 4 \\
\text { telephone calls, Tailored internet aid }\end{array}$ & Nurse/case managers \\
\hline & Irizarry, $2015^{102}$ & $\begin{array}{l}\text { Patient experience and/or ways that patients may be supported to make } \\
\text { competent health care decisions and act on those decisions using patient portal } \\
\text { functionality. }\end{array}$ & Patient Portal \\
\hline & McIntyre, $2015^{105}$ & $\begin{array}{l}\text { computer-based learning systems (CBLSs) providing patient education about } \\
\text { decision making, treatment options. A Web-based communication aid for } \\
\text { patients with cancer, The impact of an empowering Internet-based Breast Cancer } \\
\text { Patient Pathway program. }\end{array}$ & Other technology \\
\hline & Friedrichs, $2016^{107}$ & $\begin{array}{l}\text { Physician led counseling and SDM sessions with paper prompts; computerized } \\
\text { tailored decisional support tool; }\end{array}$ & Education/Counseling \\
\hline & van Weert, $2016^{109}$ & $\begin{array}{l}\text { Decision aids were defined as "interventions designed to help people make } \\
\text { specific and deliberative choices among options (including the status quo) by } \\
\text { making the decision explicit and by providing (at the minimum) information on the } \\
\text { options and outcomes }\end{array}$ & Education/Counseling \\
\hline & Nathan, $2016^{99}$ & $\begin{array}{l}\text { Many of the trials attempted to test their SDM intervention through multiple } \\
\text { modalities (Table 3). Eight studies utilized more than one method to deliver their } \\
\text { intervention (e.g., print and phone counseling), and the remaining ten studies } \\
\text { used one method }\end{array}$ & Multiple modalities \\
\hline & Stovell, $2016^{114}$ & $\begin{array}{l}\text { Interventions designed to support shared decision-making in relation to current } \\
\text { and future treatment. Paper-based or web-based decision or communication } \\
\text { aids. one evaluated a group intervention;30 another evaluated the effects of } \\
\text { training clinicians in a }\end{array}$ & Multiple modalities \\
\hline & Porter, $2016^{115}$ & $\begin{array}{l}\text { multi-component diabetes management strategy- dietary data was recorded in } \\
\text { addition to a range of other medical information (e.g., blood glucose levels, } \\
\text { medications, physical activity); Interventions were delivered via a mobile phone } \\
\text { applications }\end{array}$ & Multiple technologies \\
\hline & Stacey D, $2017^{119}$ & $\begin{array}{l}\text { Patient decision aids that included information about the options and outcomes } \\
\text { and provided at least implicit clarification of values. Guidance and coaching for } \\
\text { patients and training for physicians as well. }\end{array}$ & Nurse/case managers \\
\hline & Morrell, 2016 116 & $\begin{array}{l}\text { Decision aids in form of Self-paced audio and video, Self-guided and interactive } \\
\text { computerized and surrogate involvement such as clinician, staff, parents, family } \\
\text { members (Only for patients with dementia or without decision-making capacity). } \\
\text { Other: patient }\end{array}$ & Nurse/case managers \\
\hline
\end{tabular}




\begin{tabular}{|c|c|c|c|}
\hline Type of Strategy & Author, Year & Description of Intervention & Modality \\
\hline & Jain, $2015^{104}$ & $\begin{array}{l}\text { video decision aids to assist with ACP e.g: a narrative description of CPR, and } \\
\text { likelihood of its success in patients with advanced cancer or Verbal description by } \\
\text { research staff followed by video. }\end{array}$ & Other technology \\
\hline \multirow{4}{*}{$\begin{array}{l}\text { Shared Decision } \\
\text { Making - Adults and } \\
\text { Children }\end{array}$} & Clayman, $2015^{97}$ & $\begin{array}{l}\text { physician training and decision aid use for patients. Patient physician treatment } \\
\text { discussions and SDM. }\end{array}$ & Education/Counseling \\
\hline & Kew, $2017^{96}$ & $\begin{array}{l}\text { SDM intervention for patients and HCPS. SDM seminars for HCPS. SDM portal } \\
\text { and SDM online tool for patients/parents and HCPs. Two pediatric studies } \\
\text { involved use of an online portal, followed by face-to-face consultations. clinical } \\
\text { decision-making intervention }\end{array}$ & Education/Counseling \\
\hline & Winston, $2017^{98}$ & Video based DAs & Other technology \\
\hline & Voruganti, $2017^{95}$ & $\begin{array}{l}\text { Of the } 47 \text { tools identified, the majority }(74.5 \%, 35 / 47) \text { were Internet-enabled } \\
\text { applications accessible from a Web browser, whereas } 9(19 \%, 9 / 47) \text { were native } \\
\text { applications developed as computer software or for use on a mobile phone. }\end{array}$ & Multiple technologies \\
\hline \multirow{4}{*}{$\begin{array}{l}\text { Shared Decision } \\
\text { Making - Children } \\
\text { Only }\end{array}$} & Wyatt, $2015^{94}$ & Online, web-based; in person sessions; paper formats & multiple modalities \\
\hline & Malone, $2019^{91}$ & & Education/Counseling \\
\hline & Cheng, $2017^{92}$ & $\begin{array}{l}\text { therapeutic techniques, decision aids, psychoeducational information, action } \\
\text { planning or goal setting, discussion prompts, and mobilizing patients to engage }\end{array}$ & multiple modalities \\
\hline & Coyne, $2016^{93}$ & $\begin{array}{l}\text { Interventions that promote SDM between children with cancer and parents and } \\
\text { healthcare professionals }\end{array}$ & Education/Counseling \\
\hline
\end{tabular}


Evidence Table 4. Outcomes of included systematic reviews addressing direct patient care

\begin{tabular}{|c|c|c|c|c|c|c|c|c|c|c|c|c|c|c|c|c|}
\hline Type of Strategy & Author, Year & 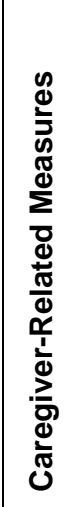 & 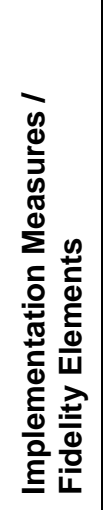 & 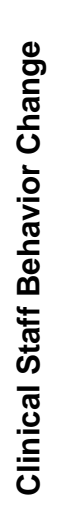 & $\begin{array}{l}\frac{0}{\frac{0}{\pi}} \\
\frac{\pi}{\pi} \\
\tilde{N} \\
0\end{array}$ & $\begin{array}{l}\frac{0}{0} \\
\frac{0}{0} \\
\frac{0}{0} \\
\frac{1}{0} \\
\frac{0}{0} \\
\frac{0}{0} \\
0 \\
\frac{0}{0} \\
. \frac{\pi}{0} \\
\frac{0}{0}\end{array}$ & 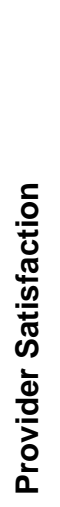 & 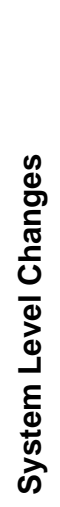 & 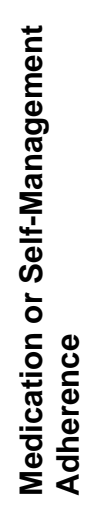 & 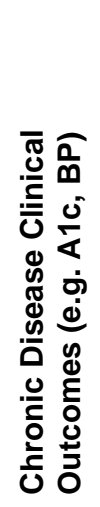 & 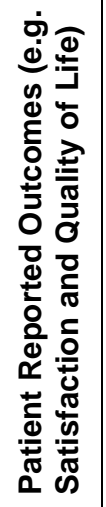 & 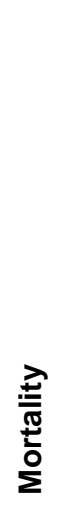 & 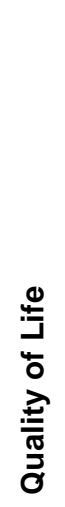 & 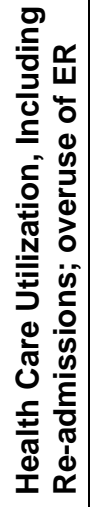 & 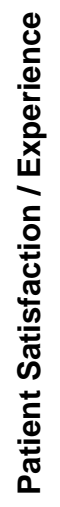 & 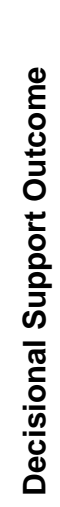 \\
\hline Advanced Care Planning - Adults Only & Coulter, $2015^{1}$ & & & & & & & & $\mathrm{X}$ & $\mathrm{X}$ & & & & $\mathrm{X}$ & & \\
\hline Health Literacy - Adults Only & Schaepe, $2015^{2}$ & & & & & & & & & $\mathrm{X}$ & & & $\mathrm{X}$ & & $\mathrm{X}$ & \\
\hline \multirow[t]{25}{*}{ Self Management Support - Adults Only } & Kruse, $2015^{89}$ & & & & & & & & & $\mathrm{X}$ & & & & & $\mathrm{X}$ & \\
\hline & \begin{tabular}{|l|} 
Yin, $2019^{90}$ \\
\end{tabular} & $\mathrm{X}$ & & & & & & & $\mathrm{X}$ & $\mathrm{X}$ & & & $\mathrm{X}$ & & $\mathrm{X}$ & \\
\hline & Debon, $2019^{48}$ & & & & & & & & $\mathrm{X}$ & $\mathrm{X}$ & & & $\mathrm{X}$ & & & \\
\hline & Luedke, $2019^{49}$ & & & & & & & & $\mathrm{X}$ & $\mathrm{X}$ & & & $\mathrm{X}$ & $\mathrm{X}$ & & \\
\hline & \begin{tabular}{|l|} 
Warner, $2019^{50}$ \\
\end{tabular} & & & & & & & & $\mathrm{X}$ & $\mathrm{X}$ & & & $\mathrm{X}$ & & & \\
\hline & Han, $2019^{51}$ & & & & $\mathrm{x}$ & & & & $\mathrm{X}$ & $\mathrm{X}$ & $\mathrm{X}$ & & & & & \\
\hline & Levengood, $2019^{52}$ & & & & & & & & & $\mathrm{X}$ & $\mathrm{X}$ & $\mathrm{X}$ & & $\mathrm{X}$ & & \\
\hline & Lederle, $2019^{54}$ & & & & & & & & & & & & & $\mathrm{X}$ & & \\
\hline & Nazarov, $2019^{55}$ & & & & & & & & & & & & $X$ & & & \\
\hline & \begin{tabular}{|l} 
Sangrar, $2019^{56}$ \\
\end{tabular} & & & & & & & & $\mathrm{X}$ & $\mathrm{X}$ & & & $\mathrm{X}$ & & & \\
\hline & \begin{tabular}{|l} 
Dounavi, $2019^{69}$ \\
\end{tabular} & & & & & & & & $\mathrm{X}$ & $\mathrm{X}$ & & & & & $\mathrm{x}$ & \\
\hline & Skrabal Ross, $2018^{58}$ & & $\mathrm{X}$ & & & & & & $\mathrm{X}$ & & & & & & & \\
\hline & Alessa, $2018^{47}$ & & & & & & & & & $\mathrm{X}$ & & & & & $\mathrm{X}$ & \\
\hline & \begin{tabular}{|l} 
Ko, $2018^{60}$ \\
\end{tabular} & & & & & & & & $\mathrm{X}$ & $\mathrm{X}$ & & & & $\mathrm{X}$ & & \\
\hline & Changizi, $2017^{61}$ & & & & & & & & $\mathrm{X}$ & $\mathrm{X}$ & & & & & & \\
\hline & Kelly, $2018^{62}$ & & & & & & & & & $\mathrm{X}$ & & & $\mathrm{X}$ & & & \\
\hline & Jeddi, $2017^{63}$ & & & & & & & & $\mathrm{X}$ & $\mathrm{X}$ & & & & & & \\
\hline & Conway, $2017^{64}$ & & & & & & & & $\mathrm{X}$ & & & & & & & \\
\hline & Kim, $2017^{65}$ & & & & & & & & $\mathrm{X}$ & $\mathrm{X}$ & & & & & & $\mathrm{X}$ \\
\hline & Cho, $2017^{66}$ & & & & & & & & & & & & $\mathrm{X}$ & & & \\
\hline & Clarkesmith, $2017^{67}$ & & & & & & & & $\mathrm{X}$ & $\mathrm{X}$ & & & & & $X$ & $\mathrm{X}$ \\
\hline & Massimi, $2017^{68}$ & & & & & & & & $\mathrm{X}$ & $X$ & & & $X$ & & & \\
\hline & Zomahoun, $2016^{37}$ & & & & & & & & $x$ & & & & & & & \\
\hline & Whiteman, $2016^{35}$ & & & & & & & & $\mathrm{X}$ & $\mathrm{X}$ & & & & $x$ & & \\
\hline & Zhao, $2016^{57}$ & & & & & & & & $\mathrm{X}$ & $\mathrm{X}$ & & & $\mathrm{X}$ & & & \\
\hline
\end{tabular}




\begin{tabular}{|c|c|c|c|c|c|c|c|c|c|c|c|c|c|c|c|c|}
\hline \multirow[t]{27}{*}{ Type of Strategy } & Author, Year & 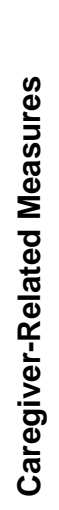 & 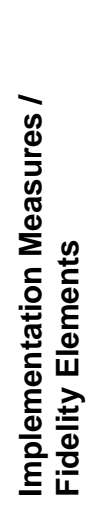 & 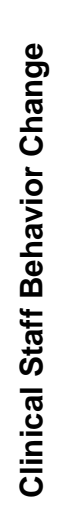 & 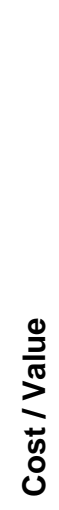 & 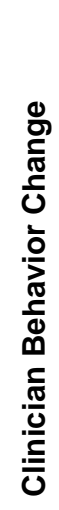 & 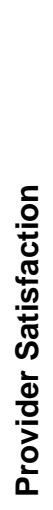 & 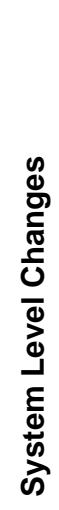 & 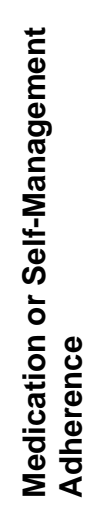 & 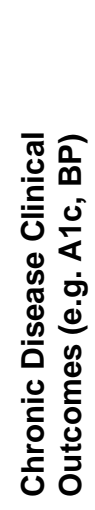 & 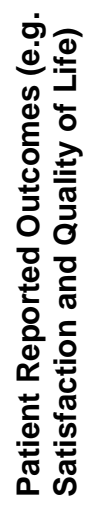 & $\begin{array}{l}\stackrel{\lambda}{\frac{\lambda}{\sigma}} \\
\frac{ \pm}{0} \\
\sum\end{array}$ & 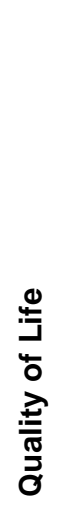 & 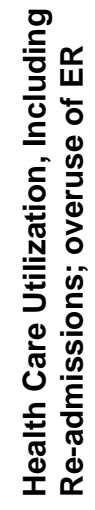 & 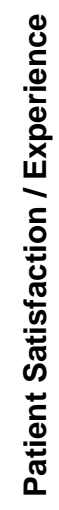 & 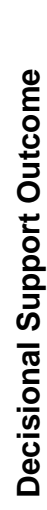 \\
\hline & Teljeur, $2016^{27}$ & & & & & & & & $\mathrm{X}$ & $\mathrm{X}$ & & & & $\mathrm{X}$ & & \\
\hline & Hecke, $2016^{28}$ & & & & & & & & $\mathrm{X}$ & $X$ & & & & $\mathrm{X}$ & & \\
\hline & Hooft, $2016^{29}$ & & & & & & & & $\mathrm{X}$ & $\mathrm{X}$ & & & & & & \\
\hline & Stenberg, $2016^{30}$ & & & & & & & & $\mathrm{X}$ & $\mathrm{X}$ & & & $x$ & & & \\
\hline & Plow, $2016^{31}$ & & & & & & & & $\mathrm{X}$ & $\mathrm{X}$ & & & & & & \\
\hline & Noonan, $2019^{32}$ & & & & & & & & $\mathrm{X}$ & & & & $X$ & & & \\
\hline & Sakakibara, $2016^{34}$ & & & & & & & & $\mathrm{X}$ & $\mathrm{X}$ & & & & & & \\
\hline & $\begin{array}{l}\text { Bolscher-Niehuis, } \\
2016^{46}\end{array}$ & & & & & & & & $\mathrm{X}$ & & & & $x$ & & & \\
\hline & Sokol, $2016^{36}$ & & & $\mathrm{X}$ & & & & & $\mathrm{X}$ & & & & & & $\mathrm{X}$ & \\
\hline & Whitehead, $2016^{25}$ & & & & & & & & $\mathrm{X}$ & $\mathrm{X}$ & & & & & & \\
\hline & Palacio, $2016^{38}$ & & & & & & & & $\mathrm{X}$ & & & & & & & \\
\hline & Eeden, $2016^{39}$ & & & & & & & & $\mathrm{X}$ & & & & $X$ & $X$ & & \\
\hline & Kuo, $2016^{33}$ & & & & & & & & & $\mathrm{X}$ & & & & & & \\
\hline & Jacelon, $2016^{40}$ & & & & & & & & $\mathrm{X}$ & & & & & & & \\
\hline & Smith, $2017^{41}$ & & & & $\mathrm{X}$ & $\mathrm{X}$ & & $\mathrm{X}$ & $\mathrm{X}$ & $\mathrm{X}$ & & & $\mathrm{X}$ & $X$ & & \\
\hline & Laukner, $2016^{76}$ & & & & & & & & $\mathrm{X}$ & $\mathrm{X}$ & & & & & $\mathrm{X}$ & \\
\hline & Kim, $2016^{43}$ & & & & $\mathrm{X}$ & & & & & $\mathrm{X}$ & & & & & & \\
\hline & Ha Dinh, $2016^{59}$ & & & & & & & & $\mathrm{X}$ & & & & $\mathrm{X}$ & $\mathrm{X}$ & & \\
\hline & Thakkar, $2016^{44}$ & & & & & & & & $\mathrm{X}$ & & & & & & & \\
\hline & Deek, $2016^{45}$ & & & & $\mathrm{X}$ & & & & $\mathrm{X}$ & & & $\mathrm{X}$ & $\mathrm{X}$ & $\mathrm{X}$ & & \\
\hline & Kim, $2015^{26}$ & & & & & & & & & $\mathrm{X}$ & & & $x$ & & & \\
\hline & $\begin{array}{l}\text { Peytremann-Bridevaux, } \\
2015^{75}\end{array}$ & & & & $\mathrm{X}$ & & & & $X$ & $X$ & & & $X$ & $x$ & & \\
\hline & Jones, $2015^{87}$ & & & & & & & & $\mathrm{X}$ & & & & $\mathrm{X}$ & & $\mathrm{X}$ & \\
\hline & Price, $2015^{70}$ & & & & & & & & & $\mathrm{X}$ & & & & & $\mathrm{X}$ & \\
\hline & Wildevuur, $2015^{86}$ & & & & $\mathrm{X}$ & & & & $\mathrm{X}$ & & & & $\mathrm{X}$ & $\mathrm{X}$ & & \\
\hline & Hamine, $2015^{85}$ & & $\mathrm{X}$ & & & & & & $\mathrm{X}$ & $\mathrm{X}$ & & & & & & \\
\hline
\end{tabular}




\begin{tabular}{|c|c|c|c|c|c|c|c|c|c|c|c|c|c|c|c|c|}
\hline Type of Strategy & Author, Year & 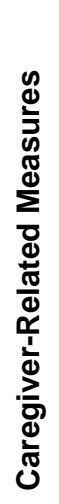 & 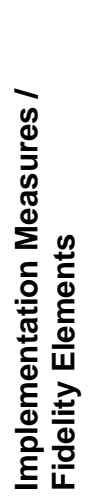 & 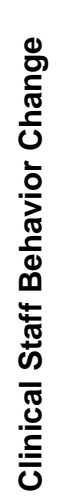 & 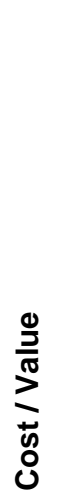 & 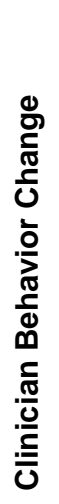 & 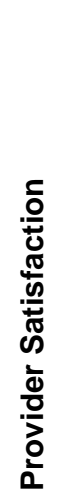 & 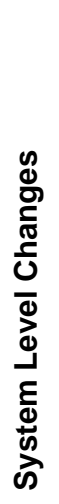 & 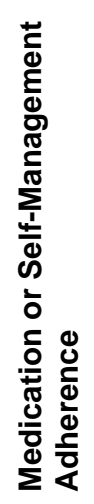 & 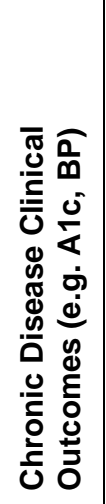 & 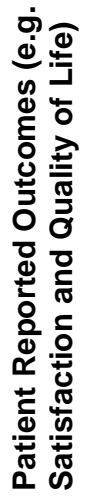 & 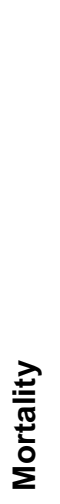 & 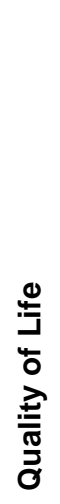 & 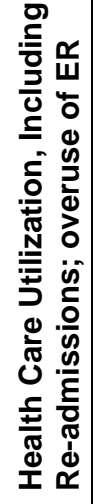 & 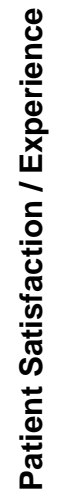 & 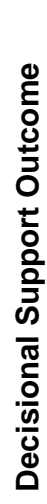 \\
\hline & Hammer, $2015^{42}$ & & & & & & & & & $X$ & & & $\mathrm{X}$ & & & \\
\hline & Zhao, $2015^{53}$ & & & & & & & & $\mathrm{X}$ & $X$ & & & & & & \\
\hline & Ammenwerth, $2019^{72}$ & & & & & & & & $\mathrm{X}$ & & & & & & $\mathrm{X}$ & \\
\hline & Dendere, $2019^{81}$ & & $\mathrm{X}$ & & & & & & & $\mathrm{X}$ & & & & & $\mathrm{X}$ & \\
\hline & Warrington, $2019^{78}$ & & & & & & & & $\mathrm{X}$ & $x$ & & & $\mathrm{X}$ & & $\mathrm{X}$ & \\
\hline & Boulley, $2018^{74}$ & & & & & & & & & & & & $\mathrm{X}$ & $\mathrm{X}$ & $\mathrm{X}$ & \\
\hline & Kelly, $2018^{83}$ & & & & & & & & & & & & & & & \\
\hline & Aquino, $2017^{71}$ & & & & & & & & $\mathrm{X}$ & $\mathrm{X}$ & & & $\mathrm{X}$ & & & \\
\hline & Donald, $2018^{73}$ & & & & & & & & $\mathrm{X}$ & & & & $\mathrm{X}$ & & $\mathrm{X}$ & \\
\hline & Lee, $2018^{77}$ & & & & & & & & $\mathrm{X}$ & $\mathrm{X}$ & & & $\mathrm{X}$ & & & \\
\hline & Risling, $2017^{84}$ & & & & & & & & & & & & & & $\mathrm{X}$ & \\
\hline & Palacios, $2017^{79}$ & & & & & & & & $\mathrm{X}$ & $\mathrm{X}$ & & & $\mathrm{X}$ & & & \\
\hline & Pereira, $2015^{88}$ & & & & & & & & $\mathrm{X}$ & $\mathrm{X}$ & & & & & & \\
\hline & Warner, $2015^{80}$ & & & & & & & & $\mathrm{X}$ & & & & $\mathrm{X}$ & $\mathrm{X}$ & $\mathrm{X}$ & \\
\hline & Almutairi, $2019^{82}$ & & & & & & & & $\mathrm{X}$ & $\mathrm{X}$ & & & & & & \\
\hline \multirow{12}{*}{$\begin{array}{l}\text { Self Management Support - Adults and } \\
\text { Children }\end{array}$} & Yin, $2019^{20}$ & & & & & & & & $\mathrm{x}$ & $x$ & & & & & & \\
\hline & Lancaster, $2018^{17}$ & & & & & & & & $\mathrm{X}$ & $\mathrm{X}$ & & & & $\mathrm{X}$ & $\mathrm{X}$ & \\
\hline & Lycett, $2018^{16}$ & & & & & & & & $\mathrm{X}$ & $\mathrm{X}$ & & & $\mathrm{X}$ & $\mathrm{X}$ & & \\
\hline & Bashi, $2018^{15}$ & & & & & & & & $\mathrm{X}$ & $\mathrm{X}$ & & & & & & \\
\hline & Niznik, $2018^{13}$ & & & & & & & & $\mathrm{X}$ & $\mathrm{X}$ & & & & & & \\
\hline & Kew, $2016^{18}$ & & & & & & & & & $\mathrm{x}$ & & & $\mathrm{X}$ & & & \\
\hline & Graham, 2016 & & & & & & & & $\mathrm{X}$ & & & & $\mathrm{X}$ & & & \\
\hline & Kruse, $2015^{24}$ & & & & & & $\mathrm{X}$ & & & & & & & & $\mathrm{X}$ & \\
\hline & Chi, $2015^{21}$ & & & & & & & & $\mathrm{X}$ & & & & $\mathrm{X}$ & & & \\
\hline & Hill, $2015^{22}$ & & & & & & & & $\mathrm{X}$ & $\mathrm{X}$ & & & $\mathrm{X}$ & & & \\
\hline & Pamungkas, $2017^{23}$ & & & & & & & & $\mathrm{X}$ & $\mathrm{X}$ & & & & & & \\
\hline & Barello, $2016^{14}$ & & & & & & & & $\mathrm{X}$ & & & & & & $\mathrm{X}$ & \\
\hline Self-Management Support - Children Only & Low, $2019^{4}$ & & & & & & & & $\mathrm{X}$ & & & & $\mathrm{X}$ & & $\mathrm{X}$ & \\
\hline
\end{tabular}




\begin{tabular}{|c|c|c|c|c|c|c|c|c|c|c|c|c|c|c|c|c|}
\hline Type of Strategy & Author, Year & 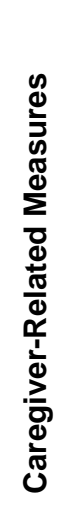 & 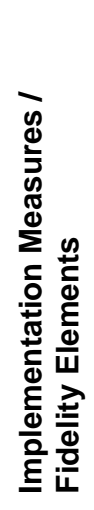 & 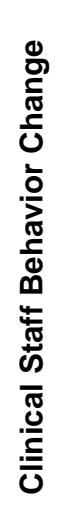 & 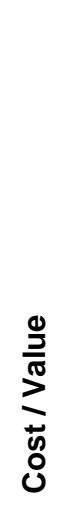 & 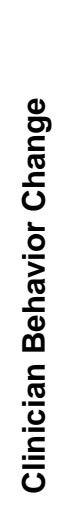 & 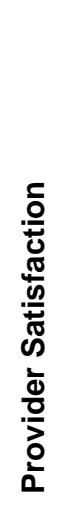 & 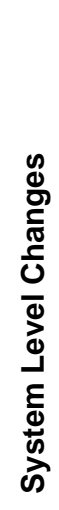 & 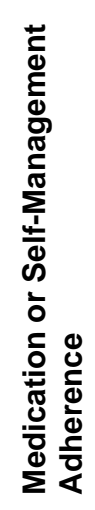 & 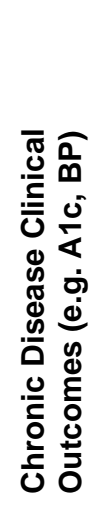 & 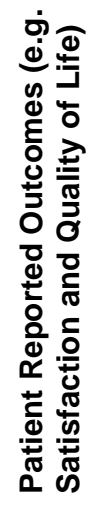 & 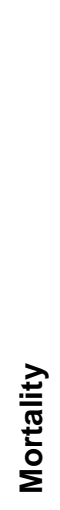 & 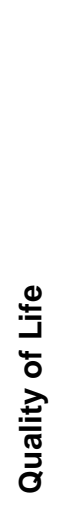 & 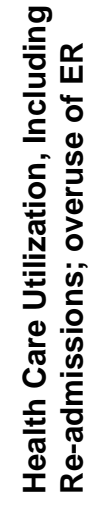 & 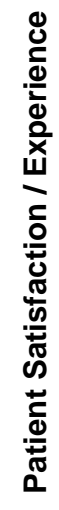 & 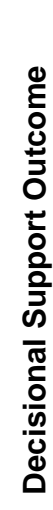 \\
\hline & Saxby, $2018^{5}$ & & & & & & & & $\mathrm{X}$ & & & & $\mathrm{X}$ & & & \\
\hline & Knafl, $2017^{6}$ & & & & & & & & $\mathrm{X}$ & $\mathrm{X}$ & & & $\mathrm{X}$ & & & \\
\hline & Clemente, $2016^{12}$ & & & & & & & & $\mathrm{X}$ & $\mathrm{X}$ & & & $\mathrm{X}$ & & & \\
\hline & Campbell, $2016^{7}$ & & & & & & & & $\mathrm{X}$ & $\mathrm{X}$ & & & $\mathrm{X}$ & & & \\
\hline & Bal, $2015^{3}$ & & & & & & & & $\mathrm{X}$ & $\mathrm{X}$ & & & $\mathrm{X}$ & & & \\
\hline & Majeed-Ariss, $2015^{8}$ & & & & & & & & $\mathrm{X}$ & $\mathrm{X}$ & & & & & & \\
\hline & Charlier, $2015^{9}$ & & & & & & & & $\mathrm{X}$ & & & & & & & \\
\hline & Sattoe, $2015^{10}$ & & & & & & & & & $\mathrm{X}$ & & & $\mathrm{X}$ & & & \\
\hline & Hamline, $2018^{11}$ & & & & & & & & & & & & & $\mathrm{X}$ & & \\
\hline \multirow[t]{16}{*}{ Shared Decision Making - Adults Only } & Vermunt, $2017^{120}$ & & & & & $\mathrm{X}$ & & & & & & & & & & $\mathrm{X}$ \\
\hline & Kashaf, $2017^{121}$ & & & & & & & & $\mathrm{X}$ & $\mathrm{X}$ & & & $\mathrm{X}$ & & & $\mathrm{X}$ \\
\hline & Wagner, $2019^{100}$ & & & & & $\mathrm{X}$ & $\mathrm{X}$ & & & & & & & & $\mathrm{X}$ & $\mathrm{X}$ \\
\hline & Goodridge, $2019^{101}$ & & & & & & & & $\mathrm{X}$ & & & & & & $\mathrm{X}$ & $\mathrm{X}$ \\
\hline & Baik, 2018 & $\mathrm{X}$ & & & & & & & & & & & & & $\mathrm{X}$ & $\mathrm{X}$ \\
\hline & Berlin, 2018 ${ }^{110}$ & & & & & & & & & & & & & & $\mathrm{X}$ & $x$ \\
\hline & Johnson, $2018^{111}$ & & & $\mathrm{X}$ & & & & & & $\mathrm{X}$ & & & & & & $\mathrm{X}$ \\
\hline & $\begin{array}{l}\text { Martínez-González, } \\
2018^{124}\end{array}$ & & & & & & & & & & & & $X$ & & $\mathrm{X}$ & $\mathrm{X}$ \\
\hline & Légaré, $2018^{113}$ & & & & & $\mathrm{X}$ & & & & $\mathrm{X}$ & & & $\mathrm{X}$ & $\mathrm{X}$ & & $\mathrm{X}$ \\
\hline & Baptista, $2018^{117}$ & & & & & & & & & & & & & & & $\mathrm{x}$ \\
\hline & Spronk, $2018^{118}$ & & & & & & & & & $\mathrm{X}$ & & & & & $\mathrm{X}$ & $x$ \\
\hline & Samalin, $2018^{122}$ & & & & & & & & $\mathrm{X}$ & & & & $x$ & & $\mathrm{X}$ & $\mathrm{X}$ \\
\hline & $\begin{array}{l}\text { Martínez-González, } \\
2018^{123}\end{array}$ & & & & & & & & & & & & & & & $X$ \\
\hline & $\begin{array}{l}\text { Martínez-Alonso, } \\
2017^{112}\end{array}$ & & & & & & & & & & & & & & & $x$ \\
\hline & Stacey $D, 2017^{119}$ & & & & & $\mathrm{X}$ & & & $\mathrm{X}$ & & & & & & & $\mathrm{X}$ \\
\hline & Morrell, 2016 ${ }^{116}$ & & & & & & & & & & & & & & $\mathrm{X}$ & $x$ \\
\hline
\end{tabular}




\begin{tabular}{|c|c|c|c|c|c|c|c|c|c|c|c|c|c|c|c|c|}
\hline Type of Strategy & Author, Year & 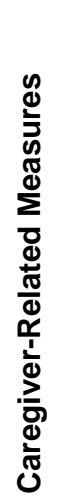 & 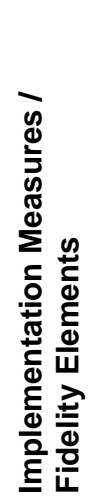 & 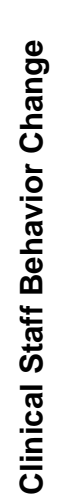 & 离 & 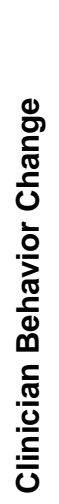 & 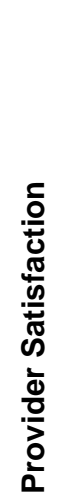 & 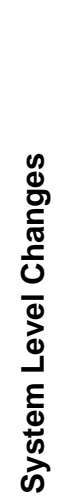 & 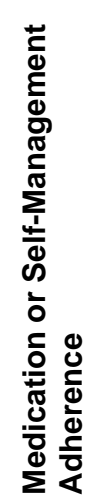 & 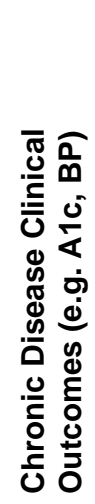 & 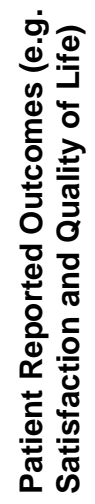 & 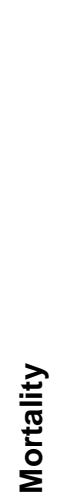 & 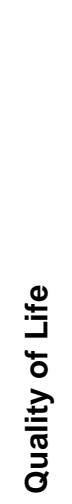 & 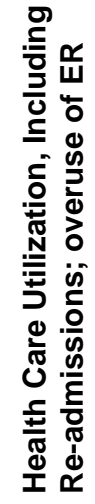 & 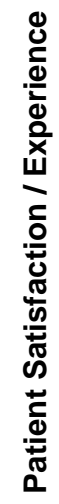 & 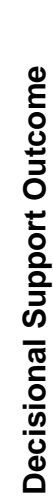 \\
\hline & Porter, 2016 & & & & & & & & $\mathrm{X}$ & $\mathrm{X}$ & & & & & $\mathrm{X}$ & \\
\hline & Stovell, $2016^{114}$ & & & & & & & & $\mathrm{X}$ & & & & & & & $\mathrm{X}$ \\
\hline & Nathan, $2016^{99}$ & & & & & & & & & $\mathrm{X}$ & & & & & & $x$ \\
\hline & van Weert, $2016^{109}$ & & & & & & & & $\mathrm{X}$ & & & & & & $\mathrm{X}$ & $x$ \\
\hline & Friedrichs, $2016^{107}$ & & & & & & & & & $\mathrm{X}$ & & & & & $\mathrm{X}$ & $x$ \\
\hline & Kashaf, $2015^{106}$ & & & & & & & & & & & & $X$ & & & $\mathrm{X}$ \\
\hline & Mclntyre, $2015^{105}$ & & & & & & & & $\mathrm{X}$ & & & & & & $\mathrm{X}$ & $\mathrm{X}$ \\
\hline & Jain, $2015^{104}$ & & & & & & & & & & & & & & & $x$ \\
\hline & Irizarry, $2015^{102}$ & & & & & & $\mathrm{X}$ & & & & & & & & & $x$ \\
\hline & Violette, $2015^{103}$ & & & & & & & & & $X$ & & & $\mathrm{X}$ & $X$ & & $\mathrm{X}$ \\
\hline & Voruganti, $2017^{95}$ & & & & & & & & & $\mathrm{X}$ & & & & & $\mathrm{X}$ & \\
\hline & Winston, $2017^{98}$ & & & & & & & & $\mathrm{X}$ & $\mathrm{X}$ & & & & & & $\mathrm{X}$ \\
\hline & Kew, $2017^{96}$ & & & & & & & & $\mathrm{X}$ & & & & $\mathrm{X}$ & $\mathrm{X}$ & $\mathrm{X}$ & \\
\hline & Clayman, $2015^{97}$ & & & & & & & & $\mathrm{X}$ & $\mathrm{X}$ & & & $\mathrm{X}$ & & & $\mathrm{X}$ \\
\hline \multirow[t]{4}{*}{ Shared Decision Making - Children Only } & Malone, $2019^{91}$ & & & & & & & & & & & & & & & \\
\hline & Cheng, $2017^{92}$ & & & & & & & & & & & & & & & $\mathrm{X}$ \\
\hline & Coyne, $2016^{93}$ & & & & & & & & & & & & & & & \\
\hline & Wyatt, $2015^{94}$ & & & & & & & & & & & & $\mathrm{X}$ & $\mathrm{X}$ & $\mathrm{X}$ & $\mathrm{X}$ \\
\hline \multirow[t]{2}{*}{ Transitional Care - Adults Only } & Berre, $2017^{126}$ & & & & & & & & & & & $\mathrm{X}$ & $\mathrm{X}$ & $\mathrm{X}$ & & \\
\hline & Sendall, $2016^{125}$ & & & & & & $\mathrm{X}$ & & & & & & $\mathrm{X}$ & $\mathrm{X}$ & & \\
\hline
\end{tabular}


Evidence Table 5. Findings of included systematic reviews addressing direct patient care

\begin{tabular}{|c|c|c|c|c|c|}
\hline $\begin{array}{l}\text { Type of } \\
\text { Strategy }\end{array}$ & Author, Year & $\begin{array}{l}\text { Overall } \\
\text { Findings }\end{array}$ & $\begin{array}{l}\text { Strength of } \\
\text { Evidence } \\
\text { Reported? }\end{array}$ & Findings & Conclusion Sentence \\
\hline $\begin{array}{l}\text { Advanced Care } \\
\text { Planning - } \\
\text { Adult Only }\end{array}$ & Coulter, $2015^{1}$ & $\begin{array}{l}\text { Positive } \\
\text { benefit }\end{array}$ & Yes & $\begin{array}{l}\text { Nine studies measured glycated hemoglobin } \\
\text { (HbA1c), giving a combined mean difference } \\
\text { (MD) between intervention and control of - } \\
0.24 \% \text { ( } 95 \% \text { confidence interval }(\mathrm{Cl})-0.35 \text { to - } \\
0.14) \text {, a small positive effect in favor of } \\
\text { personalized care planning }\end{array}$ & $\begin{array}{l}\text { Personalized care planning leads to } \\
\text { improvements in certain indicators of } \\
\text { physical and psychological health status, } \\
\text { and people's capability to self-manage } \\
\text { their condition when compared to usual } \\
\text { care. The effects are not large }\end{array}$ \\
\hline $\begin{array}{l}\text { Health Literacy } \\
\text { - Adult Only }\end{array}$ & $\begin{array}{l}\text { Schaepe, } \\
2015^{2}\end{array}$ & $\begin{array}{l}\text { Unclear } \\
\text { benefit }\end{array}$ & No & $\begin{array}{l}\text { Most articles focused on educational } \\
\text { intervention programs for people undergoing } \\
\text { PD. Findings on the link between the PD } \\
\text { trainer's background and peritonitis rates } \\
\text { among individuals undergoing PD are } \\
\text { inconsistent. PD learners should be taught } \\
\text { self-management }\end{array}$ & $\begin{array}{l}\text { Educational interventions for PD remain an } \\
\text { under-researched area, despite the } \\
\text { potential they have to make this type of } \\
\text { therapy more successful. Further research } \\
\text { on education and training for people } \\
\text { receiving PD and for PD nurses is needed }\end{array}$ \\
\hline \multirow[t]{2}{*}{$\begin{array}{l}\text { Transitional } \\
\text { Care - Adult } \\
\text { Only }\end{array}$} & Berre, $2017^{126}$ & $\begin{array}{l}\text { Positive } \\
\text { benefit }\end{array}$ & Yes & $\begin{array}{l}\text { Compared to usual care, significantly better } \\
\text { outcomes were observed: a lower mortality at } \\
3 \text { (RD: } 0.02 \text { [0.05, 0.00]; NNT: } 50), 6,12 \text { and } \\
18 \text { months post-discharge, a lower rate of ED } \\
\text { visits at } 3 \text { months (RD: } 0.08 \text { [ } 0.15,0.01] \text {; } \\
\text { NNT: } 13 \text { ) }\end{array}$ & $\begin{array}{l}\text { Transitional care for older patients with CD } \\
\text { discharged from hospital to home leads to } \\
\text { better outcomes in mortality, readmission } \\
\text { and readmission days. Decision-makers, } \\
\text { managers and clinicians should take these } \\
\text { results into account when developing } \\
\text { policies }\end{array}$ \\
\hline & $\begin{array}{l}\text { Sendall, } \\
2016^{125}\end{array}$ & $\begin{array}{l}\text { Positive } \\
\text { benefit }\end{array}$ & Yes & $\begin{array}{l}\text { this study found intervention patients had } \\
\text { lower rehospitalization rates at } 30 \text { days ( } 8.3 \\
\text { vs. } 11.9, \mathrm{p} V 40.048) \text { and at } 90 \text { days ( } 16.7 \text { vs. } \\
22.5, \mathrm{p} V 40.04) \text { than control patients, and } \\
\text { lower hospital costs than intervention patients }\end{array}$ & $\begin{array}{l}\text { This structured review found that } \\
\text { community linkages, clinical information } \\
\text { sharing, delivery system design, self- } \\
\text { management and clinical decision support } \\
\text { are common components of a model of } \\
\text { care for older people with two or more } \\
\text { chronic disease. }\end{array}$ \\
\hline \multirow[t]{2}{*}{$\begin{array}{l}\text { Self } \\
\text { Management } \\
\text { Support - Adult } \\
\text { Only }\end{array}$} & $\begin{array}{l}\text { Jacelon, } \\
2016^{40}\end{array}$ & $\begin{array}{l}\text { Unclear } \\
\text { benefit }\end{array}$ & & $\begin{array}{l}\text { The most frequently employed system } \\
\text { components were peripheral sensors, } \\
\text { feedback on data provided to the individual } \\
\text { with the disease, a user diary, provider access } \\
\text { to data, provider consultation with the } \\
\text { individual who is self managing and web- } \\
\text { based education }\end{array}$ & $\begin{array}{l}\text { For individuals to effectively use systems } \\
\text { to maintain maximum wellness, the } \\
\text { systems must have a strong component of } \\
\text { self-management and provide the user } \\
\text { with meaningful information regarding their } \\
\text { health states. }\end{array}$ \\
\hline & $\begin{array}{l}\text { Bolscher- } \\
\text { Niehuis, } 2016^{46}\end{array}$ & $\begin{array}{l}\text { Positive } \\
\text { benefit }\end{array}$ & Yes & $\begin{array}{l}\text { Although there was considerable variation in } \\
\text { study population, intervention characteristics } \\
\text { and measurement instruments used, in seven } \\
\text { of the } 12 \text { included studies (both with a low and } \\
\text { high risk of bias) }\end{array}$ & $\begin{array}{l}\text { There is a moderate level of evidence that } \\
\text { self-management support programs with a } \\
\text { multi-component structure, containing } \\
\text { disease-specific information, education of } \\
\text { knowledge and skills and, in particular, } \\
\text { individually tailored coaching }\end{array}$ \\
\hline
\end{tabular}




\begin{tabular}{|c|c|c|c|c|c|}
\hline $\begin{array}{l}\text { Type of } \\
\text { Strategy }\end{array}$ & Author, Year & $\begin{array}{l}\text { Overall } \\
\text { Findings }\end{array}$ & $\begin{array}{l}\text { Strength of } \\
\text { Evidence } \\
\text { Reported? }\end{array}$ & Findings & Conclusion Sentence \\
\hline & Deek, $2016^{45}$ & $\begin{array}{l}\text { Potential } \\
\text { benefit }\end{array}$ & Yes & $\begin{array}{l}\text { Significant reductions in the readmission rates } \\
\text { at } 30,90 \text { and } 180 \text { days: adjusted odds ratios } \\
0.52 \text { at } 30 \text { days } 0.43 \text { at } 90 \text { days } 0.57 \text { at } 180 \\
\text { days and a significant prolongation in time to } \\
\text { rehospitalization (one study) }\end{array}$ & $\begin{array}{l}\text { Involving the family in self-care has shown } \\
\text { improved out- comes in patients with } \\
\text { chronic conditions. Coping with a long-term } \\
\text { illness may be facilitated when a family } \\
\text { caregiver is involved but prospective, } \\
\text { systematic and accountable processes are } \\
\text { lacking }\end{array}$ \\
\hline & $\begin{array}{l}\text { Thakkar, } \\
2016^{44}\end{array}$ & $\begin{array}{l}\text { Positive } \\
\text { benefit }\end{array}$ & Yes & $\begin{array}{l}\text { In the pooled analysis of } 2742 \text { patients } \\
\text { (median age, } 39 \text { years and } 50.3 \% \text { [1380 of } \\
2742 \text { ] female), text messaging significantly } \\
\text { improved medication adherence (odds ratio, } \\
2.11 ; 95 \% \mathrm{Cl}, 1.52-2.93 ; \mathrm{P}<.001 \text { ). The effect } \\
\text { was not sensitive to study characteristics }\end{array}$ & $\begin{array}{l}\text { Mobile phone text messaging } \\
\text { approximately doubles the odds of } \\
\text { medication adherence. This increase } \\
\text { translates into adherence rates improving } \\
\text { from } 50 \% \text { (assuming this baseline rate in } \\
\text { patients with chronic disease) to } 67.8 \% \text {, or } \\
\text { an absolute increase of } 17.8 \% \text {. }\end{array}$ \\
\hline & $\begin{array}{l}\text { Ha Dinh, } \\
2016^{59}\end{array}$ & $\begin{array}{l}\text { Potential } \\
\text { benefit }\end{array}$ & Yes & $\begin{array}{l}\text { Four studies confirmed improved disease- } \\
\text { specific knowledge in intervention } \\
\text { participants. One study showed a statistically } \\
\text { significant improvement in adherence to } \\
\text { medication and diet among type } 2 \text { diabetics } \\
\text { patients in the intervention group }\end{array}$ & $\begin{array}{l}\text { Overall, the teach-back method showed } \\
\text { positive effects in a wide range of health } \\
\text { care outcomes although these were not } \\
\text { always statistically significant. Studies in } \\
\text { this systematic review revealed improved } \\
\text { outcomes in disease-specific knowledge, } \\
\text { adherence }\end{array}$ \\
\hline & Kim, $2016^{43}$ & $\begin{array}{l}\text { Positive } \\
\text { benefit }\end{array}$ & Yes & $\begin{array}{l}\text { Of the } 30 \text { studies that tested the effect of a } \\
\text { CBHW-led intervention on cancer control, } 21 \\
\text { studies ( } 70 \%) \text { found improvements in cancer } \\
\text { screening behaviors; Sixteen studies ( } 62 \%) \\
\text { included in the review found a significant } \\
\text { effect of CBHW intervention on CVD }\end{array}$ & $\begin{array}{l}\text { In conclusion, our review of } 67 \text { articles } \\
\text { shows that CBHWs can be an effective } \\
\text { intervention model that is also cost- } \\
\text { effective for certain health conditions (e.g., } \\
\text { high blood pressure, diabetes) or } \\
\text { behaviors (e.g., mammogram and Pap test } \\
\text { use) }\end{array}$ \\
\hline & Smith, $2017^{41}$ & $\begin{array}{l}\text { Unclear } \\
\text { benefit }\end{array}$ & Yes & $\begin{array}{l}\text { Overall the results regarding the effectiveness } \\
\text { of interventions were mixed. There were no } \\
\text { clear positive improvements in clinical } \\
\text { outcomes, health service use, medication } \\
\text { adherence, patient-related health behaviors, } \\
\text { health professional behaviors }\end{array}$ & $\begin{array}{l}\text { The review suggests that interventions that } \\
\text { are designed to target specific risk factors } \\
\text { (for example treatment for depression) or } \\
\text { interventions that focus on difficulties that } \\
\text { people experience with daily functioning } \\
\text { (for example, physiotherapy treatment }\end{array}$ \\
\hline & Jones, $2015^{87}$ & $\begin{array}{l}\text { Unclear } \\
\text { benefit }\end{array}$ & Yes & $\begin{array}{l}\text { Three studies showed favorable physical } \\
\text { activity outcomes following self-management } \\
\text { interventions for stroke; however, risk of bias } \\
\text { was high, and overall efficacy remains } \\
\text { unclear. Although not used in isolation from } \\
\text { face-to-face delivery, remote delivery }\end{array}$ & $\begin{array}{l}\text { The efficacy of self-management programs } \\
\text { in increasing physical activity levels in } \\
\text { community-dwelling adults following } \\
\text { acquired brain injury }(\mathrm{ABI}) \text { is still unknown. } \\
\text { Research into the efficacy of self- } \\
\text { management programs }\end{array}$ \\
\hline
\end{tabular}




\begin{tabular}{|c|c|c|c|c|c|}
\hline $\begin{array}{l}\text { Type of } \\
\text { Strategy }\end{array}$ & Author, Year & $\begin{array}{l}\text { Overall } \\
\text { Findings }\end{array}$ & $\begin{array}{l}\text { Strength of } \\
\text { Evidence } \\
\text { Reported? }\end{array}$ & Findings & Conclusion Sentence \\
\hline & Kuo, $2016^{33}$ & $\begin{array}{l}\text { Potential } \\
\text { benefit }\end{array}$ & no & $\begin{array}{l}\text { Evidence from } 7 \text { of the } 11 \text { included studies } \\
\text { suggests significant improvement in patients' } \\
\text { hemoglobin A1c (HbA1c) with the use of } \\
\text { secure messaging. }\end{array}$ & $\begin{array}{l}\text { Further work must be done to determine } \\
\text { how to best maximize the potential of } \\
\text { available tools such as secure messaging } \\
\text { and EHRs to improve patient outcomes }\end{array}$ \\
\hline & Eeden, $2016^{39}$ & $\begin{array}{l}\text { Positive } \\
\text { benefit }\end{array}$ & Yes & $\begin{array}{l}\text { Eighteen studies found the self-management } \\
\text { intervention(s) to be cost-effective compared } \\
\text { with other interventions, either due to } \\
\text { increased costs and effects in comparison } \\
\text { with the control intervention, or decreased } \\
\text { costs and increased effects in comparison }\end{array}$ & $\begin{array}{l}\text { Self-management interventions for adult } \\
\text { chronic patients were heterogeneous and } \\
\text { there was no clear, well-considered } \\
\text { definition of self-management. Eighteen } \\
\text { studies found the self-management } \\
\text { intervention(s) to be cost-effective } \\
\text { compared with other interventions }\end{array}$ \\
\hline & Palacio, $2016^{38}$ & $\begin{array}{l}\text { Positive } \\
\text { benefit }\end{array}$ & Yes & $\begin{array}{l}\text { For studies reporting a categorical measure ( } \mathrm{n} \\
=11) \text {, the pooled RR for medication } \\
\text { adherence was higher for } \mathrm{Ml} \text { compared with } \\
\text { control }(1.17 ; 95 \% \mathrm{Cl} 1.05-1.31 ; \mathrm{p}<0.01) \text {. For } \\
\text { studies reporting a continuous measure } \\
\text { ( } \mathrm{n}=11) \text {, the pooled SMD for medication } \\
\text { adherence }\end{array}$ & $\begin{array}{l}\text { Ml improves medication adherence at } \\
\text { different exposure times and counselors' } \\
\text { educational level. However, the evaluation } \\
\text { of MI characteristics associated with } \\
\text { success had inconsistent results. }\end{array}$ \\
\hline & $\begin{array}{l}\text { Whitehead, } \\
2016^{25}\end{array}$ & $\begin{array}{l}\text { Potential } \\
\text { benefit }\end{array}$ & Yes & $\begin{array}{l}\text { Of the } 9 \text { papers we reviewed, } 6 \text { of the } \\
\text { interventions demonstrated a statistically } \\
\text { significant improvement in the primary } \\
\text { measure of clinical outcome in mobile apps } \\
\text { user. Significant improvements in diabetes- } \\
\text { specific clinical outcomes (HbA1c) were } \\
\text { reported }\end{array}$ & $\begin{array}{l}\text { The evidence indicates the potential of } \\
\text { mobile apps in improving symptom } \\
\text { management through self-management } \\
\text { interventions. The use of apps in mHealth } \\
\text { has the potential to improve health } \\
\text { outcomes among those living with chronic } \\
\text { diseases }\end{array}$ \\
\hline & Yin, $2019^{90}$ & $\begin{array}{l}\text { Positive } \\
\text { benefit }\end{array}$ & No & & $\begin{array}{l}\text { Significant benefits have been seen in } \\
\text { trials with use of Mobile Health APPs for } \\
\text { IBD relating to education, quality of life, } \\
\text { quality of care, treatment adherence, and } \\
\text { medication management. No studies have } \\
\text { reported a negative impact. }\end{array}$ \\
\hline & $\begin{array}{l}\text { Laukner, } \\
2016^{76}\end{array}$ & $\begin{array}{l}\text { Positive } \\
\text { benefit }\end{array}$ & No & $\begin{array}{l}\text { Of the nine studies that reported on program } \\
\text { outcomes, eight reported positive outcomes } \\
\text { with one study reporting mixed results. Overall } \\
\text { program success, participants valuing the } \\
\text { social aspects of the programs, improved } \\
\text { activity or weight loss, and participation }\end{array}$ & $\begin{array}{l}\text { The key findings of this scoping review are } \\
\text { as follows: } 1 \text {. A combination of } \\
\text { telecommunications with some face-to- } \\
\text { face meetings can support the accessibility } \\
\text { of peer support programs in rural areas. } 2 \text {. } \\
\text { Core elements of these programs are the } \\
\text { provision of } s\end{array}$ \\
\hline
\end{tabular}




\begin{tabular}{|c|c|c|c|c|c|}
\hline $\begin{array}{l}\text { Type of } \\
\text { Strategy }\end{array}$ & Author, Year & $\begin{array}{l}\text { Overall } \\
\text { Findings }\end{array}$ & $\begin{array}{l}\text { Strength of } \\
\text { Evidence } \\
\text { Reported? }\end{array}$ & Findings & Conclusion Sentence \\
\hline & $\begin{array}{l}\text { Ammenwerth, } \\
2019^{72}\end{array}$ & $\begin{array}{l}\text { Unclear } \\
\text { benefit }\end{array}$ & No & $\begin{array}{l}\text { studies found no or only a small, clinically } \\
\text { non-relevant effect of patient portals. }\end{array}$ & $\begin{array}{l}\text { Future research should develop a } \\
\text { taxonomy to describe patient portal } \\
\text { functionalities to facilitate the aggregation } \\
\text { of evidence. }\end{array}$ \\
\hline & Lee, $2018^{77}$ & $\begin{array}{l}\text { Positive } \\
\text { benefit }\end{array}$ & Yes & $\begin{array}{l}\text { Of the } 12 \text { RCTs reviewed, } 10 \text { of the mHealth } \\
\text { interventions demonstrated statistically } \\
\text { significant improvement in some health } \\
\text { outcomes by incorporating mobile applications } \\
\text { in managing chronic diseases. }\end{array}$ & $\begin{array}{l}\text { Findings from mHealth intervention studies } \\
\text { for chronic disease management have } \\
\text { shown promising aspects, particularly in } \\
\text { improving self-management and some } \\
\text { health outcomes. }\end{array}$ \\
\hline & Donald, $2018^{73}$ & $\begin{array}{l}\text { Unclear } \\
\text { benefit }\end{array}$ & No & $\begin{array}{l}\text { The most common intervention topic was } \\
\text { diet/nutrition and interventions were regularly } \\
\text { delivered face to face. Interventions were } \\
\text { administered by a variety of providers, with } \\
\text { nursing professionals the most common } \\
\text { health professional group. }\end{array}$ & $\begin{array}{l}\text { We were unable to draw conclusions } \\
\text { regarding the most effective self- } \\
\text { management intervention for adult patients } \\
\text { with chronic kidney disease, keeping in } \\
\text { mind that our aim was to review the } \\
\text { breadth of the current literature and } \\
\text { present the gaps that exist. }\end{array}$ \\
\hline & Aquino, $2017^{71}$ & $\begin{array}{l}\text { No } \\
\text { benefit }\end{array}$ & Yes & $\begin{array}{l}\text { Five studies (45.5\%) showed significant } \\
\text { improvements in HbA1c reduction, } \\
\text { improvements in self-efficacy }(18.2 \%), \\
\text { knowledge levels of DM (18.2\%), quality of life } \\
(18.2 \%) \text {. However, after meta-analysis, no } \\
\text { statistically significant improvement was found }\end{array}$ & $\begin{array}{l}\text { This systematic review showed that } \\
\text { individual strategies for DM empowerment } \\
\text { were not effective in reducing HbA1c, } \\
\text { despite contributing to improvements in } \\
\text { psychosocial parameters. Therefore, } \\
\text { individual strategies need to be reviewed } \\
\text { so that they become effective }\end{array}$ \\
\hline & Kelly, $2018^{83}$ & $\begin{array}{l}\text { Unclear } \\
\text { benefit }\end{array}$ & No & $\begin{array}{l}\text { Evidence supporting the role of inpatient } \\
\text { portals in improving patient and caregiver } \\
\text { engagement, knowledge, communication, and } \\
\text { care quality and safety is limited. Providers } \\
\text { had concerns about using inpatient portals }\end{array}$ & $\begin{array}{l}\text { Further investigation is needed to optimally } \\
\text { design inpatient portals to maximize } \\
\text { potential benefits or hospitalized patients } \\
\text { and caregivers while minimizing } \\
\text { unintended consequences for healthcare } \\
\text { teams. }\end{array}$ \\
\hline & Boulley, $2018^{74}$ & $\begin{array}{l}\text { Potential } \\
\text { benefit }\end{array}$ & Yes & $\begin{array}{l}\text { There was considerable heterogeneity in } \\
\text { study methods, in outcome definitions, in } \\
\text { measures for engagement with DI and in } \\
\text { psychosocial variables assessed. Results } \\
\text { from the studies showed a high level of } \\
\text { engagement. }\end{array}$ & $\begin{array}{l}\text { The present review showed that despite } \\
\text { the heterogeneity in the studies assessed } \\
\text { and inconsistent results, DI may constitute } \\
\text { an excellent means to help cancer patients } \\
\text { and survivors cope better with the disease } \\
\text { and with treatment side effects }\end{array}$ \\
\hline
\end{tabular}




\begin{tabular}{|c|c|c|c|c|c|}
\hline $\begin{array}{l}\text { Type of } \\
\text { Strategy }\end{array}$ & Author, Year & $\begin{array}{l}\text { Overall } \\
\text { Findings }\end{array}$ & $\begin{array}{l}\text { Strength of } \\
\text { Evidence } \\
\text { Reported? }\end{array}$ & Findings & Conclusion Sentence \\
\hline & Kim, $2015^{26}$ & $\begin{array}{l}\text { Positive } \\
\text { benefit }\end{array}$ & Yes & $\begin{array}{l}\text { Compared to conventional interventions the } \\
\text { web-based interventions provide diverse } \\
\text { health information in various forms by } \\
\text { providing links to rich information sources. } \\
\text { Another unique feature of automated } \\
\text { functions of the web-based interventions is } \\
\text { the tail }\end{array}$ & $\begin{array}{l}\text { Web-based self-management support } \\
\text { interventions for cancer survivors were } \\
\text { found to be effective in improving fatigue, } \\
\text { depression, anxiety, and overall quality of } \\
\text { life, with the benefits of the automated and } \\
\text { communicative functions available on the } \\
\text { Web }\end{array}$ \\
\hline & $\begin{array}{l}\text { Dendere, } \\
2019^{81}\end{array}$ & $\begin{array}{l}\text { Unclear } \\
\text { benefit }\end{array}$ & No & $\begin{array}{l}\text { some but not all studies found patient portals } \\
\text { improved patient engagement; patients } \\
\text { perceived some portal functions as } \\
\text { inadequate but others as useful; patients and } \\
\text { staff thought portals may improve patient care } \\
\text { but could cause anxiety in some patients }\end{array}$ & $\begin{array}{l}\text { While the evidence is currently immature, } \\
\text { inpatient portals have demonstrated } \\
\text { benefit by enabling the discovery of } \\
\text { medical errors, improving adherence to } \\
\text { medications, and providing patient-provider } \\
\text { communication, etc. High-quality studies } \\
\text { are needed }\end{array}$ \\
\hline & $\begin{array}{l}\text { Peytremann- } \\
\text { Bridevaux, } \\
2015^{75}\end{array}$ & $\begin{array}{l}\text { Positive } \\
\text { benefit }\end{array}$ & Yes & $\begin{array}{l}\text { Compared with usual care, chronic disease } \\
\text { management program resulted in } \\
\text { improvements in asthma-specific quality of life } \\
\text { (SMD 0.22, 95\% confidence interval (Cl) } 0.08 \\
\text { to } 0.37 \text { ), asthma severity scores (SMD } 0.18 \text {, } \\
95 \% \mathrm{Cl} 0.05 \text { to } 0.30 \text { ) }\end{array}$ & $\begin{array}{l}\text { There is moderate to low quality evidence } \\
\text { that chronic disease management program } \\
\text { for adults with asthma can improve } \\
\text { asthma-specific quality of life, asthma } \\
\text { severity, and lung function tests. Overall, } \\
\text { these results provide encouraging } \\
\text { evidence }\end{array}$ \\
\hline & Zhao, $2015^{53}$ & $\begin{array}{l}\text { No } \\
\text { benefit }\end{array}$ & Yes & $\begin{array}{l}\text { The meta-analysis of six eligible studies } \\
\text { revealed no significant difference in asthma } \\
\text { symptom score change between the } \\
\text { telemedicine and control groups (pooled } \\
\text { Hedges's } g=0.34,95 \% \text { confidence interval= - } \\
0.05 \text { to } 0.74, \mathrm{Z}=1.69, p=0.090 \text { ). }\end{array}$ & $\begin{array}{l}\text { Telemedicine interventions do not appear } \\
\text { to improve asthma function scores, but } \\
\text { other benefits may be present. }\end{array}$ \\
\hline & Kruse, $2015^{89}$ & $\begin{array}{l}\text { Unclear } \\
\text { benefit }\end{array}$ & No & $\begin{array}{l}\text { Very few studies associated use of the patient } \\
\text { portal, or its features, to improved outcomes; } \\
37 \%(10 / 27) \text { of papers reported improvements } \\
\text { in medication adherence, disease awareness, } \\
\text { self-management of disease, a decrease of } \\
\text { office visits }\end{array}$ & $\begin{array}{l}\text { More studies needed that assess effect of } \\
\text { patient portals on patient outcomes quality } \\
\text { of care. Few articles analyzed the full } \\
\text { patient portal but instead analyzed features } \\
\text { of a portal such as secure messaging, as } \\
\text { well as disease management and } \\
\text { monitoring. }\end{array}$ \\
\hline & $\begin{array}{l}\text { Hammer, } \\
2015^{42}\end{array}$ & $\begin{array}{l}\text { Potential } \\
\text { benefit }\end{array}$ & No & $\begin{array}{l}\text { Overall findings from these in-person } \\
\text { educational sessions were positive with an } \\
\text { overarching quality of life and/or symptom- } \\
\text { driven quality-of-life theme. More definitive } \\
\text { findings between specific interventions and } \\
\text { focused outcomes were not found }\end{array}$ & $\begin{array}{l}\text { Current interventions that direct patients in } \\
\text { self-care management of symptoms and } \\
\text { associated challenges with } \\
\text { cancer/survivorship are helpful, but } \\
\text { incomplete. No one intervention can be } \\
\text { recommended over another }\end{array}$ \\
\hline
\end{tabular}




\begin{tabular}{|c|c|c|c|c|c|}
\hline $\begin{array}{l}\text { Type of } \\
\text { Strategy }\end{array}$ & Author, Year & $\begin{array}{l}\text { Overall } \\
\text { Findings }\end{array}$ & $\begin{array}{l}\text { Strength of } \\
\text { Evidence } \\
\text { Reported? }\end{array}$ & Findings & Conclusion Sentence \\
\hline & $\begin{array}{l}\text { Hamine, } \\
2015^{85}\end{array}$ & $\begin{array}{l}\text { Potential } \\
\text { benefit }\end{array}$ & No & $\begin{array}{l}\text { Short message service was the most } \\
\text { commonly used mAdherence tool in } 40.2 \% \\
\text { (43/107) of studies. Usability, feasibility, and } \\
\text { acceptability or patient preferences for } \\
\text { mAdherence interventions were assessed in } \\
57.9 \%(62 / 107) \text { of studies and found to be } \\
\text { general }\end{array}$ & $\begin{array}{l}\text { There is potential for mHealth tools to } \\
\text { better facilitate adherence to chronic } \\
\text { disease management, but the evidence } \\
\text { supporting its current effectiveness is } \\
\text { mixed. Further research should focus on } \\
\text { understanding and improving how mHealth } \\
\text { tools can overcome }\end{array}$ \\
\hline & $\begin{array}{l}\text { Wildevuur, } \\
2015^{86}\end{array}$ & $\begin{array}{l}\text { Potential } \\
\text { benefit }\end{array}$ & No & $\begin{array}{l}\text { The type of ICT mostly used by persons with } \\
\text { a chronic condition for interacting with health } \\
\text { care providers is ICT for self-measurement of } \\
\text { the body ( } n=143 \text { ) (Table } 9 \text { ); the highest } \\
\text { rankings were found in studies on diabetes } \\
(n=48) \text { and cardiovascular }(n=47)\end{array}$ & $\begin{array}{l}\text { Persons with a chronic disease could } \\
\text { benefit from an ICT-enabled PCC } \\
\text { approach, but ICT-PCC also yields } \\
\text { organizational paybacks. It could lead to } \\
\text { an increase in health care usage, as } \\
\text { reported in some studies. Few } \\
\text { interventions could be regarded as "fully" }\end{array}$ \\
\hline & Price, $2015^{70}$ & $\begin{array}{l}\text { Positive } \\
\text { benefit }\end{array}$ & No & $\begin{array}{l}\text { Diabetes outcomes related to PHR most } \\
\text { studied - } 11 \text { of the } 12 \text { studies in DM showed } \\
\text { benefit. }\end{array}$ & $\begin{array}{l}\text { Small body of condition specific evidence } \\
\text { that has been published. Conditions with } \\
\text { evidence of benefit when using PHRs } \\
\text { tended to be chronic conditions with a } \\
\text { feedback loop between monitoring in the } \\
\text { PHR and direct behaviors that could be } \\
\text { self-managed }\end{array}$ \\
\hline & $\begin{array}{l}\text { Warrington, } \\
2019^{78}\end{array}$ & $\begin{array}{l}\text { Unclear } \\
\text { benefit }\end{array}$ & Yes & $\begin{array}{l}\text { Only publications that included some data on } \\
\text { patient engagement or patient-centered } \\
\text { outcomes were included }(\mathrm{N}=29) \text {. A lack of } \\
\text { consistency between studies in how } \\
\text { engagement was defined, measured, or } \\
\text { reported, and a wide range of methods } \\
\text { chosen to evaluate }\end{array}$ & $\begin{array}{l}\text { Electronic systems have the potential to } \\
\text { help patients manage side effects of } \\
\text { cancer treatment, with some evidence to } \\
\text { suggest a positive effect on patient- } \\
\text { centered outcomes. However, comparison } \\
\text { across studies is difficult due to the wide } \\
\text { range of assessment }\end{array}$ \\
\hline & $\begin{array}{l}\text { Nazarov, } \\
2019^{55}\end{array}$ & $\begin{array}{l}\text { Positive } \\
\text { benefit }\end{array}$ & Yes & $\begin{array}{l}\text { We found that workplace-based interventions } \\
\text { can lead to positive changes in employment } \\
\text { status, work ability, RTW, and sick leave rates } \\
\text { for people with various chronic conditions; } \\
\text { Here, four of the seven studies on } \\
\text { multidisciplinary interventions reported }\end{array}$ & $\begin{array}{l}\text { We found that workplace-oriented and } \\
\text { multidisciplinary programs are the most } \\
\text { supportive to RTW and reducing the } \\
\text { absence due to illness. In addition, } \\
\text { cognitive behavioral therapies achieve } \\
\text { positive results on RTW and sick leave. }\end{array}$ \\
\hline & Sokol, $2016^{36}$ & $\begin{array}{l}\text { Potential } \\
\text { benefit }\end{array}$ & No & $\begin{array}{l}\text { Forty-four studies (94\%) reported significant } \\
\text { changes favoring peer support. Eleven } \\
\text { strategies emerged for engaging and retaining } \\
\text { hardly reached individuals. Among them, } \\
\text { programs that reported a strategy of trust and } \\
\text { respect had higher participant retention }\end{array}$ & $\begin{array}{l}\text { Peer support is a broad and robust } \\
\text { strategy for reaching groups that health } \\
\text { services too often fail to engage. The wide } \\
\text { range of audiences and health concerns } \\
\text { among which peer support is successful } \\
\text { suggests that a basis for its success may } \\
\text { be its flexible }\end{array}$ \\
\hline
\end{tabular}




\begin{tabular}{|c|c|c|c|c|c|}
\hline $\begin{array}{l}\text { Type of } \\
\text { Strategy }\end{array}$ & Author, Year & $\begin{array}{l}\text { Overall } \\
\text { Findings }\end{array}$ & $\begin{array}{l}\text { Strength of } \\
\text { Evidence } \\
\text { Reported? }\end{array}$ & Findings & Conclusion Sentence \\
\hline & $\begin{array}{l}\text { Changizi, } \\
2017^{61}\end{array}$ & $\begin{array}{l}\text { Positive } \\
\text { benefit }\end{array}$ & No & $\begin{array}{l}\text { The mHealth technology can help change and } \\
\text { develop health behaviors (increased } \\
\text { consumption of fruits and vegetables, stress } \\
\text { management, reduced consumption of salt } \\
\text { and improved quality of diet and sleep) and } \\
\text { improve self- efficacy in elderly individuals }\end{array}$ & $\begin{array}{l}\text { Findings showed that mHealth can } \\
\text { improve care, self-management, self- } \\
\text { efficacy, behavior promotion (quality of } \\
\text { sleep, diet, physical activity mental health) } \\
\text { and medication adherence. The mHealth } \\
\text { technology has proven effective for } \\
\text { disease prevention }\end{array}$ \\
\hline & Ko, $2018^{60}$ & $\begin{array}{l}\text { Unclear } \\
\text { benefit }\end{array}$ & No & $\begin{array}{l}\text { This review found that self management has } \\
\text { been assessed through prerequisites of self- } \\
\text { management and behaviors involved in self- } \\
\text { management. }\end{array}$ & $\begin{array}{l}\text { This review revealed that current literature } \\
\text { does not operationalize self-management } \\
\text { in multiple chronic conditions as a process, } \\
\text { indicating incomplete assessments of self- } \\
\text { management. Future studies should } \\
\text { consider self-management as an iterative } \\
\text { process }\end{array}$ \\
\hline & Alessa, $2018^{47}$ & $\begin{array}{l}\text { Potential } \\
\text { benefit }\end{array}$ & Yes & $\begin{array}{l}\text { As shown in Table } 3,6 / 9(67 \%) \text { studies } \\
\text { demonstrated positive effects on BP; The } \\
\text { decrease in the intervention arm ranged from } \\
8.7 \text { to } 34.8 \mathrm{~mm} \text { Hg. Significant decreases in } \\
\text { DBP were reported in } 2 / 6(33 \%) \text { studies, } \\
\text { ranging from } 4.9 \text { to } 12 \mathrm{~mm} \mathrm{Hg} \text {; }\end{array}$ & $\begin{array}{l}\text { Most of the studies reported that apps } \\
\text { might be effective in lowering blood } \\
\text { pressure and are accepted by users. } \\
\text { However, these findings should be } \\
\text { interpreted with caution, as most of the } \\
\text { studies had a high risk of bias. }\end{array}$ \\
\hline & $\begin{array}{l}\text { Skrabal Ross, } \\
2018^{58}\end{array}$ & $\begin{array}{l}\text { Unclear } \\
\text { benefit }\end{array}$ & No & $\begin{array}{l}\text { The } 2 \text { experimental studies in this review } \\
\text { (Spoelstra et al's study and Greer's et al's } \\
\text { CORA experimental study) did not find } \\
\text { statistically significant differences between the } \\
\text { experimental and control groups. }\end{array}$ & $\begin{array}{l}\text { Despite the increasing body of evidence on } \\
\text { the use of mobile phones to deliver } \\
\text { medication adherence- enhancing } \\
\text { interventions in chronic diseases, literature } \\
\text { on the oral chemotherapy context is } \\
\text { lacking. }\end{array}$ \\
\hline & Jeddi, $2017^{63}$ & $\begin{array}{l}\text { Positive } \\
\text { benefit }\end{array}$ & Yes & $\begin{array}{l}\text { The studies assessed } 15 \text { outcomes, including } \\
\text { eight clinical out- comes and seven process of } \\
\text { care outcomes. In } 12(80 \%) \text { of the } 15 \\
\text { outcomes, the studies had revealed the } \\
\text { effects of the interventions as statistically } \\
\text { significant positive. }\end{array}$ & $\begin{array}{l}\text { This systematic review combined the } \\
\text { results of RCTs that evaluated the effect of } \\
\text { IT-based interventions on self- } \\
\text { management outcomes in CKD patients. } \\
\text { The results (eight studies, } 1637 \text { patients) } \\
\text { showed that IT-based interventions had } \\
\text { improved self-management }\end{array}$ \\
\hline & $\begin{array}{l}\text { Sangrar, } \\
2019^{56}\end{array}$ & $\begin{array}{l}\text { Potential } \\
\text { benefit }\end{array}$ & No & $\begin{array}{l}\text { The most prominent consideration when } \\
\text { designing blended learning patient self- } \\
\text { management programs is to ensure that the } \\
\text { educational program is suitable for the target } \\
\text { population and the individual patient. }\end{array}$ & $\begin{array}{l}\text { This review identified that most research } \\
\text { related to chronic disease self- } \\
\text { management programs that blend face-to- } \\
\text { face educational components with } \\
\text { online/computer-based educational } \\
\text { components remains at the level of pilot or } \\
\text { feasibility studies. }\end{array}$ \\
\hline
\end{tabular}




\begin{tabular}{|c|c|c|c|c|c|}
\hline $\begin{array}{l}\text { Type of } \\
\text { Strategy }\end{array}$ & Author, Year & $\begin{array}{l}\text { Overall } \\
\text { Findings }\end{array}$ & $\begin{array}{l}\text { Strength of } \\
\text { Evidence } \\
\text { Reported? }\end{array}$ & Findings & Conclusion Sentence \\
\hline & $\begin{array}{l}\text { Conway, } \\
2017^{64}\end{array}$ & $\begin{array}{l}\text { Unclear } \\
\text { benefit }\end{array}$ & No & $\begin{array}{l}\text { There are positive benefits of the use of one- } \\
\text { way and two-way digital health messages to } \\
\text { engage patients in timely self-management to } \\
\text { improve medication adherence. Strategies } \\
\text { such as IVR and SMS text messaging provide } \\
\text { evidence of improved short-term }\end{array}$ & $\begin{array}{l}\text { The } 13 \text { studies included in this review } \\
\text { found no conclusive evidence of improved } \\
\text { medication adherence using digital health } \\
\text { interventions such as interactive voice } \\
\text { response (IVR), short message service } \\
\text { (SMS) text messaging, telemonitoring }\end{array}$ \\
\hline & Lederle, $2019^{54}$ & $\begin{array}{l}\text { Potential } \\
\text { benefit }\end{array}$ & No & $\begin{array}{l}\text { The meta- analysis showed mixed results, } \\
\text { and many of the overall effect sizes were not } \\
\text { statistically significant. The participants of a } \\
\text { lay-led self-management program had fewer } \\
\text { emergency department visits (SMD: }-0.08 \text {; } \\
95 \% \mathrm{Cl}:-0.15 \text { to }-0.01 ; p=0.02)\end{array}$ & $\begin{array}{l}\text { Although the statistically significant effects } \\
\text { of the meta-analysis are low, our overall } \\
\text { findings show that only a small number of } \\
\text { the included studies tackled the task of } \\
\text { comprehensively investigating self- } \\
\text { management skills in the context of health } \\
\text { care }\end{array}$ \\
\hline & $\begin{array}{l}\text { Levengood, } \\
2019^{52}\end{array}$ & $\begin{array}{l}\text { Positive } \\
\text { benefit }\end{array}$ & No & $\begin{array}{l}\text { Com- pared with usual care, TBC reduced } \\
\text { HbA1c levels by an additional } 0.5 \%(95 \% \mathrm{Cl}= \\
-0.7,-0.3, \mathrm{I} 2=84.8 \%)\end{array}$ & $\begin{array}{l}\text { The available evidence demonstrates that } \\
\text { for patients with diabetes, TBC improves } \\
\text { their blood glucose, BP, and lipid levels. } \\
\text { TBC interventions also increase the pro- } \\
\text { portion of patients who reach target blood } \\
\text { glucose, BP, and lipid levels }\end{array}$ \\
\hline & Han, $2019^{51}$ & $\begin{array}{l}\text { Positive } \\
\text { benefit }\end{array}$ & Yes & $\begin{array}{l}\text { CHC interventions were generally effective in } \\
\text { HbA1c reduction either via individual } \\
\text { education, group education or both although } \\
\text { insignificant HbA1c reductions were also } \\
\text { noted in nine studies. CHC interventions } \\
\text { were also effective in improving lipids }\end{array}$ & $\begin{array}{l}\text { CHC interventions are in general effective } \\
\text { in promoting glucose control when using } \\
\text { face-to-face interactions in low-income, } \\
\text { underserved, and racial and ethnic minority } \\
\text { patients with diabetes. }\end{array}$ \\
\hline & Warner, $2019^{50}$ & $\begin{array}{l}\text { Potential } \\
\text { benefit }\end{array}$ & Yes & $\begin{array}{l}\text { The } 31 \text { included studies demonstrated } \\
\text { community-based SMP programs actively } \\
\text { engaged participants and provided strategies } \\
\text { to improve health behavior or care of their } \\
\text { condition. Seventy-nine percent of studies } \\
\text { reported significant differences }\end{array}$ & $\begin{array}{l}\text { Self Management Programs are not } \\
\text { supporting older adults to use strategies to } \\
\text { address the impact of conditions on their } \\
\text { everyday lives, addressing the needs of } \\
\text { older adults with multiple conditions, nor } \\
\text { assessing outcomes that align with the } \\
\text { strategies }\end{array}$ \\
\hline & Luedke, $2019^{49}$ & $\begin{array}{l}\text { Unclear } \\
\text { benefit }\end{array}$ & Yes & $\begin{array}{l}\text { Limited evidence for benefit on selected } \\
\text { primary or secondary outcomes. Educational } \\
\text { self-management interventions may improve } \\
\text { the use of self-management practices, and } \\
\text { quality of life may improve with therapy-based } \\
\text { self-management approaches. }\end{array}$ & $\begin{array}{l}\text { These self-management interventions } \\
\text { showed clinically important benefit for only } \\
\text { a limited number of outcomes, but the } \\
\text { confidence in these findings was mostly } \\
\text { low. Further, there is unexplained } \\
\text { variability in the effect of education } \\
\text { interventions on quality }\end{array}$ \\
\hline
\end{tabular}




\begin{tabular}{|c|c|c|c|c|c|}
\hline $\begin{array}{l}\text { Type of } \\
\text { Strategy }\end{array}$ & Author, Year & $\begin{array}{l}\text { Overall } \\
\text { Findings }\end{array}$ & $\begin{array}{l}\text { Strength of } \\
\text { Evidence } \\
\text { Reported? }\end{array}$ & Findings & Conclusion Sentence \\
\hline & Debon, $2019^{48}$ & $\begin{array}{l}\text { Positive } \\
\text { benefit }\end{array}$ & No & $\begin{array}{l}\text { The main lifestyle changes were the reduction } \\
\text { of body weight, promotion of healthy eating, } \\
\text { and adherence to the regular practice of } \\
\text { physical exercises. Another significant result } \\
\text { is the decrease in blood pressure and } \\
\text { glycemic levels. }\end{array}$ & $\begin{array}{l}\text { Technology can facilitate health care with } \\
\text { simple messages and alerts that aid in } \\
\text { adherence to treatment. Changes in } \\
\text { lifestyle with the use of applications are } \\
\text { remarkable }\end{array}$ \\
\hline & $\begin{array}{l}\text { Dounavi, } \\
2019^{69}\end{array}$ & $\begin{array}{l}\text { Positive } \\
\text { benefit }\end{array}$ & Yes & $\begin{array}{l}\text { mHealth apps can be effective in the self- } \\
\text { management of weight, such as in reducing } \\
\text { weight, and improving health indicators, such } \\
\text { as glucose levels and blood pressure; one } \\
\text { study found no significant improvements as a } \\
\text { result of using a PDA for self-monitoring }\end{array}$ & $\begin{array}{l}\text { There is sufficient consensus across } \\
\text { studies that mHealth apps are acceptable } \\
\text { by patients and effective in producing } \\
\text { weight loss through lifestyle changes in } \\
\text { eating behaviors and physical activity } \\
\text { patterns. }\end{array}$ \\
\hline & Zhao, $2016^{57}$ & $\begin{array}{l}\text { Positive } \\
\text { benefit }\end{array}$ & Yes & $\begin{array}{l}\text { The pooled main outcomes by random-effects } \\
\text { model showed significant improvements in } \\
\text { HbA1c, self-efficacy, and diabetes knowledge, } \\
\text { but not in BMI. we cannot draw any real } \\
\text { conclusions regarding QoL due to } \\
\text { heterogeneity. }\end{array}$ & $\begin{array}{l}\text { To get theory-based interventions to } \\
\text { produce more effects, the role of patients } \\
\text { should be more involved and stronger and } \\
\text { the education team should be trained } \\
\text { beyond the primary preparation for the } \\
\text { self-management education program. }\end{array}$ \\
\hline & $\begin{array}{l}\text { Sakakibara, } \\
2016^{34}\end{array}$ & $\begin{array}{l}\text { Positive } \\
\text { benefit }\end{array}$ & Yes & $\begin{array}{l}\text { The model estimating an effect averaged } \\
\text { across all stroke risk factors was not } \\
\text { significant, but became significant when four } \\
\text { low-quality studies were removed (SMD = } \\
0.10 \text { [95\% } \% \text { Cl = } 0.02 \text { to } 0.17], 12=0 \%, p= \\
0.01) \text {. }\end{array}$ & $\begin{array}{l}\text { Self-management interventions appear to } \\
\text { be effective at improving overall risk factor } \\
\text { control; however, more high-quality } \\
\text { research is needed to corroborate this } \\
\text { observation. Self-management has a } \\
\text { greater effect on lifestyle behavior risk } \\
\text { factors }\end{array}$ \\
\hline & $\begin{array}{l}\text { Noonan, } \\
2019^{32}\end{array}$ & $\begin{array}{l}\text { No } \\
\text { benefit }\end{array}$ & Yes & $\begin{array}{l}\text { Compared with usual care, there was similar } \\
\text { magnitude in mean improvement in patient } \\
\text { health-related quality of life with self } \\
\text { management interventions in trials involving } \\
\text { caregivers (SMD: } 0.23,95 \% \text { confidence } \\
\text { interval: }-0.15-0.61 \text { ) }\end{array}$ & $\begin{array}{l}\text { Within the methodological constraints of } \\
\text { this study, our results indicate that } \\
\text { involving caregivers in self-management } \\
\text { interventions does not result in additional } \\
\text { improvement in patient health-related } \\
\text { quality of life in heart failure or chronic } \\
\text { obstructive }\end{array}$ \\
\hline & Plow, $2016^{31}$ & $\begin{array}{l}\text { Potential } \\
\text { benefit }\end{array}$ & No & $\begin{array}{l}\text { Reported intervention effects on health and } \\
\text { function included statistically significant } \\
\text { improvements across time or between groups } \\
\text { in patient-reported physical function }(n=5) \text {, } \\
\text { fatigue }(n=3) \text {, patient-reported mental health } \\
(n=3)\end{array}$ & $\begin{array}{l}\text { To advance the science of tailoring self- } \\
\text { management interventions, we } \\
\text { recommend conducting comparative } \\
\text { effectiveness research and further } \\
\text { developing a taxonomy to standardize } \\
\text { descriptions of tailoring. }\end{array}$ \\
\hline
\end{tabular}




\begin{tabular}{|c|c|c|c|c|c|}
\hline $\begin{array}{l}\text { Type of } \\
\text { Strategy }\end{array}$ & Author, Year & $\begin{array}{l}\text { Overall } \\
\text { Findings }\end{array}$ & $\begin{array}{l}\text { Strength of } \\
\text { Evidence } \\
\text { Reported? }\end{array}$ & Findings & Conclusion Sentence \\
\hline & $\begin{array}{l}\text { Stenberg, } \\
2016^{30}\end{array}$ & $\begin{array}{l}\text { Positive } \\
\text { benefit }\end{array}$ & Yes & $\begin{array}{l}\text { The participants experienced the programs as } \\
\text { beneficial according to less symptom distress } \\
\text { and greater awareness of their own health, } \\
\text { improved self-management strategies, peer } \\
\text { support, learning and hope. }\end{array}$ & $\begin{array}{l}\text { A substantial evidence base (Quantitative } \\
\text { and Qualitative studies) supports the } \\
\text { conclusion that participating in a group } \\
\text { based patient education programs aimed } \\
\text { at promoting self management in different } \\
\text { ways have been experienced as beneficial } \\
\text { for the participant }\end{array}$ \\
\hline & Hooft, $2016^{29}$ & $\begin{array}{l}\text { Potential } \\
\text { benefit }\end{array}$ & Yes & $\begin{array}{l}\text { Nurse-led interventions focusing on patients' } \\
\text { intrinsic motivation and self-efficacy were } \\
\text { most successful. Least successful were } \\
\text { interventions providing solely education aimed } \\
\text { at changing patients' behavior. }\end{array}$ & $\begin{array}{l}\text { Successful interventions focus on patients' } \\
\text { intrinsic processes (i.e. motivation or self- } \\
\text { efficacy). This would guide nurses to } \\
\text { decide what self-management support } \\
\text { intervention they can best use in their } \\
\text { specific setting and patient group. }\end{array}$ \\
\hline & Kelly, $2018^{62}$ & $\begin{array}{l}\text { Unclear } \\
\text { benefit }\end{array}$ & Yes & $\begin{array}{l}\text { Primary QOL: Mean SGRQ Total scores in } \\
\text { both groups improved over time but were not } \\
\text { significantly different at six weeks', three and } \\
12 \text { months' fol- low-up ( } 6 \text { weeks MD }-12.70 \text {, } \\
95 \% \mathrm{Cl}-30.39 \text { to } 4.99 ; 3 \text { months MD }-9.15 \text {, } \\
95 \% \mathrm{Cl}-28.08 \text { to } 9.78 ; 12 \text { months MD }-1\end{array}$ & $\begin{array}{l}\text { There is inadequate published evidence to } \\
\text { guide clinical decisions as to the potential } \\
\text { benefits and risks of self-management } \\
\text { interventions for people with non-cystic } \\
\text { fibrosis bronchiectasis. The evidence was } \\
\text { limited by a number of factors }\end{array}$ \\
\hline & Teljeur, $2016^{27}$ & $\begin{array}{l}\text { Positive } \\
\text { benefit }\end{array}$ & Yes & $\begin{array}{l}\text { There was reasonably consistent evidence } \\
\text { across } 22 \text { studies evaluating education self- } \\
\text { management support programs suggesting } \\
\text { these interventions are cost-effective or } \\
\text { superior to usual care. Telemedicine-type } \\
\text { interventions were more expensive than usual }\end{array}$ & $\begin{array}{l}\text { Self-management support education } \\
\text { programs may be cost-effective. There } \\
\text { was limited evidence regarding other } \\
\text { formats of self-management support } \\
\text { interventions. The poor quality of many of } \\
\text { the studies undermines the evidence base }\end{array}$ \\
\hline & Pereira, $2015^{88}$ & $\begin{array}{l}\text { Positive } \\
\text { benefit }\end{array}$ & No & $\begin{array}{l}\text { DSME delivered via the Internet is effective at } \\
\text { improving measures of glycemic control and } \\
\text { diabetes knowledge compared with usual care } \\
\text { (printed written interventions). In addition, } \\
\text { results demonstrate that improved eating } \\
\text { habits and increased attendance }\end{array}$ & $\begin{array}{l}\text { Internet-delivered diabetes education has } \\
\text { the added benefit of easier access for } \\
\text { many individuals, and patients can self- } \\
\text { pace themselves through materials. More } \\
\text { research on the cost-benefits of Internet } \\
\text { diabetes education }\end{array}$ \\
\hline & $\begin{array}{l}\text { Whiteman, } \\
2016^{35}\end{array}$ & $\begin{array}{l}\text { Potential } \\
\text { benefit }\end{array}$ & Yes & $\begin{array}{l}\text { Most studies demonstrated feasibility, } \\
\text { acceptability, and preliminary effectiveness; } \\
\text { however, clinical effectiveness could not be } \\
\text { established in most of the studies due to } \\
\text { methodological limitations. }\end{array}$ & $\begin{array}{l}\text { Integrated medical and psychiatric illness } \\
\text { self-management interventions appear } \\
\text { feasible and acceptable, with high potential } \\
\text { for clinical effectiveness. However, } \\
\text { implementation considerations were rarely } \\
\text { considered in intervention development }\end{array}$ \\
\hline
\end{tabular}




\begin{tabular}{|c|c|c|c|c|c|}
\hline $\begin{array}{l}\text { Type of } \\
\text { Strategy }\end{array}$ & Author, Year & $\begin{array}{l}\text { Overall } \\
\text { Findings }\end{array}$ & $\begin{array}{l}\text { Strength of } \\
\text { Evidence } \\
\text { Reported? }\end{array}$ & Findings & Conclusion Sentence \\
\hline & $\begin{array}{l}\text { Zomahoun, } \\
2016^{37}\end{array}$ & $\begin{array}{l}\text { Positive } \\
\text { benefit }\end{array}$ & Yes & $\begin{array}{l}\text { Interventions that were based on } \mathrm{Ml} \text { only }[\mathrm{b} 1 / 4 \\
\left.0.183,95 \% \mathrm{Cl}^{1 / 4}(0.004,0.362)\right] \text { or those in } \\
\text { which interventionists were coached during } \\
\text { intervention implementation }[\mathrm{b} 1 / 40.465,95 \% \\
\mathrm{Cl} 1 / 4(0.028,0.902)] \text { were the most effective. } \\
\mathrm{Ml} \text { interventions that were delivered }\end{array}$ & $\begin{array}{l}\text { This synthesis of RCTs suggests that } \\
\text { pooled MI intervention effect size on } \\
\text { medication adherence is positive yet very } \\
\text { small. MI interventions might be effective } \\
\text { at enhancing of medication adherence in } \\
\text { adults treated for chronic diseases }\end{array}$ \\
\hline & $\begin{array}{l}\text { Massimi, } \\
2017^{68}\end{array}$ & $\begin{array}{l}\text { Positive } \\
\text { benefit }\end{array}$ & Yes & $\begin{array}{l}\text { Meta-analyses on systolic (SBP) and diastolic } \\
\text { (DBP) blood pressure reduction (10 } \\
\text { studiesĐ3,881 patients) and HbA1c reduction } \\
\text { ( } 7 \text { studiesĐ2,669 patients) were carried-out. } \\
\text { The pooled MD were: SBP }-3.04(95 \% \mathrm{Cl}- \\
5.01 Đ-1.06), \text { DBP }-1.42(95 \% \mathrm{Cl}-1.42 Đ-0.49) \\
\text { an }\end{array}$ & $\begin{array}{l}\text { Meta-analyses of subgroups showed, } \\
\text { among others, a statistically significant } \\
\text { effect if the interventions were delivered to } \\
\text { patients with diabetes (SBP) or CVD } \\
\text { (DBP), if the nurses were specifically } \\
\text { trained, if the studies had a sample size } \\
\text { higher than } 200\end{array}$ \\
\hline & $\begin{array}{l}\text { Clarkesmith, } \\
2017^{67}\end{array}$ & $\begin{array}{l}\text { Unclear } \\
\text { benefit }\end{array}$ & Yes & $\begin{array}{l}\text { There was uncertainty about the effect of self- } \\
\text { monitoring plus education on the percentage } \\
\text { of time the INR was within the therapeutic } \\
\text { range. Small and positive effects on anxiety } \\
\text { and depression in individuals who received } \\
\text { education compared to those who r }\end{array}$ & $\begin{array}{l}\text { Patients participating in both educational } \\
\text { interventions and self monitoring } \\
\text { interventions (with education) appear to } \\
\text { spend more time within the therapeutic } \\
\text { INR range, but pooled analyses of the AF } \\
\text { data did not significantly favor self- } \\
\text { monitoring plus education }\end{array}$ \\
\hline & Cho, $2017^{66}$ & $\begin{array}{l}\text { Unclear } \\
\text { benefit }\end{array}$ & Yes & $\begin{array}{l}\text { Four studies of technology-mediated } \\
\text { interventions resulted in improvement in QoL. } \\
\text { Four studies considered QoL as a secondary } \\
\text { outcome neutral impact on QoL and one study } \\
\text { resulted in a negative. Overall, four studies } \\
\text { had a low risk of bias }\end{array}$ & $\begin{array}{l}\text { The evidence to support the improvement } \\
\text { of QoL using technology-mediated } \\
\text { interventions is insufficient. This lack of } \\
\text { research highlights the need for increased } \\
\text { study of QoL as an outcome measure and } \\
\text { the need for consistent measures }\end{array}$ \\
\hline & Kim, $2017^{65}$ & $\begin{array}{l}\text { Unclear } \\
\text { benefit }\end{array}$ & No & $\begin{array}{l}\text { Data from } 13 \text { RCTs showed that patients with } \\
\text { heart failure and asthma reported improved } \\
\text { quality of life in } 4 \text { trials, the most frequently } \\
\text { reported health improvement was HbA1c } \\
\text { control among patients with diabetes in } 3 \text { trials }\end{array}$ & $\begin{array}{l}\text { Limitations of current research included a } \\
\text { lack of gerontological focus, dominance of } \\
\text { preexperimental design, narrow research } \\
\text { scope, inadequate support for participants, } \\
\text { and insufficient evidence for clinical } \\
\text { outcome. }\end{array}$ \\
\hline & Hecke, $2016^{28}$ & $\begin{array}{l}\text { Unclear } \\
\text { benefit }\end{array}$ & Yes & $\begin{array}{l}\text { Two high-quality studies showed inconsistent } \\
\text { results regarding reduction of death rates, } \\
\text { emergency department admissions and } \\
\text { hospitalization. Although with acceptable } \\
\text { quality of studies, there is no clear evidence } \\
\text { for the effectiveness }\end{array}$ & $\begin{array}{l}\text { Limited evidence was found for self- } \\
\text { management support interventions in } \\
\text { chronically ill patients with low socio- } \\
\text { economic status. Essential characteristics } \\
\text { and component(s) of effective self- } \\
\text { management support interventions for } \\
\text { these patients }\end{array}$ \\
\hline
\end{tabular}




\begin{tabular}{|c|c|c|c|c|c|}
\hline $\begin{array}{l}\text { Type of } \\
\text { Strategy }\end{array}$ & Author, Year & $\begin{array}{l}\text { Overall } \\
\text { Findings }\end{array}$ & $\begin{array}{l}\text { Strength of } \\
\text { Evidence } \\
\text { Reported? }\end{array}$ & Findings & Conclusion Sentence \\
\hline & Risling, $2017^{84}$ & $\begin{array}{l}\text { Unclear } \\
\text { benefit }\end{array}$ & No & $\begin{array}{l}4 \text { of } 19 \text { (mostly non quantitative studies) were } \\
\text { found to have used specific patient } \\
\text { empowerment measures. }\end{array}$ & $\begin{array}{l}\text { Few non-qualitative studies; overall, } \\
\text { studies lack of conceptual clarity in patient } \\
\text { empowerment research, and this has } \\
\text { extended to study within the eHealth } \\
\text { context }\end{array}$ \\
\hline & $\begin{array}{l}\text { Palacios, } \\
2017^{79}\end{array}$ & $\begin{array}{l}\text { Potential } \\
\text { benefit }\end{array}$ & Yes & $\begin{array}{l}\text { Statistically significant difference/ } \\
\text { improvement in intervention group for wt loss, } \\
\text { angina symptoms and frequency ( } 2 \text { trials), in } \\
\text { dietary outcomes and increased physical } \\
\text { activity ( } 5 \text { trials), improved QoL over longer } \\
\text { f/up ( } 3 \text { trials), and mood symptoms }\end{array}$ & $\begin{array}{l}\text { Internet-delivered self-management } \\
\text { support for improving CHD, well-being and } \\
\text { self-management related outcomes is } \\
\text { potentially effective, but existing trial } \\
\text { evidence is insufficient to draw definitive } \\
\text { conclusions. There is a lack of evidence on } \\
\text { the impact }\end{array}$ \\
\hline \multirow[t]{4}{*}{$\begin{array}{l}\text { Self } \\
\text { Management } \\
\text { Support - } \\
\text { Adults and } \\
\text { Children }\end{array}$} & Barello, $2016^{14}$ & $\begin{array}{l}\text { Unclear } \\
\text { benefit }\end{array}$ & No & $\begin{array}{l}\text { On the one hand, we described the main } \\
\text { patient engagement outcomes of eHealth } \\
\text { interventions; they appear to confirm how } \\
\text { internet technologies in healthcare are able to } \\
\text { give patients a starring role in their own } \\
\text { healthcare. }\end{array}$ & $\begin{array}{l}\text { To sum up, the eHealth interventions } \\
\text { reviewed were mainly devoted to foster } \\
\text { only one or two experiential dimensions of } \\
\text { patient engagement (i.e., alternatively } \\
\text { cognitive, emotional or behavioral } \\
\text { experiential dimensions related to the } \\
\text { healthcare management }\end{array}$ \\
\hline & $\begin{array}{l}\text { Lancaster, } \\
2018^{17}\end{array}$ & $\begin{array}{l}\text { Positive } \\
\text { benefit }\end{array}$ & Yes & $\begin{array}{l}\text { 14 RCTs found statistically significant } \\
\text { increases in positive medication changes as a } \\
\text { result of using eHealth tools, as did the single } \\
\text { open-label study. Moreover, } 8 \text { RCTs found } \\
\text { improvement in patient symptoms following } \\
\text { eHealth tool use, especially in adolescents }\end{array}$ & $\begin{array}{l}\text { Patients generally found eHealth tools } \\
\text { useful in improving communication with } \\
\text { health care providers. Moreover, health- } \\
\text { related outcomes among frequent eHealth } \\
\text { tool users improved in comparison with } \\
\text { individuals who did not use eHealth tools } \\
\text { frequently. }\end{array}$ \\
\hline & Lycett, $2018^{16}$ & $\begin{array}{l}\text { Positive } \\
\text { benefit }\end{array}$ & Yes & $\begin{array}{l}\text { A total of } 3 \text { studies reported a significant } \\
\text { positive effect of the intervention on } \\
\text { adherence; } 3 \text { studies reported a significant } \\
\text { positive effect of the intervention on self- } \\
\text { management behavior [54], } 2 \text { studies reported } \\
\text { a significant positive effect on asthma }\end{array}$ & $\begin{array}{l}\text { Our findings suggest that theory-based } \\
\text { digital interventions to enhance asthma } \\
\text { self-management can be effective at } \\
\text { improving adherence and self- } \\
\text { management and that more extensive use } \\
\text { of theory in the development and } \\
\text { application of digital interventions }\end{array}$ \\
\hline & Bashi, $2018^{15}$ & $\begin{array}{l}\text { Positive } \\
\text { benefit }\end{array}$ & Yes & $\begin{array}{l}\text { 4 RCTs showed statistically significant effects } \\
\text { of smartphone-based interventions on health } \\
\text { outcomes, including the patient engagement } \\
\text { level, hemoglobin A, weight loss, and } \\
\text { depression while } 3 \text { RCTS showed no } \\
\text { difference on cardiorespiratory fitness }\end{array}$ & $\begin{array}{l}\text { this review generally support that patients } \\
\text { with diverse conditions benefit from } \\
\text { mobile-based educational interventions. } \\
\text { However, we were unable to identify any } \\
\text { effective specific structure or strategy for } \\
\text { the delivery of such interventions }\end{array}$ \\
\hline
\end{tabular}




\begin{tabular}{|c|c|c|c|c|c|}
\hline $\begin{array}{l}\text { Type of } \\
\text { Strategy }\end{array}$ & Author, Year & $\begin{array}{l}\text { Overall } \\
\text { Findings }\end{array}$ & $\begin{array}{l}\text { Strength of } \\
\text { Evidence } \\
\text { Reported? }\end{array}$ & Findings & Conclusion Sentence \\
\hline & Niznik, $2018^{13}$ & $\begin{array}{l}\text { Positive } \\
\text { benefit }\end{array}$ & No & $\begin{array}{l}\text { Studies reporting adherence outcomes had an } \\
\text { overall positive rate of } 62.5 \% \text { ( } 5 \text { of } 8 \text { ). Studies } \\
\text { reporting clinical disease management } \\
\text { outcomes had an overall positive rate of } \\
67.8 \% \text { (19 of } 28) \text {. }\end{array}$ & $\begin{array}{l}\text { Clinical pharmacy telemedicine } \\
\text { interventions in the outpatient or } \\
\text { ambulatory setting, primarily via phone, } \\
\text { have an overall positive impact on } \\
\text { outcomes related to clinical disease } \\
\text { management, patient self-management, } \\
\text { and adherence }\end{array}$ \\
\hline & Kew, $2016^{18}$ & $\begin{array}{l}\text { Unclear } \\
\text { benefit }\end{array}$ & Yes & $\begin{array}{l}\text { Evidence from these studies did not show } \\
\text { clearly whether asthma telemonitoring with } \\
\text { feedback from a healthcare professional } \\
\text { increases or decreases the odds of } \\
\text { exacerbations that require a course of oral } \\
\text { steroids (OR 0.93, 95\% confidence Interval } \\
\text { (Cl) } 0 \text {. }\end{array}$ & $\begin{array}{l}\text { Current evidence does not support the } \\
\text { widespread implementation of } \\
\text { telemonitoring with healthcare provider } \\
\text { feedback between asthma clinic visits. } \\
\text { Studies have not yet proven that additional } \\
\text { telemonitoring strategies lead to better } \\
\text { symptom control or reduction }\end{array}$ \\
\hline & $\begin{array}{l}\text { Graham, } \\
2016^{19}\end{array}$ & $\begin{array}{l}\text { Potential } \\
\text { benefit }\end{array}$ & Yes & $\begin{array}{l}\text { In summary, with one exception (Nordin \& } \\
\text { Rorsman, 2012), ACT interventions were } \\
\text { consistently associated with post-intervention } \\
\text { improvements (i.e., reductions) in distress. } \\
\text { However, bar one highly- supportive but } \\
\text { lower-quality study (Rost et al., 2012) }\end{array}$ & $\begin{array}{l}\text { ACT has been applied in many different } \\
\text { ways within a range of long-term } \\
\text { conditions. However, there have been no } \\
\text { trials of ACT for improving medication non- } \\
\text { adherence. Most of the included studies } \\
\text { were of low quality and there were very } \\
\text { few RCTs. }\end{array}$ \\
\hline & Kruse, $2015^{24}$ & $\begin{array}{l}\text { Unclear } \\
\text { benefit }\end{array}$ & No & $\begin{array}{l}\text { Patient portals showed significant } \\
\text { improvements in patient self-management of } \\
\text { chronic disease and improve the quality of } \\
\text { care provided by providers. Patient-provider } \\
\text { communication improved in } 10 \text { of } 27 \text { articles } \\
\text { as reported by both patients and providers. }\end{array}$ & $\begin{array}{l}\text { Mixed attitudes from patients and their } \\
\text { providers regarding the use of patient } \\
\text { portals to manage their chronic disease. }\end{array}$ \\
\hline & Chi, $2015^{21}$ & $\begin{array}{l}\text { Positive } \\
\text { benefit }\end{array}$ & Yes & $\begin{array}{l}\text { Sixty-two articles (95\%) reported that } \\
\text { caregivers had significant improvements in } \\
\text { outcomes. The outcomes included enhanced } \\
\text { psychological health (less anxiety, } \\
\text { depression, stress, burden, irritation and } \\
\text { isolation) (44\%), higher } \\
\text { satisfaction/confidence }\end{array}$ & $\begin{array}{l}\text { The review showed that telehealth tools } \\
\text { can enhance care not only to patients but } \\
\text { also to family caregivers. As caregivers } \\
\text { take care of a loved one, they may benefit } \\
\text { from increased and more efficient } \\
\text { communication with health care providers } \\
\text { or other caregivers }\end{array}$ \\
\hline & $\begin{array}{l}\text { Pamungkas, } \\
2017^{23}\end{array}$ & $\begin{array}{l}\text { Positive } \\
\text { benefit }\end{array}$ & No & $\begin{array}{l}\text { The combination of didactic with other } \\
\text { methods such as participatory learning, goal } \\
\text { setting, action planning and problem-solving } \\
\text { had a positive impact on health outcomes and } \\
\text { improved health behaviors ( } 4 \text { trials). }\end{array}$ & $\begin{array}{l}\text { Overall, family support had a positive } \\
\text { impact on healthy diet, increased } \\
\text { perceived support, higher self-efficacy, } \\
\text { improved psychological well-being and } \\
\text { better glycemic control. No meta-analysis } \\
\text { done. }\end{array}$ \\
\hline
\end{tabular}




\begin{tabular}{|c|c|c|c|c|c|}
\hline $\begin{array}{l}\text { Type of } \\
\text { Strategy }\end{array}$ & Author, Year & $\begin{array}{l}\text { Overall } \\
\text { Findings }\end{array}$ & $\begin{array}{l}\text { Strength of } \\
\text { Evidence } \\
\text { Reported? }\end{array}$ & Findings & Conclusion Sentence \\
\hline & Yin, $2019^{20}$ & $\begin{array}{l}\text { Unclear } \\
\text { benefit }\end{array}$ & No & $\begin{array}{l}\text { No findings presented for the self-monitoring } \\
\text { or self-management outcomes; Only } 3 \text { studies } \\
\text { reported the impact of the intervention on } \\
\text { health-related measures }[12,14] \text {. Specifically, } \\
\text { Bächlin [17] found that the intervention had a } \\
\text { sensitivity of } 73.1 \%\end{array}$ & $\begin{array}{l}\text { The emerging nature of the field is } \\
\text { reflected in the small number of included } \\
\text { studies, their recent time of publication (all } \\
\text { after 2010), and the predominance of } \\
\text { quasi-experimental study designs. RCTs } \\
\text { are needed to evaluate the effectiveness }\end{array}$ \\
\hline & Hill, $2015^{22}$ & $\begin{array}{l}\text { Positive } \\
\text { benefit }\end{array}$ & Yes & $\begin{array}{l}\text { Fifteen of } 16 \text { eligible studies reported a } \\
\text { positive intervention effect in at least one } \\
\text { outcome. (Improved wt loss, self efficacy, SM, } \\
\text { life style change, QoL) There was } \\
\text { heterogeneity in terms of health coaching } \\
\text { interventions with different emthodology }\end{array}$ & $\begin{array}{l}\text { Health coaching is a promising strategy for } \\
\text { health improvements; however, future } \\
\text { research should ensure clarity in reporting } \\
\text { intervention details, clearer definitions of } \\
\text { health coaching/theoretical bases, } \\
\text { consistency in reporting BCTs }\end{array}$ \\
\hline \multirow[t]{4}{*}{$\begin{array}{l}\text { Self } \\
\text { Management } \\
\text { Support - } \\
\text { Children Only }\end{array}$} & Low, $2019^{4}$ & $\begin{array}{l}\text { No } \\
\text { benefit }\end{array}$ & Yes & $\begin{array}{l}\text { The combined data for meta-analysis of } 6 \\
\text { trails showed that there was no statistically } \\
\text { significant group difference in quality of life } \\
\text { ( } n=3 \text {, standardized mean difference }-0.15 \text {, } \\
95 \% \mathrm{Cl}-0.52 \text { to } 0.22 ; \mathrm{P}=.43 \text { ), self-efficacy } \\
(\mathrm{n}=3 \text {, standardized mean difference) }\end{array}$ & $\begin{array}{l}\text { AYAs were receptive to receiving } \\
\text { information through a website or mobile } \\
\text { app, which is a first step to engaging them } \\
\text { in their own care. Although no conclusion } \\
\text { can be drawn on an effective intervention } \\
\text { design because of the lack of intervention } \\
\text { efficacy }\end{array}$ \\
\hline & Saxby, $2018^{5}$ & $\begin{array}{l}\text { Positive } \\
\text { benefit }\end{array}$ & Yes & $\begin{array}{l}\text { The matching of teaching approaches to } \\
\text { children's age/ developmental stage was } \\
\text { identified as an overarching concept in CCSM } \\
\text { educational interventions. It is important that } \\
\text { knowledge and skills are taught to the right } \\
\text { level of complexity. }\end{array}$ & $\begin{array}{l}\text { Self-management education interventions } \\
\text { that are tailored to a child's } \\
\text { age/developmental stage help children } \\
\text { with asthma, T1DM, and CF to develop } \\
\text { effective skills, attitudes, and behaviors to } \\
\text { manage their health. }\end{array}$ \\
\hline & Knafl, $2017^{6}$ & $\begin{array}{l}\text { Potential } \\
\text { benefit }\end{array}$ & No & $\begin{array}{l}\text { Interventions focusing on family functioning } \\
\text { targeted changing family roles, relationships, } \\
\text { or processes. In } 89 \% \text { of the interventions, } \\
\text { changes in these areas were linked to } \\
\text { improved condition control, adherence, or } \\
\text { child well-being. }\end{array}$ & $\begin{array}{l}\text { Investigators most often sought to improve } \\
\text { condition control or management, with } \\
\text { parent engagement focused on improving } \\
\text { capacity to manage the treatment regimen. } \\
\text { Few investigators addressed capacity } \\
\text { building in the context of family functioning. }\end{array}$ \\
\hline & $\begin{array}{l}\text { Clemente, } \\
2016^{12}\end{array}$ & $\begin{array}{l}\text { Potential } \\
\text { benefit }\end{array}$ & Yes & $\begin{array}{l}\text { The "Growing up and moving on" program } \\
\text { achieved improvements in health-related } \\
\text { quality of life, arthritis- related knowledge and } \\
\text { satisfaction with rheumatology care in both } \\
\text { adolescents and parents, and in vocational } \\
\text { readiness markers }\end{array}$ & $\begin{array}{l}\text { We have found several common core } \\
\text { features amongst the evaluated programs. } \\
\text { Firstly, a written transition policy, that } \\
\text { incorporates the views of the pediatric and } \\
\text { adult team, as well as the wider } \\
\text { multidisciplinary team as appropriate. }\end{array}$ \\
\hline
\end{tabular}




\begin{tabular}{|c|c|c|c|c|c|}
\hline $\begin{array}{l}\text { Type of } \\
\text { Strategy }\end{array}$ & Author, Year & $\begin{array}{l}\text { Overall } \\
\text { Findings }\end{array}$ & $\begin{array}{l}\text { Strength of } \\
\text { Evidence } \\
\text { Reported? }\end{array}$ & Findings & Conclusion Sentence \\
\hline & $\begin{array}{l}\text { Campbell, } \\
2016^{7}\end{array}$ & $\begin{array}{l}\text { Unclear } \\
\text { benefit }\end{array}$ & Yes & $\begin{array}{l}\text { There were positive outcomes in the patients' } \\
\text { knowledge of their condition following a nurse- } \\
\text { led, one- on-one intervention. The results also } \\
\text { suggested that interventions that use } \\
\text { technology may have a beneficial effect on } \\
\text { participants' self-efficacy }\end{array}$ & $\begin{array}{l}\text { The available evidence (four small studies; } \\
\mathrm{N}=238 \text { ), covers a limited range of } \\
\text { interventions developed to facilitate } \\
\text { transition in a limited number of clinical } \\
\text { conditions, with only four to } 12 \text { months } \\
\text { follow-up. }\end{array}$ \\
\hline & Bal, $2015^{3}$ & $\begin{array}{l}\text { Potential } \\
\text { benefit }\end{array}$ & Yes & $\begin{array}{l}\text { Our findings suggests that pediatric SMI might } \\
\text { be effective at influencing disease knowledge, } \\
\text { adherence, but not at symptom severity and } \\
\text { school attendance. Conflicting evidence was } \\
\text { found for effectiveness of SMI on dealing with } \\
\text { the chronic condition }\end{array}$ & $\begin{array}{l}\text { Adherence could be improved through } \\
\text { interventions focused on medical } \\
\text { management, provided individually in a } \\
\text { clinical or home setting by a mono- } \\
\text { disciplinary team. Interventions focused on } \\
\text { dealing with a chronic condition might be } \\
\text { provided individually }\end{array}$ \\
\hline & $\begin{array}{l}\text { Majeed-Ariss, } \\
2015^{8}\end{array}$ & $\begin{array}{l}\text { Unclear } \\
\text { benefit }\end{array}$ & Yes & $\begin{array}{l}\text { The one study that was a randomized } \\
\text { controlled trial did not report any quantitative } \\
\text { results as only } 3 \text { of the } 4 \text { participants } \\
\text { completed the trial. Cafazzo et al reported } \\
\text { means and standard deviations before and } \\
\text { after their intervention }\end{array}$ & $\begin{array}{l}\text { A key finding of the review is the paucity of } \\
\text { evidence-based apps that exist, in contrast } \\
\text { to the thousands of apps available on the } \\
\text { app market that are not evidence-based or } \\
\text { user or professional informed. }\end{array}$ \\
\hline & Charlier, $2015^{9}$ & $\begin{array}{l}\text { Potential } \\
\text { benefit }\end{array}$ & Yes & $\begin{array}{l}\text { Two studies reported significantly better self- } \\
\text { management in the game group than in the } \\
\text { control group after the intervention. For self- } \\
\text { management, pooled estimate of Hedges' gu } \\
\text { was } 0.310 \text { ( } 95 \% \text { confidence intervals, } 0.122- \\
0.497)\end{array}$ & $\begin{array}{l}\text { Two studies reported significantly better } \\
\text { self-management in the game group than } \\
\text { in the control group after the intervention. } \\
\text { Our meta-analysis showed that } \\
\text { educational video games are effective in } \\
\text { improving knowledge and self- } \\
\text { management of young persons }\end{array}$ \\
\hline & Sattoe, $2015^{10}$ & $\begin{array}{l}\text { Unclear } \\
\text { benefit }\end{array}$ & No & $\begin{array}{l}\text { This review revealed that most interventions } \\
\text { for young people represented in the literature } \\
\text { solely aim at medical management, like } \\
\text { interventions for adults. Six self-management } \\
\text { skills match the tasks of medical, role and } \\
\text { emotion management }\end{array}$ & $\begin{array}{l}\text { SMI relate to self-management tasks and } \\
\text { skill-building. Yet, conceptualizations of } \\
\text { self-management support often remained } \\
\text { unclear and content focuses predominantly } \\
\text { on the medical domain, neglecting psycho- } \\
\text { social challenges for chronically ill young } \\
\text { people }\end{array}$ \\
\hline & $\begin{array}{l}\text { Hamline, } \\
2018^{11}\end{array}$ & $\begin{array}{l}\text { Positive } \\
\text { benefit }\end{array}$ & Yes & $\begin{array}{l}\text { The overall risk ratio of readmissions for } \\
\text { studies in which an FEl alone was conducted } \\
\text { was } 0.78 \text { ( } 95 \% \mathrm{Cl}: 0.63-0.97 ; \mathrm{I} 2=66 \% \text {; } \\
\text { Supplemental Fig 3, Table 3). Overall,43\% of } \\
\text { FEl studies ( } 13 \text { of } 30) \text { were associated with } \\
\text { reduced readmissions. }\end{array}$ & $\begin{array}{l}\text { Specifically, the meta- analysis revealed } \\
\text { that pooling of CCIs and FEls in both } \\
\text { chronically ill and neonatal patient } \\
\text { populations was associated with a } \\
\text { decrease in readmissions, whereas single- } \\
\text { category interventions alone did not } \\
\text { decrease readmissions. }\end{array}$ \\
\hline
\end{tabular}




\begin{tabular}{|c|c|c|c|c|c|}
\hline $\begin{array}{l}\text { Type of } \\
\text { Strategy }\end{array}$ & Author, Year & $\begin{array}{l}\text { Overall } \\
\text { Findings }\end{array}$ & $\begin{array}{l}\text { Strength of } \\
\text { Evidence } \\
\text { Reported? }\end{array}$ & Findings & Conclusion Sentence \\
\hline & $\begin{array}{l}\text { Almutairi, } \\
2019^{82}\end{array}$ & $\begin{array}{l}\text { Positive } \\
\text { benefit }\end{array}$ & No & $\begin{array}{l}\text { Seven intervention demonstrated a significant } \\
\text { reduction in } \mathrm{HbA} 1 \mathrm{c} \text {, ranged from } 0.36 \text { to } \\
0.80 \% \text {. All interventions presented an } \\
\text { improvement in at least one self-management } \\
\text { behavior. }\end{array}$ & $\begin{array}{l}\text { Patient activation intervention showed a } \\
\text { significant positive effect on T2DM } \\
\text { glycemic control and SMBs, particularly } \\
\text { physical activity, healthy diet, foot care and } \\
\text { blood glucose self-monitoring. }\end{array}$ \\
\hline & Warner, $2015^{80}$ & $\begin{array}{l}\text { Potential } \\
\text { benefit }\end{array}$ & Yes & $\begin{array}{l}\text { Six of the nine studies assessed participation } \\
\text { in everyday life activities or functional ability, } \\
\text { two of the six demonstrated statistically } \\
\text { significant differences between the self- } \\
\text { management interventions and the controls } \\
\text { over time in stroke patients. }\end{array}$ & $\begin{array}{l}\text { There are indications that self- } \\
\text { management programs in stroke patients } \\
\text { can significantly increase participation and } \\
\text { functional ability. However, the high level } \\
\text { of clinical heterogeneity in program } \\
\text { delivery, outcomes and level of stroke } \\
\text { severity }\end{array}$ \\
\hline \multirow[t]{4}{*}{$\begin{array}{l}\text { Shared } \\
\text { Decision } \\
\text { Making - Adults } \\
\text { Only }\end{array}$} & $\begin{array}{l}\text { Kashaf, } \\
2017^{121}\end{array}$ & $\begin{array}{l}\text { Positive } \\
\text { benefit }\end{array}$ & Yes & Same as conclusion & $\begin{array}{l}\text { We found substantial evidence of an } \\
\text { association between SDM and improved } \\
\text { decision quality, patient knowledge and } \\
\text { patient risk perception. We found little } \\
\text { evidence of an association between SDM } \\
\text { and glycemic control, patient satisfaction, } \\
\text { quality of life }\end{array}$ \\
\hline & $\begin{array}{l}\text { Légaré, } \\
2018^{113}\end{array}$ & $\begin{array}{l}\text { Unclear } \\
\text { benefit }\end{array}$ & Yes & $\begin{array}{l}\text { It is uncertain if interventions targeting } \\
\text { patients when compared with usual care } \\
\text { increase SDM whether measured by } \\
\text { observation or reported by patients, reduce } \\
\text { decision regret, improve physical or mental } \\
\text { health-related quality of life, affect } \\
\text { consultation }\end{array}$ & $\begin{array}{l}\text { It is uncertain whether any interventions for } \\
\text { increasing the use of SDM by healthcare } \\
\text { professionals are effective because the } \\
\text { certainty of the evidence is low or very low. }\end{array}$ \\
\hline & $\begin{array}{l}\text { Martínez- } \\
\text { González, } \\
2018^{124}\end{array}$ & $\begin{array}{l}\text { No } \\
\text { benefit }\end{array}$ & Yes & $\begin{array}{l}\text { SDM improved knowledge (SMD } 0.23,95 \% \mathrm{Cl} \\
0.02 \text { to } 0.43 ; 2 \text { RCTs), but was not different to } \\
\text { usual care in reducing either patient } \\
\text { participation in prostate-specific antigen (PSA) } \\
\text { testing (RR } 1.03,95 \% \mathrm{Cl} 0.90 \text { to } 1.19 ; 2 \\
\text { RCTs) or decisional conflict }\end{array}$ & $\begin{array}{l}\text { There is currently insufficient evidence to } \\
\text { support a clear association of SDM on } \\
\text { patient- and SDM related outcomes for } \\
\text { decisions about PSA testing. Further } \\
\text { research needs to assess the clinical } \\
\text { effectiveness of SDM using well-defined } \\
\text { SDM interventions }\end{array}$ \\
\hline & $\begin{array}{l}\text { Johnson, } \\
2018^{111}\end{array}$ & $\begin{array}{l}\text { Unclear } \\
\text { benefit }\end{array}$ & Yes & $\begin{array}{l}\text { Four studies, none at low risk of bias, reported } \\
\text { a measure of shared decision making; the } \\
\text { intervention increased shared decision } \\
\text { making in one study. Four studies reported } \\
\text { blood pressure between } 6 \text { months and } 3 \\
\text { years after the intervention }\end{array}$ & $\begin{array}{l}\text { Despite widespread calls for shared } \\
\text { decision making to be embedded in health } \\
\text { care, there is little evidence to inform } \\
\text { shared decision making for hypertension, } \\
\text { one of the most common conditions } \\
\text { managed in primary care. }\end{array}$ \\
\hline
\end{tabular}




\begin{tabular}{|c|c|c|c|c|c|}
\hline $\begin{array}{l}\text { Type of } \\
\text { Strategy }\end{array}$ & Author, Year & $\begin{array}{l}\text { Overall } \\
\text { Findings }\end{array}$ & $\begin{array}{l}\text { Strength of } \\
\text { Evidence } \\
\text { Reported? }\end{array}$ & Findings & Conclusion Sentence \\
\hline & Berlin, 2018 & $\begin{array}{l}\text { Positive } \\
\text { benefit }\end{array}$ & Yes & $\begin{array}{l}\text { Studies suggest that DAs reduce patient- } \\
\text { reported decisional conflict (MD, }-4.55 \text { [ } 95 \% \\
\mathrm{Cl},-8.65 \text { to }-0.45], \mathrm{P}=0.03 \text { in the fixed- } \\
\text { effects model and } \mathrm{MD},-4.70 \text { [ } 95 \% \mathrm{Cl},-10.75 \\
\text { to } 1.34], \mathrm{P}=0.13 \text { in the random-effects } \\
\text { model). }\end{array}$ & $\begin{array}{l}\text { The existing literature suggests that DAs } \\
\text { reduce decisional conflict, improve self- } \\
\text { reported satisfaction with information, and } \\
\text { improve perceived involvement in the } \\
\text { decision-making process for women } \\
\text { considering breast reconstruction. }\end{array}$ \\
\hline & Baik, $2018^{108}$ & $\begin{array}{l}\text { Unclear } \\
\text { benefit }\end{array}$ & Yes & $\begin{array}{l}\text { Across studies, there were inconsistent } \\
\text { findings about whether the SDM intervention } \\
\text { improved patient/caregiver outcomes. The } \\
\text { quality of the studies was modest and } \\
\text { technology-enabled delivery modes (e.g., } \\
\text { video, DVD, web-based tool) were most } \\
\text { commonly used }\end{array}$ & $\begin{array}{l}\text { The findings from the studies that } \\
\text { examined the effects of the SDM } \\
\text { intervention on patient outcomes were } \\
\text { inconsistent, highlighting the need for } \\
\text { further SDM intervention studies among } \\
\text { diverse patient populations using } \\
\text { consistent measures. }\end{array}$ \\
\hline & $\begin{array}{l}\text { Wagner, } \\
2019^{100}\end{array}$ & $\begin{array}{l}\text { Unclear } \\
\text { benefit }\end{array}$ & Yes & $\begin{array}{l}\text { The results from the studies on patient } \\
\text { education showed a significant increase in } \\
\text { patient activation. Ten studies showed } \\
\text { statistically significant and, in part, clinically } \\
\text { relevant improvements in the target } \\
\text { parameters following the intervention. }\end{array}$ & $\begin{array}{l}\text { The differences found in the studies made } \\
\text { it difficult to compare the interventions and } \\
\text { the results. There is a need for studies that } \\
\text { systematically evaluate and further } \\
\text { develop interventions in this area to } \\
\text { promote shared decision-making. }\end{array}$ \\
\hline & $\begin{array}{l}\text { Vermunt, } \\
2017^{120}\end{array}$ & $\begin{array}{l}\text { Positive } \\
\text { benefit }\end{array}$ & Yes & $\begin{array}{l}\text { The four multifactorial interventions ( Guided } \\
\text { Care Program for Family and Friends, Guided } \\
\text { Care for patients and Helping Older People } \\
\text { Experience Success) had significant effects } \\
\text { on the application of goal setting, the number } \\
\text { of advance directives }\end{array}$ & $\begin{array}{l}\text { A specific focus on collaborative goal } \\
\text { setting and/or priority setting was mostly } \\
\text { found in a multifactorial intervention, which } \\
\text { seems to improve the application of goal } \\
\text { setting and the numbers of agreed upon } \\
\text { goals and advance directives. }\end{array}$ \\
\hline & $\begin{array}{l}\text { Goodridge, } \\
2019^{101}\end{array}$ & $\begin{array}{l}\text { Potential } \\
\text { benefit }\end{array}$ & No & $\begin{array}{l}\text { Qualitative synthesis: Interventions directed at } \\
\text { building patient capacity to participate in care } \\
\text { while hospitalized were categorized as those } \\
\text { related to improving: patient safety (20.9\%); } \\
\text { care coordination (5.7\%); effective treatment } \\
(5.7 \%)\end{array}$ & $\begin{array}{l}\text { The majority of studies to build capacity for } \\
\text { participation in care report one or more } \\
\text { positive outcomes, although a more } \\
\text { comprehensive analysis is warranted. }\end{array}$ \\
\hline & Porter, $2016^{115}$ & $\begin{array}{l}\text { Unclear } \\
\text { benefit }\end{array}$ & Yes & $\begin{array}{l}\text { There was significantly greater improvement } \\
\text { in } \mathrm{HbA} 1 \mathrm{c} \text { in the intervention group compared } \\
\text { to the control group in four of the nine studies; } \\
\text { Due to the multiple and varied components of } \\
\text { the intervention and usual care }\end{array}$ & $\begin{array}{l}\text { It is notable that in all four studies where } \\
\text { there were significant benefits for HbA1c, } \\
\text { the intervention involved providing the } \\
\text { patient with analysis and/or feedback from } \\
\text { a clinician on data captured. }\end{array}$ \\
\hline
\end{tabular}




\begin{tabular}{|c|c|c|c|c|c|}
\hline $\begin{array}{l}\text { Type of } \\
\text { Strategy }\end{array}$ & Author, Year & $\begin{array}{l}\text { Overall } \\
\text { Findings }\end{array}$ & $\begin{array}{l}\text { Strength of } \\
\text { Evidence } \\
\text { Reported? }\end{array}$ & Findings & Conclusion Sentence \\
\hline & $\begin{array}{l}\text { Mclntyre, } \\
2015^{105}\end{array}$ & $\begin{array}{l}\text { Potential } \\
\text { benefit }\end{array}$ & No & $\begin{array}{l}\text { Results are conflicting. Although CBLSs } \\
\text { scored highly in certain outcomes, standard } \\
\text { education was more suited to some patient } \\
\text { characteristics. Additionally, subgroups of } \\
\text { society (older and low socioeconomic } \\
\text { populations) were poorly represented in } \\
\text { studies. }\end{array}$ & $\begin{array}{l}\text { Up-to-date research on cancer patient } \\
\text { education is lacking. CBLSs proved to be } \\
\text { beneficial across the outcomes of } \\
\text { knowledge, satisfaction, and decision } \\
\text { making and also showed promise for } \\
\text { tailoring of information in some cancer } \\
\text { populations. }\end{array}$ \\
\hline & Jain, $2015^{104}$ & $\begin{array}{l}\text { Potential } \\
\text { benefit }\end{array}$ & Yes & $\begin{array}{l}\text { Low-quality evidence suggests that patients } \\
\text { who use a video decision aid are less likely to } \\
\text { indicate a preference for cardiopulmonary } \\
\text { resuscitation (pooled risk ratio, } 0.50(95 \% \mathrm{Cl} \\
0.27 \text { to } 0.95) ; 12=65 \%) \text {. }\end{array}$ & $\begin{array}{l}\text { Video decision aids may improve some } \\
\text { ACP-related outcomes. Before } \\
\text { recommending their use in clinical practice, } \\
\text { more evidence is needed to confirm these } \\
\text { findings and to evaluate the impact of } \\
\text { video decision aids when integrated into } \\
\text { patient care. }\end{array}$ \\
\hline & Irizarry, $2015^{102}$ & $\begin{array}{l}\text { Unclear } \\
\text { benefit }\end{array}$ & no & $\begin{array}{l}\text { research shows mixed results leading } \\
\text { researchers to believe that the relationship } \\
\text { between SM and utilization is more complex } \\
\text { than the simple substitution of online for in- } \\
\text { person care suggests. }\end{array}$ & $\begin{array}{l}\text { Health care delivery factors, mainly } \\
\text { provider endorsement and patient portal } \\
\text { usability also contribute to patient's ability } \\
\text { to engage through and with the patient } \\
\text { portal. Future directions of research should } \\
\text { focus on identifying specific populations }\end{array}$ \\
\hline & $\begin{array}{l}\text { Violette, } \\
2015^{103}\end{array}$ & $\begin{array}{l}\text { Unclear } \\
\text { benefit }\end{array}$ & Yes & $\begin{array}{l}\text { Two trials out of } 14 \text { suggested a modest } \\
\text { positive impact on decisional regret. Results } \\
\text { across studies varied widely for decisional } \\
\text { conflict ( } 4 \text { studies), satisfaction with decision } \\
\text { ( } 2 \text { studies), and knowledge ( } 2 \text { studies). }\end{array}$ & $\begin{array}{l}\text { scant evidence at high risk of bias } \\
\text { suggests the variable impact of existing } \\
\text { decision aids on a limited set of decisional } \\
\text { processes and outcomes. Because current } \\
\text { decision aids provide information but do } \\
\text { not directly facilitate shared decision } \\
\text { making }\end{array}$ \\
\hline & $\begin{array}{l}\text { Friedrichs, } \\
2016^{107}\end{array}$ & $\begin{array}{l}\text { Potential } \\
\text { benefit }\end{array}$ & Yes & $\begin{array}{l}\text { Two studies found that patients with SUD } \\
\text { preferred to be actively involved in treatment } \\
\text { decisions. Treatment preferences were } \\
\text { assessed in } n=18 \text { studies, where the majority } \\
\text { of patients preferred outpatient compared with } \\
\text { inpatient treatment. }\end{array}$ & $\begin{array}{l}\text { Given the evidence and recommendations } \\
\text { of existing guidelines (e.g. Nice guidelines } \\
\text { [5] or the German guideline for screening, } \\
\text { diagnosis and treatment of alcohol-related } \\
\text { disorders [25]) as well as legislation }[3,4]\end{array}$ \\
\hline & $\begin{array}{l}\text { van Weert, } \\
2016^{109}\end{array}$ & $\begin{array}{l}\text { Potential } \\
\text { benefit }\end{array}$ & Yes & $\begin{array}{l}\text { This review shows promising results on the } \\
\text { effectiveness of decision aids for older adults. } \\
\text { Decision aids have the potential to increase } \\
\text { older adults' risk perception, improve } \\
\text { knowledge, decrease decisional conflict, and } \\
\text { improve patient participation }\end{array}$ & $\begin{array}{l}\text { This review shows promising results on the } \\
\text { effectiveness of decision aids for older } \\
\text { adults. Decision aids improve older adults' } \\
\text { knowledge, increase their risk perception, } \\
\text { decrease decisional conflict and seem to } \\
\text { enhance participation in SDM. }\end{array}$ \\
\hline
\end{tabular}




\begin{tabular}{|c|c|c|c|c|c|}
\hline $\begin{array}{l}\text { Type of } \\
\text { Strategy }\end{array}$ & Author, Year & $\begin{array}{l}\text { Overall } \\
\text { Findings }\end{array}$ & $\begin{array}{l}\text { Strength of } \\
\text { Evidence } \\
\text { Reported? }\end{array}$ & Findings & Conclusion Sentence \\
\hline & $\begin{array}{l}\text { Baptista, } \\
2018^{117}\end{array}$ & $\begin{array}{l}\text { Potential } \\
\text { benefit }\end{array}$ & Yes & $\begin{array}{l}\text { Compared to usual care, Web-based decision } \\
\text { aids increased knowledge (SMD 0.46; } 95 \% \mathrm{Cl} \\
0.18-0.75 \text { ), reduced decisional conflict (MD - } \\
7.07 \% ; 95 \% \mathrm{Cl}-9.44 \text { to }-4.71 \text { ), and reduced } \\
\text { the practitioner control role in the decision- } \\
\text { making process (RR } 0.50 ; 95 \% \mathrm{Cl} 0.3\end{array}$ & $\begin{array}{l}\text { According to this analysis, Web-based } \\
\text { decision aids performed similarly to } \\
\text { alternative formats (ie, printed, video) for } \\
\text { the assessed decision-quality outcomes. } \\
\text { The low cost, readiness, availability, and } \\
\text { anonymity of the Web can be an } \\
\text { advantage }\end{array}$ \\
\hline & Stovell, $2016^{114}$ & $\begin{array}{l}\text { Potential } \\
\text { benefit }\end{array}$ & Yes & $\begin{array}{l}\text { Small beneficial effects of increased shared } \\
\text { decision-making were found on indices of } \\
\text { treatment related empowerment ( } 6 \text { RCTs; } g= \\
0.30,95 \% \mathrm{Cl} 0.09-0.51) \text {,although the effect } \\
\text { was smaller if trials with } 425 \% \text { missing data } \\
\text { were excluded. }\end{array}$ & $\begin{array}{l}\text { For people with psychosis the } \\
\text { implementation of shared treatment } \\
\text { decision-making appears to have small } \\
\text { beneficial effects on indices of treatment- } \\
\text { related empowerment, but more direct } \\
\text { evidence is required. }\end{array}$ \\
\hline & $\begin{array}{l}\text { Kashaf, } \\
2015^{106}\end{array}$ & $\begin{array}{l}\text { Potential } \\
\text { benefit }\end{array}$ & Yes & $\begin{array}{l}\text { The review found weak, but suggestive, } \\
\text { evidence for a positive association between } \\
\text { perceived patient involvement in decision } \\
\text { making, a central dimension of SDM, and } \\
\text { QOL outcomes in cancer. No evidence for an } \\
\text { inverse association between SDM and QOL. }\end{array}$ & $\begin{array}{l}\text { There is weak evidence that aspects of } \\
\text { shared decision-making approaches are } \\
\text { positively associated with QOL outcomes } \\
\text { and very little evidence of a negative } \\
\text { association. The extant literature largely } \\
\text { assessed patient involvement, only } \\
\text { capturing one aspect }\end{array}$ \\
\hline & Morrell, $2016^{116}$ & $\begin{array}{l}\text { Positive } \\
\text { benefit }\end{array}$ & Yes & $\begin{array}{l}\text { Current low-level evidence suggests that DAs } \\
\text { at the end of life are generally acceptable by } \\
\text { users, and appear to increase knowledge and } \\
\text { reduce decisional conflict, so their refinement } \\
\text { and use in routine practice to better document } \\
\text { advance care planning }\end{array}$ & $\begin{array}{l}\text { Compared with other ill-health situations, } \\
\text { there is a shortage of decision aids for End } \\
\text { of Life treatment involving initiation or } \\
\text { discontinuation. Overall the available } \\
\text { decision aids seemed to enhance patients } \\
\text { or surrogate decision-makers' knowledge }\end{array}$ \\
\hline & $\begin{array}{l}\text { Stacey D, } \\
2017^{119}\end{array}$ & $\begin{array}{l}\text { Positive } \\
\text { benefit }\end{array}$ & Yes & $\begin{array}{l}\text { Compared to usual care, decision aids } \\
\text { decreased decisional conflict related to feeling } \\
\text { uninformed (MD }-9.28 / 100 ; 95 \% \mathrm{Cl}-12.20 \text { to } \\
-6.36 ; 27 \text { studies; } \mathrm{N}=5707 \text {; high-quality } \\
\text { evidence), indecision about personal values } \\
\text { (MD }-8.81 / 100 ; 95 \% \mathrm{Cl}-11.99 \text { to }-5.63 \text {; }\end{array}$ & $\begin{array}{l}\text { Compared to usual care across a wide } \\
\text { variety of decision contexts, people } \\
\text { exposed to decision aids feel more } \\
\text { knowledgeable, better informed, and } \\
\text { clearer about their values, and they } \\
\text { probably have a more active role in } \\
\text { decision making }\end{array}$ \\
\hline & $\begin{array}{l}\text { Martínez- } \\
\text { Alonso, 2017112 }\end{array}$ & $\begin{array}{l}\text { Potential } \\
\text { benefit }\end{array}$ & Yes & $\begin{array}{l}\text { The use of DAs increased the proportion of } \\
\text { women making an informed decision by } 14 \% \text {, } \\
95 \% \mathrm{Cl}(2 \% \text { to } 27 \%) \text { and the proportion of } \\
\text { women with adequate knowledge by } 12 \% \text {, } \\
95 \% \mathrm{Cl}(7 \% \text { to } 16 \%) \text {. We observed } \\
\text { heterogeneity among the studies in } \\
\text { confidence in the decision }\end{array}$ & $\begin{array}{l}\text { Tools to aid decision making in screening } \\
\text { for breast cancer improve knowledge and } \\
\text { promote informed decision; however, we } \\
\text { found divergent results on decisional } \\
\text { conflict and confidence in the decision. } \\
\text { Under the current paradigm change }\end{array}$ \\
\hline
\end{tabular}




\begin{tabular}{|c|c|c|c|c|c|}
\hline $\begin{array}{l}\text { Type of } \\
\text { Strategy }\end{array}$ & Author, Year & $\begin{array}{l}\text { Overall } \\
\text { Findings }\end{array}$ & $\begin{array}{l}\text { Strength of } \\
\text { Evidence } \\
\text { Reported? }\end{array}$ & Findings & Conclusion Sentence \\
\hline & $\begin{array}{l}\text { Martínez- } \\
\text { González, } \\
2018^{123}\end{array}$ & $\begin{array}{l}\text { Unclear } \\
\text { benefit }\end{array}$ & No & & $\begin{array}{l}\text { There is significant variation in the extent } \\
\text { of SDM implementation among studies } \\
\text { addressing SDM for prostate cancer } \\
\text { screening and treatment. Further } \\
\text { evaluation of these results on patient } \\
\text { outcomes }\end{array}$ \\
\hline & $\begin{array}{l}\text { Samalin, } \\
2018^{122}\end{array}$ & $\begin{array}{l}\text { Positive } \\
\text { benefit }\end{array}$ & No & $\begin{array}{l}\text { Selected } 3 \text { RCTs showed that the intervention } \\
\text { effectively improved patient satisfaction and } \\
\text { engagement in the decision-making process } \\
\text { in patients with depression. Only one study in } \\
\text { patients with bipolar disorder (BD) showed } \\
\text { improvement of depressive symptoms }\end{array}$ & $\begin{array}{l}\text { SDM interventions using decision aids and } \\
\text { collaborative care showed evidence of } \\
\text { improvements in the management of } \\
\text { depression. Stronger evidence of SDM } \\
\text { interest in BD is needed. }\end{array}$ \\
\hline & $\begin{array}{l}\text { Spronk, } \\
2018^{118}\end{array}$ & $\begin{array}{l}\text { Potential } \\
\text { benefit }\end{array}$ & Yes & $\begin{array}{l}\text { Tools were mainly applicable across the care } \\
\text { process, and usable for decisions on } \\
\text { supportive care with or without chemotherapy. } \\
\text { All tools were designed for patients to be used } \\
\text { before a consultation with the physician. }\end{array}$ & $\begin{array}{l}\text { Despite its recognized importance, only } \\
\text { two tools were positively evaluated on } \\
\text { effectiveness and are available to support } \\
\text { patients with metastatic breast cancer in } \\
\text { SDM. These tools show promising results } \\
\text { in pilot studies and focus on different } \\
\text { aspects }\end{array}$ \\
\hline & Nathan, $2016^{99}$ & $\begin{array}{l}\text { Positive } \\
\text { benefit }\end{array}$ & Yes & $\begin{array}{l}\text { Most of the trials studied racial }(78 \%) \text { or } \\
\text { ethnic }(17 \%) \text { minorities with only one trial } \\
\text { focused on sexual minorities and none on } \\
\text { gender minorities. Ten studies tailored their } \\
\text { interventions for their minority populations. }\end{array}$ & $\begin{array}{l}\text { DAs have been effective in improving } \\
\text { patient-doctor communication and decision } \\
\text { quality outcomes in minority populations } \\
\text { and could help address health disparities. } \\
\text { However, the existing literature is almost } \\
\text { non-existent for sexual and gender } \\
\text { minorities }\end{array}$ \\
\hline \multirow[t]{2}{*}{$\begin{array}{l}\text { Shared } \\
\text { Decision } \\
\text { Making - Adults } \\
\text { and Children }\end{array}$} & $\begin{array}{l}\text { Clayman, } \\
2015^{97}\end{array}$ & $\begin{array}{l}\text { Positive } \\
\text { benefit }\end{array}$ & Yes & $\begin{array}{l}\text { Interventions increased patient involvement in } \\
10(91 \%) \text { of the } 11 \text { RCTs. At least one } \\
\text { positive outcome was detected in } 5(50 \%) \text { of } \\
\text { the } 10 \text { RCTs reporting increased participation; } \\
\text { the ratio of positive results among all outcome } \\
\text { variables measured }\end{array}$ & $\begin{array}{l}\text { Very few RCTs in the field have measures } \\
\text { of participation in decision making and at } \\
\text { least one health outcome. Moreover, } \\
\text { extant studies exhibit little consistency in } \\
\text { measurement of these variables, and } \\
\text { results are mixed. }\end{array}$ \\
\hline & Kew, $2017^{96}$ & $\begin{array}{l}\text { Potential } \\
\text { benefit }\end{array}$ & Yes & $\begin{array}{l}\text { Because these studies were conducted in } \\
\text { different ways, we were unable to combine } \\
\text { their findings. We found evidence from } \\
\text { individual studies indicating that shared } \\
\text { decision-making may improve quality of life, } \\
\text { inhaler adherence and asthma control }\end{array}$ & $\begin{array}{l}\text { Substantial differences between the four } \\
\text { included RCTs indicate that we cannot } \\
\text { provide meaningful overall conclusions. } \\
\text { Individual studies demonstrated some } \\
\text { benefits of SDM over control, in terms of } \\
\text { quality of life; patient and parent } \\
\text { satisfaction; adherence }\end{array}$ \\
\hline
\end{tabular}




\begin{tabular}{|c|c|c|c|c|c|}
\hline $\begin{array}{l}\text { Type of } \\
\text { Strategy }\end{array}$ & Author, Year & $\begin{array}{l}\text { Overall } \\
\text { Findings }\end{array}$ & $\begin{array}{l}\text { Strength of } \\
\text { Evidence } \\
\text { Reported? }\end{array}$ & Findings & Conclusion Sentence \\
\hline & $\begin{array}{l}\text { Winston, } \\
2017^{98}\end{array}$ & $\begin{array}{l}\text { Potential } \\
\text { benefit }\end{array}$ & No & $\begin{array}{l}\text { The most common decisions addressed were } \\
\text { cancer screening, risk reduction, advance } \\
\text { care planning, and adherence to provider } \\
\text { recommendations. Most studies had sample } \\
\text { sizes of fewer than } 300 \text {, and most were } \\
\text { performed in the United State. }\end{array}$ & $\begin{array}{l}\text { Video based patient decision aids are } \\
\text { largely a positive addition to the traditional } \\
\text { process of health care provision. They are } \\
\text { used primarily to assist patients in making } \\
\text { an informed decision about screening, to } \\
\text { promote adherence to medical } \\
\text { recommendation }\end{array}$ \\
\hline & $\begin{array}{l}\text { Voruganti, } \\
2017^{95}\end{array}$ & $\begin{array}{l}\text { Positive } \\
\text { benefit }\end{array}$ & No & $\begin{array}{l}\text { These tools were predominantly accessed } \\
\text { from websites as opposed to Internet-linked } \\
\text { native apps and mainly functioned as part of a } \\
\text { multifunction platform such as patient-facing } \\
\text { portals. Few tools enabled patients to } \\
\text { communicate with multiple health care } \\
\text { professionals }\end{array}$ & $\begin{array}{l}\text { Web-based tools for text-based patient- } \\
\text { provider communication were identified } \\
\text { from a wide variety of clinical contexts and } \\
\text { with varied functionality. Tools were most } \\
\text { prevalent in contexts where intended use } \\
\text { was self-management. }\end{array}$ \\
\hline \multirow{4}{*}{$\begin{array}{l}\text { Shared } \\
\text { Decision } \\
\text { Making - } \\
\text { Children Only }\end{array}$} & Malone, $2019^{91}$ & $\begin{array}{l}\text { Unclear } \\
\text { benefit }\end{array}$ & & & \\
\hline & Cheng, $2017^{92}$ & $\begin{array}{l}\text { Positive } \\
\text { benefit }\end{array}$ & Yes & $\begin{array}{l}\text { Of the records included in this review, } 12 \text { were } \\
\text { aimed at parents, eight were aimed at children } \\
\text { or young people, and two were aimed at both } \\
\text { parents and young people. Approaches that } \\
\text { scored higher on the quality assessment } \\
\text { frame- work tended to be decision }\end{array}$ & $\begin{array}{l}\text { Despite these limitations, evidence from } \\
\text { the present review suggests that six } \\
\text { different approaches are being } \\
\text { implemented to facilitate SDM in child and } \\
\text { youth mental health. These consist of } \\
\text { therapeutic techniques, decision aids, } \\
\text { psychoeducational information }\end{array}$ \\
\hline & Coyne, $2016^{93}$ & $\begin{array}{l}\text { Unclear } \\
\text { benefit }\end{array}$ & & & \\
\hline & Wyatt, $2015^{94}$ & $\begin{array}{l}\text { Potential } \\
\text { benefit }\end{array}$ & Yes & $\begin{array}{l}\text { Heterogeneity across studies was high. Meta- } \\
\text { analysis revealed SDM interventions } \\
\text { significantly improved knowledge } \\
\text { (standardized mean difference [SMD] 1.21, } \\
95 \% \text { confidence interval [Cl] } 0.26 \text { to } 2.17, \mathrm{P} \\
\text { V4 .01) and reduced decisional conflict }\end{array}$ & $\begin{array}{l}\text { A limited evidence base suggests that } \\
\text { pediatric SDM interventions improve } \\
\text { knowledge and decisional conflict, but their } \\
\text { impact on other outcomes is unclear. The } \\
\text { research enterprise to promote SDM has } \\
\text { left children behind. }\end{array}$ \\
\hline
\end{tabular}




\section{Healthcare Organization and Systems}

\begin{tabular}{|c|c|c|c|c|c|c|}
\hline $\begin{array}{l}\text { Author, } \\
\text { Year }\end{array}$ & $\begin{array}{l}\text { Objective of } \\
\text { Review }\end{array}$ & $\begin{array}{l}\text { Literature } \\
\text { Start - } \\
\text { End Year }\end{array}$ & Inclusion Criteria & Exclusion Criteria & $\begin{array}{l}\text { Number of } \\
\text { Included } \\
\text { Studies } \\
\end{array}$ & Settings \\
\hline $\begin{array}{l}\text { Oldfield, } \\
2017^{127}\end{array}$ & $\begin{array}{l}\text { To characterize } \\
\text { the impact of } \\
\text { PFACs on health } \\
\text { systems }\end{array}$ & 1946-2016 & $\begin{array}{l}\text { "involved an organization of patients, } \\
\text { family members, or community members } \\
\text { (possibly including clinicians or hospital } \\
\text { staff) that advised a health care system on } \\
\text { the level of direct care, organizational } \\
\text { design and quality improvement, policy- } \\
\text { making, or research, but did not require } \\
\text { the term PFAC"; had a comparison group, } \\
\text { including RTC, parallel cohort, or historical } \\
\text { control }\end{array}$ & $\begin{array}{l}\text { No comparison group; } \\
\text { Not in English }\end{array}$ & $\begin{array}{l}\text { Total: } 18 \text { (16 } \\
\text { unique) } \\
\text { RCTs:5 } \\
\text { Other: } \\
7 \text { cohort, } 1 \text { case } \\
\text { study with pre- } \\
\text { post evaluations, } \\
3 \text { cross-sectional }\end{array}$ & $\begin{array}{l}\text { Multiple including: } \\
\text { Canadian Health } \\
\text { and Social } \\
\text { Services Centers, } \\
\text { US primary health } \\
\text { care centers, US } \\
\text { Health Systems } \\
\text { Agencies, } \\
\text { European } \\
\text { hospitals, US state } \\
\text { newborn } \\
\text { screening } \\
\text { programs, others } \\
\text { not specified }\end{array}$ \\
\hline $\begin{array}{l}\text { Sharma, } \\
2019^{128}\end{array}$ & $\begin{array}{l}\text { To investigate } \\
\text { whether patient } \\
\text { engagement in } \\
\text { patient advisory } \\
\text { councils is linked } \\
\text { to improvement in } \\
\text { clinical quality, } \\
\text { patient safety, or } \\
\text { patient satisfaction }\end{array}$ & $2002-2015$ & $\begin{array}{l}\text { patient input on an advisory council, } \\
\text { board, or committee in any healthcare } \\
\text { settings, written in English, activities } \\
\text { address organization/system-level } \\
\text { changes, including assessment of impact }\end{array}$ & $\begin{array}{l}\text { Patient engagement } \\
\text { within their own } \\
\text { individual care, patient } \\
\text { engagement within } \\
\text { research studies, any } \\
\text { studies not meeting } \\
\text { inclusion criteria }\end{array}$ & $\begin{array}{l}\text { Total: } 32 \\
\text { RCTs:1 } \\
\text { Other: } \\
\text { Quasi- } \\
\text { experimental:4 } \\
\text { Qualitative: } 9 \\
\text { Case studies: } 16 \\
\text { Other designs: } 2\end{array}$ & Not well described \\
\hline $\begin{array}{l}\text { McCarron, } \\
2018^{129}\end{array}$ & $\begin{array}{l}\text { To understand } \\
\text { how health } \\
\text { systems are } \\
\text { intentionally } \\
\text { investing in the } \\
\text { training and skill } \\
\text { development of } \\
\text { patients and family } \\
\text { members }\end{array}$ & $2000-2016$ & $\begin{array}{l}\text { Studies were included if they (1) had an } \\
\text { adult patient/Studies were included if they } \\
\text { (1) had an adult patient/ consumer focus, } \\
\text { (2) contained a description of an } \\
\text { investment, (3) focused on } \\
\text { programs/activities/events that were } \\
\text { determined to have an impact on the } \\
\text { participation of patients in healthcare, (4) } \\
\text { showcased how patients/ consumers } \\
\text { engaged with other patients or the health } \\
\text { system, and (5) incorporated investments } \\
\text { that enable patients/consumers to } \\
\text { participate in various healthcare roles. }\end{array}$ & $\begin{array}{l}\text { Studies were excluded } \\
\text { if they (1) focused on } \\
\text { in- vestments to } \\
\text { improve self-care; (2) } \\
\text { did not involve or } \\
\text { engage patients; (3) } \\
\text { focused on children, } \\
\text { animals, or family } \\
\text { members; (4) did not } \\
\text { report outcomes; or (5) } \\
\text { were opinion pieces or } \\
\text { letters to the editor. }\end{array}$ & $\begin{array}{l}\text { Total: } 15 \\
\text { RCTs:1 } \\
\text { Other: } \\
9 \text { qualitative; } \\
3 \text { mixed } \\
\text { methods; } 2 \\
\text { quantitative non- } \\
\text { randomized } \\
\text { designs }\end{array}$ & Not well described \\
\hline
\end{tabular}




\begin{tabular}{|c|c|c|c|c|c|c|}
\hline $\begin{array}{l}\text { Author, } \\
\text { Year }\end{array}$ & $\begin{array}{l}\text { Objective of } \\
\text { Review }\end{array}$ & $\begin{array}{l}\text { Literature } \\
\text { Start - } \\
\text { End Year }\end{array}$ & Inclusion Criteria & Exclusion Criteria & $\begin{array}{l}\text { Number of } \\
\text { Included } \\
\text { Studies }\end{array}$ & Settings \\
\hline $\begin{array}{l}\text { Bombard, } \\
2018^{130}\end{array}$ & $\begin{array}{l}\text { To identify } \\
\text { effective strategies } \\
\text { for engaging } \\
\text { patients in the } \\
\text { design or delivery } \\
\text { of health care and } \\
\text { the contextual } \\
\text { factors enabling } \\
\text { their outcomes }\end{array}$ & $1990-2016$ & $\begin{array}{l}\text { Empirical articles that explicitly } \\
\text { investigated the participation of patients, } \\
\text { caregivers, or families in the design, } \\
\text { delivery, and evaluation of health services }\end{array}$ & $\begin{array}{l}\text { Did not explicitly } \\
\text { address patient } \\
\text { engagement, did not } \\
\text { pertain to the design, } \\
\text { delivery, or evaluation } \\
\text { of health services, did } \\
\text { not describe outcomes } \\
\text { of engagement, or } \\
\text { outcomes did not } \\
\text { measure impact on } \\
\text { design, delivery, or } \\
\text { evaluation of health } \\
\text { services }\end{array}$ & $\begin{array}{l}\text { Total: } 48 \\
\text { Other: } \\
27 \text { qualitative; } \\
3 \text { quantitative; } \\
13 \text { mixed } \\
\text { methods; } 5 \text { user } \\
\text { panels or } \\
\text { advisory } \\
\text { meetings }\end{array}$ & No restriction \\
\hline $\begin{array}{l}\text { Liang, } \\
2018^{131}\end{array}$ & $\begin{array}{l}\text { To describe } \\
\text { patient } \\
\text { engagement in } \\
\text { hospital health } \\
\text { service } \\
\text { improvement }\end{array}$ & 2006-2016 & $\begin{array}{l}\text { Published original studies on engagement } \\
\text { of adult patients or providers in hospital } \\
\text { health service improvement activities; } \\
\text { English language }\end{array}$ & $\begin{array}{l}\text { Studies not based in } \\
\text { hospitals; providers not } \\
\text { hospital based; more } \\
\text { than half of providers } \\
\text { were trainees; } \\
\text { engagement of } \\
\text { children, youth, or their } \\
\text { surrogates }\end{array}$ & $\begin{array}{l}\text { Total: } 10 \\
\text { Other: } \\
7 \text { qualitative; } \\
3 \text { cross-sectional }\end{array}$ & Hospital \\
\hline
\end{tabular}


Evidence Table 2. Population characteristics of included systematic reviews addressing healthcare organization and systems

\begin{tabular}{|c|c|c|}
\hline Author, Year & Study Populations & Medical Conditions \\
\hline Oldfield, $2017^{127}$ & $\begin{array}{l}\text { Patients (Adult only); } \\
\text { Family care givers; } \\
\text { Health care providers; } \\
\text { Community members }\end{array}$ & $\begin{array}{l}\text { Diabetes } \\
\text { Cancer or cancer screening } \\
\text { Mental health } \\
\text { Neonatal mortality, newborn screening } \\
\text { Ranking of health concerns and priority setting, not specified for a specific disease Development of a } \\
\text { research volunteer registry } \\
\text { Recommendations for clinical practice guidelines } \\
\text { Others not specified }\end{array}$ \\
\hline Sharma, $2019^{128}$ & $\begin{array}{l}\text { Patients (Adult only); } \\
\text { Health care providers; } \\
\text { Community members, not } \\
\text { specified }\end{array}$ & $\begin{array}{l}\text { Asthma } \\
\text { Blood pressure, not disease specific (e.g., altered visiting hours) } \\
\text { Not specified }\end{array}$ \\
\hline $\begin{array}{l}\text { McCarron, } \\
2018^{129}\end{array}$ & $\begin{array}{l}\text { Patients (age not specified); } \\
\text { "Consumers" }\end{array}$ & $\begin{array}{l}\text { Cancer or cancer screening } \\
\text { Mental health } \\
\text { Pharmacy, not specified }\end{array}$ \\
\hline $\begin{array}{l}\text { Bombard, } \\
2018^{130}\end{array}$ & $\begin{array}{l}\text { Patients (Adults and Children); } \\
\text { Family care givers }\end{array}$ & $\begin{array}{l}\text { Diabetes } \\
\text { Cancer or cancer screening } \\
\text { Mental health } \\
\text { Neurologic conditions } \\
\text { General health } \\
\text { Pediatric or maternity care } \\
\text { Emergency or acute care } \\
\text { Substance abuse } \\
\text { Disability }\end{array}$ \\
\hline Liang, $2018^{131}$ & $\begin{array}{l}\text { Patients (Adult only); } \\
\text { Family care givers; } \\
\text { Health care providers; } \\
\text { Well members of the public }\end{array}$ & Not specified \\
\hline
\end{tabular}




\begin{tabular}{|c|c|c|c|}
\hline $\begin{array}{l}\text { Author, } \\
\text { Year }\end{array}$ & Intervention Focus & Description of Intervention & Strategies \\
\hline $\begin{array}{l}\text { Oldfield, } \\
2017^{127}\end{array}$ & $\begin{array}{l}\text { Direct care (three studies) - care } \\
\text { strategies or educational materials, } \\
\text { disease specific practice guidelines; } \\
\text { Organizational design (three studies) - } \\
\text { diabetes centers, pediatric clinic for } \\
\text { families with limited English proficiency, } \\
\text { primary care clinics; } \\
\text { Policy-making (five studies); } \\
\text { Research (five studies) - four in } \\
\text { recruitment and retention of research } \\
\text { participants among marginalized } \\
\text { populations }\end{array}$ & $\begin{array}{l}\text { "an organization of patients, family } \\
\text { members, or community members (possibly } \\
\text { including clinicians or hospital staff) that } \\
\text { advised a health care system on the level of } \\
\text { direct care, organizational design and } \\
\text { quality improvement, policy-making, or } \\
\text { research" }\end{array}$ & $\begin{array}{l}\text { Groups that involved patients, family members, or } \\
\text { community members only } \\
\text { Groups that engaged staff or clinicians in addition to } \\
\text { patients, family members, or community members }\end{array}$ \\
\hline $\begin{array}{l}\text { Sharma, } \\
2019^{128}\end{array}$ & $\begin{array}{l}\text { Health promotion - colon cancer } \\
\text { screening, asthma and blood pressure } \\
\text { management; } \\
\text { Patient safety; Patient satisfaction }\end{array}$ & $\begin{array}{l}\text { "a group of patients or consumers working } \\
\text { with healthcare staff in order to provide input } \\
\text { on healthcare services or delivery" }\end{array}$ & $\begin{array}{l}\text { Community Advisory council, community focus } \\
\text { groups, town halls }\end{array}$ \\
\hline $\begin{array}{l}\text { McCarron, } \\
2018^{129}\end{array}$ & $\begin{array}{l}\text { Enhanced care or service delivery; } \\
\text { Development of specific policy or } \\
\text { planning documents; } \\
\text { Enhanced governance; } \\
\text { Education or tool development } \\
\end{array}$ & & Forums, Patient instructors, Workshops, Co-design \\
\hline $\begin{array}{l}\text { Bombard, } \\
2018^{130}\end{array}$ & $\begin{array}{l}\text { Patient engagement in the design, } \\
\text { delivery, and evaluation of health services }\end{array}$ & & $\begin{array}{l}\text { Consultative activities (i.e., where patients } \\
\text { provided input on research design or measures as } \\
\text { part of the research or administrative team); } \\
\text { "Co-design (i.e., deliberative, } \\
\text { reflexive processes where patients and providers } \\
\text { work together to create solutions)" }\end{array}$ \\
\hline $\begin{array}{l}\text { Liang, } \\
2018^{131}\end{array}$ & Hospital service improvement & & $\begin{array}{l}\text { "Consultation activities: questionnaires, interviews, } \\
\text { mass media and suggestion boxes;" } \\
\text { "Involvement activities: members of standing } \\
\text { committees, advisory bodies, project teams or } \\
\text { providing education to other patients;" } \\
\text { Partnership: citizen advisory panel }\end{array}$ \\
\hline
\end{tabular}


Evidence Table 4. Outcomes of included systematic reviews addressing healthcare organization and systems

\begin{tabular}{|c|c|c|}
\hline Author, Year & Intermediate Outcomes & Patient Outcomes \\
\hline $\begin{array}{l}\text { Oldfield, } \\
2017^{127}\end{array}$ & $\begin{array}{l}\text { Organizational priority setting } \\
\text { Implementation of quality improvement } \\
\text { Recruitment/retention of research subjects } \\
\text { Conduct of research } \\
\text { Direct care services } \\
\text { Clinical outcomes } \\
\text { Recruitment strategies }\end{array}$ & $\begin{array}{l}\text { Clinical outcomes } \\
\text { Recruitment strategies }\end{array}$ \\
\hline $\begin{array}{l}\text { Sharma, } \\
2019^{128}\end{array}$ & $\begin{array}{l}\text { Clinical care - increased intention to engage in colorectal cancer screening, increased use of } \\
\text { inhalers and asthma action plans, improved blood pressure control rates } \\
\text { Patient safety - } 1 \text { report of multiple case studies. One case report attributed } 62 \% \text { reduction in } \\
\text { medical errors to redesign including patient advisors } \\
\text { Patient satisfaction - } 4 \text { papers with "case based results" - "patient advisory councils had a role } \\
\text { in affecting patient satisfaction." }\end{array}$ & \\
\hline $\begin{array}{l}\text { McCarron, } \\
2018^{129}\end{array}$ & $\begin{array}{l}\text { Study themes - strategies for engagement } \\
\text { Patient engagement in healthcare decision making }\end{array}$ & \\
\hline $\begin{array}{l}\text { Bombard, } \\
2018^{130}\end{array}$ & $\begin{array}{l}\text { Strategies for optimal patient engagement } \\
\text { Outcomes of engaging patients } \\
\text { Patients' experiences of being engaged }\end{array}$ & \\
\hline Liang, $2018^{131}$ & $\begin{array}{l}\text { Extent of patient engagement } \\
\text { Determinants of engagement } \\
\text { Interventions to increase patient engagement } \\
\text { Impact }\end{array}$ & \\
\hline
\end{tabular}




\begin{tabular}{|c|c|c|c|c|}
\hline $\begin{array}{l}\text { Author, } \\
\text { Year }\end{array}$ & $\begin{array}{l}\text { Overall } \\
\text { Findings }\end{array}$ & Findings & Recommendations & Conclusion Sentence \\
\hline $\begin{array}{l}\text { Oldfield, } \\
2017^{127}\end{array}$ & $\begin{array}{l}\text { Unclear } \\
\text { benefit }\end{array}$ & $\begin{array}{l}\text { Impact:"'In person deliberation with health system } \\
\text { leadership was most effective, and studies that } \\
\text { involved patient engagement in research focused } \\
\text { on increasing study participation but not in } \\
\text { research design or the dissemination of research } \\
\text { findings. Programs varied in: structural makeup, } \\
\text { terminology used to describe organizations and } \\
\text { their components, strategies of recruitment of } \\
\text { individuals, and types of outcomes measured. All } \\
\text { studies suggested important benefits of community } \\
\text { engagement to health systems and research, but } \\
\text { some described drawbacks, including greater } \\
\text { costs, longer time to achieve consensus among } \\
\text { groups, and the generation of lower quality plans } \\
\text { when patients are involved." "In-person and } \\
\text { collective deliberation, particularly in community- } \\
\text { based settings (such as community organizations), } \\
\text { is more effective than using mailed surveys or } \\
\text { telephone calls, or one-on-one meetings. } \\
\text {...patients with greater community credibility, such } \\
\text { as leaders of community organizations, are more } \\
\text { effective participants in PFACs than those } \\
\text { without." } \\
\text { Other: Recruitment: by clinical/administrative staff, } \\
\text { recruited by community outreach, elected, and } \\
\text { unknown }\end{array}$ & $\begin{array}{l}\text { Standardized tools for } \\
\text { measuring engagement; } \\
\text { Transparency in and evaluation } \\
\text { of recruiting of PFAC members, } \\
\text { trainings for communities } \\
\text { members without leadership } \\
\text { experience }\end{array}$ & $\begin{array}{l}\text { PFACs engage communities through } \\
\text { individual projects but evidence of their } \\
\text { impact on outcomes is lacking. A paucity of } \\
\text { randomized controlled trials or high-quality } \\
\text { observational studies guide strategies for } \\
\text { engagement through PFACs. Standardized } \\
\text { measurement tools for engagement are } \\
\text { needed. Strategies for PFAC recruitment } \\
\text { should be investigated and reported. }\end{array}$ \\
\hline $\begin{array}{l}\text { Sharma, } \\
2019^{128}\end{array}$ & $\begin{array}{l}\text { Unclear } \\
\text { benefit }\end{array}$ & $\begin{array}{l}\text { Impact: "We did not find any rigorous, prospective } \\
\text { RCTs that assessed our primary outcomes of } \\
\text { patient clinical care, patient safety, or patient } \\
\text { satisfaction... We found one cluster randomized } \\
\text { trial showing patient advisors helped clinics set } \\
\text { priorities that were better aligned with the PCMH } \\
\text { and chronic care model." "The few experimental } \\
\text { studies did show improvements in some clinical } \\
\text { care metrics, clinic priorities, and staff awareness } \\
\text { of patient engagement; however, more concrete } \\
\text { outcome measures were lacking." }\end{array}$ & & $\begin{array}{l}\text { "Five included studies demonstrate } \\
\text { promising methods for evaluating patient } \\
\text { engagement in healthcare delivery and } \\
\text { describe impacts on clinical outcomes and } \\
\text { priority setting. Based on the case studies } \\
\text { found, patient advisors tend to contribute to } \\
\text { patient-facing services that may affect } \\
\text { clinical care but are not easily evaluated. As } \\
\text { clinics and hospitals implement patient } \\
\text { advisory councils, rigorous evaluation of } \\
\text { their programs is needed to support the } \\
\text { expansion of system-level patient } \\
\text { engagement." }\end{array}$ \\
\hline
\end{tabular}




\begin{tabular}{|c|c|c|c|c|}
\hline $\begin{array}{l}\text { Author, } \\
\text { Year }\end{array}$ & $\begin{array}{l}\text { Overall } \\
\text { Findings }\end{array}$ & Findings & Recommendations & Conclusion Sentence \\
\hline $\begin{array}{l}\text { McCarron, } \\
2018^{129}\end{array}$ & $\begin{array}{l}\text { Unclear } \\
\text { benefit }\end{array}$ & $\begin{array}{l}\text { Impact: While significant research exists that } \\
\text { highlights how health systems are working with } \\
\text { patients to better manage their own care, studies } \\
\text { that explore other dimensions of patient } \\
\text { engagement are largely absent. }\end{array}$ & & $\begin{array}{l}\text { "An evidence base around programs to } \\
\text { advance patient engagement is largely } \\
\text { absent. An opportunity exists for further } \\
\text { research to identify strategies and } \\
\text { measures to support patient engagement in } \\
\text { healthcare decision-making. " }\end{array}$ \\
\hline $\begin{array}{l}\text { Bombard, } \\
2018^{130}\end{array}$ & $\begin{array}{l}\text { Possible } \\
\text { benefit }\end{array}$ & $\begin{array}{l}\text { Impact: Most studies noted more than one type of } \\
\text { outcome on the quality of care, including } \\
\text { enhanced care or service delivery, development of } \\
\text { specific policy or planning documents, and } \\
\text { enhanced governance and education or tool } \\
\text { development. Engaging patients can also change } \\
\text { the culture of staff and care settings. } \\
\text { Barriers: Thematically grouped barriers to (1) } \\
\text { design (e.g., lack of role clarity), (2) recruitment } \\
\text { (e.g., bias in selection), (3) involvement, (4) } \\
\text { context (e.g., provider skepticism), and (5) } \\
\text { leadership actions. }\end{array}$ & & $\begin{array}{l}\text { "Patient engagement can inform patient and } \\
\text { provider education and policies, as well as } \\
\text { enhance service delivery and governance. } \\
\text { Additional evidence is needed to } \\
\text { understand patients' experiences of the } \\
\text { engagement process and whether these } \\
\text { outcomes translate into improved quality of } \\
\text { care." }\end{array}$ \\
\hline $\begin{array}{l}\text { Liang, } \\
2018^{131}\end{array}$ & $\begin{array}{l}\text { Unclear } \\
\text { benefit }\end{array}$ & $\begin{array}{l}\text { Impact: "Only one study evaluated how patient } \\
\text { engagement impacted hospital services and no } \\
\text { study evaluated the impact of patient engagement } \\
\text { on clinical outcomes." } \\
\text { Barriers: "Barriers to patient engagement were } \\
\text { primarily at the provider level including negative } \\
\text { beliefs and attitudes about patient roles and input, } \\
\text { lack of knowledge and skills, provider dysfunction } \\
\text { and hierarchies, and uncertainty about how to } \\
\text { resolve differences between patient and senior } \\
\text { management priorities." } \\
\text { Other: Extent of patient engagement: "Patient } \\
\text { input and influence on decisions was minimal." }\end{array}$ & & $\begin{array}{l}\text { "Given the important role of PE in improving } \\
\text { hospital services and the paucity of } \\
\text { research on this topic, future research } \\
\text { should develop and evaluate behavioural } \\
\text { interventions for PE directed at patients and } \\
\text { providers informed by the PE barriers and } \\
\text { facilitators identified here. } \\
\text { Future studies should also assess the } \\
\text { impact on various individual and } \\
\text { organisational outcomes." }\end{array}$ \\
\hline
\end{tabular}




\section{References}

1. Coulter A, Entwistle VA, Eccles A, et al. Personalised care planning for adults with chronic or long-term health conditions. The Cochrane database of systematic reviews. 2015 Mar 3(3):Cd010523. doi: 10.1002/14651858.CD010523.pub2. PMID: 25733495.

2. Schaepe C, Bergjan M. Educational interventions in peritoneal dialysis: a narrative review of the literature. International journal of nursing studies. 2015 Apr;52(4):882-98. doi: 10.1016/j.ijnurstu.2014.12.009. PMID: 25616708.

3. Bal MI, Sattoe JN, Roelofs PD, et al. Exploring effectiveness and effective components of self-management interventions for young people with chronic physical conditions: A systematic review. Patient education and counseling. 2016 Aug;99(8):1293-309. doi: 10.1016/j.pec.2016.02.012. PMID: 26954345.

4. Low JK, Manias E. Use of Technology-Based Tools to Support Adolescents and Young Adults With Chronic Disease: Systematic Review and Meta-Analysis. JMIR mHealth and uHealth. 2019 Jul 18;7(7):e12042. doi: 10.2196/12042. PMID: 31322129.

5. Saxby N, Beggs S, Battersby M, et al. What are the components of effective chronic condition self-management education interventions for children with asthma, cystic fibrosis, and diabetes? A systematic review. Patient education and counseling. 2019 Apr;102(4):607-22. doi: 10.1016/j.pec.2018.11.001. PMID: 30471988.

6. Knafl KA, Havill NL, Leeman J, et al. The Nature of Family Engagement in Interventions for Children With Chronic Conditions. Western journal of nursing research. 2017 May;39(5):690-723. doi: 10.1177/0193945916664700. PMID: 27596106.

7. Campbell F, Biggs K, Aldiss SK, et al. Transition of care for adolescents from paediatric services to adult health services. The Cochrane database of systematic reviews. 2016 Apr 29;4:Cd009794. doi: 10.1002/14651858.CD009794.pub2. PMID: 27128768.

8. Majeed-Ariss R, Baildam E, Campbell M, et al. Apps and Adolescents: A Systematic Review of Adolescents' Use of Mobile Phone and Tablet Apps That Support Personal Management of Their Chronic or LongTerm Physical Conditions. Journal of medical Internet research. 2015 Dec 23;17(12):e287. doi: 10.2196/jmir.5043. PMID: 26701961.
9. Charlier N, Zupancic N, Fieuws S, et al. Serious games for improving knowledge and self-management in young people with chronic conditions: a systematic review and meta-analysis. Journal of the American Medical Informatics Association : JAMIA. 2016 Jan;23(1):230-9. doi: 10.1093/jamia/ocv100. PMID: 26186934.

10. Sattoe JN, Bal MI, Roelofs PD, et al. Self-management interventions for young people with chronic conditions: A systematic overview. Patient education and counseling. 2015 Jun;98(6):704-15. doi: 10.1016/j.pec.2015.03.004. PMID: 25819373.

11. Hamline MY, Speier RL, Vu PD, et al. Hospital-to-Home Interventions, Use, and Satisfaction: A Meta-analysis. Pediatrics. 2018 Nov;142(5). doi: 10.1542/peds.2018-0442. PMID: 30352792.

12. Clemente D, Leon L, Foster H, et al. Systematic review and critical appraisal of transitional care programmes in rheumatology. Seminars in arthritis and rheumatism. 2016 Dec;46(3):372-9. doi: 10.1016/j.semarthrit.2016.06.003. PMID: 27496195.

13. Niznik JD, He H, Kane-Gill SL. Impact of clinical pharmacist services delivered via telemedicine in the outpatient or ambulatory care setting: A systematic review. Research in social \& administrative pharmacy : RSAP. 2018 Aug;14(8):707-17. doi: 10.1016/j.sapharm.2017.10.011. PMID: 29100941.

14. Barello S, Triberti S, Graffigna G, et al. eHealth for Patient Engagement: A Systematic Review. Frontiers in psychology. 2015;6:2013. doi: 10.3389/fpsyg.2015.02013. PMID: 26779108.

15. Bashi N, Fatehi F, Fallah M, et al. Self-Management Education Through mHealth: Review of Strategies and Structures. JMIR mHealth and uHealth. 2018 Oct 19;6(10):e10771. doi: 10.2196/10771. PMID: 30341042

16. Lycett HJ, Raebel EM, Wildman EK, et al. Theory-Based Digital Interventions to Improve Asthma Self-Management Outcomes: Systematic Review. Journal of medical Internet research. 2018 Dec 12;20(12):e293. doi: 10.2196/jmir.9666. PMID: 30541741. 
17. Lancaster K, Abuzour A, Khaira M, et al. The Use and Effects of Electronic Health Tools for Patient Self-Monitoring and Reporting of Outcomes Following Medication Use: Systematic Review. Journal of medical Internet research. 2018 Dec 18;20(12):e294. doi: 10.2196/jmir.9284. PMID: 30563822.

18. Kew KM, Cates CJ. Home telemonitoring and remote feedback between clinic visits for asthma. The Cochrane database of systematic reviews. 2016 Aug 3(8):Cd011714. doi:

10.1002/14651858.CD011714.pub2. PMID: 27486836.

19. Graham CD, Gouick J, Krahe C, et al. A systematic review of the use of Acceptance and Commitment Therapy (ACT) in chronic disease and long-term conditions. Clinical psychology review. 2016 Jun;46:46-58. doi: 10.1016/j.cpr.2016.04.009. PMID: 27176925.

20. Yin K, Laranjo L, Tong HL, et al. Context-Aware Systems for Chronic Disease Patients: Scoping Review. Journal of medical Internet research. 2019 Jun 17;21(6):e10896. doi: 10.2196/10896. PMID: 31210138.

21. Chi NC, Demiris G. A systematic review of telehealth tools and interventions to support family caregivers. Journal of telemedicine and telecare. 2015 Jan;21(1):37-44. doi: 10.1177/1357633x14562734. PMID: 25475220.

22. Hill B, Richardson B, Skouteris H. Do we know how to design effective health coaching interventions: a systematic review of the state of the literature. American journal of health promotion : AJHP. 2015 May-Jun;29(5):e158-68. doi: 10.4278/ajhp.130510-LIT-238. PMID: 24720388.

23. Pamungkas RA, Chamroonsawasdi K, Vatanasomboon P. A Systematic Review: Family Support Integrated with Diabetes Self-Management among Uncontrolled Type II Diabetes Mellitus Patients. Behavioral sciences (Basel, Switzerland). 2017 Sep 15;7(3). doi: 10.3390/bs7030062. PMID: 28914815.

24. Kruse CS, Argueta DA, Lopez L, et al. Patient and provider attitudes toward the use of patient portals for the management of chronic disease: a systematic review. Journal of medical Internet research. 2015 Feb 20;17(2):e40. doi: 10.2196/jmir.3703. PMID: 25707035.
25. Whitehead L, Seaton P. The Effectiveness of Self-Management Mobile Phone and Tablet Apps in Long-term Condition Management: A Systematic Review. Journal of medical Internet research. 2016 May 16;18(5):e97. doi: 10.2196/jmir.4883. PMID: 27185295.

26. Kim AR, Park HA. Web-based Self-management Support Interventions for Cancer Survivors: A Systematic Review and Meta-analyses. Studies in health technology and informatics. 2015;216:142-7. PMID: 26262027.

27. Teljeur C, Moran PS, Walshe S, et al. Economic evaluation of chronic disease self-management for people with diabetes: a systematic review. Diabetic medicine : a journal of the British Diabetic Association. 2017 Aug;34(8):1040-9. doi: 10.1111/dme.13281. PMID: 27770591.

28. Van Hecke A, Heinen M, Fernandez-Ortega P, et al. Systematic literature review on effectiveness of self-management support interventions in patients with chronic conditions and low socioeconomic status. Journal of advanced nursing. 2017 Apr;73(4):775-93. doi: 10.1111/jan.13159. PMID: 27653960

29. van Hooft SM, Been-Dahmen JMJ, Ista E, et al. A realist review: what do nurse-led self-management interventions achieve for outpatients with a chronic condition? Journal of advanced nursing. 2017 Jun;73(6):1255-71. doi: 10.1111/jan.13189. PMID: 27754557.

30. Stenberg U, Haaland-Overby M, Fredriksen K, et al. A scoping review of the literature on benefits and challenges of participating in patient education programs aimed at promoting self-management for people living with chronic illness. Patient education and counseling. 2016 Nov;99(11):1759-71. doi: 10.1016/j.pec.2016.07.027. PMID: 27461944.

31. Plow M, Mangal S, Geither K, et al. A Scoping Review of Tailored Self-management Interventions among Adults with Mobility Impairing Neurological and Musculoskeletal Conditions. Frontiers in public health. 2016;4:165. doi: 10.3389/fpubh.2016.00165. PMID: 27672633. 
32. Noonan MC, Wingham J, Dalal HM, et al. Involving caregivers in selfmanagement interventions for patients with heart failure and chronic obstructive pulmonary disease. A systematic review and metaanalysis. Journal of advanced nursing. 2019 Aug 22. doi: 10.1111/jan.14172. PMID: 31441088.

33. Kuo A, Dang S. Secure Messaging in Electronic Health Records and Its Impact on Diabetes Clinical Outcomes: A Systematic Review. Telemedicine journal and e-health : the official journal of the American Telemedicine Association. 2016 Sep;22(9):769-77. doi: 10.1089/tmj.2015.0207. PMID: 27027337.

34. Sakakibara BM, Kim AJ, Eng JJ. A Systematic Review and MetaAnalysis on Self-Management for Improving Risk Factor Control in Stroke Patients. International journal of behavioral medicine. 2017 Feb;24(1):42-53. doi: 10.1007/s12529-016-9582-7. PMID: 27469998.

35. Whiteman KL, Naslund JA, DiNapoli EA, et al. Systematic Review of Integrated General Medical and Psychiatric Self-Management Interventions for Adults With Serious Mental Illness. Psychiatric services (Washington, DC). 2016 Nov 1;67(11):1213-25. doi: 10.1176/appi.ps.201500521. PMID: 27301767.

36. Sokol R, Fisher E. Peer Support for the Hardly Reached: A Systematic Review. American journal of public health. 2016 Jul;106(7):e1-8. doi: 10.2105/ajph.2016.303180. PMID: 27196645.

37. Zomahoun HTV, Guenette L, Gregoire JP, et al. Effectiveness of motivational interviewing interventions on medication adherence in adults with chronic diseases: a systematic review and meta-analysis. International journal of epidemiology. 2017 Apr 1;46(2):589-602. doi: 10.1093/ije/dyw273. PMID: 27864410.

38. Palacio A, Garay D, Langer B, et al. Motivational Interviewing Improves Medication Adherence: a Systematic Review and Metaanalysis. Journal of general internal medicine. 2016 Aug;31(8):929-40. doi: 10.1007/s11606-016-3685-3. PMID: 27160414.

39. van Eeden M, van Heugten CM, van Mastrigt GA, et al. ECONOMIC EVALUATION STUDIES OF SELF-MANAGEMENT INTERVENTIONS IN CHRONIC DISEASES: A SYSTEMATIC REVIEW. International journal of technology assessment in health care. 2016 Jan;32(1-2):16-28. doi: 10.1017/s0266462316000027. PMID: 27125195.
40. Jacelon CS, Gibbs MA, Ridgway JV. Computer technology for selfmanagement: a scoping review. Journal of clinical nursing. 2016 May;25(9-10):1179-92. doi: 10.1111/jocn.13221. PMID: 26990364.

41. Smith SM, Wallace E, O'Dowd T, et al. Interventions for improving outcomes in patients with multimorbidity in primary care and community settings. The Cochrane database of systematic reviews. 2016 Mar 14;3:Cd006560. doi: 10.1002/14651858.CD006560.pub3. PMID: 26976529.

42. Hammer MJ, Ercolano EA, Wright F, et al. Self-management for adult patients with cancer: an integrative review. Cancer nursing. 2015 MarApr;38(2):E10-26. doi: 10.1097/ncc.0000000000000122. PMID: 25692736.

43. Kim K, Choi JS, Choi E, et al. Effects of Community-Based Health Worker Interventions to Improve Chronic Disease Management and Care Among Vulnerable Populations: A Systematic Review. American journal of public health. 2016 Apr;106(4):e3-e28. doi: 10.2105/ajph.2015.302987. PMID: 26890177.

44. Thakkar J, Kurup R, Laba TL, et al. Mobile Telephone Text Messaging for Medication Adherence in Chronic Disease: A Meta-analysis.

JAMA internal medicine. 2016 Mar;176(3):340-9. doi: 10.1001/jamainternmed.2015.7667. PMID: 26831740.

45. Deek H, Hamilton S, Brown N, et al. Family-centred approaches to healthcare interventions in chronic diseases in adults: a quantitative systematic review. Journal of advanced nursing. 2016 May;72(5):96879. doi: 10.1111/jan.12885. PMID: 26751971.

46. van Het Bolscher-Niehuis MJ, den Ouden ME, de Vocht HM, et al. Effects of self-management support programmes on activities of daily living of older adults: A systematic review. International journal of nursing studies. 2016 Sep;61:230-47. doi: 10.1016/j.ijnurstu.2016.06.014. PMID: 27400029.

47. Alessa T, Abdi S, Hawley MS, et al. Mobile Apps to Support the SelfManagement of Hypertension: Systematic Review of Effectiveness, Usability, and User Satisfaction. JMIR mHealth and uHealth. 2018 Jul 23;6(7):e10723. doi: 10.2196/10723. PMID: 30037787. 
48. Debon R, Coleone JD, Bellei EA, et al. Mobile health applications for chronic diseases: A systematic review of features for lifestyle improvement. Diabetes \& metabolic syndrome. 2019 Jul Aug;13(4):2507-12. doi: 10.1016/j.dsx.2019.07.016. PMID: 31405669 .

49. Luedke MW, Blalock DV, Lewinski AA, et al. VA Evidence-based Synthesis Program Reports. Self-management of Epilepsy: A Systematic Review. Washington (DC): Department of Veterans Affairs (US); 2019.

50. Warner G, Packer TL, Kervin E, et al. A systematic review examining whether community-based self-management programs for older adults with chronic conditions actively engage participants and teach them patient-oriented self-management strategies. Patient education and counseling. 2019 Jul 2. doi: 10.1016/j.pec.2019.07.002. PMID: 31301922.

51. Chan WCH, Chan SO, Wong ALY, et al. Understanding family involvement in body donation in Hong Kong: A qualitative study of registered donors and bereaved family members. Health \& social care in the community. 2019 Sep 30. doi: 10.1111/hsc.12861. PMID: 31566842.

52. Levengood TW, Peng Y, Xiong KZ, et al. Team-Based Care to Improve Diabetes Management: A Community Guide Meta-analysis. American journal of preventive medicine. 2019 Jul;57(1):e17-e26. doi: 10.1016/j.amepre.2019.02.005. PMID: 31227069.

53. Zhao J, Zhai YK, Zhu WJ, et al. Effectiveness of Telemedicine for Controlling Asthma Symptoms: A Systematic Review and Metaanalysis. Telemedicine journal and e-health : the official journal of the American Telemedicine Association. 2015 Jun;21(6):484-92. doi: 10.1089/tmj.2014.0119. PMID: 25393915.

54. Lederle M, Bitzer EM. A close look at lay-led self-management programs for chronic diseases and health care utilisation: A systematic review and meta-analysis. German medical science : GMS e-journal. 2019;17:Doc03. doi: 10.3205/000269. PMID: 31148954
55. Nazarov S, Manuwald U, Leonardi M, et al. Chronic Diseases and Employment: Which Interventions Support the Maintenance of Work and Return to Work among Workers with Chronic Illnesses? A Systematic Review. International journal of environmental research and public health. 2019 May 27;16(10). doi: 10.3390/ijerph16101864. PMID: 31137817.

56. Sangrar R, Docherty-Skippen SM, Beattie K. Blended face-to-face and online/computer-based education approaches in chronic disease selfmanagement: A critical interpretive synthesis. Patient education and counseling. 2019 Oct;102(10):1822-32. doi: 10.1016/j.pec.2019.05.009. PMID: 31101427.

57. Zhao FF, Suhonen R, Koskinen S, et al. Theory-based self-management educational interventions on patients with type 2 diabetes: a systematic review and meta-analysis of randomized controlled trials. Journal of advanced nursing. 2017 Apr;73(4):812-33. doi: 10.1111/jan.13163. PMID: 27681948.

58. Skrabal Ross X, Gunn KM, Patterson P, et al. Mobile-Based Oral Chemotherapy Adherence-Enhancing Interventions: Scoping Review. JMIR mHealth and uHealth. 2018 Dec 21;6(12):e11724. doi: 10.2196/11724. PMID: 30578182.

59. Ha Dinh TT, Bonner A, Clark R, et al. The effectiveness of the teachback method on adherence and self-management in health education for people with chronic disease: a systematic review. JBI database of systematic reviews and implementation reports. 2016 Jan;14(1):21047. doi: 10.11124/jbisrir-2016-2296. PMID: 26878928.

60. Ko D, Bratzke LC, Roberts T. Self-management assessment in multiple chronic conditions: A narrative review of literature. International journal of nursing studies. 2018 Jul;83:83-90. doi: 10.1016/j.ijnurstu.2018.04.009. PMID: 29709734.

61. Changizi M, Kaveh MH. Effectiveness of the mHealth technology in improvement of healthy behaviors in an elderly population-a systematic review. mHealth. 2017;3:51. doi: 10.21037/mhealth.2017.08.06. PMID: 29430455.

62. Kelly C, Grundy S, Lynes D, et al. Self-management for bronchiectasis. The Cochrane database of systematic reviews. 2018 Feb 7;2:Cd012528. doi: 10.1002/14651858.CD012528.pub2. PMID: 29411860 . 
63. Jeddi FR, Nabovati E, Amirazodi S. Features and Effects of Information Technology-Based Interventions to Improve SelfManagement in Chronic Kidney Disease Patients: a Systematic Review of the Literature. Journal of medical systems. 2017 Sep 18;41(11):170. doi: 10.1007/s10916-017-0820-6. PMID: 28920174.

64. Conway CM, Kelechi TJ. Digital Health for Medication Adherence in Adult Diabetes or Hypertension: An Integrative Review. JMIR diabetes. 2017 Aug 16;2(2):e20. doi: 10.2196/diabetes.8030. PMID: 30291093.

65. Kim BY, Lee J. Smart Devices for Older Adults Managing Chronic Disease: A Scoping Review. JMIR mHealth and uHealth. 2017 May 23;5(5):e69. doi: 10.2196/mhealth.7141. PMID: 28536089.

66. Cho H, Iribarren S, Schnall R. Technology-Mediated Interventions and Quality of Life for Persons Living with HIV/AIDS. A Systematic Review. Applied clinical informatics. 2017 Apr 12;8(2):348-68. doi: 10.4338/aci-2016-10-r-0175. PMID: 28401246.

67. Clarkesmith DE, Pattison HM, Khaing PH, et al. Educational and behavioural interventions for anticoagulant therapy in patients with atrial fibrillation. The Cochrane database of systematic reviews. 2017 Apr 5;4:Cd008600. doi: 10.1002/14651858.CD008600.pub3. PMID: 28378924.

68. Massimi A, De Vito C, Brufola I, et al. Are community-based nurse-led self-management support interventions effective in chronic patients? Results of a systematic review and meta-analysis. PloS one. 2017;12(3):e0173617. doi: 10.1371/journal.pone.0173617. PMID: 28282465.

69. Dounavi K, Tsoumani O. Mobile Health Applications in Weight Management: A Systematic Literature Review. American journal of preventive medicine. 2019 Jun;56(6):894-903. doi: 10.1016/j.amepre.2018.12.005. PMID: 31003801.

70. Price M, Bellwood P, Kitson N, et al. Conditions potentially sensitive to a personal health record (PHR) intervention, a systematic review. BMC medical informatics and decision making. 2015 Apr 18;15:32. doi: 10.1186/s12911-015-0159-1. PMID: 25927384.
71. Aquino JA, Baldoni NR, Flor CR, et al. Effectiveness of individual strategies for the empowerment of patients with diabetes mellitus: A systematic review with meta-analysis. Primary care diabetes. 2018 Apr;12(2):97-110. doi: 10.1016/j.pcd.2017.10.004. PMID: 29162491.

72. Ammenwerth E, Hoerbst A, Lannig S, et al. Effects of Adult Patient Portals on Patient Empowerment and Health-Related Outcomes: A Systematic Review. Studies in health technology and informatics. 2019 Aug 21;264:1106-10. doi: 10.3233/shti190397. PMID: 31438096.

73. Donald M, Kahlon BK, Beanlands H, et al. Self-management interventions for adults with chronic kidney disease: a scoping review. BMJ open. 2018 Mar 22;8(3):e019814. doi: 10.1136/bmjopen-2017019814. PMID: 29567848.

74. Escriva Boulley G, Leroy T, Bernetiere C, et al. Digital health interventions to help living with cancer: A systematic review of participants' engagement and psychosocial effects. Psycho-oncology. 2018 Dec;27(12):2677-86. doi: 10.1002/pon.4867. PMID: 30152074

75. Peytremann-Bridevaux I, Arditi C, Gex G, et al. Chronic disease management programmes for adults with asthma. The Cochrane database of systematic reviews. 2015 May 27(5):Cd007988. doi: 10.1002/14651858.CD007988.pub2. PMID: 26014500.

76. Lauckner HM, Hutchinson SL. Peer support for people with chronic conditions in rural areas: a scoping review. Rural and remote health. 2016 Jan-Mar;16(1):3601. PMID: 26943760.

77. Lee JA, Choi M, Lee SA, et al. Effective behavioral intervention strategies using mobile health applications for chronic disease management: a systematic review. BMC medical informatics and decision making. 2018 Feb 20;18(1):12. doi: 10.1186/s12911-0180591-0. PMID: 29458358.

78. Warrington L, Absolom K, Conner M, et al. Electronic Systems for Patients to Report and Manage Side Effects of Cancer Treatment: Systematic Review. Journal of medical Internet research. 2019 Jan 24;21(1):e10875. doi: 10.2196/10875. PMID: 30679145. 
79. Palacios J, Lee GA, Duaso M, et al. Internet-Delivered Selfmanagement Support for Improving Coronary Heart Disease and Selfmanagement-Related Outcomes: A Systematic Review. The Journal of cardiovascular nursing. 2017 Jul/Aug;32(4):E9-e23. doi: 10.1097/jcn.0000000000000392. PMID: 28107251.

80. Warner G, Packer T, Villeneuve $\mathrm{M}$, et al. A systematic review of the effectiveness of stroke self-management programs for improving function and participation outcomes: self-management programs for stroke survivors. Disability and rehabilitation. 2015;37(23):2141-63. doi: 10.3109/09638288.2014.996674. PMID: 25579669.

81. Dendere R, Slade C, Burton-Jones A, et al. Patient Portals Facilitating Engagement With Inpatient Electronic Medical Records: A Systematic Review. Journal of medical Internet research. 2019 Apr 11;21(4):e12779. doi: 10.2196/12779. PMID: 30973347.

82. Almutairi N, Hosseinzadeh H, Gopaldasani V. The effectiveness of patient activation intervention on type 2 diabetes mellitus glycemic control and self-management behaviors: A systematic review of RCTs. Primary care diabetes. 2019 Sep 19. doi: 10.1016/j.pcd.2019.08.009. PMID: 31543458.

83. Kelly MM, Coller RJ, Hoonakker PL. Inpatient Portals for Hospitalized Patients and Caregivers: A Systematic Review. Journal of hospital medicine. 2018 Jun 1;13(6):405-12. doi: 10.12788/jhm.2894. PMID: 29261819.

84. Risling T, Martinez J, Young J, et al. Evaluating Patient Empowerment in Association With eHealth Technology: Scoping Review. Journal of medical Internet research. 2017 Sep 29;19(9):e329. doi: 10.2196/jmir.7809. PMID: 28963090.

85. Hamine S, Gerth-Guyette E, Faulx D, et al. Impact of mHealth chronic disease management on treatment adherence and patient outcomes: a systematic review. Journal of medical Internet research. 2015 Feb 24;17(2):e52. doi: 10.2196/jmir.3951. PMID: 25803266.

86. Wildevuur SE, Simonse LW. Information and communication technology-enabled person-centered care for the "big five" chronic conditions: scoping review. Journal of medical Internet research. 2015 Mar 27;17(3):e77. doi: 10.2196/jmir.3687. PMID: 25831199.
87. Jones TM, Dean CM, Hush JM, et al. A systematic review of the efficacy of self-management programs for increasing physical activity in community-dwelling adults with acquired brain injury (ABI). Systematic reviews. 2015 Apr 19;4:51. doi: 10.1186/s13643-0150039-x. PMID: 25927591.

88. Pereira K, Phillips B, Johnson C, et al. Internet delivered diabetes selfmanagement education: a review. Diabetes technology \& therapeutics. 2015 Jan;17(1):55-63. doi: 10.1089/dia.2014.0155. PMID: 25238257.

89. Kruse CS, Bolton K, Freriks G. The effect of patient portals on quality outcomes and its implications to meaningful use: a systematic review. Journal of medical Internet research. 2015 Feb 10;17(2):e44. doi: 10.2196/jmir.3171. PMID: 25669240.

90. Yin AL, Hachuel D, Pollak JP, et al. Digital Health Apps in the Clinical Care of Inflammatory Bowel Disease: Scoping Review. Journal of medical Internet research. 2019 Aug 19;21(8):e14630. doi: 10.2196/14630. PMID: 31429410.

91. Malone H, Biggar S, Javadpour S, et al. Interventions for promoting participation in shared decision-making for children and adolescents with cystic fibrosis. The Cochrane database of systematic reviews. 2019 May 23;5:Cd012578. doi: 10.1002/14651858.CD012578.pub2. PMID: 31119726

92. Cheng H, Hayes D, Edbrooke-Childs J, et al. What approaches for promoting shared decision-making are used in child mental health? A scoping review. Clinical psychology \& psychotherapy. 2017 Nov;24(6):O1495-o511. doi: 10.1002/cpp.2106. PMID: 28752631.

93. Coyne I, O'Mathuna DP, Gibson F, et al. Interventions for promoting participation in shared decision-making for children with cancer. The Cochrane database of systematic reviews. 2016 Nov 29;11:Cd008970. doi: 10.1002/14651858.CD008970.pub3. PMID: 27898175.

94. Wyatt KD, List B, Brinkman WB, et al. Shared Decision Making in Pediatrics: A Systematic Review and Meta-analysis. Academic pediatrics. 2015 Nov-Dec;15(6):573-83. doi: 10.1016/j.acap.2015.03.011. PMID: 25983006. 
95. Voruganti T, Grunfeld E, Makuwaza T, et al. Web-Based Tools for Text-Based Patient-Provider Communication in Chronic Conditions: Scoping Review. Journal of medical Internet research. 2017 Oct 27;19(10):e366. doi: 10.2196/jmir.7987. PMID: 29079552.

96. Kew KM, Malik P, Aniruddhan K, et al. Shared decision-making for people with asthma. The Cochrane database of systematic reviews. 2017 Oct 3;10:Cd012330. doi: 10.1002/14651858.CD012330.pub2. PMID: 28972652.

97. Clayman ML, Bylund CL, Chewning B, et al. The Impact of Patient Participation in Health Decisions Within Medical Encounters: A Systematic Review. Medical decision making : an international journal of the Society for Medical Decision Making. 2016 May;36(4):427-52. doi: 10.1177/0272989x15613530. PMID: 26585293.

98. Winston K, Grendarova P, Rabi D. Video-based patient decision aids: A scoping review. Patient education and counseling. 2018 Apr;101(4):558-78. doi: 10.1016/j.pec.2017.10.009. PMID: 29102063.

99. Nathan AG, Marshall IM, Cooper JM, et al. Use of Decision Aids with Minority Patients: a Systematic Review. Journal of general internal medicine. 2016 Jun;31(6):663-76. doi: 10.1007/s11606-016-3609-2. PMID: 26988981.

100. Wagner A, Radionova N, Rieger MA, et al. Patient Education and Continuing Medical Education to Promote Shared Decision-Making. A Systematic Literature Review. International journal of environmental research and public health. 2019 Jul 12;16(14). doi: 10.3390/ijerph16142482. PMID: 31336828.

101. Goodridge D, McDonald M, New L, et al. Building patient capacity to participate in care during hospitalisation: a scoping review. BMJ open. 2019 Jul 3;9(7):e026551. doi: 10.1136/bmjopen-2018-026551. PMID: 31272973.

102. Irizarry T, DeVito Dabbs A, Curran CR. Patient Portals and Patient Engagement: A State of the Science Review. Journal of medical Internet research. 2015 Jun 23;17(6):e148. doi: 10.2196/jmir.4255. PMID: 26104044.
103. Violette PD, Agoritsas T, Alexander P, et al. Decision aids for localized prostate cancer treatment choice: Systematic review and meta-analysis. CA: a cancer journal for clinicians. 2015 MayJun;65(3):239-51. doi: 10.3322/caac.21272. PMID: 25772796.

104. Jain A, Corriveau S, Quinn K, et al. Video decision aids to assist with advance care planning: a systematic review and meta-analysis. BMJ open. 2015 Jun 24;5(6):e007491. doi: 10.1136/bmjopen-2014-007491. PMID: 26109115.

105. McIntyre R, Craig A. A Literature Review of Patient Education: Is IT Time to Move Forward? Journal of medical imaging and radiation sciences. 2015 Sep;46(3s):S75-s85. doi: 10.1016/j.jmir.2015.04.010. PMID: 31052112

106. Kashaf MS, McGill E. Does Shared Decision Making in Cancer Treatment Improve Quality of Life? A Systematic Literature Review. Medical decision making : an international journal of the Society for Medical Decision Making. 2015 Nov;35(8):1037-48. doi: 10.1177/0272989x15598529. PMID: 26246515.

107. Friedrichs A, Spies M, Harter M, et al. Patient Preferences and Shared Decision Making in the Treatment of Substance Use Disorders: A Systematic Review of the Literature. PloS one. 2016;11(1):e0145817. doi: 10.1371/journal.pone.0145817. PMID: 26731679

108. Baik D, Cho H, Masterson Creber RM. Examining Interventions Designed to Support Shared Decision Making and Subsequent Patient Outcomes in Palliative Care: A Systematic Review of the Literature. The American journal of hospice \& palliative care. 2019 Jan;36(1):7688. doi: 10.1177/1049909118783688. PMID: 29925244.

109. van Weert JC, van Munster BC, Sanders R, et al. Decision aids to help older people make health decisions: a systematic review and metaanalysis. BMC medical informatics and decision making. $2016 \mathrm{Apr}$ 21;16:45. doi: 10.1186/s12911-016-0281-8. PMID: 27098100.

110. Berlin NL, Tandon VJ, Hawley ST, et al. Feasibility and Efficacy of Decision Aids to Improve Decision Making for Postmastectomy Breast Reconstruction: A Systematic Review and Meta-analysis. Medical decision making : an international journal of the Society for Medical Decision Making. 2019 Jan;39(1):5-20. doi: 10.1177/0272989x18803879. PMID: 30799692. 
111. Johnson RA, Huntley A, Hughes RA, et al. Interventions to support shared decision making for hypertension: A systematic review of controlled studies. Health expectations : an international journal of public participation in health care and health policy. 2018 Dec;21(6):1191-207. doi: 10.1111/hex.12826. PMID: 30221454.

112. Martinez-Alonso M, Carles-Lavila M, Perez-Lacasta MJ, et al. Assessment of the effects of decision aids about breast cancer screening: a systematic review and meta-analysis. BMJ open. 2017 Oct 6;7(10):e016894. doi: 10.1136/bmjopen-2017-016894. PMID: 28988175.

113. Legare F, Adekpedjou R, Stacey D, et al. Interventions for increasing the use of shared decision making by healthcare professionals. The Cochrane database of systematic reviews. 2018 Jul 19;7:Cd006732. doi: 10.1002/14651858.CD006732.pub4. PMID: 30025154.

114. Stovell D, Morrison AP, Panayiotou M, et al. Shared treatment decision-making and empowerment-related outcomes in psychosis: systematic review and meta-analysis. The British journal of psychiatry : the journal of mental science. 2016 Jul;209(1):23-8. doi: 10.1192/bjp.bp.114.158931. PMID: 27198483.

115. Porter J, Huggins CE, Truby H, et al. The Effect of Using Mobile Technology-Based Methods That Record Food or Nutrient Intake on Diabetes Control and Nutrition Outcomes: A Systematic Review. Nutrients. 2016 Dec 17;8(12). doi: 10.3390/nu8120815. PMID: 27999302.

116. Cardona-Morrell M, Benfatti-Olivato G, Jansen J, et al. A systematic review of effectiveness of decision aids to assist older patients at the end of life. Patient education and counseling. 2017 Mar;100(3):42535. doi: 10.1016/j.pec.2016.10.007. PMID: 27765378.

117. Baptista S, Teles Sampaio E, Heleno B, et al. Web-Based Versus Usual Care and Other Formats of Decision Aids to Support Prostate Cancer Screening Decisions: Systematic Review and Meta-Analysis. Journal of medical Internet research. 2018 Jun 26;20(6):e228. doi: 10.2196/jmir.9070. PMID: 29945857.

118. Spronk I, Burgers JS, Schellevis FG, et al. The availability and effectiveness of tools supporting shared decision making in metastatic breast cancer care: a review. BMC palliative care. 2018 May 11;17(1):74. doi: 10.1186/s12904-018-0330-4. PMID: 29747628.
119. Stacey D, Legare F, Lewis K, et al. Decision aids for people facing health treatment or screening decisions. The Cochrane database of systematic reviews. 2017 Apr 12;4:Cd001431. doi: 10.1002/14651858.CD001431.pub5. PMID: 28402085.

120. Vermunt N, Harmsen M, Westert GP, et al. Collaborative goal setting with elderly patients with chronic disease or multimorbidity: a systematic review. BMC geriatrics. 2017 Jul 31;17(1):167. doi: 10.1186/s12877-017-0534-0. PMID: 28760149.

121. Saheb Kashaf M, McGill ET, Berger ZD. Shared decision-making and outcomes in type 2 diabetes: A systematic review and meta-analysis. Patient education and counseling. 2017 Dec;100(12):2159-71. doi: 10.1016/j.pec.2017.06.030. PMID: 28693922.

122. Samalin L, Genty JB, Boyer L, et al. Shared Decision-Making: a Systematic Review Focusing on Mood Disorders. Current psychiatry reports. 2018 Mar 27;20(4):23. doi: 10.1007/s11920-018-0892-0. PMID: 29589129.

123. Martinez-Gonzalez NA, Plate A, Senn O, et al. Shared decisionmaking for prostate cancer screening and treatment: a systematic review of randomised controlled trials. Swiss medical weekly. 2018;148:w14584. doi: 10.4414/smw.2018.14584. PMID: 29473938.

124. Martinez-Gonzalez NA, Neuner-Jehle S, Plate A, et al. The effects of shared decision-making compared to usual care for prostate cancer screening decisions: a systematic review and meta-analysis. BMC cancer. 2018 Oct 22;18(1):1015. doi: 10.1186/s12885-018-4794-7. PMID: 30348120.

125. Sendall M, McCosker L, Crossley K, et al. A structured review of chronic care model components supporting transition between healthcare service delivery types for older people with multiple chronic diseases. Health information management : journal of the Health Information Management Association of Australia. 2017 May;46(2):58-68. doi: 10.1177/1833358316681687. PMID: 27923916.

126. Le Berre M, Maimon G, Sourial N, et al. Impact of Transitional Care Services for Chronically Ill Older Patients: A Systematic Evidence Review. Journal of the American Geriatrics Society. 2017 Jul;65(7):1597-608. doi: 10.1111/jgs.14828. PMID: 28403508. 
127. Oldfield BJ, Harrison MA, Genao I, et al. Patient, Family, and Community Advisory Councils in Health Care and Research: a Systematic Review. Journal of general internal medicine. 2019 Jul;34(7):1292-303. doi: 10.1007/s11606-018-4565-9. PMID: 30051331.

128. Sharma AE, Knox M, Mleczko VL, et al. The impact of patient advisors on healthcare outcomes: a systematic review. BMC health services research. 2017 Oct 23;17(1):693. doi: 10.1186/s12913-0172630-4. PMID: 29058625.
129. McCarron TL, Moffat K, Wilkinson G, et al. Understanding patient engagement in health system decision-making: a co-designed scoping review. Systematic reviews. 2019 Apr 18;8(1):97. doi: 10.1186/s13643-019-0994-8. PMID: 30999950.

130. Bombard Y, Baker GR, Orlando E, et al. Engaging patients to improve quality of care: a systematic review. Implementation science : IS. 2018 Jul 26;13(1):98. doi: 10.1186/s13012-018-0784-z. PMID: 30045735.

131. Liang L, Cako A, Urquhart R, et al. Patient engagement in hospital health service planning and improvement: a scoping review. BMJ open. 2018 Jan 30;8(1):e018263. doi: 10.1136/bmjopen-2017-018263. PMID: 29382676. 


\section{Appendix C. Summary Table for Gray Literature Search}

Summary table of patient and family engagement strategies found in the gray literature

\begin{tabular}{|c|c|c|}
\hline Strategy/Resources & Description of Strategy & Evidence/Implementation \\
\hline $\begin{array}{l}\text { Institute for Healthcare Improvement (IHI) } \\
\text { Patient Activation } \\
\text { http://www.ihi.org/resources/Pages/Tools } \\
\text { /Ask-Me-3-Good-Questions-for-Your-Good- } \\
\text { Health.aspx }\end{array}$ & $\begin{array}{l}\text { Ask Me } 3 \circledR \text { is an educational tool, designed by health literacy experts } \\
\text { consisting of } 3 \text { questions, that encourages patients and families to } \\
\text { ask their providers about their health condition and have better } \\
\text { communication with their providers. } \\
\text { 1. What is my main problem? } \\
\text { 2. What do I need to do? } \\
\text { 3. Why is it important for me to do this? }\end{array}$ & $\begin{array}{l}\text { Tested through few pretest-posttest } \\
\text { studies. Low significant effect or } \\
\text { mixed results on patient activation, } \\
\text { patient satisfaction and medication } \\
\text { reconciliation. } \\
\text { https://www.dhs.wisconsin.gov/libra } \\
\text { ry/P-00867.htm } \\
\text { https://www.ncbi.nlm.nih.gov/pubm } \\
\text { ed/19019809 } \\
\text { https://www.ncbi.nlm.nih.gov/pubm } \\
\text { ed/20586368 }\end{array}$ \\
\hline $\begin{array}{l}\text { Institute for Healthcare Improvement (IHI) } \\
\text { Patient-administered self-care } \\
\text { http://www.ihi.org/resources/Pages/Publications/Teach } \\
\text { ing-Patients-to-Administer-Their-Own-Care.aspx } \\
\text { http://www.ihi.org/resources/Pages/Publications/Patien } \\
\text { t-Administered-Self-Care.aspx }\end{array}$ & $\begin{array}{l}\text { A 90-day innovation project evaluating the two primary approaches to } \\
\text { patient-administered self-care i-e at healthcare facility and at home } \\
\text { and the experiences of two healthcare settings with implementing } \\
\text { self-care for dialysis and intravenous antibiotics. } \\
\text { Four components of a successful patient-administered self-care } \\
\text { system. } \\
\text { 1. Activated, capable patient and/or caregiver } \\
\text { 2. Protocolized procedures } \\
\text { 3. Supportive health care system } \\
\text { 4. Practitioners trained in patient-administered self-care. }\end{array}$ & $\begin{array}{l}\text { "When comparing the } 944 \text { patients } \\
\text { in the at-home self-care program to } \\
224 \text { patients who received } \\
\text { traditional in-hospital IV antibiotics, } \\
\text { the home-based self-care patients } \\
\text { had a } 47 \text { percent lower readmission } \\
\text { rate and a statistically similar } \\
\text { mortality rate." }\end{array}$ \\
\hline $\begin{array}{l}\text { Institute for Healthcare Improvement (IHI) } \\
\text { Family/Care giver support } \\
\text { http://www.ihi.org/resources/Pages/Publications/Suppo } \\
\text { rting-Supporters-What-Family-Caregivers-Need- } \\
\text { Cancer-Care.aspx }\end{array}$ & $\begin{array}{l}\text { Supporting the Supporters: What Family } \\
\text { Caregivers Need to Care for a Loved One with Cancer } \\
\text { (Recommendation/education material) } \\
\text { Four-part framework for supporting family caregivers: } \\
\text { (1) Assess caregivers' needs using formal measures, just as the } \\
\text { cancer patient's own needs are assessed. } \\
\text { (2) Educate caregivers for their caregiving roles, most notably, with } \\
\text { training in the low-level medical support that cancer patients require } \\
\text { at home. } \\
\text { (3) Empower caregivers to become full-fledged members of the } \\
\text { patient's cancer team, all working toward common goals. (4) Assist } \\
\text { caregivers proactively in their duties, so that they retain a sense of } \\
\text { control and self-efficacy rather than having to react to imminent } \\
\text { medical crises without sufficient resources at their disposal. }\end{array}$ & \\
\hline
\end{tabular}




\begin{tabular}{|c|c|c|}
\hline Strategy/Resources & Description of Strategy & Evidence/Implementation \\
\hline $\begin{array}{l}\text { Institute for Healthcare Improvement (IHI) } \\
\text { ACP/End of life care } \\
\text { http://www.ihi.org/resources/Pages/IHIWhitePapers } \\
\text { /ConversationReadyEndofLifeCare.aspx }\end{array}$ & $\begin{array}{l}\text { "Conversation Ready": A Framework for Improving End- of-Life Care } \\
\text { IHI's "Conversation Ready" approach to help health care } \\
\text { organizations and clinicians provide respectful end-of-life care that is } \\
\text { concordant with patients' stated goals, values, and preferences and } \\
\text { defines five interconnected Conversation Ready principles. }\end{array}$ & \\
\hline $\begin{array}{l}\text { Institute for Healthcare Improvement (IHI) } \\
\text { Patient education } \\
\text { http://lippincottsolutions.Iww.com/blog.entry.html } \\
\text { /2017/08/22/5_strategies_forpro-kDDq.html }\end{array}$ & $\begin{array}{l}5 \text { Strategies for Providing Effective Patient Education } \\
\text { This web article describes five strategies for success in patient } \\
\text { education and engagement. } \\
\text { 1.Use of Technology } \\
\text { 2. Determine the patient's learning style. } \\
\text { 3.Stimulate the patient's interest } \\
\text { 4.Consider the patient's limitations and strengths } \\
\text { 5. Include family members in patient care. }\end{array}$ & \\
\hline $\begin{array}{l}\text { Planetree International } \\
\text { Patient Activation and Engagement } \\
\text { https://resources.planetree.org/50-ways-to-be-an- } \\
\text { engaged-patient-2/ }\end{array}$ & $\begin{array}{l}50 \text { Ways to be an Engaged Patient. A resource for patients and } \\
\text { families. } \\
\text { A list of specific actions and behaviors patients and family members } \\
\text { can adopt to be more active participants in their healthcare. }\end{array}$ & \\
\hline $\begin{array}{l}\text { Planetree International } \\
6 \text { Steps to Creating a Culture of Person and Family } \\
\text { Engagement in Healthcare. } \\
\text { https://resources.planetree.org/6-steps-to-creating-a- } \\
\text { culture-of-person-and-family-engagement-in-health- } \\
\text { care/ }\end{array}$ & $\begin{array}{l}\text { "This toolkit provides guidance to physician practices on creating a } \\
\text { practice culture that emphasizes and incorporates the patient and } \\
\text { family perspective in every aspect of care, to ultimately improve } \\
\text { quality of care" }\end{array}$ & \\
\hline $\begin{array}{l}\text { Planetree International } \\
\text { Patient Activation and Engagement } \\
\text { https://resources.planetree.org/questions-to-ask-your- } \\
\text { doctor-about-person-centered-care/ }\end{array}$ & $\begin{array}{l}\text { Questions to Ask Your Doctor About Person-Centered Care } \\
\text { "12 questions framework patients and family members can ask their } \\
\text { doctor to better understand opportunities for partnership." }\end{array}$ & \\
\hline $\begin{array}{l}\text { Open Notes } \\
\text { OpenNotes - Patient portals/EHR }\end{array}$ & $\begin{array}{l}\text { OpenNotes is an international movement and platform for patients } \\
\text { and Healthcare providers committed to spreading the availability of } \\
\text { open visit notes and studying the effects. }\end{array}$ & $\begin{array}{l}\text { Multiple studies showing significant } \\
\text { positive impact on patient centered } \\
\text { outcomes and engagement. }\end{array}$ \\
\hline
\end{tabular}




\begin{tabular}{|c|c|c|}
\hline Strategy/Resources & Description of Strategy & Evidence/Implementation \\
\hline $\begin{array}{l}\text { Institute for Patient- and Family-Centered Care } \\
\text { (IPFCC) } \\
\text { Individual and Family Engagement in the Medicaid } \\
\text { Population } \\
\text { https://www.ipfcc.org/bestpractices/Individual- } \\
\text { Engagement.pdf }\end{array}$ & $\begin{array}{l}\text { Literature review and key interviews } \\
4 \text { broad recommendation categories: } \\
\text { 1. Advance Individual and Family Engagement Best Practices as a } \\
\text { Strategic Priority Within the Medicaid Agency } \\
\text { 2. Expand Opportunities for Individuals and Families to Engage with } \\
\text { Medicaid Staff and Managed } \\
\text { Care Entities to Influence Policies, Programs, and Practices. } \\
\text { 3. Support Direct Care Service Providers in Acquiring the Knowledge } \\
\text { and Skills to Develop Effective Partnerships with Individuals and } \\
\text { Families. } \\
\text { 4. Encourage and Support Individuals and Families to Engage More } \\
\text { Fully in Their Health and with Their Health Care Team. }\end{array}$ & \\
\hline $\begin{array}{l}\text { Institute for Patient- and Family-Centered Care } \\
\text { (IPFCC) } \\
\text { Patient and Family Advisory Councils } \\
\text { https://www.ipfcc.org/bestpractices/NYSHF_2018_PF } \\
\text { ACOnline_v3.pdf }\end{array}$ & $\begin{array}{l}\text { Strategically Advancing Patient and Family Advisory Councils in New } \\
\text { York State Hospitals; Survey of NY hospitals. } \\
\text { Recommendations: } \\
\text { 1. Build partnerships with patients and families into State and } \\
\text { regional quality and safety initiatives. } \\
\text { 2. Create opportunities for shared learning and mentorship around } \\
\text { PFAC work. } \\
\text { 3. Develop guidance to help hospitals access existing PFAC training } \\
\text { resources in ways that address the need for tailored information. } \\
\text { 4. Conduct additional research about the evolution and impact of } \\
\text { PFACs and expand work to other states and settings. } \\
\text { 5. Disseminate PFAC study results to share learnings within and } \\
\text { outside of New York State. }\end{array}$ & \\
\hline $\begin{array}{l}\text { American Institutes for Research (AIR) } \\
\text { Strategic Vision Roadmap for Person and Family } \\
\text { Engagement (PFE) }\end{array}$ & $\begin{array}{l}\text { Achieving the PFE Metrics to Improve Patient Safety and Health } \\
\text { Equity. } \\
\text { Six overarching strategies that are designed to help hospitals } \\
\text { implement PFE practices-including the five PFE metrics-in ways } \\
\text { that reflect and operationalize the core PFE principles. } \\
\text { 1. Organizational partnership } \\
\text { 2. Patient and family preparation } \\
\text { 3. Clinician and leadership preparation } \\
\text { 4. Care, policy, and practice redesign } \\
\text { 5. Measurement and research } \\
\text { 6. Transparency and accountability }\end{array}$ & \\
\hline
\end{tabular}




\begin{tabular}{|c|c|c|}
\hline Strategy/Resources & Description of Strategy & Evidence/Implementation \\
\hline $\begin{array}{l}\text { American Institutes for Research (AIR) } \\
\text { Innovative Patient Engagement Strategies } \\
\text { https://www.air.org/project/innovative-patient- } \\
\text { engagement-strategies }\end{array}$ & $\begin{array}{l}\text { AIR health experts conducted site visits to several accountable care } \\
\text { organizations (ACOs - groups of doctors, hospitals, and other health } \\
\text { care providers, who come together voluntarily to give coordinated } \\
\text { high quality care to their Medicare patients) and primary care settings } \\
\text { around the country to observe efforts by providers to increase patient } \\
\text { involvement and engagement in their health care. Innovative patient } \\
\text { engagement strategies observed included: } \\
\text { - Visiting patients in their homes allows providers to focus on } \\
\text { providing efficient and effective care. One ACO achieved a } 15 \\
\text { percent reduction in hospital admissions along with very high patient } \\
\text { satisfaction scores. } \\
\text { - Meeting end-stage renal disease patients at dialysis centers allows } \\
\text { providers to support patients in following recommendations between } \\
\text { appointments and coordinate care. This helped an ACO engage } \\
\text { patients in ways that improved follow-up on important primary care } \\
\text { needs. } \\
\text { - Using a "nurse navigator" to follow up with patients over the phone } \\
\text { helps providers understand patient needs, answer questions, and } \\
\text { develop effective approaches. This increases patients' understanding } \\
\text { about their conditions and helped an ACO ensure it was providing } \\
\text { proper at-home follow-up care. } \\
\text { - Including patients and family caregivers in care teams and in } \\
\text { developing and reviewing care plans to work through medical, social, } \\
\text { psychological, and logistical issues helps providers better care for } \\
\text { patients with multiple chronic issues, such as dementia or end-stage } \\
\text { cancer. An AcO that developed integrated plans with patient and } \\
\text { family input and engagement correlated this to more effective care } \\
\text { and greater patient satisfaction. }\end{array}$ & \\
\hline $\begin{array}{l}\text { Agency for Healthcare Research and Quality } \\
\text { (AHRQ) } \\
\text { Engaging Patients and Families in Their Health Care } \\
\text { https://www.ahrq.gov/patient-safety/resources/patient- } \\
\text { family-engagement/index.html }\end{array}$ & $\begin{array}{l}\text { The variety of resources offered by AHRQ in form of guides and tools } \\
\text { for medical staff and patients and their caregiver to improve } \\
\text { healthcare quality and safety in hospital and the primary care setting. } \\
\text { Following are some examples of such guides and tools. }\end{array}$ & \\
\hline
\end{tabular}




\begin{tabular}{|c|c|c|}
\hline Strategy/Resources & Description of Strategy & Evidence/Implementation \\
\hline $\begin{array}{l}\text { Agency for Healthcare Research and Quality } \\
\text { (AHRQ) } \\
\text { Guide to Patient and Family Engagement in Hospital } \\
\text { Quality and Safety } \\
\text { https://www.ahrq.gov/professionals/systems/ } \\
\text { hospital/engagingfamilies/guide.html }\end{array}$ & $\begin{array}{l}\text { The guide: } \\
\text { o } \begin{array}{l}\text { Describes critical opportunities for hospitals to engage } \\
\text { patients and families and to create partnerships between } \\
\text { patients, families, and hospitals around the same goals. }\end{array} \\
\text { o } \\
\text { Addresses real-world challenges. The Guide was } \\
\text { developed, implemented, and evaluated with the input of } \\
\text { patients, family members, clinicians, hospital staff, and } \\
\text { hospital leaders. } \\
\text { Helps hospitals engage patients and families, which in turn } \\
\text { can help improve quality and safety, respond to health care } \\
\text { reform and accreditation standards, improve CAHPS }{ }^{8} \\
\text { Hospital Survey scores, improve financial performance, and } \\
\text { enhance market share and competitiveness. } \\
\text { Facilitates implementation and evaluation of each strategy } \\
\text { with detailed guidance and customizable tools. } \\
\text { The Guide to Patient and Family Engagement in Hospital Quality and } \\
\text { Safety is a tested, evidence-based resource to help hospitals work as } \\
\text { partners with patients and families to improve quality and safety. }\end{array}$ & \\
\hline $\begin{array}{l}\text { Agency for Healthcare Research and Quality } \\
\text { (AHRQ) } \\
\text { Toolkit To Educate and Engage Residents and Family } \\
\text { Members } \\
\text { https://www.ahrq.gov/nhguide/toolkits/educate-and- } \\
\text { engage/index.html }\end{array}$ & $\begin{array}{l}\text { The Resident and Family Member Education toolkit helps the nursing } \\
\text { home (1) encourage an open and respectful dialogue between } \\
\text { nurses and prescribing clinicians and residents and their family } \\
\text { members, and (2) help residents and family members participate in } \\
\text { their care. }\end{array}$ & \\
\hline $\begin{array}{l}\text { Agency for Healthcare Research and Quality } \\
\text { (AHRQ) } \\
\text { Guide to Improving Patient Safety in Primary Care } \\
\text { Settings by } \\
\text { Engaging Patients and Families } \\
\text { https://www.ahrq.gov/patientsafety/reports/engage.htm } \\
\text { | }\end{array}$ & $\begin{array}{l}\text { The Guide to Improving Patient Safety in Primary Care Settings by } \\
\text { Engaging Patients and Families (the Guide) is a resource to help } \\
\text { primary care practices partner with patients and their families to } \\
\text { improve patient safety. The Guide is composed of materials and } \\
\text { resources to help primary care practices implement patient and family } \\
\text { engagement to improve patient safety. }\end{array}$ & \\
\hline
\end{tabular}




\begin{tabular}{|c|c|c|}
\hline Strategy/Resources & Description of Strategy & Evidence/Implementation \\
\hline $\begin{array}{l}\text { Healthwise } \\
\text { Patient Response: Giving Voice to the Patients (White } \\
\text { paper) } \\
\text { https://www.healthwise.org/resources/patient- } \\
\text { responsevoicetopatient.aspx?back=\%2fresources.asp } \\
\text { x\%3fsmartsearchfilter\%3d4\%3b }\end{array}$ & $\begin{array}{l}\text { This white paper highlights the significance of patient responses and } \\
\text { voices. Some quotes from paper: } \\
\text { "While clinicians can now deliver good information to patients through } \\
\text { their EMRs, there is no accepted use of IT standards that allows the } \\
\text { patient's voice to get back to the clinician. } \\
\text { Patient response is a new way (and a new use of existing standards) } \\
\text { to ensure that the patient's voice can be well heard in creating care } \\
\text { plans, in deciding among treatment options, and in advancing the } \\
\text { doctor-patient partnership. By providing an easier way to pay more } \\
\text { attention to the choices of our patients, we can finally put them at the } \\
\text { center of care. } \\
\text { In short, patient response provides the mechanism to routinely } \\
\text { capture patients' input in their electronic records so that physicians } \\
\text { have easy access to the information." }\end{array}$ & \\
\hline $\begin{array}{l}\text { Healthwise } \\
6 \text { Keys to Keeping Your Diabetes Patients Engaged } \\
\text { (Infographic information) } \\
\text { https://www.healthwise.org/resources/keep-diabetes- } \\
\text { patients-engaged.aspx?back=\%2fresources.aspx }\end{array}$ & $\begin{array}{l}\text { This infographic mentions the } 6 \text { key steps to engage diabetic patients } \\
\text { in their care to improve clinical outcomes. } \\
\text { 1. Use technology to engage people } \\
\text { 2. Touchbase frequently, both in and out of the clinic } \\
\text { 3. Encourage people to treat themselves-the right way } \\
\text { 4. Identify what's in it for them } \\
\text { 5. Create and celebrate milestones } \\
\text { 6. Help patients focus on the future }\end{array}$ & \\
\hline $\begin{array}{l}\text { Healthwise } \\
\text { Engaging Patients Within the Workflow Through } \\
\text { Standards-Based Interoperability (eBrief) } \\
\text { https://www.healthwise.org/resources/engaging- } \\
\text { patientswithinworkflow.aspx?back=\%2fresources.aspx } \\
\% 3 f p a g e \% 3 d 4\end{array}$ & $\begin{array}{l}\text { This eBrief describes the ways of chronic care management services } \\
\text { and engaging patients through EHR. } \\
\text { "Using two reimbursement programs by Centers for Medicare and } \\
\text { Medicaid Services (CMS), this eBrief will demonstrate how open APIs } \\
\text { and standards-based interoperability offer a seamless medium for } \\
\text { delivering health education and receiving important information back } \\
\text { from the patient, such as a completed health risk assessment (HRA) } \\
\text { or the patient's preferences." }\end{array}$ & \\
\hline $\begin{array}{l}\text { Healthwise } \\
8 \text { Ways to Engage Your Hispanic Population } \\
\text { (Infographic information) } \\
\text { https://www.healthwise.org/resources/engage-your- } \\
\text { hispanicpopulation.aspx?back=\%2fresources.aspx\%3f } \\
\text { page\%3d4 }\end{array}$ & $\begin{array}{l}\text { 1. Record member language preference in your system. } \\
\text { 2. Increase physician awareness for coaching programs. } \\
\text { 3. Implement a system that makes prescribing } \\
\text { education easy. } \\
\text { 4. Provide basic education first } \\
\text { 5. Make sure key self-management materials are in English and } \\
\text { Spanish. } \\
\text { 6. Include easy to find Spanish education at your portal. } \\
\text { 7. Reinforce self-management skills on an ongoing basis. } \\
\text { 8. Track adherence to standards of care. }\end{array}$ & \\
\hline
\end{tabular}




\begin{tabular}{|c|c|c|}
\hline Strategy/Resources & Description of Strategy & Evidence/Implementation \\
\hline $\begin{array}{l}\text { Healthwise } \\
\text { e-Coaching for Boomer Health (White paper) } \\
\text { https://www.healthwise.org/resources/e-coaching-for- } \\
\text { boomerhealth.aspx?back=\%2fresources.aspx\%3fsmar } \\
\text { tsearchfilter\%3d4 }\end{array}$ & $\begin{array}{l}\text { This gray/white paper describes how Information Therapy }(I X ®) \text { Tools } \\
\text { can give boomers the personalized and efficient health care } \\
\text { experience they want and need. It highlights the need of disease } \\
\text { specific personalized virtual/online coaching that help them in self- } \\
\text { management and making better decisions for better health. }\end{array}$ & \\
\hline $\begin{array}{l}\text { Healthwise } \\
8 \text { Steps to Shared Decision Making Success for Care } \\
\text { Management (Infographic information) } \\
\\
\text { https://www.healthwise.org/resources/shared-decision- } \\
\text { makingsuccesscm.aspx?back=\%2fresources.aspx\%3f } \\
\text { smartsearchfilter\%3d3\%3b\%26page\%3d2 }\end{array}$ & $\begin{array}{l}\text { 1.Engage clinicians and staff } \\
\text { 2. Target individuals or populations for SDM } \\
\text { 3. Identify and involve members for intervention } \\
\text { 4. Distribute decision aids to members } \\
\text { 5. Encourage decision aid viewing } \\
\text { 6. Support SDM conversations } \\
\text { 7. Measure the impact } \\
\text { 8. Provide multilevel feedback }\end{array}$ & \\
\hline $\begin{array}{l}\text { Lucile Packard Foundation for Children's Health } \\
\text { Five Top Tips for Engaging Families in Advisory Roles: } \\
\text { Advice from a Family Leader } \\
\text { https://www.lpfch.org/sites/default/files/field/publication } \\
\text { s/five_top_tips_12.19.18.pdf }\end{array}$ & $\begin{array}{l}\text { A factsheet mentioning the advice and five tips from a family leader } \\
\text { and PFAC chair for meaningfully engaging a group of family advisors. } \\
\text { 1. Organization Values Families' Time and Input -compensation for } \\
\text { family member in form of stipends, food etc } \\
\text { 2. Organization Offers Ongoing Training and Support to Family } \\
\text { Members } \\
\text { 3. Organization Provides Support to Committee Chair } \\
\text { 4. Chair Models Appropriate Facilitation and Behavior } \\
\text { 5. Chair Builds Community, Cohesion, and Trust }\end{array}$ & \\
\hline $\begin{array}{l}\text { Lucile Packard Foundation for Children's Health } \\
\text { A Framework for Assessing Family Engagement in } \\
\text { Systems Change } \\
\text { https://www.lpfch.org/sites/default/files/field/publication } \\
\text { s/assessing_family_engagement_4.10.18.pdf }\end{array}$ & $\begin{array}{l}\text { This Issue Brief summarizes strategies for ensuring, enhancing, and } \\
\text { supporting the meaningful engagement of families at the systems } \\
\text { level of health care and the barriers to effective family engagement - } \\
\text { findings from existing literature and a series of key informant } \\
\text { interviews with family leaders and professionals. Further mentioned } \\
\text { are four domains of family engagement in systems -Representation, } \\
\text { Transparency, Impact and Commitment. }\end{array}$ & \\
\hline $\begin{array}{l}\text { Lucile Packard Foundation for Children's Health } \\
\text { Engaging Families in Improving the Health Care } \\
\text { System for Children with Special Health Care Needs } \\
\text { https://www.lpfch.org/sites/default/files/field/publication } \\
\text { s/engaging_families_in_improving_the_health_care_s } \\
\text { ystem.pdf }\end{array}$ & $\begin{array}{l}\text { This factsheet describes the findings from literature on evidence that } \\
\text { patient and family engagement is essential and beneficial to get } \\
\text { better patient outcomes. Further mentioned are the barriers to family } \\
\text { engagement, strategies for improving organizations and health } \\
\text { systems to engage family members effectively, and the role of Lucile } \\
\text { Packard Foundation for Children's Health to support such activities to } \\
\text { improve the process of family engagement for better health care } \\
\text { system for children with special health care needs. }\end{array}$ & \\
\hline
\end{tabular}




\begin{tabular}{|c|c|c|}
\hline Strategy/Resources & Description of Strategy & Evidence/Implementation \\
\hline $\begin{array}{l}\text { Lucile Packard Foundation for Children's Health } \\
\text { Creating and Sustaining Effective Hospital Family } \\
\text { Advisory Councils: } \\
\text { Findings from the California Patient and Family } \\
\text { Centered Care Network of Pediatric Hospitals } \\
\text { https://www.Ipfch.org/sites/default/files/field/publication } \\
\text { s/creating_and_sustaining_effective_hospital_family_a } \\
\text { dvisory_councils.pdf }\end{array}$ & $\begin{array}{l}\text { To support the development of effective FACs in pediatric settings, } \\
\text { the Lucile Packard Foundation for Children's Health provided grant } \\
\text { funding in } 2012 \text { to create the California Patient \& Family Centered } \\
\text { Care Network (CA-PFCC). The primary goal of the Network is to } \\
\text { share ideas and resources to facilitate the formation of sustainable } \\
\text { Family Advisory Councils in health care settings. To achieve this } \\
\text { goal, Network members participated in a range of activities } \\
\text { (webinars, ideation sessions, and work groups) to gather information } \\
\text { about the current state of FACs in California. This report shares the } \\
\text { results of analyzed data that helped in identification of foundational } \\
\text { elements of FACs: function, venue, authority, and membership } \\
\text { configuration. Collectively, this work resulted in a checklist of key } \\
\text { activities intended to guide the creation of new FACs and to enhance } \\
\text { and expand the work of existing Councils. }\end{array}$ & \\
\hline $\begin{array}{l}\text { Lucile Packard Foundation for Children's Health } \\
\text { A Guide to Establishing Effective Hospital Family } \\
\text { Advisory Councils } \\
\text { https://www.Ipfch.org/sites/default/files/field/publication } \\
\text { s/ } \\
\text { a_guide_to_establishing_effective_hospital_family_ad } \\
\text { visory_councils_0.pdf }\end{array}$ & $\begin{array}{l}\text { This fact sheet describes the shared experiences of network } \\
\text { members of California Patient \& Family Centered Care Network with } \\
\text { FACs and a checklist for establishing effective Councils. }\end{array}$ & \\
\hline $\begin{array}{l}\text { Children's Hospital Association } \\
\text { Invoking the Power of Family Partnerships to Improve } \\
\text { Outcomes for Children with Medical Complexity } \\
\text { https://www.childrenshospitals.org/- } \\
\text { /media/Files/CHA/Main/Programs_and_Services/Quali } \\
\text { ty_Safety_and_Performance/ } \\
\text { CARE/care_081318_invoking_power_family_partners } \\
\text { hips.pdf?la=en\&hash= } \\
\text { C0B173FA84AEF23E63A4744575376BDAE447293D }\end{array}$ & $\begin{array}{l}\text { This paper mentions briefly the } 10 \text { vital actions for effective family } \\
\text { partnerships and levels of family involvement. It also reports the } \\
\text { CARE Award Model (The Coordinating All Resources Effectively) } \\
\text { designed to transform care through the provision of appropriate, } \\
\text { coordinated care in the right setting, and develop alternative payment } \\
\text { models that more effectively align with the new care model. The } \\
\text { CARE Award called for the direct participation of families on quality } \\
\text { improvement (QI) teams, and in the design and evaluation of clinical } \\
\text { methods and tools grounded in shared decision-making with families } \\
\text { and members of their health care team. Highlighted are findings of } \\
\text { different QI projects by member organizations. }\end{array}$ & \\
\hline
\end{tabular}




\begin{tabular}{|c|c|c|}
\hline Strategy/Resources & Description of Strategy & Evidence/Implementation \\
\hline $\begin{array}{l}\text { Family Voices } \\
\text { Family Engagement in Systems: A Literature Review } \\
\text { http://familyvoices.org/wp- } \\
\text { content/uploads/2018/10/Engagement-Assessment- } \\
\text { literature-4.10.18_r4.26.18.pdf }\end{array}$ & $\begin{array}{l}\text { This literature review used various peer-reviewed articles and grey } \\
\text { literature resources to describe wide ranging and sophisticated } \\
\text { approaches to patient and family engagement being practiced today, } \\
\text { and provide a picture of a vibrant and increasingly evidence-based } \\
\text { field of study. According to author, "the Family Voices project, } \\
\text { Framework for Assessing Family Engagement, addresses the topic of } \\
\text { ensuring and enhancing the role and participation of families in all } \\
\text { aspects of the systems on which CYSHCN depend. The purpose of } \\
\text { the work was to develop a brief that } 1 \text { ) establishes key characteristics } \\
\text { of effective family engagement in systems level programs and 2) } \\
\text { outlines specific actions to build an assessment based on those key } \\
\text { criteria." }\end{array}$ & \\
\hline $\begin{array}{l}\text { Family Voices } \\
\text { Family Voices }\end{array}$ & $\begin{array}{l}\text { Family Voices is a national family-led organization of families and } \\
\text { friends of children and youth with special health care needs } \\
\text { (CYSHCN) and disabilities. Multiple resources related to patient and } \\
\text { family engagement including white papers and reports listed at } \\
\text { Family Voices site. } \\
\text { https://familyvoices.org/resources/ }\end{array}$ & \\
\hline $\begin{array}{l}\text { American Cancer Society } \\
\text { Caregiver Resource Guide } \\
\text { https://www.cancer.org/treatment/caregivers/caregiver- } \\
\text { resource-guide.html\# }\end{array}$ & $\begin{array}{l}\text { "The American Cancer Society Caregiver Resource Guide is a tool } \\
\text { for people who are caring for someone with cancer. It can help you: } \\
\text { learn how to care for yourself as a caregiver, better understand what } \\
\text { your loved one is going through, develop skills for coping and caring, } \\
\text { and take steps to help protect your health and well-being." }\end{array}$ & \\
\hline $\begin{array}{l}\text { American Cancer Society } \\
\text { The Doctor-Patient Relationship } \\
\text { https://www.cancer.org/treatment/finding-and-paying- } \\
\text { for-treatment/choosing-your-treatment-team/the- } \\
\text { doctor-patient-relationship.html }\end{array}$ & $\begin{array}{l}\text { Information and guide on patient activation. What cancer patients } \\
\text { should expect and ask from physicians to make informed decisions } \\
\text { for their health. }\end{array}$ & \\
\hline $\begin{array}{l}\text { American Heart Association } \\
\text { Caregiver Support } \\
\text { https://www.heart.org/en/health-topics/caregiver- } \\
\text { support }\end{array}$ & $\begin{array}{l}\text { Multiple resources addressing the needs of caregivers and tips for } \\
\text { them to stay healthy and provide better care to their loved ones with } \\
\text { various cardiac diseases. Resources and educational material for } \\
\text { specific disease also available. }\end{array}$ & \\
\hline
\end{tabular}




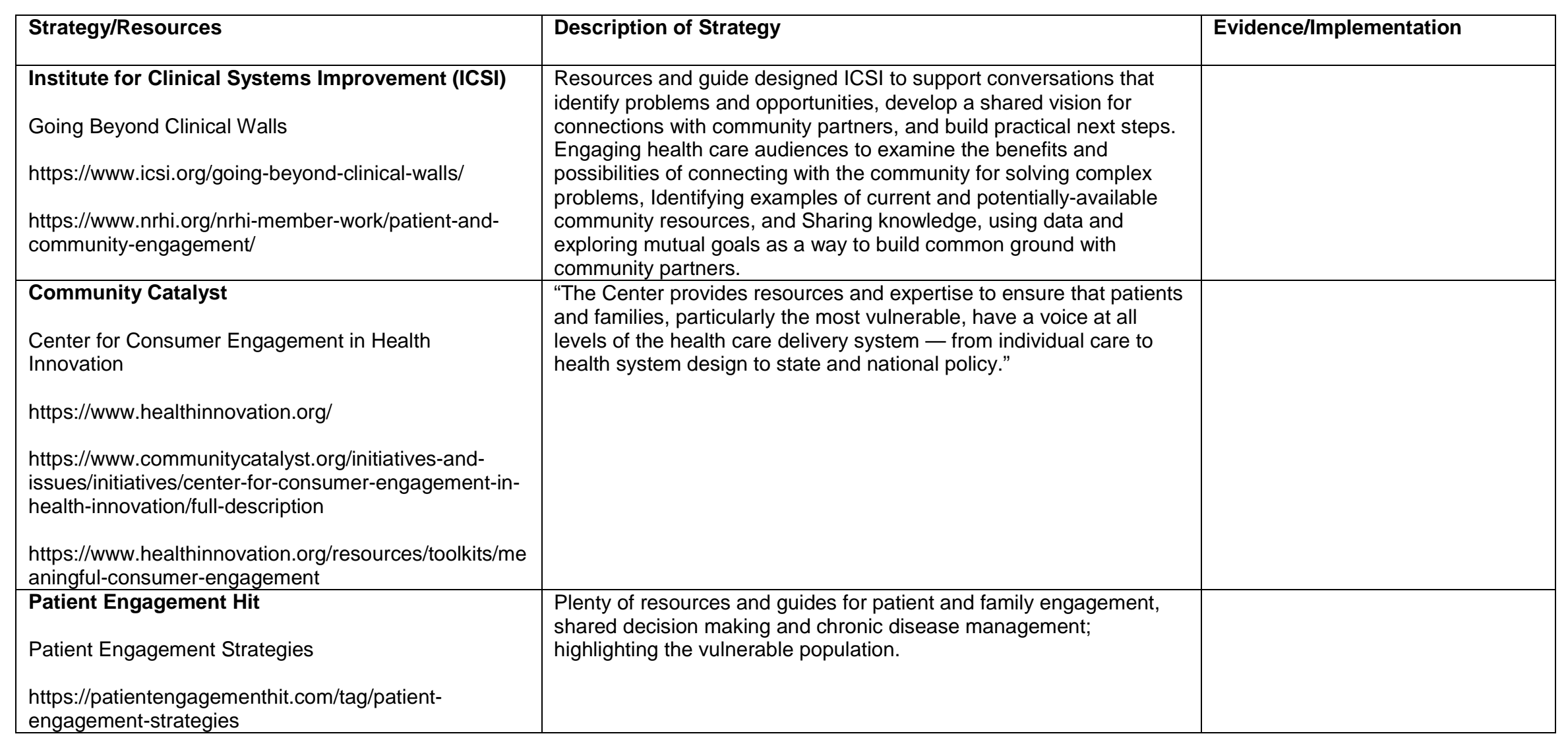




\section{Appendix D. List of Excluded Studies for Systematic Reviews}

Studies are sorted by the reason for exclusion.

\section{Does not apply to the Key Question}

1. Lombardero A, Hansen CD, Richie AE, et al. A Narrative Review of the Literature on Insufficient Sleep, Insomnia, and Health Correlates in American Indian/Alaska Native Populations. Journal of environmental and public health. 2019;2019:4306463. PMID: 31360174.

2. Miller WR. The Projected Care Trajectory for Persons with Epilepsy. The Nursing clinics of North America. 2019 Sep;54(3):425-35. PMID: 31331628.

3. Sousa H, Ribeiro O, Paul C, et al. Social support and treatment adherence in patients with end-stage renal disease: A systematic review. Seminars in dialysis. 2019 Jul 15. PMID: 31309612.

4. Vo V, Auroy L, Sarradon-Eck A. Patients' Perceptions of mHealth Apps: Meta-Ethnographic Review of Qualitative Studies. JMIR mHealth and uHealth. 2019 Jul 10;7(7):e13817. PMID: 31293246.

5. Mulligan H, Wilkinson A, Chen D, et al. Components of community rehabilitation programme for adults with chronic conditions: A systematic review. International journal of nursing studies. 2019 May 31;97:114-29. PMID: 31234105.

6. Reiners F, Sturm J, Bouw LJW, et al. Sociodemographic Factors Influencing the Use of eHealth in People with Chronic Diseases. International journal of environmental research and public health. 2019 Feb 21;16(4). PMID: 30795623.

7. McGilton KS, Vellani S, Yeung L, et al. Identifying and understanding the health and social care needs of older adults with multiple chronic conditions and their caregivers: a scoping review. BMC geriatrics. 2018 Oct 1;18(1):231. PMID: 30285641.

8. Kuo AM, Thavalathil B, Elwyn G, et al. The Promise of Electronic Health Records to Promote Shared Decision Making: A Narrative Review and a Look Ahead. Medical decision making : an international journal of the Society for Medical Decision Making. 2018 Nov;38(8):1040-5. PMID: 30226100.

9. Han HR, Hong H, Starbird LE, et al. eHealth Literacy in People Living with HIV: Systematic Review. JMIR public health and surveillance. 2018 Sep 10;4(3):e64. PMID: 30201600.

10. McCabe E, Miciak M, Dennett L, et al. Measuring therapeutic relationship in the care of patients with haemophilia: A scoping review. Health expectations : an international journal of public participation in health care and health policy. 2018 Dec;21(6):1208-30. PMID: 30160003.

11. Nazir MA, AlGhamdi L, AlKadi M, et al. The burden of Diabetes, Its Oral Complications and Their Prevention and Management. Open access Macedonian journal of medical sciences. 2018 Aug 20;6(8):1545-53. PMID: 30159091.

12. Budhwani S, Wodchis WP, Zimmermann C, et al. Self-management, self-management support needs and interventions in advanced cancer: a scoping review. BMJ supportive \& palliative care. 2019 Mar;9(1):12-25. PMID: 30121581. 
13. Kusnanto H, Agustian D, Hilmanto D. Biopsychosocial model of illnesses in primary care: A hermeneutic literature review. Journal of family medicine and primary care. 2018 MayJun;7(3):497-500. PMID: 30112296.

14. Hart T, Driver S, Sander A, et al. Traumatic brain injury education for adult patients and families: a scoping review. Brain injury. 2018;32(11):1295-306. PMID: 30084694.

15. Miranda S, Marques A. Pilates in noncommunicable diseases: A systematic review of its effects. Complementary therapies in medicine. 2018 Aug;39:114-30. PMID: 30012382.

16. Mabweazara SZ, Ley C, Leach LL. Physical activity, social support and socio-economic status amongst persons living with HIV and AIDS: a review. African journal of AIDS research : AJAR. 2018 Jul;17(2):203-12. PMID: 30003848.

17. De Angelis G, Wells GA, Davies B, et al. The use of social media among health professionals to facilitate chronic disease self-management with their patients: A systematic review. Digital health. 2018 Jan-Dec;4:2055207618771416. PMID: 29942633.

18. Poitras ME, Maltais ME, Bestard-Denomme L, et al. What are the effective elements in patient-centered and multimorbidity care? A scoping review. BMC health services research. 2018 Jun 14;18(1):446. PMID: 29898713.

19. Contreras I, Vehi J. Artificial Intelligence for Diabetes Management and Decision Support: Literature Review. Journal of medical Internet research. 2018 May 30;20(5):e10775. PMID: 29848472.

20. Santos T, Lovell J, Shiell K, et al. The impact of cognitive impairment in dementia on selfcare domains in diabetes: A systematic search and narrative review. Diabetes/metabolism research and reviews. 2018 Sep;34(6):e3013. PMID: 29707902.

21. de Kok BC, Widdicombe S, Pilnick A, et al. Doing patient-centredness versus achieving public health targets: A critical review of interactional dilemmas in ART adherence support. Social science \& medicine (1982). 2018 May;205:17-25. PMID: 29631198.

22. Gierisch JM, Hughes JM, Edelman D, et al. VA Evidence-based Synthesis Program Reports. The Effectiveness of Health Coaching. Washington (DC): Department of Veterans Affairs (US); 2017.

23. Chan RJ, Marx W, Bradford N, et al. Clinical and economic outcomes of nurse-led services in the ambulatory care setting: A systematic review. International journal of nursing studies. 2018 May;81:61-80. PMID: 29518623.

24. McBrien KA, Ivers N, Barnieh L, et al. Patient navigators for people with chronic disease: A systematic review. PloS one. 2018;13(2):e0191980. PMID: 29462179.

25. Baker JM, Grant RW, Gopalan A. A systematic review of care management interventions targeting multimorbidity and high care utilization. BMC health services research. 2018 Jan 30;18(1):65. PMID: 29382327.

26. Tabassum R, Froeschl G, Cruz JP, et al. Untapped aspects of mass media campaigns for changing health behaviour towards non-communicable diseases in Bangladesh. Globalization and health. 2018 Jan 18;14(1):7. PMID: 29347986. 
27. Marcolino MS, Oliveira JAQ, D'Agostino M, et al. The Impact of mHealth Interventions: Systematic Review of Systematic Reviews. JMIR mHealth and uHealth. 2018 Jan 17;6(1):e23. PMID: 29343463.

28. Kish AM, Newcombe PA, Haslam DM. Working and caring for a child with chronic illness: A review of current literature. Child: care, health and development. 2018 May;44(3):343-54. PMID: 29341191.

29. King L, Harrington A, Linedale E, et al. A mixed methods thematic review: Health-related decision-making by the older person. Journal of clinical nursing. 2018 Apr;27(7-8):e1327-e43. PMID: 29322576.

30. Packer TL, Fracini A, Audulv A, et al. What we know about the purpose, theoretical foundation, scope and dimensionality of existing self-management measurement tools: A scoping review. Patient education and counseling. 2018 Apr;101(4):579-95. PMID: 29239734.

31. Warner MM, Kelly JT, Reidlinger DP, et al. Reporting of Telehealth-Delivered Dietary Intervention Trials in Chronic Disease: Systematic Review. Journal of medical Internet research. 2017 Dec 11;19(12):e410. PMID: 29229588.

32. Lenzen SA, Daniels R, van Bokhoven MA, et al. Disentangling self-management goal setting and action planning: A scoping review. PloS one. 2017;12(11):e0188822. PMID: 29176800 .

33. Horrell LN, Kneipp SM. Strategies for recruiting populations to participate in the chronic disease self-management program (CDSMP): A systematic review. Health marketing quarterly. 2017 Oct-Dec;34(4):268-83. PMID: 29173109.

34. Mileski M, Kruse CS, Catalani J, et al. Adopting Telemedicine for the Self-Management of Hypertension: Systematic Review. JMIR medical informatics. 2017 Oct 24;5(4):e41. PMID: 29066424.

35. Lawn S, Zhi X, Morello A. An integrative review of e-learning in the delivery of selfmanagement support training for health professionals. BMC medical education. 2017 Oct 10;17(1):183. PMID: 29017521.

36. Roland KB, Milliken EL, Rohan EA, et al. Use of Community Health Workers and Patient Navigators to Improve Cancer Outcomes Among Patients Served by Federally Qualified Health Centers: A Systematic Literature Review. Health equity. 2017;1(1):61-76. PMID: 28905047.

37. McCarroll R, Eyles H, Ni Mhurchu C. Effectiveness of mobile health (mHealth) interventions for promoting healthy eating in adults: A systematic review. Preventive medicine. 2017 Dec;105:156-68. PMID: 28882743.

38. Lamore K, Montalescot L, Untas A. Treatment decision-making in chronic diseases: What are the family members' roles, needs and attitudes? A systematic review. Patient education and counseling. 2017 Dec;100(12):2172-81. PMID: 28838630.

39. Allison RL. Back to Basics: The Effect of Healthy Diet and Exercise on Chronic Disease Management. South Dakota medicine : the journal of the South Dakota State Medical Association. 2017 Spec;Spec No:10-8. PMID: 28817856. 
40. Bassola B, Lusignani M. Self-care in People With Motor Neuron Disease: An Integrative Review. The Journal of neuroscience nursing : journal of the American Association of Neuroscience Nurses. 2017 Oct;49(5):311-7. PMID: 28817497.

41. Wierenga KL, Lehto RH, Given B. Emotion Regulation in Chronic Disease Populations: An Integrative Review. Research and theory for nursing practice. 2017 Aug 1;31(3):247-71. PMID: 28793948.

42. Murphy LA, Harrington P, Taylor SJ, et al. Clinical-effectiveness of self-management interventions in chronic obstructive pulmonary disease: An overview of reviews. Chronic respiratory disease. 2017 Aug;14(3):276-88. PMID: 28774200.

43. Duff OM, Walsh DM, Furlong BA, et al. Behavior Change Techniques in Physical Activity eHealth Interventions for People With Cardiovascular Disease: Systematic Review. Journal of medical Internet research. 2017 Aug 2;19(8):e281. PMID: 28768610.

44. Birkhoff SD, Smeltzer SC. Perceptions of Smartphone User-Centered Mobile Health Tracking Apps Across Various Chronic Illness Populations: An Integrative Review. Journal of nursing scholarship : an official publication of Sigma Theta Tau International Honor Society of Nursing. 2017 Jul;49(4):371-8. PMID: 28605151.

45. Napoles TM, Burke NJ, Shim JK, et al. Assessing Patient Activation among High-Need, High-Cost Patients in Urban Safety Net Care Settings. Journal of urban health : bulletin of the New York Academy of Medicine. 2017 Dec;94(6):803-13. PMID: 28597203.

46. Morsa M, Gagnayre R, Deccache C, et al. Factors influencing the transition from pediatric to adult care: A scoping review of the literature to conceptualize a relevant education program. Patient education and counseling. 2017 Oct;100(10):1796-806. PMID: 28528694.

47. Xu X, Mishra GD, Jones M. Evidence on multimorbidity from definition to intervention: An overview of systematic reviews. Ageing research reviews. 2017 Aug;37:53-68. PMID: 28511964.

48. Wang Y, Xue H, Huang Y, et al. A Systematic Review of Application and Effectiveness of mHealth Interventions for Obesity and Diabetes Treatment and Self-Management. Advances in nutrition (Bethesda, Md). 2017 May;8(3):449-62. PMID: 28507010.

49. Guo Y, Albright D. The effectiveness of telehealth on self-management for older adults with a chronic condition: A comprehensive narrative review of the literature. Journal of telemedicine and telecare. 2018 Jul;24(6):392-403. PMID: 28449619.

50. Janssen-Niemeijer AJ, Visse M, Van Leeuwen R, et al. The Role of Spirituality in Lifestyle Changing Among Patients with Chronic Cardiovascular Diseases: A Literature Review of Qualitative Studies. Journal of religion and health. 2017 Aug;56(4):1460-77. PMID: 28349298.

51. Bland V, Sharma M. Physical activity interventions in African American women: A systematic review. Health promotion perspectives. 2017;7(2):52-9. PMID: 28326284.

52. Whitehead L, Jacob E, Towell A, et al. The role of the family in supporting the selfmanagement of chronic conditions: A qualitative systematic review. Journal of clinical nursing. 2018 Jan;27(1-2):22-30. PMID: 28231630. 
53. Ulbrich EM, Mattei AT, de Fatima Mantovani M, et al. Care models for people with chronic diseases: integrative review. Investigacion y educacion en enfermeria. 2017 Jan;35(1):8-16.

PMID: 29767919.

54. Reisinho MD, Gomes BP. Nursing interventions in monitoring the adolescent with Cystic Fibrosis: a literature review. Revista latino-americana de enfermagem. 2016 Dec 8;24:e2845. PMID: 27982311.

55. Damery S, Flanagan S, Combes G. Does integrated care reduce hospital activity for patients with chronic diseases? An umbrella review of systematic reviews. BMJ open. 2016 Nov 21;6(11):e011952. PMID: 27872113.

56. Thombs BD, Kwakkenbos L, Riehm KE, et al. Comparison of Self-Efficacy for Managing Chronic Disease between patients with systemic sclerosis and other chronic conditions: a systematic review. Rheumatology international. 2017 Feb;37(2):281-92. PMID: 27866246.

57. Rao A, Hickman LD, Sibbritt D, et al. Is energy healing an effective non-pharmacological therapy for improving symptom management of chronic illnesses? A systematic review. Complementary therapies in clinical practice. 2016 Nov;25:26-41. PMID: 27863608.

58. Lewis J, Ray P, Liaw ST. Recent Worldwide Developments in eHealth and mHealth to more Effectively Manage Cancer and other Chronic Diseases - A Systematic Review. Yearbook of medical informatics. 2016 Nov 10(1):93-108. PMID: 27830236.

59. Jornten-Karlsson M, Pintat S, Molloy-Bland M, et al. Patient-Centered Interventions to Improve Adherence to Statins: A Narrative Synthesis of Systematically Identified Studies. Drugs. 2016 Oct;76(15):1447-65. PMID: 27677773.

60. Bartlett Ellis RJ, Welch JL. Medication-taking behaviours in chronic kidney disease with multiple chronic conditions: a meta-ethnographic synthesis of qualitative studies. Journal of clinical nursing. 2017 Mar;26(5-6):586-98. PMID: 27648739.

61. Boehmer KR, Gionfriddo MR, Rodriguez-Gutierrez R, et al. Patient capacity and constraints in the experience of chronic disease: a qualitative systematic review and thematic synthesis. BMC family practice. 2016 Sep 1;17:127. PMID: 27585439.

62. Totten AM, Womack DM, Eden KB, et al. AHRQ Comparative Effectiveness Technical Briefs. Telehealth: Mapping the Evidence for Patient Outcomes From Systematic Reviews. Rockville (MD): Agency for Healthcare Research and Quality (US); 2016.

63. Jiang D, Kong W, Jiang JJ. Patient Engagement in Randomized Controlled Tai Chi Clinical Trials among the Chronically Ill. Reviews on recent clinical trials. 2017;12(1):24-33. PMID: 27527892.

64. Brzan PP, Rotman E, Pajnkihar M, et al. Mobile Applications for Control and Self Management of Diabetes: A Systematic Review. Journal of medical systems. 2016 Sep;40(9):210. PMID: 27520615.

65. Ershad Sarabi R, Sadoughi F, Jamshidi Orak R, et al. The Effectiveness of Mobile Phone Text Messaging in Improving Medication Adherence for Patients with Chronic Diseases: A Systematic Review. Iranian Red Crescent medical journal. 2016 May;18(5):e25183. PMID: 27437126. 
66. Gucciardi E, Jean-Pierre N, Karam G, et al. Designing and delivering facilitated storytelling interventions for chronic disease self-management: a scoping review. BMC health services research. 2016 Jul 11;16:249. PMID: 27401836.

67. Matthew-Maich N, Harris L, Ploeg J, et al. Designing, Implementing, and Evaluating Mobile Health Technologies for Managing Chronic Conditions in Older Adults: A Scoping Review. JMIR mHealth and uHealth. 2016 Jun 9;4(2):e29. PMID: 27282195.

68. van Schie D, Castelein S, van der Bijl J, et al. Systematic review of self-management in patients with schizophrenia: psychometric assessment of tools, levels of self-management and associated factors. Journal of advanced nursing. 2016 Nov;72(11):2598-611. PMID: 27200500.

69. Scarton LJ, de Groot M. Emotional and Behavioral Aspects of Diabetes in American Indians/Alaska Natives. Health education \& behavior : the official publication of the Society for Public Health Education. 2017 Feb;44(1):70-82. PMID: 27179289.

70. Totten AM, White-Chu EF, Wasson N, et al. AHRQ Comparative Effectiveness Reviews. Home-Based Primary Care Interventions. Rockville (MD): Agency for Healthcare Research and Quality (US); 2016.

71. Yasmin F, Banu B, Zakir SM, et al. Positive influence of short message service and voice call interventions on adherence and health outcomes in case of chronic disease care: a systematic review. BMC medical informatics and decision making. 2016 Apr 22;16:46. PMID: 27106263.

72. Vernooij RW, Willson M, Gagliardi AR. Characterizing patient-oriented tools that could be packaged with guidelines to promote self-management and guideline adoption: a meta-review. Implementation science : IS. 2016 Apr 14;11:52. PMID: 27079375.

73. Morgan HM, Entwistle VA, Cribb A, et al. We need to talk about purpose: a critical interpretive synthesis of health and social care professionals' approaches to self-management support for people with long-term conditions. Health expectations : an international journal of public participation in health care and health policy. 2017 Apr;20(2):243-59. PMID: 27075246.

74. Mackey LM, Doody C, Werner EL, et al. Self-Management Skills in Chronic Disease Management: What Role Does Health Literacy Have? Medical decision making : an international journal of the Society for Medical Decision Making. 2016 Aug;36(6):741-59. PMID: 27053527.

75. Allen C, Vassilev I, Kennedy A, et al. Long-Term Condition Self-Management Support in Online Communities: A Meta-Synthesis of Qualitative Papers. Journal of medical Internet research. 2016 Mar 10;18(3):e61. PMID: 26965990.

76. Duprez V, Van Hooft SM, Dwarswaard J, et al. The development and psychometric validation of the self-efficacy and performance in self-management support (SEPSS) Instrument. Journal of advanced nursing. 2016 Jun;72(6):1381-95. PMID: 26913585.

77. Wiering B, de Boer D, Delnoij D. Patient involvement in the development of patientreported outcome measures: a scoping review. Health expectations : an international journal of public participation in health care and health policy. 2017 Feb;20(1):11-23. PMID: 26889874.

78. de Ridder M, Kim J, Jing Y, et al. A systematic review on incentive-driven mobile health technology: As used in diabetes management. Journal of telemedicine and telecare. 2017 Jan;23(1):26-35. PMID: 26888421. 
79. Willis EA, Szabo-Reed AN, Ptomey LT, et al. Do weight management interventions delivered by online social networks effectively improve body weight, body composition, and chronic disease risk factors? A systematic review. Journal of telemedicine and telecare. 2017 Feb;23(2):263-72. PMID: 26880695.

80. Arnold LM, Gebke KB, Choy EH. Fibromyalgia: management strategies for primary care providers. International journal of clinical practice. 2016 Feb;70(2):99-112. PMID: 26817567.

81. Benyamina A, Reynaud M. [Management of alcohol use disorders in ambulatory care: Which follow-up and for how long?]. L'Encephale. 2016 Feb;42(1):67-73. PMID: 26796554.

82. Kogan AC, Wilber K, Mosqueda L. Person-Centered Care for Older Adults with Chronic Conditions and Functional Impairment: A Systematic Literature Review. Journal of the American Geriatrics Society. 2016 Jan;64(1):e1-7. PMID: 26626408.

83. Cruz J, Marques A, Figueiredo D. Impacts of COPD on family carers and supportive interventions: a narrative review. Health \& social care in the community. 2017 Jan;25(1):11-25. PMID: 26499310.

84. Altin SV, Halbach S, Ernstmann N, et al. [How can we measure cancer literacy?--A systematic review on the quality of available measurement tools]. Zeitschrift fur Evidenz, Fortbildung und Qualitat im Gesundheitswesen. 2015;109(6):466-82. PMID: 26474652.

85. Jamieson NJ, Hanson CS, Josephson MA, et al. Motivations, Challenges, and Attitudes to Self-management in Kidney Transplant Recipients: A Systematic Review of Qualitative Studies. American journal of kidney diseases : the official journal of the National Kidney Foundation. 2016 Mar;67(3):461-78. PMID: 26372087.

86. Keim-Malpass J, Letzkus LC, Kennedy C. Parent/caregiver health literacy among children with special health care needs: a systematic review of the literature. BMC pediatrics. 2015 Aug 5;15:92. PMID: 26242306.

87. Wu S, Kutlubaev MA, Chun HY, et al. Interventions for post-stroke fatigue. The Cochrane database of systematic reviews. 2015 Jul 2(7):Cd007030. PMID: 26133313.

88. Radhakrishnan K, Xie B, Berkley A, et al. Barriers and Facilitators for Sustainability of Tele-Homecare Programs: A Systematic Review. Health services research. 2016 Feb;51(1):4875. PMID: 26119048.

89. Spaling MA, Currie K, Strachan PH, et al. Improving support for heart failure patients: a systematic review to understand patients' perspectives on self-care. Journal of advanced nursing. 2015 Nov;71(11):2478-89. PMID: 26084885.

90. Fradgley EA, Paul CL, Bryant J. A systematic review of barriers to optimal outpatient specialist services for individuals with prevalent chronic diseases: what are the unique and common barriers experienced by patients in high income countries? International journal for equity in health. 2015 Jun 9;14:52. PMID: 26051244.

91. Bogetz JF, Rassbach CE, Bereknyei S, et al. Training health care professionals for 21stcentury practice: a systematic review of educational interventions on chronic care. Academic medicine : journal of the Association of American Medical Colleges. 2015 Nov;90(11):1561-72. PMID: 26039140. 
92. Davy C, Bleasel J, Liu H, et al. Effectiveness of chronic care models: opportunities for improving healthcare practice and health outcomes: a systematic review. BMC health services research. 2015 May 10;15:194. PMID: 25958128.

93. Khangura SD, Karaceper MD, Trakadis Y, et al. Scoping review of patient- and familyoriented outcomes and measures for chronic pediatric disease. BMC pediatrics. $2015 \mathrm{Feb}$ 13;15:7. PMID: 25886474.

94. Zanchetta MS, Maheu C, Kolisnyk O, et al. Canadian Men's Self-Management of Chronic Diseases: A Literature Analysis of Strategies for Dealing With Risks and Promoting Wellness. American journal of men's health. 2017 Jul;11(4):1077-95. PMID: 25804217.

95. Dwarswaard J, Bakker EJ, van Staa A, et al. Self-management support from the perspective of patients with a chronic condition: a thematic synthesis of qualitative studies. Health expectations : an international journal of public participation in health care and health policy. 2016 Apr;19(2):194-208. PMID: 25619975.

96. Mbuagbaw L, Mursleen S, Lytvyn L, et al. Mobile phone text messaging interventions for HIV and other chronic diseases: an overview of systematic reviews and framework for evidence transfer. BMC health services research. 2015 Jan 22;15:33. PMID: 25609559.

97. Anglada-Martinez H, Riu-Viladoms G, Martin-Conde M, et al. Does mHealth increase adherence to medication? Results of a systematic review. International journal of clinical practice. 2015 Jan;69(1):9-32. PMID: 25472682.

98. Bratzke LC, Muehrer RJ, Kehl KA, et al. Self-management priority setting and decisionmaking in adults with multimorbidity: a narrative review of literature. International journal of nursing studies. 2015 Mar;52(3):744-55. PMID: 25468131.

99. Koch G, Wakefield BJ, Wakefield DS. Barriers and facilitators to managing multiple chronic conditions: a systematic literature review. Western journal of nursing research. 2015 Apr;37(4):498-516. PMID: 25193613.

100. Hynes L, Byrne M, Dinneen SF, et al. Barriers and facilitators associated with attendance at hospital diabetes clinics among young adults (15-30 years) with type 1 diabetes mellitus: a systematic review. Pediatric diabetes. 2016 Nov;17(7):509-18. PMID: 25080975.

101. Harkness K, Spaling MA, Currie K, et al. A systematic review of patient heart failure selfcare strategies. The Journal of cardiovascular nursing. 2015 Mar-Apr;30(2):121-35. PMID: 24651683.

102. Ducasse D, Fond G. [Acceptance and commitment therapy]. L'Encephale. 2015 Feb;41(1):1-9. PMID: 24262333.

103. Vat LE, Finlay T, Jan Schuitmaker-Warnaar T, et al. Evaluating the "return on patient engagement initiatives" in medicines research and development: A literature review. Health expectations : an international journal of public participation in health care and health policy. 2019 Sep 6. PMID: 31489988.

104. Pham C, Lizarondo L, Karnon J, et al. Strategies for implementing shared decision making in elective surgery by health care practitioners: A systematic review. Journal of evaluation in clinical practice. 2019 Sep 6. PMID: 31490593. 
105. Harris J, Haltbakk J, Dunning T, et al. How patient and community involvement in diabetes research influences health outcomes: A realist review. Health expectations : an international journal of public participation in health care and health policy. 2019 Jul 8. PMID: 31286639.

106. Vromans RD, van Eenbergen MC, Pauws SC, et al. Communicative aspects of decision aids for localized prostate cancer treatment - A systematic review. Urologic oncology. 2019 Jul;37(7):409-29. PMID: 31053529.

107. Chegini Z, Arab-Zozani M, Janati A. Patient and Health Professional Perspectives about Engaging Patients in Addressing Patient Safety: A Systematic Review Protocol. Open access Macedonian journal of medical sciences. 2019 May 15;7(9):1561-5. PMID: 31198473.

108. Yiu A, Bajorek B. Patient-focused interventions to support vulnerable people using oral anticoagulants: a narrative review. Therapeutic advances in drug safety.

2019;10:2042098619847423. PMID: 31205676.

109. Covvey JR, Kamal KM, Gorse EE, et al. Barriers and facilitators to shared decision-making in oncology: a systematic review of the literature. Supportive care in cancer : official journal of the Multinational Association of Supportive Care in Cancer. 2019 May;27(5):1613-37. PMID: 30737578.

110. Manias E, Bucknall T, Hughes C, et al. Family involvement in managing medications of older patients across transitions of care: a systematic review. BMC geriatrics. 2019 Mar 29;19(1):95. PMID: 30925899.

111. Larsen MH, Hagen KB, Krogstad AL, et al. Shared Decision Making in Psoriasis: A Systematic Review of Quantitative and Qualitative Studies. American journal of clinical dermatology. 2019 Feb;20(1):13-29. PMID: 30324563.

112. de Mik SML, Stubenrouch FE, Balm R, et al. Systematic review of shared decision-making in surgery. The British journal of surgery. 2018 Dec;105(13):1721-30. PMID: 30357815.

113. Martinez-Millana A, Jarones E, Fernandez-Llatas C, et al. App Features for Type 1 Diabetes Support and Patient Empowerment: Systematic Literature Review and Benchmark Comparison. JMIR mHealth and uHealth. 2018 Nov 21;6(11):e12237. PMID: 30463839.

114. Odgers HL, Tong A, Lopez-Vargas P, et al. Research priority setting in childhood chronic disease: a systematic review. Archives of disease in childhood. 2018 Oct;103(10):942-51. PMID: 29643102.

115. Dukhanin V, Topazian R, DeCamp M. Metrics and Evaluation Tools for Patient Engagement in Healthcare Organization- and System-Level Decision-Making: A Systematic Review. International journal of health policy and management. 2018 Oct 1;7(10):889-903. PMID: 30316241.

116. Rochfort A, Beirne S, Doran G, et al. Does patient self-management education of primary care professionals improve patient outcomes: a systematic review. BMC family practice. 2018 Sep 29;19(1):163. PMID: 30268092.

117. Simblett S, Greer B, Matcham F, et al. Barriers to and Facilitators of Engagement With Remote Measurement Technology for Managing Health: Systematic Review and Content Analysis of Findings. Journal of medical Internet research. 2018 Jul 12;20(7):e10480. PMID: 30001997. 
118. Menichetti J, Graffigna G, Steinsbekk A. What are the contents of patient engagement interventions for older adults? A systematic review of randomized controlled trials. Patient education and counseling. 2018 Jun;101(6):995-1005. PMID: 29246493.

119. Grant S, Hazlewood GS, Peay HL, et al. Practical Considerations for Using Online Methods to Engage Patients in Guideline Development. The patient. 2018 Apr;11(2):155-66. PMID: 29030831.

120. Manafo E, Petermann L, Vandall-Walker V, et al. Patient and public engagement in priority setting: A systematic rapid review of the literature. PloS one. 2018;13(3):e0193579. PMID: 29499043.

121. Kim C, Wright FC, Look Hong NJ, et al. Patient and provider experiences with active surveillance: A scoping review. PloS one. 2018;13(2):e0192097. PMID: 29401514.

122. Baines RL, Regan de Bere S. Optimizing patient and public involvement (PPI): Identifying its "essential" and "desirable" principles using a systematic review and modified Delphi methodology. Health expectations : an international journal of public participation in health care and health policy. 2018 Feb;21(1):327-35. PMID: 28929554.

123. Aronson PL, Shapiro ED, Niccolai LM, et al. Shared Decision-Making with Parents of Acutely Ill Children: A Narrative Review. Academic pediatrics. 2018 Jan - Feb;18(1):3-7. PMID: 28723588.

124. Young A, Menon D, Street J, et al. Exploring patient and family involvement in the lifecycle of an orphan drug: a scoping review. Orphanet journal of rare diseases. 2017 Dec 22;12(1):188. PMID: 29273068.

125. Land V, Parry R, Seymour J. Communication practices that encourage and constrain shared decision making in health-care encounters: Systematic review of conversation analytic research. Health expectations : an international journal of public participation in health care and health policy. 2017 Dec;20(6):1228-47. PMID: 28520201.

126. Williams N, Fleming C, Doubleday A. Patient and provider perspectives on shared decision making: a systematic review of the peer-reviewed literature. Journal of comparative effectiveness research. 2017 Nov;6(8):683-92. PMID: 29148283.

127. Sharpe EE, Karasouli E, Meyer C. Examining Factors of Engagement With Digital Interventions for Weight Management: Rapid Review. JMIR research protocols. 2017 Oct 23;6(10):e205. PMID: 29061557.

128. Doody O, Butler MP, Lyons R, et al. Families' experiences of involvement in care planning in mental health services: an integrative literature review. Journal of psychiatric and mental health nursing. 2017 Aug;24(6):412-30. PMID: 28102020.

129. Zandstra D, Busser JAS, Aarts JWM, et al. Interventions to support shared decisionmaking for women with heavy menstrual bleeding: A systematic review. European journal of obstetrics, gynecology, and reproductive biology. 2017 Apr;211:156-63. PMID: 28273646.

130. Doherr H, Christalle E, Kriston L, et al. Use of the 9-item Shared Decision Making Questionnaire (SDM-Q-9 and SDM-Q-Doc) in intervention studies-A systematic review. PloS one. 2017;12(3):e0173904. PMID: 28358864. 
131. Alvarado MM, Kum HC, Gonzalez Coronado K, et al. Barriers to Remote Health Interventions for Type 2 Diabetes: A Systematic Review and Proposed Classification Scheme. Journal of medical Internet research. 2017 Feb 13;19(2):e28. PMID: 28193598.

132. Olding M, McMillan SE, Reeves S, et al. Patient and family involvement in adult critical and intensive care settings: a scoping review. Health expectations : an international journal of public participation in health care and health policy. 2016 Dec;19(6):1183-202. PMID: 27878937.

133. Singer AE, Ash T, Ochotorena C, et al. A Systematic Review of Family Meeting Tools in Palliative and Intensive Care Settings. The American journal of hospice \& palliative care. 2016 Sep;33(8):797-806. PMID: 26213225.

134. Miller LM, Whitlatch CJ, Lyons KS. Shared decision-making in dementia: A review of patient and family carer involvement. Dementia (London, England). 2016 Sep;15(5):1141-57. PMID: 25370075.

135. Chaet AV, Morshedi B, Wells KJ, et al. Spanish-Language Consumer Health Information Technology Interventions: A Systematic Review. Journal of medical Internet research. 2016 Aug 10;18(8):e214. PMID: 27511437.

136. Barnes GD, Izzo B, Conte ML, et al. Use of decision aids for shared decision making in venous thromboembolism: A systematic review. Thrombosis research. 2016 Jul;143:71-5. PMID: 27203185.

137. Walsh S, Golden E, Priebe S. Systematic review of patients' participation in and experiences of technology-based monitoring of mental health symptoms in the community. BMJ open. 2016 Jun 21;6(6):e008362. PMID: 27329437.

138. Peek ME, Lopez FY, Williams HS, et al. Development of a Conceptual Framework for Understanding Shared Decision making Among African-American LGBT Patients and their Clinicians. Journal of general internal medicine. 2016 Jun;31(6):677-87. PMID: 27008649.

139. Oczkowski SJ, Chung HO, Hanvey L, et al. Communication tools for end-of-life decisionmaking in the intensive care unit: a systematic review and meta-analysis. Critical care (London, England). 2016 Apr 9;20:97. PMID: 27059989.

140. Otte-Trojel T, de Bont A, Rundall TG, et al. What do we know about developing patient portals? a systematic literature review. Journal of the American Medical Informatics Association : JAMIA. 2016 Apr;23(e1):e162-8. PMID: 26335985.

141. Kynoch K, Chang A, Coyer F, et al. The effectiveness of interventions to meet family needs of critically ill patients in an adult intensive care unit: a systematic review update. JBI database of systematic reviews and implementation reports. 2016 Mar;14(3):181-234. PMID: 27532144.

142. Gagliardi AR, Legare F, Brouwers MC, et al. Patient-mediated knowledge translation (PKT) interventions for clinical encounters: a systematic review. Implementation science : IS. 2016 Feb 29;11:26. PMID: 26923462.

143. Phillips NM, Street M, Haesler E. A systematic review of reliable and valid tools for the measurement of patient participation in healthcare. BMJ quality \& safety. 2016 Feb;25(2):110-7. PMID: 26415751. 
144. Syrowatka A, Kromker D, Meguerditchian AN, et al. Features of Computer-Based Decision Aids: Systematic Review, Thematic Synthesis, and Meta-Analyses. Journal of medical Internet research. 2016 Jan 26;18(1):e20. PMID: 26813512.

145. Snyder H, Engstrom J. The antecedents, forms and consequences of patient involvement: A narrative review of the literature. International journal of nursing studies. 2016 Jan;53:351-78. PMID: 26602069.

146. Coxeter P, Del Mar CB, McGregor L, et al. Interventions to facilitate shared decision making to address antibiotic use for acute respiratory infections in primary care. The Cochrane database of systematic reviews. 2015 Nov 12(11):Cd010907. PMID: 26560888.

147. Calvillo J, Roman I, Roa LM. How technology is empowering patients? A literature review. Health expectations : an international journal of public participation in health care and health policy. 2015 Oct;18(5):643-52. PMID: 23711169.

148. Couet N, Desroches S, Robitaille H, et al. Assessments of the extent to which health-care providers involve patients in decision making: a systematic review of studies using the OPTION instrument. Health expectations : an international journal of public participation in health care and health policy. 2015 Aug;18(4):542-61. PMID: 23451939.

149. Welch JL, Johnson M, Zimmerman L, et al. Self-management interventions in stages 1 to 4 chronic kidney disease: an integrative review. Western journal of nursing research. 2015 May;37(5):652-78. PMID: 25239136.

150. Vaismoradi M, Jordan S, Kangasniemi M. Patient participation in patient safety and nursing input - a systematic review. Journal of clinical nursing. 2015 Mar;24(5-6):627-39. PMID: 25178172.

151. Menon D, Stafinski T, Dunn A, et al. Involving patients in reducing decision uncertainties around orphan and ultra-orphan drugs: a rare opportunity? The patient. 2015 Feb;8(1):29-39. PMID: 25516506.

152. Shay LA, Lafata JE. Where is the evidence? A systematic review of shared decision making and patient outcomes. Medical decision making : an international journal of the Society for Medical Decision Making. 2015 Jan;35(1):114-31. PMID: 25351843.

\section{No comparison group}

1. De Nunzio C, Presicce F, Lombardo R, et al. Patient centred care for the medical treatment of lower urinary tract symptoms in patients with benign prostatic obstruction: a key point to improve patients' care - a systematic review. BMC urology. 2018 Jun 26;18(1):62. PMID: 29940928.

2. Slater H, Campbell JM, Stinson JN, et al. End User and Implementer Experiences of mHealth Technologies for Noncommunicable Chronic Disease Management in Young Adults: Systematic Review. Journal of medical Internet research. 2017 Dec 12;19(12):e406. PMID: 29233804.

3. Fernandes LTB, Nobrega VMD, Silva MEA, et al. Supported self-care for children and adolescents with chronic disease and their families. Revista brasileira de enfermagem. 2017 NovDec;70(6):1318-29. PMID: 29160496. 
4. Morton K, Dennison L, May C, et al. Using digital interventions for self-management of chronic physical health conditions: A meta-ethnography review of published studies. Patient education and counseling. 2017 Apr;100(4):616-35. PMID: 28029572.

5. Reisinho MD, Gomes BP. Nursing interventions in monitoring the adolescent with Cystic Fibrosis: a literature review. Revista latino-americana de enfermagem. 2016 Dec 8;24:e2845. PMID: 27982311.

6. Boehmer KR, Gionfriddo MR, Rodriguez-Gutierrez R, et al. Patient capacity and constraints in the experience of chronic disease: a qualitative systematic review and thematic synthesis. BMC family practice. 2016 Sep 1;17:127. PMID: 27585439.

7. Allen C, Vassilev I, Kennedy A, et al. Long-Term Condition Self-Management Support in Online Communities: A Meta-Synthesis of Qualitative Papers. Journal of medical Internet research. 2016 Mar 10;18(3):e61. PMID: 26965990.

8. Jamieson NJ, Hanson CS, Josephson MA, et al. Motivations, Challenges, and Attitudes to Self-management in Kidney Transplant Recipients: A Systematic Review of Qualitative Studies. American journal of kidney diseases : the official journal of the National Kidney Foundation. 2016 Mar;67(3):461-78. PMID: 26372087.

9. Fu Y, McNichol E, Marczewski K, et al. Patient-professional partnerships and chronic back pain self-management: a qualitative systematic review and synthesis. Health \& social care in the community. 2016 May;24(3):247-59. PMID: 25809204.

10. Zanchetta MS, Maheu C, Kolisnyk O, et al. Canadian Men's Self-Management of Chronic Diseases: A Literature Analysis of Strategies for Dealing With Risks and Promoting Wellness. American journal of men's health. 2017 Jul;11(4):1077-95. PMID: 25804217.

11. Dwarswaard J, Bakker EJ, van Staa A, et al. Self-management support from the perspective of patients with a chronic condition: a thematic synthesis of qualitative studies. Health expectations : an international journal of public participation in health care and health policy. 2016 Apr;19(2):194-208. PMID: 25619975.

12. Bratzke LC, Muehrer RJ, Kehl KA, et al. Self-management priority setting and decisionmaking in adults with multimorbidity: a narrative review of literature. International journal of nursing studies. 2015 Mar;52(3):744-55. PMID: 25468131.

13. Harkness K, Spaling MA, Currie K, et al. A systematic review of patient heart failure selfcare strategies. The Journal of cardiovascular nursing. 2015 Mar-Apr;30(2):121-35. PMID: 24651683.

14. Lepore M, Scales K, Anderson RA, et al. Person-directed care planning in nursing homes: A scoping review. International journal of older people nursing. 2018 Dec;13(4):e12212. PMID: 30358099.

15. Davis S, Roudsari A, Raworth R, et al. Shared decision-making using personal health record technology: a scoping review at the crossroads. Journal of the American Medical Informatics Association : JAMIA. 2017 Jul 1;24(4):857-66. PMID: 28158573.

16. Alvarado MM, Kum HC, Gonzalez Coronado K, et al. Barriers to Remote Health Interventions for Type 2 Diabetes: A Systematic Review and Proposed Classification Scheme. Journal of medical Internet research. 2017 Feb 13;19(2):e28. PMID: 28193598. 
17. Rose A, Rosewilliam S, Soundy A. Shared decision making within goal setting in rehabilitation settings: A systematic review. Patient education and counseling. 2017 Jan;100(1):65-75. PMID: 27486052.

\section{Not in English}

1. Ulbrich EM, Mattei AT, de Fatima Mantovani M, et al. Care models for people with chronic diseases: integrative review. Investigacion y educacion en enfermeria. 2017 Jan;35(1):8-16. PMID: 29767919.

2. Ducasse D, Fond G. [Acceptance and commitment therapy]. L'Encephale. 2015 Feb;41(1):19. PMID: 24262333.

\section{Non-USA based studies included in this systematic review}

1. Navodia N, Wahoush O, Tang T, et al. Culturally Tailored Self-Management Interventions for South Asians With Type 2 Diabetes: A Systematic Review. Canadian journal of diabetes. 2019 Aug;43(6):445-52. PMID: 31375180.

2. Tabassum R, Froeschl G, Cruz JP, et al. Untapped aspects of mass media campaigns for changing health behaviour towards non-communicable diseases in Bangladesh. Globalization and health. 2018 Jan 18;14(1):7. PMID: 29347986.

3. Lee MC, Wu SV, Hsieh NC, et al. Self-Management Programs on eGFR, Depression, and Quality of Life among Patients with Chronic Kidney Disease: A Meta-Analysis. Asian nursing research. 2016 Dec;10(4):255-62. PMID: 28057311.

4. Milavec Kapun M, Sustersic O, Rajkovic V. The Integrated Patient's Self-Care Process Model. Studies in health technology and informatics. 2016;225:108-12. PMID: 27332172.

5. Wakefield D, Bayly J, Selman LE, et al. Patient empowerment, what does it mean for adults in the advanced stages of a life-limiting illness: A systematic review using critical interpretive synthesis. Palliative medicine. 2018 Sep;32(8):1288-304. PMID: 29956568.

\section{Not a systematic review}

1. Lombardero A, Hansen CD, Richie AE, et al. A Narrative Review of the Literature on Insufficient Sleep, Insomnia, and Health Correlates in American Indian/Alaska Native Populations. Journal of environmental and public health. 2019;2019:4306463. PMID: 31360174.

2. Miller WR. The Projected Care Trajectory for Persons with Epilepsy. The Nursing clinics of North America. 2019 Sep;54(3):425-35. PMID: 31331628.

3. Mollard E, Michaud K. Mobile Apps for Rheumatoid Arthritis: Opportunities and Challenges. Rheumatic diseases clinics of North America. 2019 May;45(2):197-209. PMID: 30952393.

4. Bertoncello C, Colucci M, Baldovin T, et al. How does it work? Factors involved in telemedicine home-interventions effectiveness: A review of reviews. PloS one. 2018;13(11):e0207332. PMID: 30440004. 
5. Pinchera B, DelloIacono D, Lawless CA. Best Practices for Patient Self-Management: Implications for Nurse Educators, Patient Educators, and Program Developers. Journal of continuing education in nursing. 2018 Sep 1;49(9):432-40. PMID: 30148541.

6. Mabweazara SZ, Ley C, Leach LL. Physical activity, social support and socio-economic status amongst persons living with HIV and AIDS: a review. African journal of AIDS research : AJAR. 2018 Jul;17(2):203-12. PMID: 30003848.

7. Atreja A, Otobo E, Ramireddy K, et al. Remote Patient Monitoring in IBD: Current State and Future Directions. Current gastroenterology reports. 2018 Mar 7;20(2):6. PMID: 29516186.

8. Kahan SI. Practical Strategies for Engaging Individuals With Obesity in Primary Care. Mayo Clinic proceedings. 2018 Mar;93(3):351-9. PMID: 29502565.

9. Pugh P, Hemingway P, Christian M, et al. Children's, parents' and other stakeholders' perspectives on early dietary self-management to delay disease progression of chronic disease in children: a protocol for a mixed studies systematic review with a narrative synthesis. Systematic reviews. 2018 Jan 25;7(1):20. PMID: 29370832.

10. Flanagan S, Damery S, Combes G. The effectiveness of integrated care interventions in improving patient quality of life (QoL) for patients with chronic conditions. An overview of the systematic review evidence. Health and quality of life outcomes. 2017 Sep 29;15(1):188. PMID: 28962570.

11. Bassola B, Lusignani M. Self-care in People With Motor Neuron Disease: An Integrative Review. The Journal of neuroscience nursing : journal of the American Association of Neuroscience Nurses. 2017 Oct;49(5):311-7. PMID: 28817497.

12. Ghazisaeidi M, Safdari R, Goodini A, et al. Digital games as an effective approach for cancer management: Opportunities and challenges. Journal of education and health promotion. 2017;6:30. PMID: 28584830.

13. Hanlon P, Daines L, Campbell C, et al. Telehealth Interventions to Support SelfManagement of Long-Term Conditions: A Systematic Metareview of Diabetes, Heart Failure, Asthma, Chronic Obstructive Pulmonary Disease, and Cancer. Journal of medical Internet research. 2017 May 17;19(5):e172. PMID: 28526671.

14. Wilson MG, Lavis JN, Gauvin FP. Designing Integrated Approaches to Support People with Multimorbidity: Key Messages from Systematic Reviews, Health System Leaders and Citizens. Healthcare policy = Politiques de sante. 2016 Nov;12(2):91-104. PMID: 28032827.

15. Kessler D, Liddy C. An integrative literature review to examine the provision of selfmanagement support following transient ischaemic attack. Journal of clinical nursing. 2017 Nov;26(21-22):3256-70. PMID: 28001339.

16. Brzan PP, Rotman E, Pajnkihar M, et al. Mobile Applications for Control and Self Management of Diabetes: A Systematic Review. Journal of medical systems. 2016 Sep;40(9):210. PMID: 27520615.

17. Kitsiou S, Pare G, Jaana M. Effects of home telemonitoring interventions on patients with chronic heart failure: an overview of systematic reviews. Journal of medical Internet research. 2015 Mar 12;17(3):e63. PMID: 25768664. 
18. Mbuagbaw L, Mursleen S, Lytvyn L, et al. Mobile phone text messaging interventions for HIV and other chronic diseases: an overview of systematic reviews and framework for evidence transfer. BMC health services research. 2015 Jan 22;15:33. PMID: 25609559.

19. Chegini Z, Arab-Zozani M, Janati A. Patient and Health Professional Perspectives about Engaging Patients in Addressing Patient Safety: A Systematic Review Protocol. Open access Macedonian journal of medical sciences. 2019 May 15;7(9):1561-5. PMID: 31198473.

20. Finset A. Special Section: Review articles on decision making and patient education interventions. Patient education and counseling. 2017 Dec;100(12):2157-8. PMID: 29032951.

21. Doody O, Butler MP, Lyons R, et al. Families' experiences of involvement in care planning in mental health services: an integrative literature review. Journal of psychiatric and mental health nursing. 2017 Aug;24(6):412-30. PMID: 28102020.

22. Roberts S, Chaboyer W, Gonzalez R, et al. Using technology to engage hospitalised patients in their care: a realist review. BMC health services research. 2017 Jun 6;17(1):388. PMID: 28587640.

23. Scholz B, Gordon S, Happell B. Consumers in mental health service leadership: A systematic review. International journal of mental health nursing. 2017 Feb;26(1):20-31. PMID: 28093883.

24. Welch JL, Johnson M, Zimmerman L, et al. Self-management interventions in stages 1 to 4 chronic kidney disease: an integrative review. Western journal of nursing research. 2015 May;37(5):652-78. PMID: 25239136.

\section{Others}

1. Vo V, Auroy L, Sarradon-Eck A. Patients' Perceptions of mHealth Apps: Meta-Ethnographic Review of Qualitative Studies. JMIR mHealth and uHealth. 2019 Jul 10;7(7):e13817. PMID: 31293246.

2. Mollard E, Michaud K. Mobile Apps for Rheumatoid Arthritis: Opportunities and Challenges. Rheumatic diseases clinics of North America. 2019 May;45(2):197-209. PMID: 30952393.

3. Sample D, Turner J. Improving gluten free diet adherence by youth with celiac disease. International journal of adolescent medicine and health. 2019 Mar 15. PMID: 30875325.

4. Maddison R, Cartledge S, Rogerson M, et al. Usefulness of Wearable Cameras as a Tool to Enhance Chronic Disease Self-Management: Scoping Review. JMIR mHealth and uHealth. 2019 Jan 3;7(1):e10371. PMID: 30609985.

5. Pinchera B, DelloIacono D, Lawless CA. Best Practices for Patient Self-Management: Implications for Nurse Educators, Patient Educators, and Program Developers. Journal of continuing education in nursing. 2018 Sep 1;49(9):432-40. PMID: 30148541.

6. Kusnanto H, Agustian D, Hilmanto D. Biopsychosocial model of illnesses in primary care: A hermeneutic literature review. Journal of family medicine and primary care. 2018 MayJun;7(3):497-500. PMID: 30112296.

7. Byambasuren O, Sanders S, Beller E, et al. Prescribable mHealth apps identified from an overview of systematic reviews. NPJ digital medicine. 2018;1:12. PMID: 31304297. 
8. Marcolino MS, Oliveira JAQ, D'Agostino M, et al. The Impact of mHealth Interventions: Systematic Review of Systematic Reviews. JMIR mHealth and uHealth. 2018 Jan 17;6(1):e23. PMID: 29343463.

9. Lamore K, Montalescot L, Untas A. Treatment decision-making in chronic diseases: What are the family members' roles, needs and attitudes? A systematic review. Patient education and counseling. 2017 Dec;100(12):2172-81. PMID: 28838630.

10. Ghazisaeidi M, Safdari R, Goodini A, et al. Digital games as an effective approach for cancer management: Opportunities and challenges. Journal of education and health promotion. 2017;6:30. PMID: 28584830.

11. Bland V, Sharma M. Physical activity interventions in African American women: A systematic review. Health promotion perspectives. 2017;7(2):52-9. PMID: 28326284.

12. Wilson MG, Lavis JN, Gauvin FP. Designing Integrated Approaches to Support People with Multimorbidity: Key Messages from Systematic Reviews, Health System Leaders and Citizens. Healthcare policy = Politiques de sante. 2016 Nov;12(2):91-104. PMID: 28032827.

13. Morton K, Dennison L, May C, et al. Using digital interventions for self-management of chronic physical health conditions: A meta-ethnography review of published studies. Patient education and counseling. 2017 Apr;100(4):616-35. PMID: 28029572.

14. Ershad Sarabi R, Sadoughi F, Jamshidi Orak R, et al. The Effectiveness of Mobile Phone Text Messaging in Improving Medication Adherence for Patients with Chronic Diseases: A Systematic Review. Iranian Red Crescent medical journal. 2016 May;18(5):e25183. PMID: 27437126.

15. Matthew-Maich N, Harris L, Ploeg J, et al. Designing, Implementing, and Evaluating Mobile Health Technologies for Managing Chronic Conditions in Older Adults: A Scoping Review. JMIR mHealth and uHealth. 2016 Jun 9;4(2):e29. PMID: 27282195.

16. Ganguli A, Clewell J, Shillington AC. The impact of patient support programs on adherence, clinical, humanistic, and economic patient outcomes: a targeted systematic review. Patient preference and adherence. 2016;10:711-25. PMID: 27175071.

17. Flodgren G, Rachas A, Farmer AJ, et al. Interactive telemedicine: effects on professional practice and health care outcomes. The Cochrane database of systematic reviews. 2015 Sep 7(9):Cd002098. PMID: 26343551.

18. Button K, Roos PE, Spasic I, et al. The clinical effectiveness of self-care interventions with an exercise component to manage knee conditions: A systematic review. The Knee. 2015 Oct;22(5):360-71. PMID: 26056046.

19. Dukhanin V, Topazian R, DeCamp M. Metrics and Evaluation Tools for Patient Engagement in Healthcare Organization- and System-Level Decision-Making: A Systematic Review. International journal of health policy and management. 2018 Oct 1;7(10):889-903. PMID: 30316241.

20. Finset A. Special Section: Review articles on decision making and patient education interventions. Patient education and counseling. 2017 Dec;100(12):2157-8. PMID: 29032951.

21. Olding M, McMillan SE, Reeves S, et al. Patient and family involvement in adult critical and intensive care settings: a scoping review. Health expectations : an international journal of 
public participation in health care and health policy. 2016 Dec;19(6):1183-202. PMID: 27878937.

22. Oczkowski SJ, Chung HO, Hanvey L, et al. Communication tools for end-of-life decisionmaking in the intensive care unit: a systematic review and meta-analysis. Critical care (London, England). 2016 Apr 9;20:97. PMID: 27059989.

23. Kynoch K, Chang A, Coyer F, et al. The effectiveness of interventions to meet family needs of critically ill patients in an adult intensive care unit: a systematic review update. JBI database of systematic reviews and implementation reports. 2016 Mar;14(3):181-234. PMID: 27532144.

24. Gagliardi AR, Legare F, Brouwers MC, et al. Patient-mediated knowledge translation (PKT) interventions for clinical encounters: a systematic review. Implementation science : IS. 2016 Feb 29;11:26. PMID: 26923462.

25. Snyder H, Engstrom J. The antecedents, forms and consequences of patient involvement: A narrative review of the literature. International journal of nursing studies. 2016 Jan;53:351-78. PMID: 26602069.

\section{Does not report any of the outcomes of interest (QoL, Patient satisfaction/experience, Medication adherence, Healthcare utilization, Chronic disease management outcome, Implementation measure)}

1. Tricco AC, Ashoor HM, Cardoso R, et al. Sustainability of knowledge translation interventions in healthcare decision-making: a scoping review. Implementation science : IS. 2016 Apr 21;11:55. PMID: 27097827.

2. Kogan AC, Wilber K, Mosqueda L. Person-Centered Care for Older Adults with Chronic Conditions and Functional Impairment: A Systematic Literature Review. Journal of the American Geriatrics Society. 2016 Jan;64(1):e1-7. PMID: 26626408.

3. Vromans RD, van Eenbergen MC, Pauws SC, et al. Communicative aspects of decision aids for localized prostate cancer treatment - A systematic review. Urologic oncology. 2019 Jul;37(7):409-29. PMID: 31053529.

4. Dogba MJ, Dossa AR, Breton E, et al. Using information and communication technologies to involve patients and the public in health education in rural and remote areas: a scoping review. BMC health services research. 2019 Feb 19;19(1):128. PMID: 30782147.

5. Simblett S, Greer B, Matcham F, et al. Barriers to and Facilitators of Engagement With Remote Measurement Technology for Managing Health: Systematic Review and Content Analysis of Findings. Journal of medical Internet research. 2018 Jul 12;20(7):e10480. PMID: 30001997.

6. Menichetti J, Graffigna G, Steinsbekk A. What are the contents of patient engagement interventions for older adults? A systematic review of randomized controlled trials. Patient education and counseling. 2018 Jun;101(6):995-1005. PMID: 29246493.

7. Young A, Menon D, Street J, et al. Exploring patient and family involvement in the lifecycle of an orphan drug: a scoping review. Orphanet journal of rare diseases. 2017 Dec 22;12(1):188. PMID: 29273068. 
8. Schmidt K, Damm K, Prenzler A, et al. Preferences of lung cancer patients for treatment and decision-making: a systematic literature review. European journal of cancer care. 2016 Jul;25(4):580-91. PMID: 26676876.

9. Walsh S, Golden E, Priebe S. Systematic review of patients' participation in and experiences of technology-based monitoring of mental health symptoms in the community. BMJ open. 2016 Jun 21;6(6):e008362. PMID: 27329437.

10. Menon D, Stafinski T, Dunn A, et al. Involving patients in reducing decision uncertainties around orphan and ultra-orphan drugs: a rare opportunity? The patient. 2015 Feb;8(1):29-39. PMID: 25516506.

11. Waid J, Kelly M. Supporting family engagement with child and adolescent mental health services: A scoping review. Health Soc Care Community. 2020 Jan 17. doi: 10.1111/hsc.12947. Epub ahead of print. PMID: 31951087.

12. Menear M, Dugas M, Careau E, Chouinard MC, Dogba MJ, Gagnon MP, Gervais M, Gilbert M, Houle J, Kates N, Knowles S, Martin N, Nease DE Jr, Zomahoun HTV, Légaré F. Strategies for engaging patients and families in collaborative care programs for depression and anxiety disorders: A systematic review. J Affect Disord. 2020 Feb 15;263:528-539. doi: 10.1016/j.jad.2019.11.008. Epub 2019 Nov 4. PMID: 31744737. 


\section{Appendix E. List of Excluded Studies for Original Articles}

Studies are sorted by the reason for exclusion.

\section{Does not apply to the Key Question}

1. Gordon WJ, Bates DW, Fuchs D, et al. Comparing Characteristics of Patients Who Connect Their iPhones to an Electronic Health Records System Versus Patients Who Connect Without Personal Devices: Cohort Study. Journal of medical Internet research. 2019 Aug 22;21(8):e14871. PMID: 31441430.

2. Carmel AS, Cornelius-Schecter A, Frankel B, et al. Evaluation of the Patient Activated Learning System (PALS) to improve knowledge acquisition, retention, and medication decision making among hypertensive adults: Results of a pilot randomized controlled trial. Patient education and counseling. 2019 Aug;102(8):1467-74. PMID: 30928344.

3. Bajracharya AS, Crotty BH, Kowoloff HB, et al. Patient experience with family history tool: analysis of patients' experience sharing their family health history through patient-computer dialogue in a patient portal. Journal of the American Medical Informatics Association : JAMIA. 2019 Jul 1;26(7):603-9. PMID: 30946464.

4. Minneci PC, Cooper JN, Leonhart K, et al. Effects of a Patient Activation Tool on Decision Making Between Surgery and Nonoperative Management for Pediatric Appendicitis: A Randomized Clinical Trial. JAMA network open. 2019 Jun 5;2(6):e195009. PMID: 31173118.

5. Brice YN, Joynt KE, Tompkins CP, et al. Meaningful Use and Hospital Performance on PostAcute Utilization Indicators. Health services research. 2018 Apr;53(2):803-23. PMID: 28255995.

6. Jiang S, Hong YA. Mobile-based patient-provider communication in cancer survivors: The roles of health literacy and patient activation. Psycho-oncology. 2018 Mar;27(3):886-91. PMID: 29193503.

7. Gibson B, Butler J, Doyon K, et al. Veterans Like Me: Formative evaluation of a patient decision aid design. Journal of biomedical informatics. 2017 Jul;71s:S46-s52. PMID: 27623534.

8. Aslakson RA, Isenberg SR, Crossnohere NL, et al. Utilising advance care planning videos to empower perioperative cancer patients and families: a study protocol of a randomised controlled trial. BMJ open. 2017 Jun 6;7(5):e016257. PMID: 28592584.

9. Taylor LJ, Rathouz PJ, Berlin A, et al. Navigating high-risk surgery: protocol for a multisite, stepped wedge, cluster-randomised trial of a question prompt list intervention to empower older adults to ask questions that inform treatment decisions. BMJ open. 2017 May 29;7(5):e014002. PMID: 28554911.

10. Lawrence D, Miller JH, C WF. Medication Adherence. Journal of clinical pharmacology. 2017 Apr;57(4):422-7. PMID: 28105688. 
11. An J. The Impact of Patient-Centered Medical Homes on Quality of Care and Medication Adherence in Patients with Diabetes Mellitus. Journal of managed care \& specialty pharmacy. 2016 Nov;22(11):1272-84. PMID: 27783547.

12. Simmons K, Gibson S, White JM. Drivers Advancing Oral Health in a Large Group Dental Practice Organization. The journal of evidence-based dental practice. 2016 Jun;16 Suppl:104-12. PMID: 27237003.

13. Tothy AS, Limper HM, Driscoll J, et al. The Ask Me to Explain Campaign: A 90-Day Intervention to Promote Patient and Family Involvement in Care in a Pediatric Emergency Department. Joint Commission journal on quality and patient safety. 2016 Jun;42(6):281-5. PMID: 27184244.

14. Purkaple BA, Mold JW, Chen S. Encouraging Patient-Centered Care by Including Qualityof-Life Questions on Pre-Encounter Forms. Annals of family medicine. 2016 May;14(3):221-6. PMID: 27184992.

15. Altshuler L, Plaksin J, Zabar S, et al. Transforming the Patient Role to Achieve Better Outcomes Through a Patient Empowerment Program: A Randomized Wait-List Control Trial Protocol. JMIR research protocols. 2016 Apr 21;5(2):e68. PMID: 27103306.

16. Serpico V, Liepert AE, Boucher K, et al. The Effect of Previsit Education in Breast Cancer Patients: A Study of a Shared-decision-making Tool. The American surgeon. 2016 Mar;82(3):259-65. PMID: 27099063.

17. Hayes RM, Wickline A, Hensley C, et al. A Quality Improvement Project to Improve Family Recognition of Medical Team Member Roles. Hospital pediatrics. 2015 Sep;5(9):480-6. PMID: 26330247.

18. Spertus JA, Bach R, Bethea C, et al. Improving the process of informed consent for percutaneous coronary intervention: patient outcomes from the Patient Risk Information Services Manager (ePRISM) study. American heart journal. 2015 Feb;169(2):234-41.e1. PMID: 25641532.

19. Tassone C, Keshavjee K, Paglialonga A, Moreira N, Pinto J, Quintana Y. Evaluation of mobile apps for treatment of patients at risk of developing gestational diabetes. Health Informatics J. 2020 Jan 8:1460458219896639. doi: 10.1177/1460458219896639. Epub ahead of print. PMID: 31912754.

\section{No comparison group}

1. Quinn CC, Staub S, Barr E, et al. Mobile Support for Older Adults and Their Caregivers: Dyad Usability Study. JMIR aging. 2019 May 23;2(1):e12276. PMID: 31518271.

2. Kata A, Sudore R, Finlayson E, et al. Increasing Advance Care Planning Using a Surgical Optimization Program for Older Adults. Journal of the American Geriatrics Society. 2018 Oct;66(10):2017-21. PMID: 30289968. 
3. Selter A, Tsangouri C, Ali SB, et al. An mHealth App for Self-Management of Chronic Lower Back Pain (Limbr): Pilot Study. JMIR mHealth and uHealth. 2018 Sep 17;6(9):e179. PMID: 30224333.

4. Jiang S, Hong YA. Mobile-based patient-provider communication in cancer survivors: The roles of health literacy and patient activation. Psycho-oncology. 2018 Mar;27(3):886-91. PMID: 29193503.

5. Woolf SH, Krist AH, Lafata JE, et al. Engaging Patients in Decisions About Cancer Screening: Exploring the Decision Journey Through the Use of a Patient Portal. American journal of preventive medicine. 2018 Feb;54(2):237-47. PMID: 29241715.

6. Forcino RC, Barr PJ, O'Malley AJ, et al. Using CollaboRATE, a brief patient-reported measure of shared decision making: Results from three clinical settings in the United States. Health expectations : an international journal of public participation in health care and health policy. 2018 Feb;21(1):82-9. PMID: 28678426.

7. Hines AL, Roter D, Ghods Dinoso BK, et al. Informed and patient-centered decision-making in the primary care visits of African Americans with depression. Patient education and counseling. 2018 Feb;101(2):233-40. PMID: 28779910.

8. Byrne C, Kurmas N, Burant CJ, et al. Cooking Classes: A Diabetes Self-Management Support Intervention Enhancing Clinical Values. The Diabetes educator. 2017 Dec;43(6):600-7. PMID: 29047323.

9. Whitney E, Kindred E, Pratt A, et al. Culturally Tailoring a Patient Empowerment and Diabetes Education Curriculum for the African American Church. The Diabetes educator. 2017 Oct;43(5):441-8. PMID: 28793835.

10. Gibson B, Butler J, Doyon K, et al. Veterans Like Me: Formative evaluation of a patient decision aid design. Journal of biomedical informatics. 2017 Jul;71s:S46-s52. PMID: 27623534.

11. Marshall AP, Lemieux M, Dhaliwal R, et al. Novel, Family-Centered Intervention to Improve Nutrition in Patients Recovering From Critical Illness: A Feasibility Study. Nutrition in clinical practice : official publication of the American Society for Parenteral and Enteral Nutrition. 2017 Jun;32(3):392-9. PMID: 28537514.

12. de Jong M, van der Meulen-de Jong A, Romberg-Camps M, et al. Development and Feasibility Study of a Telemedicine Tool for All Patients with IBD: MyIBDcoach. Inflammatory bowel diseases. 2017 Apr;23(4):485-93. PMID: 28267047.

13. Tothy AS, Limper HM, Driscoll J, et al. The Ask Me to Explain Campaign: A 90-Day Intervention to Promote Patient and Family Involvement in Care in a Pediatric Emergency Department. Joint Commission journal on quality and patient safety. 2016 Jun;42(6):281-5. PMID: 27184244. 
14. Gibson A, Cooper M, Rae J, Hayes J. Clients' experiences of shared decision making in an integrative psychotherapy for depression. J Eval Clin Pract. 2019 Dec 1. doi: 10.1111/jep.13320. Epub ahead of print. PMID: 31788932.

\section{Not in English}

1. Pons Rafols JM, Moharra Frances M. [Shared decision making]. Medicina clinica. 2016 Mar 4;146(5):205-6. PMID: 26589733.

2. Gröger S, Mäder-Porombka C, Stang C, Wallacher S. Partizipative Entscheidungsfindung bei Prostatakrebspatienten [Shared decision-making in prostate cancer patients]. Urologe A. 2019 Nov;58(11):1324-1330. German. doi: 10.1007/s00120-018-0774-4. PMID: 30238132.

\section{Non-USA based study}

1. Vankan E, Schoorel E, van Kuijk S, et al. The effect of the use of a decision aid with individual risk estimation on the mode of delivery after a caesarean section: A prospective cohort study. PloS one. 2019;14(9):e0222499. PMID: 31557177.

2. Clarke AL, Roscoe J, Appleton R, et al. Promoting integrated care in prostate cancer through online prostate cancer-specific holistic needs assessment: a feasibility study in primary care. Supportive care in cancer : official journal of the Multinational Association of Supportive Care in Cancer. 2019 Jul 23. PMID: 31338642.

3. Guo Y, Lane DA, Wang L, et al. Mobile Health (mHealth) technology for improved screening, patient involvement and optimising integrated care in atrial fibrillation: The mAFA (mAF-App) II randomised trial. International journal of clinical practice. 2019 Jul;73(7):e13352. PMID: 31002434.

4. Burns K, McBride CA, Patel B, et al. Creating Consumer-Generated Health Data: Interviews and a Pilot Trial Exploring How and Why Patients Engage. Journal of medical Internet research. 2019 Jun 13;21(6):e12367. PMID: 31199312.

5. Mata J, Pecorelli N, Kaneva P, et al. A mobile device application (app) to improve adherence to an enhanced recovery program for colorectal surgery: a randomized controlled trial. Surgical endoscopy. 2019 May 13. PMID: 31087175.

6. Chao DY, Lin TM, Ma WY. Enhanced Self-Efficacy and Behavioral Changes Among Patients With Diabetes: Cloud-Based Mobile Health Platform and Mobile App Service. JMIR diabetes. 2019 May 10;4(2):e11017. PMID: 31094324.

7. Zuidema R, van Dulmen S, Nijhuis-van der Sanden M, et al. Efficacy of a Web-Based SelfManagement Enhancing Program for Patients with Rheumatoid Arthritis: Explorative Randomized Controlled Trial. Journal of medical Internet research. 2019 Apr 30;21(4):e12463. PMID: 31038461.

8. Reumkens K, de Die-Smulders CEM, van Osch L. Exploring the preferences of involved health professionals regarding the implementation of an online decision aid to support couples 
during reproductive decision-making in hereditary cancer: a mixed methods approach. Familial cancer. 2019 Apr;18(2):285-91. PMID: 30656480.

9. Luhr K, Eldh AC, Theander K, et al. Effects of a self-management programme on patient participation in patients with chronic heart failure or chronic obstructive pulmonary disease: A randomized controlled trial. European journal of cardiovascular nursing : journal of the Working Group on Cardiovascular Nursing of the European Society of Cardiology. 2019 Mar;18(3):18593. PMID: 30277807.

10. McCusker J, Lambert SD, Haggerty J, et al. Self-management support in primary care is associated with improvement in patient activation. Patient education and counseling. 2019 Mar;102(3):571-7. PMID: 30497799.

11. Henselmans I, van Laarhoven HWM, de Haes H, et al. Training for Medical Oncologists on Shared Decision-Making About Palliative Chemotherapy: A Randomized Controlled Trial. The oncologist. 2019 Feb;24(2):259-65. PMID: 29959285.

12. Withidpanyawong U, Lerkiatbundit S, Saengcharoen W. Family-based intervention by pharmacists for type 2 diabetes: A randomised controlled trial. Patient education and counseling. 2019 Jan;102(1):85-92. PMID: 30150128.

13. Nost TH, Steinsbekk A, Bratas O, et al. Twelve-month effect of chronic pain selfmanagement intervention delivered in an easily accessible primary healthcare service - a randomised controlled trial. BMC health services research. 2018 Dec 29;18(1):1012. PMID: 30594190.

14. Bonin K, McGuffin M, Lechtman E, et al. Evaluation of an Online Education Resource on Radiation Therapy Created for Patients with Postprostatectomy Prostate Cancer and Their Caregivers. Journal of medical imaging and radiation sciences. 2018 Dec;49(4):365-70. PMID: 30514552.

15. Davins Riu M, Borras Perez X, Artigas Raventos V, et al. Use of Telehealth as a New Model for Following Intermittent Claudication and Promoting Patient Expertise. Telemedicine journal and e-health : the official journal of the American Telemedicine Association. 2018 Oct;24(10):773-81. PMID: 29323628.

16. Kapell Brown C, Kryworuchko J, Martin W. Evaluation of the CPR video decision aid with patients with end stage renal disease. BMC nephrology. 2018 Sep 12;19(1):226. PMID: 30208850.

17. Risling T, Martinez J, Young J, et al. Defining Empowerment and Supporting Engagement Using Patient Views From the Citizen Health Information Portal: Qualitative Study. JMIR medical informatics. 2018 Sep 10;6(3):e43. PMID: 30201603.

18. Amundsen A, Bergvik S, Butow P, et al. Supporting doctor-patient communication: Providing a question prompt list and audio recording of the consultation as communication aids to outpatients in a cancer clinic. Patient education and counseling. 2018 Sep;101(9):1594-600. PMID: 29703493. 
19. Au SS, Roze des Ordons AL, Parsons Leigh J, et al. A Multicenter Observational Study of Family Participation in ICU Rounds. Critical care medicine. 2018 Aug;46(8):1255-62. PMID: 29742590.

20. Tinsel I, Siegel A, Schmoor C, et al. Encouraging Self-Management in Cardiovascular Disease Prevention. Deutsches Arzteblatt international. 2018 Jul 9;115(27-28):469-76. PMID: 30064627.

21. Baijens SWE, Huppelschoten AG, Van Dillen J, et al. Improving shared decision-making in a clinical obstetric ward by using the three questions intervention, a pilot study. BMC pregnancy and childbirth. 2018 Jul 4;18(1):283. PMID: 29973187.

22. Melissant HC, Verdonck-de Leeuw IM, Lissenberg-Witte BI, et al. 'Oncokompas', a webbased self-management application to support patient activation and optimal supportive care: a feasibility study among breast cancer survivors. Acta oncologica (Stockholm, Sweden). 2018 Jul;57(7):924-34. PMID: 29451059.

23. Jegan NRA, Kurwitz SA, Kramer LK, et al. The effect of a new lifetime-cardiovascular-risk display on patients' motivation to participate in shared decision-making. BMC family practice. 2018 Jun 9;19(1):84. PMID: 29885661.

24. Li LC, Shaw CD, Lacaille D, et al. Effects of a Web-Based Patient Decision Aid on Biologic and Small-Molecule Agents for Rheumatoid Arthritis: Results From a Proof-of-Concept Study. Arthritis care \& research. 2018 Mar;70(3):343-52. PMID: 28544648.

25. Nafradi L, Nakamoto K, Csabai M, et al. An empirical test of the Health Empowerment Model: Does patient empowerment moderate the effect of health literacy on health status? Patient education and counseling. 2018 Mar;101(3):511-7. PMID: 28899712.

26. Ruiz-Baques A, Contreras-Porta J, Marques-Mejias M, et al. Evaluation of an Online Educational Program for Parents and Caregivers of Children With Food Allergies. Journal of investigational allergology \& clinical immunology. 2018;28(1):37-41. PMID: 29461207.

27. Dinius J, Gaupp R, Becker S, et al. Patient Safety in Hospitals: What We Do and What We Need-Focus Groups With Stakeholders of Hospitals in Southern Germany. Journal of patient safety. 2017 Dec 15. PMID: 29252966.

28. Ryu B, Kim N, Heo E, et al. Impact of an Electronic Health Record-Integrated Personal Health Record on Patient Participation in Health Care: Development and Randomized Controlled Trial of MyHealthKeeper. Journal of medical Internet research. 2017 Dec 7;19(12):e401. PMID: 29217503.

29. Guo Y, Chen Y, Lane DA, et al. Mobile Health Technology for Atrial Fibrillation Management Integrating Decision Support, Education, and Patient Involvement: mAF App Trial. The American journal of medicine. 2017 Dec;130(12):1388-96.e6. PMID: 28847546. 
30. Boland L, Taljaard M, Dervin G, et al. Effect of patient decision aid was influenced by presurgical evaluation among patients with osteoarthritis of the knee. Canadian journal of surgery Journal canadien de chirurgie. 2017 Dec;60(6):3316. PMID: 29171829.

31. Fridman S, Saposnik G, Sposato LA. Visual Aids for Improving Patient Decision Making in Severe Symptomatic Carotid Stenosis. Journal of stroke and cerebrovascular diseases : the official journal of National Stroke Association. 2017 Dec;26(12):2888-92. PMID: 28797613.

32. Geiger F, Liethmann K, Reitz D, et al. Efficacy of the doktormitSDM training module in supporting shared decision making - Results from a multicenter double-blind randomized controlled trial. Patient education and counseling. 2017 Dec;100(12):2331-8. PMID: 28647064.

33. Flode M, Iversen MM, Aarflot $M$, et al. Lasting impact of an implemented self-management programme for people with type 2 diabetes referred from primary care: a one-group, before-after design. Scandinavian journal of caring sciences. 2017 Dec;31(4):789-95. PMID: 28145104.

34. Early F, Young JS, Robinshaw E, et al. A case series of an off-the-shelf online health resource with integrated nurse coaching to support self-management in COPD. International journal of chronic obstructive pulmonary disease. 2017;12:2955-67. PMID: 29070947.

35. Harcourt D, Paraskeva N, White P, et al. A study protocol of the effectiveness of PEGASUS: a multi-centred study comparing an intervention to promote shared decision making about breast reconstruction with treatment as usual. BMC medical informatics and decision making. 2017 Oct 2;17(1):143. PMID: 28969622.

36. Perestelo-Perez L, Rivero-Santana A, Sanchez-Afonso JA, et al. Effectiveness of a decision aid for patients with depression: A randomized controlled trial. Health expectations : an international journal of public participation in health care and health policy. 2017 Oct;20(5):1096-105. PMID: 28295915.

37. Sundaresan P, Ager B, Turner S, et al. A randomised controlled trial evaluating the utility of a patient Decision Aid to improve clinical trial (RAVES 08.03) related decision-making. Radiotherapy and oncology : journal of the European Society for Therapeutic Radiology and Oncology. 2017 Oct;125(1):124-9. PMID: 28844330.

38. Marshall AP, Lemieux M, Dhaliwal R, et al. Novel, Family-Centered Intervention to Improve Nutrition in Patients Recovering From Critical Illness: A Feasibility Study. Nutrition in clinical practice : official publication of the American Society for Parenteral and Enteral Nutrition. 2017 Jun;32(3):392-9. PMID: 28537514.

39. Akca A, Corbacioglu Esmer A, Ozyurek ES, et al. The influence of the systematic birth preparation program on childbirth satisfaction. Archives of gynecology and obstetrics. 2017 May;295(5):1127-33. PMID: 28303340.

40. Wang RH, Hsu HC, Kao CC, et al. Associations of changes in psychosocial factors and their interactions with diabetes distress in patients with type 2 diabetes: a longitudinal study. Journal of advanced nursing. 2017 May;73(5):1137-46. PMID: 27862194. 
41. de Jong M, van der Meulen-de Jong A, Romberg-Camps M, et al. Development and Feasibility Study of a Telemedicine Tool for All Patients with IBD: MyIBDcoach. Inflammatory bowel diseases. 2017 Apr;23(4):485-93. PMID: 28267047.

42. Stegmann ME, Schuling J, Hiltermann TJ, et al. Study protocol for the OPTion randomised controlled trial on the effect of prioritising treatment goals among older patients with cancer in a palliative setting. Maturitas. 2017 Feb;96:84-8. PMID: 28041600.

43. Wolderslund M, Kofoed PE, Holst R, et al. Digital audio recordings improve the outcomes of patient consultations: A randomised cluster trial. Patient education and counseling. 2017 Feb;100(2):242-9. PMID: 27593087.

44. Zisman-Ilani Y, Roe D, Scholl I, et al. Shared Decision Making During Active Psychiatric Hospitalization: Assessment and Psychometric Properties. Health communication. 2017 Jan;32(1):126-30. PMID: 27168160.

45. Grainger R, Townsley H, Langlotz T, et al. Patient-Clinician Co-Design Co-Participation in Design of an App for Rheumatoid Arthritis Management via Telehealth Yields an App with High Usability and Acceptance. Studies in health technology and informatics. 2017;245:1223. PMID: 29295310.

46. Hoskins G, Williams B, Abhyankar P, et al. Achieving Good Outcomes for Asthma Living (GOAL): mixed methods feasibility and pilot cluster randomised controlled trial of a practical intervention for eliciting, setting and achieving goals for adults with asthma. Trials. 2016 Dec 8;17(1):584. PMID: 27931242.

47. Foley NM, O'Connell EP, Lehane EA, et al. PATI: Patient accessed tailored information: A pilot study to evaluate the effect on preoperative breast cancer patients of information delivered via a mobile application. Breast (Edinburgh, Scotland). 2016 Dec;30:54-8. PMID: 27611236.

48. Due-Christensen M, Hommel E, Ridderstrale M. Potential positive impact of group-based diabetes dialogue meetings on diabetes distress and glucose control in people with type 1 diabetes. Patient education and counseling. 2016 Dec;99(12):1978-83. PMID: 27444233.

49. McBride E, Hacking B, O'Carroll R, et al. Increasing patient involvement in the diabetic foot pathway: a pilot randomized controlled trial. Diabetic medicine : a journal of the British Diabetic Association. 2016 Nov;33(11):1483-92. PMID: 27223310.

50. Wong CK, Lam CL, Wan EY, et al. Evaluation of patient-reported outcomes data in structured diabetes education intervention: 2-year follow-up data of patient empowerment programme. Endocrine. 2016 Nov;54(2):422-32. PMID: 27623970.

51. Nota I, Drossaert CH, Taal E, et al. Evaluation of a patient decision aid for initiating disease modifying anti-rheumatic drugs. Arthritis research \& therapy. 2016 Oct 28;18(1):252. PMID: 27793171.

52. Wong CK, Wong WC, Wan YF, et al. Effect of a structured diabetes education programme in primary care on hospitalizations and emergency department visits among people with Type 2 
diabetes mellitus: results from the Patient Empowerment Programme. Diabetic medicine : a journal of the British Diabetic Association. 2016 Oct;33(10):1427-36. PMID: 26433212.

53. Gardner T, Refshauge K, McAuley J, et al. Patient-led Goal Setting: A Pilot Study Investigating a Promising Approach for the Management of Chronic Low Back Pain. Spine. 2016 Sep 15;41(18):1405-13. PMID: 26937604.

54. Ruiz-Garcia J, Diez-Villanueva P, Ayesta A, et al. End-of-life care in a cardiology department: have we improved? Journal of geriatric cardiology : JGC. 2016 Jul;13(7):587-92. PMID: 27605939.

55. Lara-Cabrera ML, Gjerden M, Grawe RW, et al. Short-term effects of a peer co-led educational programme delivered before mental health treatment: A randomised controlled trial. Patient education and counseling. 2016 Jul;99(7):1257-61. PMID: 26905956.

56. Morrison D, Wyke S, Saunderson K, et al. Findings from a pilot Randomised trial of an Asthma Internet Self-management Intervention (RAISIN). BMJ open. 2016 May 12;6(5):e009254. PMID: 27173807.

57. Kuijpers W, Groen WG, Oldenburg HS, et al. eHealth for Breast Cancer Survivors: Use, Feasibility and Impact of an Interactive Portal. JMIR cancer. 2016 May 10;2(1):e3. PMID: 28410178.

58. Beg S, Curtis S, Shariff M. Patient education and its effect on self-management in cirrhosis: a pilot study. European journal of gastroenterology \& hepatology. 2016 May;28(5):582-7. PMID: 27015016.

59. Nicolai J, Buchholz A, Seefried N, et al. When do cancer patients regret their treatment decision? A path analysis of the influence of clinicians' communication styles and the match of decision-making styles on decision regret. Patient education and counseling. 2016 May;99(5):739-46. PMID: 26658703.

60. van Tol-Geerdink JJ, Leer JW, Wijburg CJ, et al. Does a decision aid for prostate cancer affect different aspects of decisional regret, assessed with new regret scales? A randomized, controlled trial. Health expectations : an international journal of public participation in health care and health policy. 2016 Apr;19(2):459-70. PMID: 25940277.

61. Modin L, Walsted AM, Rittig CS, et al. Follow-up in Childhood Functional Constipation: A Randomized, Controlled Clinical Trial. Journal of pediatric gastroenterology and nutrition. 2016 Apr;62(4):594-9. PMID: 26348685.

62. O'Donnell M, Alvarez-Iglesias A, McGuire BE, et al. The impact of sharing personalised clinical information with people with type 2 diabetes prior to their consultation: A pilot randomised controlled trial. Patient education and counseling. 2016 Apr;99(4):591-9. PMID: 26654869. 
63. Stacey D, Taljaard M, Dervin G, et al. Impact of patient decision aids on appropriate and timely access to hip or knee arthroplasty for osteoarthritis: a randomized controlled trial. Osteoarthritis and cartilage. 2016 Jan;24(1):99-107. PMID: 26254238.

64. Couet N, Labrecque M, Robitaille $\mathrm{H}$, et al. The impact of DECISION+2 on patient intention to engage in shared decision making: secondary analysis of a multicentre clustered randomized trial. Health expectations : an international journal of public participation in health care and health policy. 2015 Dec;18(6):2629-37. PMID: 25041071.

65. Riippa I, Linna M, Ronkko I. A Patient Portal With Electronic Messaging: Controlled Before-and-After Study. Journal of medical Internet research. 2015 Nov 9;17(11):e250. PMID: 26553595.

66. MacInnes JA, Salkovskis PM, Wroe A, et al. Helping patients to reach decisions regarding their treatment: Do 'non-directive' approaches cause systematic bias? British journal of health psychology. 2015 Nov;20(4):877-88. PMID: 26286684.

67. Aljumah K, Hassali MA. Impact of pharmacist intervention on adherence and measurable patient outcomes among depressed patients: a randomised controlled study. BMC psychiatry. 2015 Sep 16;15:219. PMID: 26376830.

68. Moral RR, Torres LA, Ortega LP, et al. Effectiveness of motivational interviewing to improve therapeutic adherence in patients over 65 years old with chronic diseases: A cluster randomized clinical trial in primary care. Patient education and counseling. 2015 Aug;98(8):97783. PMID: 25858633.

69. Cingi C, Yorgancioglu A, Cingi CC, et al. The "physician on call patient engagement trial" (POPET): measuring the impact of a mobile patient engagement application on health outcomes and quality of life in allergic rhinitis and asthma patients. International forum of allergy \& rhinology. 2015 Jun;5(6):487-97. PMID: 25856270.

70. Labrie NH, Schulz PJ. Exploring the relationships between participatory decision-making, visit duration, and general practitioners' provision of argumentation to support their medical advice: results from a content analysis. Patient education and counseling. 2015 May;98(5):572-7. PMID: 25746127.

71. Heijmans N, van Lieshout J, Wensing M. Improving participation rates by providing choice of participation mode: two randomized controlled trials. BMC medical research methodology. 2015 Apr 2;15:29. PMID: 25886757.

72. Weymann N, Dirmaier J, von Wolff A, et al. Effectiveness of a Web-based tailored interactive health communication application for patients with type 2 diabetes or chronic low back pain: randomized controlled trial. Journal of medical Internet research. $2015 \mathrm{Mar}$ 3;17(3):e53. PMID: 25736340.

73. Strauss K, Benvenuto A, Battan B, et al. Promoting Shared Decision Making to strengthen outcome of young children with Autism Spectrum Disorders: the role of staff competence. Research in developmental disabilities. 2015 Mar;38:48-63. PMID: 25544428. 
74. Turner A, Anderson JK, Wallace LM, et al. An evaluation of a self-management program for patients with long-term conditions. Patient education and counseling. 2015 Feb;98(2):213-9. PMID: 25441096.

75. Allam A, Kostova Z, Nakamoto K, et al. The effect of social support features and gamification on a Web-based intervention for rheumatoid arthritis patients: randomized controlled trial. Journal of medical Internet research. 2015 Jan 9;17(1):e14. PMID: 25574939.

76. Mata J, Pecorelli N, Kaneva P, Moldoveanu D, Gosselin-Tardiff A, Alhashemi M, Robitaille S, Balvardi S, Lee L, Stein BL, Liberman S, Charlebois P, Fiore JF Jr, Feldman LS. A mobile device application (app) to improve adherence to an enhanced recovery program for colorectal surgery: a randomized controlled trial. Surg Endosc. 2020 Feb;34(2):742-751. doi: 10.1007/s00464-019-06823-w. Epub 2019 May 13. PMID: 31087175.

77. Granados-Santiago M, Valenza MC, López-López L, Prados-Román E, Rodríguez- Torres J, Cabrera-Martos I. Shared decision-making and patient engagement program during acute exacerbation of COPD hospitalization: A randomized control trial. Patient Educ Couns. 2019 Dec 11:S0738-3991(19)30548-8. doi: 10.1016/j.pec.2019.12.004. Epub ahead of print. PMID: 31859121.

78. Henselmans I, van Laarhoven HWM, van Maarschalkerweerd P, de Haes HCJM, Dijkgraaf MGW, Sommeijer DW, Ottevanger PB, Fiebrich HB, Dohmen S, Creemers GJ, de Vos FYFL, Smets EMA. Effect of a Skills Training for Oncologists and a Patient Communication Aid on Shared Decision Making About Palliative Systemic Treatment: A Randomized Clinical Trial. Oncologist. 2019 Nov 26:theoncologist.2019-0453. doi: 10.1634/theoncologist.2019-0453. Epub ahead of print. PMID: 31771987.

79. Knudsen MV, Petersen AK, Angel S, Hjortdal VE, Maindal HT, Laustsen S. Telerehabilitation and hospital-based cardiac rehabilitation are comparable in increasing patient activation and health literacy: A pilot study. Eur J Cardiovasc Nurs. 2019 Nov 8:1474515119885325. doi: 10.1177/1474515119885325. Epub ahead of print. PMID: 31702397.

80. Singer S, Danker H, Meixensberger J, Briest S, Dietz A, Kortmann RD, Stolzenburg JU, Kersting A, Roick J. Structured multi-disciplinary psychosocial care for cancer patients and the perceived quality of care from the patient perspective: a cluster-randomized trial. J Cancer Res Clin Oncol. 2019 Nov;145(11):2845-2854. doi: 10.1007/s00432-019-03018-7. Epub 2019 Sep 10. PMID: 31506739.

81. Kim SH, Utz S. Effectiveness of a Social Media-Based, Health Literacy- Sensitive Diabetes Self-Management Intervention: A Randomized Controlled Trial. J Nurs Scholarsh. 2019 Nov;51(6):661-669. doi: 10.1111/jnu.12521. Epub 2019 Oct 17. PMID: 31622033.

82. Qaderi SM, Vromen H, Dekker HM, Stommel MWJ, Bremers AJA, de Wilt JHW. Development and implementation of a remote follow-up plan for colorectal cancer patients. Eur J Surg Oncol. 2020 Mar;46(3):429-432. doi: 10.1016/j.ejso.2019.10.014. Epub 2019 Oct 11. PMID: 31668976. 


\section{Not an original article}

1. Rosenbloom ST, Steitz BD, Warner JL. Window of Opportunity: Patient Portals and Cancer. Journal of oncology practice. 2018 Nov;14(11):639-41. PMID: 30423264.

2. Gander JC, Basu M, McPherson L, et al. iChoose Kidney for Treatment Options: Updated Models for Shared Decision Aid. Transplantation. 2018 Sep;102(9):e370-e1. PMID: 29979349.

3. King TL, Likis FE, Wilson-Liverman AM. Shared Decision Making: Misconstrued and Misused. Journal of midwifery \& women's health. 2018 May;63(3):257-8. PMID: 29733504.

4. Tomlinson JP. Shifting the focus of shared decision making to human relationships. BMJ (Clinical research ed). 2018 Jan 8;360:k53. PMID: 29311057.

5. Lang E, Bell NR, Dickinson JA, et al. Eliciting patient values and preferences to inform shared decision making in preventive screening. Canadian family physician Medecin de famille canadien. 2018 Jan;64(1):28-31. PMID: 29358246.

6. Grad R, Legare F, Bell NR, et al. Shared decision making in preventive health care: What it is; what it is not. Canadian family physician Medecin de famille canadien. 2017 Sep;63(9):682-4. PMID: 28904031.

7. Ivatury SJ, Durand MA, Elwyn G. Shared Decision-Making for Rectal Cancer Treatment: A Path Forward. Dis Colon Rectum. 2019 Dec;62(12):1412-1413. doi:

10.1097/DCR.0000000000001454. PMID: 31725575.

\section{Does not report any of the outcomes of interest (QoL, Patient satisfaction/experience, Medication adherence, Healthcare utilization, Chronic disease management outcome, Implementation measure)}

1. Moore BA, Buono FD, Printz DMB, et al. Customized recommendations and reminder text messages for automated, computer-based treatment during methadone. Experimental and clinical psychopharmacology. 2017 Dec;25(6):485-95. PMID: 29251978.

2. Long AC, Downey L, Engelberg RA, et al. Understanding Response Rates to Surveys About Family Members' Psychological Symptoms After Patients' Critical Illness. Journal of pain and symptom management. 2017 Jul;54(1):96-104. PMID: 28552830.

3. Simmons K, Gibson S, White JM. Drivers Advancing Oral Health in a Large Group Dental Practice Organization. The journal of evidence-based dental practice. 2016 Jun;16 Suppl:104-12. PMID: 27237003.

4. Jack B, Bickmore T, Hempstead M, et al. Reducing Preconception Risks Among African American Women with Conversational Agent Technology. Journal of the American Board of Family Medicine : JABFM. 2015 Jul-Aug;28(4):441-51. PMID: 26152434.

5. Spertus JA, Bach R, Bethea C, et al. Improving the process of informed consent for percutaneous coronary intervention: patient outcomes from the Patient Risk Information Services 
Manager (ePRISM) study. American heart journal. 2015 Feb;169(2):234-41.e1. PMID: 25641532.

\section{Other}

1. Minneci PC, Cooper JN, Leonhart K, et al. Effects of a Patient Activation Tool on Decision Making Between Surgery and Nonoperative Management for Pediatric Appendicitis: A Randomized Clinical Trial. JAMA network open. 2019 Jun 5;2(6):e195009. PMID: 31173118.

2. Caccavale LJ, Corona R, LaRose JG, et al. Exploring the role of motivational interviewing in adolescent patient-provider communication about type 1 diabetes. Pediatric diabetes. 2019 Mar;20(2):217-25. PMID: 30575237.

3. Aslakson RA, Isenberg SR, Crossnohere NL, et al. Utilising advance care planning videos to empower perioperative cancer patients and families: a study protocol of a randomised controlled trial. BMJ open. 2017 Jun 6;7(5):e016257. PMID: 28592584.

4. Taylor LJ, Rathouz PJ, Berlin A, et al. Navigating high-risk surgery: protocol for a multisite, stepped wedge, cluster-randomised trial of a question prompt list intervention to empower older adults to ask questions that inform treatment decisions. BMJ open. 2017 May 29;7(5):e014002. PMID: 28554911.

5. Altshuler L, Plaksin J, Zabar S, et al. Transforming the Patient Role to Achieve Better Outcomes Through a Patient Empowerment Program: A Randomized Wait-List Control Trial Protocol. JMIR research protocols. 2016 Apr 21;5(2):e68. PMID: 27103306.

6. Matthew AG, Yang ZG. Online interventions for sexual health in cancer. Curr Opin Support Palliat Care. 2020 Mar;14(1):80-86. doi: 10.1097/SPC.0000000000000477. PMID: 31789945. 


\title{
Appendix F. List of Included Studies for Original Articles
}

\author{
Original articles search - List of articles included for direct patient care stragegy
}

1. Subramanian L, Zhao J, Zee J, et al. Use of a Decision Aid for Patients Considering Peritoneal Dialysis and In-Center Hemodialysis: A Randomized Controlled Trial. American journal of kidney diseases : the official journal of the National Kidney Foundation. 2019 Sep;74(3):351-60. doi: 10.1053/j.ajkd.2019.01.030. PMID: 30954312.

2. Carroll JK, Tobin JN, Luque A, et al. "Get Ready and Empowered About Treatment" (GREAT) Study: a Pragmatic Randomized Controlled Trial of Activation in Persons Living with HIV. Journal of general internal medicine. 2019 Sep;34(9):1782-9. doi: 10.1007/s11606-01905102-7. PMID: 31240605.

3. Kamal AH, Wolf S, Nicolla JM, et al. Usability of PCforMe in Patients With Advanced Cancer Referred to Outpatient Palliative Care: Results of a Randomized, Active-Controlled Pilot Trial. Journal of pain and symptom management. 2019 Sep;58(3):382-9. doi: 10.1016/j.jpainsymman.2019.05.007. PMID: 31163259.

4. Nahm ES, Miller K, McQuaige M, et al. Testing the Impact of a Cancer Survivorship Patient Engagement Toolkit on Selected Health Outcomes. Oncology nursing forum. 2019 Sep 1;46(5):572-84. doi: 10.1188/19.onf.572-584. PMID: 31424456.

5. Moin T, Duru OK, Turk N, et al. Effectiveness of Shared Decision-making for Diabetes Prevention: 12-Month Results from the Prediabetes Informed Decision and Education (PRIDE) Trial. Journal of general internal medicine. 2019 Aug 30. doi: 10.1007/s11606-019-05238-6. PMID: 31471729.

6. Yakovchenko V, Hogan TP, Houston TK, et al. Automated Text Messaging With Patients in Department of Veterans Affairs Specialty Clinics: Cluster Randomized Trial. Journal of medical Internet research. 2019 Aug 4;21(8):e14750. doi: 10.2196/14750. PMID: 31444872.

7. Harman SM, Blankenburg R, Satterfield JM, et al. Promoting Shared Decision-Making Behaviors During Inpatient Rounds: A Multimodal Educational Intervention. Academic medicine : journal of the Association of American Medical Colleges. 2019 Jul;94(7):1010-8. doi: 10.1097/acm.0000000000002715. PMID: 30893066.

8. Biederman J, Fried R, DiSalvo M, et al. A Novel Text Message Intervention to Improve Adherence to Stimulants in Adults With Attention Deficit/Hyperactivity Disorder. Journal of clinical psychopharmacology. 2019 Jul/Aug;39(4):351-6. doi: 10.1097/jcp.0000000000001055. PMID: 31162154.

9. Potter JE, Duthely LM, Diaz-Mendez N, et al. Implementing CenteringPregnancy Group Prenatal Care for Minority Women Living with HIV at an Urban University Hospital. Journal of midwifery \& women's health. 2019 Jul;64(4):451-61. doi: 10.1111/jmwh.12987. PMID: 31222899.

10. Struwe LA, Schmaderer MS, Zimmerman L. Changes in Patient Activation in a SelfManagement Intervention. Western journal of nursing research. 2019 May 16:193945919848091. doi: 10.1177/0193945919848091. PMID: 31092139. 
11. Barnason S, Zimmerman L, Schulz P, et al. Weight management telehealth intervention for overweight and obese rural cardiac rehabilitation participants: A randomised trial. Journal of clinical nursing. 2019 May;28(9-10):1808-18. doi: 10.1111/jocn.14784. PMID: 30667588.

12. Gassaway J, Jones ML, Sweatman WM, et al. Peer-led, transformative learning approaches increase classroom engagement in care self-management classes during inpatient rehabilitation of individuals with spinal cord injury. The journal of spinal cord medicine. 2019 May;42(3):33846. doi: 10.1080/10790268.2017.1385992. PMID: 29037112.

13. Lauffenburger JC, Ghazinouri R, Jan S, et al. Impact of a novel pharmacist-delivered behavioral intervention for patients with poorly-controlled diabetes: The ENhancing outcomes through Goal Assessment and Generating Engagement in Diabetes Mellitus (ENGAGE-DM) pragmatic randomized trial. PloS one. 2019;14(4):e0214754. doi: 10.1371/journal.pone.0214754. PMID: 30939143.

14. Akyar I, Dionne-Odom JN, Bakitas MA. Using Patients and Their Caregivers Feedback to Develop ENABLE CHF-PC: An Early Palliative Care Intervention for Advanced Heart Failure. Journal of palliative care. 2019 Apr;34(2):103-10. doi: 10.1177/0825859718785231. PMID: 29952216.

15. Hutyra CA, Smiley S, Taylor DC, et al. Efficacy of a Preference-Based Decision Tool on Treatment Decisions for a First-Time Anterior Shoulder Dislocation: A Randomized Controlled Trial of At-Risk Patients. Medical decision making : an international journal of the Society for Medical Decision Making. 2019 Apr;39(3):253-63. doi: 10.1177/0272989x19832915. PMID: 30834817.

16. Liaw KR, Cho J, Devins L, et al. Co-designed PICU Family Stress Screening and Response System to Improve Experience, Quality, and Safety. Pediatric quality \& safety. 2019 MarApr;4(2):e145. doi: 10.1097/pq9.0000000000000145. PMID: 31321362.

17. Tai-Seale M, Downing NL, Jones VG, et al. Technology-Enabled Consumer Engagement: Promising Practices At Four Health Care Delivery Organizations. Health affairs (Project Hope). 2019 Mar;38(3):383-90. doi: 10.1377/hlthaff.2018.05027. PMID: 30830826.

18. Kripalani S, Hart K, Schaninger C, et al. Use of a tablet computer application to engage patients in updating their medication list. American journal of health-system pharmacy : AJHP : official journal of the American Society of Health-System Pharmacists. 2019 Feb 9;76(5):293300. doi: 10.1093/ajhp/zxy047. PMID: 30753287.

19. Doll JA, Jones WS, Lokhnygina Y, et al. PREPARED Study: A Study of Shared DecisionMaking for Coronary Artery Disease. Circulation Cardiovascular quality and outcomes. 2019 Feb;12(2):e005244. doi: 10.1161/circoutcomes.118.005244. PMID: 30764651.

20. Moore BA, Buono FD, Lloyd DP, et al. A randomized clinical trial of the Recovery Line among methadone treatment patients with ongoing illicit drug use. Journal of substance abuse treatment. 2019 Feb;97:68-74. doi: 10.1016/j.jsat.2018.11.011. PMID: 30577901.

21. Bluml BM, Kolb LE, Lipman R. Evaluating the Impact of Year-Long, Augmented Diabetes Self-Management Support. Population health management. 2019 Jan 22. doi: 10.1089/pop.2018.0175. PMID: 30668228. 
22. Krebs P, Burkhalter J, Fiske J, et al. The QuitIT Coping Skills Game for Promoting Tobacco Cessation Among Smokers Diagnosed With Cancer: Pilot Randomized Controlled Trial. JMIR mHealth and uHealth. 2019 Jan 10;7(1):e10071. doi: 10.2196/10071. PMID: 30632971.

23. Schaeffer AM, Jolles D. Not Missing the Opportunity: Improving Depression Screening and Follow-Up in a Multicultural Community. Joint Commission journal on quality and patient safety. 2019 Jan;45(1):31-9. doi: 10.1016/j.jcjq.2018.06.002. PMID: 30139563.

24. Muralidharan A, Brown CH, J EP, et al. Living Well: An Intervention to Improve Medical Illness Self-Management Among Individuals With Serious Mental Illness. Psychiatric services (Washington, DC). 2019 Jan 1;70(1):19-25. doi: 10.1176/appi.ps.201800162. PMID: 30353790.

25. Greysen SR, Harrison JD, Rareshide C, et al. A randomized controlled trial to improve engagement of hospitalized patients with their patient portals. Journal of the American Medical Informatics Association : JAMIA. 2018 Dec 1;25(12):1626-33. doi: 10.1093/jamia/ocy125. PMID: 30346543.

26. Turner BJ, Liang Y, Rodriguez N, et al. Randomized Trial of a Low-Literacy Chronic Pain Self-Management Program: Analysis of Secondary Pain and Psychological Outcome Measures. The journal of pain : official journal of the American Pain Society. 2018 Dec;19(12):1471-9. doi: 10.1016/j.jpain.2018.06.010. PMID: 30012494.

27. McIlvennan CK, Matlock DD, Thompson JS, et al. Caregivers of Patients Considering a Destination Therapy Left Ventricular Assist Device and a Shared Decision-Making Intervention: The DECIDE-LVAD Trial. JACC Heart failure. 2018 Nov;6(11):904-13. doi: 10.1016/j.jchf.2018.06.019. PMID: 30316931.

28. Mirro M, Daley C, Wagner S, et al. Delivering remote monitoring data to patients with implantable cardioverter-defibrillators: Does medium matter? Pacing and clinical electrophysiology : PACE. 2018 Nov;41(11):1526-35. doi: 10.1111/pace.13505. PMID: 30225880 .

29. Watterson JL, Rodriguez HP, Shortell SM, et al. Improved Diabetes Care Management Through a Text-Message Intervention for Low-Income Patients: Mixed-Methods Pilot Study. JMIR diabetes. 2018 Oct 30;3(4):e15. doi: 10.2196/diabetes.8645. PMID: 30377141.

30. Probst MA, Tschatscher CF, Lohse CM, et al. Factors Associated With Patient Involvement in Emergency Care Decisions: A Secondary Analysis of the Chest Pain Choice Multicenter Randomized Trial. Academic emergency medicine : official journal of the Society for Academic Emergency Medicine. 2018 Oct;25(10):1107-17. doi: 10.1111/acem.13503. PMID: 29904986.

31. Michaud TL, Siahpush M, Schwab RJ, et al. Remote Patient Monitoring and Clinical Outcomes for Postdischarge Patients with Type 2 Diabetes. Population health management. 2018 Oct;21(5):387-94. doi: 10.1089/pop.2017.0175. PMID: 29583057.

32. Sullivan M, Langford DJ, Davies PS, et al. A Controlled Pilot Trial of PainTracker SelfManager, a Web-Based Platform Combined With Patient Coaching, to Support Patients' SelfManagement of Chronic Pain. The journal of pain : official journal of the American Pain Society. 2018 Sep;19(9):996-1005. doi: 10.1016/j.jpain.2018.03.009. PMID: 29605691.

33. Treichler EBH, Avila A, Evans EA, et al. Collaborative decision skills training: Feasibility and preliminary outcomes of a novel intervention. Psychological services. 2018 Aug 13. doi: 10.1037/ser0000275. PMID: 30102050. 
34. Cabral HJ, Davis-Plourde K, Sarango M, et al. Peer Support and the HIV Continuum of Care: Results from a Multi-Site Randomized Clinical Trial in Three Urban Clinics in the United States. AIDS and behavior. 2018 Aug;22(8):2627-39. doi: 10.1007/s10461-017-1999-8. PMID: 29306990.

35. Hood KK, Iturralde E, Rausch J, et al. Preventing Diabetes Distress in Adolescents With Type 1 Diabetes: Results 1 Year After Participation in the STePS Program. Diabetes care. 2018 Aug;41(8):1623-30. doi: 10.2337/dc17-2556. PMID: 29921624.

36. Freeman K, Hanlon M, Denslow S, et al. Patient Engagement in Type 2 Diabetes: A Collaborative Community Health Initiative. The Diabetes educator. 2018 Aug;44(4):395-404. doi: 10.1177/0145721718784262. PMID: 29972097.

37. Ehde DM, Arewasikporn A, Alschuler KN, et al. Moderators of Treatment Outcomes After Telehealth Self-Management and Education in Adults With Multiple Sclerosis: A Secondary Analysis of a Randomized Controlled Trial. Archives of physical medicine and rehabilitation. 2018 Jul;99(7):1265-72. doi: 10.1016/j.apmr.2017.12.012. PMID: 29337024.

38. Spencer MS, Kieffer EC, Sinco B, et al. Outcomes at 18 Months From a Community Health Worker and Peer Leader Diabetes Self-Management Program for Latino Adults. Diabetes care. 2018 Jul;41(7):1414-22. doi: 10.2337/dc17-0978. PMID: 29703724.

39. Ledford CJW, Womack JJ, Rider HA, et al. Unexpected Effects of a System-Distributed Mobile Application in Maternity Care: A Randomized Controlled Trial. Health education \& behavior : the official publication of the Society for Public Health Education. 2018 Jun;45(3):323-30. doi: 10.1177/1090198117732110. PMID: 28918669.

40. Taylor YJ, Tapp H, Shade LE, et al. Impact of shared decision making on asthma quality of life and asthma control among children. The Journal of asthma : official journal of the Association for the Care of Asthma. 2018 Jun;55(6):675-83. doi: 10.1080/02770903.2017.1362423. PMID: 28800266.

41. Hudon C, Chouinard MC, Dubois MF, et al. Case Management in Primary Care for Frequent Users of Health Care Services: A Mixed Methods Study. Annals of family medicine. 2018 May;16(3):232-9. doi: 10.1370/afm.2233. PMID: 29760027.

42. Elsabrout K. Increasing diabetic patient engagement and self-reported medication adherence using a web-based multimedia program. Journal of the American Association of Nurse Practitioners. 2018 May;30(5):293-8. doi: 10.1097/jxx.0000000000000045. PMID: 29757846.

43. Turner BJ, Liang Y, Simmonds MJ, et al. Randomized Trial of Chronic Pain SelfManagement Program in the Community or Clinic for Low-Income Primary Care Patients. Journal of general internal medicine. 2018 May;33(5):668-77. doi: 10.1007/s11606-017-4244-2. PMID: 29299814.

44. Collinsworth AW, Brown RM, James CS, et al. The impact of patient education and shared decision making on hospital readmissions for COPD. International journal of chronic obstructive pulmonary disease. 2018;13:1325-32. doi: 10.2147/copd.s154414. PMID: 29731620.

45. Wang J, Cai C, Padhye N, et al. A Behavioral Lifestyle Intervention Enhanced With Multiple-Behavior Self-Monitoring Using Mobile and Connected Tools for Underserved Individuals With Type 2 Diabetes and Comorbid Overweight or Obesity: Pilot Comparative 
Effectiveness Trial. JMIR mHealth and uHealth. 2018 Apr 10;6(4):e92. doi: 10.2196/mhealth.4478. PMID: 29636320.

46. Perry TT, Halterman JS, Brown RH, et al. Results of an asthma education program delivered via telemedicine in rural schools. Annals of allergy, asthma \& immunology : official publication of the American College of Allergy, Asthma, \& Immunology. 2018 Apr;120(4):401-8. doi: 10.1016/j.anai.2018.02.013. PMID: 29471032.

47. Dumitrascu AG, Burton MC, Dawson NL, et al. Patient portal use and hospital outcomes. Journal of the American Medical Informatics Association : JAMIA. 2018 Apr 1;25(4):447-53. doi: 10.1093/jamia/ocx149. PMID: 29300961.

48. Allen LA, McIlvennan CK, Thompson JS, et al. Effectiveness of an Intervention Supporting Shared Decision Making for Destination Therapy Left Ventricular Assist Device: The DECIDELVAD Randomized Clinical Trial. JAMA internal medicine. 2018 Apr 1;178(4):520-9. doi: 10.1001/jamainternmed.2017.8713. PMID: 29482225.

49. Alegria M, Nakash O, Johnson K, et al. Effectiveness of the DECIDE Interventions on Shared Decision Making and Perceived Quality of Care in Behavioral Health With Multicultural Patients: A Randomized Clinical Trial. JAMA psychiatry. 2018 Apr 1;75(4):325-35. doi: 10.1001/jamapsychiatry.2017.4585. PMID: 29466533.

50. Rocque GB, Williams CP, Halilova KI, et al. Improving shared decision-making in chronic lymphocytic leukemia through multidisciplinary education. Translational behavioral medicine. 2018 Mar 1;8(2):175-82. doi: 10.1093/tbm/ibx034. PMID: 29390159.

51. Quinn CC, Butler EC, Swasey KK, et al. Mobile Diabetes Intervention Study of Patient Engagement and Impact on Blood Glucose: Mixed Methods Analysis. JMIR mHealth and uHealth. 2018 Feb 2;6(2):e31. doi: 10.2196/mhealth.9265. PMID: 29396389.

52. Egeter J, Hufner K, Sztankay M, et al. Implementation of an electronic routine outcome monitoring at an inpatient unit for psychosomatic medicine. Journal of psychosomatic research. 2018 Feb;105:64-71. doi: 10.1016/j.jpsychores.2017.12.009. PMID: 29332636.

53. Wittink MN, Walsh P, Yilmaz S, et al. Patient priorities and the doorknob phenomenon in primary care: Can technology improve disclosure of patient stressors? Patient education and counseling. 2018 Feb;101(2):214-20. doi: 10.1016/j.pec.2017.08.004. PMID: 28844522.

54. Hantsoo L, Criniti S, Khan A, et al. A Mobile Application for Monitoring and Management of Depressed Mood in a Vulnerable Pregnant Population. Psychiatric services (Washington, DC). 2018 Jan 1;69(1):104-7. doi: 10.1176/appi.ps.201600582. PMID: 29032705.

55. Barlow SE, Butte NF, Hoelscher DM, et al. Strategies to Recruit a Diverse Low-Income Population to Child Weight Management Programs From Primary Care Practices. Preventing chronic disease. 2017 Dec 21;14:E138. doi: 10.5888/pcd14.170301. PMID: 29267156.

56. Parker Oliver D, Demiris G, Washington K, et al. Hospice Family Caregiver Involvement in Care Plan Meetings: A Mixed-Methods Randomized Controlled Trial. The American journal of hospice \& palliative care. 2017 Nov;34(9):849-59. doi: 10.1177/1049909116661816. PMID: 27465403. 
57. Kaplan AL, Cohen ER, Zimlichman E. Improving patient engagement in self-measured blood pressure monitoring using a mobile health technology. Health information science and systems. 2017 Dec;5(1):4. doi: 10.1007/s13755-017-0026-9. PMID: 29081974.

58. Kangovi S, Mitra N, Grande D, et al. Community Health Worker Support for Disadvantaged Patients With Multiple Chronic Diseases: A Randomized Clinical Trial. American journal of public health. 2017 Oct;107(10):1660-7. doi: 10.2105/ajph.2017.303985. PMID: 28817334.

59. Rick R, Hoye RE, Thron RW, et al. Marketplace Clinics Complementing Diabetes Care for Urban Residing American Indians. Journal of primary care \& community health. 2017 Oct;8(4):198-205. doi: 10.1177/2150131917720556. PMID: 28707507.

60. Dillon EC, Stults CD, Wilson C, et al. An evaluation of two interventions to enhance patient-physician communication using the observer OPTION(5) measure of shared decision making. Patient education and counseling. 2017 Oct;100(10):1910-7. doi: 10.1016/j.pec.2017.04.020. PMID: 28532861.

61. Lv N, Xiao L, Simmons ML, et al. Personalized Hypertension Management Using PatientGenerated Health Data Integrated With Electronic Health Records (EMPOWER-H): Six-Month Pre-Post Study. Journal of medical Internet research. 2017 Sep 19;19(9):e311. doi: 10.2196/jmir.7831. PMID: 28928111.

62. Bakitas M, Dionne-Odom JN, Pamboukian SV, et al. Engaging patients and families to create a feasible clinical trial integrating palliative and heart failure care: results of the ENABLE CHF-PC pilot clinical trial. BMC palliative care. 2017 Aug 31;16(1):45. doi: 10.1186/s12904017-0226-8. PMID: 28859648.

63. Peters RM, Lui M, Patel K, et al. Improving Glycemic Control With a Standardized TextMessage and Phone-Based Intervention: A Community Implementation. JMIR diabetes. 2017 Jul 25;2(2):e15. doi: 10.2196/diabetes.7910. PMID: 30291063.

64. Page-Reeves J, Regino L, Murray-Krezan C, et al. A comparative effectiveness study of two culturally competent models of diabetes self-management programming for Latinos from lowincome households. BMC endocrine disorders. 2017 Jul 24;17(1):46. doi: 10.1186/s12902-0170192-4. PMID: 28738902.

65. Frias J, Virdi N, Raja P, et al. Effectiveness of Digital Medicines to Improve Clinical Outcomes in Patients with Uncontrolled Hypertension and Type 2 Diabetes: Prospective, OpenLabel, Cluster-Randomized Pilot Clinical Trial. Journal of medical Internet research. $2017 \mathrm{Jul}$ 11;19(7):e246. doi: 10.2196/jmir.7833. PMID: 28698169.

66. Barnato AE, Moore R, Moore CG, et al. Financial Incentives to Increase Advance Care Planning Among Medicaid Beneficiaries: Lessons Learned From Two Pragmatic Randomized Trials. Journal of pain and symptom management. 2017 Jul;54(1):85-95.e1. doi: 10.1016/j.jpainsymman.2017.02.016. PMID: 28450218.

67. Magnani JW, Schlusser CL, Kimani E, et al. The Atrial Fibrillation Health Literacy Information Technology System: Pilot Assessment. JMIR cardio. 2017 Jul-Dec;1(2):e7. doi: 10.2196/cardio.8543. PMID: 29473644.

68. Downing J, Gleeson H, Clayton PE, et al. Communication with young people in paediatric and adult endocrine consultations: an intervention development and feasibility study. BMC endocrine disorders. 2017 Jun 15;17(1):33. doi: 10.1186/s12902-017-0182-6. PMID: 28619024. 
69. Houlihan BV, Brody M, Everhart-Skeels S, et al. Randomized Trial of a Peer-Led, Telephone-Based Empowerment Intervention for Persons With Chronic Spinal Cord Injury Improves Health Self-Management. Archives of physical medicine and rehabilitation. 2017 Jun;98(6):1067-76.e1. doi: 10.1016/j.apmr.2017.02.005. PMID: 28284835.

70. Rocque GB, Halilova KI, Varley AL, et al. Feasibility of a Telehealth Educational Program on Self-Management of Pain and Fatigue in Adult Cancer Patients. Journal of pain and symptom management. 2017 Jun;53(6):1071-8. doi: 10.1016/j.jpainsymman.2016.12.345. PMID: 28185891.

71. Haas K, Martin A, Park KT. Text Message Intervention (TEACH) Improves Quality of Life and Patient Activation in Celiac Disease: A Randomized Clinical Trial. The Journal of pediatrics. 2017 Jun;185:62-7.e2. doi: 10.1016/j.jpeds.2017.02.062. PMID: 28343658.

72. Schumacher JR, Lutz BJ, Hall AG, et al. Feasibility of an ED-to-Home Intervention to Engage Patients: A Mixed-Methods Investigation. The western journal of emergency medicine. 2017 Jun;18(4):743-51. doi: 10.5811/westjem.2017.2.32570. PMID: 28611897.

73. Tremont G, Davis JD, Ott BR, et al. Randomized Trial of the Family Intervention: Telephone Tracking-Caregiver for Dementia Caregivers: Use of Community and Healthcare Resources. Journal of the American Geriatrics Society. 2017 May;65(5):924-30. doi: 10.1111/jgs.14684. PMID: 28008609.

74. Kelly MM, Dean SM, Carayon P, et al. Healthcare Team Perceptions of a Portal for Parents of Hospitalized Children Before and After Implementation. Applied clinical informatics. 2017 Mar 15;8(1):265-78. doi: 10.4338/aci-2016-11-ra-0194. PMID: 28293685.

75. Gimbel R, Shi L, Williams JE, et al. Enhancing mHealth Technology in the Patient-Centered Medical Home Environment to Activate Patients With Type 2 Diabetes: A Multisite Feasibility Study Protocol. JMIR research protocols. 2017 Mar 6;6(3):e38. doi: 10.2196/resprot.6993. PMID: 28264792.

76. Selman LE, Daveson BA, Smith M, et al. How empowering is hospital care for older people with advanced disease? Barriers and facilitators from a cross-national ethnography in England, Ireland and the USA. Age and ageing. 2017 Mar 1;46(2):300-9. doi: 10.1093/ageing/afw193. PMID: 27810850.

77. Reddy A, Huseman TL, Canamucio A, et al. Patient and Partner Feedback Reports to Improve Statin Medication Adherence: A Randomized Control Trial. Journal of general internal medicine. 2017 Mar;32(3):256-61. doi: 10.1007/s11606-016-3858-0. PMID: 27612487.

78. Dunn AS, Reyna M, Radbill B, et al. The Impact of Bedside Interdisciplinary Rounds on Length of Stay and Complications. Journal of hospital medicine. 2017 Mar;12(3):137-42. doi: 10.12788/jhm.2695. PMID: 28272588.

79. Dingemann J, Szczepanski R, Ernst G, et al. Transition of Patients with Esophageal Atresia to Adult Care: Results of a Transition-Specific Education Program. European journal of pediatric surgery : official journal of Austrian Association of Pediatric Surgery [et al] = Zeitschrift fur Kinderchirurgie. 2017 Feb;27(1):61-7. doi: 10.1055/s-0036-1587334. PMID: 27522121.

80. Smallwood AJ, Schapira MM, Fedders M, et al. A pilot randomized controlled trial of a decision aid with tailored fracture risk tool delivered via a patient portal. Osteoporosis international : a journal established as result of cooperation between the European Foundation 
for Osteoporosis and the National Osteoporosis Foundation of the USA. 2017 Feb;28(2):567-76. doi: 10.1007/s00198-016-3767-4. PMID: 27647529.

81. Song L, Tyler C, Clayton MF, et al. Patient and family communication during consultation visits: The effects of a decision aid for treatment decision-making for localized prostate cancer. Patient education and counseling. 2017 Feb;100(2):267-75. doi: 10.1016/j.pec.2016.09.012. PMID: 27692491.

82. Milani RV, Lavie CJ, Bober RM, et al. Improving Hypertension Control and Patient Engagement Using Digital Tools. The American journal of medicine. 2017 Jan;130(1):14-20. doi: 10.1016/j.amjmed.2016.07.029. PMID: 27591179.

83. Saver BG, Mazor KM, Luckmann R, et al. Persuasive Interventions for Controversial Cancer Screening Recommendations: Testing a Novel Approach to Help Patients Make Evidence-Based Decisions. Annals of family medicine. 2017 Jan;15(1):48-55. doi: 10.1370/afm.1996. PMID: 28376460.

84. Epstein RM, Duberstein PR, Fenton JJ, et al. Effect of a Patient-Centered Communication Intervention on Oncologist-Patient Communication, Quality of Life, and Health Care Utilization in Advanced Cancer: The VOICE Randomized Clinical Trial. JAMA oncology. 2017 Jan 1;3(1):92-100. doi: 10.1001/jamaoncol.2016.4373. PMID: 27612178.

85. Hess EP, Hollander JE, Schaffer JT, et al. Shared decision making in patients with low risk chest pain: prospective randomized pragmatic trial. BMJ (Clinical research ed). 2016 Dec 5;355:i6165. doi: 10.1136/bmj.i6165. PMID: 27919865.

86. O'Leary KJ, Killarney A, Hansen LO, et al. Effect of patient-centred bedside rounds on hospitalised patients' decision control, activation and satisfaction with care. BMJ quality \& safety. 2016 Dec;25(12):921-8. doi: 10.1136/bmjqs-2015-004561. PMID: 26628552.

87. Politi MC, Kuzemchak MD, Kaphingst KA, et al. Decision Aids Can Support Cancer Clinical Trials Decisions: Results of a Randomized Trial. The oncologist. 2016 Dec;21(12):1461-70. doi: 10.1634/theoncologist.2016-0068. PMID: 27511904.

88. Coylewright M, Dick S, Zmolek B, et al. PCI Choice Decision Aid for Stable Coronary Artery Disease: A Randomized Trial. Circulation Cardiovascular quality and outcomes. 2016 Nov;9(6):767-76. doi: 10.1161/circoutcomes.116.002641. PMID: 27803090.

89. Kvale EA, Huang CS, Meneses KM, et al. Patient-centered support in the survivorship care transition: Outcomes from the Patient-Owned Survivorship Care Plan Intervention. Cancer. 2016 Oct 15;122(20):3232-42. doi: 10.1002/cncr.30136. PMID: 27387096.

90. Young L, Hertzog M, Barnason S. Effects of a home-based activation intervention on selfmanagement adherence and readmission in rural heart failure patients: the PATCH randomized controlled trial. BMC cardiovascular disorders. 2016 Sep 8;16(1):176. doi: 10.1186/s12872-0160339-7. PMID: 27608624.

91. Doorenbos AZ, Levy WC, Curtis JR, et al. An Intervention to Enhance Goals-of-Care Communication Between Heart Failure Patients and Heart Failure Providers. Journal of pain and symptom management. 2016 Sep;52(3):353-60. doi: 10.1016/j.jpainsymman.2016.03.018. PMID: 27401505. 
92. Olomu A, Hart-Davidson W, Luo Z, et al. Implementing shared decision making in federally qualified health centers, a quasi-experimental design study: the Office-Guidelines Applied to Practice (Office-GAP) program. BMC health services research. 2016 Aug 2;16(a):334. doi: 10.1186/s12913-016-1603-3. PMID: 27484348.

93. Weisner CM, Chi FW, Lu Y, et al. Examination of the Effects of an Intervention Aiming to Link Patients Receiving Addiction Treatment With Health Care: The LINKAGE Clinical Trial. JAMA psychiatry. 2016 Aug 1;73(8):804-14. doi: 10.1001/jamapsychiatry.2016.0970. PMID: 27332703.

94. Zeng-Treitler Q, Gibson B, Hill B, et al. The effect of simulated narratives that leverage EMR data on shared decision-making: a pilot study. BMC research notes. 2016 Jul 22;9:359. doi: 10.1186/s13104-016-2152-x. PMID: 27448407.

95. Henry SL, Shen E, Ahuja A, et al. The Online Personal Action Plan: A Tool to Transform Patient-Enabled Preventive and Chronic Care. American journal of preventive medicine. 2016 Jul;51(1):71-7. doi: 10.1016/j.amepre.2015.11.014. PMID: 26826751.

96. Barton JL, Trupin L, Schillinger D, et al. Use of Low-Literacy Decision Aid to Enhance Knowledge and Reduce Decisional Conflict Among a Diverse Population of Adults With Rheumatoid Arthritis: Results of a Pilot Study. Arthritis care \& research. 2016 Jul;68(7):889-98. doi: 10.1002/acr.22801. PMID: 26605752.

97. Pillemer F, Price RA, Paone S, et al. Direct Release of Test Results to Patients Increases Patient Engagement and Utilization of Care. PloS one. 2016;11(6):e0154743. doi: 10.1371/journal.pone.0154743. PMID: 27337092.

98. Kim JY, Wineinger NE, Steinhubl SR. The Influence of Wireless Self-Monitoring Program on the Relationship Between Patient Activation and Health Behaviors, Medication Adherence, and Blood Pressure Levels in Hypertensive Patients: A Substudy of a Randomized Controlled Trial. Journal of medical Internet research. 2016 Jun 22;18(6):e116. doi: 10.2196/jmir.5429. PMID: 27334418.

99. Griffin A, Skinner A, Thornhill J, et al. Patient Portals: Who uses them? What features do they use? And do they reduce hospital readmissions? Applied clinical informatics. 2016;7(2):489-501. doi: 10.4338/aci-2016-01-ra-0003. PMID: 27437056.

100. Rivo J, Page TF, Arrieta A, et al. The Impact of Comprehensive Pre-visit Preparation on Patient Engagement and Quality of Care in a Population of Underserved Patients with Diabetes: Evidence from the Care Management Medical Home Center Model. Population health management. 2016 Jun;19(3):171-7. doi: 10.1089/pop.2015.0063. PMID: 26440513.

101. Luan A, Hui KJ, Remington AC, et al. Effects of A Novel Decision Aid for Breast Reconstruction: A Randomized Prospective Trial. Annals of plastic surgery. 2016 May;76 Suppl 3:S249-54. doi: 10.1097/sap.0000000000000722. PMID: 27070681.

102. Patzer RE, Basu M, Mohan S, et al. A Randomized Controlled Trial of a Mobile Clinical Decision Aid to Improve Access to Kidney Transplantation: iChoose Kidney. Kidney international reports. 2016 May;1(1):34-42. doi: 10.1016/j.ekir.2016.04.001. PMID: 27610423.

103. Fraenkel L, Peters E, Tyra S, et al. Shared Medical Decision Making in Lung Cancer Screening: Experienced versus Descriptive Risk Formats. Medical decision making : an 
international journal of the Society for Medical Decision Making. 2016 May;36(4):518-25. doi: 10.1177/0272989x15611083. PMID: 26442791.

104. Krouse RS, Grant M, McCorkle R, et al. A chronic care ostomy self-management program for cancer survivors. Psycho-oncology. 2016 May;25(5):574-81. doi: 10.1002/pon.4078. PMID: 26804708.

105. Hawley ST, Newman L, Griggs JJ, et al. Evaluating a Decision Aid for Improving Decision Making in Patients with Early-stage Breast Cancer. The patient. 2016 Apr;9(2):161-9. doi: 10.1007/s40271-015-0135-y. PMID: 26178202.

106. Kearing S, Berg SZ, Lurie JD. Can Decision Support Help Patients With Spinal Stenosis Make a Treatment Choice?: A Prospective Study Assessing the Impact of a Patient Decision Aid and Health Coaching. Spine. 2016 Apr;41(7):563-7. doi: 10.1097/brs.0000000000001272. PMID: 27018897.

107. Pugliese L, Woodriff M, Crowley O, et al. Feasibility of the "Bring Your Own Device" Model in Clinical Research: Results from a Randomized Controlled Pilot Study of a Mobile Patient Engagement Tool. Cureus. 2016 Mar 16;8(3):e535. doi: 10.7759/cureus.535. PMID: 27096135.

108. Masterson Creber R, Prey J, Ryan B, et al. Engaging hospitalized patients in clinical care: Study protocol for a pragmatic randomized controlled trial. Contemporary clinical trials. 2016 Mar;47:165-71. doi: 10.1016/j.cct.2016.01.005. PMID: 26795675.

109. Backhaus S, Ibarra S, Parrott D, et al. Comparison of a Cognitive-Behavioral Coping Skills Group to a Peer Support Group in a Brain Injury Population. Archives of physical medicine and rehabilitation. 2016 Feb;97(2):281-91. doi: 10.1016/j.apmr.2015.10.097. PMID: 26551230.

110. Bailey RA, Pfeifer M, Shillington AC, et al. Effect of a patient decision aid (PDA) for type 2 diabetes on knowledge, decisional self-efficacy, and decisional conflict. BMC health services research. 2016 Jan 14;16:10. doi: 10.1186/s12913-016-1262-4. PMID: 26762150.

111. Toscos T, Daley C, Heral L, et al. Impact of electronic personal health record use on engagement and intermediate health outcomes among cardiac patients: a quasi-experimental study. Journal of the American Medical Informatics Association : JAMIA. 2016 Jan;23(1):11928. doi: 10.1093/jamia/ocv164. PMID: 26912538.

112. Graumlich JF, Wang H, Madison A, et al. Effects of a Patient-Provider, Collaborative, Medication-Planning Tool: A Randomized, Controlled Trial. Journal of diabetes research. 2016;2016:2129838. doi: 10.1155/2016/2129838. PMID: 27699179.

113. Somers TJ, Kelleher SA, Westbrook KW, et al. A Small Randomized Controlled Pilot Trial Comparing Mobile and Traditional Pain Coping Skills Training Protocols for Cancer Patients with Pain. Pain research and treatment. 2016;2016:2473629. doi: 10.1155/2016/2473629. PMID: 27891252.

114. Lau YK, Caverly TJ, Cao P, et al. Evaluation of a Personalized, Web-Based Decision Aid for Lung Cancer Screening. American journal of preventive medicine. 2015 Dec;49(6):e125-9. doi: 10.1016/j.amepre.2015.07.027. PMID: 26456873.

115. Song MK, Ward SE, Fine JP, et al. Advance care planning and end-of-life decision making in dialysis: a randomized controlled trial targeting patients and their surrogates. American 
journal of kidney diseases : the official journal of the National Kidney Foundation. 2015 Nov;66(5):813-22. doi: 10.1053/j.ajkd.2015.05.018. PMID: 26141307.

116. Fraenkel L, Matzko CK, Webb DE, et al. Use of Decision Support for Improved Knowledge, Values Clarification, and Informed Choice in Patients With Rheumatoid Arthritis. Arthritis care \& research. 2015 Nov;67(11):1496-502. doi: 10.1002/acr.22659. PMID: 26195173.

117. Fleisher L, Wen KY, Miller SM, et al. Development and utilization of complementary communication channels for treatment decision making and survivorship issues among cancer patients: The CIS Research Consortium Experience. Internet interventions. 2015 Nov 1;2(4):3928. doi: 10.1016/j.invent.2015.09.002. PMID: 26855885.

118. LeBlanc A, Herrin J, Williams MD, et al. Shared Decision Making for Antidepressants in Primary Care: A Cluster Randomized Trial. JAMA internal medicine. 2015 Nov;175(11):176170. doi: 10.1001/jamainternmed.2015.5214. PMID: 26414670.

119. Brito JP, Castaneda-Guarderas A, Gionfriddo MR, et al. Development and Pilot Testing of an Encounter Tool for Shared Decision Making About the Treatment of Graves' Disease. Thyroid : official journal of the American Thyroid Association. 2015 Nov;25(11):1191-8. doi: 10.1089/thy.2015.0277. PMID: 26413979.

120. Fiscella K, Boyd M, Brown J, et al. Activation of persons living with HIV for treatment, the great study. BMC public health. 2015 Oct 16;15:1056. doi: 10.1186/s12889-015-2382-1. PMID: 26474979.

121. Tung J, Grunow JE, Jacobs N. Pilot Development of an Electronic Pediatric Inflammatory Bowel Disease Quiz Game. Journal of pediatric gastroenterology and nutrition. 2015 Sep;61(3):292-6. doi: 10.1097/mpg.0000000000000788. PMID: 25793902.

122. Wang ML, Gallivan L, Lemon SC, et al. Navigating to health: Evaluation of a community health center patient navigation program. Preventive medicine reports. 2015;2:664-8. doi: 10.1016/j.pmedr.2015.08.002. PMID: 26844134.

123. Safford MM, Andreae S, Cherrington AL, et al. Peer Coaches to Improve Diabetes Outcomes in Rural Alabama: A Cluster Randomized Trial. Annals of family medicine. 2015 Aug;13 Suppl 1:S18-26. doi: 10.1370/afm.1798. PMID: 26304967.

124. Wilson M, Roll JM, Corbett C, et al. Empowering Patients with Persistent Pain Using an Internet-based Self-Management Program. Pain management nursing : official journal of the American Society of Pain Management Nurses. 2015 Aug;16(4):503-14. doi:

10.1016/j.pmn.2014.09.009. PMID: 26088940.

125. Greenwood DA, Blozis SA, Young HM, et al. Overcoming Clinical Inertia: A Randomized Clinical Trial of a Telehealth Remote Monitoring Intervention Using Paired Glucose Testing in Adults With Type 2 Diabetes. Journal of medical Internet research. 2015 Jul 21;17(7):e178. doi: 10.2196/jmir.4112. PMID: 26199142.

126. LeBlanc A, Wang AT, Wyatt K, et al. Encounter Decision Aid vs. Clinical Decision Support or Usual Care to Support Patient-Centered Treatment Decisions in Osteoporosis: The Osteoporosis Choice Randomized Trial II. PloS one. 2015;10(5):e0128063. doi: 10.1371/journal.pone.0128063. PMID: 26010755. 
127. Shah VO, Carroll C, Mals R, et al. A Home-Based Educational Intervention Improves Patient Activation Measures and Diabetes Health Indicators among Zuni Indians. PloS one. 2015;10(5):e0125820. doi: 10.1371/journal.pone.0125820. PMID: 25954817.

128. Chabrera C, Zabalegui A, Bonet M, et al. A Decision Aid to Support Informed Choices for Patients Recently Diagnosed With Prostate Cancer: A Randomized Controlled Trial. Cancer nursing. 2015 May-Jun;38(3):E42-50. doi: 10.1097/ncc.0000000000000170. PMID: 25010250.

129. Aikens JE, Rosland AM, Piette JD. Improvements in illness self-management and psychological distress associated with telemonitoring support for adults with diabetes. Primary care diabetes. 2015 Apr;9(2):127-34. doi: 10.1016/j.pcd.2014.06.003. PMID: 25065270.

130. Irvine AB, Russell $\mathrm{H}$, Manocchia $\mathrm{M}$, et al. Mobile-Web app to self-manage low back pain: randomized controlled trial. Journal of medical Internet research. 2015 Jan 2;17(1):e1. doi: 10.2196/jmir.3130. PMID: 25565416.

131. Caine K, Kohn S, Lawrence C, et al. Designing a patient-centered user interface for access decisions about EHR data: implications from patient interviews. Journal of general internal medicine. 2015 Jan;30 Suppl 1:S7-16. doi: 10.1007/s11606-014-3049-9. PMID: 25480719.

132. Tomko C, Davis KM, Luta G, et al. A comparison of web-based versus print-based decision AIDS for prostate cancer screening: participants' evaluation and utilization. Journal of general internal medicine. 2015 Jan;30(1):33-42. doi: 10.1007/s11606-014-2994-7. PMID: 25183475.

133. Le TK, Chang M, Nelson C, et al. Upstream discussion provided in the ambulatory setting to assist patients with chronic kidney disease considering dialysis. The Permanente journal. 2015 Winter;19(1):30-3. doi: 10.7812/tpp/14-053. PMID: 25432000.

134. DeCamp LR, Polk S, Chrismer MC, et al. Health Care Engagement of Limited English Proficient Latino Families: Lessons Learned from Advisory Board Development. Progress in community health partnerships : research, education, and action. 2015 Winter;9(4):521-30. doi: 10.1353/cpr.2015.0068. PMID: 26639378.

135. Iezzoni LI, Heaphy D, Warsett KS, et al. Description of YESHealth: A consumer-directed intervention in a randomized trial of methods to improve quality of care for persons with disability. Disability and health journal. 2018 Oct;11(4):545-54. PMID: 29983376.

136. Khan A, Spector ND, Baird JD, et al. Patient safety after implementation of a coproduced family centered communication programme: multicenter before and after intervention study. BMJ (Clinical research ed). 2018 Dec 5;363:k4764. PMID: 30518517

137. Johns SA, Beck-Coon K, Stutz PV, Talib TL, Chinh K, Cottingham AH, Schmidt K, Shields C, Stout ME, Stump TE, Monahan PO, Torke AM, Helft PR. Mindfulness Training Supports Quality of Life and Advance Care Planning in Adults With Metastatic Cancer and Their Caregivers: Results of a Pilot Study. Am J Hosp Palliat Care. 2020 Feb;37(2):88-99. doi: 10.1177/1049909119862254. Epub 2019 Aug 4. PubMed PMID: 31378080.

138. Dyck DG, Weeks DL, Smith CL, Shaw M. Multiple family group intervention for spinal cord injury: Quantitative and qualitative comparison with standard education. J Spinal Cord Med. 2020 Jan 21:1-11. doi: 10.1080/10790268.2019.1710946. [Epub ahead of print] PubMed PMID: 31961284. 
139. Christiansen TL, Lipsitz S, Scanlan M, Yu SP, Lindros ME, Leung WY, Adelman J, Bates DW, Dykes PC. Patient Activation Related to Fall Prevention: A Multisite Study. Jt Comm J Qual Patient Saf. 2020 Mar;46(3):129-135. doi: 10.1016/j.jcjq.2019.11.010. Epub 2020 Jan 13. PubMed PMID: 31948814.

140. Glenn LE, Nichols M, Enriquez M, Jenkins C. Impact of a community-based approach to patient engagement in rural, low-income adults with type 2 diabetes. Public Health Nurs. 2020 Mar;37(2):178-187. doi: 10.1111/phn.12693. Epub 2019 Dec 13. PubMed PMID: 31833102; PubMed Central PMCID: PMC7067669.

141. Chen J, Kaye L, Tuffli M, Barrett MA, Jones-Ford S, Shenouda T, Gondalia R, Henderson K, Combs V, Van Sickle D, Stempel DA. Passive Monitoring of Short-Acting Beta-Agonist Use via Digital Platform in Patients With Chronic Obstructive Pulmonary Disease: Quality Improvement Retrospective Analysis. JMIR Form Res. 2019 Oct 23;3(4):e13286. doi: 10.2196/13286. PubMed PMID: 31647471; PubMed Central PMCID: PMC7010108. 


\section{Appendix G. Word Cloud}

The strategies, barriers, and facilitators related to patient and family engagement suggested by our Key Informants using Poll Everywhere.

- $\quad$ Status Quo Bias- Hard To Start Something New - Resistance To Change/Too Much Work To Add Another Thing

- Need To Streamline For Families When Need To Engage For Multiple Family Members

- No Requirement For Clinics And Hospitals To Engage Patients And Families

- Patient Confidence In Decision Making (E.G., Using A Tool Such As Sure)

- Medical Culture That Does Not Prioritize/Value Patient And Family Input

- Patient Use Of Portal, Apps, Other Electronic Engagement Tools

- Difficulty Selecting To Right Tools And Standardizing Protocols

- Look Upstream To See How Well You Capture The Population

- Portal Helps Input And Share Data With Multiple Providers

- $\quad$ Lack Of Trust/Evidence That It Will Be Helpful/Useful

- Structure Of Care System Doesn't Accommodate PFE

- $\quad$ Lack Of Payer And Health System Support

- Patient Understanding Using Teach-Back

- Patient-Reported Outcome Measures

- Ability To Provide Useful Analytics

- No Dedicated Funding/Resources

- Is The Patient Ready To Engage?

- Myths/Misperceptions Of PFE

- Understandable Language

- Fear Of Reputation Drop

- Managing Expectations

- Standardized Methods

- Lack Of ROI Evidence

- Telephonic Discussions

- Family Preparedness

- Medical Paternalism

- Contact Information

- Not Enough Time

- Response

- Willingness

- Informed

- Cost 